\title{
AS-RUN NEUTRONICS EVALUATION FOR THE CSM-10584 EXPERIMENT IN THE ATR
}

\author{
Jill R Mitchell
}

October 2020

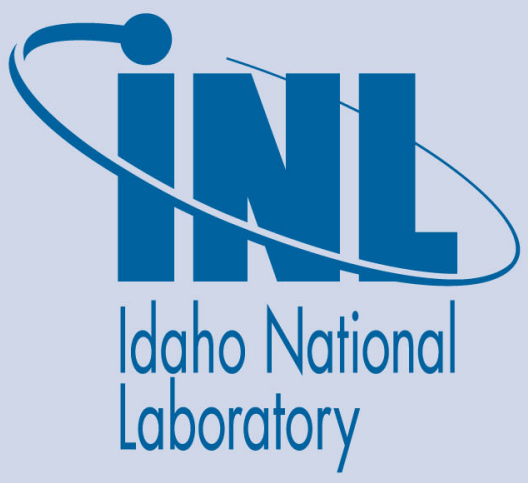

The INL is a U.S. Department of Energy National Laboratory operated by Battelle Energy Alliance 


\section{AS-RUN NEUTRONICS EVALUATION FOR THE CSM- 10584 EXPERIMENT IN THE ATR}

Jill R Mitchell

October 2020

Idaho National Laboratory Idaho Falls, Idaho 83415

http://www.inl.gov

Prepared for the

U.S. Department of Energy

Under DOE Idaho Operations Office

Contract DE-AC07-05ID14517 
TEM-10200-1

$12 / 19 / 17$

Rev.08
ENGINEERING CALCULATIONS AND

ANALYSIS

Title: $\quad$ AS-RUN NEUTRONICS EVALUATION FOR THE CSM-10584 EXPERIMENT IN

\begin{tabular}{llllllll} 
& \multicolumn{1}{l}{ THE ATR } & & & & \\
\cline { 2 - 6 } ECAR NO.: & 4496 & REV. NO.: & 1 & PROJECT NO.: & 32501 & Date: & $10 / 19 / 2020$ \\
\hline
\end{tabular}

\begin{tabular}{|c|c|c|c|}
\hline 1. & $\begin{array}{l}\text { Does this ECAR involve a } \\
\text { Safety SSC? }\end{array}$ & N/A & \multirow{6}{*}{$\begin{array}{l}\text { Professional Engineer's Stamp } \\
\text { N/A } \\
\text { See LWP-10010 for requirements }\end{array}$} \\
\hline 2. & $\begin{array}{l}\text { Safety SSC Determination } \\
\text { Document ID }\end{array}$ & N/A & \\
\hline 3. & Engineering Job (EJ) No. & 2315 & \\
\hline 4. & SSC ID & N/A & \\
\hline 5. & Building & TRA-670 & \\
\hline 6. & Site Area & ATR Complex & \\
\hline 7. & \multicolumn{3}{|c|}{$\begin{array}{l}\text { Objective/Purpose: } \\
\text { This engineering calculations and analysis report (ECAR) documents the results of the Advanced } \\
\text { Test Reactor (ATR) detailed Monte Carlo N-Particle (MCNP) code full-core model as-run physics } \\
\text { analysis performed to support the Colorado School of Mines (CSM) experiment in the B-5 } \\
\text { position. }\end{array}$} \\
\hline & \multicolumn{3}{|c|}{ The purpose of this analysis is to calculate the following: } \\
\hline & \multicolumn{3}{|c|}{$\begin{array}{l}\text { - The heat-generation rates of the test specimens and associated components as the } \\
\text { result of irradiation for the currently planned cycles. } \\
\text { - The DPA for each capsule as a result of irradiation for the currently planned cycles. } \\
\text { - Decay heat (watts) at shutdown and after } 30 \text { minutes, } 5 \text { hours, } 1 \text { day, } 7 \text { days, } 14 \text { days, } \\
30 \text { days, } 60 \text { days, and } 90 \text { days of cooling for each capsule. } \\
\text { - Source terms (curies) at shutdown and after } 30 \text { minutes, } 5 \text { hours, } 1 \text { day, } 7 \text { days, } 14 \text { days, } \\
30 \text { days, } 60 \text { days, and } 90 \text { days of cooling for each capsule. }\end{array}$} \\
\hline & \multicolumn{3}{|c|}{$\begin{array}{l}\text { These calculations were performed using the computer codes MCNP and ORIGEN2. The } \\
\text { neutronics model description, analysis details, and results are presented in the attached report. } \\
\text { As part of the physics analysis, this ECAR was formalized as requested by the project manager; } \\
\text { see Appendix A- 'Physics Analysis Request Form' for details. }\end{array}$} \\
\hline
\end{tabular}


TEM-10200-1

$12 / 19 / 17$

Rev.08

Title: AS-RUN NEUTRONICS EVALUATION FOR THE CSM-10584 EXPERIMENT IN

THE ATR

\begin{tabular}{llllllll}
\cline { 2 - 6 } ECAR NO.: & 4496 & REV. NO.: & 1 & PROJECT NO.: & 32501 & Date: & $10 / 19 / 2020$ \\
\hline
\end{tabular}

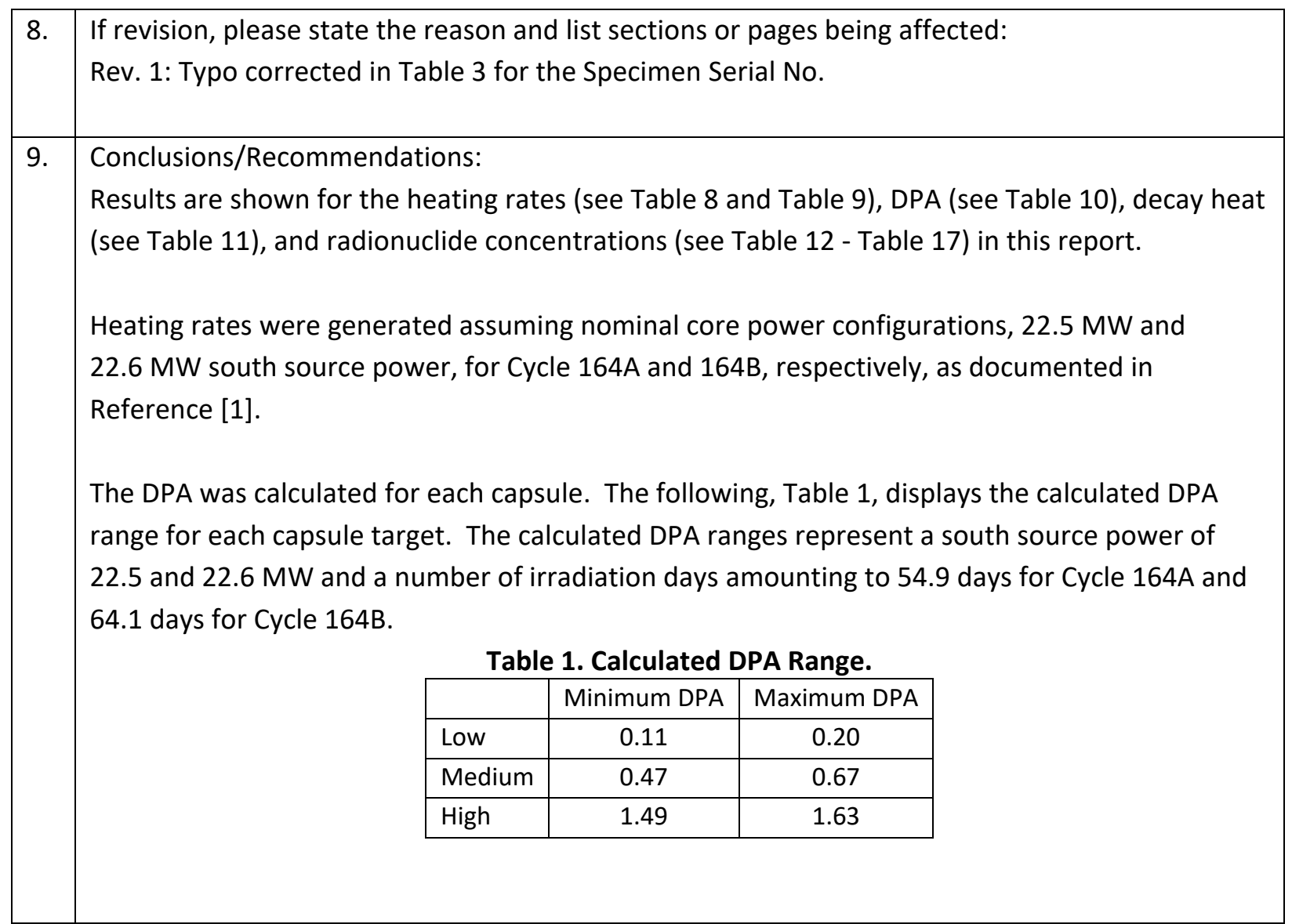


TEM-10200-1

$12 / 19 / 17$

Rev.08

Title:
ENGINEERING CALCULATIONS AND

ANALYSIS

\begin{tabular}{llllllll} 
ECAR NO.: & 4496 & REV. NO.: & 1 & PROJECT NO.: & 32501 & Date: & $10 / 19 / 2020$ \\
\hline
\end{tabular}

\section{Contents}

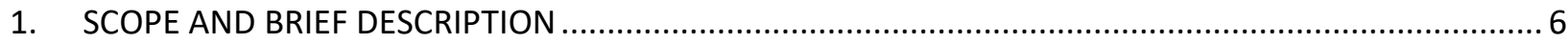

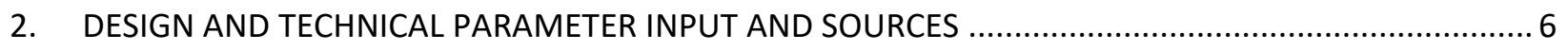

3. RESULTS OF LITERATURE SEARCHES AND OTHER BACKGROUND DATA …................................... 17

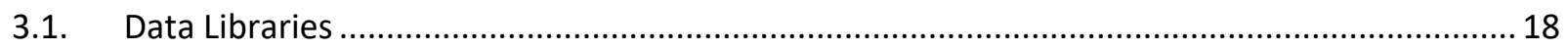

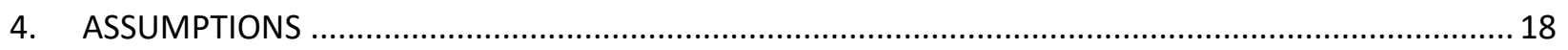

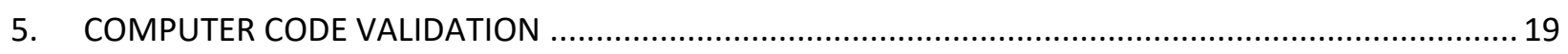

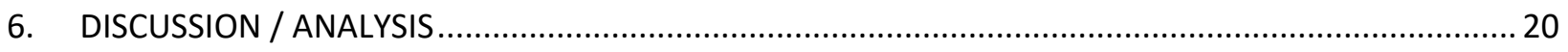

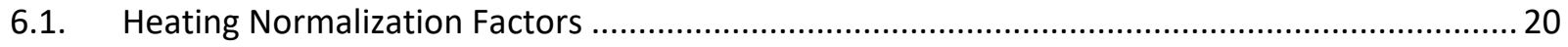

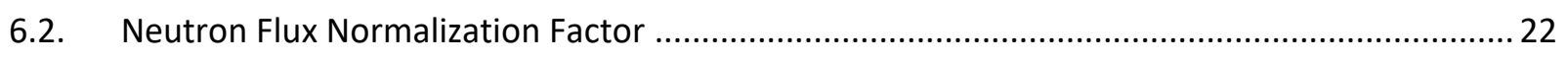

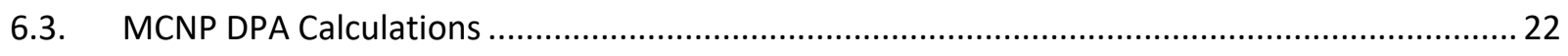

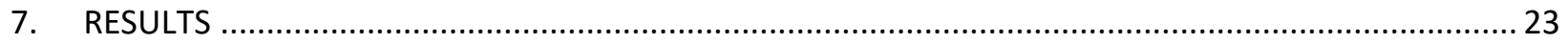

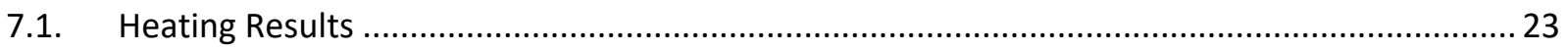

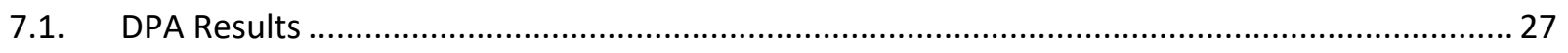

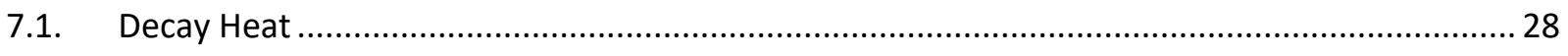

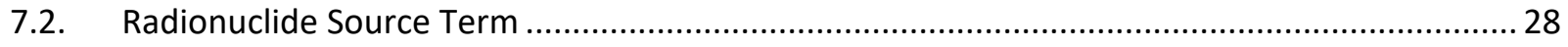

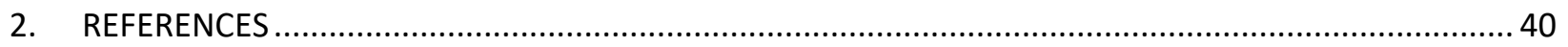

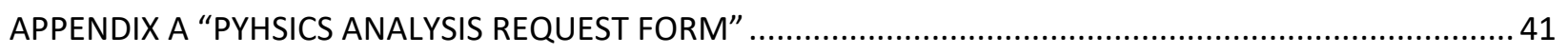

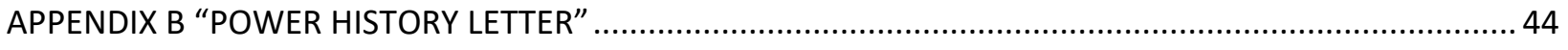

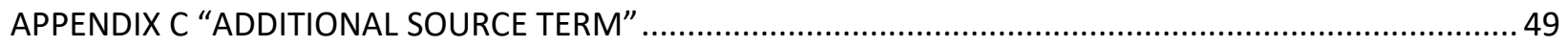


TEM-10200-1

$12 / 19 / 17$

Rev.08

Title:
ENGINEERING CALCULATIONS AND

ANALYSIS

THE ATR

AS-RUN NEUTRONICS EVALUATION FOR THE CSM-10584 EXPERIMENT IN

ECAR NO.: 4496

REV. NO.: 1 PROJECT NO.: 32501

Date: $10 / 19 / 2020$

\section{List of Tables}

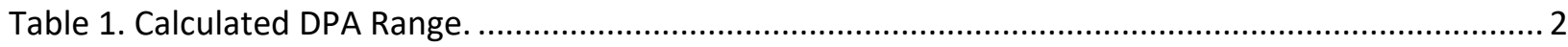

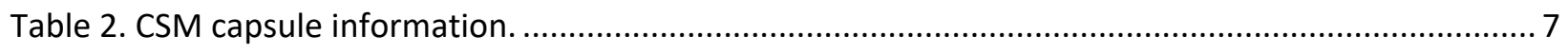

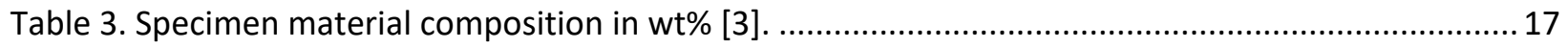

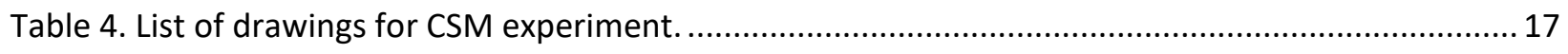

Table 5. INL qualified analysis software, version, and EA ID. ............................................................ 19

Table 6. Computer configurations for INL-qualified MCNP5 and ORIGEN2 installations..........................20

Table 7. Heating in CSM specimen and capsule components. ................................................................. 24

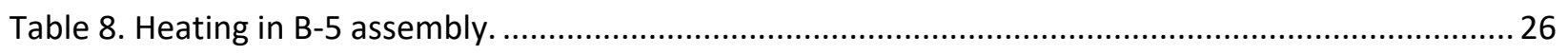

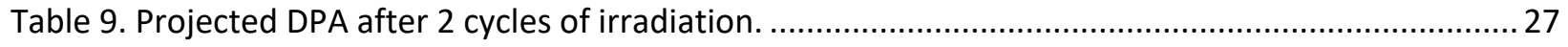

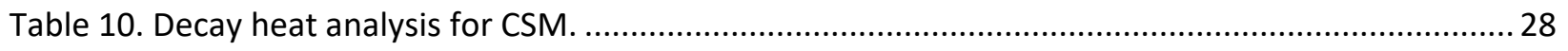

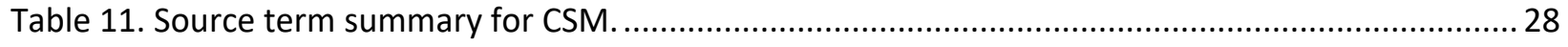

Table 12. Radionuclide source term for CSM capsule A after 54.9 days of irradiation (Ci)......................28

Table 13. Radionuclide source term for CSM capsule B after 119 days of irradiation (Ci).......................31

Table 14. Radionuclide source term for CSM capsule $\mathrm{C}$ after 119 days of irradiation (Ci).......................33

Table 15. Radionuclide source term for CSM capsule D after 119 days of irradiation (Ci). ...................... 35

Table 16. Radionuclide source term for CSM capsule E after 54.9 days of irradiation (Ci)......................37 
TEM-10200-1

$12 / 19 / 17$

Rev.08
ENGINEERING CALCULATIONS AND

ANALYSIS

Title: AS-RUN NEUTRONICS EVALUATION FOR THE CSM-10584 EXPERIMENT IN

THE ATR

\begin{tabular}{llllllll} 
ECAR NO.: & 4496 & REV. NO.: & 1 & PROJECT NO.: & 32501 & Date: & $10 / 19 / 2020$ \\
\hline
\end{tabular}

\section{PROJECT ROLES AND RESPONSIBILITIES}

\begin{tabular}{|c|c|c|c|}
\hline Project Role & Name (Typed) & Organization & Pages Covered (if applicable) \\
\hline Performer(s) & Jill Mitchell & C-130 & All \\
\hline Checker $^{\mathrm{a}}$ & Jason Brookman & C-130 & All \\
\hline Independent & $N / A$ & -- & -- \\
\hline Reviewer $^{\mathrm{b}}$ & & & \\
\hline CUI Reviewer ${ }^{\mathrm{C}}$ & TBD & -- & -- \\
\hline Manager $^{d}$ & Misti Lillo & C-130 & All \\
\hline Requestor ${ }^{\mathrm{e}}$ & Katie Anderson & $C-630$ & All \\
\hline Nuclear Safety & N/A & -- & -- \\
\hline Document Ownere & Donna Guillen & B-120 & All \\
\hline
\end{tabular}

\section{Responsibilities:}

a. Confirmation of completeness, mathematical accuracy, and correctness of data and appropriateness of assumptions.

b. Concurrence of method or approach. See definition, LWP-10106.

c. Concurrence with the document's markings in accordance with LWP-11202.

d. Concurrence of procedure compliance. Concurrence with method/approach and conclusion.

e. Concurrence with the document's assumptions and input information. See definition of Acceptance, LWP-10200.

NOTE: $\quad$ Delete or mark "N/A" for project roles not engaged. Include ALL personnel and their roles listed above in the eCR system. The list of the roles above is not allinclusive. If needed, the list can be extended or reduced. 
TEM-10200-1

$12 / 19 / 17$

Rev.08

Title:
ENGINEERING CALCULATIONS AND

ANALYSIS

\begin{tabular}{llllllll}
\cline { 2 - 6 } ECAR NO.: & 4496 & REV. NO.: & 1 & PROJECT NO.: & 32501 & Date: & 10/19/2020 \\
\hline
\end{tabular}

\section{SCOPE AND BRIEF DESCRIPTION}

This engineering calculations and analysis report (ECAR) documents the results of the Advanced Test Reactor (ATR) physics analysis performed to support the irradiation of the Colorado School of Mines (CSM) Experiment in a small B position (B-5) located in the south region of the ATR. The purpose of this analysis is to calculate the following:

- The heat-generation rates of the test specimens and associated components as the result of irradiation for the currently planned cycles.

- The DPA for each capsule as a result of irradiation for the currently planned cycles.

- Decay heat (watts) at shutdown and after 30 minutes, 5 hours, 1 day, 7 days, 14 days, 30 days, 60 days, and 90 days of cooling for each capsule.

- Source terms (curies) at shutdown and after 30 minutes, 5 hours, 1 day, 7 days, 14 days, 30 days, 60 days, and 90 days of cooling for each capsule.

\section{DESIGN AND TECHNICAL PARAMETER INPUT AND SOURCES}

Three types of specimen geometries were manufactured using additive manufacturing techniques. The sample materials, 316 stainless steel and Inconel, were fabricated in the form of miniature tensile bars $(16 \mathrm{~mm} \times 4 \mathrm{~mm} \times 1 \mathrm{~mm}$ ), PIE (TEM discs intended for PIE testing) disks ( $3 \mathrm{~mm}$ diameter, $0.3 \mathrm{~mm}$ thick), and TPP (discs intended for thermophysical property testing) disks ( $6 \mathrm{~mm}$ diameter, $1 \mathrm{~mm}$ thick). The specimens were irradiated in a total of five capsules in the B-5 position in the south region of the ATR at three different levels of intensity to evaluate DPA (displacement per atom), see Figure 1.

The CSM irradiation test used an aluminum basket and the experiment components were located in stainless steel capsules. The configuration for each capsule with materials and specimen information is illustrated in Table 2. Table 3 identifies the experiment specimen capsule location. Detailed material compositions for the specimens are listed in Table 4 from which the maximum value of any range listed was used in the MCNP model. 
TEM-10200-1

$12 / 19 / 17$

Rev.08
ENGINEERING CALCULATIONS AND

ANALYSIS

Title: $\quad$ AS-RUN NEUTRONICS EVALUATION FOR THE CSM-10584 EXPERIMENT IN

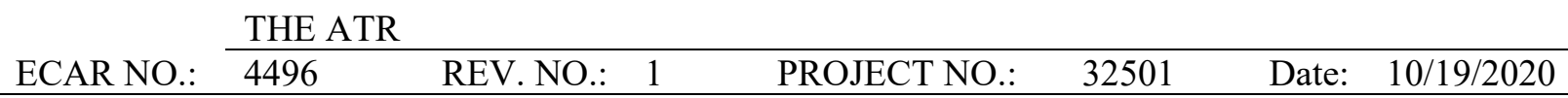

Table 2. CSM capsule information.

\begin{tabular}{|c|c|c|c|c|c|c|}
\hline $\begin{array}{c}\text { DPA } \\
\text { Target }\end{array}$ & $\begin{array}{l}\text { ATR } \\
\text { Cycle }\end{array}$ & Capsule & $\begin{array}{c}\text { Elevation } \\
\text { (cm from } \\
\text { core } \\
\text { center } \\
\text { line) }\end{array}$ & $\begin{array}{l}\text { Specimen } \\
\text { Holder }\end{array}$ & $\begin{array}{l}\text { Specimen } \\
\text { Material }\end{array}$ & Specimen Geometry \\
\hline \multirow{2}{*}{ low } & \multirow{2}{*}{$164 \mathrm{~A}$} & \multirow{2}{*}{$E$} & -60.73 & E2 & Inconel & $\begin{array}{c}16 \text { PIE and } 16 \text { TPP } \\
\text { disk }\end{array}$ \\
\hline & & & -57.23 & E1 & $316 s s t l$ & $\begin{array}{c}16 \text { PIE and } 16 \text { TPP } \\
\text { disk }\end{array}$ \\
\hline \multirow{2}{*}{ medium } & \multirow{2}{*}{$\begin{array}{c}164 A \\
\& \\
164 B\end{array}$} & \multirow{2}{*}{ D } & -53.73 & D2 & Inconel & $\begin{array}{c}16 \text { PIE and } 16 \text { TPP } \\
\text { disk }\end{array}$ \\
\hline & & & -50.23 & D1 & $316 s s t l$ & $\begin{array}{c}16 \text { PIE and } 16 \text { TPP } \\
\text { disk }\end{array}$ \\
\hline \multirow{4}{*}{ high } & \multirow{4}{*}{$\begin{array}{c}164 A \\
\& \\
164 B\end{array}$} & \multirow{4}{*}{ C } & -3.80 & $\mathrm{C} 4$ & Inconel & $\begin{array}{c}16 \text { PIE and } 16 \text { TPP } \\
\text { disk }\end{array}$ \\
\hline & & & -0.51 & $\mathrm{C3}$ & Inconel & 16 tensile \\
\hline & & & 2.54 & $\mathrm{C} 2$ & 316 sstl & 16 tensile \\
\hline & & & 5.80 & C1 & $316 s s t \mid$ & $\begin{array}{c}16 \text { PIE and } 16 \text { TPP } \\
\text { disk }\end{array}$ \\
\hline \multirow[b]{2}{*}{ medium } & \multirow{2}{*}{$\begin{array}{c}164 \mathrm{~A} \\
\& \\
164 \mathrm{~B}\end{array}$} & \multirow[b]{2}{*}{ B } & 52.23 & B2 & Inconel & 16 tensile \\
\hline & & & 55.73 & B1 & $316 s s t \mid$ & 16 tensile \\
\hline \multirow{2}{*}{ low } & \multirow{2}{*}{$164 \mathrm{~A}$} & \multirow{2}{*}{ A } & 59.23 & $\mathrm{~A} 2$ & Inconel & 16 tensile \\
\hline & & & 62.74 & A1 & 316 sstl & 16 tensile \\
\hline
\end{tabular}

Table 3. CSM-10584 Specimen Identification.

\begin{tabular}{|cc|c|}
\hline Specimen Type & Specimen Serial No & Heating Identification \\
\hline SS Tensile Batch 1 & 1 & \\
SS Tensile Batch 1 & 2 & \\
SS Tensile Batch 1 & 3 & \\
SS Tensile Batch 1 & 4 & \\
SS Tensile Batch 3 & 145 & A-1 Tensile sample smear \\
SS Tensile Batch 3 & 146 & \\
SS Tensile Batch 3 & 147 & \\
SS Tensile Batch 3 & 148 & \\
SS Tensile Batch 3 & 161 & \\
\end{tabular}


TEM-10200-1

$12 / 19 / 17$

Rev.08
ENGINEERING CALCULATIONS AND

ANALYSIS

Title: $\quad$ AS-RUN NEUTRONICS EVALUATION FOR THE CSM-10584 EXPERIMENT IN

\begin{tabular}{llllllll} 
& \multicolumn{1}{l}{ THE ATR } & & & \\
\cline { 2 - 6 } ECAR NO.: & 4496 & REV. NO.: & 1 & PROJECT NO.: & 32501 & Date: & $10 / 19 / 2020$ \\
\hline
\end{tabular}

\begin{tabular}{|c|c|c|}
\hline Specimen Type & Specimen Serial No & Heating Identification \\
\hline SS Tensile Batch 3 & 162 & \\
\hline SS Tensile Batch 3 & 163 & \\
\hline SS Tensile Batch 3 & 164 & \\
\hline SS Tensile Batch 4 & 217 & \\
\hline SS Tensile Batch 4 & 218 & \\
\hline SS Tensile Batch 4 & 219 & \\
\hline SS Tensile Batch 4 & 220 & \\
\hline Inconel Tensile Batch 1 & 13 & \multirow{16}{*}{ A-2 Tensile sample smear } \\
\hline Inconel Tensile Batch 1 & 14 & \\
\hline Inconel Tensile Batch 1 & 15 & \\
\hline Inconel Tensile Batch 1 & 16 & \\
\hline Inconel Tensile Batch 2 & 73 & \\
\hline Inconel Tensile Batch 2 & 74 & \\
\hline Inconel Tensile Batch 2 & 75 & \\
\hline Inconel Tensile Batch 2 & 76 & \\
\hline Inconel Tensile Batch 2 & 89 & \\
\hline Inconel Tensile Batch 2 & 90 & \\
\hline Inconel Tensile Batch 2 & 91 & \\
\hline Inconel Tensile Batch 2 & 92 & \\
\hline Inconel Tensile Batch 4 & 229 & \\
\hline Inconel Tensile Batch 4 & 230 & \\
\hline Inconel Tensile Batch 4 & 231 & \\
\hline Inconel Tensile Batch 4 & 232 & \\
\hline SS Tensile Batch 1 & 5 & \multirow{13}{*}{ B-1 Tensile sample smear } \\
\hline SS Tensile Batch 1 & 6 & \\
\hline SS Tensile Batch 1 & 7 & \\
\hline SS Tensile Batch 1 & 8 & \\
\hline SS Tensile Batch 3 & 153 & \\
\hline SS Tensile Batch 3 & 154 & \\
\hline SS Tensile Batch 3 & 155 & \\
\hline SS Tensile Batch 3 & 156 & \\
\hline SS Tensile Batch 3 & 157 & \\
\hline SS Tensile Batch 3 & 158 & \\
\hline SS Tensile Batch 3 & 159 & \\
\hline SS Tensile Batch 3 & 160 & \\
\hline SS Tensile Batch 4 & 221 & \\
\hline
\end{tabular}


TEM-10200-1

$12 / 19 / 17$

Rev.08
ENGINEERING CALCULATIONS AND

ANALYSIS

Title: $\quad$ AS-RUN NEUTRONICS EVALUATION FOR THE CSM-10584 EXPERIMENT IN

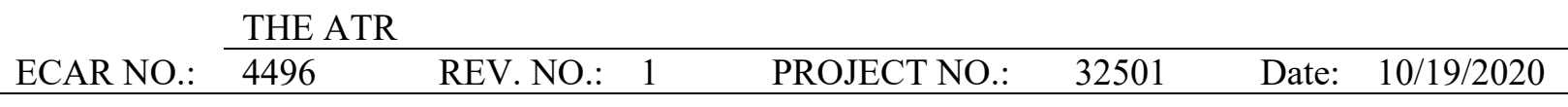

\begin{tabular}{|c|c|c|}
\hline Specimen Type & Specimen Serial No & Heating Identification \\
\hline SS Tensile Batch 4 & 222 & \\
\hline SS Tensile Batch 4 & 223 & \\
\hline SS Tensile Batch 4 & 224 & \\
\hline Inconel Tensile Batch 1 & 17 & \multirow{16}{*}{ B-2 Tensile sample smear } \\
\hline Inconel Tensile Batch 1 & 18 & \\
\hline Inconel Tensile Batch 1 & 19 & \\
\hline Inconel Tensile Batch 1 & 20 & \\
\hline Inconel Tensile Batch 2 & 81 & \\
\hline Inconel Tensile Batch 2 & 82 & \\
\hline Inconel Tensile Batch 2 & 83 & \\
\hline Inconel Tensile Batch 2 & 84 & \\
\hline Inconel Tensile Batch 2 & 85 & \\
\hline Inconel Tensile Batch 2 & 86 & \\
\hline Inconel Tensile Batch 2 & 87 & \\
\hline Inconel Tensile Batch 2 & 88 & \\
\hline Inconel Tensile Batch 4 & 233 & \\
\hline Inconel Tensile Batch 4 & 234 & \\
\hline Inconel Tensile Batch 4 & 235 & \\
\hline Inconel Tensile Batch 4 & 236 & \\
\hline Small SS TEM Batch 1 & 49 & \multirow{4}{*}{ C-1 PIE disk stack 1} \\
\hline Small SS TEM Batch 1 & 50 & \\
\hline Small SS TEM Batch 1 & 51 & \\
\hline Small SS TEM Batch 1 & 52 & \\
\hline Small SS TEM Batch 3 & 289 & \multirow{4}{*}{ C-1 PIE disk stack 2} \\
\hline Small SS TEM Batch 3 & 290 & \\
\hline Small SS TEM Batch 3 & 206 & \\
\hline Small SS TEM Batch 3 & 208 & \\
\hline Small SS TEM Batch 3 & 201 & \multirow{4}{*}{ C-1 PIE disk stack 3} \\
\hline Small SS TEM Batch 3 & 202 & \\
\hline Small SS TEM Batch 3 & 203 & \\
\hline Small SS TEM Batch 3 & 204 & \\
\hline Small SS TEM Batch 4 & 265 & \multirow{4}{*}{ C-1 PIE disk stack 4} \\
\hline Small SS TEM Batch 4 & 266 & \\
\hline Small SS TEM Batch 4 & 267 & \\
\hline Small SS TEM Batch 4 & 268 & \\
\hline Large SS TEM Batch 1 & 25 & C-1 TPP disk \\
\hline
\end{tabular}


TEM-10200-1

$12 / 19 / 17$

Rev.08
ENGINEERING CALCULATIONS AND

ANALYSIS

Title: $\quad$ AS-RUN NEUTRONICS EVALUATION FOR THE CSM-10584 EXPERIMENT IN

\begin{tabular}{|c|c|c|c|c|}
\hline & $\mathrm{IF} \wedge \mathrm{T}$ & & & \\
\hline CAR NO.: & REV. NO.: & PROJECT NO.: & 32501 & Date: $10 / 19 / 2020$ \\
\hline
\end{tabular}

\begin{tabular}{|c|c|c|}
\hline Specimen Type & Specimen Serial No & Heating Identification \\
\hline Large SS TEM Batch 1 & 26 & \\
\hline Large SS TEM Batch 1 & 27 & \\
\hline Large SS TEM Batch 1 & 28 & \\
\hline Large SS TEM Batch 3 & 181 & \\
\hline Large SS TEM Batch 3 & 182 & \\
\hline Large SS TEM Batch 3 & 183 & \\
\hline Large SS TEM Batch 3 & 184 & \\
\hline Large SS TEM Batch 3 & 177 & \\
\hline Large SS TEM Batch 3 & 178 & \\
\hline Large SS TEM Batch 3 & 179 & \\
\hline Large SS TEM Batch 3 & 180 & \\
\hline Large SS TEM Batch 4 & 241 & \\
\hline Large SS TEM Batch 4 & 242 & \\
\hline Large SS TEM Batch 4 & 243 & \\
\hline Large SS TEM Batch 4 & 244 & \\
\hline SS Tensile Batch 1 & 9 & \multirow{16}{*}{ C-2 SS Tensile sample smear } \\
\hline SS Tensile Batch 1 & 10 & \\
\hline SS Tensile Batch 1 & 11 & \\
\hline SS Tensile Batch 1 & 12 & \\
\hline SS Tensile Batch 3 & 149 & \\
\hline SS Tensile Batch 3 & 150 & \\
\hline SS Tensile Batch 3 & 151 & \\
\hline SS Tensile Batch 3 & 152 & \\
\hline SS Tensile Batch 3 & 165 & \\
\hline SS Tensile Batch 3 & 166 & \\
\hline SS Tensile Batch 3 & 167 & \\
\hline SS Tensile Batch 3 & 168 & \\
\hline SS Tensile Batch 4 & 225 & \\
\hline SS Tensile Batch 4 & 226 & \\
\hline SS Tensile Batch 4 & 227 & \\
\hline SS Tensile Batch 4 & 228 & \\
\hline Inconel Tensile Batch 1 & 21 & \multirow{5}{*}{$\begin{array}{c}\text { C-3 Inconel Tensile sample } \\
\text { smear }\end{array}$} \\
\hline Inconel Tensile Batch 1 & 22 & \\
\hline Inconel Tensile Batch 1 & 23 & \\
\hline Inconel Tensile Batch 1 & 24 & \\
\hline Inconel Tensile Batch 2 & 77 & \\
\hline
\end{tabular}


TEM-10200-1

$12 / 19 / 17$

Rev.08
ENGINEERING CALCULATIONS AND

ANALYSIS

Title: $\quad$ AS-RUN NEUTRONICS EVALUATION FOR THE CSM-10584 EXPERIMENT IN

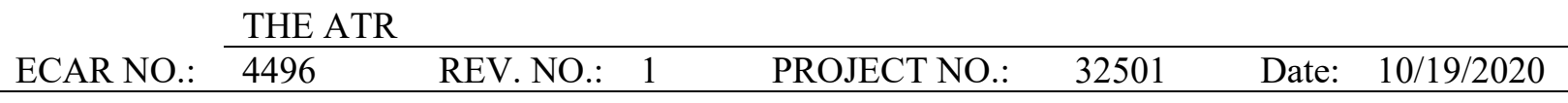

\begin{tabular}{|c|c|c|}
\hline Specimen Type & Specimen Serial No & Heating Identification \\
\hline Inconel Tensile Batch 2 & 78 & \\
\hline Inconel Tensile Batch 2 & 79 & \\
\hline Inconel Tensile Batch 2 & 80 & \\
\hline Inconel Tensile Batch 2 & 93 & \\
\hline Inconel Tensile Batch 2 & 94 & \\
\hline Inconel Tensile Batch 2 & 95 & \\
\hline Inconel Tensile Batch 2 & 96 & \\
\hline Inconel Tensile Batch 4 & 237 & \\
\hline Inconel Tensile Batch 4 & 238 & \\
\hline Inconel Tensile Batch 4 & 239 & \\
\hline Inconel Tensile Batch 4 & 240 & \\
\hline Small IN TEM Batch 1 & 61 & \multirow{4}{*}{ C4 - PIE disk stack 1} \\
\hline Small IN TEM Batch 1 & 62 & \\
\hline Small IN TEM Batch 1 & 63 & \\
\hline Small IN TEM Batch 1 & 64 & \\
\hline Small IN TEM Batch 2 & 121 & \multirow{4}{*}{ C4 - PIE disk stack 2} \\
\hline Small IN TEM Batch 2 & 122 & \\
\hline Small IN TEM Batch 2 & 123 & \\
\hline Small IN TEM Batch 2 & 124 & \\
\hline Small IN TEM Batch 2 & 137 & \multirow{4}{*}{ C4 - PIE disk stack 3} \\
\hline Small IN TEM Batch 2 & 138 & \\
\hline Small IN TEM Batch 2 & 139 & \\
\hline Small IN TEM Batch 2 & 140 & \\
\hline Small IN TEM Batch 4 & 281 & \multirow{4}{*}{ C4 - PIE disk stack 4} \\
\hline Small IN TEM Batch 4 & 282 & \\
\hline Small IN TEM Batch 4 & 283 & \\
\hline Small IN TEM Batch 4 & 284 & \\
\hline Large IN TEM Batch 1 & 37 & \multirow{9}{*}{ C-4 TPP disk } \\
\hline Large IN TEM Batch 1 & 38 & \\
\hline Large IN TEM Batch 1 & 39 & \\
\hline Large IN TEM Batch 1 & 40 & \\
\hline Large IN TEM Batch 2 & 97 & \\
\hline Large IN TEM Batch 2 & 98 & \\
\hline Large IN TEM Batch 2 & 99 & \\
\hline Large IN TEM Batch 2 & 100 & \\
\hline Large IN TEM Batch 2 & 113 & \\
\hline
\end{tabular}


TEM-10200-1

$12 / 19 / 17$

Rev.08
ENGINEERING CALCULATIONS AND

ANALYSIS

Title: $\quad$ AS-RUN NEUTRONICS EVALUATION FOR THE CSM-10584 EXPERIMENT IN

\begin{tabular}{llllllll} 
& \multicolumn{4}{l}{ THE ATR } & & & \\
\cline { 2 - 6 } ECAR NO.: & 4496 & REV. NO.: & 1 & PROJECT NO.: & 32501 & Date: & $10 / 19 / 2020$ \\
\hline
\end{tabular}

\begin{tabular}{|c|c|c|}
\hline Specimen Type & Specimen Serial No & Heating Identification \\
\hline Large IN TEM Batch 2 & 114 & \\
\hline Large IN TEM Batch 2 & 115 & \\
\hline Large IN TEM Batch 2 & 116 & \\
\hline Large IN TEM Batch 4 & 253 & \\
\hline Large IN TEM Batch 4 & 254 & \\
\hline Large IN TEM Batch 4 & 255 & \\
\hline Large IN TEM Batch 4 & 256 & \\
\hline Small SS TEM Batch 1 & 53 & \multirow{4}{*}{ D1 - PIE disk stack 1} \\
\hline Small SS TEM Batch 1 & 54 & \\
\hline Small SS TEM Batch 1 & 55 & \\
\hline Small SS TEM Batch 1 & 56 & \\
\hline Small SS TEM Batch 3 & 193 & \multirow{4}{*}{ D1 - PIE disk stack 2} \\
\hline Small SS TEM Batch 3 & 194 & \\
\hline Small SS TEM Batch 3 & 195 & \\
\hline Small SS TEM Batch 3 & 196 & \\
\hline Small SS TEM Batch 3 & 209 & \multirow{4}{*}{ D1 - PIE disk stack 3} \\
\hline Small SS TEM Batch 3 & 210 & \\
\hline Small SS TEM Batch 3 & 211 & \\
\hline Small SS TEM Batch 3 & 212 & \\
\hline Small SS TEM Batch 4 & 269 & \multirow{4}{*}{ D1 - PIE disk stack 4} \\
\hline Small SS TEM Batch 4 & 270 & \\
\hline Small SS TEM Batch 4 & 271 & \\
\hline Small SS TEM Batch 4 & 272 & \\
\hline Large SS TEM Batch 1 & 29 & \multirow{13}{*}{ D1 - TPP disk } \\
\hline Large SS TEM Batch 1 & 30 & \\
\hline Large SS TEM Batch 1 & 31 & \\
\hline Large SS TEM Batch 1 & 32 & \\
\hline Large SS TEM Batch 3 & 169 & \\
\hline Large SS TEM Batch 3 & 170 & \\
\hline Large SS TEM Batch 3 & 171 & \\
\hline Large SS TEM Batch 3 & 172 & \\
\hline Large SS TEM Batch 3 & 185 & \\
\hline Large SS TEM Batch 3 & 186 & \\
\hline Large SS TEM Batch 3 & 187 & \\
\hline Large SS TEM Batch 3 & 188 & \\
\hline Large SS TEM Batch 4 & 245 & \\
\hline
\end{tabular}


TEM-10200-1

$12 / 19 / 17$

Rev.08
ENGINEERING CALCULATIONS AND

ANALYSIS

Title: $\quad$ AS-RUN NEUTRONICS EVALUATION FOR THE CSM-10584 EXPERIMENT IN

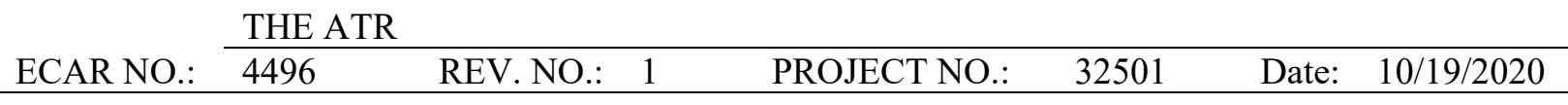

\begin{tabular}{|c|c|c|}
\hline Specimen Type & Specimen Serial No & Heating Identification \\
\hline Large SS TEM Batch 4 & 246 & \\
\hline Large SS TEM Batch 4 & 247 & \\
\hline Large SS TEM Batch 4 & 248 & \\
\hline Small IN TEM Batch 1 & 65 & \multirow{4}{*}{ D2 - PIE disk stack 1} \\
\hline Small IN TEM Batch 1 & 66 & \\
\hline Small IN TEM Batch 1 & 67 & \\
\hline Small IN TEM Batch 1 & 68 & \\
\hline Small IN TEM Batch 2 & 125 & \multirow{4}{*}{ D2 - PIE disk stack 2} \\
\hline Small IN TEM Batch 2 & 126 & \\
\hline Small IN TEM Batch 2 & 127 & \\
\hline Small IN TEM Batch 2 & 128 & \\
\hline Small IN TEM Batch 2 & 141 & \multirow{4}{*}{ D2 - PIE disk stack 3} \\
\hline Small IN TEM Batch 2 & 142 & \\
\hline Small IN TEM Batch 2 & 143 & \\
\hline Small IN TEM Batch 2 & 144 & \\
\hline Small IN TEM Batch 4 & 277 & \multirow{4}{*}{ D2 - PIE disk stack 4} \\
\hline Small IN TEM Batch 4 & 278 & \\
\hline Small IN TEM Batch 4 & 279 & \\
\hline Small IN TEM Batch 4 & 280 & \\
\hline Large IN TEM Batch 1 & 41 & \multirow{16}{*}{ D2 - TPP disk } \\
\hline Large IN TEM Batch 1 & 42 & \\
\hline Large IN TEM Batch 1 & 43 & \\
\hline Large IN TEM Batch 1 & 44 & \\
\hline Large IN TEM Batch 2 & 101 & \\
\hline Large IN TEM Batch 2 & 102 & \\
\hline Large IN TEM Batch 2 & 103 & \\
\hline Large IN TEM Batch 2 & 104 & \\
\hline Large IN TEM Batch 2 & 117 & \\
\hline Large IN TEM Batch 2 & 118 & \\
\hline Large IN TEM Batch 2 & 119 & \\
\hline Large IN TEM Batch 2 & 120 & \\
\hline Large IN TEM Batch 4 & 257 & \\
\hline Large IN TEM Batch 4 & 258 & \\
\hline Large IN TEM Batch 4 & 259 & \\
\hline Large IN TEM Batch 4 & 260 & \\
\hline Small SS TEM Batch 1 & 57 & E1 - PIE disk stack 1 \\
\hline
\end{tabular}


TEM-10200-1

$12 / 19 / 17$

Rev.08
ENGINEERING CALCULATIONS AND

ANALYSIS

Title: $\quad$ AS-RUN NEUTRONICS EVALUATION FOR THE CSM-10584 EXPERIMENT IN

\begin{tabular}{llllllll} 
& \multicolumn{1}{l}{ THE ATR } & & & & \\
\cline { 2 - 6 } ECAR NO.: & 4496 & REV. NO.: & 1 & PROJECT NO.: & 32501 & Date: & $10 / 19 / 2020$ \\
\hline
\end{tabular}

\begin{tabular}{|c|c|c|}
\hline Specimen Type & Specimen Serial No & Heating Identification \\
\hline Small SS TEM Batch 1 & 58 & \\
\hline Small SS TEM Batch 1 & 59 & \\
\hline Small SS TEM Batch 1 & 60 & \\
\hline Small SS TEM Batch 3 & 197 & \multirow{4}{*}{ E1 - PIE disk stack 2} \\
\hline Small SS TEM Batch 3 & 198 & \\
\hline Small SS TEM Batch 3 & 199 & \\
\hline Small SS TEM Batch 3 & 200 & \\
\hline Small SS TEM Batch 3 & 213 & \multirow{4}{*}{ E1 - PIE disk stack 3} \\
\hline Small SS TEM Batch 3 & 214 & \\
\hline Small SS TEM Batch 3 & 215 & \\
\hline Small SS TEM Batch 3 & 216 & \\
\hline Small SS TEM Batch 4 & 273 & \multirow{4}{*}{ E1 - PIE disk stack 4} \\
\hline Small SS TEM Batch 4 & 274 & \\
\hline Small SS TEM Batch 4 & 275 & \\
\hline Small SS TEM Batch 4 & 276 & \\
\hline Large SS TEM Batch 1 & 33 & \multirow{16}{*}{ E1 - TPP disk } \\
\hline Large SS TEM Batch 1 & 34 & \\
\hline Large SS TEM Batch 1 & 35 & \\
\hline Large SS TEM Batch 1 & 36 & \\
\hline Large SS TEM Batch 3 & 173 & \\
\hline Large SS TEM Batch 3 & 174 & \\
\hline Large SS TEM Batch 3 & 175 & \\
\hline Large SS TEM Batch 3 & 176 & \\
\hline Large SS TEM Batch 3 & 189 & \\
\hline Large SS TEM Batch 3 & 190 & \\
\hline Large SS TEM Batch 3 & 191 & \\
\hline Large SS TEM Batch 3 & 192 & \\
\hline Large SS TEM Batch 4 & 249 & \\
\hline Large SS TEM Batch 4 & 250 & \\
\hline Large SS TEM Batch 4 & 251 & \\
\hline Large SS TEM Batch 4 & 252 & \\
\hline Small IN TEM Batch 1 & 69 & \multirow{4}{*}{ E2 - PIE disk stack 1} \\
\hline Small IN TEM Batch 1 & 70 & \\
\hline Small IN TEM Batch 1 & 71 & \\
\hline Small IN TEM Batch 1 & 72 & \\
\hline Small IN TEM Batch 2 & 129 & E2 - PIE disk stack 2 \\
\hline
\end{tabular}


TEM-10200-1

$12 / 19 / 17$

Rev.08
ENGINEERING CALCULATIONS AND

ANALYSIS

Title: $\quad$ AS-RUN NEUTRONICS EVALUATION FOR THE CSM-10584 EXPERIMENT IN

\begin{tabular}{llllllll} 
& \multicolumn{1}{l}{ THE ATR } & & & & \\
\cline { 2 - 6 } ECAR NO.: & 4496 & REV. NO.: & 1 & PROJECT NO.: & 32501 & Date: & $10 / 19 / 2020$ \\
\hline
\end{tabular}

\begin{tabular}{|cc|c|}
\hline Specimen Type & Specimen Serial No & Heating Identification \\
\hline Small IN TEM Batch 2 & 130 & \\
Small IN TEM Batch 2 & 131 & \\
Small IN TEM Batch 2 & 132 & \\
\hline Small IN TEM Batch 2 & 133 & E2 - PIE disk stack 3 \\
Small IN TEM Batch 2 & 134 & \\
Small IN TEM Batch 2 & 135 & \\
Small IN TEM Batch 2 & 136 & E2 - PIE disk stack 4 \\
\hline Small IN TEM Batch 4 & 285 & \\
Small IN TEM Batch 4 & 286 & \\
Small IN TEM Batch 4 & 287 & \\
Small IN TEM Batch 4 & 288 & \\
Large IN TEM Batch 1 & 45 & \\
Large IN TEM Batch 1 & 46 & \\
Large IN TEM Batch 1 & 47 & \\
Large IN TEM Batch 1 & 48 & \\
Large IN TEM Batch 2 & 105 & \\
Large IN TEM Batch 2 & 106 & \\
Large IN TEM Batch 2 & 107 & \\
Large IN TEM Batch 2 & 108 & \\
Large IN TEM Batch 2 & 109 & \\
Large IN TEM Batch 2 & 110 & \\
Large IN TEM Batch 2 & 111 & \\
Large IN TEM Batch 2 & 112 & \\
Large IN TEM Batch 4 & 261 & \\
Large IN TEM Batch 4 & 262 & \\
Large IN TEM Batch 4 & 263 & \\
Large IN TEM Batch 4 & 264 & \\
\hline & & \\
\hline
\end{tabular}


TEM-10200-1

$12 / 19 / 17$

Rev.08

Title:

AS-RUN NEUTRONICS EVALUATION FOR THE CSM-10584 EXPERIMENT IN THE ATR

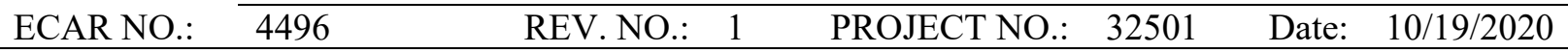

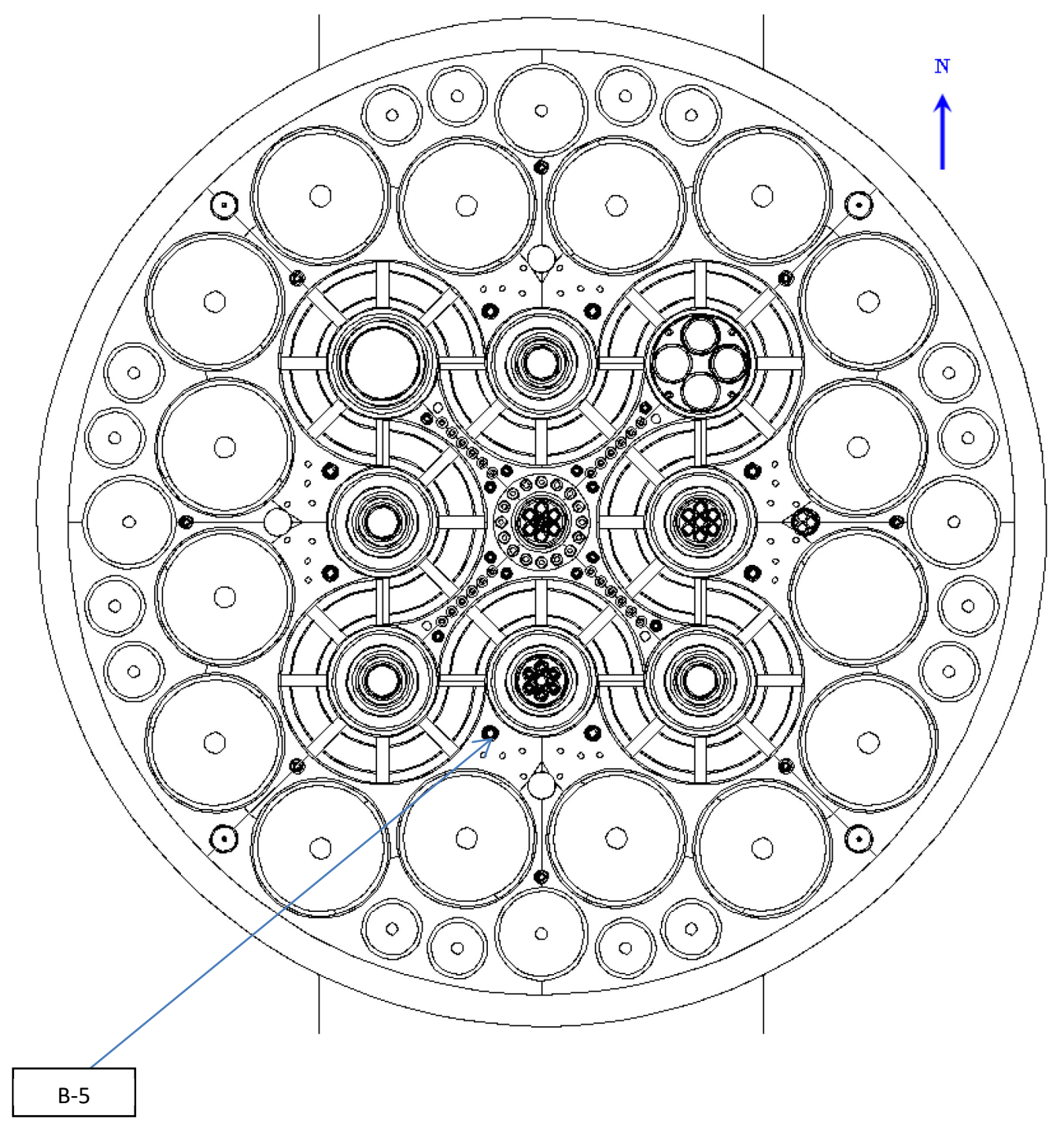

Figure 1. Radial cross section view of the ATR core, B-5 irradiation test position. 
TEM-10200-1

$12 / 19 / 17$

ENGINEERING CALCULATIONS AND ANALYSIS

Page 17 of 81

Rev.08

Title: $\quad$ AS-RUN NEUTRONICS EVALUATION FOR THE CSM-10584 EXPERIMENT IN THE ATR

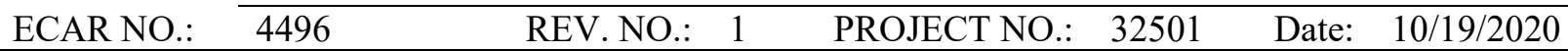

Table 4. Specimen material composition in wt\% [3].

\begin{tabular}{|c|c|c|}
\hline Element & 316 stainless steel & Inconel \\
\hline $\mathrm{Ni}$ & 12 & $50-55$ \\
\hline $\mathrm{Cr}$ & 17 & $17-21$ \\
\hline $\mathrm{Fe}$ & balance & balance \\
\hline $\mathrm{Nb} \mathrm{\&} \mathrm{Ta}$ & $\mathrm{n} / \mathrm{a}$ & $4.75-5.50$ \\
\hline $\mathrm{Mo}$ & 2.5 & $2.8-3.3$ \\
\hline $\mathrm{Ti}$ & $\mathrm{n} / \mathrm{a}$ & $0.65-1.15$ \\
\hline $\mathrm{Al}$ & $\mathrm{n} / \mathrm{a}$ & $0.2-0.8$ \\
\hline $\mathrm{Co}$ & $\mathrm{n} / \mathrm{a}$ & $1 \mathrm{max}$ \\
\hline $\mathrm{C}$ & 0.08 & $0.08 \max$ \\
\hline $\mathrm{Mn}$ & 2 & $0.35 \max$ \\
\hline $\mathrm{Si}$ & 1 & $0.35 \max$ \\
\hline $\mathrm{K}$ & $\mathrm{n} / \mathrm{a}$ & $0.015 \max$ \\
\hline $\mathrm{S}$ & 0.030 & $0.015 \max$ \\
\hline $\mathrm{B}$ & $\mathrm{n} / \mathrm{a}$ & $0.006 \max$ \\
\hline $\mathrm{Cu}$ & $\mathrm{n} / \mathrm{a}$ & $0.30 \max$ \\
\hline $\mathrm{P}$ & 0.045 & $\mathrm{n} / \mathrm{a}$ \\
\hline
\end{tabular}

\section{RESULTS OF LITERATURE SEARCHES AND OTHER BACKGROUND DATA}

The general purpose Monte $\underline{\text { Carlo }} \underline{\mathrm{N}}$-Particle transport code, MCNP [4][5], was used to model and evaluate the CSM experiment. ORIGEN2 [6] was used to calculate the source term for the experiment. The model for the CSM experiment is based on the drawings listed in Table 5. Nominal dimensions for the specimen, holders, and capsules are used in the model.

Table 5. List of drawings for CSM experiment.

\begin{tabular}{|c|l|}
\hline INL Drawing & Drawing Title \\
\hline 605840 REV. 0 & $\begin{array}{l}\text { ATR NATIONAL SCIENTIFIC USER FACILITIES (NSUF) COLORADO SCHOOL OF MINES } \\
\text { (CSM-10584) CYCLE 164A-1 STACKUP INSTALLATION }\end{array}$ \\
\hline 605842 REV. 0 & $\begin{array}{l}\text { ATR NATIONAL SCIENTIFIC USER FACILITIES (NSUF) COLORADO SCHOOL OF MINES } \\
\text { (CSM-10584) B5 EXPERIMENT TRANSPORT CAPSULES ASSEMBLY AND DETAILS }\end{array}$ \\
\hline
\end{tabular}


TEM-10200-1

$12 / 19 / 17$

Rev.08

Title: AS-RUN NEUTRONICS EVALUATION FOR THE CSM-10584 EXPERIMENT IN THE ATR

\begin{tabular}{llllllll}
\cline { 2 - 5 } ECAR NO.: & 4496 & REV. NO.: & 1 & PROJECT NO.: & 32501 & Date: & 10/19/2020 \\
\hline
\end{tabular}

\begin{tabular}{|c|l|}
\hline INL Drawing & Drawing Title \\
\hline 605843 REV. 0 & $\begin{array}{l}\text { ATR NATIONAL SCIENTIFIC USER FACILITIES (NSUF) COLORADO SCHOOL OF MINES } \\
\text { (CSM-10584) B5 EXPERIMENT SPECIMEN HOLDERS AND BASKETS ASSMEBLY AND } \\
\text { DETAILS }\end{array}$ \\
\hline 605844 REV. 0 & $\begin{array}{l}\text { ATR NATIONAL SCIENTIFIC USER FACILITIES (NSUF) COLORADO SCHOOL OF MINES } \\
\text { (CSM-10584) TENSILE, PIE AND TPP TEM SPECIMEN DETAILS }\end{array}$ \\
\hline 605845 REV. 0 & $\begin{array}{l}\text { ATR NATIONAL SCIENTIFIC USER FACILITIES (NSUF) COLORADO SCHOOL OF MINES } \\
\text { (CSM-10584) CYCLE 164B-1 STACKUP INSTALLATION }\end{array}$ \\
\hline 035140 & ATR REAC “O”\& “Y”FLOW RESTRICTORS ASSEMBLY AND DETAILS \\
\hline
\end{tabular}

\subsection{Data Libraries}

The standard MCNP cross-section data libraries [4][5], provided with MCNP, were used to calculate the reactivity worth, heating rates, neutron flux, and DPA for the CSM experiment. The ATRXS library [6] was used in the ORIGEN2 calculations. The ENDF/B-VII library was used in the MCNP models.

\section{ASSUMPTIONS}

The following assumptions were used in this analysis:

1. The as-run analysis was performed based on nominal lobe powers provided in Appendix B for $164 \mathrm{~A}$ and 164B.

2. An increase in lobe power for any lobe affecting the south region of the reactor will result in a change in test heating. Therefore, any power changes must be accounted for as a function of the ratio of the actual south lobe power to the analyzed south lobe source power.

3. The south lobe power is defined by the average of the SW, SE, and Clobe powers; $\mathrm{S}=(\mathrm{SW}+\mathrm{SE}+\mathrm{C}) / 3$.

4. Heating rate values reported for CSM include energy deposition from prompt neutrons, prompt gammas, and delayed fission product gammas.

5. MCNP models describing the average core loading and control drum/neck shim positioning were used for this analysis. 
TEM-10200-1

$12 / 19 / 17$

ENGINEERING CALCULATIONS AND ANALYSIS

Page 19 of 81

Rev.08

Title:

AS-RUN NEUTRONICS EVALUATION FOR THE CSM-10584 EXPERIMENT IN THE ATR

ECAR NO

4496

REV. NO.: 1 PROJECT NO.: 32501

Date: $10 / 19 / 2020$

\section{COMPUTER CODE VALIDATION}

The computer code, MCNP, is listed in the INL Enterprise Architecture (EA) Repository and is accepted as a qualified scientific and engineering analysis software. Table 6 lists the version and EA identification (ID) for the computer code used to perform the calculations and analyses documented by this ECAR.

Table 6. INL qualified analysis software, version, and EA ID.

\begin{tabular}{|c|c|c|}
\hline Code Name & Version & V\&V Tracking Number \\
\hline MCNP & 5 (Release 1.60) & 234728 [7] \\
\hline ORIGEN2 & 2.2 & $201298[12]$ \\
\hline
\end{tabular}

MCNP has been verified and validated (V\&V'd) for use at INL, as documented by the MCNP Version 5, Release 1.60 software management report [7]. The MCNP Version 5, Release $1.60 \mathrm{~V} \& \mathrm{~V}$ process was performed and accepted on high-performance computing systems at INL. The computer configurations listed in Table 7 were used to perform the MCNP5 calculations reported in this ECAR. 
TEM-10200-1

$12 / 19 / 17$

ENGINEERING CALCULATIONS AND ANALYSIS

Page 20 of 81

Rev.08

Title: $\quad$ AS-RUN NEUTRONICS EVALUATION FOR THE CSM-10584 EXPERIMENT IN THE ATR

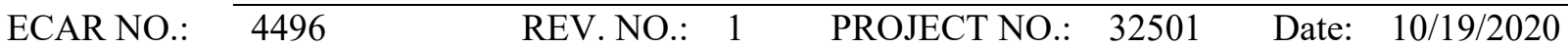

Table 7. Computer configurations for INL-qualified MCNP5 and ORIGEN2 installations.

\begin{tabular}{|c|c|c|}
\hline Computer & Processor & Operating System \\
\hline $\begin{array}{l}\text { 34,992 core } \\
\text { SGI ICE X distributed } \\
\text { memory cluster } \\
\text { (a.k.a. falcon } \\
\text { computer system) }\end{array}$ & $\begin{array}{l}2 \text { Login Nodes } \\
\text { falcon1, falcon2 } \\
\text { - } 2 \text { Intel Xeon E5-2695 v4 CPUs } \\
\circ \quad \text { Broadwell chipset } \\
\circ \quad 18 \text { cores per CPU } \\
\circ \quad 2.10 \mathrm{GHz} \\
\text { - } 128 \mathrm{~GB} \text { of RAM } \\
\text { - } \quad \text { FDR InfiniBand Interconnect } \\
972 \text { Compute Nodes with: } \\
\text { - } 2 \text { Intel Xeon E5-2695 v4 CPUs } \\
\circ \quad \text { Broadwell chipset } \\
\circ \quad 18 \text { cores per CPU } \\
\circ \quad 2.10 \mathrm{GHz} \\
\text { - } 128 \mathrm{~GB} \text { of RAM } \\
\text { FDR InfiniBand Interconnect }\end{array}$ & $\begin{array}{l}\text { SUSE Linux } \\
\text { Enterprise Server } 11 \\
\text { SP4 }\end{array}$ \\
\hline
\end{tabular}

\section{DISCUSSION / ANALYSIS}

MCNP was used to calculate the heating rates, flux, and DPA for CSM. ORIGEN2 was used to calculate the decay heat, radionuclide inventory, and radionuclide source term versus cooling time for CSM.

\subsection{Heating Normalization Factors}

MCNP reports tally results normalized per source particle. The MCNP Type 6 energy deposition tally results or Type 7 fission energy deposition tally results are used to calculate HGRs. The MCNP Tally Type 6 has units of MeV/g per source particle (fission neutron for prompt neutron, gamma heating, and fission heating). The heating normalization factor (HNF) is defined by Equation (1).

$$
H N F=\left(\frac{2.43 \text { fission neutrons }}{\text { fission }}\right)\left(\frac{\text { fission }}{200 \mathrm{MeV}}\right)\left(\frac{1 \times 10^{6} \mathrm{~W}}{1 \mathrm{MW}}\right)
$$


TEM-10200-1

$12 / 19 / 17$

ENGINEERING CALCULATIONS AND ANALYSIS

Page 21 of 81

Rev.08

Title: $\quad$ AS-RUN NEUTRONICS EVALUATION FOR THE CSM-10584 EXPERIMENT IN THE ATR

\begin{tabular}{llllllll} 
ECAR NO.: & 4496 & REV. NO.: & 1 & PROJECT NO.: & 32501 & Date: & 10/19/2020 \\
\hline
\end{tabular}

$$
H N F=1.215 \times 10^{4} \frac{\text { fission neutrons } \cdot W}{M W \cdot M e V}
$$

The HGR values are calculated using the MCNP Tally Type 6 results, the HNF, and the ATR core power. Prompt neutron and gamma heating rates (PHRs) are calculated using Equation (2).

$$
\begin{gathered}
\text { PHR }=\left(\text { type } 6 \text { tally } \frac{M e V}{g \cdot \text { fission neutron }}\right)(1.215 \\
\left.\times 10^{4} \frac{\text { fission neutrons } \cdot W}{M W \cdot M e V}\right)(\text { Core Power } M W) \\
P H R=(f 6)(H N F)(\text { Core Power }) \frac{W}{g}
\end{gathered}
$$

MCNP reports tally results normalized per source particle. The heating tallies have units of MeV/g per fission neutron. The MCNP Type 6 energy deposition tally results are used to calculate delayed gamma HGRs. The MCNP Tally Type 6 has units of MeV/g per source particle (per delayed fission product gamma for delayed fission product gamma heating). The delayed gamma heating normalization factor (DNF) is defined by Equation (3) using 8.9603 delayed fission photons per fission.

$$
\begin{gathered}
D F N=\left(\frac{8.9603 \text { delayed photons }}{\text { fission }}\right)\left(\frac{\text { fission }}{200 \mathrm{MeV}}\right)\left(\frac{1 \times 10^{6} \mathrm{~W}}{\mathrm{MW}}\right) \\
D F N=4.480 \times 10^{4} \frac{\mathrm{delayed} \mathrm{photons} \cdot \mathrm{W}}{\mathrm{M} W_{\text {core power }} \cdot \mathrm{MeV}}
\end{gathered}
$$

The HGR values are calculated using the MCNP Tally Type 6 or Type 7 results, the HNF, and the ATR core power. The delayed fission product heating rate (DHR) is calculated using Equation (4).

$$
\begin{gathered}
\text { DHR }=\left(\text { type } 6 \text { tally } \frac{M e V}{g \cdot \text { source photon }}\right)(4.480 \\
\left.\times 10^{4} \frac{\text { delayed photons } \cdot W}{M W \cdot M e V}\right)(\text { Core Power } M W) \\
D H R=(f 6)(D N F)(\text { Core Power }) \frac{W}{g}
\end{gathered}
$$


TEM-10200-1

$12 / 19 / 17$

ENGINEERING CALCULATIONS AND ANALYSIS

Page 22 of 81

Rev.08

Title:

AS-RUN NEUTRONICS EVALUATION FOR THE CSM-10584 EXPERIMENT IN THE ATR

REV. NO.: 1 PROJECT NO.: $32501 \quad$ Date: $10 / 19 / 2020$

\subsection{Neutron Flux Normalization Factor}

MCNP reports tally results normalized per source particle. The MCNP Type 4 flux tally results are used to generate neutron flux input values for the ORIGEN2 activation calculations. The MCNP Tally Type 4 (for neutrons) has units of neutrons $/ \mathrm{cm}^{2}$ per source neutron. The neutron flux conversion factor (NFCF) is defined by Equation (5).

$$
\begin{gathered}
\text { NFCF }=\left(\frac{2.43 \text { fission neutrons }}{\text { fission }}\right)\left(\frac{\text { fission }}{200 \mathrm{MeV}}\right)\left(\frac{6.24151 \times 10^{18} \mathrm{MeV}}{M W_{\text {Core Power }} \cdot s}\right) \\
N F C F=7.583 \times 10^{16} \frac{\text { fission neutrons }}{M W_{\text {Core Power }} \cdot \mathrm{s}}
\end{gathered}
$$

The neutron flux values are calculated using the MCNP tally type 4 results, the NFCF, and the ATR core power. The neutron flux is calculated using Equation (6).

$$
\begin{aligned}
& \phi_{\text {neutron }}=\left(\text { type } 4 \text { tally } \frac{\text { neutrons }}{\mathrm{cm}^{2}-\text { fission neutron }}\right)(7.583 \\
& \left.\times 10^{16} \frac{\text { fission neutrons }}{\mathrm{MW}_{\text {core power }}-\mathrm{s}}\right)(\text { Core Power MW }) \\
& \phi_{\text {neutron }}=(\mathrm{f} 4)\left(7.583 \times 10^{16}\right)(\text { Core Power }) \frac{\text { neutrons }}{\mathrm{cm}^{2}-\mathrm{s}}
\end{aligned}
$$

\subsection{MCNP DPA Calculations}

The DPA rate in a material is estimated by using a tally multiplier card with a standard flux tally in MCNP. The tally multiplier card applied to a flux tally calculates the reaction rate, which is defined as the quantity:

$$
C \int \Phi(E) R_{m}(E) d E
$$

Where,

$$
\begin{array}{lll}
C & = & \text { multiplicative constant } \\
\Phi(E)= & \text { energy dependent flux }
\end{array}
$$


TEM-10200-1

$12 / 19 / 17$

ENGINEERING CALCULATIONS AND ANALYSIS

Page 23 of 81

Rev.08

Title:

AS-RUN NEUTRONICS EVALUATION FOR THE CSM-10584 EXPERIMENT IN THE ATR

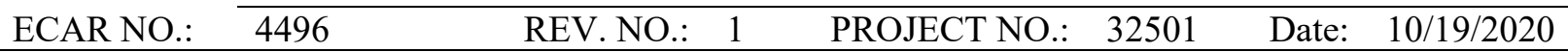

$R_{m}(E)=\quad$ energy dependent reaction rate of interest (ENDF/B-VII damage cross-section)

The quantity defined in Equation 12 is the total damage energy rate for the material. Assigning the quantity $\eta / 2 E_{d}$ to the constant $C$ in Equation (7) results in:

$$
\text { DPA rate }=C \int \Phi(E) R_{m}(E) d E
$$

Where,

$$
C=\eta / 2 E d
$$

$\int \Phi(E) R m(E) d E$ = total damage energy.

The DPA cross section would be calculated by:

$$
\sigma_{D P A}=\frac{\int \Phi(E) R_{m}(E) d E}{\int \Phi(E) d E}
$$

The resulting cross section has units of MeV-barns per atom.

Therefore, using the MCNP tallies, the DPA rate is calculated by converting the flux multiplier result to units of $\mathrm{MeV}-\mathrm{cm}^{2}$ per atom, then multiplying by the efficiency $(\eta)$ and dividing by 2 times the cutoff energy $\left(E_{d}\right)$ then using the standard tally conversion factors:

$$
\text { DPA rate }=F M n \times \frac{\eta}{2 E_{d}} \times \text { Flux Normalization Factor } \times \text { Core Power }
$$

\section{RESULTS}

\subsection{Heating Results}

Heating rates were generated assuming nominal core power configurations for cycle 164A and 164B [1]. The source power is assumed to be scaled to a nominal south power of $23.5 \mathrm{MW}$ and $22.9 \mathrm{MW}$, for ATR cycles $164 \mathrm{~A}$ and $164 \mathrm{~B}$, respectively. The heating results include prompt neutron and gamma heating, as well as delayed gamma heating. Delayed gamma heating was explicitly calculated using a separate MCNP model. The results for the heat generation rate calculations are presented in Table 8 - Table 9. 
TEM-10200-1

$12 / 19 / 17$

Rev.08

Title:

AS-RUN NEUTRONICS EVALUATION FOR THE CSM-10584 EXPERIMENT IN THE ATR

ECAR NO.: $\quad 4496$

REV. NO.: 1 PROJECT NO.: 32501

Date: $10 / 19 / 2020$

Table 8. Heating in CSM specimen and capsule components.

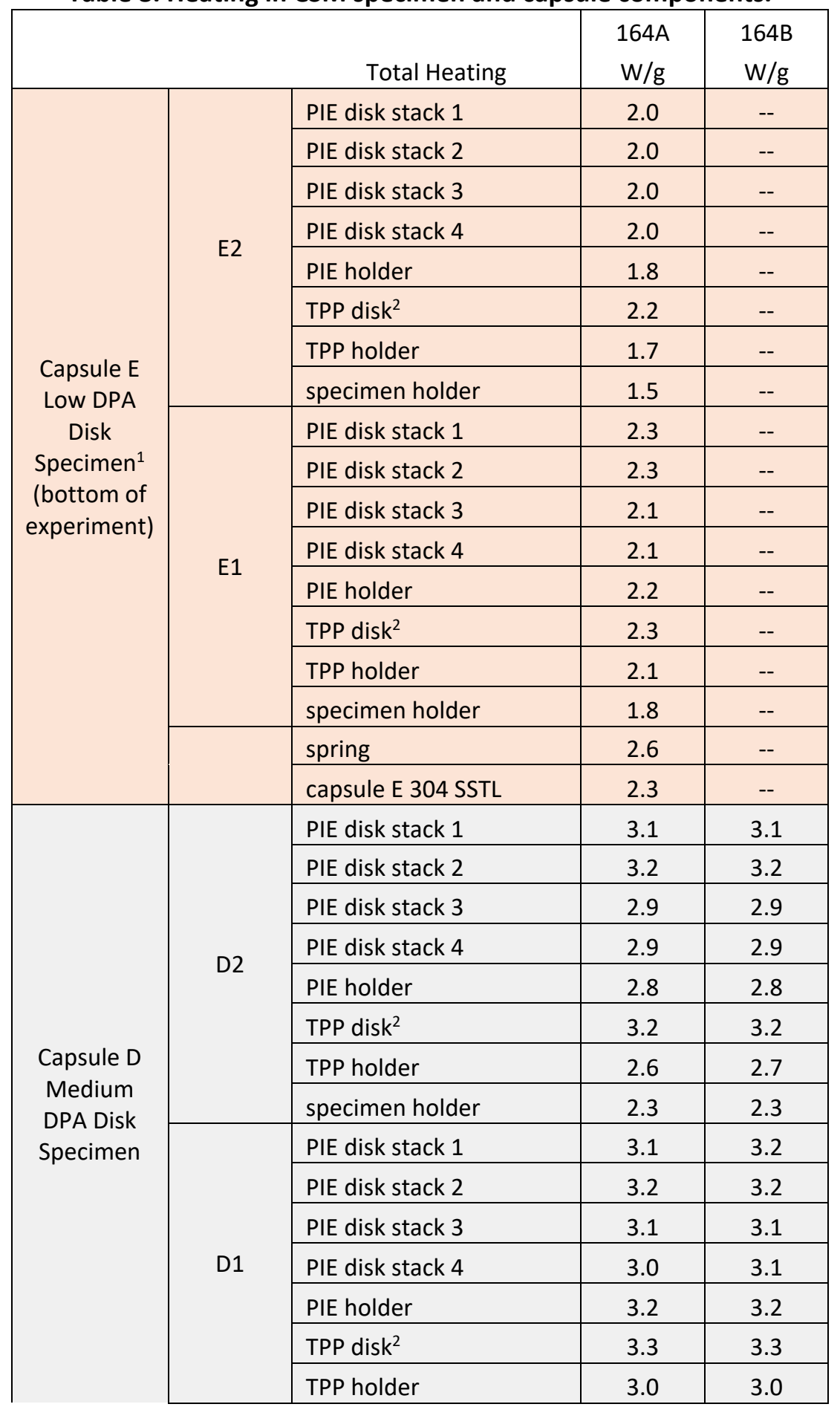


TEM-10200-1

$12 / 19 / 17$

ENGINEERING CALCULATIONS AND ANALYSIS

Page 25 of 81

Rev.08

Title:

AS-RUN NEUTRONICS EVALUATION FOR THE CSM-10584 EXPERIMENT IN THE ATR

ECAR NO.: 4496

REV. NO.: $1 \quad$ PROJECT NO.: 32501

Date: $10 / 19 / 2020$

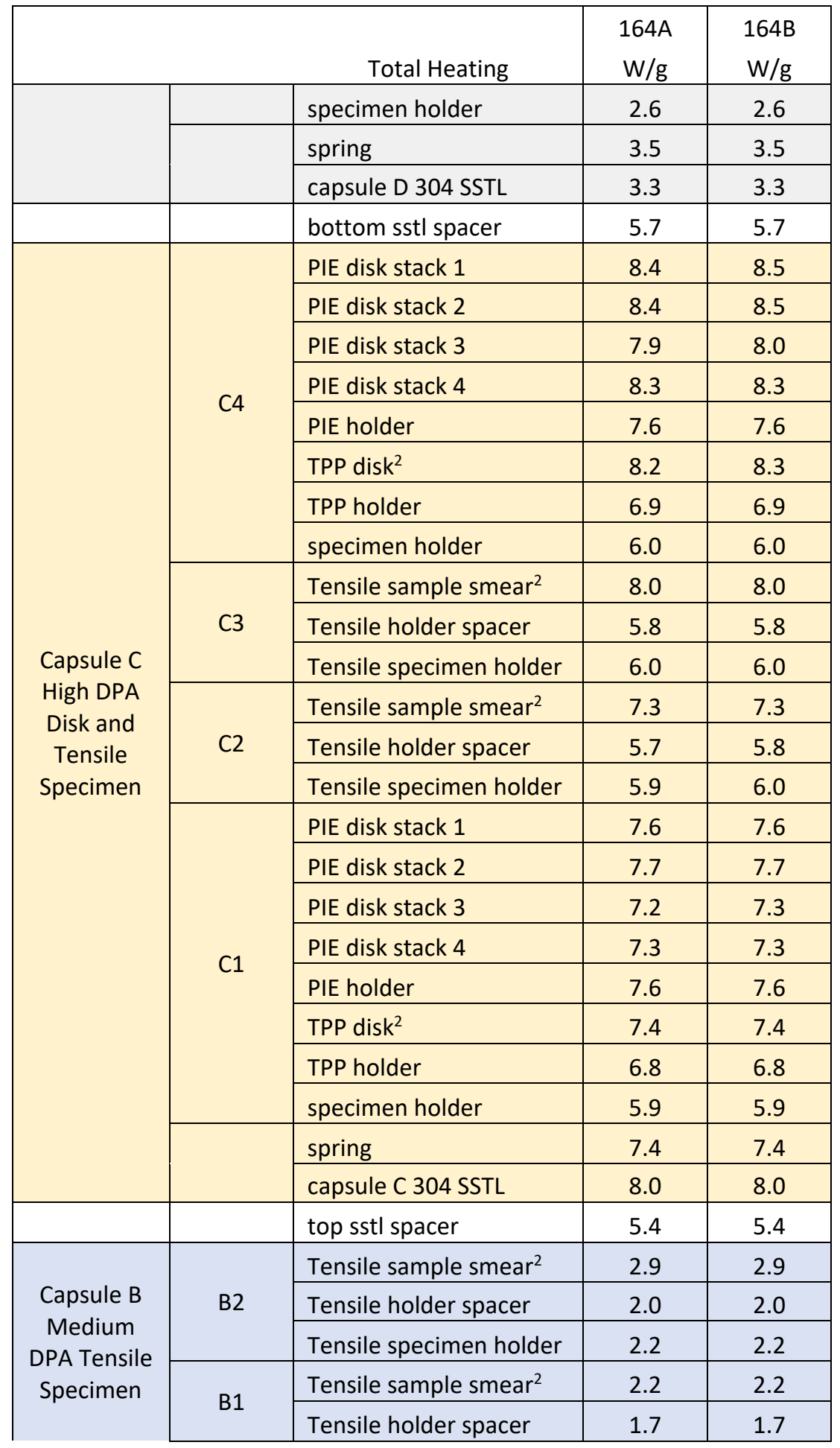


TEM-10200-1

Rev.08

Title: $\quad$ AS-RUN NEUTRONICS EVALUATION FOR THE CSM-10584 EXPERIMENT IN THE ATR

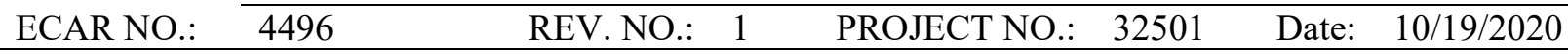

\begin{tabular}{|c|c|c|c|c|}
\hline \multicolumn{3}{|r|}{ Total Heating } & $\begin{array}{l}164 \mathrm{~A} \\
\mathrm{~W} / \mathrm{g}\end{array}$ & $\begin{array}{l}164 \mathrm{~B} \\
\mathrm{~W} / \mathrm{g}\end{array}$ \\
\hline & & Tensile specimen holder & 1.8 & 1.9 \\
\hline & & spring & 2.0 & 2.0 \\
\hline & & capsule B 304 SSTL & 2.6 & 2.6 \\
\hline \multirow{8}{*}{$\begin{array}{l}\text { Capsule A } \\
\text { Low DPA } \\
\text { Tensile } \\
\text { Specimen }{ }^{1} \\
\text { (top of } \\
\text { experiment) }\end{array}$} & \multirow{3}{*}{ A2 } & Tensile sample smear ${ }^{2}$ & 1.8 & -- \\
\hline & & Tensile holder spacer & 1.3 & -- \\
\hline & & Tensile specimen holder & 1.4 & -- \\
\hline & \multirow{3}{*}{$\mathrm{A} 1$} & Tensile sample smear ${ }^{2}$ & 1.3 & -- \\
\hline & & Tensile holder spacer & 0.9 & -- \\
\hline & & Tensile specimen holder & 1.1 & -- \\
\hline & & spring & 1.1 & -- \\
\hline & & capsule B 304 SSTL & 1.6 & -- \\
\hline \multicolumn{5}{|c|}{$\begin{array}{l}\text { 1) Capsule Removed after } 164 \mathrm{~A} \\
\text { 2) Refers to the stack of tensile samples in each capsule, it is not the } \\
\text { average }\end{array}$} \\
\hline
\end{tabular}

Table 9. Heating in B-5 assembly.

\begin{tabular}{|c|cc|cc|cc|cc|}
\hline \multirow{2}{*}{$\begin{array}{c}\text { Elevation } \\
\text { (cm from }\end{array}$} & \multicolumn{2}{|c|}{ Al Basket } & \multicolumn{2}{c|}{ H2O inside basket } & \multicolumn{2}{c|}{ H2O outside basket } & \multicolumn{2}{c|}{ 1/4in Be Reflector } \\
core center & 164A & 164B & 164A & 164B & 164A & 164B & 164A & 164B \\
\cline { 2 - 9 } line) & W/g & W/g & W/g & W/g & W/g & W/g & W/g & W/g \\
\hline-63.48 & 1.3 & 1.3 & 2.1 & 2.1 & 2.1 & 2.1 & 1.2 & 1.2 \\
-60.73 & 1.5 & 1.5 & 2.7 & 2.7 & 2.7 & 2.7 & 1.5 & 1.5 \\
-57.23 & 2.0 & 2.0 & 3.8 & 3.9 & 3.9 & 3.9 & 2.0 & 2.0 \\
-53.73 & 2.4 & 2.4 & 4.7 & 4.7 & 4.7 & 4.8 & 2.5 & 2.5 \\
\hline-50.23 & 2.7 & 2.8 & 5.5 & 5.5 & 5.5 & 5.6 & 2.8 & 2.9 \\
-27.03 & 4.9 & 4.9 & 10.1 & 10.1 & 10.1 & 10.2 & 5.2 & 5.2 \\
-3.80 & 6.2 & 6.3 & 12.8 & 12.8 & 12.8 & 12.9 & 6.5 & 6.5 \\
-0.51 & 6.3 & 6.3 & 12.8 & 12.9 & 12.9 & 12.9 & 6.5 & 6.6 \\
\hline 2.54 & 6.2 & 6.3 & 12.8 & 12.8 & 12.9 & 12.9 & 6.5 & 6.6 \\
5.80 & 6.2 & 6.2 & 12.7 & 12.8 & 12.8 & 12.8 & 6.5 & 6.5 \\
29.00 & 4.7 & 4.7 & 9.6 & 9.6 & 9.7 & 9.7 & 4.9 & 4.9 \\
52.23 & 2.3 & 2.3 & 4.6 & 4.6 & 4.6 & 4.6 & 2.4 & 2.4 \\
\hline 55.73 & 1.9 & 1.9 & 3.8 & 3.8 & 3.8 & 3.9 & 2.0 & 2.0 \\
59.23 & 1.5 & 1.5 & 3.0 & 3.0 & 3.0 & 3.0 & 1.6 & 1.6 \\
62.74 & 1.1 & 1.1 & 2.1 & 2.1 & 2.1 & 2.1 & 1.1 & 1.1 \\
65.74 & 0.8 & 0.8 & 1.4 & 1.4 & 1.4 & 1.4 & 0.8 & 0.8 \\
\hline
\end{tabular}


TEM-10200-1

$12 / 19 / 17$

Rev.08

Title:

AS-RUN NEUTRONICS EVALUATION FOR THE CSM-10584 EXPERIMENT IN THE ATR

ECAR NO.: $\quad 4496$

\subsection{DPA Results}

The projected cumulative DPA was calculated for the CSM sample specimen. The efficiency was assumed to be $80 \%$. The cutoff energy for 316 stainless steel and Inconel was calculated to be $4.03 \times 10^{-5} \mathrm{MeV}$ and $4.05 \times 10^{-5} \mathrm{MeV}$, respectively. Table 10 reports the projected DPA for the CSM experiment after two ATR cycles of irradiation. The calculated DPA ranges represent a south source power of $22.5 \mathrm{MW}(164 \mathrm{~A})$ and $22.6 \mathrm{MW}$ (164B) and a number of irradiation days amounting to 54.9 days for Cycle 164A and 64.1 days for Cycle 164B.

Table 10. Projected total DPA after irradiation.

\begin{tabular}{|c|c|c|c|c|c|c|}
\hline & $\begin{array}{l}316 \text { Stainless Steel } \\
\text { Specimen }\end{array}$ & Total DPA & Inconel Specimen & $\begin{array}{l}\text { Total } \\
\text { DPA }\end{array}$ & $\begin{array}{l}\text { Max } \\
\text { DPA }\end{array}$ & Min DPA \\
\hline \multirow{5}{*}{$\begin{array}{l}\text { Capsule } \\
\mathrm{E}^{1}\end{array}$} & PIE disk stack 1 & 0.19 & PIE disk stack 1 & 0.12 & \multirow{5}{*}{0.20} & \multirow{5}{*}{0.11} \\
\hline & PIE disk stack 2 & 0.18 & PIE disk stack 2 & 0.11 & & \\
\hline & PIE disk stack 3 & 0.18 & PIE disk stack 3 & 0.12 & & \\
\hline & PIE disk stack 4 & 0.17 & PIE disk stack 4 & 0.12 & & \\
\hline & TPP disk ${ }^{2}$ & 0.20 & TPP disk ${ }^{2}$ & 0.14 & & \\
\hline \multirow{5}{*}{$\begin{array}{c}\text { Capsule } \\
\text { D }\end{array}$} & PIE disk stack 1 & 0.65 & PIE disk stack 1 & 0.56 & \multirow{5}{*}{0.67} & \multirow{5}{*}{0.51} \\
\hline & PIE disk stack 2 & 0.65 & PIE disk stack 2 & 0.56 & & \\
\hline & PIE disk stack 3 & 0.60 & PIE disk stack 3 & 0.51 & & \\
\hline & PIE disk stack 4 & 0.62 & PIE disk stack 4 & 0.54 & & \\
\hline & TPP disk ${ }^{2}$ & 0.67 & TPP disk ${ }^{2}$ & 0.57 & & \\
\hline \multirow{6}{*}{$\begin{array}{l}\text { Capsule } \\
\text { C }\end{array}$} & Tensile sample smear ${ }^{2}$ & 1.58 & PIE disk stack 1 & 1.58 & \multirow{6}{*}{1.63} & \multirow{6}{*}{1.49} \\
\hline & PIE disk stack 1 & 1.63 & PIE disk stack 2 & 1.60 & & \\
\hline & PIE disk stack 2 & 1.59 & PIE disk stack 3 & 1.56 & & \\
\hline & PIE disk stack 3 & 1.53 & PIE disk stack 4 & 1.54 & & \\
\hline & PIE disk stack 4 & 1.49 & TPP disk ${ }^{2}$ & 1.56 & & \\
\hline & TPP disk ${ }^{2}$ & 1.55 & Tensile sample smear ${ }^{2}$ & 1.57 & & \\
\hline $\begin{array}{c}\text { Capsule } \\
\text { B }\end{array}$ & Tensile sample smear ${ }^{2}$ & 0.55 & Tensile sample smear ${ }^{2}$ & 0.47 & 0.55 & 0.47 \\
\hline $\begin{array}{l}\text { Capsule } \\
\mathrm{A}^{1}\end{array}$ & Tensile sample smear ${ }^{2}$ & 0.17 & Tensile sample smear ${ }^{2}$ & 0.11 & 0.17 & 0.11 \\
\hline \multicolumn{7}{|c|}{ 1) Capsule Removed after 164A } \\
\hline
\end{tabular}


TEM-10200-1

$12 / 19 / 17$

ENGINEERING CALCULATIONS AND ANALYSIS

Page 28 of 81

Rev.08

Title:

AS-RUN NEUTRONICS EVALUATION FOR THE CSM-10584 EXPERIMENT IN THE ATR

\begin{tabular}{llllllll} 
ECAR NO.: & 4496 & REV. NO.: & 1 & PROJECT NO.: & 32501 & Date: & 10/19/2020 \\
\hline
\end{tabular}

\subsection{Decay Heat}

Table 11 provides the decay heat (in watts) for each of the CSM capsules immediately after the applicable cycle or combined cycles end, as well as additional time periods over the subsequent 90 days.

Table 11. Decay heat analysis for CSM.

\begin{tabular}{|c|c|c|c|c|c|c|c|c|c|c|}
\hline \multirow{2}{*}{ Capsule } & \multirow{2}{*}{ \# Days of Irradiation } & EOC & 30 MINs & 5 HRS & 1 DAY & 7 DAYS & 14 DAYS & 30 DAYS & 60 DAYS & 90 DAYS \\
\cline { 2 - 11 } & & Watts & Watts & Watts & Watts & Watts & Watts & Watts & Watts & Watts \\
\hline Capsule E* & 54.9 & $3.94 \mathrm{E}+00$ & $2.93 \mathrm{E}-01$ & $1.47 \mathrm{E}-01$ & $6.88 \mathrm{E}-02$ & $4.71 \mathrm{E}-02$ & $4.21 \mathrm{E}-02$ & $3.31 \mathrm{E}-02$ & $2.21 \mathrm{E}-02$ & $1.56 \mathrm{E}-02$ \\
\hline Capsule D & 119 & $3.96 \mathrm{E}+00$ & $3.11 \mathrm{E}-01$ & $1.65 \mathrm{E}-01$ & $8.64 \mathrm{E}-02$ & $6.32 \mathrm{E}-02$ & $5.69 \mathrm{E}-02$ & $4.53 \mathrm{E}-02$ & $3.11 \mathrm{E}-02$ & $2.26 \mathrm{E}-02$ \\
\hline Capsule C & 119 & $7.32 \mathrm{E}+00$ & $5.46 \mathrm{E}-01$ & $2.92 \mathrm{E}-01$ & $1.55 \mathrm{E}-01$ & $1.12 \mathrm{E}-01$ & $1.01 \mathrm{E}-01$ & $8.06 \mathrm{E}-02$ & $5.53 \mathrm{E}-02$ & $4.04 \mathrm{E}-02$ \\
\hline Capsule B & 119 & $3.42 \mathrm{E}+00$ & $2.88 \mathrm{E}-01$ & $1.51 \mathrm{E}-01$ & $7.82 \mathrm{E}-02$ & $5.80 \mathrm{E}-02$ & $5.22 \mathrm{E}-02$ & $4.16 \mathrm{E}-02$ & $2.86 \mathrm{E}-02$ & $2.09 \mathrm{E}-02$ \\
\hline Capsule A* & 54.9 & $3.40 \mathrm{E}+00$ & $2.71 \mathrm{E}-01$ & $1.34 \mathrm{E}-01$ & $6.21 \mathrm{E}-02$ & $4.31 \mathrm{E}-02$ & $3.86 \mathrm{E}-02$ & $3.04 \mathrm{E}-02$ & $2.03 \mathrm{E}-02$ & $1.43 \mathrm{E}-02$ \\
\hline
\end{tabular}

\subsection{Radionuclide Source Term}

The source term for the CSM experiment was evaluated using the neutron flux for the experiment in the B-5 position. The peak MCNP calculated neutron flux, scaled to a south power of $22.6 \mathrm{MW}$, is $1.18 \times 10^{15} \mathrm{n} / \mathrm{cm}^{2} \cdot \mathrm{s}$. This flux value was used to calculate all radiological source terms and the decay heat generation rates for the CSM experiment.

Table 12 provides the summary of the radionuclide activity (in Curies) for each of the CSM capsules (which includes all material components: specimen, capsule, springs, and specimen holders) immediately after shutdown and for an additional 90 days. Table 13 - Table 17 display the isotopic data for each CSM capsule after their respective irradiation times.

Table 12. Source term summary for CSM.

\begin{tabular}{|c|c|c|c|c|c|c|c|c|c|c|}
\hline \multirow{2}{*}{ Capsule } & \# Days of & $\mathrm{EOC}$ & $30 \mathrm{MINs}$ & $5 \mathrm{HRS}$ & $1 \mathrm{DAY}$ & $7 \mathrm{DAYS}$ & $14 \mathrm{DAYS}$ & $30 \mathrm{DAYS}$ & $60 \mathrm{DAYS}$ & $90 \mathrm{DAYS}$ \\
\cline { 3 - 12 } & Irradiation & $\mathrm{Ci}$ & $\mathrm{Ci}$ & $\mathrm{Ci}$ & $\mathrm{Ci}$ & $\mathrm{Ci}$ & $\mathrm{Ci}$ & $\mathrm{Ci}$ & $\mathrm{Ci}$ & $\mathrm{Ci}$ \\
\hline Capsule $\mathrm{E}^{*}$ & 54.9 & $3.34 \mathrm{E}+02$ & $1.25 \mathrm{E}+02$ & $1.14 \mathrm{E}+02$ & $1.06 \mathrm{E}+02$ & $9.16 \mathrm{E}+01$ & $7.78 \mathrm{E}+01$ & $5.41 \mathrm{E}+01$ & $2.83 \mathrm{E}+01$ & $1.59 \mathrm{E}+01$ \\
\hline Capsule D & 119 & $3.66 \mathrm{E}+02$ & $1.56 \mathrm{E}+02$ & $1.45 \mathrm{E}+02$ & $1.38 \mathrm{E}+02$ & $1.19 \mathrm{E}+02$ & $1.02 \mathrm{E}+02$ & $7.16 \mathrm{E}+01$ & $3.91 \mathrm{E}+01$ & $2.33 \mathrm{E}+01$ \\
\hline Capsule C & 119 & $6.65 \mathrm{E}+02$ & $2.76 \mathrm{E}+02$ & $2.57 \mathrm{E}+02$ & $2.43 \mathrm{E}+02$ & $2.10 \mathrm{E}+02$ & $1.80 \mathrm{E}+02$ & $1.27 \mathrm{E}+02$ & $6.90 \mathrm{E}+01$ & $4.12 \mathrm{E}+01$ \\
\hline Capsule B & 119 & $3.23 \mathrm{E}+02$ & $1.43 \mathrm{E}+02$ & $1.32 \mathrm{E}+02$ & $1.25 \mathrm{E}+02$ & $1.09 \mathrm{E}+02$ & $9.27 \mathrm{E}+01$ & $6.53 \mathrm{E}+01$ & $3.56 \mathrm{E}+01$ & $2.13 \mathrm{E}+01$ \\
\hline Capsule A* & 54.9 & $2.94 \mathrm{E}+02$ & $1.14 \mathrm{E}+02$ & $1.04 \mathrm{E}+02$ & $9.69 \mathrm{E}+01$ & $8.35 \mathrm{E}+01$ & $7.09 \mathrm{E}+01$ & $4.93 \mathrm{E}+01$ & $2.58 \mathrm{E}+01$ & $1.45 \mathrm{E}+01$ \\
\hline
\end{tabular}

Table 13. Radionuclide source term for CSM capsule A after 54.9 days of irradiation (Ci).

\begin{tabular}{|c|c|c|c|c|c|c|c|c|c|}
\hline \multicolumn{10}{|c|}{ Capsule A - 54.9 days irradiation } \\
\hline & $\mathrm{EOC}$ & $30 \mathrm{MINs}$ & $5 \mathrm{HRS}$ & $1 \mathrm{DAY}$ & $7 \mathrm{DAYS}$ & $14 \mathrm{DAYS}$ & 30 DAYS & 60 DAYS & 90 DAYS \\
\hline ISOTOPE & $\mathrm{Ci}$ & $\mathrm{Ci}$ & $\mathrm{Ci}$ & $\mathrm{Ci}$ & $\mathrm{Ci}$ & $\mathrm{Ci}$ & $\mathrm{Ci}$ & $\mathrm{Ci}$ & $\mathrm{Ci}$ \\
\hline $\mathrm{H} 3$ & $7.32 \mathrm{E}-09$ & $7.32 \mathrm{E}-09$ & $7.32 \mathrm{E}-09$ & $7.32 \mathrm{E}-09$ & $7.31 \mathrm{E}-09$ & $7.31 \mathrm{E}-09$ & $7.29 \mathrm{E}-09$ & $7.25 \mathrm{E}-09$ & $7.22 \mathrm{E}-09$ \\
\hline $\mathrm{H} 4$ & $7.57 \mathrm{E}-05$ & $0.00 \mathrm{E}+00$ & $0.00 \mathrm{E}+00$ & $0.00 \mathrm{E}+00$ & $0.00 \mathrm{E}+00$ & $0.00 \mathrm{E}+00$ & $0.00 \mathrm{E}+00$ & $0.00 \mathrm{E}+00$ & $0.00 \mathrm{E}+00$ \\
\hline
\end{tabular}


TEM-10200-1

ENGINEERING CALCULATIONS AND ANALYSIS

Page 29 of 81

$12 / 19 / 17$

Rev.08

Title:

AS-RUN NEUTRONICS EVALUATION FOR THE CSM-10584 EXPERIMENT IN THE ATR

\begin{tabular}{llllllll} 
ECAR NO.: & 4496 & REV. NO.: & 1 & PROJECT NO.: & 32501 & Date: & 10/19/2020 \\
\hline
\end{tabular}

\begin{tabular}{|c|c|c|c|c|c|c|c|c|c|}
\hline \multicolumn{10}{|c|}{ Capsule A - 54.9 days irradiation } \\
\hline & EOC & $30 \mathrm{MINs}$ & $5 \mathrm{HRS}$ & $1 \mathrm{DAY}$ & 7 DAYS & 14 DAYS & 30 DAYS & 60 DAYS & 90 DAYS \\
\hline ISOTOPE & $\mathrm{Ci}$ & $\mathrm{Ci}$ & $\mathrm{Ci}$ & $\mathrm{Ci}$ & $\mathrm{Ci}$ & $\mathrm{Ci}$ & $\mathrm{Ci}$ & $\mathrm{Ci}$ & $\mathrm{Ci}$ \\
\hline HE 6 & $1.26 \mathrm{E}-05$ & $0.00 E+00$ & $0.00 \mathrm{E}+00$ & $0.00 \mathrm{E}+00$ & $0.00 \mathrm{E}+00$ & $0.00 \mathrm{E}+00$ & $0.00 \mathrm{E}+00$ & $0.00 \mathrm{E}+00$ & $0.00 \mathrm{E}+00$ \\
\hline LI 8 & $3.04 \mathrm{E}-05$ & $0.00 \mathrm{E}+00$ & $0.00 \mathrm{E}+00$ & $0.00 \mathrm{E}+00$ & $0.00 \mathrm{E}+00$ & $0.00 \mathrm{E}+00$ & $0.00 \mathrm{E}+00$ & $0.00 \mathrm{E}+00$ & $0.00 \mathrm{E}+00$ \\
\hline BE 8 & $5.21 \mathrm{E}-05$ & $0.00 \mathrm{E}+00$ & $0.00 \mathrm{E}+00$ & $0.00 \mathrm{E}+00$ & $0.00 \mathrm{E}+00$ & $0.00 \mathrm{E}+00$ & $0.00 \mathrm{E}+00$ & $0.00 \mathrm{E}+00$ & $0.00 \mathrm{E}+00$ \\
\hline BE 10 & $8.85 \mathrm{E}-11$ & $8.85 \mathrm{E}-11$ & $8.85 \mathrm{E}-11$ & $8.85 \mathrm{E}-11$ & $8.85 \mathrm{E}-11$ & $8.85 \mathrm{E}-11$ & $8.85 \mathrm{E}-11$ & $8.85 \mathrm{E}-11$ & $8.85 \mathrm{E}-11$ \\
\hline BE 11 & $7.00 \mathrm{E}-10$ & $0.00 \mathrm{E}+00$ & $0.00 \mathrm{E}+00$ & $0.00 \mathrm{E}+00$ & $0.00 \mathrm{E}+00$ & $0.00 \mathrm{E}+00$ & $0.00 \mathrm{E}+00$ & $0.00 \mathrm{E}+00$ & $0.00 \mathrm{E}+00$ \\
\hline B 12 & $2.29 \mathrm{E}-05$ & $0.00 \mathrm{E}+00$ & $0.00 \mathrm{E}+00$ & $0.00 \mathrm{E}+00$ & $0.00 \mathrm{E}+00$ & $0.00 \mathrm{E}+00$ & $0.00 \mathrm{E}+00$ & $0.00 \mathrm{E}+00$ & $0.00 \mathrm{E}+00$ \\
\hline C 14 & $3.23 \mathrm{E}-09$ & $3.23 \mathrm{E}-09$ & $3.23 \mathrm{E}-09$ & $3.23 \mathrm{E}-09$ & $3.23 \mathrm{E}-09$ & $3.23 \mathrm{E}-09$ & $3.23 \mathrm{E}-09$ & $3.23 \mathrm{E}-09$ & $3.23 \mathrm{E}-09$ \\
\hline C 15 & $3.36 \mathrm{E}-11$ & $0.00 \mathrm{E}+00$ & $0.00 \mathrm{E}+00$ & $0.00 \mathrm{E}+00$ & $0.00 \mathrm{E}+00$ & $0.00 \mathrm{E}+00$ & $0.00 \mathrm{E}+00$ & $0.00 \mathrm{E}+00$ & $0.00 \mathrm{E}+00$ \\
\hline O 19 & $2.40 \mathrm{E}-12$ & $0.00 \mathrm{E}+00$ & $0.00 \mathrm{E}+00$ & $0.00 \mathrm{E}+00$ & $0.00 \mathrm{E}+00$ & $0.00 \mathrm{E}+00$ & $0.00 \mathrm{E}+00$ & $0.00 \mathrm{E}+00$ & $0.00 \mathrm{E}+00$ \\
\hline$F 20$ & $9.06 \mathrm{E}-09$ & $0.00 \mathrm{E}+00$ & $0.00 \mathrm{E}+00$ & $0.00 \mathrm{E}+00$ & $0.00 \mathrm{E}+00$ & $0.00 \mathrm{E}+00$ & $0.00 \mathrm{E}+00$ & $0.00 \mathrm{E}+00$ & $0.00 \mathrm{E}+00$ \\
\hline NE 23 & $9.41 \mathrm{E}-03$ & $2.65 \mathrm{E}-17$ & $0.00 \mathrm{E}+00$ & $0.00 \mathrm{E}+00$ & $0.00 \mathrm{E}+00$ & $0.00 \mathrm{E}+00$ & $0.00 \mathrm{E}+00$ & $0.00 \mathrm{E}+00$ & $0.00 \mathrm{E}+00$ \\
\hline NA 24 & $1.50 \mathrm{E}+00$ & $1.46 \mathrm{E}+00$ & $1.19 \mathrm{E}+00$ & $4.93 \mathrm{E}-01$ & $6.34 \mathrm{E}-04$ & $2.69 \mathrm{E}-07$ & $5.30 \mathrm{E}-15$ & $1.88 \mathrm{E}-29$ & $0.00 \mathrm{E}+00$ \\
\hline NA 24M & $2.01 \mathrm{E}-06$ & $0.00 \mathrm{E}+00$ & $0.00 E+00$ & $0.00 \mathrm{E}+00$ & $0.00 \mathrm{E}+00$ & $0.00 \mathrm{E}+00$ & $0.00 \mathrm{E}+00$ & $0.00 \mathrm{E}+00$ & $0.00 \mathrm{E}+00$ \\
\hline NA 25 & $5.68 \mathrm{E}-03$ & $4.60 \mathrm{E}-12$ & $0.00 \mathrm{E}+00$ & $0.00 \mathrm{E}+00$ & $0.00 \mathrm{E}+00$ & $0.00 \mathrm{E}+00$ & $0.00 \mathrm{E}+00$ & $0.00 \mathrm{E}+00$ & $0.00 \mathrm{E}+00$ \\
\hline MG 27 & $7.05 \mathrm{E}+00$ & $7.83 \mathrm{E}-01$ & $2.01 \mathrm{E}-09$ & $0.00 \mathrm{E}+00$ & $0.00 \mathrm{E}+00$ & $0.00 E+00$ & $0.00 E+00$ & $0.00 \mathrm{E}+00$ & $0.00 \mathrm{E}+00$ \\
\hline MG 28 & $2.51 \mathrm{E}-08$ & $2.46 \mathrm{E}-08$ & $2.12 \mathrm{E}-08$ & $1.13 \mathrm{E}-08$ & $9.56 \mathrm{E}-11$ & $3.65 \mathrm{E}-13$ & $1.08 \mathrm{E}-18$ & $4.67 \mathrm{E}-29$ & $0.00 \mathrm{E}+00$ \\
\hline AL 28 & $1.68 \mathrm{E}+02$ & $1.56 \mathrm{E}-02$ & $2.13 \mathrm{E}-08$ & $1.13 \mathrm{E}-08$ & $9.58 \mathrm{E}-11$ & $3.65 \mathrm{E}-13$ & $1.08 \mathrm{E}-18$ & $0.00 \mathrm{E}+00$ & $0.00 \mathrm{E}+00$ \\
\hline AL 29 & 1.77E-03 & $7.31 \mathrm{E}-05$ & $2.50 \mathrm{E}-17$ & $0.00 \mathrm{E}+00$ & $0.00 \mathrm{E}+00$ & $0.00 \mathrm{E}+00$ & $0.00 \mathrm{E}+00$ & $0.00 \mathrm{E}+00$ & $0.00 \mathrm{E}+00$ \\
\hline AL 30 & $1.72 \mathrm{E}-06$ & $0.00 \mathrm{E}+00$ & $0.00 \mathrm{E}+00$ & $0.00 \mathrm{E}+00$ & $0.00 \mathrm{E}+00$ & $0.00 \mathrm{E}+00$ & $0.00 \mathrm{E}+00$ & $0.00 \mathrm{E}+00$ & $0.00 \mathrm{E}+00$ \\
\hline SI 31 & $1.72 \mathrm{E}-02$ & $1.51 \mathrm{E}-02$ & $4.59 \mathrm{E}-03$ & $3.02 \mathrm{E}-05$ & $8.82 \mathrm{E}-22$ & $0.00 \mathrm{E}+00$ & $0.00 \mathrm{E}+00$ & $0.00 \mathrm{E}+00$ & $0.00 \mathrm{E}+00$ \\
\hline SI 32 & $1.95 \mathrm{E}-12$ & $1.95 \mathrm{E}-12$ & $1.95 \mathrm{E}-12$ & $1.95 \mathrm{E}-12$ & $1.95 \mathrm{E}-12$ & $1.95 \mathrm{E}-12$ & $1.95 \mathrm{E}-12$ & $1.95 \mathrm{E}-12$ & $1.95 \mathrm{E}-12$ \\
\hline P 32 & $3.68 \mathrm{E}-03$ & $3.68 \mathrm{E}-03$ & $3.65 \mathrm{E}-03$ & $3.51 \mathrm{E}-03$ & $2.62 \mathrm{E}-03$ & 1.87E-03 & $8.60 \mathrm{E}-04$ & $2.01 \mathrm{E}-04$ & $4.70 \mathrm{E}-05$ \\
\hline P 33 & $1.15 \mathrm{E}-07$ & $1.15 \mathrm{E}-07$ & $1.14 \mathrm{E}-07$ & $1.12 \mathrm{E}-07$ & $9.44 \mathrm{E}-08$ & 7.77E-08 & 4.99E-08 & $2.17 \mathrm{E}-08$ & $9.45 \mathrm{E}-09$ \\
\hline P 34 & $8.45 \mathrm{E}-07$ & $0.00 \mathrm{E}+00$ & $0.00 \mathrm{E}+00$ & $0.00 \mathrm{E}+00$ & $0.00 \mathrm{E}+00$ & $0.00 \mathrm{E}+00$ & $0.00 \mathrm{E}+00$ & $0.00 \mathrm{E}+00$ & $0.00 \mathrm{E}+00$ \\
\hline S 35 & $3.40 \mathrm{E}-05$ & $3.40 \mathrm{E}-05$ & $3.39 \mathrm{E}-05$ & 3.37E-05 & $3.21 \mathrm{E}-05$ & $3.04 \mathrm{E}-05$ & $2.68 \mathrm{E}-05$ & $2.12 \mathrm{E}-05$ & $1.67 \mathrm{E}-05$ \\
\hline S 37 & $2.54 \mathrm{E}-07$ & $4.18 \mathrm{E}-09$ & $3.61 \mathrm{E}-25$ & $0.00 \mathrm{E}+00$ & $0.00 \mathrm{E}+00$ & $0.00 \mathrm{E}+00$ & $0.00 \mathrm{E}+00$ & $0.00 \mathrm{E}+00$ & $0.00 \mathrm{E}+00$ \\
\hline CL 36 & $1.86 \mathrm{E}-11$ & $1.86 \mathrm{E}-11$ & $1.86 \mathrm{E}-11$ & $1.86 \mathrm{E}-11$ & $1.86 \mathrm{E}-11$ & $1.86 \mathrm{E}-11$ & $1.86 \mathrm{E}-11$ & $1.86 \mathrm{E}-11$ & $1.86 \mathrm{E}-11$ \\
\hline CL 38 & $1.42 \mathrm{E}-10$ & $8.12 \mathrm{E}-11$ & $5.32 \mathrm{E}-13$ & $3.20 \mathrm{E}-22$ & $0.00 \mathrm{E}+00$ & $0.00 \mathrm{E}+00$ & $0.00 \mathrm{E}+00$ & $0.00 E+00$ & $0.00 \mathrm{E}+00$ \\
\hline CL 38M & $1.69 \mathrm{E}-12$ & $0.00 E+00$ & $0.00 \mathrm{E}+00$ & $0.00 \mathrm{E}+00$ & $0.00 \mathrm{E}+00$ & $0.00 \mathrm{E}+00$ & $0.00 \mathrm{E}+00$ & $0.00 \mathrm{E}+00$ & $0.00 \mathrm{E}+00$ \\
\hline AR 39 & $6.99 \mathrm{E}-08$ & $6.99 \mathrm{E}-08$ & $6.99 \mathrm{E}-08$ & $6.99 \mathrm{E}-08$ & $6.99 \mathrm{E}-08$ & $6.99 \mathrm{E}-08$ & $6.99 \mathrm{E}-08$ & $6.99 \mathrm{E}-08$ & $6.98 \mathrm{E}-08$ \\
\hline AR 41 & 1.13E-08 & 9.33E-09 & 1.69E-09 & 1.25E-12 & $0.00 \mathrm{E}+00$ & $0.00 \mathrm{E}+00$ & $0.00 \mathrm{E}+00$ & $0.00 \mathrm{E}+00$ & $0.00 \mathrm{E}+00$ \\
\hline K 40 & $1.56 \mathrm{E}-13$ & 1.56E-13 & $1.56 \mathrm{E}-13$ & $1.56 \mathrm{E}-13$ & $1.56 \mathrm{E}-13$ & $1.56 \mathrm{E}-13$ & $1.56 \mathrm{E}-13$ & $1.56 \mathrm{E}-13$ & $1.56 \mathrm{E}-13$ \\
\hline K 42 & 1.05E-04 & $1.02 \mathrm{E}-04$ & 7.94E-05 & $2.74 \mathrm{E}-05$ & $8.52 \mathrm{E}-09$ & $6.90 \mathrm{E}-13$ & 6.27E-18 & $6.26 \mathrm{E}-18$ & $6.25 \mathrm{E}-18$ \\
\hline K 43 & $5.08 \mathrm{E}-11$ & 5.00E-11 & 4.36E-11 & $2.43 \mathrm{E}-11$ & $2.94 \mathrm{E}-13$ & $1.70 \mathrm{E}-15$ & $1.31 \mathrm{E}-20$ & $3.36 \mathrm{E}-30$ & $0.00 \mathrm{E}+00$ \\
\hline K 44 & 8.99E-12 & 3.49E-12 & 7.06E-16 & $0.00 \mathrm{E}+00$ & $0.00 \mathrm{E}+00$ & $0.00 \mathrm{E}+00$ & $0.00 \mathrm{E}+00$ & $0.00 \mathrm{E}+00$ & $0.00 \mathrm{E}+00$ \\
\hline CA 45 & $2.74 \mathrm{E}-07$ & $2.74 \mathrm{E}-07$ & $2.74 \mathrm{E}-07$ & $2.73 \mathrm{E}-07$ & 2.66E-07 & $2.58 \mathrm{E}-07$ & $2.41 \mathrm{E}-07$ & $2.12 \mathrm{E}-07$ & 1.87E-07 \\
\hline CA 47 & 5.73E-09 & $5.72 \mathrm{E}-09$ & 5.56E-09 & 4.92E-09 & 1.97E-09 & $6.75 \mathrm{E}-10$ & $5.85 \mathrm{E}-11$ & $5.98 \mathrm{E}-13$ & $6.10 \mathrm{E}-15$ \\
\hline SC 46 & 6.16E-05 & 6.16E-05 & $6.15 \mathrm{E}-05$ & $6.11 \mathrm{E}-05$ & $5.82 \mathrm{E}-05$ & 5.49E-05 & $4.81 \mathrm{E}-05$ & $3.75 \mathrm{E}-05$ & $2.93 \mathrm{E}-05$ \\
\hline SC $46 \mathrm{M}$ & $6.93 \mathrm{E}-10$ & $0.00 \mathrm{E}+00$ & $0.00 \mathrm{E}+00$ & $0.00 \mathrm{E}+00$ & $0.00 \mathrm{E}+00$ & $0.00 \mathrm{E}+00$ & $0.00 \mathrm{E}+00$ & $0.00 \mathrm{E}+00$ & $0.00 \mathrm{E}+00$ \\
\hline SC 47 & 2.63E-04 & 2.62E-04 & $2.52 \mathrm{E}-04$ & 2.14E-04 & $6.18 \mathrm{E}-05$ & 1.45E-05 & $5.30 \mathrm{E}-07$ & 1.07E-09 & $2.17 \mathrm{E}-12$ \\
\hline SC 48 & $2.50 \mathrm{E}-05$ & $2.48 \mathrm{E}-05$ & $2.31 \mathrm{E}-05$ & $1.71 \mathrm{E}-05$ & 1.75E-06 & $1.23 \mathrm{E}-07$ & $2.82 \mathrm{E}-10$ & 3.17E-15 & $3.58 \mathrm{E}-20$ \\
\hline SC 49 & 2.11E-05 & 1.47E-05 & 5.67E-07 & $6.10 \mathrm{E}-13$ & $0.00 \mathrm{E}+00$ & $0.00 \mathrm{E}+00$ & $0.00 \mathrm{E}+00$ & $0.00 \mathrm{E}+00$ & $0.00 \mathrm{E}+00$ \\
\hline SC 50 & $4.30 \mathrm{E}-07$ & $2.22 \mathrm{E}-12$ & $0.00 \mathrm{E}+00$ & $0.00 \mathrm{E}+00$ & $0.00 \mathrm{E}+00$ & $0.00 \mathrm{E}+00$ & $0.00 \mathrm{E}+00$ & $0.00 \mathrm{E}+00$ & $0.00 \mathrm{E}+00$ \\
\hline TI 51 & $1.15 \mathrm{E}-03$ & $3.12 \mathrm{E}-05$ & $2.42 \mathrm{E}-19$ & $0.00 \mathrm{E}+00$ & $0.00 \mathrm{E}+00$ & $0.00 \mathrm{E}+00$ & $0.00 \mathrm{E}+00$ & $0.00 \mathrm{E}+00$ & $0.00 \mathrm{E}+00$ \\
\hline V 52 & $8.92 \mathrm{E}-01$ & $3.49 \mathrm{E}-03$ & $7.38 \mathrm{E}-25$ & $0.00 \mathrm{E}+00$ & $0.00 \mathrm{E}+00$ & $0.00 \mathrm{E}+00$ & $0.00 \mathrm{E}+00$ & $0.00 \mathrm{E}+00$ & $0.00 \mathrm{E}+00$ \\
\hline V 53 & $5.53 \mathrm{E}-03$ & $1.36 \mathrm{E}-08$ & $0.00 \mathrm{E}+00$ & $0.00 \mathrm{E}+00$ & $0.00 \mathrm{E}+00$ & $0.00 \mathrm{E}+00$ & $0.00 \mathrm{E}+00$ & $0.00 \mathrm{E}+00$ & $0.00 \mathrm{E}+00$ \\
\hline V 54 & $5.71 \mathrm{E}-05$ & $8.03 \mathrm{E}-15$ & $0.00 \mathrm{E}+00$ & $0.00 \mathrm{E}+00$ & $0.00 \mathrm{E}+00$ & $0.00 \mathrm{E}+00$ & $0.00 \mathrm{E}+00$ & $0.00 \mathrm{E}+00$ & $0.00 \mathrm{E}+00$ \\
\hline CR 51 & $9.07 \mathrm{E}+01$ & $9.07 \mathrm{E}+01$ & $9.02 \mathrm{E}+01$ & $8.85 \mathrm{E}+01$ & $7.61 \mathrm{E}+01$ & $6.39 \mathrm{E}+01$ & $4.28 \mathrm{E}+01$ & $2.02 \mathrm{E}+01$ & $9.55 \mathrm{E}+00$ \\
\hline CR 55 & $1.56 \mathrm{E}+00$ & $4.48 \mathrm{E}-03$ & $0.00 \mathrm{E}+00$ & $0.00 \mathrm{E}+00$ & $0.00 \mathrm{E}+00$ & $0.00 \mathrm{E}+00$ & $0.00 \mathrm{E}+00$ & $0.00 \mathrm{E}+00$ & $0.00 \mathrm{E}+00$ \\
\hline MN 54 & $8.56 \mathrm{E}-01$ & $8.55 \mathrm{E}-01$ & $8.55 \mathrm{E}-01$ & $8.54 \mathrm{E}-01$ & $8.42 \mathrm{E}-01$ & $8.29 \mathrm{E}-01$ & $8.00 \mathrm{E}-01$ & $7.49 \mathrm{E}-01$ & $7.01 \mathrm{E}-01$ \\
\hline MN 56 & $1.27 \mathrm{E}+01$ & $1.11 \mathrm{E}+01$ & $3.32 \mathrm{E}+00$ & $2.01 \mathrm{E}-02$ & $3.10 \mathrm{E}-19$ & $0.00 \mathrm{E}+00$ & $0.00 \mathrm{E}+00$ & $0.00 \mathrm{E}+00$ & $0.00 \mathrm{E}+00$ \\
\hline MN 57 & $1.79 \mathrm{E}-02$ & $4.40 \mathrm{E}-08$ & $0.00 \mathrm{E}+00$ & $0.00 \mathrm{E}+00$ & $0.00 \mathrm{E}+00$ & $0.00 \mathrm{E}+00$ & $0.00 \mathrm{E}+00$ & $0.00 \mathrm{E}+00$ & $0.00 \mathrm{E}+00$ \\
\hline MN 58 & 5.71E-05 & $2.87 \mathrm{E}-13$ & $0.00 \mathrm{E}+00$ & $0.00 \mathrm{E}+00$ & $0.00 \mathrm{E}+00$ & $0.00 \mathrm{E}+00$ & $0.00 \mathrm{E}+00$ & $0.00 \mathrm{E}+00$ & $0.00 \mathrm{E}+00$ \\
\hline FE 55 & $3.10 \mathrm{E}+00$ & $3.10 \mathrm{E}+00$ & $3.10 \mathrm{E}+00$ & $3.09 \mathrm{E}+00$ & $3.08 \mathrm{E}+00$ & $3.06 \mathrm{E}+00$ & $3.03 E+00$ & $2.96 \mathrm{E}+00$ & $2.90 \mathrm{E}+00$ \\
\hline FE 59 & $1.30 \mathrm{E}+00$ & $1.29 \mathrm{E}+00$ & $1.29 \mathrm{E}+00$ & $1.28 \mathrm{E}+00$ & $1.16 \mathrm{E}+00$ & $1.04 \mathrm{E}+00$ & $8.16 \mathrm{E}-01$ & $5.14 \mathrm{E}-01$ & $3.24 \mathrm{E}-01$ \\
\hline
\end{tabular}


TEM-10200-1

ENGINEERING CALCULATIONS AND ANALYSIS

Page 30 of 81

$12 / 19 / 17$

Rev.08

Title:

AS-RUN NEUTRONICS EVALUATION FOR THE CSM-10584 EXPERIMENT IN THE ATR

\begin{tabular}{llllllll}
\cline { 2 - 6 } ECAR NO.: & 4496 & REV. NO.: & 1 & PROJECT NO.: & 32501 & Date: & $10 / 19 / 2020$ \\
\hline
\end{tabular}

\begin{tabular}{|c|c|c|c|c|c|c|c|c|c|}
\hline \multicolumn{10}{|c|}{ Capsule A - 54.9 days irradiation } \\
\hline & EOC & $30 \mathrm{MINs}$ & $5 \mathrm{HRS}$ & $1 \mathrm{DAY}$ & 7 DAYS & 14 DAYS & 30 DAYS & 60 DAYS & 90 DAYS \\
\hline ISOTOPE & $\mathrm{Ci}$ & $\mathrm{Ci}$ & $\mathrm{Ci}$ & $\mathrm{Ci}$ & $\mathrm{Ci}$ & $\mathrm{Ci}$ & $\mathrm{Ci}$ & $\mathrm{Ci}$ & $\mathrm{Ci}$ \\
\hline $\mathrm{CO} 58$ & $2.31 \mathrm{E}+00$ & $2.31 \mathrm{E}+00$ & $2.30 \mathrm{E}+00$ & $2.29 \mathrm{E}+00$ & $2.16 \mathrm{E}+00$ & $2.01 \mathrm{E}+00$ & $1.72 \mathrm{E}+00$ & $1.28 \mathrm{E}+00$ & $9.56 \mathrm{E}-01$ \\
\hline $\mathrm{CO} 60$ & $3.17 \mathrm{E}-02$ & $3.17 \mathrm{E}-02$ & $3.17 \mathrm{E}-02$ & $3.17 \mathrm{E}-02$ & $3.16 \mathrm{E}-02$ & $3.15 \mathrm{E}-02$ & $3.14 \mathrm{E}-02$ & $3.10 \mathrm{E}-02$ & $3.07 \mathrm{E}-02$ \\
\hline CO 60M & $8.52 \mathrm{E}-01$ & $1.17 \mathrm{E}-01$ & $2.02 \mathrm{E}-09$ & $0.00 \mathrm{E}+00$ & $0.00 \mathrm{E}+00$ & $0.00 \mathrm{E}+00$ & $0.00 \mathrm{E}+00$ & $0.00 \mathrm{E}+00$ & $0.00 E+00$ \\
\hline CO 61 & $7.14 \mathrm{E}-03$ & $5.79 \mathrm{E}-03$ & $8.74 \mathrm{E}-04$ & $2.99 \mathrm{E}-07$ & $0.00 \mathrm{E}+00$ & $0.00 \mathrm{E}+00$ & $0.00 \mathrm{E}+00$ & $0.00 E+00$ & $0.00 \mathrm{E}+00$ \\
\hline $\mathrm{CO} 62$ & $8.99 \mathrm{E}-04$ & $8.57 \mathrm{E}-10$ & $0.00 \mathrm{E}+00$ & $0.00 \mathrm{E}+00$ & $0.00 \mathrm{E}+00$ & $0.00 \mathrm{E}+00$ & $0.00 \mathrm{E}+00$ & $0.00 \mathrm{E}+00$ & $0.00 \mathrm{E}+00$ \\
\hline $\mathrm{NI} 59$ & $3.25 \mathrm{E}-04$ & $3.25 \mathrm{E}-04$ & $3.25 \mathrm{E}-04$ & $3.25 \mathrm{E}-04$ & $3.25 \mathrm{E}-04$ & $3.25 \mathrm{E}-04$ & $3.25 \mathrm{E}-04$ & $3.25 \mathrm{E}-04$ & $3.25 \mathrm{E}-04$ \\
\hline $\mathrm{NI} 63$ & $4.12 \mathrm{E}-02$ & $4.12 \mathrm{E}-02$ & $4.12 \mathrm{E}-02$ & $4.12 \mathrm{E}-02$ & $4.12 \mathrm{E}-02$ & $4.12 \mathrm{E}-02$ & $4.12 \mathrm{E}-02$ & $4.11 \mathrm{E}-02$ & $4.11 \mathrm{E}-02$ \\
\hline $\mathrm{NI} 65$ & $1.04 \mathrm{E}+00$ & $9.10 \mathrm{E}-01$ & $2.64 \mathrm{E}-01$ & $1.42 \mathrm{E}-03$ & $8.91 \mathrm{E}-21$ & $0.00 \mathrm{E}+00$ & $0.00 \mathrm{E}+00$ & $0.00 \mathrm{E}+00$ & $0.00 \mathrm{E}+00$ \\
\hline NI 66 & $3.61 \mathrm{E}-05$ & $3.59 \mathrm{E}-05$ & $3.39 \mathrm{E}-05$ & $2.66 \mathrm{E}-05$ & $4.28 \mathrm{E}-06$ & 5.07E-07 & $3.88 \mathrm{E}-09$ & $4.17 \mathrm{E}-13$ & $4.48 \mathrm{E}-17$ \\
\hline CU 64 & $1.16 \mathrm{E}+00$ & $1.13 \mathrm{E}+00$ & $8.83 \mathrm{E}-01$ & $3.13 \mathrm{E}-01$ & $1.21 \mathrm{E}-04$ & $1.26 \mathrm{E}-08$ & $9.96 \mathrm{E}-18$ & $0.00 \mathrm{E}+00$ & $0.00 E+00$ \\
\hline CU 66 & $2.52 \mathrm{E}-01$ & $4.30 \mathrm{E}-03$ & 3.39E-05 & $2.66 \mathrm{E}-05$ & $4.28 \mathrm{E}-06$ & $5.08 \mathrm{E}-07$ & $3.88 \mathrm{E}-09$ & 4.17E-13 & $4.48 \mathrm{E}-17$ \\
\hline CU 67 & $1.63 \mathrm{E}-06$ & $1.62 \mathrm{E}-06$ & $1.54 \mathrm{E}-06$ & $1.24 \mathrm{E}-06$ & $2.48 \mathrm{E}-07$ & $3.77 \mathrm{E}-08$ & $5.10 \mathrm{E}-10$ & $1.60 \mathrm{E}-13$ & $5.02 \mathrm{E}-17$ \\
\hline ZN 65 & $1.73 \mathrm{E}-05$ & $1.73 \mathrm{E}-05$ & $1.72 \mathrm{E}-05$ & $1.72 \mathrm{E}-05$ & 1.69E-05 & 1.66E-05 & $1.58 \mathrm{E}-05$ & $1.46 \mathrm{E}-05$ & $1.34 \mathrm{E}-05$ \\
\hline ZN 69 & $1.18 \mathrm{E}-10$ & $8.43 \mathrm{E}-11$ & $9.47 \mathrm{E}-12$ & $2.54 \mathrm{E}-12$ & $1.80 \mathrm{E}-15$ & $3.80 \mathrm{E}-19$ & $0.00 \mathrm{E}+00$ & $0.00 \mathrm{E}+00$ & $0.00 E+00$ \\
\hline ZN 69M & $7.93 \mathrm{E}-12$ & $7.73 \mathrm{E}-12$ & $6.16 \mathrm{E}-12$ & $2.37 \mathrm{E}-12$ & $1.68 \mathrm{E}-15$ & $3.54 \mathrm{E}-19$ & $1.41 \mathrm{E}-27$ & $0.00 \mathrm{E}+00$ & $0.00 E+00$ \\
\hline SR 89 & $8.71 \mathrm{E}-12$ & $8.71 \mathrm{E}-12$ & $8.68 \mathrm{E}-12$ & $8.59 \mathrm{E}-12$ & $7.91 \mathrm{E}-12$ & $7.19 \mathrm{E}-12$ & 5.77E-12 & $3.82 \mathrm{E}-12$ & $2.53 \mathrm{E}-12$ \\
\hline SR 90 & $1.92 \mathrm{E}-14$ & $1.92 \mathrm{E}-14$ & $1.92 \mathrm{E}-14$ & $1.92 \mathrm{E}-14$ & $1.92 \mathrm{E}-14$ & $1.92 \mathrm{E}-14$ & $1.92 \mathrm{E}-14$ & $1.92 \mathrm{E}-14$ & $1.91 \mathrm{E}-14$ \\
\hline SR 91 & $6.09 \mathrm{E}-13$ & 5.87E-13 & $4.23 \mathrm{E}-13$ & $1.06 \mathrm{E}-13$ & $2.89 \mathrm{E}-18$ & $1.37 \mathrm{E}-23$ & $0.00 \mathrm{E}+00$ & $0.00 \mathrm{E}+00$ & $0.00 \mathrm{E}+00$ \\
\hline Y 89M & 9.17E-07 & 9.13E-07 & $8.78 \mathrm{E}-07$ & $7.42 \mathrm{E}-07$ & $2.08 \mathrm{E}-07$ & $4.71 \mathrm{E}-08$ & $1.58 \mathrm{E}-09$ & $2.73 \mathrm{E}-12$ & $4.72 \mathrm{E}-15$ \\
\hline Y 90 & $1.23 \mathrm{E}-05$ & $1.22 \mathrm{E}-05$ & $1.16 \mathrm{E}-05$ & $9.46 \mathrm{E}-06$ & 1.99E-06 & $3.23 \mathrm{E}-07$ & $5.04 \mathrm{E}-09$ & $2.09 \mathrm{E}-12$ & $2.00 \mathrm{E}-14$ \\
\hline Y 90M & $4.70 \mathrm{E}-13$ & $4.21 \mathrm{E}-13$ & $1.54 \mathrm{E}-13$ & $2.13 \mathrm{E}-15$ & $2.22 \mathrm{E}-29$ & $0.00 \mathrm{E}+00$ & $0.00 \mathrm{E}+00$ & $0.00 \mathrm{E}+00$ & $0.00 \mathrm{E}+00$ \\
\hline Y91 & $9.48 \mathrm{E}-10$ & $9.47 \mathrm{E}-10$ & $9.45 \mathrm{E}-10$ & $9.37 \mathrm{E}-10$ & $8.72 \mathrm{E}-10$ & $8.03 \mathrm{E}-10$ & $6.64 \mathrm{E}-10$ & $4.66 \mathrm{E}-10$ & $3.26 \mathrm{E}-10$ \\
\hline Y92 & $7.03 \mathrm{E}-11$ & $6.37 \mathrm{E}-11$ & $2.64 \mathrm{E}-11$ & $6.39 \mathrm{E}-13$ & $3.60 \mathrm{E}-25$ & $0.00 \mathrm{E}+00$ & $0.00 \mathrm{E}+00$ & $0.00 \mathrm{E}+00$ & $0.00 \mathrm{E}+00$ \\
\hline Y 94 & $4.34 \mathrm{E}-13$ & $1.46 \mathrm{E}-13$ & $8.12 \mathrm{E}-18$ & $0.00 E+00$ & $0.00 \mathrm{E}+00$ & $0.00 \mathrm{E}+00$ & $0.00 \mathrm{E}+00$ & $0.00 \mathrm{E}+00$ & $0.00 \mathrm{E}+00$ \\
\hline ZR 89 & $9.19 \mathrm{E}-07$ & $9.15 \mathrm{E}-07$ & $8.79 \mathrm{E}-07$ & $7.43 \mathrm{E}-07$ & $2.08 \mathrm{E}-07$ & $4.72 \mathrm{E}-08$ & $1.59 \mathrm{E}-09$ & $2.74 \mathrm{E}-12$ & $4.72 \mathrm{E}-15$ \\
\hline ZR 93 & $8.54 \mathrm{E}-12$ & $8.54 \mathrm{E}-12$ & $8.54 \mathrm{E}-12$ & $8.54 \mathrm{E}-12$ & $8.54 \mathrm{E}-12$ & $8.54 \mathrm{E}-12$ & $8.54 \mathrm{E}-12$ & $8.54 \mathrm{E}-12$ & $8.54 \mathrm{E}-12$ \\
\hline ZR 95 & $1.42 \mathrm{E}-06$ & $1.42 \mathrm{E}-06$ & $1.42 \mathrm{E}-06$ & $1.41 \mathrm{E}-06$ & $1.32 \mathrm{E}-06$ & $1.22 \mathrm{E}-06$ & $1.03 \mathrm{E}-06$ & $7.42 \mathrm{E}-07$ & $5.36 \mathrm{E}-07$ \\
\hline ZR 97 & $7.27 \mathrm{E}-08$ & $7.12 \mathrm{E}-08$ & $5.92 \mathrm{E}-08$ & $2.72 \mathrm{E}-08$ & $7.41 \mathrm{E}-11$ & $7.50 \mathrm{E}-14$ & $1.08 \mathrm{E}-20$ & $0.00 \mathrm{E}+00$ & $0.00 \mathrm{E}+00$ \\
\hline NB 92 & 1.79E-04 & $1.78 \mathrm{E}-04$ & $1.76 \mathrm{E}-04$ & 1.67E-04 & 1.11E-04 & 6.87E-05 & $2.31 \mathrm{E}-05$ & $2.98 \mathrm{E}-06$ & $3.85 \mathrm{E}-07$ \\
\hline NB 93M & $3.10 \mathrm{E}-14$ & $3.10 \mathrm{E}-14$ & $3.13 \mathrm{E}-14$ & $3.22 \mathrm{E}-14$ & $3.89 \mathrm{E}-14$ & $4.68 \mathrm{E}-14$ & $6.48 \mathrm{E}-14$ & $9.84 \mathrm{E}-14$ & $1.32 \mathrm{E}-13$ \\
\hline NB 94 & $1.38 \mathrm{E}-06$ & $1.38 \mathrm{E}-06$ & $1.38 \mathrm{E}-06$ & $1.38 \mathrm{E}-06$ & $1.38 \mathrm{E}-06$ & $1.38 \mathrm{E}-06$ & $1.38 \mathrm{E}-06$ & $1.38 \mathrm{E}-06$ & $1.38 \mathrm{E}-06$ \\
\hline NB 95 & $2.51 \mathrm{E}-03$ & $2.51 \mathrm{E}-03$ & $2.50 \mathrm{E}-03$ & $2.46 \mathrm{E}-03$ & $2.19 \mathrm{E}-03$ & $1.91 \mathrm{E}-03$ & $1.39 \mathrm{E}-03$ & $7.70 \mathrm{E}-04$ & 4.27E-04 \\
\hline NB 95M & $9.22 \mathrm{E}-09$ & $9.22 \mathrm{E}-09$ & $9.25 \mathrm{E}-09$ & $9.34 \mathrm{E}-09$ & $9.43 \mathrm{E}-09$ & 8.97E-09 & 7.61E-09 & $5.51 \mathrm{E}-09$ & $3.98 \mathrm{E}-09$ \\
\hline NB 96 & $1.76 \mathrm{E}-05$ & 1.73E-05 & $1.51 \mathrm{E}-05$ & $8.62 \mathrm{E}-06$ & $1.20 \mathrm{E}-07$ & $8.19 \mathrm{E}-10$ & 9.17E-15 & 4.79E-24 & $0.00 \mathrm{E}+00$ \\
\hline NB 97 & $2.57 \mathrm{E}-06$ & $1.94 \mathrm{E}-06$ & $2.03 \mathrm{E}-07$ & $2.73 \mathrm{E}-08$ & $7.45 \mathrm{E}-11$ & $7.58 \mathrm{E}-14$ & 1.17E-20 & $0.00 \mathrm{E}+00$ & $0.00 \mathrm{E}+00$ \\
\hline NB 97M & $6.88 \mathrm{E}-08$ & $6.74 \mathrm{E}-08$ & $5.61 \mathrm{E}-08$ & 2.57E-08 & $7.02 \mathrm{E}-11$ & 7.14E-14 & $1.03 \mathrm{E}-20$ & $0.00 \mathrm{E}+00$ & $0.00 \mathrm{E}+00$ \\
\hline NB 98 & $3.84 \mathrm{E}-07$ & $0.00 \mathrm{E}+00$ & $0.00 \mathrm{E}+00$ & $0.00 \mathrm{E}+00$ & $0.00 \mathrm{E}+00$ & $0.00 \mathrm{E}+00$ & $0.00 \mathrm{E}+00$ & $0.00 \mathrm{E}+00$ & $0.00 \mathrm{E}+00$ \\
\hline NB100 & $1.91 \mathrm{E}-08$ & $0.00 \mathrm{E}+00$ & $0.00 \mathrm{E}+00$ & $0.00 \mathrm{E}+00$ & $0.00 \mathrm{E}+00$ & $0.00 \mathrm{E}+00$ & $0.00 \mathrm{E}+00$ & $0.00 \mathrm{E}+00$ & $0.00 E+00$ \\
\hline MO 93M & $5.31 \mathrm{E}-05$ & $5.05 \mathrm{E}-05$ & $3.20 \mathrm{E}-05$ & $4.68 \mathrm{E}-06$ & $2.20 \mathrm{E}-12$ & $9.11 \mathrm{E}-20$ & $0.00 \mathrm{E}+00$ & $0.00 \mathrm{E}+00$ & $0.00 \mathrm{E}+00$ \\
\hline MO 93 & 4.87E-08 & $4.87 \mathrm{E}-08$ & $4.87 \mathrm{E}-08$ & $4.87 \mathrm{E}-08$ & 4.87E-08 & 4.87E-08 & 4.87E-08 & $4.87 \mathrm{E}-08$ & $4.87 \mathrm{E}-08$ \\
\hline MO 99 & $3.72 \mathrm{E}-02$ & $3.70 \mathrm{E}-02$ & $3.53 \mathrm{E}-02$ & $2.89 \mathrm{E}-02$ & $6.36 \mathrm{E}-03$ & $1.09 \mathrm{E}-03$ & $1.93 \mathrm{E}-05$ & $1.01 \mathrm{E}-08$ & $5.23 \mathrm{E}-12$ \\
\hline MO101 & $9.25 \mathrm{E}-03$ & $2.23 \mathrm{E}-03$ & $6.15 E-09$ & $0.00 E+00$ & $0.00 E+00$ & $0.00 E+00$ & $0.00 \mathrm{E}+00$ & $0.00 E+00$ & $0.00 E+00$ \\
\hline TC 99 & $2.05 \mathrm{E}-09$ & $2.05 \mathrm{E}-09$ & $2.06 \mathrm{E}-09$ & $2.09 \mathrm{E}-09$ & $2.19 \mathrm{E}-09$ & $2.21 \mathrm{E}-09$ & $2.21 \mathrm{E}-09$ & $2.21 \mathrm{E}-09$ & $2.21 \mathrm{E}-09$ \\
\hline TC100 & $2.14 \mathrm{E}-04$ & $0.00 E+00$ & $0.00 E+00$ & $0.00 E+00$ & $0.00 E+00$ & $0.00 \mathrm{E}+00$ & $0.00 \mathrm{E}+00$ & $0.00 E+00$ & $0.00 E+00$ \\
\hline TC101 & $9.25 \mathrm{E}-03$ & $5.34 \mathrm{E}-03$ & $7.76 \mathrm{E}-08$ & $0.00 \mathrm{E}+00$ & $0.00 \mathrm{E}+00$ & $0.00 \mathrm{E}+00$ & $0.00 \mathrm{E}+00$ & $0.00 \mathrm{E}+00$ & $0.00 E+00$ \\
\hline RU103 & $2.86 \mathrm{E}-08$ & $2.86 \mathrm{E}-08$ & $2.85 \mathrm{E}-08$ & $2.81 \mathrm{E}-08$ & $2.53 \mathrm{E}-08$ & $2.24 \mathrm{E}-08$ & $1.69 \mathrm{E}-08$ & $9.94 \mathrm{E}-09$ & $5.85 \mathrm{E}-09$ \\
\hline RU105 & $1.69 \mathrm{E}-13$ & $1.57 \mathrm{E}-13$ & $7.76 \mathrm{E}-14$ & $4.01 \mathrm{E}-15$ & $6.87 \mathrm{E}-25$ & $0.00 \mathrm{E}+00$ & $0.00 \mathrm{E}+00$ & $0.00 \mathrm{E}+00$ & $0.00 E+00$ \\
\hline RH104 & $1.58 \mathrm{E}-10$ & $1.13 \mathrm{E}-13$ & $0.00 E+00$ & $0.00 E+00$ & $0.00 E+00$ & $0.00 \mathrm{E}+00$ & $0.00 \mathrm{E}+00$ & $0.00 E+00$ & $0.00 E+00$ \\
\hline RH104M & $1.15 \mathrm{E}-11$ & $9.25 \mathrm{E}-14$ & $0.00 \mathrm{E}+00$ & $0.00 \mathrm{E}+00$ & $0.00 \mathrm{E}+00$ & $0.00 \mathrm{E}+00$ & $0.00 \mathrm{E}+00$ & $0.00 \mathrm{E}+00$ & $0.00 E+00$ \\
\hline RH105 & $1.28 \mathrm{E}-13$ & $1.28 \mathrm{E}-13$ & $1.27 \mathrm{E}-13$ & $9.45 \mathrm{E}-14$ & $5.65 \mathrm{E}-15$ & $2.14 \mathrm{E}-16$ & $1.15 \mathrm{E}-19$ & $8.54 \mathrm{E}-26$ & $0.00 E+00$ \\
\hline SUMTOT & $2.94 \mathrm{E}+02$ & $1.14 \mathrm{E}+02$ & $1.04 \mathrm{E}+02$ & $9.69 \mathrm{E}+01$ & $8.35 \mathrm{E}+01$ & $7.09 \mathrm{E}+01$ & $4.93 \mathrm{E}+01$ & $2.58 \mathrm{E}+01$ & $1.45 \mathrm{E}+01$ \\
\hline OTOTAL & $2.94 \mathrm{E}+02$ & $1.14 \mathrm{E}+02$ & $1.04 \mathrm{E}+02$ & $9.69 \mathrm{E}+01$ & $8.35 \mathrm{E}+01$ & $7.09 \mathrm{E}+01$ & $4.93 \mathrm{E}+01$ & $2.58 \mathrm{E}+01$ & $1.45 \mathrm{E}+01$ \\
\hline
\end{tabular}


TEM-10200-1

ENGINEERING CALCULATIONS AND ANALYSIS

Page 31 of 81

$12 / 19 / 17$

Rev.08

Title:

AS-RUN NEUTRONICS EVALUATION FOR THE CSM-10584 EXPERIMENT IN THE ATR

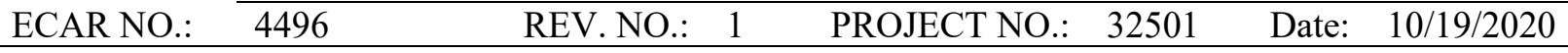

Table 14. Radionuclide source term for CSM capsule B after 119 days of irradiation (Ci).

\begin{tabular}{|c|c|c|c|c|c|c|c|c|c|}
\hline \multicolumn{10}{|c|}{ Capsule B - 119 days irradiation } \\
\hline & EOC & $30 \mathrm{MINs}$ & $5 \mathrm{HRS}$ & 1 DAY & 7 DAYS & 14 DAYS & 30 DAYS & 60 DAYS & 90 DAYS \\
\hline ISOTOPE & $\mathrm{Ci}$ & $\mathrm{Ci}$ & $\mathrm{Ci}$ & $\mathrm{Ci}$ & $\mathrm{Ci}$ & $\mathrm{Ci}$ & $\mathrm{Ci}$ & $\mathrm{Ci}$ & $\mathrm{Ci}$ \\
\hline H 3 & $6.60 \mathrm{E}-08$ & $6.60 \mathrm{E}-08$ & $6.60 \mathrm{E}-08$ & $6.60 \mathrm{E}-08$ & $6.59 \mathrm{E}-08$ & $6.58 \mathrm{E}-08$ & 6.57E-08 & $6.54 \mathrm{E}-08$ & $6.51 \mathrm{E}-08$ \\
\hline $\mathrm{H} 4$ & $8.40 \mathrm{E}-05$ & $0.00 \mathrm{E}+00$ & $0.00 \mathrm{E}+00$ & $0.00 \mathrm{E}+00$ & $0.00 \mathrm{E}+00$ & $0.00 \mathrm{E}+00$ & $0.00 \mathrm{E}+00$ & $0.00 \mathrm{E}+00$ & $0.00 \mathrm{E}+00$ \\
\hline HE 6 & $2.72 \mathrm{E}-05$ & $0.00 \mathrm{E}+00$ & $0.00 \mathrm{E}+00$ & $0.00 \mathrm{E}+00$ & $0.00 \mathrm{E}+00$ & $0.00 \mathrm{E}+00$ & $0.00 \mathrm{E}+00$ & $0.00 \mathrm{E}+00$ & $0.00 \mathrm{E}+00$ \\
\hline LI 8 & $3.38 \mathrm{E}-05$ & $0.00 \mathrm{E}+00$ & $0.00 \mathrm{E}+00$ & $0.00 \mathrm{E}+00$ & $0.00 \mathrm{E}+00$ & $0.00 \mathrm{E}+00$ & $0.00 \mathrm{E}+00$ & $0.00 \mathrm{E}+00$ & $0.00 \mathrm{E}+00$ \\
\hline BE 8 & $8.07 \mathrm{E}-05$ & $0.00 \mathrm{E}+00$ & $0.00 \mathrm{E}+00$ & $0.00 \mathrm{E}+00$ & $0.00 \mathrm{E}+00$ & $0.00 \mathrm{E}+00$ & $0.00 \mathrm{E}+00$ & $0.00 \mathrm{E}+00$ & $0.00 \mathrm{E}+00$ \\
\hline BE 10 & $1.91 \mathrm{E}-10$ & $1.91 \mathrm{E}-10$ & $1.91 \mathrm{E}-10$ & $1.91 \mathrm{E}-10$ & $1.91 \mathrm{E}-10$ & $1.91 \mathrm{E}-10$ & $1.91 \mathrm{E}-10$ & $1.91 \mathrm{E}-10$ & $1.91 \mathrm{E}-10$ \\
\hline BE 11 & $1.51 \mathrm{E}-09$ & $0.00 \mathrm{E}+00$ & $0.00 \mathrm{E}+00$ & $0.00 \mathrm{E}+00$ & $0.00 \mathrm{E}+00$ & $0.00 \mathrm{E}+00$ & $0.00 \mathrm{E}+00$ & $0.00 \mathrm{E}+00$ & $0.00 \mathrm{E}+00$ \\
\hline B 12 & $2.29 \mathrm{E}-05$ & $0.00 \mathrm{E}+00$ & $0.00 \mathrm{E}+00$ & $0.00 \mathrm{E}+00$ & $0.00 \mathrm{E}+00$ & $0.00 \mathrm{E}+00$ & $0.00 \mathrm{E}+00$ & $0.00 \mathrm{E}+00$ & $0.00 \mathrm{E}+00$ \\
\hline C 14 & $6.99 \mathrm{E}-09$ & $6.99 \mathrm{E}-09$ & $6.99 \mathrm{E}-09$ & $6.99 \mathrm{E}-09$ & $6.99 \mathrm{E}-09$ & $6.99 \mathrm{E}-09$ & $6.99 \mathrm{E}-09$ & $6.99 \mathrm{E}-09$ & $6.99 \mathrm{E}-09$ \\
\hline C 15 & $1.58 \mathrm{E}-10$ & $0.00 \mathrm{E}+00$ & $0.00 \mathrm{E}+00$ & $0.00 \mathrm{E}+00$ & $0.00 \mathrm{E}+00$ & $0.00 \mathrm{E}+00$ & $0.00 \mathrm{E}+00$ & $0.00 \mathrm{E}+00$ & $0.00 \mathrm{E}+00$ \\
\hline O 19 & $1.13 \mathrm{E}-11$ & $0.00 \mathrm{E}+00$ & $0.00 \mathrm{E}+00$ & $0.00 \mathrm{E}+00$ & $0.00 \mathrm{E}+00$ & $0.00 \mathrm{E}+00$ & $0.00 \mathrm{E}+00$ & $0.00 \mathrm{E}+00$ & $0.00 \mathrm{E}+00$ \\
\hline $\mathrm{F} 20$ & $1.96 \mathrm{E}-08$ & $0.00 \mathrm{E}+00$ & $0.00 \mathrm{E}+00$ & $0.00 \mathrm{E}+00$ & $0.00 \mathrm{E}+00$ & $0.00 \mathrm{E}+00$ & $0.00 \mathrm{E}+00$ & $0.00 \mathrm{E}+00$ & $0.00 \mathrm{E}+00$ \\
\hline NE 23 & $9.41 \mathrm{E}-03$ & $2.65 \mathrm{E}-17$ & $0.00 \mathrm{E}+00$ & $0.00 \mathrm{E}+00$ & $0.00 \mathrm{E}+00$ & $0.00 \mathrm{E}+00$ & $0.00 \mathrm{E}+00$ & $0.00 \mathrm{E}+00$ & $0.00 \mathrm{E}+00$ \\
\hline NA 24 & $1.50 \mathrm{E}+00$ & $1.46 \mathrm{E}+00$ & $1.19 \mathrm{E}+00$ & 4.93E-01 & $6.39 \mathrm{E}-04$ & $2.72 \mathrm{E}-07$ & $5.34 \mathrm{E}-15$ & $1.90 \mathrm{E}-29$ & $0.00 \mathrm{E}+00$ \\
\hline NA 24M & 4.35E-06 & $0.00 \mathrm{E}+00$ & $0.00 \mathrm{E}+00$ & $0.00 \mathrm{E}+00$ & $0.00 \mathrm{E}+00$ & $0.00 \mathrm{E}+00$ & $0.00 \mathrm{E}+00$ & $0.00 \mathrm{E}+00$ & $0.00 E+00$ \\
\hline NA 25 & $5.68 \mathrm{E}-03$ & 4.60E-12 & $0.00 \mathrm{E}+00$ & $0.00 \mathrm{E}+00$ & $0.00 \mathrm{E}+00$ & $0.00 \mathrm{E}+00$ & $0.00 \mathrm{E}+00$ & $0.00 \mathrm{E}+00$ & $0.00 \mathrm{E}+00$ \\
\hline MG 27 & $7.05 E+00$ & 7.83E-01 & $2.01 \mathrm{E}-09$ & $0.00 E+00$ & $0.00 \mathrm{E}+00$ & $0.00 \mathrm{E}+00$ & $0.00 \mathrm{E}+00$ & $0.00 \mathrm{E}+00$ & $0.00 \mathrm{E}+00$ \\
\hline MG 28 & $2.51 \mathrm{E}-08$ & $2.46 \mathrm{E}-08$ & $2.12 \mathrm{E}-08$ & $1.13 \mathrm{E}-08$ & $9.56 \mathrm{E}-11$ & $3.65 \mathrm{E}-13$ & $1.08 \mathrm{E}-18$ & 4.67E-29 & $0.00 \mathrm{E}+00$ \\
\hline AL 28 & $1.68 \mathrm{E}+02$ & $1.56 \mathrm{E}-02$ & $2.13 \mathrm{E}-08$ & $1.13 \mathrm{E}-08$ & $9.58 \mathrm{E}-11$ & $3.66 \mathrm{E}-13$ & $1.08 \mathrm{E}-18$ & $0.00 \mathrm{E}+00$ & $0.00 \mathrm{E}+00$ \\
\hline AL 29 & $1.78 \mathrm{E}-03$ & $7.32 \mathrm{E}-05$ & $2.51 \mathrm{E}-17$ & $0.00 \mathrm{E}+00$ & $0.00 \mathrm{E}+00$ & $0.00 \mathrm{E}+00$ & $0.00 \mathrm{E}+00$ & $0.00 \mathrm{E}+00$ & $0.00 \mathrm{E}+00$ \\
\hline AL 30 & $1.72 \mathrm{E}-06$ & $0.00 \mathrm{E}+00$ & $0.00 \mathrm{E}+00$ & $0.00 \mathrm{E}+00$ & $0.00 \mathrm{E}+00$ & $0.00 \mathrm{E}+00$ & $0.00 \mathrm{E}+00$ & $0.00 \mathrm{E}+00$ & $0.00 \mathrm{E}+00$ \\
\hline SI 31 & $1.72 \mathrm{E}-02$ & $1.51 \mathrm{E}-02$ & 4.59E-03 & $3.02 \mathrm{E}-05$ & $8.82 \mathrm{E}-22$ & $0.00 \mathrm{E}+00$ & $0.00 \mathrm{E}+00$ & $0.00 \mathrm{E}+00$ & $0.00 \mathrm{E}+00$ \\
\hline SI 32 & $4.24 \mathrm{E}-12$ & $4.24 \mathrm{E}-12$ & $4.24 \mathrm{E}-12$ & $4.24 \mathrm{E}-12$ & $4.24 \mathrm{E}-12$ & $4.24 \mathrm{E}-12$ & $4.24 \mathrm{E}-12$ & $4.24 \mathrm{E}-12$ & 4.24E-12 \\
\hline P 32 & 3.95E-03 & $3.95 \mathrm{E}-03$ & $3.91 \mathrm{E}-03$ & $3.76 \mathrm{E}-03$ & $2.81 \mathrm{E}-03$ & $2.00 \mathrm{E}-03$ & $9.22 \mathrm{E}-04$ & $2.16 \mathrm{E}-04$ & 5.03E-05 \\
\hline P 33 & $1.46 \mathrm{E}-07$ & $1.46 \mathrm{E}-07$ & 1.45E-07 & $1.42 \mathrm{E}-07$ & $1.20 \mathrm{E}-07$ & $9.89 \mathrm{E}-08$ & $6.35 \mathrm{E}-08$ & $2.76 \mathrm{E}-08$ & $1.20 \mathrm{E}-08$ \\
\hline P 34 & $8.45 \mathrm{E}-07$ & $0.00 \mathrm{E}+00$ & $0.00 \mathrm{E}+00$ & $0.00 \mathrm{E}+00$ & $0.00 \mathrm{E}+00$ & $0.00 \mathrm{E}+00$ & $0.00 \mathrm{E}+00$ & $0.00 \mathrm{E}+00$ & $0.00 \mathrm{E}+00$ \\
\hline S 35 & $5.89 \mathrm{E}-05$ & $5.88 \mathrm{E}-05$ & $5.88 \mathrm{E}-05$ & $5.84 \mathrm{E}-05$ & $5.57 \mathrm{E}-05$ & $5.27 \mathrm{E}-05$ & $4.65 \mathrm{E}-05$ & 3.67E-05 & $2.90 \mathrm{E}-05$ \\
\hline S 37 & $2.87 \mathrm{E}-07$ & $4.72 \mathrm{E}-09$ & $4.08 \mathrm{E}-25$ & $0.00 \mathrm{E}+00$ & $0.00 \mathrm{E}+00$ & $0.00 \mathrm{E}+00$ & $0.00 \mathrm{E}+00$ & $0.00 \mathrm{E}+00$ & $0.00 \mathrm{E}+00$ \\
\hline CL 36 & 4.04E-11 & 4.04E-11 & 4.04E-11 & $4.04 \mathrm{E}-11$ & 4.04E-11 & 4.04E-11 & 4.04E-11 & 4.04E-11 & 4.04E-11 \\
\hline CL 38 & $4.98 \mathrm{E}-10$ & $2.85 \mathrm{E}-10$ & $1.86 \mathrm{E}-12$ & $1.12 \mathrm{E}-21$ & $0.00 \mathrm{E}+00$ & $0.00 \mathrm{E}+00$ & $0.00 \mathrm{E}+00$ & $0.00 \mathrm{E}+00$ & $0.00 \mathrm{E}+00$ \\
\hline $\mathrm{CL} 38 \mathrm{M}$ & $5.92 \mathrm{E}-12$ & $0.00 \mathrm{E}+00$ & $0.00 \mathrm{E}+00$ & $0.00 \mathrm{E}+00$ & $0.00 \mathrm{E}+00$ & $0.00 \mathrm{E}+00$ & $0.00 \mathrm{E}+00$ & $0.00 \mathrm{E}+00$ & $0.00 \mathrm{E}+00$ \\
\hline AR 39 & $1.28 \mathrm{E}-07$ & $1.28 \mathrm{E}-07$ & $1.28 \mathrm{E}-07$ & $1.28 \mathrm{E}-07$ & $1.28 \mathrm{E}-07$ & $1.28 \mathrm{E}-07$ & $1.28 \mathrm{E}-07$ & $1.28 \mathrm{E}-07$ & $1.28 \mathrm{E}-07$ \\
\hline AR 41 & 4.73E-08 & $3.92 \mathrm{E}-08$ & 7.10E-09 & $5.26 \mathrm{E}-12$ & $0.00 \mathrm{E}+00$ & $0.00 \mathrm{E}+00$ & $0.00 \mathrm{E}+00$ & $0.00 \mathrm{E}+00$ & $0.00 \mathrm{E}+00$ \\
\hline K 40 & $3.15 \mathrm{E}-13$ & $3.15 \mathrm{E}-13$ & $3.15 \mathrm{E}-13$ & $3.15 \mathrm{E}-13$ & $3.15 \mathrm{E}-13$ & $3.15 \mathrm{E}-13$ & $3.15 \mathrm{E}-13$ & $3.15 \mathrm{E}-13$ & $3.15 \mathrm{E}-13$ \\
\hline K 42 & $1.05 \mathrm{E}-04$ & $1.02 \mathrm{E}-04$ & $7.94 \mathrm{E}-05$ & $2.73 \mathrm{E}-05$ & $8.51 \mathrm{E}-09$ & $6.90 \mathrm{E}-13$ & $5.83 \mathrm{E}-17$ & $5.82 \mathrm{E}-17$ & $5.81 \mathrm{E}-17$ \\
\hline K 43 & $1.10 \mathrm{E}-10$ & $1.08 \mathrm{E}-10$ & $9.45 \mathrm{E}-11$ & $5.27 \mathrm{E}-11$ & $6.37 \mathrm{E}-13$ & $3.69 \mathrm{E}-15$ & $2.84 \mathrm{E}-20$ & $7.28 \mathrm{E}-30$ & $0.00 \mathrm{E}+00$ \\
\hline K 44 & $1.95 \mathrm{E}-11$ & $7.58 \mathrm{E}-12$ & $1.53 \mathrm{E}-15$ & $0.00 \mathrm{E}+00$ & $0.00 \mathrm{E}+00$ & $0.00 \mathrm{E}+00$ & $0.00 \mathrm{E}+00$ & $0.00 \mathrm{E}+00$ & $0.00 \mathrm{E}+00$ \\
\hline CA 45 & $5.31 \mathrm{E}-07$ & $5.31 \mathrm{E}-07$ & $5.30 \mathrm{E}-07$ & $5.28 \mathrm{E}-07$ & $5.15 \mathrm{E}-07$ & $5.00 \mathrm{E}-07$ & 4.67E-07 & $4.11 \mathrm{E}-07$ & $3.62 \mathrm{E}-07$ \\
\hline CA 47 & 9.08E-09 & 9.05E-09 & $8.80 \mathrm{E}-09$ & $7.80 \mathrm{E}-09$ & $3.12 \mathrm{E}-09$ & $1.07 \mathrm{E}-09$ & $9.27 \mathrm{E}-11$ & $9.47 \mathrm{E}-13$ & $9.67 \mathrm{E}-15$ \\
\hline SC 46 & $1.06 \mathrm{E}-04$ & $1.06 \mathrm{E}-04$ & $1.05 \mathrm{E}-04$ & $1.05 \mathrm{E}-04$ & $9.96 \mathrm{E}-05$ & $9.40 \mathrm{E}-05$ & $8.24 \mathrm{E}-05$ & $6.43 \mathrm{E}-05$ & $5.01 \mathrm{E}-05$ \\
\hline SC 46M & $3.00 \mathrm{E}-09$ & $0.00 \mathrm{E}+00$ & $0.00 \mathrm{E}+00$ & $0.00 \mathrm{E}+00$ & $0.00 \mathrm{E}+00$ & $0.00 \mathrm{E}+00$ & $0.00 \mathrm{E}+00$ & $0.00 \mathrm{E}+00$ & $0.00 \mathrm{E}+00$ \\
\hline SC 47 & $2.64 \mathrm{E}-04$ & $2.63 \mathrm{E}-04$ & $2.53 \mathrm{E}-04$ & $2.14 \mathrm{E}-04$ & $6.20 \mathrm{E}-05$ & $1.46 \mathrm{E}-05$ & $5.32 \mathrm{E}-07$ & $1.08 \mathrm{E}-09$ & $2.20 \mathrm{E}-12$ \\
\hline SC 48 & $2.56 \mathrm{E}-05$ & $2.54 \mathrm{E}-05$ & $2.37 \mathrm{E}-05$ & $1.75 \mathrm{E}-05$ & 1.79E-06 & $1.26 \mathrm{E}-07$ & $2.88 \mathrm{E}-10$ & $3.25 \mathrm{E}-15$ & 3.67E-20 \\
\hline SC 49 & $2.25 \mathrm{E}-05$ & 1.57E-05 & $6.06 \mathrm{E}-07$ & $6.51 \mathrm{E}-13$ & $0.00 \mathrm{E}+00$ & $0.00 \mathrm{E}+00$ & $0.00 \mathrm{E}+00$ & $0.00 \mathrm{E}+00$ & $0.00 \mathrm{E}+00$ \\
\hline SC 50 & $4.48 \mathrm{E}-07$ & $2.31 \mathrm{E}-12$ & $0.00 \mathrm{E}+00$ & $0.00 \mathrm{E}+00$ & $0.00 \mathrm{E}+00$ & $0.00 \mathrm{E}+00$ & $0.00 \mathrm{E}+00$ & $0.00 \mathrm{E}+00$ & $0.00 \mathrm{E}+00$ \\
\hline TI 51 & $1.22 \mathrm{E}-03$ & $3.31 \mathrm{E}-05$ & $2.56 \mathrm{E}-19$ & $0.00 \mathrm{E}+00$ & $0.00 \mathrm{E}+00$ & $0.00 \mathrm{E}+00$ & $0.00 \mathrm{E}+00$ & $0.00 E+00$ & $0.00 \mathrm{E}+00$ \\
\hline V 52 & $1.21 \mathrm{E}+00$ & $4.72 \mathrm{E}-03$ & $1.00 \mathrm{E}-24$ & $0.00 \mathrm{E}+00$ & $0.00 \mathrm{E}+00$ & $0.00 \mathrm{E}+00$ & $0.00 \mathrm{E}+00$ & $0.00 \mathrm{E}+00$ & $0.00 \mathrm{E}+00$ \\
\hline V 53 & 5.57E-03 & 1.37E-08 & $0.00 \mathrm{E}+00$ & $0.00 \mathrm{E}+00$ & $0.00 \mathrm{E}+00$ & $0.00 \mathrm{E}+00$ & $0.00 \mathrm{E}+00$ & $0.00 \mathrm{E}+00$ & $0.00 \mathrm{E}+00$ \\
\hline V 54 & $5.96 \mathrm{E}-05$ & $8.38 \mathrm{E}-15$ & $0.00 \mathrm{E}+00$ & $0.00 \mathrm{E}+00$ & $0.00 \mathrm{E}+00$ & $0.00 \mathrm{E}+00$ & $0.00 \mathrm{E}+00$ & $0.00 \mathrm{E}+00$ & $0.00 \mathrm{E}+00$ \\
\hline CR 51 & $1.14 \mathrm{E}+02$ & $1.14 \mathrm{E}+02$ & $1.14 \mathrm{E}+02$ & $1.12 \mathrm{E}+02$ & $9.60 \mathrm{E}+01$ & $8.06 \mathrm{E}+01$ & $5.40 \mathrm{E}+01$ & $2.55 \mathrm{E}+01$ & $1.20 \mathrm{E}+01$ \\
\hline CR 55 & $1.63 \mathrm{E}+00$ & $4.66 \mathrm{E}-03$ & $0.00 \mathrm{E}+00$ & $0.00 \mathrm{E}+00$ & $0.00 \mathrm{E}+00$ & $0.00 \mathrm{E}+00$ & $0.00 \mathrm{E}+00$ & $0.00 \mathrm{E}+00$ & $0.00 \mathrm{E}+00$ \\
\hline MN 54 & $1.73 E+00$ & $1.73 \mathrm{E}+00$ & $1.72 \mathrm{E}+00$ & $1.72 \mathrm{E}+00$ & $1.70 \mathrm{E}+00$ & $1.67 \mathrm{E}+00$ & $1.61 \mathrm{E}+00$ & $1.51 \mathrm{E}+00$ & $1.41 \mathrm{E}+00$ \\
\hline MN 56 & $1.27 \mathrm{E}+01$ & $1.11 \mathrm{E}+01$ & $3.32 \mathrm{E}+00$ & $2.01 \mathrm{E}-02$ & $3.11 \mathrm{E}-19$ & $0.00 \mathrm{E}+00$ & $0.00 \mathrm{E}+00$ & $0.00 \mathrm{E}+00$ & $0.00 \mathrm{E}+00$ \\
\hline
\end{tabular}


TEM-10200-1

ENGINEERING CALCULATIONS AND ANALYSIS

Page 32 of 81

$12 / 19 / 17$

Rev.08

Title:

AS-RUN NEUTRONICS EVALUATION FOR THE CSM-10584 EXPERIMENT IN THE ATR

\begin{tabular}{llllllll} 
ECAR NO.: & 4496 & REV. NO.: & 1 & PROJECT NO.: & 32501 & Date: & 10/19/2020 \\
\hline
\end{tabular}

\begin{tabular}{|c|c|c|c|c|c|c|c|c|c|}
\hline \multicolumn{10}{|c|}{ Capsule B - 119 days irradiation } \\
\hline & EOC & $30 \mathrm{MINs}$ & $5 \mathrm{HRS}$ & $1 \mathrm{DAY}$ & 7 DAYS & 14 DAYS & 30 DAYS & 60 DAYS & 90 DAYS \\
\hline ISOTOPE & $\mathrm{Ci}$ & $\mathrm{Ci}$ & $\mathrm{Ci}$ & $\mathrm{Ci}$ & $\mathrm{Ci}$ & $\mathrm{Ci}$ & $\mathrm{Ci}$ & $\mathrm{Ci}$ & $\mathrm{Ci}$ \\
\hline MN 57 & $1.91 \mathrm{E}-02$ & $4.70 \mathrm{E}-08$ & $0.00 \mathrm{E}+00$ & $0.00 \mathrm{E}+00$ & $0.00 \mathrm{E}+00$ & $0.00 \mathrm{E}+00$ & $0.00 \mathrm{E}+00$ & $0.00 \mathrm{E}+00$ & $0.00 \mathrm{E}+00$ \\
\hline MN 58 & $5.78 \mathrm{E}-05$ & $2.91 \mathrm{E}-13$ & $0.00 \mathrm{E}+00$ & $0.00 \mathrm{E}+00$ & $0.00 \mathrm{E}+00$ & $0.00 \mathrm{E}+00$ & $0.00 \mathrm{E}+00$ & $0.00 \mathrm{E}+00$ & $0.00 \mathrm{E}+00$ \\
\hline FE 55 & $6.55 \mathrm{E}+00$ & $6.55 \mathrm{E}+00$ & $6.55 \mathrm{E}+00$ & $6.55 \mathrm{E}+00$ & $6.52 \mathrm{E}+00$ & $6.49 \mathrm{E}+00$ & $6.41 \mathrm{E}+00$ & $6.27 E+00$ & $6.14 \mathrm{E}+00$ \\
\hline FE 59 & $1.92 \mathrm{E}+00$ & $1.92 \mathrm{E}+00$ & $1.92 \mathrm{E}+00$ & $1.89 \mathrm{E}+00$ & $1.73 \mathrm{E}+00$ & $1.55 \mathrm{E}+00$ & $1.21 \mathrm{E}+00$ & $7.63 \mathrm{E}-01$ & $4.80 \mathrm{E}-01$ \\
\hline $\mathrm{CO} 58$ & $2.53 \mathrm{E}+00$ & $2.53 \mathrm{E}+00$ & $2.53 \mathrm{E}+00$ & $2.51 \mathrm{E}+00$ & $2.36 \mathrm{E}+00$ & $2.21 \mathrm{E}+00$ & $1.89 \mathrm{E}+00$ & $1.41 \mathrm{E}+00$ & $1.05 \mathrm{E}+00$ \\
\hline $\mathrm{CO} 60$ & $7.25 \mathrm{E}-02$ & $7.26 \mathrm{E}-02$ & $7.25 \mathrm{E}-02$ & $7.25 \mathrm{E}-02$ & $7.24 \mathrm{E}-02$ & $7.22 \mathrm{E}-02$ & $7.18 \mathrm{E}-02$ & $7.10 \mathrm{E}-02$ & $7.02 \mathrm{E}-02$ \\
\hline $\mathrm{CO} 60 \mathrm{M}$ & $9.92 \mathrm{E}-01$ & $1.36 \mathrm{E}-01$ & $2.35 \mathrm{E}-09$ & $0.00 \mathrm{E}+00$ & $0.00 \mathrm{E}+00$ & $0.00 \mathrm{E}+00$ & $0.00 \mathrm{E}+00$ & $0.00 \mathrm{E}+00$ & $0.00 \mathrm{E}+00$ \\
\hline $\mathrm{CO} 61$ & $1.04 \mathrm{E}-02$ & $8.44 \mathrm{E}-03$ & $1.27 \mathrm{E}-03$ & $4.35 \mathrm{E}-07$ & $0.00 \mathrm{E}+00$ & $0.00 \mathrm{E}+00$ & $0.00 \mathrm{E}+00$ & $0.00 \mathrm{E}+00$ & $0.00 \mathrm{E}+00$ \\
\hline $\mathrm{CO} 62$ & $8.92 \mathrm{E}-04$ & $8.51 \mathrm{E}-10$ & $0.00 \mathrm{E}+00$ & $0.00 \mathrm{E}+00$ & $0.00 \mathrm{E}+00$ & $0.00 \mathrm{E}+00$ & $0.00 \mathrm{E}+00$ & $0.00 \mathrm{E}+00$ & $0.00 \mathrm{E}+00$ \\
\hline NI 59 & $6.77 \mathrm{E}-04$ & $6.77 \mathrm{E}-04$ & $6.77 \mathrm{E}-04$ & $6.77 \mathrm{E}-04$ & $6.77 \mathrm{E}-04$ & $6.77 \mathrm{E}-04$ & $6.77 \mathrm{E}-04$ & $6.77 \mathrm{E}-04$ & $6.77 \mathrm{E}-04$ \\
\hline $\mathrm{NI} 63$ & $8.82 \mathrm{E}-02$ & $8.82 \mathrm{E}-02$ & $8.82 \mathrm{E}-02$ & $8.82 \mathrm{E}-02$ & $8.82 \mathrm{E}-02$ & $8.82 \mathrm{E}-02$ & $8.82 \mathrm{E}-02$ & $8.81 \mathrm{E}-02$ & $8.81 \mathrm{E}-02$ \\
\hline NI 65 & $1.04 \mathrm{E}+00$ & $9.10 \mathrm{E}-01$ & $2.64 \mathrm{E}-01$ & $1.42 \mathrm{E}-03$ & $8.92 \mathrm{E}-21$ & $0.00 \mathrm{E}+00$ & $0.00 \mathrm{E}+00$ & $0.00 \mathrm{E}+00$ & $0.00 \mathrm{E}+00$ \\
\hline $\mathrm{NI} 66$ & $3.58 \mathrm{E}-05$ & $3.56 \mathrm{E}-05$ & $3.36 \mathrm{E}-05$ & $2.64 \mathrm{E}-05$ & $4.24 \mathrm{E}-06$ & $5.03 \mathrm{E}-07$ & 3.84E-09 & $4.13 \mathrm{E}-13$ & $4.44 \mathrm{E}-17$ \\
\hline CU 64 & $1.16 \mathrm{E}+00$ & $1.13 \mathrm{E}+00$ & $8.80 \mathrm{E}-01$ & $3.12 \mathrm{E}-01$ & $1.21 \mathrm{E}-04$ & $1.26 \mathrm{E}-08$ & $9.93 \mathrm{E}-18$ & $0.00 \mathrm{E}+00$ & $0.00 \mathrm{E}+00$ \\
\hline CU 66 & $2.53 \mathrm{E}-01$ & $4.32 \mathrm{E}-03$ & $3.36 \mathrm{E}-05$ & $2.64 \mathrm{E}-05$ & $4.25 \mathrm{E}-06$ & $5.04 \mathrm{E}-07$ & $3.85 \mathrm{E}-09$ & $4.14 \mathrm{E}-13$ & $4.45 \mathrm{E}-17$ \\
\hline CU 67 & $1.64 \mathrm{E}-06$ & $1.63 \mathrm{E}-06$ & $1.55 \mathrm{E}-06$ & $1.25 \mathrm{E}-06$ & $2.49 \mathrm{E}-07$ & $3.79 \mathrm{E}-08$ & $5.13 \mathrm{E}-10$ & $1.61 \mathrm{E}-13$ & $5.04 \mathrm{E}-17$ \\
\hline ZN 65 & 7.70E-05 & $7.69 \mathrm{E}-05$ & $7.69 \mathrm{E}-05$ & 7.67E-05 & 7.54E-05 & $7.39 \mathrm{E}-05$ & 7.07E-05 & $6.49 \mathrm{E}-05$ & $5.96 \mathrm{E}-05$ \\
\hline ZN 69 & $1.18 \mathrm{E}-09$ & $8.44 \mathrm{E}-10$ & $9.48 \mathrm{E}-11$ & $2.54 \mathrm{E}-11$ & $1.80 \mathrm{E}-14$ & $3.81 \mathrm{E}-18$ & $0.00 \mathrm{E}+00$ & $0.00 \mathrm{E}+00$ & $0.00 \mathrm{E}+00$ \\
\hline ZN 69M & $7.94 \mathrm{E}-11$ & $7.74 \mathrm{E}-11$ & $6.17 \mathrm{E}-11$ & 2.37E-11 & $1.68 \mathrm{E}-14$ & $3.54 \mathrm{E}-18$ & $1.41 \mathrm{E}-26$ & $0.00 \mathrm{E}+00$ & $0.00 \mathrm{E}+00$ \\
\hline GA 70 & 1.87E-12 & $6.97 \mathrm{E}-13$ & $9.80 \mathrm{E}-17$ & $0.00 \mathrm{E}+00$ & $0.00 \mathrm{E}+00$ & $0.00 \mathrm{E}+00$ & $0.00 \mathrm{E}+00$ & $0.00 \mathrm{E}+00$ & $0.00 \mathrm{E}+00$ \\
\hline SR 89 & 3.69E-11 & 3.69E-11 & $3.68 \mathrm{E}-11$ & 3.64E-11 & 3.35E-11 & 3.04E-11 & $2.44 \mathrm{E}-11$ & $1.62 \mathrm{E}-11$ & 1.07E-11 \\
\hline SR 90 & $9.00 \mathrm{E}-14$ & 9.00E-14 & $9.00 \mathrm{E}-14$ & $9.00 \mathrm{E}-14$ & $9.00 \mathrm{E}-14$ & 8.99E-14 & $8.98 \mathrm{E}-14$ & $8.97 \mathrm{E}-14$ & $8.95 \mathrm{E}-14$ \\
\hline SR 91 & $1.35 \mathrm{E}-12$ & $1.30 \mathrm{E}-12$ & $9.38 \mathrm{E}-13$ & $2.35 \mathrm{E}-13$ & $6.41 \mathrm{E}-18$ & $3.05 \mathrm{E}-23$ & $0.00 \mathrm{E}+00$ & $0.00 \mathrm{E}+00$ & $0.00 \mathrm{E}+00$ \\
\hline Y 89M & 9.17E-07 & $9.13 \mathrm{E}-07$ & $8.78 \mathrm{E}-07$ & $7.42 \mathrm{E}-07$ & $2.08 \mathrm{E}-07$ & $4.71 \mathrm{E}-08$ & $1.58 \mathrm{E}-09$ & $2.74 \mathrm{E}-12$ & $4.72 \mathrm{E}-15$ \\
\hline Y 90 & $1.22 \mathrm{E}-05$ & $1.22 \mathrm{E}-05$ & $1.16 \mathrm{E}-05$ & $9.43 \mathrm{E}-06$ & $1.98 \mathrm{E}-06$ & $3.22 \mathrm{E}-07$ & 5.02E-09 & $2.15 \mathrm{E}-12$ & $9.04 \mathrm{E}-14$ \\
\hline Y 90M & 1.07E-12 & $9.58 \mathrm{E}-13$ & $3.50 \mathrm{E}-13$ & $5.04 \mathrm{E}-15$ & $5.24 \mathrm{E}-29$ & $0.00 \mathrm{E}+00$ & $0.00 \mathrm{E}+00$ & $0.00 \mathrm{E}+00$ & $0.00 \mathrm{E}+00$ \\
\hline Y 91 & 1.54E-09 & 1.54E-09 & 1.54E-09 & $1.52 \mathrm{E}-09$ & $1.42 \mathrm{E}-09$ & $1.31 \mathrm{E}-09$ & $1.08 \mathrm{E}-09$ & $7.57 \mathrm{E}-10$ & $5.31 \mathrm{E}-10$ \\
\hline Y 92 & $1.66 \mathrm{E}-10$ & $1.50 \mathrm{E}-10$ & $6.22 \mathrm{E}-11$ & $1.51 \mathrm{E}-12$ & $8.48 \mathrm{E}-25$ & $0.00 \mathrm{E}+00$ & $0.00 \mathrm{E}+00$ & $0.00 E+00$ & $0.00 \mathrm{E}+00$ \\
\hline Y 94 & $9.58 \mathrm{E}-13$ & $3.23 \mathrm{E}-13$ & $1.79 \mathrm{E}-17$ & $0.00 \mathrm{E}+00$ & $0.00 \mathrm{E}+00$ & $0.00 \mathrm{E}+00$ & $0.00 \mathrm{E}+00$ & $0.00 \mathrm{E}+00$ & $0.00 \mathrm{E}+00$ \\
\hline ZR 89 & 9.19E-07 & 9.15E-07 & 8.79E-07 & 7.43E-07 & $2.08 \mathrm{E}-07$ & $4.72 \mathrm{E}-08$ & 1.59E-09 & $2.74 \mathrm{E}-12$ & $4.72 \mathrm{E}-15$ \\
\hline ZR 93 & $1.84 \mathrm{E}-11$ & $1.84 \mathrm{E}-11$ & $1.84 \mathrm{E}-11$ & $1.84 \mathrm{E}-11$ & $1.84 \mathrm{E}-11$ & $1.84 \mathrm{E}-11$ & $1.84 \mathrm{E}-11$ & $1.84 \mathrm{E}-11$ & $1.84 \mathrm{E}-11$ \\
\hline ZR 95 & $2.30 \mathrm{E}-06$ & $2.30 \mathrm{E}-06$ & $2.29 \mathrm{E}-06$ & 2.27E-06 & $2.13 \mathrm{E}-06$ & 1.97E-06 & 1.66E-06 & $1.20 \mathrm{E}-06$ & $8.67 \mathrm{E}-07$ \\
\hline ZR 97 & $7.26 \mathrm{E}-08$ & 7.11E-08 & $5.91 \mathrm{E}-08$ & $2.71 \mathrm{E}-08$ & $7.40 \mathrm{E}-11$ & 7.57E-14 & 1.09E-20 & $0.00 \mathrm{E}+00$ & $0.00 \mathrm{E}+00$ \\
\hline NB 92 & $1.83 \mathrm{E}-04$ & 1.83E-04 & $1.80 \mathrm{E}-04$ & $1.71 \mathrm{E}-04$ & $1.13 \mathrm{E}-04$ & 7.03E-05 & $2.36 \mathrm{E}-05$ & $3.05 \mathrm{E}-06$ & $3.94 \mathrm{E}-07$ \\
\hline NB 93M & $1.45 \mathrm{E}-13$ & 1.45E-13 & $1.46 \mathrm{E}-13$ & 1.47E-13 & $1.62 \mathrm{E}-13$ & $1.79 \mathrm{E}-13$ & $2.18 \mathrm{E}-13$ & $2.90 \mathrm{E}-13$ & $3.62 \mathrm{E}-13$ \\
\hline NB 94 & 2.94E-06 & 2.94E-06 & $2.94 \mathrm{E}-06$ & $2.94 \mathrm{E}-06$ & 2.94E-06 & 2.94E-06 & $2.94 \mathrm{E}-06$ & 2.94E-06 & $2.94 \mathrm{E}-06$ \\
\hline NB 95 & $8.48 \mathrm{E}-03$ & $8.48 \mathrm{E}-03$ & 8.44E-03 & $8.31 \mathrm{E}-03$ & 7.39E-03 & $6.43 \mathrm{E}-03$ & 4.69E-03 & $2.60 \mathrm{E}-03$ & $1.44 \mathrm{E}-03$ \\
\hline NB 95M & 1.57E-08 & 1.57E-08 & 1.57E-08 & $1.58 \mathrm{E}-08$ & 1.55E-08 & $1.46 \mathrm{E}-08$ & $1.23 \mathrm{E}-08$ & $8.90 \mathrm{E}-09$ & $6.43 \mathrm{E}-09$ \\
\hline NB 96 & 4.44E-05 & 4.38E-05 & 3.83E-05 & $2.18 \mathrm{E}-05$ & 3.03E-07 & 2.07E-09 & 2.32E-14 & $1.21 \mathrm{E}-23$ & $0.00 \mathrm{E}+00$ \\
\hline NB 97 & $2.58 \mathrm{E}-06$ & 1.95E-06 & 2.03E-07 & $2.73 \mathrm{E}-08$ & $7.43 \mathrm{E}-11$ & $7.61 \mathrm{E}-14$ & $1.18 \mathrm{E}-20$ & $0.00 \mathrm{E}+00$ & $0.00 \mathrm{E}+00$ \\
\hline NB 97M & 6.87E-08 & $6.74 \mathrm{E}-08$ & 5.60E-08 & 2.57E-08 & 7.01E-11 & 7.17E-14 & 1.04E-20 & $0.00 \mathrm{E}+00$ & $0.00 \mathrm{E}+00$ \\
\hline NB 98 & 3.84E-07 & $0.00 \mathrm{E}+00$ & $0.00 E+00$ & $0.00 \mathrm{E}+00$ & $0.00 \mathrm{E}+00$ & $0.00 \mathrm{E}+00$ & $0.00 \mathrm{E}+00$ & $0.00 \mathrm{E}+00$ & $0.00 \mathrm{E}+00$ \\
\hline NB100 & $1.91 \mathrm{E}-08$ & $0.00 \mathrm{E}+00$ & $0.00 \mathrm{E}+00$ & $0.00 \mathrm{E}+00$ & $0.00 \mathrm{E}+00$ & $0.00 \mathrm{E}+00$ & $0.00 E+00$ & $0.00 \mathrm{E}+00$ & $0.00 \mathrm{E}+00$ \\
\hline MO 93M & $5.31 \mathrm{E}-05$ & $5.05 \mathrm{E}-05$ & $3.20 \mathrm{E}-05$ & $4.68 \mathrm{E}-06$ & $2.20 \mathrm{E}-12$ & $9.10 \mathrm{E}-20$ & $0.00 \mathrm{E}+00$ & $0.00 \mathrm{E}+00$ & $0.00 \mathrm{E}+00$ \\
\hline MO 93 & 1.06E-07 & 1.06E-07 & 1.06E-07 & 1.06E-07 & 1.06E-07 & 1.06E-07 & 1.06E-07 & $1.06 \mathrm{E}-07$ & $1.06 \mathrm{E}-07$ \\
\hline MO 99 & $3.72 \mathrm{E}-02$ & $3.70 \mathrm{E}-02$ & $3.53 \mathrm{E}-02$ & $2.89 \mathrm{E}-02$ & $6.37 \mathrm{E}-03$ & 1.09E-03 & $1.93 \mathrm{E}-05$ & $1.01 \mathrm{E}-08$ & $5.23 \mathrm{E}-12$ \\
\hline MO101 & $9.24 \mathrm{E}-03$ & $2.23 \mathrm{E}-03$ & $6.15 \mathrm{E}-09$ & $0.00 \mathrm{E}+00$ & $0.00 \mathrm{E}+00$ & $0.00 \mathrm{E}+00$ & $0.00 \mathrm{E}+00$ & $0.00 \mathrm{E}+00$ & $0.00 \mathrm{E}+00$ \\
\hline TC 99 & 4.49E-09 & 4.49E-09 & $4.50 \mathrm{E}-09$ & $4.53 \mathrm{E}-09$ & $4.63 \mathrm{E}-09$ & $4.65 \mathrm{E}-09$ & $4.65 \mathrm{E}-09$ & $4.65 \mathrm{E}-09$ & $4.65 \mathrm{E}-09$ \\
\hline TC100 & 4.70E-04 & $0.00 \mathrm{E}+00$ & $0.00 \mathrm{E}+00$ & $0.00 \mathrm{E}+00$ & $0.00 \mathrm{E}+00$ & $0.00 \mathrm{E}+00$ & $0.00 \mathrm{E}+00$ & $0.00 \mathrm{E}+00$ & $0.00 \mathrm{E}+00$ \\
\hline TC101 & $9.24 \mathrm{E}-03$ & $5.33 \mathrm{E}-03$ & $7.75 \mathrm{E}-08$ & $0.00 \mathrm{E}+00$ & $0.00 \mathrm{E}+00$ & $0.00 \mathrm{E}+00$ & $0.00 \mathrm{E}+00$ & $0.00 \mathrm{E}+00$ & $0.00 \mathrm{E}+00$ \\
\hline RU103 & $2.31 \mathrm{E}-07$ & $2.31 \mathrm{E}-07$ & $2.30 \mathrm{E}-07$ & $2.27 \mathrm{E}-07$ & 2.04E-07 & $1.80 \mathrm{E}-07$ & $1.36 \mathrm{E}-07$ & $8.00 \mathrm{E}-08$ & $4.71 \mathrm{E}-08$ \\
\hline RU105 & $3.09 \mathrm{E}-12$ & $2.86 \mathrm{E}-12$ & $1.41 \mathrm{E}-12$ & $7.27 \mathrm{E}-14$ & $1.25 \mathrm{E}-23$ & $0.00 \mathrm{E}+00$ & $0.00 \mathrm{E}+00$ & $0.00 \mathrm{E}+00$ & $0.00 \mathrm{E}+00$ \\
\hline RH104 & $2.73 \mathrm{E}-09$ & $1.96 \mathrm{E}-12$ & $0.00 \mathrm{E}+00$ & $0.00 \mathrm{E}+00$ & $0.00 \mathrm{E}+00$ & $0.00 \mathrm{E}+00$ & $0.00 \mathrm{E}+00$ & $0.00 \mathrm{E}+00$ & $0.00 \mathrm{E}+00$ \\
\hline RH104M & $1.98 \mathrm{E}-10$ & $1.64 \mathrm{E}-12$ & $0.00 \mathrm{E}+00$ & $0.00 \mathrm{E}+00$ & $0.00 \mathrm{E}+00$ & $0.00 \mathrm{E}+00$ & $0.00 \mathrm{E}+00$ & $0.00 \mathrm{E}+00$ & $0.00 \mathrm{E}+00$ \\
\hline RH105 & $2.33 \mathrm{E}-12$ & $2.34 \mathrm{E}-12$ & $2.31 \mathrm{E}-12$ & $1.72 \mathrm{E}-12$ & $1.03 \mathrm{E}-13$ & $3.83 \mathrm{E}-15$ & $2.06 \mathrm{E}-18$ & $1.53 \mathrm{E}-24$ & $1.14 \mathrm{E}-30$ \\
\hline
\end{tabular}


TEM-10200-1

ENGINEERING CALCULATIONS AND ANALYSIS

Page 33 of 81

$12 / 19 / 17$

Rev.08

Title:

AS-RUN NEUTRONICS EVALUATION FOR THE CSM-10584 EXPERIMENT IN THE ATR

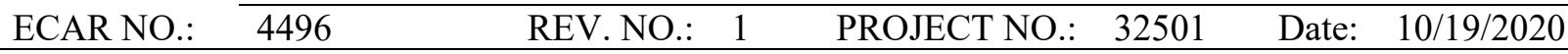

\begin{tabular}{|c|c|c|c|c|c|c|c|c|c|}
\hline \multicolumn{9}{|c|}{ Capsule B - 119 days irradiation } \\
\hline & EOC & $30 \mathrm{MINs}$ & $5 \mathrm{HRS}$ & $1 \mathrm{DAY}$ & $7 \mathrm{DAYS}$ & $14 \mathrm{DAYS}$ & 30 DAYS & 60 DAYS & 90 DAYS \\
\hline ISOTOPE & $\mathrm{Ci}$ & $\mathrm{Ci}$ & $\mathrm{Ci}$ & $\mathrm{Ci}$ & $\mathrm{Ci}$ & $\mathrm{Ci}$ & $\mathrm{Ci}$ & $\mathrm{Ci}$ & $\mathrm{Ci}$ \\
\hline RH105M & $8.65 \mathrm{E}-13$ & $8.02 \mathrm{E}-13$ & $3.97 \mathrm{E}-13$ & $2.04 \mathrm{E}-14$ & $3.50 \mathrm{E}-24$ & $0.00 \mathrm{E}+00$ & $0.00 \mathrm{E}+00$ & $0.00 \mathrm{E}+00$ & $0.00 \mathrm{E}+00$ \\
\hline RH106 & $5.14 \mathrm{E}-13$ & $1.01 \mathrm{E}-18$ & $1.01 \mathrm{E}-18$ & $1.01 \mathrm{E}-18$ & $1.01 \mathrm{E}-18$ & $1.01 \mathrm{E}-18$ & $1.01 \mathrm{E}-18$ & $9.52 \mathrm{E}-19$ & $8.98 \mathrm{E}-19$ \\
\hline RH106M & $2.50 \mathrm{E}-13$ & $2.13 \mathrm{E}-13$ & $5.16 \mathrm{E}-14$ & $7.24 \mathrm{E}-17$ & $0.00 \mathrm{E}+00$ & $0.00 \mathrm{E}+00$ & $0.00 \mathrm{E}+00$ & $0.00 \mathrm{E}+00$ & $0.00 \mathrm{E}+00$ \\
\hline SUMTOT & $3.23 \mathrm{E}+02$ & $1.43 \mathrm{E}+02$ & $1.32 \mathrm{E}+02$ & $1.25 \mathrm{E}+02$ & $1.09 \mathrm{E}+02$ & $9.27 \mathrm{E}+01$ & $6.53 \mathrm{E}+01$ & $3.56 \mathrm{E}+01$ & $2.13 \mathrm{E}+01$ \\
\hline OTOTAL & $3.23 \mathrm{E}+02$ & $1.43 \mathrm{E}+02$ & $1.32 \mathrm{E}+02$ & $1.25 \mathrm{E}+02$ & $1.09 \mathrm{E}+02$ & $9.27 \mathrm{E}+01$ & $6.53 \mathrm{E}+01$ & $3.56 \mathrm{E}+01$ & $2.13 \mathrm{E}+01$ \\
\hline
\end{tabular}

Table 15. Radionuclide source term for CSM capsule C after 119 days of irradiation (Ci).

\begin{tabular}{|c|c|c|c|c|c|c|c|c|c|}
\hline \multicolumn{10}{|c|}{ Capsule C - 119 days irradiation } \\
\hline & EOC & $30 \mathrm{MINs}$ & $5 \mathrm{HRS}$ & 1 DAY & 7 DAYS & 14 DAYS & 30 DAYS & 60 DAYS & 90 DAYS \\
\hline ISOTOPE & $\mathrm{Ci}$ & $\mathrm{Ci}$ & $\mathrm{Ci}$ & $\mathrm{Ci}$ & $\mathrm{Ci}$ & $\mathrm{Ci}$ & $\mathrm{Ci}$ & $\mathrm{Ci}$ & $\mathrm{Ci}$ \\
\hline H 3 & $1.29 \mathrm{E}-07$ & $1.29 \mathrm{E}-07$ & $1.29 \mathrm{E}-07$ & $1.29 \mathrm{E}-07$ & $1.29 \mathrm{E}-07$ & $1.29 \mathrm{E}-07$ & $1.28 \mathrm{E}-07$ & $1.28 \mathrm{E}-07$ & $1.27 \mathrm{E}-07$ \\
\hline H 4 & $1.46 \mathrm{E}-04$ & $0.00 \mathrm{E}+00$ & $0.00 \mathrm{E}+00$ & $0.00 \mathrm{E}+00$ & $0.00 \mathrm{E}+00$ & $0.00 \mathrm{E}+00$ & $0.00 \mathrm{E}+00$ & $0.00 \mathrm{E}+00$ & $0.00 \mathrm{E}+00$ \\
\hline HE 6 & $5.31 \mathrm{E}-05$ & $0.00 \mathrm{E}+00$ & $0.00 \mathrm{E}+00$ & $0.00 \mathrm{E}+00$ & $0.00 \mathrm{E}+00$ & $0.00 \mathrm{E}+00$ & $0.00 \mathrm{E}+00$ & $0.00 \mathrm{E}+00$ & $0.00 \mathrm{E}+00$ \\
\hline $\mathrm{LI} 8$ & $5.86 \mathrm{E}-05$ & $0.00 \mathrm{E}+00$ & $0.00 \mathrm{E}+00$ & $0.00 \mathrm{E}+00$ & $0.00 \mathrm{E}+00$ & $0.00 \mathrm{E}+00$ & $0.00 \mathrm{E}+00$ & $0.00 \mathrm{E}+00$ & $0.00 \mathrm{E}+00$ \\
\hline BE 8 & $1.50 \mathrm{E}-04$ & $0.00 \mathrm{E}+00$ & $0.00 \mathrm{E}+00$ & $0.00 \mathrm{E}+00$ & $0.00 \mathrm{E}+00$ & $0.00 \mathrm{E}+00$ & $0.00 \mathrm{E}+00$ & $0.00 \mathrm{E}+00$ & $0.00 \mathrm{E}+00$ \\
\hline BE 10 & $3.73 \mathrm{E}-10$ & $3.73 \mathrm{E}-10$ & $3.73 \mathrm{E}-10$ & $3.73 \mathrm{E}-10$ & $3.73 \mathrm{E}-10$ & $3.73 \mathrm{E}-10$ & $3.73 \mathrm{E}-10$ & $3.73 \mathrm{E}-10$ & $3.73 \mathrm{E}-10$ \\
\hline BE 11 & 2.95E-09 & $0.00 \mathrm{E}+00$ & $0.00 \mathrm{E}+00$ & $0.00 \mathrm{E}+00$ & $0.00 \mathrm{E}+00$ & $0.00 \mathrm{E}+00$ & $0.00 \mathrm{E}+00$ & $0.00 \mathrm{E}+00$ & $0.00 \mathrm{E}+00$ \\
\hline B 12 & $3.98 \mathrm{E}-05$ & $0.00 \mathrm{E}+00$ & $0.00 \mathrm{E}+00$ & $0.00 \mathrm{E}+00$ & $0.00 \mathrm{E}+00$ & $0.00 \mathrm{E}+00$ & $0.00 \mathrm{E}+00$ & $0.00 \mathrm{E}+00$ & $0.00 \mathrm{E}+00$ \\
\hline C 14 & 1.37E-08 & $1.37 \mathrm{E}-08$ & 1.37E-08 & $1.37 \mathrm{E}-08$ & 1.37E-08 & $1.37 \mathrm{E}-08$ & 1.37E-08 & 1.37E-08 & 1.37E-08 \\
\hline C 15 & $3.41 \mathrm{E}-10$ & $0.00 \mathrm{E}+00$ & $0.00 \mathrm{E}+00$ & $0.00 \mathrm{E}+00$ & $0.00 \mathrm{E}+00$ & $0.00 \mathrm{E}+00$ & $0.00 \mathrm{E}+00$ & $0.00 \mathrm{E}+00$ & $0.00 \mathrm{E}+00$ \\
\hline 019 & $2.44 \mathrm{E}-11$ & $0.00 \mathrm{E}+00$ & $0.00 \mathrm{E}+00$ & $0.00 \mathrm{E}+00$ & $0.00 \mathrm{E}+00$ & $0.00 \mathrm{E}+00$ & $0.00 \mathrm{E}+00$ & $0.00 \mathrm{E}+00$ & $0.00 \mathrm{E}+00$ \\
\hline $\mathrm{F} 20$ & $4.25 \mathrm{E}-08$ & $0.00 \mathrm{E}+00$ & $0.00 \mathrm{E}+00$ & $0.00 \mathrm{E}+00$ & $0.00 \mathrm{E}+00$ & $0.00 \mathrm{E}+00$ & $0.00 \mathrm{E}+00$ & $0.00 \mathrm{E}+00$ & $0.00 \mathrm{E}+00$ \\
\hline NE 23 & $2.04 \mathrm{E}-02$ & $5.74 \mathrm{E}-17$ & $0.00 \mathrm{E}+00$ & $0.00 \mathrm{E}+00$ & $0.00 \mathrm{E}+00$ & $0.00 \mathrm{E}+00$ & $0.00 \mathrm{E}+00$ & $0.00 \mathrm{E}+00$ & $0.00 \mathrm{E}+00$ \\
\hline NA 24 & $3.24 \mathrm{E}+00$ & $3.17 \mathrm{E}+00$ & $2.57 \mathrm{E}+00$ & $1.07 \mathrm{E}+00$ & $1.38 \mathrm{E}-03$ & $5.86 \mathrm{E}-07$ & $1.15 \mathrm{E}-14$ & 4.09E-29 & $0.00 \mathrm{E}+00$ \\
\hline NA 24M & $9.42 \mathrm{E}-06$ & $0.00 \mathrm{E}+00$ & $0.00 \mathrm{E}+00$ & $0.00 \mathrm{E}+00$ & $0.00 \mathrm{E}+00$ & $0.00 \mathrm{E}+00$ & $0.00 \mathrm{E}+00$ & $0.00 \mathrm{E}+00$ & $0.00 \mathrm{E}+00$ \\
\hline NA 25 & $1.23 \mathrm{E}-02$ & $9.96 \mathrm{E}-12$ & $0.00 \mathrm{E}+00$ & $0.00 \mathrm{E}+00$ & $0.00 \mathrm{E}+00$ & $0.00 \mathrm{E}+00$ & $0.00 \mathrm{E}+00$ & $0.00 \mathrm{E}+00$ & $0.00 \mathrm{E}+00$ \\
\hline MG 27 & $1.53 \mathrm{E}+01$ & $1.70 \mathrm{E}+00$ & 4.36E-09 & $0.00 \mathrm{E}+00$ & $0.00 \mathrm{E}+00$ & $0.00 \mathrm{E}+00$ & $0.00 \mathrm{E}+00$ & $0.00 \mathrm{E}+00$ & $0.00 \mathrm{E}+00$ \\
\hline MG 28 & $5.43 \mathrm{E}-08$ & $5.34 \mathrm{E}-08$ & 4.60E-08 & $2.45 \mathrm{E}-08$ & 2.07E-10 & $7.90 \mathrm{E}-13$ & $2.34 \mathrm{E}-18$ & $1.01 \mathrm{E}-28$ & $0.00 \mathrm{E}+00$ \\
\hline AL 28 & $3.64 \mathrm{E}+02$ & $3.39 \mathrm{E}-02$ & $4.61 \mathrm{E}-08$ & $2.46 \mathrm{E}-08$ & $2.08 \mathrm{E}-10$ & $7.92 \mathrm{E}-13$ & $2.35 \mathrm{E}-18$ & $0.00 \mathrm{E}+00$ & $0.00 \mathrm{E}+00$ \\
\hline AL 29 & $3.72 \mathrm{E}-03$ & $1.53 \mathrm{E}-04$ & $5.23 \mathrm{E}-17$ & $0.00 \mathrm{E}+00$ & $0.00 \mathrm{E}+00$ & $0.00 \mathrm{E}+00$ & $0.00 \mathrm{E}+00$ & $0.00 \mathrm{E}+00$ & $0.00 \mathrm{E}+00$ \\
\hline AL 30 & $3.59 \mathrm{E}-06$ & $0.00 \mathrm{E}+00$ & $0.00 \mathrm{E}+00$ & $0.00 \mathrm{E}+00$ & $0.00 \mathrm{E}+00$ & $0.00 \mathrm{E}+00$ & $0.00 \mathrm{E}+00$ & $0.00 \mathrm{E}+00$ & $0.00 \mathrm{E}+00$ \\
\hline SI 31 & $3.50 \mathrm{E}-02$ & $3.07 \mathrm{E}-02$ & 9.33E-03 & $6.14 \mathrm{E}-05$ & 1.79E-21 & $0.00 \mathrm{E}+00$ & $0.00 \mathrm{E}+00$ & $0.00 \mathrm{E}+00$ & $0.00 \mathrm{E}+00$ \\
\hline SI 32 & $8.61 \mathrm{E}-12$ & $8.61 \mathrm{E}-12$ & $8.61 \mathrm{E}-12$ & $8.61 \mathrm{E}-12$ & $8.61 \mathrm{E}-12$ & $8.61 \mathrm{E}-12$ & $8.61 \mathrm{E}-12$ & $8.60 \mathrm{E}-12$ & $8.60 \mathrm{E}-12$ \\
\hline P 32 & 5.10E-03 & $5.10 \mathrm{E}-03$ & 5.05E-03 & $4.86 \mathrm{E}-03$ & $3.64 \mathrm{E}-03$ & $2.59 \mathrm{E}-03$ & 1.19E-03 & $2.78 \mathrm{E}-04$ & $6.50 \mathrm{E}-05$ \\
\hline P 33 & $1.92 \mathrm{E}-07$ & $1.92 \mathrm{E}-07$ & $1.91 \mathrm{E}-07$ & $1.87 \mathrm{E}-07$ & $1.58 \mathrm{E}-07$ & 1.30E-07 & $8.36 \mathrm{E}-08$ & $3.64 \mathrm{E}-08$ & $1.58 \mathrm{E}-08$ \\
\hline P 34 & $1.11 \mathrm{E}-06$ & $0.00 \mathrm{E}+00$ & $0.00 \mathrm{E}+00$ & $0.00 \mathrm{E}+00$ & $0.00 \mathrm{E}+00$ & $0.00 \mathrm{E}+00$ & $0.00 \mathrm{E}+00$ & $0.00 \mathrm{E}+00$ & $0.00 \mathrm{E}+00$ \\
\hline S35 & $7.75 \mathrm{E}-05$ & $7.75 \mathrm{E}-05$ & $7.74 \mathrm{E}-05$ & $7.69 \mathrm{E}-05$ & $7.33 \mathrm{E}-05$ & $6.94 \mathrm{E}-05$ & $6.12 \mathrm{E}-05$ & $4.83 \mathrm{E}-05$ & $3.81 \mathrm{E}-05$ \\
\hline S 37 & 3.96E-07 & $6.51 \mathrm{E}-09$ & $5.63 \mathrm{E}-25$ & $0.00 \mathrm{E}+00$ & $0.00 \mathrm{E}+00$ & $0.00 \mathrm{E}+00$ & $0.00 \mathrm{E}+00$ & $0.00 \mathrm{E}+00$ & $0.00 \mathrm{E}+00$ \\
\hline $\mathrm{CL} 36$ & $6.99 \mathrm{E}-11$ & $6.99 \mathrm{E}-11$ & $6.99 \mathrm{E}-11$ & $6.99 \mathrm{E}-11$ & 6.99E-11 & $6.99 \mathrm{E}-11$ & $6.99 \mathrm{E}-11$ & $6.99 \mathrm{E}-11$ & $6.99 \mathrm{E}-11$ \\
\hline $\mathrm{CL} 38$ & $8.10 \mathrm{E}-10$ & $4.64 \mathrm{E}-10$ & 3.04E-12 & $1.83 \mathrm{E}-21$ & $0.00 \mathrm{E}+00$ & $0.00 \mathrm{E}+00$ & $0.00 \mathrm{E}+00$ & $0.00 \mathrm{E}+00$ & $0.00 \mathrm{E}+00$ \\
\hline CL 38M & $9.65 \mathrm{E}-12$ & $0.00 \mathrm{E}+00$ & $0.00 \mathrm{E}+00$ & $0.00 \mathrm{E}+00$ & $0.00 \mathrm{E}+00$ & $0.00 \mathrm{E}+00$ & $0.00 \mathrm{E}+00$ & $0.00 \mathrm{E}+00$ & $0.00 \mathrm{E}+00$ \\
\hline AR 39 & $2.22 \mathrm{E}-07$ & $2.22 \mathrm{E}-07$ & $2.22 \mathrm{E}-07$ & $2.22 \mathrm{E}-07$ & $2.22 \mathrm{E}-07$ & $2.22 \mathrm{E}-07$ & $2.22 \mathrm{E}-07$ & $2.22 \mathrm{E}-07$ & $2.22 \mathrm{E}-07$ \\
\hline AR 41 & $8.21 \mathrm{E}-08$ & $6.79 \mathrm{E}-08$ & $1.23 \mathrm{E}-08$ & $9.11 \mathrm{E}-12$ & $0.00 \mathrm{E}+00$ & $0.00 \mathrm{E}+00$ & $0.00 \mathrm{E}+00$ & $0.00 \mathrm{E}+00$ & $0.00 \mathrm{E}+00$ \\
\hline K 40 & $5.46 \mathrm{E}-13$ & $5.46 \mathrm{E}-13$ & $5.46 \mathrm{E}-13$ & $5.46 \mathrm{E}-13$ & $5.46 \mathrm{E}-13$ & $5.46 \mathrm{E}-13$ & $5.46 \mathrm{E}-13$ & $5.46 \mathrm{E}-13$ & $5.46 \mathrm{E}-13$ \\
\hline K 42 & $1.82 \mathrm{E}-04$ & 1.77E-04 & $1.38 \mathrm{E}-04$ & $4.74 \mathrm{E}-05$ & $1.48 \mathrm{E}-08$ & $1.20 \mathrm{E}-12$ & $1.01 \mathrm{E}-16$ & $1.01 \mathrm{E}-16$ & $1.01 \mathrm{E}-16$ \\
\hline K 43 & $1.91 \mathrm{E}-10$ & $1.88 \mathrm{E}-10$ & $1.64 \mathrm{E}-10$ & $9.16 \mathrm{E}-11$ & $1.11 \mathrm{E}-12$ & $6.39 \mathrm{E}-15$ & $4.91 \mathrm{E}-20$ & $1.26 \mathrm{E}-29$ & $0.00 \mathrm{E}+00$ \\
\hline K 44 & $3.39 \mathrm{E}-11$ & $1.32 \mathrm{E}-11$ & $2.66 \mathrm{E}-15$ & $0.00 \mathrm{E}+00$ & $0.00 \mathrm{E}+00$ & $0.00 \mathrm{E}+00$ & $0.00 \mathrm{E}+00$ & $0.00 \mathrm{E}+00$ & $0.00 \mathrm{E}+00$ \\
\hline CA 45 & $9.21 \mathrm{E}-07$ & $9.21 \mathrm{E}-07$ & $9.20 \mathrm{E}-07$ & $9.17 \mathrm{E}-07$ & $8.94 \mathrm{E}-07$ & $8.68 \mathrm{E}-07$ & $8.11 \mathrm{E}-07$ & $7.14 \mathrm{E}-07$ & $6.28 \mathrm{E}-07$ \\
\hline CA 47 & $1.58 \mathrm{E}-08$ & $1.58 \mathrm{E}-08$ & $1.53 \mathrm{E}-08$ & $1.36 \mathrm{E}-08$ & $5.43 \mathrm{E}-09$ & $1.86 \mathrm{E}-09$ & $1.62 \mathrm{E}-10$ & $1.65 \mathrm{E}-12$ & $1.68 \mathrm{E}-14$ \\
\hline SC 46 & $1.83 \mathrm{E}-04$ & $1.83 \mathrm{E}-04$ & $1.83 \mathrm{E}-04$ & $1.82 \mathrm{E}-04$ & $1.73 \mathrm{E}-04$ & $1.63 \mathrm{E}-04$ & $1.43 \mathrm{E}-04$ & $1.12 \mathrm{E}-04$ & $8.70 \mathrm{E}-05$ \\
\hline SC 46M & $5.21 \mathrm{E}-09$ & $0.00 \mathrm{E}+00$ & $0.00 \mathrm{E}+00$ & $0.00 \mathrm{E}+00$ & $0.00 \mathrm{E}+00$ & $0.00 \mathrm{E}+00$ & $0.00 \mathrm{E}+00$ & $0.00 \mathrm{E}+00$ & $0.00 \mathrm{E}+00$ \\
\hline SC 47 & $4.58 \mathrm{E}-04$ & $4.56 \mathrm{E}-04$ & $4.39 \mathrm{E}-04$ & $3.73 \mathrm{E}-04$ & $1.08 \mathrm{E}-04$ & $2.53 \mathrm{E}-05$ & $9.25 \mathrm{E}-07$ & 1.87E-09 & $3.83 \mathrm{E}-12$ \\
\hline SC 48 & $4.47 \mathrm{E}-05$ & $4.43 \mathrm{E}-05$ & $4.13 \mathrm{E}-05$ & $3.06 \mathrm{E}-05$ & $3.13 \mathrm{E}-06$ & $2.19 \mathrm{E}-07$ & $5.03 \mathrm{E}-10$ & 5.67E-15 & $6.40 \mathrm{E}-20$ \\
\hline
\end{tabular}


TEM-10200-1

ENGINEERING CALCULATIONS AND ANALYSIS

Page 34 of 81

$12 / 19 / 17$

Rev.08

Title:

AS-RUN NEUTRONICS EVALUATION FOR THE CSM-10584 EXPERIMENT IN THE ATR

\begin{tabular}{llllllll} 
ECAR NO.: & 4496 & REV. NO.: & 1 & PROJECT NO.: & 32501 & Date: & 10/19/2020 \\
\hline
\end{tabular}

\begin{tabular}{|c|c|c|c|c|c|c|c|c|c|}
\hline \multicolumn{10}{|c|}{ Capsule C - 119 days irradiation } \\
\hline & EOC & $30 \mathrm{MINs}$ & $5 \mathrm{HRS}$ & $1 \mathrm{DAY}$ & 7 DAYS & 14 DAYS & 30 DAYS & 60 DAYS & 90 DAYS \\
\hline ISOTOPE & $\mathrm{Ci}$ & $\mathrm{Ci}$ & $\mathrm{Ci}$ & $\mathrm{Ci}$ & $\mathrm{Ci}$ & $\mathrm{Ci}$ & $\mathrm{Ci}$ & $\mathrm{Ci}$ & $\mathrm{Ci}$ \\
\hline SC 49 & $3.92 \mathrm{E}-05$ & $2.73 \mathrm{E}-05$ & $1.05 \mathrm{E}-06$ & $1.13 \mathrm{E}-12$ & $0.00 \mathrm{E}+00$ & $0.00 \mathrm{E}+00$ & $0.00 \mathrm{E}+00$ & $0.00 \mathrm{E}+00$ & $0.00 \mathrm{E}+00$ \\
\hline SC 50 & 7.83E-07 & 4.05E-12 & $0.00 \mathrm{E}+00$ & $0.00 \mathrm{E}+00$ & $0.00 \mathrm{E}+00$ & $0.00 \mathrm{E}+00$ & $0.00 \mathrm{E}+00$ & $0.00 \mathrm{E}+00$ & $0.00 \mathrm{E}+00$ \\
\hline TI 51 & $2.28 \mathrm{E}-03$ & $6.16 \mathrm{E}-05$ & $4.77 \mathrm{E}-19$ & $0.00 \mathrm{E}+00$ & $0.00 \mathrm{E}+00$ & $0.00 \mathrm{E}+00$ & $0.00 \mathrm{E}+00$ & $0.00 \mathrm{E}+00$ & $0.00 E+00$ \\
\hline$V 52$ & $2.34 \mathrm{E}+00$ & $9.15 \mathrm{E}-03$ & $1.94 \mathrm{E}-24$ & $0.00 \mathrm{E}+00$ & $0.00 \mathrm{E}+00$ & $0.00 \mathrm{E}+00$ & $0.00 \mathrm{E}+00$ & $0.00 \mathrm{E}+00$ & $0.00 \mathrm{E}+00$ \\
\hline V 53 & $1.08 \mathrm{E}-02$ & $2.66 \mathrm{E}-08$ & $0.00 \mathrm{E}+00$ & $0.00 \mathrm{E}+00$ & $0.00 \mathrm{E}+00$ & $0.00 \mathrm{E}+00$ & $0.00 \mathrm{E}+00$ & $0.00 \mathrm{E}+00$ & $0.00 \mathrm{E}+00$ \\
\hline V 54 & $1.16 \mathrm{E}-04$ & $1.62 \mathrm{E}-14$ & $0.00 \mathrm{E}+00$ & $0.00 \mathrm{E}+00$ & $0.00 \mathrm{E}+00$ & $0.00 \mathrm{E}+00$ & $0.00 \mathrm{E}+00$ & $0.00 \mathrm{E}+00$ & $0.00 E+00$ \\
\hline CR 51 & $2.22 \mathrm{E}+02$ & $2.22 \mathrm{E}+02$ & $2.21 \mathrm{E}+02$ & $2.16 \mathrm{E}+02$ & $1.86 \mathrm{E}+02$ & $1.56 \mathrm{E}+02$ & $1.05 \mathrm{E}+02$ & $4.94 \mathrm{E}+01$ & $2.33 \mathrm{E}+01$ \\
\hline CR 55 & $3.16 \mathrm{E}+00$ & $9.04 \mathrm{E}-03$ & $0.00 \mathrm{E}+00$ & $0.00 \mathrm{E}+00$ & $0.00 \mathrm{E}+00$ & $0.00 \mathrm{E}+00$ & $0.00 \mathrm{E}+00$ & $0.00 \mathrm{E}+00$ & $0.00 \mathrm{E}+00$ \\
\hline MN 54 & $3.35 \mathrm{E}+00$ & $3.35 \mathrm{E}+00$ & $3.35 \mathrm{E}+00$ & $3.34 \mathrm{E}+00$ & $3.30 \mathrm{E}+00$ & $3.25 \mathrm{E}+00$ & $3.13 \mathrm{E}+00$ & $2.93 \mathrm{E}+00$ & $2.74 \mathrm{E}+00$ \\
\hline MN 56 & $2.29 \mathrm{E}+01$ & $2.00 \mathrm{E}+01$ & $5.96 \mathrm{E}+00$ & $3.61 \mathrm{E}-02$ & $5.58 \mathrm{E}-19$ & $0.00 \mathrm{E}+00$ & $0.00 \mathrm{E}+00$ & $0.00 \mathrm{E}+00$ & $0.00 \mathrm{E}+00$ \\
\hline MN 57 & $3.71 \mathrm{E}-02$ & $9.12 \mathrm{E}-08$ & $0.00 \mathrm{E}+00$ & $0.00 \mathrm{E}+00$ & $0.00 \mathrm{E}+00$ & $0.00 \mathrm{E}+00$ & $0.00 \mathrm{E}+00$ & $0.00 \mathrm{E}+00$ & $0.00 \mathrm{E}+00$ \\
\hline MN 58 & $1.12 \mathrm{E}-04$ & $5.65 \mathrm{E}-13$ & $0.00 \mathrm{E}+00$ & $0.00 \mathrm{E}+00$ & $0.00 \mathrm{E}+00$ & $0.00 \mathrm{E}+00$ & $0.00 \mathrm{E}+00$ & $0.00 \mathrm{E}+00$ & $0.00 \mathrm{E}+00$ \\
\hline FE 55 & $1.27 \mathrm{E}+01$ & $1.27 \mathrm{E}+01$ & 1.27E+01 & 1.27E+01 & $1.27 \mathrm{E}+01$ & $1.26 \mathrm{E}+01$ & $1.24 \mathrm{E}+01$ & $1.22 \mathrm{E}+01$ & $1.19 \mathrm{E}+01$ \\
\hline FE 59 & $3.73 \mathrm{E}+00$ & $3.73 \mathrm{E}+00$ & $3.72 \mathrm{E}+00$ & $3.67 \mathrm{E}+00$ & $3.35 \mathrm{E}+00$ & $3.01 \mathrm{E}+00$ & $2.35 \mathrm{E}+00$ & $1.48 \mathrm{E}+00$ & $9.32 \mathrm{E}-01$ \\
\hline CO 58 & $4.90 \mathrm{E}+00$ & $4.90 \mathrm{E}+00$ & $4.89 \mathrm{E}+00$ & $4.86 \mathrm{E}+00$ & $4.58 \mathrm{E}+00$ & $4.28 \mathrm{E}+00$ & $3.66 \mathrm{E}+00$ & $2.72 \mathrm{E}+00$ & $2.03 E+00$ \\
\hline $\mathrm{CO} 60$ & $1.29 \mathrm{E}-01$ & $1.29 \mathrm{E}-01$ & $1.29 \mathrm{E}-01$ & $1.29 \mathrm{E}-01$ & $1.28 \mathrm{E}-01$ & $1.28 \mathrm{E}-01$ & 1.27E-01 & $1.26 \mathrm{E}-01$ & $1.25 \mathrm{E}-01$ \\
\hline CO 60M & $1.77 \mathrm{E}+00$ & $2.43 \mathrm{E}-01$ & $4.20 \mathrm{E}-09$ & $0.00 \mathrm{E}+00$ & $0.00 \mathrm{E}+00$ & $0.00 \mathrm{E}+00$ & $0.00 \mathrm{E}+00$ & $0.00 \mathrm{E}+00$ & $0.00 \mathrm{E}+00$ \\
\hline CO 61 & $1.93 \mathrm{E}-02$ & $1.56 \mathrm{E}-02$ & $2.36 \mathrm{E}-03$ & $8.05 \mathrm{E}-07$ & $0.00 \mathrm{E}+00$ & $0.00 \mathrm{E}+00$ & $0.00 \mathrm{E}+00$ & $0.00 \mathrm{E}+00$ & $0.00 \mathrm{E}+00$ \\
\hline $\mathrm{CO} 62$ & $1.74 \mathrm{E}-03$ & 1.66E-09 & $0.00 \mathrm{E}+00$ & $0.00 \mathrm{E}+00$ & $0.00 \mathrm{E}+00$ & $0.00 \mathrm{E}+00$ & $0.00 \mathrm{E}+00$ & $0.00 \mathrm{E}+00$ & $0.00 \mathrm{E}+00$ \\
\hline NI 59 & $1.31 \mathrm{E}-03$ & $1.31 \mathrm{E}-03$ & $1.31 \mathrm{E}-03$ & $1.31 \mathrm{E}-03$ & $1.31 \mathrm{E}-03$ & $1.31 \mathrm{E}-03$ & $1.31 \mathrm{E}-03$ & $1.31 \mathrm{E}-03$ & $1.31 \mathrm{E}-03$ \\
\hline NI 63 & $1.71 \mathrm{E}-01$ & $1.71 \mathrm{E}-01$ & $1.71 \mathrm{E}-01$ & $1.71 \mathrm{E}-01$ & $1.71 \mathrm{E}-01$ & $1.71 \mathrm{E}-01$ & $1.71 \mathrm{E}-01$ & $1.71 \mathrm{E}-01$ & $1.71 \mathrm{E}-01$ \\
\hline NI 65 & $2.02 \mathrm{E}+00$ & $1.76 \mathrm{E}+00$ & $5.12 \mathrm{E}-01$ & $2.75 \mathrm{E}-03$ & $1.73 \mathrm{E}-20$ & $0.00 \mathrm{E}+00$ & $0.00 \mathrm{E}+00$ & $0.00 \mathrm{E}+00$ & $0.00 \mathrm{E}+00$ \\
\hline NI 66 & $6.93 \mathrm{E}-05$ & $6.89 \mathrm{E}-05$ & $6.51 \mathrm{E}-05$ & $5.11 \mathrm{E}-05$ & $8.22 \mathrm{E}-06$ & $9.75 \mathrm{E}-07$ & $7.45 \mathrm{E}-09$ & $8.01 \mathrm{E}-13$ & $8.60 \mathrm{E}-17$ \\
\hline CU 64 & $2.50 \mathrm{E}+00$ & $2.43 \mathrm{E}+00$ & $1.90 \mathrm{E}+00$ & $6.73 \mathrm{E}-01$ & $2.60 \mathrm{E}-04$ & $2.71 \mathrm{E}-08$ & $2.14 \mathrm{E}-17$ & $0.00 \mathrm{E}+00$ & $0.00 \mathrm{E}+00$ \\
\hline CU 66 & $5.45 \mathrm{E}-01$ & $9.31 \mathrm{E}-03$ & $6.52 \mathrm{E}-05$ & $5.12 \mathrm{E}-05$ & $8.23 \mathrm{E}-06$ & $9.76 \mathrm{E}-07$ & 7.46E-09 & $8.02 \mathrm{E}-13$ & $8.61 \mathrm{E}-17$ \\
\hline CU 67 & $3.52 \mathrm{E}-06$ & $3.50 \mathrm{E}-06$ & 3.33E-06 & $2.69 \mathrm{E}-06$ & 5.36E-07 & $8.16 \mathrm{E}-08$ & $1.11 \mathrm{E}-09$ & $3.46 \mathrm{E}-13$ & $1.09 \mathrm{E}-16$ \\
\hline ZN 65 & 1.66E-04 & $1.66 \mathrm{E}-04$ & $1.66 \mathrm{E}-04$ & $1.66 \mathrm{E}-04$ & 1.63E-04 & 1.60E-04 & $1.52 \mathrm{E}-04$ & 1.40E-04 & $1.29 \mathrm{E}-04$ \\
\hline ZN 69 & 2.55E-09 & 1.82E-09 & 2.04E-10 & 5.49E-11 & $3.88 \mathrm{E}-14$ & $8.21 \mathrm{E}-18$ & $0.00 \mathrm{E}+00$ & $0.00 \mathrm{E}+00$ & $0.00 \mathrm{E}+00$ \\
\hline ZN 69M & $1.71 \mathrm{E}-10$ & 1.67E-10 & $1.33 \mathrm{E}-10$ & $5.11 \mathrm{E}-11$ & $3.62 \mathrm{E}-14$ & 7.64E-18 & $3.04 \mathrm{E}-26$ & $0.00 \mathrm{E}+00$ & $0.00 \mathrm{E}+00$ \\
\hline GA 70 & 4.03E-12 & 1.50E-12 & $2.11 \mathrm{E}-16$ & $0.00 \mathrm{E}+00$ & $0.00 \mathrm{E}+00$ & $0.00 \mathrm{E}+00$ & $0.00 \mathrm{E}+00$ & $0.00 \mathrm{E}+00$ & $0.00 \mathrm{E}+00$ \\
\hline SR 89 & $6.39 \mathrm{E}-11$ & $6.38 \mathrm{E}-11$ & 6.37E-11 & 6.30E-11 & $5.80 \mathrm{E}-11$ & 5.27E-11 & $4.23 \mathrm{E}-11$ & $2.80 \mathrm{E}-11$ & $1.86 \mathrm{E}-11$ \\
\hline SR 90 & $1.56 \mathrm{E}-13$ & 1.56E-13 & $1.56 \mathrm{E}-13$ & $1.56 \mathrm{E}-13$ & $1.56 \mathrm{E}-13$ & $1.56 \mathrm{E}-13$ & $1.56 \mathrm{E}-13$ & 1.55E-13 & $1.55 \mathrm{E}-13$ \\
\hline SR 91 & $2.34 \mathrm{E}-12$ & $2.26 \mathrm{E}-12$ & $1.63 \mathrm{E}-12$ & $4.06 \mathrm{E}-13$ & 1.11E-17 & $5.28 \mathrm{E}-23$ & $0.00 \mathrm{E}+00$ & $0.00 \mathrm{E}+00$ & $0.00 E+00$ \\
\hline Y 89M & $1.59 \mathrm{E}-06$ & $1.58 \mathrm{E}-06$ & $1.52 \mathrm{E}-06$ & 1.29E-06 & $3.60 \mathrm{E}-07$ & 8.16E-08 & 2.74E-09 & $4.73 E-12$ & 8.14E-15 \\
\hline Y 90 & $2.12 \mathrm{E}-05$ & $2.11 \mathrm{E}-05$ & $2.01 \mathrm{E}-05$ & 1.64E-05 & $3.44 \mathrm{E}-06$ & 5.57E-07 & $8.71 \mathrm{E}-09$ & $3.73 \mathrm{E}-12$ & $1.57 \mathrm{E}-13$ \\
\hline Y 90M & $1.86 \mathrm{E}-12$ & $1.66 \mathrm{E}-12$ & $6.06 \mathrm{E}-13$ & $8.73 E-15$ & $9.07 \mathrm{E}-29$ & $0.00 \mathrm{E}+00$ & $0.00 \mathrm{E}+00$ & $0.00 \mathrm{E}+00$ & $0.00 \mathrm{E}+00$ \\
\hline Y 91 & 2.67E-09 & 2.67E-09 & 2.67E-09 & 2.64E-09 & 2.46E-09 & $2.26 \mathrm{E}-09$ & 1.87E-09 & $1.31 \mathrm{E}-09$ & $9.20 \mathrm{E}-10$ \\
\hline Y92 & 2.87E-10 & 2.60E-10 & $1.08 \mathrm{E}-10$ & $2.61 \mathrm{E}-12$ & 1.47E-24 & $0.00 \mathrm{E}+00$ & $0.00 \mathrm{E}+00$ & $0.00 \mathrm{E}+00$ & $0.00 \mathrm{E}+00$ \\
\hline Y94 & 1.66E-12 & 5.59E-13 & 3.10E-17 & $0.00 \mathrm{E}+00$ & $0.00 \mathrm{E}+00$ & $0.00 \mathrm{E}+00$ & $0.00 \mathrm{E}+00$ & $0.00 \mathrm{E}+00$ & $0.00 \mathrm{E}+00$ \\
\hline ZR 89 & $1.59 \mathrm{E}-06$ & $1.58 \mathrm{E}-06$ & $1.52 \mathrm{E}-06$ & 1.29E-06 & 3.61E-07 & 8.17E-08 & $2.75 \mathrm{E}-09$ & 4.73E-12 & $8.14 \mathrm{E}-15$ \\
\hline ZR 93 & $3.20 \mathrm{E}-11$ & $3.20 \mathrm{E}-11$ & $3.20 \mathrm{E}-11$ & 3.20E-11 & 3.20E-11 & $3.20 \mathrm{E}-11$ & 3.20E-11 & 3.20E-11 & 3.20E-11 \\
\hline ZR 95 & $3.98 \mathrm{E}-06$ & $3.98 \mathrm{E}-06$ & 3.97E-06 & $3.94 \mathrm{E}-06$ & 3.69E-06 & $3.42 \mathrm{E}-06$ & $2.88 \mathrm{E}-06$ & $2.08 \mathrm{E}-06$ & $1.50 \mathrm{E}-06$ \\
\hline ZR 97 & $1.26 \mathrm{E}-07$ & $1.23 \mathrm{E}-07$ & $1.02 \mathrm{E}-07$ & 4.70E-08 & $1.28 \mathrm{E}-10$ & $1.31 \mathrm{E}-13$ & $1.89 \mathrm{E}-20$ & $0.00 \mathrm{E}+00$ & $0.00 \mathrm{E}+00$ \\
\hline NB 92 & 3.17E-04 & $3.16 \mathrm{E}-04$ & $3.12 \mathrm{E}-04$ & $2.96 \mathrm{E}-04$ & 1.96E-04 & $1.22 \mathrm{E}-04$ & 4.09E-05 & $5.28 \mathrm{E}-06$ & $6.82 \mathrm{E}-07$ \\
\hline NB 93M & $2.51 \mathrm{E}-13$ & $2.51 \mathrm{E}-13$ & $2.52 \mathrm{E}-13$ & $2.56 \mathrm{E}-13$ & $2.81 \mathrm{E}-13$ & $3.10 \mathrm{E}-13$ & $3.77 \mathrm{E}-13$ & $5.02 \mathrm{E}-13$ & $6.27 \mathrm{E}-13$ \\
\hline NB 94 & 5.09E-06 & 5.09E-06 & 5.09E-06 & 5.09E-06 & 5.09E-06 & 5.09E-06 & 5.09E-06 & 5.09E-06 & $5.09 \mathrm{E}-06$ \\
\hline NB 95 & $1.47 \mathrm{E}-02$ & $1.47 \mathrm{E}-02$ & $1.46 \mathrm{E}-02$ & $1.44 \mathrm{E}-02$ & $1.28 \mathrm{E}-02$ & $1.12 \mathrm{E}-02$ & $8.14 \mathrm{E}-03$ & $4.50 \mathrm{E}-03$ & $2.49 \mathrm{E}-03$ \\
\hline NB 95M & $2.72 \mathrm{E}-08$ & $2.72 \mathrm{E}-08$ & $2.73 \mathrm{E}-08$ & $2.73 \mathrm{E}-08$ & $2.68 \mathrm{E}-08$ & $2.52 \mathrm{E}-08$ & $2.13 \mathrm{E}-08$ & $1.54 \mathrm{E}-08$ & $1.11 \mathrm{E}-08$ \\
\hline NB 96 & $7.70 \mathrm{E}-05$ & $7.59 \mathrm{E}-05$ & $6.64 \mathrm{E}-05$ & $3.78 \mathrm{E}-05$ & $5.26 \mathrm{E}-07$ & $3.59 \mathrm{E}-09$ & $4.02 \mathrm{E}-14$ & $2.10 \mathrm{E}-23$ & $0.00 \mathrm{E}+00$ \\
\hline NB 97 & 4.47E-06 & $3.38 \mathrm{E}-06$ & $3.52 \mathrm{E}-07$ & $4.72 \mathrm{E}-08$ & $1.29 \mathrm{E}-10$ & $1.31 \mathrm{E}-13$ & $2.03 \mathrm{E}-20$ & $0.00 \mathrm{E}+00$ & $0.00 E+00$ \\
\hline NB 97M & 1.19E-07 & 1.17E-07 & $9.70 \mathrm{E}-08$ & 4.45E-08 & $1.21 \mathrm{E}-10$ & $1.24 \mathrm{E}-13$ & $1.79 \mathrm{E}-20$ & $0.00 \mathrm{E}+00$ & $0.00 \mathrm{E}+00$ \\
\hline NB 98 & $6.65 \mathrm{E}-07$ & $0.00 \mathrm{E}+00$ & $0.00 \mathrm{E}+00$ & $0.00 \mathrm{E}+00$ & $0.00 \mathrm{E}+00$ & $0.00 \mathrm{E}+00$ & $0.00 \mathrm{E}+00$ & $0.00 \mathrm{E}+00$ & $0.00 E+00$ \\
\hline NB100 & $3.30 \mathrm{E}-08$ & $0.00 \mathrm{E}+00$ & $0.00 \mathrm{E}+00$ & $0.00 \mathrm{E}+00$ & $0.00 \mathrm{E}+00$ & $0.00 \mathrm{E}+00$ & $0.00 \mathrm{E}+00$ & $0.00 \mathrm{E}+00$ & $0.00 E+00$ \\
\hline MO 93M & $9.20 \mathrm{E}-05$ & $8.74 \mathrm{E}-05$ & $5.55 \mathrm{E}-05$ & $8.11 \mathrm{E}-06$ & $3.81 \mathrm{E}-12$ & $1.58 \mathrm{E}-19$ & $0.00 \mathrm{E}+00$ & $0.00 \mathrm{E}+00$ & $0.00 \mathrm{E}+00$ \\
\hline MO 93 & $1.83 \mathrm{E}-07$ & $1.83 \mathrm{E}-07$ & $1.83 \mathrm{E}-07$ & $1.83 \mathrm{E}-07$ & $1.83 \mathrm{E}-07$ & $1.83 \mathrm{E}-07$ & $1.83 \mathrm{E}-07$ & $1.83 \mathrm{E}-07$ & $1.83 \mathrm{E}-07$ \\
\hline
\end{tabular}


TEM-10200-1

ENGINEERING CALCULATIONS AND ANALYSIS

Page 35 of 81

$12 / 19 / 17$

Rev.08

Title:

AS-RUN NEUTRONICS EVALUATION FOR THE CSM-10584 EXPERIMENT IN THE ATR

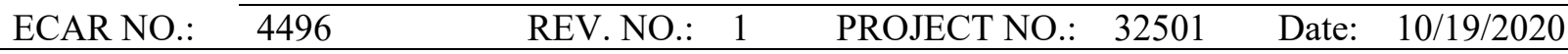

\begin{tabular}{|c|c|c|c|c|c|c|c|c|c|}
\hline \multicolumn{19}{|c|}{ Capsule C - 119 days irradiation } \\
\hline & $\mathrm{EOC}$ & $30 \mathrm{MINs}$ & $5 \mathrm{HRS}$ & $1 \mathrm{DAY}$ & $7 \mathrm{DAYS}$ & $14 \mathrm{DAYS}$ & 30 DAYS & 60 DAYS & $90 \mathrm{DAYS}$ \\
\hline ISOTOPE & $\mathrm{Ci}$ & $\mathrm{Ci}$ & $\mathrm{Ci}$ & $\mathrm{Ci}$ & $\mathrm{Ci}$ & $\mathrm{Ci}$ & $\mathrm{Ci}$ & $\mathrm{Ci}$ & $\mathrm{Ci}$ \\
\hline MO 99 & $6.44 \mathrm{E}-02$ & $6.40 \mathrm{E}-02$ & $6.11 \mathrm{E}-02$ & $5.00 \mathrm{E}-02$ & $1.10 \mathrm{E}-02$ & $1.89 \mathrm{E}-03$ & $3.35 \mathrm{E}-05$ & $1.74 \mathrm{E}-08$ & $9.05 \mathrm{E}-12$ \\
\hline MO101 & $1.60 \mathrm{E}-02$ & $3.86 \mathrm{E}-03$ & $1.07 \mathrm{E}-08$ & $0.00 \mathrm{E}+00$ & $0.00 \mathrm{E}+00$ & $0.00 \mathrm{E}+00$ & $0.00 \mathrm{E}+00$ & $0.00 \mathrm{E}+00$ & $0.00 \mathrm{E}+00$ \\
\hline TC 99 & $7.78 \mathrm{E}-09$ & $7.78 \mathrm{E}-09$ & $7.79 \mathrm{E}-09$ & $7.84 \mathrm{E}-09$ & $8.01 \mathrm{E}-09$ & $8.05 \mathrm{E}-09$ & $8.06 \mathrm{E}-09$ & $8.06 \mathrm{E}-09$ & $8.06 \mathrm{E}-09$ \\
\hline TC100 & $8.13 \mathrm{E}-04$ & $0.00 \mathrm{E}+00$ & $0.00 \mathrm{E}+00$ & $0.00 \mathrm{E}+00$ & $0.00 \mathrm{E}+00$ & $0.00 \mathrm{E}+00$ & $0.00 \mathrm{E}+00$ & $0.00 \mathrm{E}+00$ & $0.00 \mathrm{E}+00$ \\
\hline TC101 & $1.60 \mathrm{E}-02$ & $9.24 \mathrm{E}-03$ & $1.34 \mathrm{E}-07$ & $0.00 \mathrm{E}+00$ & $0.00 \mathrm{E}+00$ & $0.00 \mathrm{E}+00$ & $0.00 \mathrm{E}+00$ & $0.00 \mathrm{E}+00$ & $0.00 \mathrm{E}+00$ \\
\hline RU103 & $4.00 \mathrm{E}-07$ & $4.00 \mathrm{E}-07$ & $3.98 \mathrm{E}-07$ & $3.93 \mathrm{E}-07$ & $3.53 \mathrm{E}-07$ & $3.12 \mathrm{E}-07$ & $2.35 \mathrm{E}-07$ & $1.39 \mathrm{E}-07$ & $8.17 \mathrm{E}-08$ \\
\hline RU105 & $5.35 \mathrm{E}-12$ & $4.95 \mathrm{E}-12$ & $2.45 \mathrm{E}-12$ & $1.26 \mathrm{E}-13$ & $2.16 \mathrm{E}-23$ & $0.00 \mathrm{E}+00$ & $0.00 \mathrm{E}+00$ & $0.00 \mathrm{E}+00$ & $0.00 \mathrm{E}+00$ \\
\hline RH104 & $4.73 \mathrm{E}-09$ & $3.39 \mathrm{E}-12$ & $0.00 \mathrm{E}+00$ & $0.00 \mathrm{E}+00$ & $0.00 \mathrm{E}+00$ & $0.00 \mathrm{E}+00$ & $0.00 \mathrm{E}+00$ & $0.00 \mathrm{E}+00$ & $0.00 \mathrm{E}+00$ \\
\hline RH104M & $3.42 \mathrm{E}-10$ & $2.84 \mathrm{E}-12$ & $0.00 \mathrm{E}+00$ & $0.00 \mathrm{E}+00$ & $0.00 \mathrm{E}+00$ & $0.00 \mathrm{E}+00$ & $0.00 \mathrm{E}+00$ & $0.00 \mathrm{E}+00$ & $0.00 \mathrm{E}+00$ \\
\hline RH105 & $4.04 \mathrm{E}-12$ & $4.05 \mathrm{E}-12$ & $4.01 \mathrm{E}-12$ & $2.98 \mathrm{E}-12$ & $1.79 \mathrm{E}-13$ & $6.64 \mathrm{E}-15$ & $3.57 \mathrm{E}-18$ & $2.65 \mathrm{E}-24$ & $1.97 \mathrm{E}-30$ \\
\hline RH105M & $1.50 \mathrm{E}-12$ & $1.39 \mathrm{E}-12$ & $6.88 \mathrm{E}-13$ & $3.54 \mathrm{E}-14$ & $6.07 \mathrm{E}-24$ & $0.00 \mathrm{E}+00$ & $0.00 \mathrm{E}+00$ & $0.00 \mathrm{E}+00$ & $0.00 \mathrm{E}+00$ \\
\hline RH106 & $8.91 \mathrm{E}-13$ & $1.91 \mathrm{E}-18$ & $1.91 \mathrm{E}-18$ & $1.91 \mathrm{E}-18$ & $1.91 \mathrm{E}-18$ & $1.91 \mathrm{E}-18$ & $1.85 \mathrm{E}-18$ & $1.75 \mathrm{E}-18$ & $1.65 \mathrm{E}-18$ \\
\hline RH106M & $4.33 \mathrm{E}-13$ & $3.70 \mathrm{E}-13$ & $8.96 \mathrm{E}-14$ & $1.25 \mathrm{E}-16$ & $0.00 \mathrm{E}+00$ & $0.00 \mathrm{E}+00$ & $0.00 \mathrm{E}+00$ & $0.00 \mathrm{E}+00$ & $0.00 \mathrm{E}+00$ \\
\hline SUMTOT & $6.65 \mathrm{E}+02$ & $2.76 \mathrm{E}+02$ & $2.57 \mathrm{E}+02$ & $2.43 \mathrm{E}+02$ & $2.10 \mathrm{E}+02$ & $1.80 \mathrm{E}+02$ & $1.27 \mathrm{E}+02$ & $6.90 \mathrm{E}+01$ & $4.12 \mathrm{E}+01$ \\
\hline 0TOTAL & $6.65 \mathrm{E}+02$ & $2.76 \mathrm{E}+02$ & $2.57 \mathrm{E}+02$ & $2.43 \mathrm{E}+02$ & $2.10 \mathrm{E}+02$ & $1.80 \mathrm{E}+02$ & $1.27 \mathrm{E}+02$ & $6.90 \mathrm{E}+01$ & $4.12 \mathrm{E}+01$ \\
\hline
\end{tabular}

Table 16. Radionuclide source term for CSM capsule D after 119 days of irradiation (Ci).

\begin{tabular}{|c|c|c|c|c|c|c|c|c|c|}
\hline \multicolumn{10}{|c|}{ Capsule D - 119 days irradiation } \\
\hline & EOC & $30 \mathrm{MINs}$ & 5 HRS & $1 \mathrm{DAY}$ & 7 DAYS & 14 DAYS & 30 DAYS & 60 DAYS & 90 DAYS \\
\hline ISOTOPE & $\mathrm{Ci}$ & $\mathrm{Ci}$ & $\mathrm{Ci}$ & $\mathrm{Ci}$ & $\mathrm{Ci}$ & $\mathrm{Ci}$ & $\mathrm{Ci}$ & $\mathrm{Ci}$ & $\mathrm{Ci}$ \\
\hline H 3 & $7.25 \mathrm{E}-08$ & $7.25 \mathrm{E}-08$ & $7.25 \mathrm{E}-08$ & $7.25 \mathrm{E}-08$ & $7.24 \mathrm{E}-08$ & $7.23 \mathrm{E}-08$ & $7.21 \mathrm{E}-08$ & $7.18 \mathrm{E}-08$ & $7.15 \mathrm{E}-08$ \\
\hline H 4 & $6.18 \mathrm{E}-05$ & $0.00 \mathrm{E}+00$ & $0.00 \mathrm{E}+00$ & $0.00 \mathrm{E}+00$ & $0.00 \mathrm{E}+00$ & $0.00 \mathrm{E}+00$ & $0.00 \mathrm{E}+00$ & $0.00 \mathrm{E}+00$ & $0.00 \mathrm{E}+00$ \\
\hline HE 6 & $2.99 \mathrm{E}-05$ & $0.00 \mathrm{E}+00$ & $0.00 \mathrm{E}+00$ & $0.00 \mathrm{E}+00$ & $0.00 \mathrm{E}+00$ & $0.00 \mathrm{E}+00$ & $0.00 \mathrm{E}+00$ & $0.00 \mathrm{E}+00$ & $0.00 \mathrm{E}+00$ \\
\hline LI 8 & $2.48 \mathrm{E}-05$ & $0.00 \mathrm{E}+00$ & $0.00 \mathrm{E}+00$ & $0.00 \mathrm{E}+00$ & $0.00 \mathrm{E}+00$ & $0.00 \mathrm{E}+00$ & $0.00 \mathrm{E}+00$ & $0.00 \mathrm{E}+00$ & $0.00 \mathrm{E}+00$ \\
\hline BE 8 & 7.63E-05 & $0.00 \mathrm{E}+00$ & $0.00 \mathrm{E}+00$ & $0.00 \mathrm{E}+00$ & $0.00 \mathrm{E}+00$ & $0.00 \mathrm{E}+00$ & $0.00 \mathrm{E}+00$ & $0.00 \mathrm{E}+00$ & $0.00 \mathrm{E}+00$ \\
\hline BE 10 & $2.10 \mathrm{E}-10$ & $2.10 \mathrm{E}-10$ & $2.10 \mathrm{E}-10$ & $2.10 \mathrm{E}-10$ & $2.10 \mathrm{E}-10$ & $2.10 \mathrm{E}-10$ & $2.10 \mathrm{E}-10$ & $2.10 \mathrm{E}-10$ & $2.10 \mathrm{E}-10$ \\
\hline BE 11 & 1.66E-09 & $0.00 \mathrm{E}+00$ & $0.00 \mathrm{E}+00$ & $0.00 \mathrm{E}+00$ & $0.00 \mathrm{E}+00$ & $0.00 \mathrm{E}+00$ & $0.00 \mathrm{E}+00$ & $0.00 \mathrm{E}+00$ & $0.00 \mathrm{E}+00$ \\
\hline B 12 & 1.69E-05 & $0.00 \mathrm{E}+00$ & $0.00 \mathrm{E}+00$ & $0.00 \mathrm{E}+00$ & $0.00 \mathrm{E}+00$ & $0.00 \mathrm{E}+00$ & $0.00 \mathrm{E}+00$ & $0.00 \mathrm{E}+00$ & $0.00 \mathrm{E}+00$ \\
\hline C 14 & $7.68 \mathrm{E}-09$ & $7.68 \mathrm{E}-09$ & $7.68 \mathrm{E}-09$ & $7.68 \mathrm{E}-09$ & $7.68 \mathrm{E}-09$ & $7.68 \mathrm{E}-09$ & $7.68 \mathrm{E}-09$ & $7.68 \mathrm{E}-09$ & $7.68 \mathrm{E}-09$ \\
\hline C 15 & $1.84 \mathrm{E}-10$ & $0.00 \mathrm{E}+00$ & $0.00 \mathrm{E}+00$ & $0.00 \mathrm{E}+00$ & $0.00 \mathrm{E}+00$ & $0.00 \mathrm{E}+00$ & $0.00 \mathrm{E}+00$ & $0.00 \mathrm{E}+00$ & $0.00 \mathrm{E}+00$ \\
\hline O 19 & $1.31 \mathrm{E}-11$ & $0.00 \mathrm{E}+00$ & $0.00 \mathrm{E}+00$ & $0.00 \mathrm{E}+00$ & $0.00 \mathrm{E}+00$ & $0.00 \mathrm{E}+00$ & $0.00 \mathrm{E}+00$ & $0.00 \mathrm{E}+00$ & $0.00 \mathrm{E}+00$ \\
\hline $\mathrm{F} 20$ & $2.29 \mathrm{E}-08$ & $0.00 \mathrm{E}+00$ & $0.00 \mathrm{E}+00$ & $0.00 \mathrm{E}+00$ & $0.00 E+00$ & $0.00 \mathrm{E}+00$ & $0.00 \mathrm{E}+00$ & $0.00 \mathrm{E}+00$ & $0.00 \mathrm{E}+00$ \\
\hline NE 23 & 1.10E-02 & 3.09E-17 & $0.00 \mathrm{E}+00$ & $0.00 \mathrm{E}+00$ & $0.00 \mathrm{E}+00$ & $0.00 \mathrm{E}+00$ & $0.00 \mathrm{E}+00$ & $0.00 \mathrm{E}+00$ & $0.00 \mathrm{E}+00$ \\
\hline NA 24 & $1.75 \mathrm{E}+00$ & $1.71 \mathrm{E}+00$ & $1.39 \mathrm{E}+00$ & $5.76 \mathrm{E}-01$ & $7.45 \mathrm{E}-04$ & $3.17 \mathrm{E}-07$ & $6.23 \mathrm{E}-15$ & $2.21 \mathrm{E}-29$ & $0.00 \mathrm{E}+00$ \\
\hline NA 24M & 5.07E-06 & $0.00 \mathrm{E}+00$ & $0.00 \mathrm{E}+00$ & $0.00 \mathrm{E}+00$ & $0.00 \mathrm{E}+00$ & $0.00 \mathrm{E}+00$ & $0.00 \mathrm{E}+00$ & $0.00 \mathrm{E}+00$ & $0.00 \mathrm{E}+00$ \\
\hline NA 25 & $6.62 \mathrm{E}-03$ & 5.36E-12 & $0.00 \mathrm{E}+00$ & $0.00 \mathrm{E}+00$ & $0.00 \mathrm{E}+00$ & $0.00 \mathrm{E}+00$ & $0.00 \mathrm{E}+00$ & $0.00 \mathrm{E}+00$ & $0.00 \mathrm{E}+00$ \\
\hline MG 27 & $8.23 E+00$ & $9.14 \mathrm{E}-01$ & $2.35 \mathrm{E}-09$ & $0.00 \mathrm{E}+00$ & $0.00 E+00$ & $0.00 \mathrm{E}+00$ & $0.00 \mathrm{E}+00$ & $0.00 \mathrm{E}+00$ & $0.00 \mathrm{E}+00$ \\
\hline MG 28 & $2.93 \mathrm{E}-08$ & $2.88 \mathrm{E}-08$ & $2.48 \mathrm{E}-08$ & $1.32 \mathrm{E}-08$ & $1.12 \mathrm{E}-10$ & $4.26 \mathrm{E}-13$ & $1.26 \mathrm{E}-18$ & $5.45 \mathrm{E}-29$ & $0.00 \mathrm{E}+00$ \\
\hline AL 28 & $1.96 \mathrm{E}+02$ & $1.83 \mathrm{E}-02$ & $2.48 \mathrm{E}-08$ & $1.32 \mathrm{E}-08$ & $1.12 \mathrm{E}-10$ & $4.27 \mathrm{E}-13$ & $1.27 \mathrm{E}-18$ & $0.00 E+00$ & $0.00 E+00$ \\
\hline AL 29 & $2.02 \mathrm{E}-03$ & $8.34 \mathrm{E}-05$ & $2.85 \mathrm{E}-17$ & $0.00 \mathrm{E}+00$ & $0.00 E+00$ & $0.00 \mathrm{E}+00$ & $0.00 E+00$ & $0.00 \mathrm{E}+00$ & $0.00 E+00$ \\
\hline AL 30 & $1.96 \mathrm{E}-06$ & $0.00 \mathrm{E}+00$ & $0.00 \mathrm{E}+00$ & $0.00 \mathrm{E}+00$ & $0.00 E+00$ & $0.00 \mathrm{E}+00$ & $0.00 \mathrm{E}+00$ & $0.00 \mathrm{E}+00$ & $0.00 \mathrm{E}+00$ \\
\hline $\mathrm{SI} 31$ & 1.93E-02 & 1.69E-02 & 5.15E-03 & 3.39E-05 & $9.90 \mathrm{E}-22$ & $0.00 \mathrm{E}+00$ & $0.00 E+00$ & $0.00 \mathrm{E}+00$ & $0.00 E+00$ \\
\hline $\mathrm{SI} 32$ & $4.75 \mathrm{E}-12$ & $4.75 \mathrm{E}-12$ & $4.75 \mathrm{E}-12$ & $4.75 \mathrm{E}-12$ & $4.75 \mathrm{E}-12$ & $4.75 \mathrm{E}-12$ & $4.75 \mathrm{E}-12$ & $4.75 \mathrm{E}-12$ & $4.75 \mathrm{E}-12$ \\
\hline P 32 & $3.53 \mathrm{E}-03$ & $3.53 \mathrm{E}-03$ & $3.50 \mathrm{E}-03$ & $3.37 \mathrm{E}-03$ & $2.52 \mathrm{E}-03$ & $1.79 \mathrm{E}-03$ & $8.25 \mathrm{E}-04$ & 1.93E-04 & 4.50E-05 \\
\hline P 33 & $1.29 \mathrm{E}-07$ & $1.29 \mathrm{E}-07$ & $1.28 \mathrm{E}-07$ & $1.26 \mathrm{E}-07$ & $1.06 \mathrm{E}-07$ & $8.76 \mathrm{E}-08$ & $5.62 \mathrm{E}-08$ & $2.45 \mathrm{E}-08$ & $1.06 \mathrm{E}-08$ \\
\hline P 34 & $7.48 \mathrm{E}-07$ & $0.00 \mathrm{E}+00$ & $0.00 \mathrm{E}+00$ & $0.00 \mathrm{E}+00$ & $0.00 \mathrm{E}+00$ & $0.00 \mathrm{E}+00$ & $0.00 \mathrm{E}+00$ & $0.00 \mathrm{E}+00$ & $0.00 \mathrm{E}+00$ \\
\hline S 35 & $5.21 \mathrm{E}-05$ & $5.21 \mathrm{E}-05$ & $5.20 \mathrm{E}-05$ & $5.17 \mathrm{E}-05$ & $4.93 E-05$ & $4.67 \mathrm{E}-05$ & $4.11 \mathrm{E}-05$ & $3.25 \mathrm{E}-05$ & $2.56 \mathrm{E}-05$ \\
\hline S 37 & $2.48 \mathrm{E}-07$ & 4.07E-09 & $3.52 \mathrm{E}-25$ & $0.00 \mathrm{E}+00$ & $0.00 \mathrm{E}+00$ & $0.00 \mathrm{E}+00$ & $0.00 E+00$ & $0.00 \mathrm{E}+00$ & $0.00 \mathrm{E}+00$ \\
\hline $\mathrm{CL} 36$ & $2.97 \mathrm{E}-11$ & $2.97 \mathrm{E}-11$ & $2.97 \mathrm{E}-11$ & $2.97 \mathrm{E}-11$ & $2.97 \mathrm{E}-11$ & $2.97 \mathrm{E}-11$ & $2.97 \mathrm{E}-11$ & $2.97 \mathrm{E}-11$ & $2.97 \mathrm{E}-11$ \\
\hline $\mathrm{CL} 38$ & $3.84 \mathrm{E}-10$ & $2.20 \mathrm{E}-10$ & $1.44 \mathrm{E}-12$ & $8.65 \mathrm{E}-22$ & $0.00 \mathrm{E}+00$ & $0.00 \mathrm{E}+00$ & $0.00 \mathrm{E}+00$ & $0.00 \mathrm{E}+00$ & $0.00 \mathrm{E}+00$ \\
\hline $\mathrm{CL} 38 \mathrm{M}$ & $4.58 \mathrm{E}-12$ & $0.00 \mathrm{E}+00$ & $0.00 \mathrm{E}+00$ & $0.00 \mathrm{E}+00$ & $0.00 \mathrm{E}+00$ & $0.00 \mathrm{E}+00$ & $0.00 \mathrm{E}+00$ & $0.00 \mathrm{E}+00$ & $0.00 \mathrm{E}+00$ \\
\hline AR 39 & $9.41 \mathrm{E}-08$ & $9.41 \mathrm{E}-08$ & $9.41 \mathrm{E}-08$ & $9.41 \mathrm{E}-08$ & $9.41 \mathrm{E}-08$ & $9.41 \mathrm{E}-08$ & $9.41 \mathrm{E}-08$ & $9.41 \mathrm{E}-08$ & $9.41 \mathrm{E}-08$ \\
\hline AR 41 & $3.48 \mathrm{E}-08$ & $2.88 \mathrm{E}-08$ & $5.21 \mathrm{E}-09$ & $3.86 \mathrm{E}-12$ & $0.00 \mathrm{E}+00$ & $0.00 \mathrm{E}+00$ & $0.00 \mathrm{E}+00$ & $0.00 \mathrm{E}+00$ & $0.00 \mathrm{E}+00$ \\
\hline
\end{tabular}


TEM-10200-1

ENGINEERING CALCULATIONS AND ANALYSIS

Page 36 of 81

$12 / 19 / 17$

Rev.08

Title:

AS-RUN NEUTRONICS EVALUATION FOR THE CSM-10584 EXPERIMENT IN THE ATR

\begin{tabular}{llllllll} 
ECAR NO.: & 4496 & REV. NO.: & 1 & PROJECT NO.: & 32501 & Date: & 10/19/2020 \\
\hline
\end{tabular}

\begin{tabular}{|c|c|c|c|c|c|c|c|c|c|}
\hline \multicolumn{10}{|c|}{ Capsule D - 119 days irradiation } \\
\hline & EOC & $30 \mathrm{MINs}$ & $5 \mathrm{HRS}$ & $1 \mathrm{DAY}$ & 7 DAYS & 14 DAYS & 30 DAYS & 60 DAYS & 90 DAYS \\
\hline ISOTOPE & $\mathrm{Ci}$ & $\mathrm{Ci}$ & $\mathrm{Ci}$ & $\mathrm{Ci}$ & $\mathrm{Ci}$ & $\mathrm{Ci}$ & $\mathrm{Ci}$ & $\mathrm{Ci}$ & $\mathrm{Ci}$ \\
\hline $\mathrm{K} 40$ & $2.31 \mathrm{E}-13$ & $2.31 \mathrm{E}-13$ & $2.31 \mathrm{E}-13$ & $2.31 \mathrm{E}-13$ & $2.31 \mathrm{E}-13$ & $2.31 \mathrm{E}-13$ & $2.31 \mathrm{E}-13$ & $2.31 \mathrm{E}-13$ & $2.31 \mathrm{E}-13$ \\
\hline K 42 & $7.71 \mathrm{E}-05$ & $7.50 \mathrm{E}-05$ & 5.83E-05 & $2.01 \mathrm{E}-05$ & $6.25 \mathrm{E}-09$ & $5.06 \mathrm{E}-13$ & $4.28 \mathrm{E}-17$ & 4.27E-17 & $4.27 \mathrm{E}-17$ \\
\hline K 43 & $8.10 \mathrm{E}-11$ & $7.98 \mathrm{E}-11$ & $6.95 \mathrm{E}-11$ & $3.88 \mathrm{E}-11$ & $4.69 \mathrm{E}-13$ & $2.72 \mathrm{E}-15$ & $2.09 \mathrm{E}-20$ & $5.36 \mathrm{E}-30$ & $0.00 E+00$ \\
\hline K 44 & $1.44 \mathrm{E}-11$ & $5.58 \mathrm{E}-12$ & $1.13 \mathrm{E}-15$ & $0.00 \mathrm{E}+00$ & $0.00 \mathrm{E}+00$ & $0.00 \mathrm{E}+00$ & $0.00 \mathrm{E}+00$ & $0.00 \mathrm{E}+00$ & $0.00 \mathrm{E}+00$ \\
\hline CA 45 & $3.91 \mathrm{E}-07$ & $3.91 \mathrm{E}-07$ & $3.90 \mathrm{E}-07$ & $3.89 \mathrm{E}-07$ & $3.79 \mathrm{E}-07$ & $3.68 \mathrm{E}-07$ & $3.44 \mathrm{E}-07$ & $3.03 \mathrm{E}-07$ & $2.66 \mathrm{E}-07$ \\
\hline CA 47 & $6.79 \mathrm{E}-09$ & $6.77 \mathrm{E}-09$ & $6.57 \mathrm{E}-09$ & $5.82 \mathrm{E}-09$ & $2.33 \mathrm{E}-09$ & $7.99 \mathrm{E}-10$ & $6.93 \mathrm{E}-11$ & $7.07 \mathrm{E}-13$ & $7.22 \mathrm{E}-15$ \\
\hline SC 46 & $7.77 \mathrm{E}-05$ & $7.77 \mathrm{E}-05$ & $7.76 \mathrm{E}-05$ & $7.70 \mathrm{E}-05$ & $7.33 \mathrm{E}-05$ & $6.92 \mathrm{E}-05$ & $6.06 \mathrm{E}-05$ & $4.73 \mathrm{E}-05$ & $3.69 \mathrm{E}-05$ \\
\hline SC 46M & $2.21 \mathrm{E}-09$ & $0.00 \mathrm{E}+00$ & $0.00 \mathrm{E}+00$ & $0.00 \mathrm{E}+00$ & $0.00 \mathrm{E}+00$ & $0.00 \mathrm{E}+00$ & $0.00 \mathrm{E}+00$ & $0.00 \mathrm{E}+00$ & $0.00 \mathrm{E}+00$ \\
\hline SC 47 & $1.95 \mathrm{E}-04$ & $1.94 \mathrm{E}-04$ & $1.86 \mathrm{E}-04$ & $1.58 \mathrm{E}-04$ & $4.57 \mathrm{E}-05$ & $1.08 \mathrm{E}-05$ & $3.93 \mathrm{E}-07$ & $7.93 \mathrm{E}-10$ & $1.63 \mathrm{E}-12$ \\
\hline SC 48 & $1.92 \mathrm{E}-05$ & $1.91 \mathrm{E}-05$ & $1.78 \mathrm{E}-05$ & $1.32 \mathrm{E}-05$ & $1.35 \mathrm{E}-06$ & $9.45 \mathrm{E}-08$ & $2.17 \mathrm{E}-10$ & $2.45 \mathrm{E}-15$ & $2.76 \mathrm{E}-20$ \\
\hline SC 49 & 1.67E-05 & $1.16 \mathrm{E}-05$ & $4.49 \mathrm{E}-07$ & $4.83 \mathrm{E}-13$ & $0.00 \mathrm{E}+00$ & $0.00 \mathrm{E}+00$ & $0.00 \mathrm{E}+00$ & $0.00 \mathrm{E}+00$ & $0.00 \mathrm{E}+00$ \\
\hline SC 50 & $3.41 \mathrm{E}-07$ & $1.76 \mathrm{E}-12$ & $0.00 \mathrm{E}+00$ & $0.00 \mathrm{E}+00$ & $0.00 \mathrm{E}+00$ & $0.00 \mathrm{E}+00$ & $0.00 \mathrm{E}+00$ & $0.00 \mathrm{E}+00$ & $0.00 \mathrm{E}+00$ \\
\hline TI 51 & 1.17E-03 & 3.17E-05 & $2.46 \mathrm{E}-19$ & $0.00 \mathrm{E}+00$ & $0.00 \mathrm{E}+00$ & $0.00 \mathrm{E}+00$ & $0.00 \mathrm{E}+00$ & $0.00 \mathrm{E}+00$ & $0.00 \mathrm{E}+00$ \\
\hline V52 & $1.33 \mathrm{E}+00$ & 5.19E-03 & $1.10 \mathrm{E}-24$ & $0.00 \mathrm{E}+00$ & $0.00 \mathrm{E}+00$ & $0.00 \mathrm{E}+00$ & $0.00 \mathrm{E}+00$ & $0.00 \mathrm{E}+00$ & $0.00 \mathrm{E}+00$ \\
\hline$V 53$ & $6.12 \mathrm{E}-03$ & $1.50 \mathrm{E}-08$ & $0.00 \mathrm{E}+00$ & $0.00 \mathrm{E}+00$ & $0.00 \mathrm{E}+00$ & $0.00 \mathrm{E}+00$ & $0.00 E+00$ & $0.00 \mathrm{E}+00$ & $0.00 \mathrm{E}+00$ \\
\hline V54 & $6.54 \mathrm{E}-05$ & $9.19 \mathrm{E}-15$ & $0.00 \mathrm{E}+00$ & $0.00 \mathrm{E}+00$ & $0.00 \mathrm{E}+00$ & $0.00 \mathrm{E}+00$ & $0.00 \mathrm{E}+00$ & $0.00 \mathrm{E}+00$ & $0.00 \mathrm{E}+00$ \\
\hline CR 51 & $1.26 \mathrm{E}+02$ & $1.26 \mathrm{E}+02$ & $1.25 \mathrm{E}+02$ & $1.22 \mathrm{E}+02$ & $1.05 \mathrm{E}+02$ & $8.84 \mathrm{E}+01$ & $5.93 \mathrm{E}+01$ & $2.80 \mathrm{E}+01$ & $1.32 \mathrm{E}+01$ \\
\hline CR 55 & $1.79 \mathrm{E}+00$ & $5.12 \mathrm{E}-03$ & $0.00 \mathrm{E}+00$ & $0.00 \mathrm{E}+00$ & $0.00 \mathrm{E}+00$ & $0.00 \mathrm{E}+00$ & $0.00 \mathrm{E}+00$ & $0.00 \mathrm{E}+00$ & $0.00 \mathrm{E}+00$ \\
\hline $\mathrm{MN} 54$ & $1.90 \mathrm{E}+00$ & $1.90 \mathrm{E}+00$ & $1.90 \mathrm{E}+00$ & $1.89 \mathrm{E}+00$ & $1.87 \mathrm{E}+00$ & $1.84 \mathrm{E}+00$ & $1.77 \mathrm{E}+00$ & $1.66 \mathrm{E}+00$ & $1.55 \mathrm{E}+00$ \\
\hline MN 56 & $1.34 \mathrm{E}+01$ & $1.17 \mathrm{E}+01$ & $3.49 \mathrm{E}+00$ & $2.11 \mathrm{E}-02$ & 3.27E-19 & $0.00 \mathrm{E}+00$ & $0.00 \mathrm{E}+00$ & $0.00 \mathrm{E}+00$ & $0.00 \mathrm{E}+00$ \\
\hline MN 57 & $2.10 \mathrm{E}-02$ & 5.17E-08 & $0.00 \mathrm{E}+00$ & $0.00 \mathrm{E}+00$ & $0.00 \mathrm{E}+00$ & $0.00 \mathrm{E}+00$ & $0.00 \mathrm{E}+00$ & $0.00 \mathrm{E}+00$ & $0.00 \mathrm{E}+00$ \\
\hline $\mathrm{MN} 58$ & $6.35 \mathrm{E}-05$ & $3.20 \mathrm{E}-13$ & $0.00 \mathrm{E}+00$ & $0.00 \mathrm{E}+00$ & $0.00 \mathrm{E}+00$ & $0.00 \mathrm{E}+00$ & $0.00 \mathrm{E}+00$ & $0.00 \mathrm{E}+00$ & $0.00 \mathrm{E}+00$ \\
\hline FE 55 & $7.20 \mathrm{E}+00$ & $7.20 \mathrm{E}+00$ & $7.20 \mathrm{E}+00$ & $7.19 \mathrm{E}+00$ & $7.16 \mathrm{E}+00$ & $7.13 \mathrm{E}+00$ & $7.04 \mathrm{E}+00$ & $6.89 \mathrm{E}+00$ & $6.74 \mathrm{E}+00$ \\
\hline FE 59 & $2.11 \mathrm{E}+00$ & $2.11 \mathrm{E}+00$ & $2.11 \mathrm{E}+00$ & $2.08 \mathrm{E}+00$ & $1.90 \mathrm{E}+00$ & $1.70 \mathrm{E}+00$ & $1.33 \mathrm{E}+00$ & $8.38 \mathrm{E}-01$ & $5.28 \mathrm{E}-01$ \\
\hline CO 58 & $2.76 \mathrm{E}+00$ & $2.76 \mathrm{E}+00$ & $2.76 \mathrm{E}+00$ & $2.74 \mathrm{E}+00$ & $2.58 \mathrm{E}+00$ & $2.41 \mathrm{E}+00$ & $2.06 \mathrm{E}+00$ & $1.53 \mathrm{E}+00$ & $1.14 \mathrm{E}+00$ \\
\hline $\mathrm{CO} 60$ & $5.81 \mathrm{E}-02$ & $5.81 \mathrm{E}-02$ & $5.81 \mathrm{E}-02$ & $5.81 \mathrm{E}-02$ & $5.79 \mathrm{E}-02$ & $5.78 \mathrm{E}-02$ & $5.75 \mathrm{E}-02$ & $5.69 \mathrm{E}-02$ & $5.62 \mathrm{E}-02$ \\
\hline $\mathrm{CO} 60 \mathrm{M}$ & $8.19 \mathrm{E}-01$ & $1.12 \mathrm{E}-01$ & 1.94E-09 & $0.00 \mathrm{E}+00$ & $0.00 \mathrm{E}+00$ & $0.00 \mathrm{E}+00$ & $0.00 \mathrm{E}+00$ & $0.00 \mathrm{E}+00$ & $0.00 \mathrm{E}+00$ \\
\hline CO 61 & 9.76E-03 & 7.91E-03 & $1.20 \mathrm{E}-03$ & $4.08 \mathrm{E}-07$ & $0.00 \mathrm{E}+00$ & $0.00 \mathrm{E}+00$ & $0.00 \mathrm{E}+00$ & $0.00 \mathrm{E}+00$ & $0.00 \mathrm{E}+00$ \\
\hline $\mathrm{CO} 62$ & 9.77E-04 & $9.32 \mathrm{E}-10$ & $0.00 \mathrm{E}+00$ & $0.00 \mathrm{E}+00$ & $0.00 \mathrm{E}+00$ & $0.00 \mathrm{E}+00$ & $0.00 \mathrm{E}+00$ & $0.00 \mathrm{E}+00$ & $0.00 \mathrm{E}+00$ \\
\hline NI 59 & $7.38 \mathrm{E}-04$ & $7.38 \mathrm{E}-04$ & $7.38 \mathrm{E}-04$ & $7.38 \mathrm{E}-04$ & $7.38 \mathrm{E}-04$ & $7.38 \mathrm{E}-04$ & $7.38 \mathrm{E}-04$ & $7.38 \mathrm{E}-04$ & $7.38 \mathrm{E}-04$ \\
\hline NI 63 & $9.63 \mathrm{E}-02$ & $9.63 \mathrm{E}-02$ & $9.63 \mathrm{E}-02$ & $9.63 \mathrm{E}-02$ & $9.63 \mathrm{E}-02$ & $9.63 \mathrm{E}-02$ & $9.62 \mathrm{E}-02$ & $9.62 \mathrm{E}-02$ & $9.61 \mathrm{E}-02$ \\
\hline NI 65 & $1.14 \mathrm{E}+00$ & 9.93E-01 & $2.88 \mathrm{E}-01$ & 1.55E-03 & $9.73 \mathrm{E}-21$ & $0.00 \mathrm{E}+00$ & $0.00 \mathrm{E}+00$ & $0.00 \mathrm{E}+00$ & $0.00 \mathrm{E}+00$ \\
\hline $\mathrm{NI} 66$ & $3.91 \mathrm{E}-05$ & $3.88 \mathrm{E}-05$ & 3.67E-05 & $2.88 \mathrm{E}-05$ & 4.63E-06 & $5.49 \mathrm{E}-07$ & 4.20E-09 & $4.51 \mathrm{E}-13$ & 4.84E-17 \\
\hline CU 64 & $1.34 \mathrm{E}+00$ & $1.30 \mathrm{E}+00$ & $1.02 \mathrm{E}+00$ & $3.61 \mathrm{E}-01$ & $1.40 \mathrm{E}-04$ & $1.45 \mathrm{E}-08$ & 1.15E-17 & $0.00 \mathrm{E}+00$ & $0.00 \mathrm{E}+00$ \\
\hline CU 66 & $2.92 \mathrm{E}-01$ & 5.00E-03 & 3.67E-05 & $2.88 \mathrm{E}-05$ & 4.64E-06 & $5.50 \mathrm{E}-07$ & 4.20E-09 & $4.52 \mathrm{E}-13$ & $4.85 \mathrm{E}-17$ \\
\hline CU 67 & $1.89 \mathrm{E}-06$ & $1.88 \mathrm{E}-06$ & 1.79E-06 & $1.45 \mathrm{E}-06$ & $2.88 \mathrm{E}-07$ & $4.38 \mathrm{E}-08$ & $5.93 \mathrm{E}-10$ & $1.86 \mathrm{E}-13$ & 5.83E-17 \\
\hline ZN 65 & 8.91E-05 & 8.90E-05 & 8.90E-05 & $8.88 \mathrm{E}-05$ & 8.73E-05 & 8.56E-05 & $8.18 \mathrm{E}-05$ & 7.51E-05 & $6.90 \mathrm{E}-05$ \\
\hline ZN 69 & 1.37E-09 & 9.76E-10 & 1.10E-10 & 2.94E-11 & $2.08 \mathrm{E}-14$ & $4.40 \mathrm{E}-18$ & $0.00 \mathrm{E}+00$ & $0.00 \mathrm{E}+00$ & $0.00 \mathrm{E}+00$ \\
\hline ZN 69M & $9.18 \mathrm{E}-11$ & 8.95E-11 & 7.14E-11 & $2.74 \mathrm{E}-11$ & $1.94 \mathrm{E}-14$ & 4.10E-18 & 1.63E-26 & $0.00 \mathrm{E}+00$ & $0.00 \mathrm{E}+00$ \\
\hline GA 70 & $2.16 \mathrm{E}-12$ & 8.07E-13 & 1.13E-16 & $0.00 \mathrm{E}+00$ & $0.00 \mathrm{E}+00$ & $0.00 \mathrm{E}+00$ & $0.00 \mathrm{E}+00$ & $0.00 \mathrm{E}+00$ & $0.00 \mathrm{E}+00$ \\
\hline SR 89 & $2.70 \mathrm{E}-11$ & $2.70 \mathrm{E}-11$ & 2.69E-11 & $2.66 \mathrm{E}-11$ & $2.45 \mathrm{E}-11$ & $2.23 \mathrm{E}-11$ & 1.79E-11 & 1.18E-11 & $7.85 \mathrm{E}-12$ \\
\hline SR 90 & $6.60 \mathrm{E}-14$ & $6.60 \mathrm{E}-14$ & $6.60 \mathrm{E}-14$ & $6.60 \mathrm{E}-14$ & $6.60 \mathrm{E}-14$ & $6.60 \mathrm{E}-14$ & $6.59 \mathrm{E}-14$ & $6.58 \mathrm{E}-14$ & $6.56 \mathrm{E}-14$ \\
\hline SR 91 & $9.89 \mathrm{E}-13$ & $9.54 \mathrm{E}-13$ & $6.87 \mathrm{E}-13$ & $1.72 \mathrm{E}-13$ & $4.70 \mathrm{E}-18$ & $2.23 \mathrm{E}-23$ & $0.00 \mathrm{E}+00$ & $0.00 \mathrm{E}+00$ & $0.00 \mathrm{E}+00$ \\
\hline Y $89 M$ & $6.72 \mathrm{E}-07$ & $6.69 \mathrm{E}-07$ & $6.43 \mathrm{E}-07$ & $5.43 \mathrm{E}-07$ & $1.52 \mathrm{E}-07$ & $3.45 \mathrm{E}-08$ & 1.16E-09 & $2.00 \mathrm{E}-12$ & $3.45 \mathrm{E}-15$ \\
\hline Y 90 & $8.98 \mathrm{E}-06$ & $8.93 \mathrm{E}-06$ & $8.50 \mathrm{E}-06$ & $6.92 \mathrm{E}-06$ & $1.46 \mathrm{E}-06$ & $2.36 \mathrm{E}-07$ & 3.69E-09 & $1.58 \mathrm{E}-12$ & $6.63 \mathrm{E}-14$ \\
\hline Y 90M & $7.84 \mathrm{E}-13$ & $7.01 \mathrm{E}-13$ & $2.56 \mathrm{E}-13$ & 3.69E-15 & $3.83 \mathrm{E}-29$ & $0.00 \mathrm{E}+00$ & $0.00 \mathrm{E}+00$ & $0.00 \mathrm{E}+00$ & $0.00 \mathrm{E}+00$ \\
\hline Y 91 & $1.13 \mathrm{E}-09$ & $1.13 \mathrm{E}-09$ & $1.13 \mathrm{E}-09$ & $1.12 \mathrm{E}-09$ & 1.04E-09 & $9.58 \mathrm{E}-10$ & $7.93 \mathrm{E}-10$ & $5.56 \mathrm{E}-10$ & $3.89 \mathrm{E}-10$ \\
\hline Y 92 & $1.21 \mathrm{E}-10$ & $1.10 \mathrm{E}-10$ & $4.56 \mathrm{E}-11$ & $1.10 \mathrm{E}-12$ & $6.21 \mathrm{E}-25$ & $0.00 \mathrm{E}+00$ & $0.00 \mathrm{E}+00$ & $0.00 \mathrm{E}+00$ & $0.00 E+00$ \\
\hline Y 94 & $7.01 \mathrm{E}-13$ & $2.36 \mathrm{E}-13$ & $1.31 \mathrm{E}-17$ & $0.00 \mathrm{E}+00$ & $0.00 \mathrm{E}+00$ & $0.00 \mathrm{E}+00$ & $0.00 \mathrm{E}+00$ & $0.00 \mathrm{E}+00$ & $0.00 \mathrm{E}+00$ \\
\hline ZR 89 & $6.73 \mathrm{E}-07$ & $6.70 \mathrm{E}-07$ & $6.44 \mathrm{E}-07$ & $5.44 \mathrm{E}-07$ & $1.52 \mathrm{E}-07$ & $3.45 \mathrm{E}-08$ & $1.16 \mathrm{E}-09$ & $2.00 \mathrm{E}-12$ & $3.44 \mathrm{E}-15$ \\
\hline ZR 93 & $1.35 \mathrm{E}-11$ & $1.35 \mathrm{E}-11$ & 1.35E-11 & 1.35E-11 & $1.35 \mathrm{E}-11$ & $1.35 \mathrm{E}-11$ & $1.35 \mathrm{E}-11$ & 1.35E-11 & $1.35 \mathrm{E}-11$ \\
\hline ZR 95 & $1.68 \mathrm{E}-06$ & $1.68 \mathrm{E}-06$ & $1.68 \mathrm{E}-06$ & $1.66 \mathrm{E}-06$ & $1.56 \mathrm{E}-06$ & $1.45 \mathrm{E}-06$ & $1.22 \mathrm{E}-06$ & $8.78 \mathrm{E}-07$ & $6.35 \mathrm{E}-07$ \\
\hline ZR 97 & $5.32 \mathrm{E}-08$ & $5.21 \mathrm{E}-08$ & $4.33 \mathrm{E}-08$ & $1.99 \mathrm{E}-08$ & $5.41 \mathrm{E}-11$ & 5.50E-14 & $7.95 \mathrm{E}-21$ & $0.00 \mathrm{E}+00$ & $0.00 E+00$ \\
\hline NB 92 & $1.34 \mathrm{E}-04$ & $1.34 \mathrm{E}-04$ & $1.32 \mathrm{E}-04$ & $1.25 \mathrm{E}-04$ & $8.30 \mathrm{E}-05$ & $5.15 \mathrm{E}-05$ & $1.73 \mathrm{E}-05$ & $2.23 \mathrm{E}-06$ & $2.88 \mathrm{E}-07$ \\
\hline NB 93M & $1.06 \mathrm{E}-13$ & $1.06 \mathrm{E}-13$ & $1.07 \mathrm{E}-13$ & $1.08 \mathrm{E}-13$ & $1.19 \mathrm{E}-13$ & $1.31 \mathrm{E}-13$ & 1.60E-13 & $2.13 \mathrm{E}-13$ & $2.65 \mathrm{E}-13$ \\
\hline
\end{tabular}


TEM-10200-1

ENGINEERING CALCULATIONS AND ANALYSIS

Page 37 of 81

$12 / 19 / 17$

Rev.08

Title:

AS-RUN NEUTRONICS EVALUATION FOR THE CSM-10584 EXPERIMENT IN THE ATR

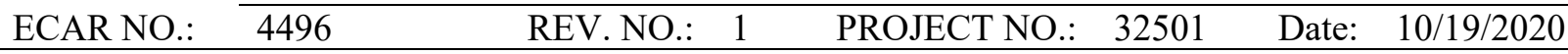

\begin{tabular}{|c|c|c|c|c|c|c|c|c|c|}
\hline \multicolumn{10}{|c|}{ Capsule D - 119 days irradiation } \\
\hline & EOC & $30 \mathrm{MINs}$ & $5 \mathrm{HRS}$ & 1 DAY & 7 DAYS & 14 DAYS & 30 DAYS & 60 DAYS & 90 DAYS \\
\hline ISOTOPE & $\mathrm{Ci}$ & $\mathrm{Ci}$ & $\mathrm{Ci}$ & $\mathrm{Ci}$ & $\mathrm{Ci}$ & $\mathrm{Ci}$ & $\mathrm{Ci}$ & $\mathrm{Ci}$ & $\mathrm{Ci}$ \\
\hline NB 94 & $2.16 \mathrm{E}-06$ & $2.16 \mathrm{E}-06$ & $2.16 \mathrm{E}-06$ & $2.16 \mathrm{E}-06$ & $2.16 \mathrm{E}-06$ & $2.16 \mathrm{E}-06$ & $2.16 \mathrm{E}-06$ & $2.16 \mathrm{E}-06$ & $2.16 \mathrm{E}-06$ \\
\hline NB 95 & $6.22 \mathrm{E}-03$ & $6.22 \mathrm{E}-03$ & $6.20 \mathrm{E}-03$ & $6.10 \mathrm{E}-03$ & $5.42 \mathrm{E}-03$ & $4.72 \mathrm{E}-03$ & $3.44 \mathrm{E}-03$ & $1.91 \mathrm{E}-03$ & $1.06 \mathrm{E}-03$ \\
\hline NB 95M & $1.15 \mathrm{E}-08$ & $1.15 \mathrm{E}-08$ & $1.15 \mathrm{E}-08$ & $1.15 \mathrm{E}-08$ & $1.13 \mathrm{E}-08$ & 1.07E-08 & $9.01 \mathrm{E}-09$ & $6.52 \mathrm{E}-09$ & $4.71 \mathrm{E}-09$ \\
\hline NB 96 & $3.26 \mathrm{E}-05$ & $3.21 \mathrm{E}-05$ & $2.81 \mathrm{E}-05$ & $1.60 \mathrm{E}-05$ & $2.23 \mathrm{E}-07$ & $1.52 \mathrm{E}-09$ & 1.70E-14 & $8.88 \mathrm{E}-24$ & $0.00 \mathrm{E}+00$ \\
\hline NB 97 & $1.89 \mathrm{E}-06$ & $1.43 \mathrm{E}-06$ & $1.49 \mathrm{E}-07$ & $2.00 \mathrm{E}-08$ & $5.44 \mathrm{E}-11$ & $5.53 \mathrm{E}-14$ & 8.57E-21 & $0.00 \mathrm{E}+00$ & $0.00 \mathrm{E}+00$ \\
\hline NB 97M & 5.03E-08 & 4.93E-08 & 4.10E-08 & $1.88 \mathrm{E}-08$ & $5.13 \mathrm{E}-11$ & $5.22 \mathrm{E}-14$ & $7.53 \mathrm{E}-21$ & $0.00 \mathrm{E}+00$ & $0.00 \mathrm{E}+00$ \\
\hline NB 98 & $2.81 \mathrm{E}-07$ & $0.00 \mathrm{E}+00$ & $0.00 \mathrm{E}+00$ & $0.00 \mathrm{E}+00$ & $0.00 \mathrm{E}+00$ & $0.00 \mathrm{E}+00$ & $0.00 \mathrm{E}+00$ & $0.00 \mathrm{E}+00$ & $0.00 \mathrm{E}+00$ \\
\hline NB100 & $1.40 \mathrm{E}-08$ & $0.00 \mathrm{E}+00$ & $0.00 \mathrm{E}+00$ & $0.00 \mathrm{E}+00$ & $0.00 \mathrm{E}+00$ & $0.00 \mathrm{E}+00$ & $0.00 \mathrm{E}+00$ & $0.00 \mathrm{E}+00$ & $0.00 \mathrm{E}+00$ \\
\hline MO 93M & $3.89 \mathrm{E}-05$ & $3.70 \mathrm{E}-05$ & $2.34 \mathrm{E}-05$ & $3.43 \mathrm{E}-06$ & $1.61 \mathrm{E}-12$ & 6.67E-20 & $0.00 \mathrm{E}+00$ & $0.00 \mathrm{E}+00$ & $0.00 \mathrm{E}+00$ \\
\hline MO 93 & $7.72 \mathrm{E}-08$ & $7.72 \mathrm{E}-08$ & $7.72 \mathrm{E}-08$ & $7.72 \mathrm{E}-08$ & $7.72 \mathrm{E}-08$ & $7.72 \mathrm{E}-08$ & $7.72 \mathrm{E}-08$ & $7.72 \mathrm{E}-08$ & $7.72 \mathrm{E}-08$ \\
\hline MO 99 & $2.72 \mathrm{E}-02$ & $2.71 \mathrm{E}-02$ & $2.58 \mathrm{E}-02$ & $2.11 \mathrm{E}-02$ & $4.66 \mathrm{E}-03$ & $7.98 \mathrm{E}-04$ & $1.42 \mathrm{E}-05$ & $7.36 \mathrm{E}-09$ & $3.83 \mathrm{E}-12$ \\
\hline M0101 & $6.77 \mathrm{E}-03$ & $1.63 \mathrm{E}-03$ & 4.50E-09 & $0.00 \mathrm{E}+00$ & $0.00 \mathrm{E}+00$ & $0.00 \mathrm{E}+00$ & $0.00 \mathrm{E}+00$ & $0.00 \mathrm{E}+00$ & $0.00 \mathrm{E}+00$ \\
\hline TC 99 & 3.29E-09 & 3.29E-09 & 3.29E-09 & $3.31 \mathrm{E}-09$ & 3.39E-09 & 3.40E-09 & $3.41 \mathrm{E}-09$ & $3.41 \mathrm{E}-09$ & $3.41 \mathrm{E}-09$ \\
\hline TC100 & 3.44E-04 & $0.00 \mathrm{E}+00$ & $0.00 \mathrm{E}+00$ & $0.00 \mathrm{E}+00$ & $0.00 \mathrm{E}+00$ & $0.00 \mathrm{E}+00$ & $0.00 \mathrm{E}+00$ & $0.00 \mathrm{E}+00$ & $0.00 E+00$ \\
\hline TC101 & $6.77 \mathrm{E}-03$ & $3.90 \mathrm{E}-03$ & $5.68 \mathrm{E}-08$ & $0.00 \mathrm{E}+00$ & $0.00 \mathrm{E}+00$ & $0.00 \mathrm{E}+00$ & $0.00 \mathrm{E}+00$ & $0.00 \mathrm{E}+00$ & $0.00 \mathrm{E}+00$ \\
\hline RU103 & $1.69 \mathrm{E}-07$ & 1.69E-07 & $1.68 \mathrm{E}-07$ & $1.66 \mathrm{E}-07$ & 1.49E-07 & $1.32 \mathrm{E}-07$ & $9.95 \mathrm{E}-08$ & $5.86 \mathrm{E}-08$ & $3.45 \mathrm{E}-08$ \\
\hline RU105 & $2.26 \mathrm{E}-12$ & $2.09 \mathrm{E}-12$ & $1.04 \mathrm{E}-12$ & $5.33 \mathrm{E}-14$ & $9.14 \mathrm{E}-24$ & $0.00 \mathrm{E}+00$ & $0.00 \mathrm{E}+00$ & $0.00 \mathrm{E}+00$ & $0.00 \mathrm{E}+00$ \\
\hline $\mathrm{RH} 104$ & 2.00E-09 & 1.43E-12 & $0.00 \mathrm{E}+00$ & $0.00 \mathrm{E}+00$ & $0.00 \mathrm{E}+00$ & $0.00 \mathrm{E}+00$ & $0.00 \mathrm{E}+00$ & $0.00 \mathrm{E}+00$ & $0.00 \mathrm{E}+00$ \\
\hline RH104M & $1.45 \mathrm{E}-10$ & 1.20E-12 & $0.00 \mathrm{E}+00$ & $0.00 \mathrm{E}+00$ & $0.00 \mathrm{E}+00$ & $0.00 \mathrm{E}+00$ & $0.00 \mathrm{E}+00$ & $0.00 E+00$ & $0.00 \mathrm{E}+00$ \\
\hline RH105 & $1.71 \mathrm{E}-12$ & $1.71 \mathrm{E}-12$ & 1.69E-12 & $1.26 \mathrm{E}-12$ & $7.54 \mathrm{E}-14$ & $2.80 \mathrm{E}-15$ & $1.51 \mathrm{E}-18$ & $1.12 \mathrm{E}-24$ & $0.00 \mathrm{E}+00$ \\
\hline RH105M & $6.33 \mathrm{E}-13$ & 5.87E-13 & $2.91 \mathrm{E}-13$ & $1.50 \mathrm{E}-14$ & $2.57 \mathrm{E}-24$ & $0.00 \mathrm{E}+00$ & $0.00 \mathrm{E}+00$ & $0.00 E+00$ & $0.00 \mathrm{E}+00$ \\
\hline RH106 & $3.77 \mathrm{E}-13$ & 7.37E-19 & 7.37E-19 & $7.37 \mathrm{E}-19$ & $7.37 \mathrm{E}-19$ & 7.37E-19 & 7.37E-19 & $6.97 \mathrm{E}-19$ & $6.57 \mathrm{E}-19$ \\
\hline SUMTOT & $3.66 \mathrm{E}+02$ & $1.56 \mathrm{E}+02$ & $1.45 \mathrm{E}+02$ & $1.38 \mathrm{E}+02$ & $1.19 \mathrm{E}+02$ & $1.02 \mathrm{E}+02$ & $7.16 \mathrm{E}+01$ & $3.91 \mathrm{E}+01$ & $2.33 \mathrm{E}+01$ \\
\hline OTOTAL & $3.66 \mathrm{E}+02$ & $1.56 \mathrm{E}+02$ & $1.45 \mathrm{E}+02$ & $1.38 \mathrm{E}+02$ & $1.19 \mathrm{E}+02$ & $1.02 \mathrm{E}+02$ & $7.16 \mathrm{E}+01$ & $3.91 \mathrm{E}+01$ & $2.33 \mathrm{E}+01$ \\
\hline
\end{tabular}

Table 17. Radionuclide source term for CSM capsule E after 54.9 days of irradiation (Ci).

\begin{tabular}{|c|c|c|c|c|c|c|c|c|c|}
\hline \multicolumn{10}{|c|}{ Capsule E - 54.9 days irradiation } \\
\hline & EOC & $30 \mathrm{MINs}$ & $5 \mathrm{HRS}$ & $1 \mathrm{DAY}$ & 7 DAYS & 14 DAYS & 30 DAYS & 60 DAYS & 90 DAYS \\
\hline ISOTOPE & $\mathrm{Ci}$ & $\mathrm{Ci}$ & $\mathrm{Ci}$ & $\mathrm{Ci}$ & $\mathrm{Ci}$ & $\mathrm{Ci}$ & $\mathrm{Ci}$ & $\mathrm{Ci}$ & $\mathrm{Ci}$ \\
\hline H 3 & $8.04 \mathrm{E}-09$ & $8.04 \mathrm{E}-09$ & $8.04 \mathrm{E}-09$ & $8.04 \mathrm{E}-09$ & $8.03 \mathrm{E}-09$ & $8.02 \mathrm{E}-09$ & $8.01 \mathrm{E}-09$ & $7.97 \mathrm{E}-09$ & $7.93 \mathrm{E}-09$ \\
\hline H 4 & $5.57 \mathrm{E}-05$ & $0.00 \mathrm{E}+00$ & $0.00 \mathrm{E}+00$ & $0.00 \mathrm{E}+00$ & $0.00 \mathrm{E}+00$ & $0.00 \mathrm{E}+00$ & $0.00 \mathrm{E}+00$ & $0.00 \mathrm{E}+00$ & $0.00 \mathrm{E}+00$ \\
\hline HE 6 & $1.38 \mathrm{E}-05$ & $0.00 \mathrm{E}+00$ & $0.00 \mathrm{E}+00$ & $0.00 \mathrm{E}+00$ & $0.00 \mathrm{E}+00$ & $0.00 \mathrm{E}+00$ & $0.00 \mathrm{E}+00$ & $0.00 \mathrm{E}+00$ & $0.00 \mathrm{E}+00$ \\
\hline LI 8 & $2.24 \mathrm{E}-05$ & $0.00 \mathrm{E}+00$ & $0.00 \mathrm{E}+00$ & $0.00 \mathrm{E}+00$ & $0.00 \mathrm{E}+00$ & $0.00 \mathrm{E}+00$ & $0.00 \mathrm{E}+00$ & $0.00 \mathrm{E}+00$ & $0.00 \mathrm{E}+00$ \\
\hline BE 8 & 4.62E-05 & $0.00 \mathrm{E}+00$ & $0.00 \mathrm{E}+00$ & $0.00 \mathrm{E}+00$ & $0.00 \mathrm{E}+00$ & $0.00 \mathrm{E}+00$ & $0.00 \mathrm{E}+00$ & $0.00 \mathrm{E}+00$ & $0.00 \mathrm{E}+00$ \\
\hline BE 10 & $9.70 \mathrm{E}-11$ & $9.70 \mathrm{E}-11$ & $9.70 \mathrm{E}-11$ & $9.70 \mathrm{E}-11$ & $9.70 \mathrm{E}-11$ & $9.70 \mathrm{E}-11$ & $9.70 \mathrm{E}-11$ & $9.70 \mathrm{E}-11$ & $9.70 \mathrm{E}-11$ \\
\hline BE 11 & $7.67 \mathrm{E}-10$ & $0.00 \mathrm{E}+00$ & $0.00 \mathrm{E}+00$ & $0.00 \mathrm{E}+00$ & $0.00 \mathrm{E}+00$ & $0.00 \mathrm{E}+00$ & $0.00 E+00$ & $0.00 \mathrm{E}+00$ & $0.00 \mathrm{E}+00$ \\
\hline B 12 & $1.69 \mathrm{E}-05$ & $0.00 \mathrm{E}+00$ & $0.00 \mathrm{E}+00$ & $0.00 \mathrm{E}+00$ & $0.00 \mathrm{E}+00$ & $0.00 \mathrm{E}+00$ & $0.00 \mathrm{E}+00$ & $0.00 \mathrm{E}+00$ & $0.00 \mathrm{E}+00$ \\
\hline C 14 & 3.54E-09 & $3.54 \mathrm{E}-09$ & $3.54 \mathrm{E}-09$ & $3.54 \mathrm{E}-09$ & 3.54E-09 & $3.54 \mathrm{E}-09$ & $3.54 \mathrm{E}-09$ & 3.54E-09 & $3.54 \mathrm{E}-09$ \\
\hline C 15 & $3.92 \mathrm{E}-11$ & $0.00 E+00$ & $0.00 E+00$ & $0.00 E+00$ & $0.00 \mathrm{E}+00$ & $0.00 \mathrm{E}+00$ & $0.00 \mathrm{E}+00$ & $0.00 \mathrm{E}+00$ & $0.00 \mathrm{E}+00$ \\
\hline O 19 & $2.79 \mathrm{E}-12$ & $0.00 \mathrm{E}+00$ & $0.00 \mathrm{E}+00$ & $0.00 \mathrm{E}+00$ & $0.00 \mathrm{E}+00$ & $0.00 \mathrm{E}+00$ & $0.00 \mathrm{E}+00$ & $0.00 \mathrm{E}+00$ & $0.00 \mathrm{E}+00$ \\
\hline$F 20$ & $1.06 \mathrm{E}-08$ & $0.00 \mathrm{E}+00$ & $0.00 \mathrm{E}+00$ & $0.00 \mathrm{E}+00$ & $0.00 \mathrm{E}+00$ & $0.00 \mathrm{E}+00$ & $0.00 \mathrm{E}+00$ & $0.00 \mathrm{E}+00$ & $0.00 \mathrm{E}+00$ \\
\hline NE 23 & 1.10E-02 & $3.09 \mathrm{E}-17$ & $0.00 \mathrm{E}+00$ & $0.00 \mathrm{E}+00$ & $0.00 \mathrm{E}+00$ & $0.00 \mathrm{E}+00$ & $0.00 \mathrm{E}+00$ & $0.00 \mathrm{E}+00$ & $0.00 \mathrm{E}+00$ \\
\hline NA 24 & $1.75 \mathrm{E}+00$ & $1.71 \mathrm{E}+00$ & $1.39 \mathrm{E}+00$ & $5.76 \mathrm{E}-01$ & $7.43 \mathrm{E}-04$ & $3.16 \mathrm{E}-07$ & $6.21 \mathrm{E}-15$ & $2.21 \mathrm{E}-29$ & $0.00 \mathrm{E}+00$ \\
\hline NA 24M & $2.34 \mathrm{E}-06$ & $0.00 \mathrm{E}+00$ & $0.00 \mathrm{E}+00$ & $0.00 \mathrm{E}+00$ & $0.00 \mathrm{E}+00$ & $0.00 \mathrm{E}+00$ & $0.00 \mathrm{E}+00$ & $0.00 \mathrm{E}+00$ & $0.00 \mathrm{E}+00$ \\
\hline NA 25 & $6.62 \mathrm{E}-03$ & $5.36 \mathrm{E}-12$ & $0.00 \mathrm{E}+00$ & $0.00 \mathrm{E}+00$ & $0.00 \mathrm{E}+00$ & $0.00 \mathrm{E}+00$ & $0.00 \mathrm{E}+00$ & $0.00 \mathrm{E}+00$ & $0.00 \mathrm{E}+00$ \\
\hline MG 27 & $8.23 \mathrm{E}+00$ & $9.14 \mathrm{E}-01$ & $2.35 \mathrm{E}-09$ & $0.00 \mathrm{E}+00$ & $0.00 \mathrm{E}+00$ & $0.00 \mathrm{E}+00$ & $0.00 \mathrm{E}+00$ & $0.00 \mathrm{E}+00$ & $0.00 \mathrm{E}+00$ \\
\hline MG 28 & $2.93 \mathrm{E}-08$ & $2.88 \mathrm{E}-08$ & $2.48 \mathrm{E}-08$ & $1.32 \mathrm{E}-08$ & $1.12 \mathrm{E}-10$ & $4.26 \mathrm{E}-13$ & $1.26 \mathrm{E}-18$ & $5.45 \mathrm{E}-29$ & $0.00 \mathrm{E}+00$ \\
\hline AL 28 & $1.96 \mathrm{E}+02$ & $1.83 \mathrm{E}-02$ & $2.48 \mathrm{E}-08$ & $1.32 \mathrm{E}-08$ & $1.12 \mathrm{E}-10$ & $4.27 \mathrm{E}-13$ & $1.27 \mathrm{E}-18$ & $0.00 \mathrm{E}+00$ & $0.00 \mathrm{E}+00$ \\
\hline AL 29 & $2.02 \mathrm{E}-03$ & $8.32 \mathrm{E}-05$ & $2.85 \mathrm{E}-17$ & $0.00 \mathrm{E}+00$ & $0.00 \mathrm{E}+00$ & $0.00 \mathrm{E}+00$ & $0.00 \mathrm{E}+00$ & $0.00 \mathrm{E}+00$ & $0.00 \mathrm{E}+00$ \\
\hline AL 30 & $1.96 \mathrm{E}-06$ & $0.00 \mathrm{E}+00$ & $0.00 \mathrm{E}+00$ & $0.00 \mathrm{E}+00$ & $0.00 \mathrm{E}+00$ & $0.00 \mathrm{E}+00$ & $0.00 \mathrm{E}+00$ & $0.00 \mathrm{E}+00$ & $0.00 \mathrm{E}+00$ \\
\hline SI 31 & $1.93 \mathrm{E}-02$ & $1.69 \mathrm{E}-02$ & $5.15 \mathrm{E}-03$ & $3.39 \mathrm{E}-05$ & $9.90 \mathrm{E}-22$ & $0.00 \mathrm{E}+00$ & $0.00 \mathrm{E}+00$ & $0.00 \mathrm{E}+00$ & $0.00 \mathrm{E}+00$ \\
\hline $\mathrm{SI} 32$ & $2.19 \mathrm{E}-12$ & $2.19 \mathrm{E}-12$ & $2.19 \mathrm{E}-12$ & $2.19 \mathrm{E}-12$ & $2.19 \mathrm{E}-12$ & $2.19 \mathrm{E}-12$ & $2.19 \mathrm{E}-12$ & $2.19 \mathrm{E}-12$ & $2.19 \mathrm{E}-12$ \\
\hline P 32 & $3.29 \mathrm{E}-03$ & $3.29 \mathrm{E}-03$ & $3.26 \mathrm{E}-03$ & $3.14 \mathrm{E}-03$ & $2.35 \mathrm{E}-03$ & $1.67 \mathrm{E}-03$ & $7.70 \mathrm{E}-04$ & $1.80 \mathrm{E}-04$ & $4.20 \mathrm{E}-05$ \\
\hline
\end{tabular}


TEM-10200-1

ENGINEERING CALCULATIONS AND ANALYSIS

Page 38 of 81

$12 / 19 / 17$

Rev.08

Title:

AS-RUN NEUTRONICS EVALUATION FOR THE CSM-10584 EXPERIMENT IN THE ATR

\begin{tabular}{llllllll} 
ECAR NO.: & 4496 & REV. NO.: & 1 & PROJECT NO.: & 32501 & Date: & 10/19/2020 \\
\hline
\end{tabular}

\begin{tabular}{|c|c|c|c|c|c|c|c|c|c|}
\hline \multicolumn{10}{|c|}{ Capsule E - 54.9 days irradiation } \\
\hline & EOC & $30 \mathrm{MINs}$ & $5 \mathrm{HRS}$ & $1 \mathrm{DAY}$ & 7 DAYS & 14 DAYS & 30 DAYS & 60 DAYS & 90 DAYS \\
\hline ISOTOPE & $\mathrm{Ci}$ & $\mathrm{Ci}$ & $\mathrm{Ci}$ & $\mathrm{Ci}$ & $\mathrm{Ci}$ & $\mathrm{Ci}$ & $\mathrm{Ci}$ & $\mathrm{Ci}$ & $\mathrm{Ci}$ \\
\hline P 33 & $1.01 \mathrm{E}-07$ & $1.01 \mathrm{E}-07$ & $1.01 \mathrm{E}-07$ & $9.87 \mathrm{E}-08$ & $8.36 \mathrm{E}-08$ & $6.88 \mathrm{E}-08$ & $4.42 \mathrm{E}-08$ & $1.92 \mathrm{E}-08$ & $8.37 \mathrm{E}-09$ \\
\hline P 34 & $7.48 \mathrm{E}-07$ & $0.00 \mathrm{E}+00$ & $0.00 \mathrm{E}+00$ & $0.00 \mathrm{E}+00$ & $0.00 \mathrm{E}+00$ & $0.00 \mathrm{E}+00$ & $0.00 \mathrm{E}+00$ & $0.00 \mathrm{E}+00$ & $0.00 \mathrm{E}+00$ \\
\hline S 35 & $3.01 \mathrm{E}-05$ & $3.01 \mathrm{E}-05$ & $3.00 \mathrm{E}-05$ & $2.98 \mathrm{E}-05$ & $2.85 \mathrm{E}-05$ & $2.69 \mathrm{E}-05$ & $2.37 \mathrm{E}-05$ & $1.87 \mathrm{E}-05$ & $1.48 \mathrm{E}-05$ \\
\hline S 37 & $2.24 \mathrm{E}-07$ & $3.67 \mathrm{E}-09$ & $3.18 \mathrm{E}-25$ & $0.00 \mathrm{E}+00$ & $0.00 \mathrm{E}+00$ & $0.00 \mathrm{E}+00$ & $0.00 \mathrm{E}+00$ & $0.00 \mathrm{E}+00$ & $0.00 \mathrm{E}+00$ \\
\hline CL 36 & $1.36 \mathrm{E}-11$ & $1.36 \mathrm{E}-11$ & $1.36 \mathrm{E}-11$ & $1.36 \mathrm{E}-11$ & $1.36 \mathrm{E}-11$ & $1.36 \mathrm{E}-11$ & $1.36 \mathrm{E}-11$ & $1.36 \mathrm{E}-11$ & $1.36 \mathrm{E}-11$ \\
\hline CL 38 & $1.13 \mathrm{E}-10$ & $6.46 \mathrm{E}-11$ & $4.23 \mathrm{E}-13$ & $2.54 \mathrm{E}-22$ & $0.00 \mathrm{E}+00$ & $0.00 \mathrm{E}+00$ & $0.00 \mathrm{E}+00$ & $0.00 \mathrm{E}+00$ & $0.00 E+00$ \\
\hline CL 38M & $1.35 \mathrm{E}-12$ & $0.00 \mathrm{E}+00$ & $0.00 \mathrm{E}+00$ & $0.00 \mathrm{E}+00$ & $0.00 \mathrm{E}+00$ & $0.00 \mathrm{E}+00$ & $0.00 \mathrm{E}+00$ & $0.00 \mathrm{E}+00$ & $0.00 \mathrm{E}+00$ \\
\hline AR 39 & $5.13 \mathrm{E}-08$ & $5.13 \mathrm{E}-08$ & $5.13 \mathrm{E}-08$ & $5.13 \mathrm{E}-08$ & $5.13 \mathrm{E}-08$ & $5.13 \mathrm{E}-08$ & $5.13 \mathrm{E}-08$ & $5.13 \mathrm{E}-08$ & $5.13 \mathrm{E}-08$ \\
\hline AR 41 & $8.28 \mathrm{E}-09$ & $6.85 \mathrm{E}-09$ & $1.24 \mathrm{E}-09$ & $9.19 \mathrm{E}-13$ & $0.00 \mathrm{E}+00$ & $0.00 \mathrm{E}+00$ & $0.00 \mathrm{E}+00$ & $0.00 \mathrm{E}+00$ & $0.00 \mathrm{E}+00$ \\
\hline K 40 & $1.15 \mathrm{E}-13$ & $1.15 \mathrm{E}-13$ & $1.15 \mathrm{E}-13$ & $1.15 \mathrm{E}-13$ & $1.15 \mathrm{E}-13$ & $1.15 \mathrm{E}-13$ & $1.15 \mathrm{E}-13$ & $1.15 \mathrm{E}-13$ & $1.15 \mathrm{E}-13$ \\
\hline K 42 & $7.71 \mathrm{E}-05$ & $7.50 \mathrm{E}-05$ & $5.83 \mathrm{E}-05$ & $2.01 \mathrm{E}-05$ & $6.25 \mathrm{E}-09$ & 5.07E-13 & 4.60E-18 & 4.60E-18 & $4.59 \mathrm{E}-18$ \\
\hline K 43 & $3.74 \mathrm{E}-11$ & $3.68 \mathrm{E}-11$ & $3.21 \mathrm{E}-11$ & $1.79 \mathrm{E}-11$ & $2.16 \mathrm{E}-13$ & $1.25 \mathrm{E}-15$ & $9.62 \mathrm{E}-21$ & $2.47 \mathrm{E}-30$ & $0.00 \mathrm{E}+00$ \\
\hline K 44 & $6.62 \mathrm{E}-12$ & $2.57 \mathrm{E}-12$ & $5.20 \mathrm{E}-16$ & $0.00 \mathrm{E}+00$ & $0.00 \mathrm{E}+00$ & $0.00 \mathrm{E}+00$ & $0.00 \mathrm{E}+00$ & $0.00 \mathrm{E}+00$ & $0.00 \mathrm{E}+00$ \\
\hline CA 45 & $2.02 \mathrm{E}-07$ & $2.02 \mathrm{E}-07$ & $2.02 \mathrm{E}-07$ & $2.01 \mathrm{E}-07$ & $1.96 \mathrm{E}-07$ & 1.90E-07 & $1.78 \mathrm{E}-07$ & $1.56 \mathrm{E}-07$ & $1.38 \mathrm{E}-07$ \\
\hline CA 47 & 4.26E-09 & $4.25 \mathrm{E}-09$ & $4.13 \mathrm{E}-09$ & $3.66 \mathrm{E}-09$ & 1.46E-09 & $5.02 \mathrm{E}-10$ & $4.35 \mathrm{E}-11$ & 4.44E-13 & $4.54 \mathrm{E}-15$ \\
\hline SC 46 & $4.54 \mathrm{E}-05$ & $4.54 \mathrm{E}-05$ & $4.53 \mathrm{E}-05$ & $4.50 \mathrm{E}-05$ & $4.28 \mathrm{E}-05$ & 4.04E-05 & $3.54 \mathrm{E}-05$ & $2.76 \mathrm{E}-05$ & $2.16 \mathrm{E}-05$ \\
\hline SC 46M & $5.10 \mathrm{E}-10$ & $0.00 \mathrm{E}+00$ & $0.00 \mathrm{E}+00$ & $0.00 \mathrm{E}+00$ & $0.00 \mathrm{E}+00$ & $0.00 \mathrm{E}+00$ & $0.00 \mathrm{E}+00$ & $0.00 \mathrm{E}+00$ & $0.00 \mathrm{E}+00$ \\
\hline SC 47 & $1.94 \mathrm{E}-04$ & $1.93 \mathrm{E}-04$ & $1.85 \mathrm{E}-04$ & 1.57E-04 & $4.55 \mathrm{E}-05$ & 1.07E-05 & $3.91 \mathrm{E}-07$ & $7.89 \mathrm{E}-10$ & $1.61 \mathrm{E}-12$ \\
\hline SC 48 & $1.85 \mathrm{E}-05$ & $1.84 \mathrm{E}-05$ & $1.71 \mathrm{E}-05$ & 1.27E-05 & 1.30E-06 & $9.08 \mathrm{E}-08$ & 2.09E-10 & $2.35 \mathrm{E}-15$ & $2.65 \mathrm{E}-20$ \\
\hline SC 49 & $1.56 \mathrm{E}-05$ & 1.09E-05 & $4.19 \mathrm{E}-07$ & $4.51 \mathrm{E}-13$ & $0.00 \mathrm{E}+00$ & $0.00 \mathrm{E}+00$ & $0.00 \mathrm{E}+00$ & $0.00 \mathrm{E}+00$ & $0.00 \mathrm{E}+00$ \\
\hline SC 50 & $3.22 \mathrm{E}-07$ & 1.66E-12 & $0.00 \mathrm{E}+00$ & $0.00 \mathrm{E}+00$ & $0.00 \mathrm{E}+00$ & $0.00 \mathrm{E}+00$ & $0.00 \mathrm{E}+00$ & $0.00 \mathrm{E}+00$ & $0.00 \mathrm{E}+00$ \\
\hline TI 51 & $1.10 \mathrm{E}-03$ & $2.97 \mathrm{E}-05$ & $2.30 \mathrm{E}-19$ & $0.00 \mathrm{E}+00$ & $0.00 \mathrm{E}+00$ & $0.00 \mathrm{E}+00$ & $0.00 \mathrm{E}+00$ & $0.00 \mathrm{E}+00$ & $0.00 \mathrm{E}+00$ \\
\hline$V 52$ & $9.79 \mathrm{E}-01$ & $3.83 \mathrm{E}-03$ & $8.10 \mathrm{E}-25$ & $0.00 \mathrm{E}+00$ & $0.00 \mathrm{E}+00$ & $0.00 \mathrm{E}+00$ & $0.00 \mathrm{E}+00$ & $0.00 \mathrm{E}+00$ & $0.00 \mathrm{E}+00$ \\
\hline$V 53$ & 6.07E-03 & 1.49E-08 & $0.00 \mathrm{E}+00$ & $0.00 \mathrm{E}+00$ & $0.00 \mathrm{E}+00$ & $0.00 \mathrm{E}+00$ & $0.00 \mathrm{E}+00$ & $0.00 \mathrm{E}+00$ & $0.00 \mathrm{E}+00$ \\
\hline$V 54$ & $6.27 \mathrm{E}-05$ & $8.81 \mathrm{E}-15$ & $0.00 \mathrm{E}+00$ & $0.00 \mathrm{E}+00$ & $0.00 \mathrm{E}+00$ & $0.00 \mathrm{E}+00$ & $0.00 \mathrm{E}+00$ & $0.00 \mathrm{E}+00$ & $0.00 \mathrm{E}+00$ \\
\hline CR 51 & $9.96 \mathrm{E}+01$ & $9.95 \mathrm{E}+01$ & $9.90 \mathrm{E}+01$ & $9.71 \mathrm{E}+01$ & $8.36 \mathrm{E}+01$ & $7.01 \mathrm{E}+01$ & $4.70 \mathrm{E}+01$ & $2.22 \mathrm{E}+01$ & $1.05 \mathrm{E}+01$ \\
\hline CR 55 & $1.72 \mathrm{E}+00$ & $4.90 \mathrm{E}-03$ & $0.00 \mathrm{E}+00$ & $0.00 \mathrm{E}+00$ & $0.00 \mathrm{E}+00$ & $0.00 \mathrm{E}+00$ & $0.00 \mathrm{E}+00$ & $0.00 \mathrm{E}+00$ & $0.00 \mathrm{E}+00$ \\
\hline MN 54 & $9.40 \mathrm{E}-01$ & 9.40E-01 & $9.40 \mathrm{E}-01$ & $9.38 \mathrm{E}-01$ & $9.26 \mathrm{E}-01$ & $9.11 \mathrm{E}-01$ & $8.80 \mathrm{E}-01$ & $8.23 \mathrm{E}-01$ & 7.70E-01 \\
\hline MN 56 & $1.34 \mathrm{E}+01$ & $1.17 \mathrm{E}+01$ & $3.48 \mathrm{E}+00$ & $2.11 \mathrm{E}-02$ & $3.26 \mathrm{E}-19$ & $0.00 \mathrm{E}+00$ & $0.00 \mathrm{E}+00$ & $0.00 \mathrm{E}+00$ & $0.00 \mathrm{E}+00$ \\
\hline MN 57 & 1.97E-02 & 4.83E-08 & $0.00 \mathrm{E}+00$ & $0.00 \mathrm{E}+00$ & $0.00 \mathrm{E}+00$ & $0.00 \mathrm{E}+00$ & $0.00 \mathrm{E}+00$ & $0.00 \mathrm{E}+00$ & $0.00 \mathrm{E}+00$ \\
\hline MN 58 & $6.27 \mathrm{E}-05$ & 3.16E-13 & $0.00 \mathrm{E}+00$ & $0.00 \mathrm{E}+00$ & $0.00 \mathrm{E}+00$ & $0.00 \mathrm{E}+00$ & $0.00 \mathrm{E}+00$ & $0.00 \mathrm{E}+00$ & $0.00 \mathrm{E}+00$ \\
\hline FE 55 & $3.40 \mathrm{E}+00$ & $3.40 \mathrm{E}+00$ & $3.40 \mathrm{E}+00$ & $3.40 \mathrm{E}+00$ & $3.39 \mathrm{E}+00$ & $3.37 \mathrm{E}+00$ & $3.33 \mathrm{E}+00$ & $3.26 \mathrm{E}+00$ & $3.19 \mathrm{E}+00$ \\
\hline FE 59 & $1.42 \mathrm{E}+00$ & $1.42 \mathrm{E}+00$ & $1.42 \mathrm{E}+00$ & $1.40 \mathrm{E}+00$ & $1.28 \mathrm{E}+00$ & $1.15 \mathrm{E}+00$ & $8.96 \mathrm{E}-01$ & $5.65 \mathrm{E}-01$ & $3.56 \mathrm{E}-01$ \\
\hline $\mathrm{CO} 58$ & $2.52 \mathrm{E}+00$ & $2.52 \mathrm{E}+00$ & $2.51 \mathrm{E}+00$ & $2.49 \mathrm{E}+00$ & $2.35 \mathrm{E}+00$ & $2.20 \mathrm{E}+00$ & $1.88 \mathrm{E}+00$ & $1.40 \mathrm{E}+00$ & $1.04 \mathrm{E}+00$ \\
\hline $\mathrm{CO} 60$ & $2.46 \mathrm{E}-02$ & $2.46 \mathrm{E}-02$ & $2.46 \mathrm{E}-02$ & $2.46 \mathrm{E}-02$ & $2.45 \mathrm{E}-02$ & $2.45 \mathrm{E}-02$ & $2.43 \mathrm{E}-02$ & $2.41 \mathrm{E}-02$ & $2.38 \mathrm{E}-02$ \\
\hline $\mathrm{CO} 60 \mathrm{M}$ & $6.57 \mathrm{E}-01$ & $9.01 \mathrm{E}-02$ & $1.56 \mathrm{E}-09$ & $0.00 \mathrm{E}+00$ & $0.00 \mathrm{E}+00$ & $0.00 \mathrm{E}+00$ & $0.00 \mathrm{E}+00$ & $0.00 \mathrm{E}+00$ & $0.00 \mathrm{E}+00$ \\
\hline $\mathrm{CO} 61$ & 7.03E-03 & $5.70 \mathrm{E}-03$ & 8.60E-04 & 2.94E-07 & $0.00 \mathrm{E}+00$ & $0.00 \mathrm{E}+00$ & $0.00 \mathrm{E}+00$ & $0.00 E+00$ & $0.00 \mathrm{E}+00$ \\
\hline $\mathrm{CO} 62$ & $9.84 \mathrm{E}-04$ & $9.38 \mathrm{E}-10$ & $0.00 \mathrm{E}+00$ & $0.00 \mathrm{E}+00$ & $0.00 \mathrm{E}+00$ & $0.00 \mathrm{E}+00$ & $0.00 \mathrm{E}+00$ & $0.00 \mathrm{E}+00$ & $0.00 \mathrm{E}+00$ \\
\hline $\mathrm{NI} 59$ & 3.55E-04 & 3.55E-04 & 3.55E-04 & 3.55E-04 & 3.55E-04 & 3.55E-04 & 3.55E-04 & 3.55E-04 & $3.55 \mathrm{E}-04$ \\
\hline $\mathrm{NI} 63$ & 4.49E-02 & 4.49E-02 & 4.49E-02 & 4.49E-02 & 4.49E-02 & 4.49E-02 & 4.49E-02 & 4.49E-02 & 4.49E-02 \\
\hline NI 65 & $1.14 \mathrm{E}+00$ & 9.93E-01 & $2.88 \mathrm{E}-01$ & 1.55E-03 & $9.73 \mathrm{E}-21$ & $0.00 \mathrm{E}+00$ & $0.00 \mathrm{E}+00$ & $0.00 E+00$ & $0.00 \mathrm{E}+00$ \\
\hline NI 66 & 3.94E-05 & 3.91E-05 & 3.69E-05 & 2.90E-05 & 4.67E-06 & $5.53 \mathrm{E}-07$ & 4.23E-09 & 4.55E-13 & $4.88 \mathrm{E}-17$ \\
\hline CU 64 & $1.34 \mathrm{E}+00$ & $1.31 \mathrm{E}+00$ & $1.02 \mathrm{E}+00$ & $3.62 \mathrm{E}-01$ & 1.40E-04 & $1.46 \mathrm{E}-08$ & 1.15E-17 & $0.00 \mathrm{E}+00$ & $0.00 \mathrm{E}+00$ \\
\hline CU 66 & 2.91E-01 & $4.97 \mathrm{E}-03$ & $3.70 \mathrm{E}-05$ & $2.91 \mathrm{E}-05$ & 4.67E-06 & 5.54E-07 & 4.24E-09 & $4.55 \mathrm{E}-13$ & $4.89 \mathrm{E}-17$ \\
\hline CU 67 & $1.88 \mathrm{E}-06$ & 1.87E-06 & $1.78 \mathrm{E}-06$ & 1.44E-06 & 2.87E-07 & $4.36 \mathrm{E}-08$ & $5.90 \mathrm{E}-10$ & $1.85 \mathrm{E}-13$ & $5.80 \mathrm{E}-17$ \\
\hline ZN 65 & $2.00 \mathrm{E}-05$ & $2.00 \mathrm{E}-05$ & $2.00 \mathrm{E}-05$ & 1.99E-05 & $1.96 \mathrm{E}-05$ & $1.92 \mathrm{E}-05$ & $1.83 \mathrm{E}-05$ & $1.68 \mathrm{E}-05$ & $1.55 \mathrm{E}-05$ \\
\hline ZN 69 & $1.37 \mathrm{E}-10$ & $9.76 \mathrm{E}-11$ & $1.10 \mathrm{E}-11$ & $2.94 \mathrm{E}-12$ & $2.08 \mathrm{E}-15$ & $4.40 \mathrm{E}-19$ & $0.00 \mathrm{E}+00$ & $0.00 \mathrm{E}+00$ & $0.00 E+00$ \\
\hline ZN 69M & $9.17 \mathrm{E}-12$ & $8.95 \mathrm{E}-12$ & $7.13 \mathrm{E}-12$ & $2.74 \mathrm{E}-12$ & $1.94 \mathrm{E}-15$ & $4.10 \mathrm{E}-19$ & $1.63 \mathrm{E}-27$ & $0.00 \mathrm{E}+00$ & $0.00 E+00$ \\
\hline SR 89 & $6.38 \mathrm{E}-12$ & $6.37 \mathrm{E}-12$ & $6.36 \mathrm{E}-12$ & $6.29 \mathrm{E}-12$ & $5.79 \mathrm{E}-12$ & $5.26 \mathrm{E}-12$ & $4.22 \mathrm{E}-12$ & $2.80 \mathrm{E}-12$ & $1.85 \mathrm{E}-12$ \\
\hline SR 91 & $4.46 \mathrm{E}-13$ & $4.30 \mathrm{E}-13$ & $3.10 \mathrm{E}-13$ & $7.74 \mathrm{E}-14$ & $2.12 \mathrm{E}-18$ & $1.01 \mathrm{E}-23$ & $0.00 \mathrm{E}+00$ & $0.00 \mathrm{E}+00$ & $0.00 E+00$ \\
\hline Y 89M & $6.72 \mathrm{E}-07$ & $6.69 \mathrm{E}-07$ & $6.43 \mathrm{E}-07$ & $5.43 \mathrm{E}-07$ & $1.52 \mathrm{E}-07$ & $3.45 \mathrm{E}-08$ & 1.16E-09 & $2.00 \mathrm{E}-12$ & $3.45 \mathrm{E}-15$ \\
\hline Y 90 & $9.01 \mathrm{E}-06$ & $8.96 \mathrm{E}-06$ & $8.53 \mathrm{E}-06$ & $6.94 \mathrm{E}-06$ & $1.46 \mathrm{E}-06$ & $2.37 \mathrm{E}-07$ & $3.70 \mathrm{E}-09$ & $1.53 \mathrm{E}-12$ & $1.47 \mathrm{E}-14$ \\
\hline Y 90M & $3.44 \mathrm{E}-13$ & $3.08 \mathrm{E}-13$ & $1.13 \mathrm{E}-13$ & $1.56 \mathrm{E}-15$ & $1.62 \mathrm{E}-29$ & $0.00 \mathrm{E}+00$ & $0.00 \mathrm{E}+00$ & $0.00 \mathrm{E}+00$ & $0.00 E+00$ \\
\hline Y 91 & $6.95 \mathrm{E}-10$ & $6.95 \mathrm{E}-10$ & $6.94 \mathrm{E}-10$ & $6.87 \mathrm{E}-10$ & $6.40 \mathrm{E}-10$ & $5.89 \mathrm{E}-10$ & $4.87 \mathrm{E}-10$ & $3.42 \mathrm{E}-10$ & $2.39 \mathrm{E}-10$ \\
\hline Y 92 & $5.15 \mathrm{E}-11$ & 4.67E-11 & $1.93 \mathrm{E}-11$ & $4.68 \mathrm{E}-13$ & $2.64 \mathrm{E}-25$ & $0.00 \mathrm{E}+00$ & $0.00 \mathrm{E}+00$ & $0.00 \mathrm{E}+00$ & $0.00 E+00$ \\
\hline
\end{tabular}


TEM-10200-1

ENGINEERING CALCULATIONS AND ANALYSIS

Page 39 of 81

$12 / 19 / 17$

Rev.08

Title:

AS-RUN NEUTRONICS EVALUATION FOR THE CSM-10584 EXPERIMENT IN THE ATR

\begin{tabular}{llllllll} 
ECAR NO.: & 4496 & REV. NO.: & 1 & PROJECT NO.: & 32501 & Date: & 10/19/2020 \\
\hline
\end{tabular}

\begin{tabular}{|c|c|c|c|c|c|c|c|c|c|}
\hline \multicolumn{10}{|c|}{ Capsule E - 54.9 days irradiation } \\
\hline & EOC & $30 \mathrm{MINs}$ & $5 \mathrm{HRS}$ & $1 \mathrm{DAY}$ & 7 DAYS & 14 DAYS & 30 DAYS & 60 DAYS & 90 DAYS \\
\hline ISOTOPE & $\mathrm{Ci}$ & $\mathrm{Ci}$ & $\mathrm{Ci}$ & $\mathrm{Ci}$ & $\mathrm{Ci}$ & $\mathrm{Ci}$ & $\mathrm{Ci}$ & $\mathrm{Ci}$ & $\mathrm{Ci}$ \\
\hline ZR 89 & $6.73 \mathrm{E}-07$ & $6.70 \mathrm{E}-07$ & $6.44 \mathrm{E}-07$ & $5.44 \mathrm{E}-07$ & $1.52 \mathrm{E}-07$ & $3.45 \mathrm{E}-08$ & $1.16 \mathrm{E}-09$ & $2.00 \mathrm{E}-12$ & $3.44 \mathrm{E}-15$ \\
\hline ZR 93 & $6.26 \mathrm{E}-12$ & $6.26 \mathrm{E}-12$ & $6.26 \mathrm{E}-12$ & $6.26 \mathrm{E}-12$ & $6.26 \mathrm{E}-12$ & $6.26 \mathrm{E}-12$ & $6.26 \mathrm{E}-12$ & $6.26 \mathrm{E}-12$ & $6.26 \mathrm{E}-12$ \\
\hline ZR 95 & $1.04 \mathrm{E}-06$ & $1.04 \mathrm{E}-06$ & $1.04 \mathrm{E}-06$ & $1.03 \mathrm{E}-06$ & $9.65 \mathrm{E}-07$ & $8.94 \mathrm{E}-07$ & $7.52 \mathrm{E}-07$ & $5.43 \mathrm{E}-07$ & $3.93 \mathrm{E}-07$ \\
\hline ZR 97 & $5.32 \mathrm{E}-08$ & $5.21 \mathrm{E}-08$ & $4.33 \mathrm{E}-08$ & $1.99 \mathrm{E}-08$ & $5.42 \mathrm{E}-11$ & $5.54 \mathrm{E}-14$ & $8.00 \mathrm{E}-21$ & $0.00 \mathrm{E}+00$ & $0.00 \mathrm{E}+00$ \\
\hline NB 92 & $1.31 \mathrm{E}-04$ & $1.31 \mathrm{E}-04$ & $1.29 \mathrm{E}-04$ & $1.22 \mathrm{E}-04$ & $8.11 \mathrm{E}-05$ & $5.03 \mathrm{E}-05$ & $1.69 \mathrm{E}-05$ & $2.18 \mathrm{E}-06$ & $2.82 \mathrm{E}-07$ \\
\hline NB 93M & $2.28 \mathrm{E}-14$ & $2.28 \mathrm{E}-14$ & $2.29 \mathrm{E}-14$ & $2.36 \mathrm{E}-14$ & $2.85 \mathrm{E}-14$ & $3.43 \mathrm{E}-14$ & $4.75 \mathrm{E}-14$ & $7.22 \mathrm{E}-14$ & $9.67 \mathrm{E}-14$ \\
\hline NB 94 & $1.01 \mathrm{E}-06$ & $1.01 \mathrm{E}-06$ & $1.01 \mathrm{E}-06$ & $1.01 \mathrm{E}-06$ & $1.01 \mathrm{E}-06$ & $1.01 \mathrm{E}-06$ & $1.01 \mathrm{E}-06$ & $1.01 \mathrm{E}-06$ & $1.01 \mathrm{E}-06$ \\
\hline NB 95 & $1.84 \mathrm{E}-03$ & $1.84 \mathrm{E}-03$ & $1.84 \mathrm{E}-03$ & $1.81 \mathrm{E}-03$ & $1.61 \mathrm{E}-03$ & $1.40 \mathrm{E}-03$ & $1.02 \mathrm{E}-03$ & $5.65 \mathrm{E}-04$ & $3.13 \mathrm{E}-04$ \\
\hline NB 95M & $6.75 \mathrm{E}-09$ & $6.75 \mathrm{E}-09$ & $6.77 \mathrm{E}-09$ & $6.84 \mathrm{E}-09$ & $6.90 \mathrm{E}-09$ & 6.57E-09 & 5.57E-09 & $4.03 E-09$ & 2.91E-09 \\
\hline NB 96 & $1.29 \mathrm{E}-05$ & $1.27 \mathrm{E}-05$ & $1.11 \mathrm{E}-05$ & $6.32 \mathrm{E}-06$ & $8.79 \mathrm{E}-08$ & $6.00 \mathrm{E}-10$ & $6.72 \mathrm{E}-15$ & $3.51 \mathrm{E}-24$ & $0.00 \mathrm{E}+00$ \\
\hline NB 97 & $1.88 \mathrm{E}-06$ & $1.42 \mathrm{E}-06$ & 1.49E-07 & $2.00 \mathrm{E}-08$ & $5.44 \mathrm{E}-11$ & $5.56 \mathrm{E}-14$ & $8.62 \mathrm{E}-21$ & $0.00 \mathrm{E}+00$ & $0.00 \mathrm{E}+00$ \\
\hline NB 97M & $5.04 \mathrm{E}-08$ & $4.94 \mathrm{E}-08$ & $4.11 \mathrm{E}-08$ & $1.88 \mathrm{E}-08$ & $5.13 \mathrm{E}-11$ & $5.24 \mathrm{E}-14$ & $7.58 \mathrm{E}-21$ & $0.00 \mathrm{E}+00$ & $0.00 \mathrm{E}+00$ \\
\hline NB 98 & $2.81 \mathrm{E}-07$ & $0.00 \mathrm{E}+00$ & $0.00 \mathrm{E}+00$ & $0.00 \mathrm{E}+00$ & $0.00 \mathrm{E}+00$ & $0.00 E+00$ & $0.00 E+00$ & $0.00 \mathrm{E}+00$ & $0.00 \mathrm{E}+00$ \\
\hline NB100 & $1.40 \mathrm{E}-08$ & $0.00 \mathrm{E}+00$ & $0.00 \mathrm{E}+00$ & $0.00 \mathrm{E}+00$ & $0.00 \mathrm{E}+00$ & $0.00 E+00$ & $0.00 \mathrm{E}+00$ & $0.00 \mathrm{E}+00$ & $0.00 \mathrm{E}+00$ \\
\hline MO 93M & $3.89 \mathrm{E}-05$ & 3.70E-05 & $2.34 \mathrm{E}-05$ & $3.43 \mathrm{E}-06$ & $1.61 \mathrm{E}-12$ & 6.67E-20 & $0.00 E+00$ & $0.00 E+00$ & $0.00 E+00$ \\
\hline MO 93 & $3.56 \mathrm{E}-08$ & $3.56 \mathrm{E}-08$ & $3.56 \mathrm{E}-08$ & $3.56 \mathrm{E}-08$ & $3.56 \mathrm{E}-08$ & $3.56 \mathrm{E}-08$ & $3.56 \mathrm{E}-08$ & $3.56 \mathrm{E}-08$ & $3.56 \mathrm{E}-08$ \\
\hline MO 99 & $2.72 \mathrm{E}-02$ & $2.71 \mathrm{E}-02$ & $2.58 \mathrm{E}-02$ & $2.11 \mathrm{E}-02$ & $4.66 \mathrm{E}-03$ & 7.98E-04 & $1.41 \mathrm{E}-05$ & $7.36 \mathrm{E}-09$ & $3.83 \mathrm{E}-12$ \\
\hline M0101 & $6.77 \mathrm{E}-03$ & $1.63 \mathrm{E}-03$ & $4.51 \mathrm{E}-09$ & $0.00 \mathrm{E}+00$ & $0.00 \mathrm{E}+00$ & $0.00 \mathrm{E}+00$ & $0.00 \mathrm{E}+00$ & $0.00 \mathrm{E}+00$ & $0.00 \mathrm{E}+00$ \\
\hline TC 99 & $1.50 \mathrm{E}-09$ & $1.50 \mathrm{E}-09$ & $1.51 \mathrm{E}-09$ & $1.53 \mathrm{E}-09$ & 1.60E-09 & 1.62E-09 & $1.62 \mathrm{E}-09$ & $1.62 \mathrm{E}-09$ & $1.62 \mathrm{E}-09$ \\
\hline TC100 & 1.57E-04 & $0.00 \mathrm{E}+00$ & $0.00 \mathrm{E}+00$ & $0.00 \mathrm{E}+00$ & $0.00 E+00$ & $0.00 E+00$ & $0.00 E+00$ & $0.00 E+00$ & $0.00 E+00$ \\
\hline TC101 & $6.77 \mathrm{E}-03$ & $3.91 \mathrm{E}-03$ & $5.68 \mathrm{E}-08$ & $0.00 \mathrm{E}+00$ & $0.00 E+00$ & $0.00 E+00$ & $0.00 E+00$ & $0.00 E+00$ & $0.00 E+00$ \\
\hline RU103 & $2.10 \mathrm{E}-08$ & $2.10 \mathrm{E}-08$ & $2.09 \mathrm{E}-08$ & $2.06 \mathrm{E}-08$ & $1.85 \mathrm{E}-08$ & $1.64 \mathrm{E}-08$ & $1.24 \mathrm{E}-08$ & $7.28 \mathrm{E}-09$ & $4.29 E-09$ \\
\hline RH104 & $1.16 \mathrm{E}-10$ & $8.29 \mathrm{E}-14$ & $0.00 \mathrm{E}+00$ & $0.00 \mathrm{E}+00$ & $0.00 \mathrm{E}+00$ & $0.00 E+00$ & $0.00 \mathrm{E}+00$ & $0.00 \mathrm{E}+00$ & $0.00 \mathrm{E}+00$ \\
\hline RH104M & $8.38 \mathrm{E}-12$ & $6.77 \mathrm{E}-14$ & $0.00 \mathrm{E}+00$ & $0.00 \mathrm{E}+00$ & $0.00 \mathrm{E}+00$ & $0.00 \mathrm{E}+00$ & $0.00 \mathrm{E}+00$ & $0.00 \mathrm{E}+00$ & $0.00 E+00$ \\
\hline SUMTOT & $3.34 \mathrm{E}+02$ & $1.25 \mathrm{E}+02$ & $1.14 \mathrm{E}+02$ & $1.06 \mathrm{E}+02$ & $9.16 \mathrm{E}+01$ & $7.78 \mathrm{E}+01$ & $5.41 \mathrm{E}+01$ & $2.83 \mathrm{E}+01$ & $1.59 \mathrm{E}+01$ \\
\hline OTOTAL & $3.34 \mathrm{E}+02$ & $1.25 \mathrm{E}+02$ & $1.14 \mathrm{E}+02$ & $1.06 \mathrm{E}+02$ & $9.16 \mathrm{E}+01$ & $7.78 \mathrm{E}+01$ & $5.41 \mathrm{E}+01$ & $2.83 \mathrm{E}+01$ & $1.59 \mathrm{E}+01$ \\
\hline
\end{tabular}


Rev.08

Title: $\quad$ AS-RUN NEUTRONICS EVALUATION FOR THE CSM-10584 EXPERIMENT IN THE ATR

\begin{tabular}{llllllll}
\cline { 2 - 4 } ECAR NO.: & 4496 & REV. NO.: & 1 & PROJECT NO.: & 32501 & Date: & $10 / 19 / 2020$ \\
\hline
\end{tabular}

\section{REFERENCES}

[1] N. H. Manwaring Letter to R. R. Little, Advanced Test Reactor Power History Through Cycle 164B-1, January 21, 2019, RE-03-19 (see Appendix B).

[2] W. C. Cook, A. C. Smith, "ATR CSAP Code Package on the Workstation Version 1," PG-T-96-002, May 1996.

[3] C.R. Tyler, "Functional and Operational Requirements Colorado School of Mines (CSM)10584", FOR-308 Rev. 1.

[4] Tim Goorley, Jeffrey S. Bull, Forrest B. Brown, et. al., "Release of MCNP5_RSICC_1.30," Trans. Am. Nucl. Soc., Vol. 91, 693-694 (2004).

[5] X-5 Monte Carlo Team, "MCNP - A General Monte Carlo N-Particle Transport Code, Version 5," Volume I, LA-UR-03-1987, Los Alamos National Laboratory, April 24, 2003 (Revised /30/2004) and Volume II, LA-CP-0245, Los Alamos National Laboratory, April 24, 2003 (Revise $6 / 30 / 2004$ ) (Vol. II is available with a licensed copy of MCNP).

[6] A. G. Croff, ORIGEN2: A Versatile Computer code for Calculating the Nuclide Compositions and Characteristics of Nuclear Materials, Nuclear Technology, Vol. 62, pp. 335-352, (1983).

[7] W. M. Cowherd, MCNP Verification and Validation For Use by the INL Neutronic Analysis Group, PLN-5021, Revision 0, January 6, 2016 (EA ID 234728).

[8] GDE-183, "Experiment Reactivities," Rev. 7, Nuclear Engineering, July 12, 2004.

[9] GDE-594, "Experiment Design and Analysis Guide - Neutronics \& Physics," Rev. 1, June 26, 2015.

[10] S. S. Kim, B. G. Schnitzler, "Advanced Test Reactor: Serpentine Arrangement of Highly Enriched Water-Moderated Uranium-Aluminide Fuel Plates Reflected by Beryllium," INL/EXT-05-00780, September 2005; published in "International Handbook of Evaluated Criticality Safety Benchmark Experiments," NEA/NSC/DOC/(95)03/11, Volume II, HEU-MET-THERM-022, September 2005 Edition.

[11] B. G. Schnitzler, "Origen2 Cross Section Library Assessment for ATR Applications," BGS-6-91, Idaho National Laboratory, April 1991.

[12] J.R. Mitchell, J.W. Nielsen, "Software Management ORIGEN2 Version 2.2," PLN-3710, Revision 0, October 2011, INL Record Number 3818329, January 25, 2012 (EA ID 201298).

[13] T.A. Tomberlin, "Advanced Test Reactor Critical Facility Measurements in Support of Advanced Test Reactor Experiments," TRA-ATRC-1546, Rev. 0, March 2000. 
TEM-10200-1

$12 / 19 / 17$

Rev.08

Title: AS-RUN NEUTRONICS EVALUATION FOR THE CSM-10584 EXPERIMENT IN THE ATR

\begin{tabular}{llllllll} 
ECAR NO.: & 4496 & REV. NO.: & 1 & PROJECT NO.: & 32501 & Date: & 10/19/2020 \\
\hline
\end{tabular}

\section{APPENDIX A “PYHSICS ANALYSIS REQUEST FORM”}

Rev. 3

\section{Experiment Analysis Request Form}

This form should be used to request analyses from the Experiment Design and Analysis (C660) department. Provide the completed form(s) to Keith Penny (Seldon.Penny@inl.gov).

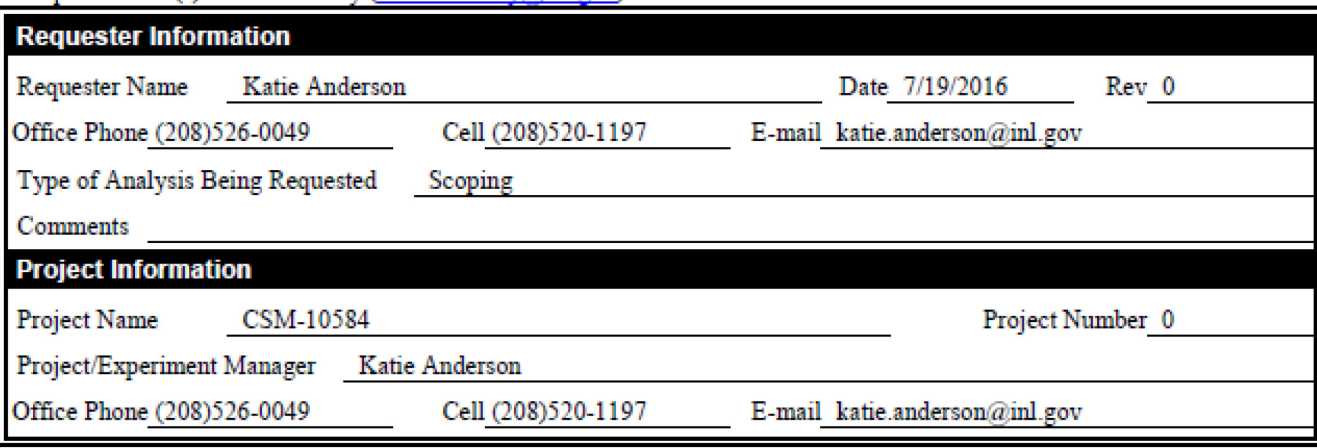

\section{Experiment Information}

Test Name $\quad$ CMS-10584

Irradiation performance testing of specimens produced by commerically available additive Test Information manufacturing techniques.

Test Location $\quad \mathrm{B}$ (Small) Insertion Cycle 164A_Final Discharge Cycle___ Duration _ _ EFPD

Specific ATR Position(s) B5, possibly the Rabbit $\quad$ ATR Cycle(s): 1 Cycle starting with 164A

Backup Test Information unknown

Is an Experiment Safety Analysis (ESA) author needed? Yes $\square$ No $\square$ Assigned

Request ESA author from ATR Experiment Engineering (Dave Schoonen). ESA Author TBD

Is this a fueled experiment? Yes $\square$ No $\$ If yes, complete "calculations for fueled test"s section.

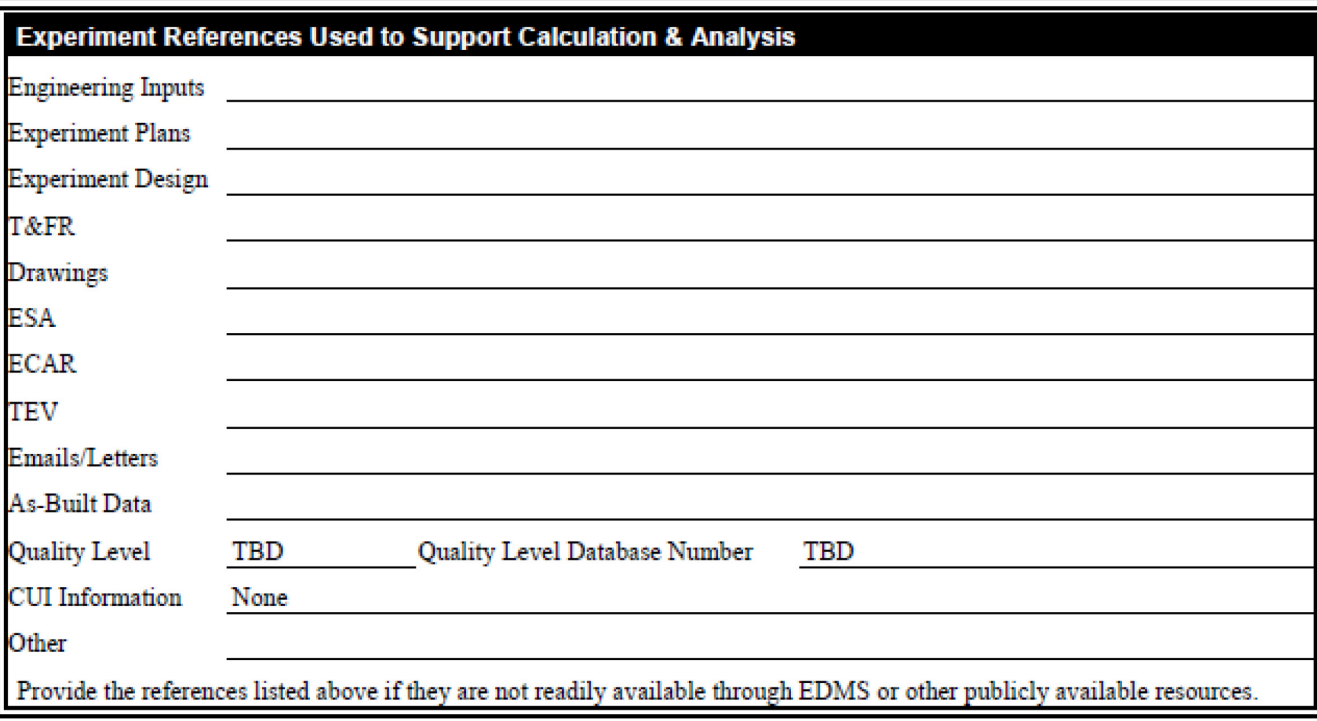


TEM-10200-1

$12 / 19 / 17$

Rev.08

Title: AS-RUN NEUTRONICS EVALUATION FOR THE CSM-10584 EXPERIMENT IN THE ATR

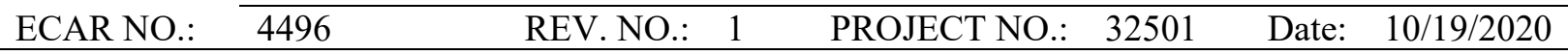

Rev. 3

\section{Experiment Analysis Request Form}

\begin{tabular}{|c|c|}
\hline \multicolumn{2}{|c|}{ Neutronics/Physics Calculation \& Analysis Request Form } \\
\hline \multicolumn{2}{|c|}{ Neutronics Analysis Request } \\
\hline \multicolumn{2}{|c|}{ Requesting a neutronics/physics analysis? Yes $\otimes \mathrm{No} \square$ If yes, please fill out the information on this page } \\
\hline Deliverable & Deliverable Title Physics Analysis of NSUF CSM-10584 Experiment \\
\hline Draft Due Date $\quad 2 / 15 / 2017$ & Final/Approved Due Date 3/15/2017 \\
\hline Comments & \\
\hline $\begin{array}{l}\text { Pupose of } \\
\text { Analysis }\end{array}$ & Aysics analysis to support CSM-10584 experiment \\
\hline \multicolumn{2}{|c|}{ Persons assigned to support calculation $\&$ analysis tasks (as applicable). } \\
\hline \multicolumn{2}{|c|}{ Performer/Author $\quad$ TBD } \\
\hline & TBD \\
\hline \multicolumn{2}{|c|}{ Independent Peer Reviewer TBD } \\
\hline \multicolumn{2}{|c|}{ Approver (Line Manager) Misti Lillo } \\
\hline \multicolumn{2}{|l|}{ Nuclear Safety Engineering_ } \\
\hline Acceptance (Owner) & Katie Anderson \\
\hline Other(s) & \\
\hline
\end{tabular}

Calculated Parameter

Scoping Design Projection As-Run Calculations for All Tests (typically required to support ESA)

Neutron Heating Rates W/g

Gamma Heating Rates W/g

Test Reactivity Worth (\$) relative to Water-Filled

Backup Test Reactivity Worth (\$) relative to Water-Filled

Axial Flux Profile (FE(s) and coolant channel(s)?)

Other

Calculations for Tests in Flux Traps (typically required to support ESA)

Temperature Coefficient of PCS (Flux Traps only) $\left(\$ /{ }^{\circ} \mathrm{F}\right)$

Other

Additional Calculations for All Tests (not necessarily required to support ESA)

\begin{tabular}{|c|c|c|c|c|c|}
\hline Activation $\mathrm{Ci} / \mathrm{cc}$ & Irradiation Time TBD days & Q & $\square$ & $\square$ & $\square$ \\
\hline Decay Heat $W / c c$ & Cooling Time 30.60 .90 days & 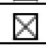 & $\square$ & $\square$ & $\square$ \\
\hline $\mathrm{DPA}$ & & $\square$ & $\square$ & $\square$ & $\square$ \\
\hline Other & & $\square$ & $\square$ & $\square$ & $\square$ \\
\hline \multicolumn{6}{|c|}{ Calculations for Fueled Tests (not necessarily required to support ESA) } \\
\hline Fuel Burnup & & $\square$ & $\square$ & $\square$ & $\square$ \\
\hline Fission Heating & Fission Density & $\square$ & $\square$ & $\square$ & $\square$ \\
\hline Flux & (Fast/Thermal) & $\square$ & $\square$ & $\square$ & $\square$ \\
\hline Fluence & (Fast/Thermal) & $\square$ & $\square$ & $\square$ & $\square$ \\
\hline Fission Products & & $\square$ & $\square$ & $\square$ & $\square$ \\
\hline Local- or Point-to- & erage Ratios & $\square$ & $\square$ & $\square$ & $\square$ \\
\hline Other & & $\square$ & $\square$ & $\square$ & $\square$ \\
\hline
\end{tabular}


TEM-10200-1

$12 / 19 / 17$

ENGINEERING CALCULATIONS AND ANALYSIS

Page 43 of 81

Rev.08

Title: $\quad$ AS-RUN NEUTRONICS EVALUATION FOR THE CSM-10584 EXPERIMENT IN THE ATR

\begin{tabular}{llllllll}
\cline { 2 - 6 } ECAR NO.: & 4496 & REV. NO.: & 1 & PROJECT NO.: & 32501 & Date: & $10 / 19 / 2020$ \\
\hline
\end{tabular} 
TEM-10200-1

Rev.08

Title: AS-RUN NEUTRONICS EVALUATION FOR THE CSM-10584 EXPERIMENT IN THE ATR

ECAR NO.: $\quad 4496$

REV. NO.: $1 \quad$ PROJECT NO.: 32501

Date: $10 / 19 / 2020$

\section{APPENDIX B “POWER HISTORY LETTER”}

\section{INTEROFFICE MEMORANDUM}

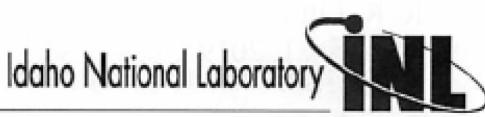

Date: January 21,2019

To: $\quad$ R. R. Little, Reactor and Nuclear Safety Engineering Manager, Advanced Test Reactor Programs

From: $\quad$ Reactor Engineering, Advanced Test Reactor Programs

Subject: $\quad$ Advanced Test Reactor Power History Through Cycle 164B-1

References: (a) A. V. Briscoe letter to J. L. Durney, ATR Power History Through Cycle 34C-1, June 7, 1977, AVB-9-77

(b) C. C. Swanson letter to J. L. Durney, ATR Power History Through Cycle 72A-1, February 3, 1986, CAS-05-86

(c) L. S. Loret letter to E. C. Anderson, Sr., ATR Power History Through Cycle 102B-1, February 28, 1994, LSL-11-94

(d) D. E. Hale letter to J. C. Chapman, ATR Power History Through Cycle 133B-1, August 18, 2004, DEH-05-04

Table 1 lists the Advanced Test Reactor (ATR) N-16 constrained power history data since the Beryllium VI Core Internals Changeout (CIC) Cycle 134A-1 through Cycle 164B-1.

Table 2 lists the accumulated N-16 lobe and total core exposure, as obtained from the ATR Data Acquisition System (RDAS) for Cycles 134A-1 through 164B-1.

The ATR power history prior to Cycle $134 \mathrm{~A}-1$ is presented in the references.

Table 1. Summary of ATR Power History by Cycle

\begin{tabular}{|c|c|c|c|c|c|c|c|c|c|c|c|c|}
\hline & \multicolumn{5}{|c|}{ Average Lobe Power (MW) } & \multicolumn{6}{|c|}{ Cycle Exposure (MWd) } & \multirow{2}{*}{$\begin{array}{l}\text { Length } \\
\text { (EFPD) }\end{array}$} \\
\hline & NW & $\mathrm{NE}$ & $\mathrm{C}$ & SW & SE & NW & NE & $\mathrm{C}$ & SW & SE & Total & \\
\hline $134 \mathrm{~A}-1$ & - & - & - & - & - & - & - & $=$ & $=$ & - & - & - \\
\hline $134 \mathrm{~A}-2$ & - & - & - & - & - & 0.21 & 0.30 & 0.39 & 0.36 & 0.36 & 1.62 & - \\
\hline $134 \mathrm{~B}-1$ & 18.0 & 18.0 & 24.5 & 23.0 & 25.0 & 452.82 & 452.24 & 615.47 & 578.48 & 628.58 & $2,727.59$ & 25.10 \\
\hline $134 B-2$ & 18.0 & 18.0 & 25.8 & 23.0 & 25.0 & 385.62 & 385.80 & 553.50 & 493.24 & 536.07 & $2,354.23$ & 21.40 \\
\hline $135 \mathrm{~A}-1$ & 18.0 & 18.0 & 29.8 & 28.1 & 35.4 & 236.16 & 235.99 & 391.07 & 368.63 & 463.98 & $1,695.83$ & 13.10 \\
\hline $135 \mathrm{~B}-1$ & 18.0 & 18.0 & 24.8 & 23.0 & 25.0 & 458.28 & 458.35 & 630.79 & 585.27 & 636.51 & $2,769.20$ & 25.50 \\
\hline $135 B-2$ & 18.0 & 18.0 & 25.2 & 23.0 & 25.0 & 405.02 & 405.70 & 567.38 & 517.82 & 562.99 & $2,458.91$ & 22.50 \\
\hline $135 \mathrm{C}-1$ & 18.0 & 18.0 & 25.0 & 23.0 & 25.0 & 729.91 & 729.70 & $1,013.45$ & 933.00 & $1,013.86$ & $4,419.92$ & 40.60 \\
\hline $136 \mathrm{~A}-1$ & 18.0 & 18.0 & 24.0 & 23.0 & 23.0 & 916.18 & 916.39 & $1,218.91$ & $1,169.47$ & $1,170.17$ & $5,391.12$ & 50.90 \\
\hline $136 \mathrm{~B}-1$ & 18.0 & 18.0 & 23.9 & 23.0 & 23.0 & 701.94 & 702.32 & 931.19 & 896.93 & 896.97 & $4,129.35$ & 39.00 \\
\hline $137 A-1$ & 18.0 & 18.0 & 24.7 & 20.0 & 25.0 & 975.35 & 974.76 & $1,336.16$ & $1,083.20$ & $1,353.31$ & $5,722.78$ & 54.10 \\
\hline $137 \mathrm{~B}-1$ & 20.0 & 17.9 & 35.5 & 56.6 & 30.4 & 241.97 & 217.01 & 429.62 & 685.38 & 367.63 & $1,941.61$ & 12.10 \\
\hline $138 \mathrm{~A}-1$ & 18.0 & 18.0 & 23.6 & 23.0 & 25.0 & $1,046.87$ & $1,047.69$ & $1,370.69$ & $1,336.39$ & $1,453.47$ & $6,255.11$ & 58.10 \\
\hline 138B-1 & 18.0 & 18.0 & 23.3 & 23.0 & 25.0 & 838.54 & 839.62 & $1,084.79$ & $1,070.94$ & $1,164.64$ & $4,998.53$ & 46.60 \\
\hline $139 \mathrm{~A}-1$ & 18.0 & 18.0 & 23.9 & 23.0 & 25.0 & 928.73 & 929.09 & $1,231.07$ & $1,186.27$ & $1,289.47$ & $5,564.63$ & 51.60 \\
\hline
\end{tabular}


TEM-10200-1

$12 / 19 / 17$

Rev.08

Title:

AS-RUN NEUTRONICS EVALUATION FOR THE CSM-10584 EXPERIMENT IN THE ATR

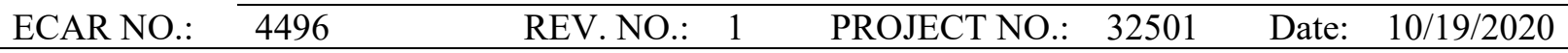

R. R. Little

January 21,2019

RE-03-19

Page 2 of 5

Table 1. Summary of ATR Power History by Cycle

\begin{tabular}{|c|c|c|c|c|c|c|c|c|c|c|c|c|}
\hline & \multicolumn{5}{|c|}{ Average Lobe Power (MW) } & \multicolumn{6}{|c|}{ Cycle Exposure (MWd) } & \multirow{2}{*}{$\begin{array}{l}\text { Length } \\
\text { (EFPD) }\end{array}$} \\
\hline & NW & NE & $\mathrm{C}$ & sw & SE & NW & NE & C & SW & SE & Total & \\
\hline 139B-1 & 18.0 & 18.0 & 23.2 & 23.0 & 23.0 & $919 . \overline{70}$ & 919.66 & $1,187.49$ & $1,174.88$ & $1,175,03$ & $5,376.76$ & 51.10 \\
\hline $140 A-1$ & 18.0 & 18.0 & 21.8 & 23.0 & 23.0 & 836.99 & 837.18 & $1,012.89$ & $1,069.69$ & $1,069.39$ & $4,826.14$ & 46.50 \\
\hline $140 \mathrm{~B}-1$ & 18.0 & 17.7 & 21.8 & 23.6 & 23.0 & 641.72 & 629.49 & 777.20 & 842.86 & 820.00 & $3,711.27$ & 35.70 \\
\hline $141 \mathrm{~A}-1$ & 18.0 & 18.0 & 23.4 & 23.0 & 23.0 & 583.25 & 583.08 & 756.83 & 745.05 & 745.45 & $3,413.66$ & 32.40 \\
\hline $142 \mathrm{~A}-1$ & 23.0 & 18.0 & 24.7 & 24.8 & 23.0 & $1,104.91$ & 864.75 & $1,186.04$ & $1,192.49$ & $1,104.04$ & $5,452.23$ & 48.00 \\
\hline $142 B-1$ & 23.0 & 18.0 & 25.4 & 25.4 & 25.0 & $1,196.93$ & 936.68 & $1,323.41$ & $1,322.45$ & $1,298.70$ & $6,078.17$ & 52.00 \\
\hline $143 A-1 / 2$ & 18.0 & 18.0 & 24.3 & 26.9 & 25.0 & 879.98 & 882.52 & $1,187.67$ & $1,315,44$ & $1,223.08$ & $5,488.69$ & 48.90 \\
\hline 143B-1 & 18.0 & 18.0 & 24.9 & 27.0 & 25.1 & $1,032.06$ & $1,032.58$ & $1,423.53$ & $1,543.74$ & $1,435.01$ & $6,466.92$ & 57.30 \\
\hline $144 A-1$ & 18.0 & 18.0 & 23.1 & 23.0 & 25.1 & 786.97 & 787.02 & $1,006.67$ & $1,004.53$ & $1,093.44$ & $4,678.63$ & 43.70 \\
\hline $144 \mathrm{~B}-1$ & 18.0 & 18.1 & 22.4 & 23.0 & 23.0 & 932.25 & 933.43 & $1,155.74$ & $1,190.72$ & $1,190.89$ & $5,403.03$ & 51.70 \\
\hline 145A-1 & 18.0 & 17.9 & 23.2 & 23.8 & 25.7 & 982.97 & 980.89 & $1,267.34$ & $1,299.49$ & $1,407.75$ & $5,938.44$ & 54.70 \\
\hline 145 & 17.8 & 17.8 & 23.0 & 24.6 & 25.8 & $1,020.46$ & $1,019.96$ & $1,321.43$ & $1,407.79$ & $1,478.25$ & $6,247.89$ & 57.30 \\
\hline 146A-1 & 18.0 & 18.0 & 24.3 & 25.8 & 26.0 & 906.76 & 906.80 & $1,225.74$ & $1,300.02$ & $1,312.55$ & $5,651.87$ & 50.50 \\
\hline 146 & 23.0 & 18.0 & 26.0 & 23.0 & 26.0 & 903.68 & 707.11 & $1,021.59$ & 903.85 & $1,020.96$ & $4,557.19$ & 39.20 \\
\hline 147 & 23.0 & 18.0 & 24.1 & 20.9 & 23.0 & $1,156.86$ & 904.42 & $1,208: 41$ & 1,$049 ; 42$ & $1,155.15$ & $5,474.26$ & 50.20 \\
\hline $148 A-1$ & 18.0 & 18.0 & 23.6 & 22.0 & 23.0 & 855.97 & 855.75 & $1,121.43$ & $1,043.79$ & $1,093.64$ & $4,970.58$ & 47.50 \\
\hline 148 & 18.0 & 18.0 & 23.0 & 23.8 & 23.0 & 927.50 & 926.72 & $1,181.60$ & $1,223.98$ & $1,185.03$ & $5,444.83$ & 51.50 \\
\hline $14 \subseteq$ & 18.0 & 18.0 & 24.2 & 24.0 & 23.0 & 662.45 & 662.65 & 891.28 & 883.28 & 846.80 & $3,946.46$ & 36.80 \\
\hline 149B-1 & 18.0 & 18.0 & 24.2 & 23.0 & 23.0 & $964: 38$ & $964: 45$ & $1,297.77$ & $1,231,63$ & $1,230.78$ & $5,689.01$ & $53: 60$ \\
\hline 150A-1 & 18.9 & 18.0 & 30.5 & 37.5 & 35.1 & 233,41 & 221.60 & 375.93 & 462.71 & 432.58 & 23 & 12,32 \\
\hline 150 & 19.9 & 18.0 & 24.2 & 23.0 & 23.1 & 832.84 & $754: 72$ & $1,014.58$ & 964.86 & 966.00 & $4,533.00$ & 41.87 \\
\hline 151A-1 & 18.9 & 14.2 & 22.0 & 23.6 & 23.0 & $1,058.63$ & 799.95 & $1,237.01$ & $1,324.39$ & $1,289.04$ & $5,709.02$ & 56.14 \\
\hline 151 & 18.9 & 14.5 & 22.1 & 23.0 & 23.0 & 971.41 & 741.75 & $1,134.87$ & $1,181.94$ & $1,179.97$ & $5,209.94$ & 51.30 \\
\hline $152 A-1 / 6$ & $=$ & $=$ & $=$ & - & - & 0.30 & 0.30 & 0.50 & 0.40 & 0.50 & 2.00 & - \\
\hline 152B-1 & 18.9 & 15.9 & 22.4 & 23.0 & 23.0 & 966.42 & 813.00 & $1,141.28$ & $1,172.08$ & $1,173.57$ & $5,266.35$ & 51.02 \\
\hline 153B-1 & 19.7 & 19.7 & 30.8 & 35.4 & 44.0 & 265.20 & 265.35 & 414.47 & 476.12 & 591.42 & $2,012.56$ & 13.45 \\
\hline 15 & 20.0 & 16.0 & 20.5 & 20.5 & 23.0 & .12 & 838.60 & $1,069.38$ & $1,071,42$ & $1,204.22$ & $5,231.74$ & 52.28 \\
\hline 154B-1 & 20.0 & 17.9 & 21.6 & 22.8 & 23.9 & $1,066.60$ & 958.47 & $1,156.40$ & $1,220.49$ & $1,275.27$ & $5,677.23$ & 53.44 \\
\hline $155 A-1$ & 17.5 & 18.0 & 21.2 & 24.9 & 23.0 & 964.46 & 992.05 & $1,169.64$ & $1,373.15$ & $1,269.33$ & $5,768.63$ & 55.14 \\
\hline 155B-1 & 18.0 & 18.7 & 22.0 & 22.9 & 23.0 & 896.41 & 933.78 & $1,097.45$ & $1,145.28$ & $1,146.21$ & $5,219.13$ & 49.92 \\
\hline $156 \mathrm{~A}-1$ & 18.0 & 18.1 & 34.4 & 47.6 & 47.3 & 258.99 & 260.10 & 495.95 & 685.85 & $681 / 77$ & $2,382,66$ & 14.40 \\
\hline 157A-1 & 18.0 & 16.9 & 20.1 & 19.9 & 24.0 & 674.80 & 636.93 & 755.89 & 749,37 & 900.30 & $3,717.29$ & 37.58 \\
\hline $157 \mathrm{C}-1$ & 18.0 & 17.0 & 21.1 & 20.1 & 25.0 & 96.61 & 91.47 & 113.15 & 107.90 & 134.07 & 543.20 & 5.37 \\
\hline 157D-1 & 18.0 & 17.0 & 21.6 & 20.9 & 25.0 & $1,072.06$ & $1,012.51$ & $1,288.16$ & $1,247.14$ & $1,490.31$ & $6,110.18$ & 59.71 \\
\hline-1 & 18.0 & 19.0 & 21.3 & 20.0 & 27.0 & 941.32 & 994.16 & $1,112.08$ & $1,044.16$ & $1,410.05$ & $5,501.77$ & 52.23 \\
\hline $158 \mathrm{~B}-1$ & 18.0 & 19.0 & 19.0 & 22.7 & 23.0 & 924.85 & 977.00 & 974.33 & $1,165.25$ & $1,183.34$ & $5,224.77$ & 51.36 \\
\hline $159 \mathrm{~A}-1$ & 22.1 & 22.9 & 29.1 & 37.4 & 35.0 & 68.55 & 71.08 & 90.14 & 116.01 & 108.61 & 454.39 & 3.10 \\
\hline & 17.5 & 21.1 & 19.8 & 20.0 & 26.1 & 924.07 & $1,1.15 .94$ & $1,050.71$ & $1,057,87$ & $1,380,71$ & $5,529.30$ & 52.94 \\
\hline $160 \mathrm{~B}-1$ & 18.0 & 21.0 & 20.9 & 23.0 & 25.6 & $1,080,05$ & $1,259.85$ & $1,252,55$ & $1,380.11$ & $1,537.65$ & $6,510.21$ & $60 ; 06$ \\
\hline $161 \mathrm{~A}-1$ & 18.0 & 20.9 & 30.6 & 42.7 & 41.5 & 230.21 & 268.17 & 392.91 & 547.87 & 532.53 & $1,971.69$ & 12.82 \\
\hline 162 & 20.1 & 17.9 & 22.8 & 24.0 & 26.2 & $1,244.31$ & $1,105.06$ & $1,410.78$ & $1,486.12$ & $1,621.56$ & $6,867.83$ & 61.90 \\
\hline 162B-1 & 20.0 & 14.5 & 19.2 & 23.9 & 23.0 & 769.04 & 560.05 & 738.98 & 918.84 & 884.90 & $3,871.81$ & 38.51 \\
\hline $163 \mathrm{~A}-1$ & 21.0 & 20.5 & 28.3 & 40.8 & 31.8 & 62.84 & 61,22 & 84.68 & 12193 & 95.13 & 425.80 & 2.99 \\
\hline & 20.0 & 16.0 & 19.4 & 22.4 & $25 ; 7$ & $1,099.06$ & 879.28 & $1,067,84$ & $1,230,00$ & $1,410: 51$ & $5,686,69$ & 54.91 \\
\hline $164 B-1$ & 19.5 & 16.4 & 19.7 & 23.1 & 25.1 & $1,246,27$ & $1,051.72$ & $1,261.5 \overline{8}$ & $1,477,80$ & $1,607,82$ & $6,645,19$ & 64.06 \\
\hline
\end{tabular}


TEM-10200-1

$12 / 19 / 17$

Rev.08

Title:

AS-RUN NEUTRONICS EVALUATION FOR THE CSM-10584 EXPERIMENT IN THE ATR

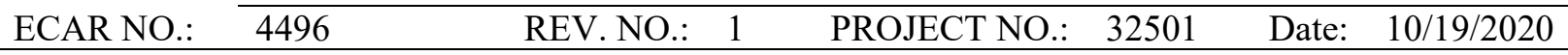

R. R. Little

January 21, 2019

RE-03-19

Page 3 of 5

Table 2. Cumulative Exposure

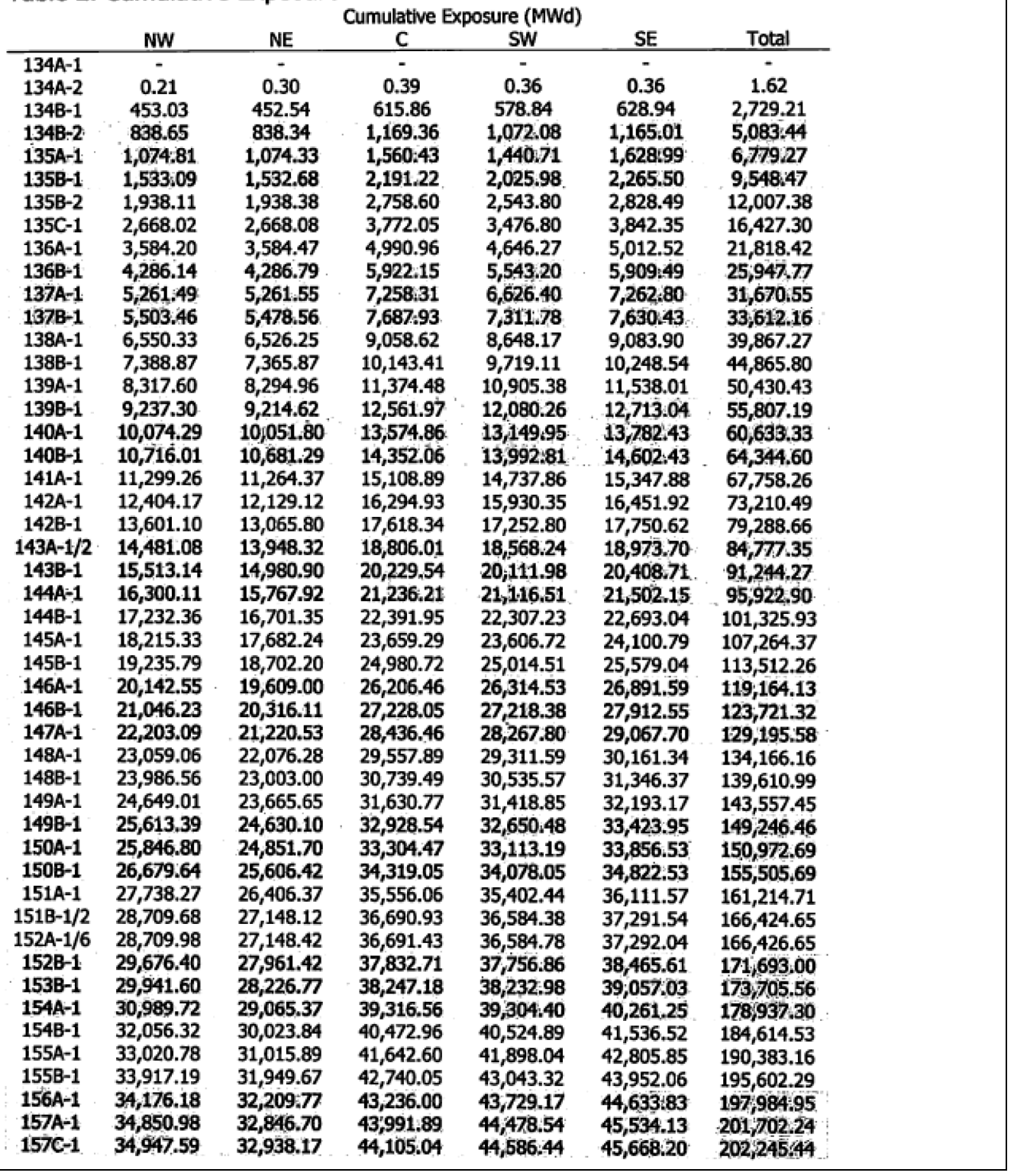


TEM-10200-1

$12 / 19 / 17$

Rev.08

Title:

AS-RUN NEUTRONICS EVALUATION FOR THE CSM-10584 EXPERIMENT IN THE ATR

ECAR NO.:

R. R. Little

January 21, 2019

RE-03-19

Page 4 of 5

Table 2. Cumulative Exposure

\begin{tabular}{|c|c|c|c|c|c|c|}
\hline & \multicolumn{6}{|c|}{ Cumulative Exposure (MWd) } \\
\hline & NW & $\mathrm{NE}$ & $\mathrm{C}$ & sw & SE & Total \\
\hline $157 \mathrm{D}-1$ & $36,019.65$ & $33,950.68$ & $45,393.20$ & $45,833.58$ & $47,158.51$ & $208,355.62$ \\
\hline $158 A-1$ & $36,960.97$ & $34,944.84$ & $46,505.28$ & $46,877.74$ & $48,568.56$ & $213,857.39$ \\
\hline 158B-1 & $37,885.82$ & $35,921.84$ & $47,479.61$ & $48,042.99$ & $49,751.90$ & $219,082.16$ \\
\hline $159 \mathrm{~A}-1$ & $37,954,37$ & 35,99292 & 47,56975 & $48,159: 00$ & $49 \%$ & 219586155 \\
\hline $160 \mathrm{~A}-1$ & 3887844 & 37,108186 & 48,620446 & $49 / 216: 87$ & $51,241,22$ & $225,065.85$ \\
\hline $160 \mathrm{~B}-1$ & $39,958: 49$ & $38,368: 71$ & $49,873.01$ & 6698 & $52,778.87$ & $231,576: 06$ \\
\hline $161 \mathrm{~A}-1$ & $40,188.70$ & $38,636.88$ & $50,265.92$ & $51,144.85$ & $53,311.40$ & $233,547.75$ \\
\hline $162 \mathrm{~A}-1$ & $41,433.01$ & $39,741.94$ & $51,676.70$ & $52,630.97$ & $54,932.96$ & $240,415.58$ \\
\hline $162 \mathrm{~B}-1$ & $42,202.05$ & $40,301.99$ & $52,415.68$ & $53,549.81$ & & $244,287.39$ \\
\hline $16 B A-1$ & 42,26489 & $40,363.21$ & & $53,67 \mathrm{~d} 74$ & 55,91299 & $244 / 713 / 9$ \\
\hline $164 A-1$ & $43,363,95$ & $41,242,49$ & $53,568,20$ & 54,901174 & $57,323.5$ & 250,39988 \\
\hline & $44,610.22$ & 42,29421 & $54,829,78$ & 56,37964 & $58 ; 931: 32$ & $257,045: 07$ \\
\hline
\end{tabular}

Prepared by

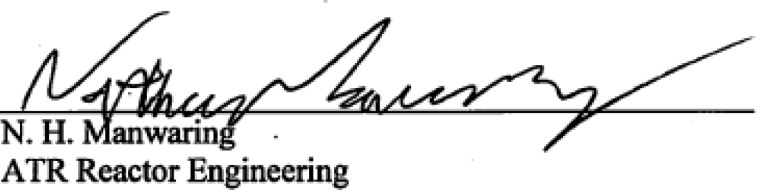

ATR Reactor Engineering

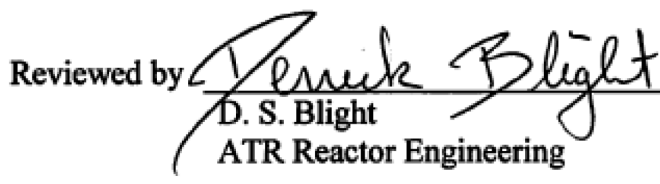

NHM:LLS 
TEM-10200-1

$12 / 19 / 17$

ENGINEERING CALCULATIONS AND ANALYSIS

Rev.08

Title:

AS-RUN NEUTRONICS EVALUATION FOR THE CSM-10584 EXPERIMENT IN THE ATR

ECAR NO

\author{
R. R. Little \\ January 21, 2019 \\ RE-03-19 \\ Page 5 of 5
}

cc:
J. O. Brower, MS 3890
H. D. Buckland, MS 7114
R. R. Bullock, MS 7117
K. H. Carlquist, MS 7104
C. D. Cooper, MS 3890
C. C. Dwight, MS 6122
K. R. Estes, MS 7114
B. J. Good, MS 7136
M. J. Guzman, MS 7117
S. A. Hill, MS 7106
M. R. Holtz, MS 7136
R. C. Howard, MS 7101
C. D. Jackson, MS 7106
W. F. Jones, MS 3818
T. L. Julius, MS 7104
V. C. Kirkpatrick, MS 7106
M. A. Lillo, MS 3860
R. R. Little, MS 7136
S. G. Louk, MS 7111
N. J. Lybeck, MS 3818

.

N. H. Manwaring, MS 7136

C. A. McPhie, FMP, MS 7103

R. M. Murphy, MS 9202

D. R. Norman, 7136

D. Ogden, MS 3553

S. O'Kelly, MS 7117

D. G. Robinson, MS 7104

D. L. Rowsell, MS 7104

D. J. Schoonen, MS 7117

K. A. Smith, MS 7101

L. D. Smith, MS 7111

K. D. Stueve, MS 7117

E. T. Swain, MS 3818

D. J. Utterbeck, MS 3870

K. L. Zimmer, MS 7106

ATR Document Management, MS 7116, email: Jennifer.eversull@inl.gov

Cycle 164B-1 Surveillance File

Reactor Engineering Letter File (RE-03-19)

Uniform File Code: $\underline{8153}$

Disposition Authority: A17-32-b-1

Retention Schedule: Retain in accordance with current regulatory requirements or for nuclear facilities, 6 years after plant or item is put into operation.

NOTE: Original disposition authority, retention schedule, and Uniform Filing Code applied by the sender may not be appropriate for all recipients. Make adjustments as needed. 
TEM-10200-1

ENGINEERING CALCULATIONS AND ANALYSIS

Page 49 of 81

$12 / 19 / 17$

Rev.08

Title:

AS-RUN NEUTRONICS EVALUATION FOR THE CSM-10584 EXPERIMENT IN THE ATR

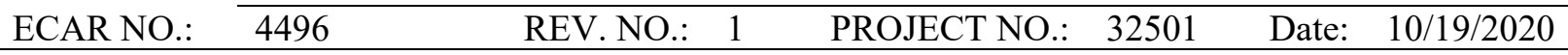

\section{APPENDIX C “ADDITIONAL SOURCE TERM”}

The CSM-10584 experiment consisted of two sample materials. To support disassembly and future PIE of the CSM-10584 experiment the source term for 1 gram of each material scaled to the maximum experiment flux $(1.18 \mathrm{E}+15)$ was calculated. This conservative source term can be scaled to the total mass for each sample geometry located in each capsule. The materials reported for the 54.9 EFPD irradiation should be applied to the specimen that were irradiated for only one cycle. The materials reported for the 119 EFPD irradiation should be applied to the remaining specimen.

\begin{tabular}{|c|c|c|c|c|c|c|c|c|c|}
\hline \multicolumn{10}{|c|}{1 gram of $316 S S$ irradiated for 54.9 EFPDs } \\
\hline & EOC & $30 \mathrm{MINs}$ & $5 \mathrm{HRS}$ & 1 DAY & 7 DAYS & 14 DAYS & 30 DAYS & 60 DAYS & 1 YEAR \\
\hline & $\mathrm{Ci} / \mathrm{g}$ & $\mathrm{Ci} / \mathrm{g}$ & $\mathrm{Ci} / \mathrm{g}$ & $\mathrm{Ci} / \mathrm{g}$ & $\mathrm{Ci} / \mathrm{g}$ & $\mathrm{Ci} / \mathrm{g}$ & $\mathrm{Ci} / \mathrm{g}$ & $\mathrm{Ci} / \mathrm{g}$ & $\mathrm{Ci} / \mathrm{g}$ \\
\hline H 3 & $6.15 \mathrm{E}-11$ & $6.15 \mathrm{E}-11$ & $6.15 \mathrm{E}-11$ & $6.15 \mathrm{E}-11$ & $6.15 \mathrm{E}-11$ & $6.14 \mathrm{E}-11$ & $6.12 \mathrm{E}-11$ & $6.10 \mathrm{E}-11$ & 5.82E-11 \\
\hline HE 6 & $1.05 \mathrm{E}-07$ & $0.00 \mathrm{E}+00$ & $0.00 \mathrm{E}+00$ & $0.00 \mathrm{E}+00$ & $0.00 \mathrm{E}+00$ & $0.00 \mathrm{E}+00$ & $0.00 \mathrm{E}+00$ & $0.00 \mathrm{E}+00$ & $0.00 \mathrm{E}+00$ \\
\hline $\mathrm{BE} 8$ & $1.82 \mathrm{E}-07$ & $0.00 \mathrm{E}+00$ & $0.00 \mathrm{E}+00$ & $0.00 \mathrm{E}+00$ & $0.00 \mathrm{E}+00$ & $0.00 \mathrm{E}+00$ & $0.00 \mathrm{E}+00$ & $0.00 \mathrm{E}+00$ & $0.00 \mathrm{E}+00$ \\
\hline BE 10 & 7.37E-13 & $7.37 \mathrm{E}-13$ & 7.37E-13 & $7.37 \mathrm{E}-13$ & $7.37 \mathrm{E}-13$ & $7.37 \mathrm{E}-13$ & $7.37 \mathrm{E}-13$ & 7.37E-13 & $7.37 \mathrm{E}-13$ \\
\hline BE 11 & $5.83 \mathrm{E}-12$ & $0.00 \mathrm{E}+00$ & $0.00 \mathrm{E}+00$ & $0.00 \mathrm{E}+00$ & $0.00 \mathrm{E}+00$ & $0.00 \mathrm{E}+00$ & $0.00 \mathrm{E}+00$ & $0.00 \mathrm{E}+00$ & $0.00 \mathrm{E}+00$ \\
\hline C 14 & $2.71 \mathrm{E}-11$ & $2.71 \mathrm{E}-11$ & $2.71 \mathrm{E}-11$ & $2.71 \mathrm{E}-11$ & $2.71 \mathrm{E}-11$ & $2.71 \mathrm{E}-11$ & $2.71 \mathrm{E}-11$ & $2.71 \mathrm{E}-11$ & $2.71 \mathrm{E}-11$ \\
\hline NE 23 & $3.23 \mathrm{E}-09$ & $9.08 \mathrm{E}-24$ & $0.00 \mathrm{E}+00$ & $0.00 \mathrm{E}+00$ & $0.00 \mathrm{E}+00$ & $0.00 \mathrm{E}+00$ & $0.00 \mathrm{E}+00$ & $0.00 \mathrm{E}+00$ & $0.00 \mathrm{E}+00$ \\
\hline NA 24 & $8.05 \mathrm{E}-12$ & 7.87E-12 & $6.39 \mathrm{E}-12$ & $2.66 \mathrm{E}-12$ & $3.42 \mathrm{E}-15$ & $1.45 \mathrm{E}-18$ & $2.85 \mathrm{E}-26$ & $0.00 \mathrm{E}+00$ & $0.00 \mathrm{E}+00$ \\
\hline NA 24M & $3.44 \mathrm{E}-13$ & $0.00 \mathrm{E}+00$ & $0.00 \mathrm{E}+00$ & $0.00 \mathrm{E}+00$ & $0.00 \mathrm{E}+00$ & $0.00 \mathrm{E}+00$ & $0.00 \mathrm{E}+00$ & $0.00 \mathrm{E}+00$ & $0.00 \mathrm{E}+00$ \\
\hline NA 25 & 4.89E-09 & $3.96 \mathrm{E}-18$ & $0.00 \mathrm{E}+00$ & $0.00 \mathrm{E}+00$ & $0.00 \mathrm{E}+00$ & $0.00 \mathrm{E}+00$ & $0.00 \mathrm{E}+00$ & $0.00 \mathrm{E}+00$ & $0.00 \mathrm{E}+00$ \\
\hline MG 27 & $6.51 \mathrm{E}-06$ & $7.23 \mathrm{E}-07$ & $1.86 \mathrm{E}-15$ & $0.00 \mathrm{E}+00$ & $0.00 \mathrm{E}+00$ & $0.00 \mathrm{E}+00$ & $0.00 \mathrm{E}+00$ & $0.00 \mathrm{E}+00$ & $0.00 \mathrm{E}+00$ \\
\hline MG 28 & $2.31 \mathrm{E}-14$ & $2.28 \mathrm{E}-14$ & $1.96 \mathrm{E}-14$ & $1.05 \mathrm{E}-14$ & $9.64 \mathrm{E}-17$ & $8.73 \mathrm{E}-18$ & $2.59 \mathrm{E}-23$ & $0.00 \mathrm{E}+00$ & $0.00 \mathrm{E}+00$ \\
\hline AL 28 & $7.84 \mathrm{E}-03$ & $7.29 E-07$ & 1.97E-14 & $1.05 \mathrm{E}-14$ & $9.65 \mathrm{E}-17$ & $8.73 \mathrm{E}-18$ & $2.59 \mathrm{E}-23$ & $0.00 \mathrm{E}+00$ & $0.00 \mathrm{E}+00$ \\
\hline AL 29 & 2.65E-04 & 1.09E-05 & $3.73 \mathrm{E}-18$ & $0.00 \mathrm{E}+00$ & $0.00 \mathrm{E}+00$ & $0.00 \mathrm{E}+00$ & $0.00 \mathrm{E}+00$ & $0.00 \mathrm{E}+00$ & $0.00 \mathrm{E}+00$ \\
\hline AL 30 & 2.57E-07 & $0.00 \mathrm{E}+00$ & $0.00 \mathrm{E}+00$ & $0.00 \mathrm{E}+00$ & $0.00 \mathrm{E}+00$ & $0.00 \mathrm{E}+00$ & $0.00 \mathrm{E}+00$ & $0.00 \mathrm{E}+00$ & $0.00 \mathrm{E}+00$ \\
\hline SI 31 & 5.04E-03 & $4.42 \mathrm{E}-03$ & $1.35 \mathrm{E}-03$ & 8.85E-06 & $2.58 \mathrm{E}-22$ & $0.00 \mathrm{E}+00$ & $0.00 \mathrm{E}+00$ & $0.00 \mathrm{E}+00$ & $0.00 \mathrm{E}+00$ \\
\hline $\mathrm{SI} 32$ & $5.72 \mathrm{E}-13$ & $5.72 \mathrm{E}-13$ & $5.72 \mathrm{E}-13$ & $5.72 \mathrm{E}-13$ & $5.72 \mathrm{E}-13$ & $5.72 \mathrm{E}-13$ & $5.72 \mathrm{E}-13$ & $5.72 \mathrm{E}-13$ & $5.71 \mathrm{E}-13$ \\
\hline P 32 & $7.21 \mathrm{E}-03$ & 7.20E-03 & 7.13E-03 & $6.87 \mathrm{E}-03$ & $5.13 \mathrm{E}-03$ & $3.66 \mathrm{E}-03$ & $1.68 \mathrm{E}-03$ & 3.93E-04 & $1.48 \mathrm{E}-10$ \\
\hline P 33 & $1.98 \mathrm{E}-07$ & $1.98 \mathrm{E}-07$ & 1.97E-07 & $1.92 \mathrm{E}-07$ & $1.63 \mathrm{E}-07$ & $1.34 \mathrm{E}-07$ & $8.61 \mathrm{E}-08$ & 3.75E-08 & $7.91 \mathrm{E}-12$ \\
\hline P 34 & 1.46E-06 & $0.00 \mathrm{E}+00$ & $0.00 E+00$ & $0.00 \mathrm{E}+00$ & $0.00 \mathrm{E}+00$ & $0.00 \mathrm{E}+00$ & $0.00 E+00$ & $0.00 \mathrm{E}+00$ & $0.00 \mathrm{E}+00$ \\
\hline S 35 & 5.86E-05 & 5.86E-05 & $5.85 \mathrm{E}-05$ & $5.82 \mathrm{E}-05$ & $5.55 \mathrm{E}-05$ & $5.25 \mathrm{E}-05$ & 4.63E-05 & 3.66E-05 & $3.30 \mathrm{E}-06$ \\
\hline S 37 & 4.21E-07 & $6.92 \mathrm{E}-09$ & $5.98 \mathrm{E}-25$ & $0.00 \mathrm{E}+00$ & $0.00 \mathrm{E}+00$ & $0.00 \mathrm{E}+00$ & $0.00 \mathrm{E}+00$ & $0.00 \mathrm{E}+00$ & $0.00 \mathrm{E}+00$ \\
\hline CL 36 & 8.13E-14 & 8.13E-14 & 8.13E-14 & $8.13 \mathrm{E}-14$ & $8.13 E-14$ & $8.13 E-14$ & 8.13E-14 & 8.13E-14 & 8.13E-14 \\
\hline $\mathrm{CL} 38$ & 9.97E-11 & $5.70 \mathrm{E}-11$ & 3.73E-13 & $2.24 \mathrm{E}-22$ & $0.00 \mathrm{E}+00$ & $0.00 \mathrm{E}+00$ & $0.00 \mathrm{E}+00$ & $0.00 \mathrm{E}+00$ & $0.00 \mathrm{E}+00$ \\
\hline CL 38M & 1.19E-12 & $0.00 \mathrm{E}+00$ & $0.00 E+00$ & $0.00 \mathrm{E}+00$ & $0.00 \mathrm{E}+00$ & $0.00 \mathrm{E}+00$ & $0.00 E+00$ & $0.00 \mathrm{E}+00$ & $0.00 \mathrm{E}+00$ \\
\hline CA 45 & 1.00E-13 & 1.00E-13 & $1.00 \mathrm{E}-13$ & 9.96E-14 & $9.71 \mathrm{E}-14$ & $9.43 \mathrm{E}-14$ & 8.81E-14 & 7.75E-14 & $2.12 \mathrm{E}-14$ \\
\hline CA 47 & 3.63E-12 & 3.62E-12 & $3.52 \mathrm{E}-12$ & 3.12E-12 & $1.25 \mathrm{E}-12$ & $4.27 \mathrm{E}-13$ & $3.71 \mathrm{E}-14$ & 3.79E-16 & $0.00 \mathrm{E}+00$ \\
\hline SC 47 & $1.86 \mathrm{E}-08$ & $1.86 \mathrm{E}-08$ & 1.79E-08 & $1.52 \mathrm{E}-08$ & 4.38E-09 & 1.03E-09 & 3.77E-11 & 7.73E-14 & $0.00 \mathrm{E}+00$ \\
\hline SC 48 & 1.11E-08 & 1.10E-08 & $1.03 \mathrm{E}-08$ & 7.61E-09 & $7.79 \mathrm{E}-10$ & $5.46 \mathrm{E}-11$ & $1.26 \mathrm{E}-13$ & $1.42 \mathrm{E}-18$ & $0.00 \mathrm{E}+00$ \\
\hline SC 49 & 5.56E-09 & 3.87E-09 & $1.50 \mathrm{E}-10$ & $1.61 \mathrm{E}-16$ & $0.00 \mathrm{E}+00$ & $0.00 \mathrm{E}+00$ & $0.00 \mathrm{E}+00$ & $0.00 \mathrm{E}+00$ & $0.00 \mathrm{E}+00$ \\
\hline SC 50 & $4.72 \mathrm{E}-10$ & $2.44 \mathrm{E}-15$ & $0.00 \mathrm{E}+00$ & $0.00 \mathrm{E}+00$ & $0.00 \mathrm{E}+00$ & $0.00 \mathrm{E}+00$ & $0.00 \mathrm{E}+00$ & $0.00 \mathrm{E}+00$ & $0.00 \mathrm{E}+00$ \\
\hline TI 51 & $2.25 \mathrm{E}-05$ & $6.09 \mathrm{E}-07$ & $4.72 \mathrm{E}-21$ & $0.00 \mathrm{E}+00$ & $0.00 \mathrm{E}+00$ & $0.00 \mathrm{E}+00$ & $0.00 \mathrm{E}+00$ & $0.00 \mathrm{E}+00$ & $0.00 \mathrm{E}+00$ \\
\hline V 52 & 2.97E-02 & 1.16E-04 & $2.45 \mathrm{E}-26$ & $0.00 \mathrm{E}+00$ & $0.00 \mathrm{E}+00$ & $0.00 \mathrm{E}+00$ & $0.00 \mathrm{E}+00$ & $0.00 \mathrm{E}+00$ & $0.00 \mathrm{E}+00$ \\
\hline V 53 & 1.83E-04 & 4.49E-10 & $0.00 \mathrm{E}+00$ & $0.00 \mathrm{E}+00$ & $0.00 \mathrm{E}+00$ & $0.00 \mathrm{E}+00$ & $0.00 \mathrm{E}+00$ & $0.00 \mathrm{E}+00$ & $0.00 \mathrm{E}+00$ \\
\hline V 54 & $1.89 \mathrm{E}-06$ & $2.65 \mathrm{E}-16$ & $0.00 \mathrm{E}+00$ & $0.00 \mathrm{E}+00$ & $0.00 \mathrm{E}+00$ & $0.00 \mathrm{E}+00$ & $0.00 \mathrm{E}+00$ & $0.00 \mathrm{E}+00$ & $0.00 \mathrm{E}+00$ \\
\hline CR 51 & $3.00 \mathrm{E}+00$ & $2.99 \mathrm{E}+00$ & $2.98 \mathrm{E}+00$ & $2.92 \mathrm{E}+00$ & $2.51 \mathrm{E}+00$ & $2.11 \mathrm{E}+00$ & $1.41 \mathrm{E}+00$ & $6.68 \mathrm{E}-01$ & 3.22E-04 \\
\hline CR 55 & 5.41E-02 & 1.55E-04 & $0.00 \mathrm{E}+00$ & $0.00 \mathrm{E}+00$ & $0.00 \mathrm{E}+00$ & $0.00 \mathrm{E}+00$ & $0.00 \mathrm{E}+00$ & $0.00 \mathrm{E}+00$ & $0.00 \mathrm{E}+00$ \\
\hline MN 54 & $3.02 \mathrm{E}-02$ & $3.02 \mathrm{E}-02$ & $3.02 \mathrm{E}-02$ & $3.01 \mathrm{E}-02$ & $2.97 \mathrm{E}-02$ & $2.93 \mathrm{E}-02$ & $2.82 \mathrm{E}-02$ & 2.64E-02 & $1.34 \mathrm{E}-02$ \\
\hline MN 56 & $1.09 \mathrm{E}+01$ & $9.53 \mathrm{E}+00$ & $2.84 \mathrm{E}+00$ & $1.72 \mathrm{E}-02$ & $2.66 \mathrm{E}-19$ & $0.00 \mathrm{E}+00$ & $0.00 \mathrm{E}+00$ & $0.00 \mathrm{E}+00$ & $0.00 \mathrm{E}+00$ \\
\hline
\end{tabular}


TEM-10200-1

ENGINEERING CALCULATIONS AND ANALYSIS

Page 50 of 81

$12 / 19 / 17$

Rev.08

Title:

AS-RUN NEUTRONICS EVALUATION FOR THE CSM-10584 EXPERIMENT IN THE ATR

\begin{tabular}{llllllll}
\cline { 2 - 6 } ECAR NO.: & 4496 & REV. NO.: & 1 & PROJECT NO.: & 32501 & Date: & $10 / 19 / 2020$ \\
\hline
\end{tabular}

\begin{tabular}{|c|c|c|c|c|c|c|c|c|c|}
\hline \multicolumn{10}{|c|}{1 gram of $316 \mathrm{SS}$ irradiated for 54.9 EFPDs } \\
\hline & EOC & $30 \mathrm{MINs}$ & $5 \mathrm{HRS}$ & $1 \mathrm{DAY}$ & 7 DAYS & 14 DAYS & 30 DAYS & 60 DAYS & 1 YEAR \\
\hline & $\mathrm{Ci} / \mathrm{g}$ & $\mathrm{Ci} / \mathrm{g}$ & $\mathrm{Ci} / \mathrm{g}$ & $\mathrm{Ci} / \mathrm{g}$ & $\mathrm{Ci} / \mathrm{g}$ & $\mathrm{Ci} / \mathrm{g}$ & $\mathrm{Ci} / \mathrm{g}$ & $\mathrm{Ci} / \mathrm{g}$ & $\mathrm{Ci} / \mathrm{g}$ \\
\hline MN 57 & $6.31 \mathrm{E}-04$ & $1.55 \mathrm{E}-09$ & $0.00 \mathrm{E}+00$ & $0.00 \mathrm{E}+00$ & $0.00 \mathrm{E}+00$ & $0.00 \mathrm{E}+00$ & $0.00 \mathrm{E}+00$ & $0.00 \mathrm{E}+00$ & $0.00 \mathrm{E}+00$ \\
\hline $\mathrm{MN} 58$ & $2.02 \mathrm{E}-06$ & $1.01 \mathrm{E}-14$ & $0.00 \mathrm{E}+00$ & $0.00 \mathrm{E}+00$ & $0.00 \mathrm{E}+00$ & $0.00 \mathrm{E}+00$ & $0.00 \mathrm{E}+00$ & $0.00 \mathrm{E}+00$ & $0.00 \mathrm{E}+00$ \\
\hline FE 55 & $1.10 \mathrm{E}-01$ & $1.10 \mathrm{E}-01$ & $1.10 \mathrm{E}-01$ & $1.10 \mathrm{E}-01$ & $1.09 \mathrm{E}-01$ & $1.09 \mathrm{E}-01$ & 1.07E-01 & $1.05 \mathrm{E}-01$ & $8.40 \mathrm{E}-02$ \\
\hline FE 59 & $4.57 \mathrm{E}-02$ & 4.57E-02 & $4.56 \mathrm{E}-02$ & $4.50 \mathrm{E}-02$ & $4.10 \mathrm{E}-02$ & $3.68 \mathrm{E}-02$ & $2.88 \mathrm{E}-02$ & $1.81 \mathrm{E}-02$ & $1.65 \mathrm{E}-04$ \\
\hline $\cos 58$ & 1.17E-01 & $1.17 \mathrm{E}-01$ & $1.16 \mathrm{E}-01$ & $1.15 \mathrm{E}-01$ & $1.09 \mathrm{E}-01$ & $1.02 \mathrm{E}-01$ & $8.69 \mathrm{E}-02$ & $6.47 \mathrm{E}-02$ & $3.26 \mathrm{E}-03$ \\
\hline $\operatorname{co} 60$ & $1.82 \mathrm{E}-04$ & $1.82 \mathrm{E}-04$ & $1.82 \mathrm{E}-04$ & $1.82 \mathrm{E}-04$ & $1.81 \mathrm{E}-04$ & $1.81 \mathrm{E}-04$ & $1.80 \mathrm{E}-04$ & $1.78 \mathrm{E}-04$ & $1.60 \mathrm{E}-04$ \\
\hline $\mathrm{CO} 60 \mathrm{M}$ & $4.23 \mathrm{E}-03$ & $5.80 \mathrm{E}-04$ & $1.00 \mathrm{E}-11$ & $0.00 \mathrm{E}+00$ & $0.00 \mathrm{E}+00$ & $0.00 \mathrm{E}+00$ & $0.00 \mathrm{E}+00$ & $0.00 \mathrm{E}+00$ & $0.00 \mathrm{E}+00$ \\
\hline CO 61 & $2.53 \mathrm{E}-04$ & $2.05 \mathrm{E}-04$ & $3.09 \mathrm{E}-05$ & 1.06E-08 & $0.00 \mathrm{E}+00$ & $0.00 \mathrm{E}+00$ & $0.00 \mathrm{E}+00$ & $0.00 \mathrm{E}+00$ & $0.00 \mathrm{E}+00$ \\
\hline CO 62 & $4.29 \mathrm{E}-05$ & $4.09 \mathrm{E}-11$ & $0.00 \mathrm{E}+00$ & $0.00 \mathrm{E}+00$ & $0.00 \mathrm{E}+00$ & $0.00 \mathrm{E}+00$ & $0.00 \mathrm{E}+00$ & $0.00 \mathrm{E}+00$ & $0.00 \mathrm{E}+00$ \\
\hline NI 59 & $1.64 \mathrm{E}-05$ & $1.64 \mathrm{E}-05$ & $1.64 \mathrm{E}-05$ & $1.64 \mathrm{E}-05$ & $1.64 \mathrm{E}-05$ & $1.64 \mathrm{E}-05$ & $1.64 \mathrm{E}-05$ & $1.64 \mathrm{E}-05$ & $1.64 \mathrm{E}-05$ \\
\hline $\mathrm{NI} 63$ & $2.08 \mathrm{E}-03$ & $2.08 \mathrm{E}-03$ & $2.08 \mathrm{E}-03$ & $2.08 \mathrm{E}-03$ & $2.08 \mathrm{E}-03$ & $2.08 \mathrm{E}-03$ & $2.08 \mathrm{E}-03$ & $2.08 \mathrm{E}-03$ & $2.06 \mathrm{E}-03$ \\
\hline NI 65 & $5.27 \mathrm{E}-02$ & $4.59 \mathrm{E}-02$ & $1.33 \mathrm{E}-02$ & $7.16 \mathrm{E}-05$ & $4.50 \mathrm{E}-22$ & $0.00 \mathrm{E}+00$ & $0.00 \mathrm{E}+00$ & $0.00 \mathrm{E}+00$ & $0.00 \mathrm{E}+00$ \\
\hline NI 66 & $1.82 \mathrm{E}-06$ & $1.81 \mathrm{E}-06$ & $1.71 \mathrm{E}-06$ & $1.34 \mathrm{E}-06$ & $2.16 \mathrm{E}-07$ & $2.56 \mathrm{E}-08$ & $1.96 \mathrm{E}-10$ & $2.10 \mathrm{E}-14$ & $0.00 \mathrm{E}+00$ \\
\hline CU 64 & $2.89 \mathrm{E}-06$ & $2.81 \mathrm{E}-06$ & $2.20 \mathrm{E}-06$ & $7.80 \mathrm{E}-07$ & $3.01 \mathrm{E}-10$ & $3.14 \mathrm{E}-14$ & $2.48 \mathrm{E}-23$ & $0.00 \mathrm{E}+00$ & $0.00 \mathrm{E}+00$ \\
\hline CU 66 & $7.08 \mathrm{E}-05$ & $2.98 \mathrm{E}-06$ & 1.71E-06 & $1.34 \mathrm{E}-06$ & 2.16E-07 & $2.56 \mathrm{E}-08$ & 1.96E-10 & $2.11 \mathrm{E}-14$ & $0.00 \mathrm{E}+00$ \\
\hline CU 67 & $4.16 \mathrm{E}-10$ & $4.14 \mathrm{E}-10$ & $3.93 \mathrm{E}-10$ & $3.18 \mathrm{E}-10$ & $6.33 \mathrm{E}-11$ & $9.64 \mathrm{E}-12$ & $1.30 \mathrm{E}-13$ & $4.09 \mathrm{E}-17$ & $0.00 \mathrm{E}+00$ \\
\hline $\mathrm{ZN} 65$ & $7.53 \mathrm{E}-12$ & $7.53 \mathrm{E}-12$ & $7.52 \mathrm{E}-12$ & $7.51 \mathrm{E}-12$ & $7.38 \mathrm{E}-12$ & $7.23 \mathrm{E}-12$ & $6.91 \mathrm{E}-12$ & $6.35 \mathrm{E}-12$ & $2.67 \mathrm{E}-12$ \\
\hline SR 89 & $6.70 \mathrm{E}-11$ & $6.70 \mathrm{E}-11$ & $6.68 \mathrm{E}-11$ & $6.61 \mathrm{E}-11$ & $6.09 \mathrm{E}-11$ & $5.53 \mathrm{E}-11$ & 4.44E-11 & $2.94 \mathrm{E}-11$ & $4.45 \mathrm{E}-13$ \\
\hline SR 90 & 1.19E-14 & $1.19 \mathrm{E}-14$ & 1.19E-14 & $1.19 \mathrm{E}-14$ & 1.19E-14 & 1.19E-14 & $1.18 \mathrm{E}-14$ & $1.18 \mathrm{E}-14$ & $1.16 \mathrm{E}-14$ \\
\hline SR 91 & $4.61 \mathrm{E}-12$ & $4.44 \mathrm{E}-12$ & $3.20 \mathrm{E}-12$ & $7.99 \mathrm{E}-13$ & $2.19 \mathrm{E}-17$ & $1.04 \mathrm{E}-22$ & $0.00 \mathrm{E}+00$ & $0.00 \mathrm{E}+00$ & $0.00 \mathrm{E}+00$ \\
\hline Y 89M & 7.06E-06 & 7.03E-06 & $6.75 \mathrm{E}-06$ & 5.71E-06 & 1.60E-06 & $3.62 \mathrm{E}-07$ & $1.22 \mathrm{E}-08$ & $2.10 \mathrm{E}-11$ & $0.00 \mathrm{E}+00$ \\
\hline Y 90 & 4.45E-09 & 4.42E-09 & $4.21 \mathrm{E}-09$ & $3.43 \mathrm{E}-09$ & $7.21 \mathrm{E}-10$ & 1.17E-10 & $1.84 \mathrm{E}-12$ & $1.26 \mathrm{E}-14$ & $1.16 \mathrm{E}-14$ \\
\hline Y 90M & $3.62 \mathrm{E}-12$ & $3.24 \mathrm{E}-12$ & $1.18 \mathrm{E}-12$ & 1.69E-14 & $1.75 \mathrm{E}-28$ & $0.00 \mathrm{E}+00$ & $0.00 \mathrm{E}+00$ & $0.00 \mathrm{E}+00$ & $0.00 \mathrm{E}+00$ \\
\hline Y 91 & $1.26 \mathrm{E}-11$ & $1.26 \mathrm{E}-11$ & $1.26 \mathrm{E}-11$ & $1.25 \mathrm{E}-11$ & $1.16 \mathrm{E}-11$ & 1.07E-11 & $8.86 \mathrm{E}-12$ & $6.21 \mathrm{E}-12$ & $1.67 \mathrm{E}-13$ \\
\hline Y 92 & $5.30 \mathrm{E}-10$ & $4.81 \mathrm{E}-10$ & 1.99E-10 & $4.82 \mathrm{E}-12$ & $2.72 \mathrm{E}-24$ & $0.00 \mathrm{E}+00$ & $0.00 \mathrm{E}+00$ & $0.00 \mathrm{E}+00$ & $0.00 \mathrm{E}+00$ \\
\hline Y 94 & $3.29 \mathrm{E}-12$ & $1.11 \mathrm{E}-12$ & $6.16 \mathrm{E}-17$ & $0.00 \mathrm{E}+00$ & $0.00 \mathrm{E}+00$ & $0.00 \mathrm{E}+00$ & $0.00 \mathrm{E}+00$ & $0.00 \mathrm{E}+00$ & $0.00 \mathrm{E}+00$ \\
\hline ZR 89 & 7.07E-06 & 7.04E-06 & 6.76E-06 & $5.72 \mathrm{E}-06$ & $1.60 \mathrm{E}-06$ & $3.63 \mathrm{E}-07$ & $1.22 \mathrm{E}-08$ & $2.11 \mathrm{E}-11$ & $0.00 \mathrm{E}+00$ \\
\hline ZR 93 & $5.28 \mathrm{E}-12$ & $5.28 \mathrm{E}-12$ & $5.28 \mathrm{E}-12$ & $5.28 \mathrm{E}-12$ & $5.28 \mathrm{E}-12$ & $5.28 \mathrm{E}-12$ & $5.28 \mathrm{E}-12$ & $5.28 \mathrm{E}-12$ & $5.28 \mathrm{E}-12$ \\
\hline ZR 95 & $1.09 \mathrm{E}-05$ & $1.09 \mathrm{E}-05$ & $1.09 \mathrm{E}-05$ & $1.08 \mathrm{E}-05$ & $1.01 \mathrm{E}-05$ & $9.40 \mathrm{E}-06$ & 7.90E-06 & $5.71 \mathrm{E}-06$ & 2.09E-07 \\
\hline ZR 97 & $5.59 \mathrm{E}-07$ & $5.48 \mathrm{E}-07$ & 4.55E-07 & $2.09 \mathrm{E}-07$ & $5.69 \mathrm{E}-10$ & $5.71 \mathrm{E}-13$ & $8.25 \mathrm{E}-20$ & $0.00 \mathrm{E}+00$ & $0.00 \mathrm{E}+00$ \\
\hline NB 92 & $1.37 \mathrm{E}-03$ & 1.37E-03 & $1.35 \mathrm{E}-03$ & $1.28 \mathrm{E}-03$ & $8.52 \mathrm{E}-04$ & $5.28 \mathrm{E}-04$ & 1.77E-04 & 2.29E-05 & 2.07E-14 \\
\hline NB 93M & $1.91 \mathrm{E}-14$ & $1.91 \mathrm{E}-14$ & 1.93E-14 & $1.98 \mathrm{E}-14$ & $2.40 \mathrm{E}-14$ & $2.89 \mathrm{E}-14$ & 4.00E-14 & $6.08 \mathrm{E}-14$ & $2.67 \mathrm{E}-13$ \\
\hline NB 94 & $6.00 \mathrm{E}-10$ & $6.00 \mathrm{E}-10$ & $6.00 \mathrm{E}-10$ & $6.00 \mathrm{E}-10$ & $6.00 \mathrm{E}-10$ & $6.00 \mathrm{E}-10$ & $6.00 \mathrm{E}-10$ & $6.00 \mathrm{E}-10$ & $6.00 \mathrm{E}-10$ \\
\hline NB 95 & $2.64 \mathrm{E}-05$ & $2.63 \mathrm{E}-05$ & $2.63 \mathrm{E}-05$ & 2.60E-05 & $2.43 \mathrm{E}-05$ & 2.24E-05 & 1.87E-05 & $1.33 \mathrm{E}-05$ & 4.66E-07 \\
\hline NB 95M & $7.09 \mathrm{E}-08$ & 7.09E-08 & 7.11E-08 & $7.18 \mathrm{E}-08$ & $7.25 \mathrm{E}-08$ & $6.90 \mathrm{E}-08$ & $5.86 \mathrm{E}-08$ & 4.23E-08 & $1.55 \mathrm{E}-09$ \\
\hline NB 96 & 4.97E-05 & $4.90 \mathrm{E}-05$ & $4.28 \mathrm{E}-05$ & 2.44E-05 & 3.39E-07 & $2.32 \mathrm{E}-09$ & $2.60 \mathrm{E}-14$ & $1.36 \mathrm{E}-23$ & $0.00 \mathrm{E}+00$ \\
\hline NB 97 & $1.98 \mathrm{E}-05$ & $1.50 \mathrm{E}-05$ & 1.56E-06 & $2.10 \mathrm{E}-07$ & $5.72 \mathrm{E}-10$ & $5.82 \mathrm{E}-13$ & 8.89E-20 & $0.00 E+00$ & $0.00 \mathrm{E}+00$ \\
\hline NB 97M & $5.29 \mathrm{E}-07$ & 5.19E-07 & 4.31E-07 & $1.98 \mathrm{E}-07$ & $5.39 \mathrm{E}-10$ & $5.48 \mathrm{E}-13$ & $7.81 \mathrm{E}-20$ & $0.00 \mathrm{E}+00$ & $0.00 \mathrm{E}+00$ \\
\hline NB 98 & 2.95E-06 & $0.00 \mathrm{E}+00$ & $0.00 \mathrm{E}+00$ & $0.00 E+00$ & $0.00 \mathrm{E}+00$ & $0.00 \mathrm{E}+00$ & $0.00 \mathrm{E}+00$ & $0.00 \mathrm{E}+00$ & $0.00 \mathrm{E}+00$ \\
\hline NB100 & 1.47E-07 & $0.00 \mathrm{E}+00$ & $0.00 \mathrm{E}+00$ & $0.00 E+00$ & $0.00 \mathrm{E}+00$ & $0.00 \mathrm{E}+00$ & $0.00 \mathrm{E}+00$ & $0.00 \mathrm{E}+00$ & $0.00 \mathrm{E}+00$ \\
\hline MO 93M & 4.09E-04 & $3.88 \mathrm{E}-04$ & $2.46 \mathrm{E}-04$ & $3.60 \mathrm{E}-05$ & $1.69 \mathrm{E}-11$ & 7.00E-19 & $0.00 \mathrm{E}+00$ & $0.00 \mathrm{E}+00$ & $0.00 \mathrm{E}+00$ \\
\hline MO 93 & $3.74 \mathrm{E}-07$ & $3.74 \mathrm{E}-07$ & 3.74E-07 & $3.74 \mathrm{E}-07$ & $3.74 \mathrm{E}-07$ & 3.74E-07 & 3.74E-07 & 3.74E-07 & $3.74 \mathrm{E}-07$ \\
\hline MO 99 & $2.86 \mathrm{E}-01$ & $2.84 \mathrm{E}-01$ & $2.71 \mathrm{E}-01$ & $2.22 \mathrm{E}-01$ & $4.90 \mathrm{E}-02$ & $8.39 \mathrm{E}-03$ & 1.49E-04 & $7.73 \mathrm{E}-08$ & $0.00 \mathrm{E}+00$ \\
\hline M0101 & $7.12 \mathrm{E}-02$ & $1.72 \mathrm{E}-02$ & $4.73 \mathrm{E}-08$ & $0.00 \mathrm{E}+00$ & $0.00 \mathrm{E}+00$ & $0.00 \mathrm{E}+00$ & $0.00 \mathrm{E}+00$ & $0.00 \mathrm{E}+00$ & $0.00 \mathrm{E}+00$ \\
\hline TC 99 & $1.58 \mathrm{E}-08$ & $1.58 \mathrm{E}-08$ & $1.58 \mathrm{E}-08$ & 1.60E-08 & $1.68 \mathrm{E}-08$ & 1.70E-08 & 1.70E-08 & $1.70 \mathrm{E}-08$ & $1.70 \mathrm{E}-08$ \\
\hline TC100 & $1.65 \mathrm{E}-03$ & $0.00 \mathrm{E}+00$ & $0.00 \mathrm{E}+00$ & $0.00 \mathrm{E}+00$ & $0.00 \mathrm{E}+00$ & $0.00 \mathrm{E}+00$ & $0.00 \mathrm{E}+00$ & $0.00 \mathrm{E}+00$ & $0.00 \mathrm{E}+00$ \\
\hline TC101 & $7.12 \mathrm{E}-02$ & $4.11 \mathrm{E}-02$ & 5.97E-07 & $0.00 \mathrm{E}+00$ & $0.00 \mathrm{E}+00$ & $0.00 \mathrm{E}+00$ & $0.00 \mathrm{E}+00$ & $0.00 \mathrm{E}+00$ & $0.00 \mathrm{E}+00$ \\
\hline RU103 & $2.20 \mathrm{E}-07$ & $2.20 \mathrm{E}-07$ & $2.20 \mathrm{E}-07$ & $2.17 \mathrm{E}-07$ & $1.95 \mathrm{E}-07$ & $1.72 \mathrm{E}-07$ & $1.30 \mathrm{E}-07$ & $7.64 \mathrm{E}-08$ & $3.50 \mathrm{E}-10$ \\
\hline RU105 & $1.30 \mathrm{E}-12$ & $1.21 \mathrm{E}-12$ & 5.97E-13 & $3.07 \mathrm{E}-14$ & $5.27 \mathrm{E}-24$ & $0.00 \mathrm{E}+00$ & $0.00 \mathrm{E}+00$ & $0.00 \mathrm{E}+00$ & $0.00 \mathrm{E}+00$ \\
\hline RH104 & $1.22 \mathrm{E}-09$ & $8.72 \mathrm{E}-13$ & $0.00 \mathrm{E}+00$ & $0.00 \mathrm{E}+00$ & $0.00 \mathrm{E}+00$ & $0.00 \mathrm{E}+00$ & $0.00 \mathrm{E}+00$ & $0.00 \mathrm{E}+00$ & $0.00 \mathrm{E}+00$ \\
\hline RH104M & $8.80 \mathrm{E}-11$ & $7.30 \mathrm{E}-13$ & $0.00 \mathrm{E}+00$ & $0.00 \mathrm{E}+00$ & $0.00 \mathrm{E}+00$ & $0.00 \mathrm{E}+00$ & $0.00 \mathrm{E}+00$ & $0.00 \mathrm{E}+00$ & $0.00 \mathrm{E}+00$ \\
\hline RH105 & $9.84 \mathrm{E}-13$ & $9.86 \mathrm{E}-13$ & $9.76 \mathrm{E}-13$ & $7.27 \mathrm{E}-13$ & $4.35 \mathrm{E}-14$ & $1.61 \mathrm{E}-15$ & $8.65 \mathrm{E}-19$ & $6.42 \mathrm{E}-25$ & $0.00 \mathrm{E}+00$ \\
\hline RH105M & $3.65 \mathrm{E}-13$ & $3.38 \mathrm{E}-13$ & $1.68 \mathrm{E}-13$ & $8.62 \mathrm{E}-15$ & $1.48 \mathrm{E}-24$ & $0.00 \mathrm{E}+00$ & $0.00 \mathrm{E}+00$ & $0.00 \mathrm{E}+00$ & $0.00 \mathrm{E}+00$ \\
\hline RH106 & $2.17 \mathrm{E}-13$ & $1.79 \mathrm{E}-19$ & $1.79 \mathrm{E}-19$ & $1.79 \mathrm{E}-19$ & $1.79 \mathrm{E}-19$ & $1.79 \mathrm{E}-19$ & $1.79 \mathrm{E}-19$ & $1.79 \mathrm{E}-19$ & $1.01 \mathrm{E}-19$ \\
\hline RH106M & $1.05 \mathrm{E}-13$ & $9.01 \mathrm{E}-14$ & $2.18 \mathrm{E}-14$ & $1.35 \mathrm{E}-16$ & $0.00 \mathrm{E}+00$ & $0.00 \mathrm{E}+00$ & $0.00 \mathrm{E}+00$ & $0.00 \mathrm{E}+00$ & $0.00 E+00$ \\
\hline
\end{tabular}


TEM-10200-1

ENGINEERING CALCULATIONS AND ANALYSIS

Page 51 of 81

$12 / 19 / 17$

Rev.08

Title:

AS-RUN NEUTRONICS EVALUATION FOR THE CSM-10584 EXPERIMENT IN

THE ATR

\begin{tabular}{llllllll}
\cline { 2 - 6 } ECAR NO.: & 4496 & REV. NO.: & 1 & PROJECT NO.: & 32501 & Date: & $10 / 19 / 2020$ \\
\hline
\end{tabular}

\begin{tabular}{|c|c|c|c|c|c|c|c|c|c|}
\hline \multicolumn{10}{|c|}{1 gram of 316SS irradiated for 54.9 EFPDs } \\
\hline & EOC & $30 \mathrm{MINs}$ & $5 \mathrm{HRS}$ & $1 \mathrm{DAY}$ & 7 DAYS & $14 \mathrm{DAYS}$ & 30 DAYS & 60 DAYS & 1 YEAR \\
\hline & $\mathrm{Ci} / \mathrm{g}$ & $\mathrm{Ci} / \mathrm{g}$ & $\mathrm{Ci} / \mathrm{g}$ & $\mathrm{Ci} / \mathrm{g}$ & $\mathrm{Ci} / \mathrm{g}$ & $\mathrm{Ci} / \mathrm{g}$ & $\mathrm{Ci} / \mathrm{g}$ & $\mathrm{Ci} / \mathrm{g}$ & $\mathrm{Ci} / \mathrm{g}$ \\
\hline SUMTOT & $1.48 \mathrm{E}+01$ & $1.32 \mathrm{E}+01$ & $6.42 \mathrm{E}+00$ & $3.47 \mathrm{E}+00$ & $2.86 \mathrm{E}+00$ & $2.40 \mathrm{E}+00$ & $1.67 \mathrm{E}+00$ & $8.85 \mathrm{E}-01$ & $1.03 \mathrm{E}-01$ \\
\hline OTOTAL & $1.48 \mathrm{E}+01$ & $1.32 \mathrm{E}+01$ & $6.42 \mathrm{E}+00$ & $3.47 \mathrm{E}+00$ & $2.86 \mathrm{E}+00$ & $2.40 \mathrm{E}+00$ & $1.67 \mathrm{E}+00$ & $8.85 \mathrm{E}-01$ & $1.03 \mathrm{E}-01$ \\
\hline
\end{tabular}

\begin{tabular}{|c|c|c|c|c|c|c|c|c|c|}
\hline \multicolumn{10}{|c|}{1 gram of $316 S S$ irradiated for 119 EFPDs } \\
\hline & EOC & $30 \mathrm{MINs}$ & $5 \mathrm{HRS}$ & 1 DAY & 7 DAYS & 14 DAYS & 30 DAYS & 60 DAYS & 1 Year \\
\hline & $\mathrm{Ci} / \mathrm{g}$ & $\mathrm{Ci} / \mathrm{g}$ & $\mathrm{Ci} / \mathrm{g}$ & $\mathrm{Ci} / \mathrm{g}$ & $\mathrm{Ci} / \mathrm{g}$ & $\mathrm{Ci} / \mathrm{g}$ & $\mathrm{Ci} / \mathrm{g}$ & $\mathrm{Ci} / \mathrm{g}$ & $\mathrm{Ci} / \mathrm{g}$ \\
\hline H 3 & $5.54 \mathrm{E}-10$ & $5.54 \mathrm{E}-10$ & $5.54 \mathrm{E}-10$ & $5.54 \mathrm{E}-10$ & $5.54 \mathrm{E}-10$ & $5.53 \mathrm{E}-10$ & $5.52 \mathrm{E}-10$ & $5.49 \mathrm{E}-10$ & $5.24 \mathrm{E}-10$ \\
\hline HE 6 & $2.28 \mathrm{E}-07$ & $0.00 \mathrm{E}+00$ & $0.00 \mathrm{E}+00$ & $0.00 \mathrm{E}+00$ & $0.00 \mathrm{E}+00$ & $0.00 \mathrm{E}+00$ & $0.00 \mathrm{E}+00$ & $0.00 \mathrm{E}+00$ & $0.00 \mathrm{E}+00$ \\
\hline BE 8 & $3.94 \mathrm{E}-07$ & $0.00 \mathrm{E}+00$ & $0.00 \mathrm{E}+00$ & $0.00 \mathrm{E}+00$ & $0.00 \mathrm{E}+00$ & $0.00 \mathrm{E}+00$ & $0.00 \mathrm{E}+00$ & $0.00 \mathrm{E}+00$ & $0.00 \mathrm{E}+00$ \\
\hline BE 10 & $1.60 \mathrm{E}-12$ & $1.60 \mathrm{E}-12$ & $1.60 \mathrm{E}-12$ & $1.60 \mathrm{E}-12$ & $1.60 \mathrm{E}-12$ & $1.60 \mathrm{E}-12$ & $1.60 \mathrm{E}-12$ & $1.60 \mathrm{E}-12$ & $1.60 \mathrm{E}-12$ \\
\hline BE 11 & $1.26 \mathrm{E}-11$ & $0.00 \mathrm{E}+00$ & $0.00 \mathrm{E}+00$ & $0.00 \mathrm{E}+00$ & $0.00 \mathrm{E}+00$ & $0.00 \mathrm{E}+00$ & $0.00 \mathrm{E}+00$ & $0.00 \mathrm{E}+00$ & $0.00 \mathrm{E}+00$ \\
\hline C 14 & 5.87E-11 & 5.87E-11 & 5.87E-11 & 5.87E-11 & 5.87E-11 & 5.87E-11 & 5.87E-11 & 5.87E-11 & 5.87E-11 \\
\hline NE 23 & $7.00 \mathrm{E}-09$ & 1.97E-23 & $0.00 \mathrm{E}+00$ & $0.00 \mathrm{E}+00$ & $0.00 \mathrm{E}+00$ & $0.00 \mathrm{E}+00$ & $0.00 \mathrm{E}+00$ & $0.00 \mathrm{E}+00$ & $0.00 \mathrm{E}+00$ \\
\hline NA 24 & $1.96 \mathrm{E}-11$ & $1.91 \mathrm{E}-11$ & $1.55 \mathrm{E}-11$ & $6.45 \mathrm{E}-12$ & $8.31 \mathrm{E}-15$ & $3.53 \mathrm{E}-18$ & $6.95 \mathrm{E}-26$ & $0.00 \mathrm{E}+00$ & $0.00 \mathrm{E}+00$ \\
\hline NA 24M & $1.62 \mathrm{E}-12$ & $0.00 \mathrm{E}+00$ & $0.00 \mathrm{E}+00$ & $0.00 \mathrm{E}+00$ & $0.00 \mathrm{E}+00$ & $0.00 \mathrm{E}+00$ & $0.00 \mathrm{E}+00$ & $0.00 \mathrm{E}+00$ & $0.00 \mathrm{E}+00$ \\
\hline NA 25 & $1.06 \mathrm{E}-08$ & $8.58 \mathrm{E}-18$ & $0.00 \mathrm{E}+00$ & $0.00 \mathrm{E}+00$ & $0.00 \mathrm{E}+00$ & $0.00 \mathrm{E}+00$ & $0.00 \mathrm{E}+00$ & $0.00 \mathrm{E}+00$ & $0.00 \mathrm{E}+00$ \\
\hline MG 27 & $6.53 \mathrm{E}-06$ & $7.25 \mathrm{E}-07$ & $1.86 \mathrm{E}-15$ & $0.00 \mathrm{E}+00$ & $0.00 \mathrm{E}+00$ & $0.00 \mathrm{E}+00$ & $0.00 \mathrm{E}+00$ & $0.00 \mathrm{E}+00$ & $0.00 \mathrm{E}+00$ \\
\hline MG 28 & $2.32 \mathrm{E}-14$ & $2.28 \mathrm{E}-14$ & $1.97 \mathrm{E}-14$ & $1.05 \mathrm{E}-14$ & $9.66 \mathrm{E}-17$ & $8.75 \mathrm{E}-18$ & $2.59 \mathrm{E}-23$ & $0.00 \mathrm{E}+00$ & $0.00 \mathrm{E}+00$ \\
\hline AL 28 & $7.84 \mathrm{E}-03$ & $7.29 \mathrm{E}-07$ & $1.97 \mathrm{E}-14$ & $1.05 \mathrm{E}-14$ & $9.68 \mathrm{E}-17$ & $8.75 \mathrm{E}-18$ & $2.60 \mathrm{E}-23$ & $0.00 \mathrm{E}+00$ & $0.00 \mathrm{E}+00$ \\
\hline AL 29 & $2.65 \mathrm{E}-04$ & $1.09 \mathrm{E}-05$ & $3.74 \mathrm{E}-18$ & $0.00 \mathrm{E}+00$ & $0.00 \mathrm{E}+00$ & $0.00 \mathrm{E}+00$ & $0.00 \mathrm{E}+00$ & $0.00 \mathrm{E}+00$ & $0.00 \mathrm{E}+00$ \\
\hline AL 30 & $2.57 \mathrm{E}-07$ & $0.00 \mathrm{E}+00$ & $0.00 \mathrm{E}+00$ & $0.00 \mathrm{E}+00$ & $0.00 \mathrm{E}+00$ & $0.00 \mathrm{E}+00$ & $0.00 \mathrm{E}+00$ & $0.00 \mathrm{E}+00$ & $0.00 \mathrm{E}+00$ \\
\hline SI 31 & 5.04E-03 & $4.42 \mathrm{E}-03$ & $1.35 \mathrm{E}-03$ & $8.85 \mathrm{E}-06$ & $2.58 \mathrm{E}-22$ & $0.00 \mathrm{E}+00$ & $0.00 \mathrm{E}+00$ & $0.00 \mathrm{E}+00$ & $0.00 \mathrm{E}+00$ \\
\hline SI 32 & $1.24 \mathrm{E}-12$ & $1.24 \mathrm{E}-12$ & $1.24 \mathrm{E}-12$ & $1.24 \mathrm{E}-12$ & $1.24 \mathrm{E}-12$ & $1.24 \mathrm{E}-12$ & $1.24 \mathrm{E}-12$ & $1.24 \mathrm{E}-12$ & $1.24 \mathrm{E}-12$ \\
\hline P 32 & $7.72 \mathrm{E}-03$ & $7.72 \mathrm{E}-03$ & $7.65 \mathrm{E}-03$ & $7.36 \mathrm{E}-03$ & $5.50 \mathrm{E}-03$ & $3.92 \mathrm{E}-03$ & $1.80 \mathrm{E}-03$ & $4.21 \mathrm{E}-04$ & $1.59 \mathrm{E}-10$ \\
\hline P 33 & $2.52 \mathrm{E}-07$ & $2.52 \mathrm{E}-07$ & $2.50 \mathrm{E}-07$ & $2.45 \mathrm{E}-07$ & 2.07E-07 & $1.71 \mathrm{E}-07$ & 1.10E-07 & 4.77E-08 & $1.01 \mathrm{E}-11$ \\
\hline P 34 & $1.46 \mathrm{E}-06$ & $0.00 \mathrm{E}+00$ & $0.00 \mathrm{E}+00$ & $0.00 \mathrm{E}+00$ & $0.00 \mathrm{E}+00$ & $0.00 \mathrm{E}+00$ & $0.00 \mathrm{E}+00$ & $0.00 \mathrm{E}+00$ & $0.00 \mathrm{E}+00$ \\
\hline S 35 & $1.02 \mathrm{E}-04$ & $1.02 \mathrm{E}-04$ & $1.01 \mathrm{E}-04$ & $1.01 \mathrm{E}-04$ & $9.61 \mathrm{E}-05$ & $9.10 \mathrm{E}-05$ & $8.02 \mathrm{E}-05$ & $6.33 \mathrm{E}-05$ & $5.72 \mathrm{E}-06$ \\
\hline S 37 & $4.21 \mathrm{E}-07$ & $6.92 \mathrm{E}-09$ & $5.98 \mathrm{E}-25$ & $0.00 \mathrm{E}+00$ & $0.00 \mathrm{E}+00$ & $0.00 \mathrm{E}+00$ & $0.00 \mathrm{E}+00$ & $0.00 \mathrm{E}+00$ & $0.00 \mathrm{E}+00$ \\
\hline CL 36 & $7.32 \mathrm{E}-13$ & $7.32 \mathrm{E}-13$ & $7.32 \mathrm{E}-13$ & $7.32 \mathrm{E}-13$ & $7.32 \mathrm{E}-13$ & $7.32 \mathrm{E}-13$ & $7.32 \mathrm{E}-13$ & $7.32 \mathrm{E}-13$ & $7.32 \mathrm{E}-13$ \\
\hline CL 38 & $2.17 \mathrm{E}-10$ & $1.24 \mathrm{E}-10$ & $8.14 \mathrm{E}-13$ & 4.89E-22 & $0.00 \mathrm{E}+00$ & $0.00 \mathrm{E}+00$ & $0.00 \mathrm{E}+00$ & $0.00 \mathrm{E}+00$ & $0.00 \mathrm{E}+00$ \\
\hline CL 38M & $2.59 \mathrm{E}-12$ & $0.00 \mathrm{E}+00$ & $0.00 \mathrm{E}+00$ & $0.00 \mathrm{E}+00$ & $0.00 \mathrm{E}+00$ & $0.00 \mathrm{E}+00$ & $0.00 \mathrm{E}+00$ & $0.00 \mathrm{E}+00$ & $0.00 \mathrm{E}+00$ \\
\hline CA 45 & $9.53 \mathrm{E}-13$ & $9.53 \mathrm{E}-13$ & $9.52 \mathrm{E}-13$ & 9.49E-13 & $9.25 \mathrm{E}-13$ & $8.98 \mathrm{E}-13$ & $8.39 \mathrm{E}-13$ & $7.38 \mathrm{E}-13$ & $2.02 \mathrm{E}-13$ \\
\hline CA 47 & $9.31 \mathrm{E}-12$ & $9.28 \mathrm{E}-12$ & $9.02 \mathrm{E}-12$ & 7.99E-12 & 3.20E-12 & 1.10E-12 & $9.51 \mathrm{E}-14$ & $9.73 \mathrm{E}-16$ & $0.00 \mathrm{E}+00$ \\
\hline SC 47 & 4.23E-08 & $4.21 \mathrm{E}-08$ & $4.05 \mathrm{E}-08$ & $3.44 \mathrm{E}-08$ & $9.94 \mathrm{E}-09$ & $2.34 \mathrm{E}-09$ & $8.56 \mathrm{E}-11$ & $1.76 \mathrm{E}-13$ & $0.00 \mathrm{E}+00$ \\
\hline SC 48 & $3.58 \mathrm{E}-08$ & 3.55E-08 & $3.31 \mathrm{E}-08$ & $2.45 \mathrm{E}-08$ & $2.51 \mathrm{E}-09$ & $1.76 \mathrm{E}-10$ & 4.03E-13 & 4.55E-18 & $0.00 \mathrm{E}+00$ \\
\hline SC 49 & $1.20 \mathrm{E}-08$ & $8.38 \mathrm{E}-09$ & $3.24 \mathrm{E}-10$ & $3.48 \mathrm{E}-16$ & $0.00 \mathrm{E}+00$ & $0.00 \mathrm{E}+00$ & $0.00 \mathrm{E}+00$ & $0.00 \mathrm{E}+00$ & $0.00 \mathrm{E}+00$ \\
\hline SC 50 & 1.03E-09 & $5.31 \mathrm{E}-15$ & $0.00 \mathrm{E}+00$ & $0.00 \mathrm{E}+00$ & $0.00 \mathrm{E}+00$ & $0.00 \mathrm{E}+00$ & $0.00 \mathrm{E}+00$ & $0.00 \mathrm{E}+00$ & $0.00 \mathrm{E}+00$ \\
\hline TI 51 & $2.48 \mathrm{E}-05$ & $6.71 \mathrm{E}-07$ & $5.20 \mathrm{E}-21$ & $0.00 \mathrm{E}+00$ & $0.00 \mathrm{E}+00$ & $0.00 \mathrm{E}+00$ & $0.00 \mathrm{E}+00$ & $0.00 \mathrm{E}+00$ & $0.00 \mathrm{E}+00$ \\
\hline V 52 & $4.01 \mathrm{E}-02$ & 1.57E-04 & $3.32 \mathrm{E}-26$ & $0.00 \mathrm{E}+00$ & $0.00 \mathrm{E}+00$ & $0.00 \mathrm{E}+00$ & $0.00 \mathrm{E}+00$ & $0.00 \mathrm{E}+00$ & $0.00 \mathrm{E}+00$ \\
\hline V53 & $1.84 \mathrm{E}-04$ & $4.52 \mathrm{E}-10$ & $0.00 \mathrm{E}+00$ & $0.00 \mathrm{E}+00$ & $0.00 \mathrm{E}+00$ & $0.00 \mathrm{E}+00$ & $0.00 \mathrm{E}+00$ & $0.00 \mathrm{E}+00$ & $0.00 \mathrm{E}+00$ \\
\hline V 54 & 1.97E-06 & $2.77 \mathrm{E}-16$ & $0.00 \mathrm{E}+00$ & $0.00 \mathrm{E}+00$ & $0.00 \mathrm{E}+00$ & $0.00 \mathrm{E}+00$ & $0.00 \mathrm{E}+00$ & $0.00 \mathrm{E}+00$ & $0.00 \mathrm{E}+00$ \\
\hline CR 51 & $3.78 \mathrm{E}+00$ & $3.78 \mathrm{E}+00$ & $3.76 \mathrm{E}+00$ & $3.68 \mathrm{E}+00$ & $3.17 \mathrm{E}+00$ & $2.66 \mathrm{E}+00$ & $1.78 \mathrm{E}+00$ & $8.42 \mathrm{E}-01$ & $4.06 \mathrm{E}-04$ \\
\hline CR 55 & $5.64 \mathrm{E}-02$ & $1.61 \mathrm{E}-04$ & $0.00 \mathrm{E}+00$ & $0.00 \mathrm{E}+00$ & $0.00 \mathrm{E}+00$ & $0.00 \mathrm{E}+00$ & $0.00 \mathrm{E}+00$ & $0.00 \mathrm{E}+00$ & $0.00 \mathrm{E}+00$ \\
\hline MN 54 & $6.09 \mathrm{E}-02$ & $6.09 \mathrm{E}-02$ & $6.08 \mathrm{E}-02$ & $6.07 \mathrm{E}-02$ & $5.99 \mathrm{E}-02$ & $5.90 \mathrm{E}-02$ & $5.70 \mathrm{E}-02$ & $5.33 \mathrm{E}-02$ & $2.71 \mathrm{E}-02$ \\
\hline MN 56 & $1.08 \mathrm{E}+01$ & $9.44 \mathrm{E}+00$ & $2.82 \mathrm{E}+00$ & $1.70 \mathrm{E}-02$ & $2.63 \mathrm{E}-19$ & $0.00 \mathrm{E}+00$ & $0.00 \mathrm{E}+00$ & $0.00 \mathrm{E}+00$ & $0.00 \mathrm{E}+00$ \\
\hline MN 57 & $6.75 \mathrm{E}-04$ & 1.66E-09 & $0.00 \mathrm{E}+00$ & $0.00 \mathrm{E}+00$ & $0.00 \mathrm{E}+00$ & $0.00 E+00$ & $0.00 E+00$ & $0.00 \mathrm{E}+00$ & $0.00 \mathrm{E}+00$ \\
\hline MN 58 & $2.04 \mathrm{E}-06$ & $1.03 \mathrm{E}-14$ & $0.00 \mathrm{E}+00$ & $0.00 \mathrm{E}+00$ & $0.00 \mathrm{E}+00$ & $0.00 \mathrm{E}+00$ & $0.00 \mathrm{E}+00$ & $0.00 \mathrm{E}+00$ & $0.00 \mathrm{E}+00$ \\
\hline FE 55 & $2.32 \mathrm{E}-01$ & $2.32 \mathrm{E}-01$ & $2.32 \mathrm{E}-01$ & $2.32 \mathrm{E}-01$ & $2.31 \mathrm{E}-01$ & $2.30 \mathrm{E}-01$ & $2.27 \mathrm{E}-01$ & $2.22 \mathrm{E}-01$ & $1.78 \mathrm{E}-01$ \\
\hline FE 59 & $6.78 \mathrm{E}-02$ & $6.78 \mathrm{E}-02$ & $6.76 \mathrm{E}-02$ & $6.68 \mathrm{E}-02$ & $6.09 \mathrm{E}-02$ & 5.47E-02 & 4.27E-02 & $2.69 \mathrm{E}-02$ & $2.44 \mathrm{E}-04$ \\
\hline CO 58 & $1.28 \mathrm{E}-01$ & $1.28 \mathrm{E}-01$ & $1.28 \mathrm{E}-01$ & 1.27E-01 & 1.19E-01 & $1.11 \mathrm{E}-01$ & $9.52 \mathrm{E}-02$ & $7.10 \mathrm{E}-02$ & $3.57 \mathrm{E}-03$ \\
\hline $\mathrm{CO} 60$ & $6.63 \mathrm{E}-04$ & $6.63 \mathrm{E}-04$ & $6.63 \mathrm{E}-04$ & $6.62 \mathrm{E}-04$ & $6.61 \mathrm{E}-04$ & $6.59 \mathrm{E}-04$ & $6.56 \mathrm{E}-04$ & 6.49E-04 & $5.81 \mathrm{E}-04$ \\
\hline $\mathrm{CO} 60 \mathrm{M}$ & $1.21 \mathrm{E}-02$ & 1.66E-03 & 2.87E-11 & $0.00 \mathrm{E}+00$ & $0.00 \mathrm{E}+00$ & $0.00 \mathrm{E}+00$ & $0.00 \mathrm{E}+00$ & $0.00 \mathrm{E}+00$ & $0.00 \mathrm{E}+00$ \\
\hline $\mathrm{CO} 61$ & $2.98 \mathrm{E}-04$ & $2.41 \mathrm{E}-04$ & $3.65 \mathrm{E}-05$ & $1.25 \mathrm{E}-08$ & $0.00 \mathrm{E}+00$ & $0.00 \mathrm{E}+00$ & $0.00 \mathrm{E}+00$ & $0.00 \mathrm{E}+00$ & $0.00 \mathrm{E}+00$ \\
\hline
\end{tabular}


TEM-10200-1

ENGINEERING CALCULATIONS AND ANALYSIS

Page 52 of 81

$12 / 19 / 17$

Rev.08

Title:

AS-RUN NEUTRONICS EVALUATION FOR THE CSM-10584 EXPERIMENT IN THE ATR

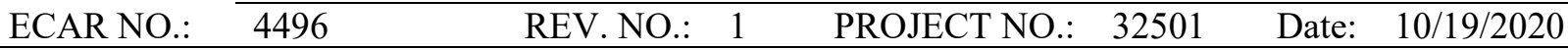

\begin{tabular}{|c|c|c|c|c|c|c|c|c|c|}
\hline \multicolumn{10}{|c|}{1 gram of $316 S S$ irradiated for 119 EFPDs } \\
\hline & EOC & $30 \mathrm{MINs}$ & $5 \mathrm{HRS}$ & $1 \mathrm{DAY}$ & 7 DAYS & 14 DAYS & 30 DAYS & 60 DAYS & 1 Year \\
\hline & $\mathrm{Ci} / \mathrm{g}$ & $\mathrm{Ci} / \mathrm{g}$ & $\mathrm{Ci} / \mathrm{g}$ & $\mathrm{Ci} / \mathrm{g}$ & $\mathrm{Ci} / \mathrm{g}$ & $\mathrm{Ci} / \mathrm{g}$ & $\mathrm{Ci} / \mathrm{g}$ & $\mathrm{Ci} / \mathrm{g}$ & $\mathrm{Ci} / \mathrm{g}$ \\
\hline CO 62 & $4.26 \mathrm{E}-05$ & $4.06 \mathrm{E}-11$ & $0.00 \mathrm{E}+00$ & $0.00 \mathrm{E}+00$ & $0.00 \mathrm{E}+00$ & $0.00 \mathrm{E}+00$ & $0.00 \mathrm{E}+00$ & $0.00 \mathrm{E}+00$ & $0.00 E+00$ \\
\hline $\mathrm{NI} 59$ & $3.42 \mathrm{E}-05$ & $3.42 \mathrm{E}-05$ & $3.42 \mathrm{E}-05$ & $3.42 \mathrm{E}-05$ & $3.42 \mathrm{E}-05$ & $3.42 \mathrm{E}-05$ & $3.42 \mathrm{E}-05$ & $3.42 \mathrm{E}-05$ & $3.42 \mathrm{E}-05$ \\
\hline NI 63 & $4.45 \mathrm{E}-03$ & $4.45 \mathrm{E}-03$ & $4.45 \mathrm{E}-03$ & $4.45 \mathrm{E}-03$ & $4.45 \mathrm{E}-03$ & $4.45 \mathrm{E}-03$ & $4.45 \mathrm{E}-03$ & $4.45 \mathrm{E}-03$ & $4.42 \mathrm{E}-03$ \\
\hline $\mathrm{NI} 65$ & 5.27E-02 & $4.59 \mathrm{E}-02$ & $1.33 \mathrm{E}-02$ & $7.16 \mathrm{E}-05$ & $4.50 \mathrm{E}-22$ & $0.00 \mathrm{E}+00$ & $0.00 \mathrm{E}+00$ & $0.00 \mathrm{E}+00$ & $0.00 \mathrm{E}+00$ \\
\hline NI 66 & $1.80 \mathrm{E}-06$ & $1.79 \mathrm{E}-06$ & $1.69 \mathrm{E}-06$ & $1.33 \mathrm{E}-06$ & $2.14 \mathrm{E}-07$ & $2.54 \mathrm{E}-08$ & $1.94 \mathrm{E}-10$ & $2.08 \mathrm{E}-14$ & $0.00 \mathrm{E}+00$ \\
\hline CU 64 & $1.33 \mathrm{E}-05$ & $1.29 \mathrm{E}-05$ & $1.01 \mathrm{E}-05$ & $3.59 \mathrm{E}-06$ & $1.39 \mathrm{E}-09$ & $1.44 \mathrm{E}-13$ & $1.14 \mathrm{E}-22$ & $0.00 \mathrm{E}+00$ & $0.00 E+00$ \\
\hline CU 66 & $1.51 \mathrm{E}-04$ & $4.33 \mathrm{E}-06$ & $1.70 \mathrm{E}-06$ & $1.33 \mathrm{E}-06$ & $2.14 \mathrm{E}-07$ & $2.54 \mathrm{E}-08$ & $1.94 \mathrm{E}-10$ & $2.09 \mathrm{E}-14$ & $0.00 \mathrm{E}+00$ \\
\hline CU 67 & $9.49 \mathrm{E}-10$ & $9.44 \mathrm{E}-10$ & $8.97 \mathrm{E}-10$ & $7.25 \mathrm{E}-10$ & $1.44 \mathrm{E}-10$ & $2.20 \mathrm{E}-11$ & $2.98 \mathrm{E}-13$ & $9.33 \mathrm{E}-17$ & $0.00 \mathrm{E}+00$ \\
\hline ZN 65 & $1.57 \mathrm{E}-10$ & $1.57 \mathrm{E}-10$ & $1.57 \mathrm{E}-10$ & $1.56 \mathrm{E}-10$ & $1.54 \mathrm{E}-10$ & $1.51 \mathrm{E}-10$ & $1.44 \mathrm{E}-10$ & $1.32 \mathrm{E}-10$ & $5.55 \mathrm{E}-11$ \\
\hline ZN 69 & $1.85 \mathrm{E}-13$ & $1.32 \mathrm{E}-13$ & $1.49 \mathrm{E}-14$ & $3.98 \mathrm{E}-15$ & $2.81 \mathrm{E}-18$ & $5.94 \mathrm{E}-22$ & $0.00 \mathrm{E}+00$ & $0.00 \mathrm{E}+00$ & $0.00 \mathrm{E}+00$ \\
\hline ZN 69M & $1.24 \mathrm{E}-14$ & $1.21 \mathrm{E}-14$ & $9.66 \mathrm{E}-15$ & $3.70 \mathrm{E}-15$ & $2.62 \mathrm{E}-18$ & $5.54 \mathrm{E}-22$ & $0.00 \mathrm{E}+00$ & $0.00 \mathrm{E}+00$ & $0.00 \mathrm{E}+00$ \\
\hline SR 89 & $2.84 \mathrm{E}-10$ & $2.84 \mathrm{E}-10$ & $2.83 \mathrm{E}-10$ & $2.80 \mathrm{E}-10$ & $2.58 \mathrm{E}-10$ & $2.34 \mathrm{E}-10$ & $1.88 \mathrm{E}-10$ & $1.24 \mathrm{E}-10$ & $1.89 \mathrm{E}-12$ \\
\hline SR 90 & $5.63 \mathrm{E}-14$ & $5.63 \mathrm{E}-14$ & $5.63 \mathrm{E}-14$ & $5.63 \mathrm{E}-14$ & $5.63 \mathrm{E}-14$ & $5.62 \mathrm{E}-14$ & $5.62 \mathrm{E}-14$ & $5.61 \mathrm{E}-14$ & $5.50 \mathrm{E}-14$ \\
\hline SR 91 & 1.00E-11 & $9.66 \mathrm{E}-12$ & $6.95 \mathrm{E}-12$ & $1.74 \mathrm{E}-12$ & $4.76 \mathrm{E}-17$ & $2.26 \mathrm{E}-22$ & $0.00 \mathrm{E}+00$ & $0.00 \mathrm{E}+00$ & $0.00 \mathrm{E}+00$ \\
\hline Y 89M & 7.06E-06 & 7.03E-06 & $6.75 \mathrm{E}-06$ & $5.71 \mathrm{E}-06$ & 1.60E-06 & $3.62 \mathrm{E}-07$ & $1.22 \mathrm{E}-08$ & $2.11 \mathrm{E}-11$ & $0.00 \mathrm{E}+00$ \\
\hline Y 90 & $1.06 \mathrm{E}-08$ & $1.05 \mathrm{E}-08$ & $1.00 \mathrm{E}-08$ & $8.17 \mathrm{E}-09$ & $1.72 \mathrm{E}-09$ & $2.78 \mathrm{E}-10$ & $4.40 \mathrm{E}-12$ & $5.79 \mathrm{E}-14$ & $5.50 \mathrm{E}-14$ \\
\hline Y 90M & $8.24 \mathrm{E}-12$ & 7.37E-12 & $2.69 \mathrm{E}-12$ & $3.84 \mathrm{E}-14$ & $3.99 \mathrm{E}-28$ & $0.00 \mathrm{E}+00$ & $0.00 \mathrm{E}+00$ & $0.00 \mathrm{E}+00$ & $0.00 \mathrm{E}+00$ \\
\hline Y91 & $4.80 \mathrm{E}-11$ & $4.80 \mathrm{E}-11$ & $4.79 \mathrm{E}-11$ & $4.75 \mathrm{E}-11$ & $4.42 \mathrm{E}-11$ & 4.07E-11 & 3.37E-11 & $2.36 \mathrm{E}-11$ & $6.35 \mathrm{E}-13$ \\
\hline Y92 & 1.26E-09 & $1.14 \mathrm{E}-09$ & $4.72 \mathrm{E}-10$ & $1.14 \mathrm{E}-11$ & $6.44 \mathrm{E}-24$ & $0.00 \mathrm{E}+00$ & $0.00 \mathrm{E}+00$ & $0.00 \mathrm{E}+00$ & $0.00 \mathrm{E}+00$ \\
\hline Y94 & $7.16 \mathrm{E}-12$ & $2.41 \mathrm{E}-12$ & $1.34 \mathrm{E}-16$ & $0.00 \mathrm{E}+00$ & $0.00 \mathrm{E}+00$ & $0.00 \mathrm{E}+00$ & $0.00 \mathrm{E}+00$ & $0.00 \mathrm{E}+00$ & $0.00 \mathrm{E}+00$ \\
\hline ZR 89 & 7.07E-06 & $7.04 \mathrm{E}-06$ & $6.76 \mathrm{E}-06$ & $5.72 \mathrm{E}-06$ & 1.60E-06 & $3.63 \mathrm{E}-07$ & $1.22 \mathrm{E}-08$ & $2.11 \mathrm{E}-11$ & $0.00 \mathrm{E}+00$ \\
\hline ZR 93 & $1.16 \mathrm{E}-11$ & 1.16E-11 & $1.16 \mathrm{E}-11$ & 1.16E-11 & $1.16 \mathrm{E}-11$ & $1.16 \mathrm{E}-11$ & $1.16 \mathrm{E}-11$ & $1.16 \mathrm{E}-11$ & $1.16 \mathrm{E}-11$ \\
\hline ZR 95 & 1.77E-05 & $1.77 \mathrm{E}-05$ & $1.76 \mathrm{E}-05$ & $1.75 \mathrm{E}-05$ & 1.64E-05 & $1.52 \mathrm{E}-05$ & $1.28 \mathrm{E}-05$ & $9.23 \mathrm{E}-06$ & $3.38 \mathrm{E}-07$ \\
\hline ZR 97 & $5.59 \mathrm{E}-07$ & 5.47E-07 & $4.55 \mathrm{E}-07$ & $2.09 \mathrm{E}-07$ & $5.68 \mathrm{E}-10$ & $5.79 \mathrm{E}-13$ & $8.36 \mathrm{E}-20$ & $0.00 \mathrm{E}+00$ & $0.00 \mathrm{E}+00$ \\
\hline NB 92 & $1.41 \mathrm{E}-03$ & 1.40E-03 & 1.39E-03 & $1.31 \mathrm{E}-03$ & $8.72 \mathrm{E}-04$ & $5.41 \mathrm{E}-04$ & $1.82 \mathrm{E}-04$ & $2.35 \mathrm{E}-05$ & $2.12 \mathrm{E}-14$ \\
\hline NB 93M & $9.02 \mathrm{E}-14$ & $9.02 \mathrm{E}-14$ & $9.05 \mathrm{E}-14$ & $9.17 \mathrm{E}-14$ & $1.01 \mathrm{E}-13$ & $1.12 \mathrm{E}-13$ & $1.36 \mathrm{E}-13$ & $1.81 \mathrm{E}-13$ & $6.33 \mathrm{E}-13$ \\
\hline NB 94 & $1.28 \mathrm{E}-09$ & $1.28 \mathrm{E}-09$ & $1.28 \mathrm{E}-09$ & $1.28 \mathrm{E}-09$ & $1.28 \mathrm{E}-09$ & $1.28 \mathrm{E}-09$ & $1.28 \mathrm{E}-09$ & $1.28 \mathrm{E}-09$ & $1.28 \mathrm{E}-09$ \\
\hline NB 95 & $4.36 \mathrm{E}-05$ & 4.36E-05 & 4.35E-05 & $4.31 \mathrm{E}-05$ & $4.02 \mathrm{E}-05$ & $3.71 \mathrm{E}-05$ & $3.08 \mathrm{E}-05$ & $2.18 \mathrm{E}-05$ & $7.53 \mathrm{E}-07$ \\
\hline NB 95M & $1.21 \mathrm{E}-07$ & $1.21 \mathrm{E}-07$ & $1.21 \mathrm{E}-07$ & $1.21 \mathrm{E}-07$ & $1.19 \mathrm{E}-07$ & $1.12 \mathrm{E}-07$ & 9.47E-08 & $6.85 \mathrm{E}-08$ & $2.51 \mathrm{E}-09$ \\
\hline NB 96 & $5.08 \mathrm{E}-05$ & $5.00 \mathrm{E}-05$ & $4.38 \mathrm{E}-05$ & $2.49 \mathrm{E}-05$ & $3.47 \mathrm{E}-07$ & $2.37 \mathrm{E}-09$ & $2.65 \mathrm{E}-14$ & $1.38 \mathrm{E}-23$ & $0.00 \mathrm{E}+00$ \\
\hline NB 97 & $1.98 \mathrm{E}-05$ & $1.50 \mathrm{E}-05$ & $1.56 \mathrm{E}-06$ & $2.10 \mathrm{E}-07$ & $5.71 \mathrm{E}-10$ & $5.82 \mathrm{E}-13$ & $9.01 \mathrm{E}-20$ & $0.00 \mathrm{E}+00$ & $0.00 \mathrm{E}+00$ \\
\hline NB 97M & $5.29 \mathrm{E}-07$ & $5.18 \mathrm{E}-07$ & $4.31 \mathrm{E}-07$ & $1.98 \mathrm{E}-07$ & $5.38 \mathrm{E}-10$ & $5.48 \mathrm{E}-13$ & $7.92 \mathrm{E}-20$ & $0.00 \mathrm{E}+00$ & $0.00 \mathrm{E}+00$ \\
\hline NB 98 & $2.96 \mathrm{E}-06$ & $0.00 \mathrm{E}+00$ & $0.00 \mathrm{E}+00$ & $0.00 \mathrm{E}+00$ & $0.00 \mathrm{E}+00$ & $0.00 \mathrm{E}+00$ & $0.00 \mathrm{E}+00$ & $0.00 \mathrm{E}+00$ & $0.00 \mathrm{E}+00$ \\
\hline NB100 & 1.47E-07 & $0.00 \mathrm{E}+00$ & $0.00 \mathrm{E}+00$ & $0.00 \mathrm{E}+00$ & $0.00 \mathrm{E}+00$ & $0.00 \mathrm{E}+00$ & $0.00 \mathrm{E}+00$ & $0.00 \mathrm{E}+00$ & $0.00 \mathrm{E}+00$ \\
\hline MO 93M & $4.08 \mathrm{E}-04$ & $3.88 \mathrm{E}-04$ & $2.46 \mathrm{E}-04$ & $3.60 \mathrm{E}-05$ & 1.69E-11 & $7.00 \mathrm{E}-19$ & $0.00 \mathrm{E}+00$ & $0.00 \mathrm{E}+00$ & $0.00 E+00$ \\
\hline MO 93 & $8.11 \mathrm{E}-07$ & $8.11 \mathrm{E}-07$ & $8.11 \mathrm{E}-07$ & $8.12 \mathrm{E}-07$ & $8.12 \mathrm{E}-07$ & $8.12 \mathrm{E}-07$ & $8.12 \mathrm{E}-07$ & $8.11 \mathrm{E}-07$ & $8.11 \mathrm{E}-07$ \\
\hline MO 99 & $2.86 \mathrm{E}-01$ & $2.84 \mathrm{E}-01$ & $2.71 \mathrm{E}-01$ & $2.22 \mathrm{E}-01$ & $4.90 \mathrm{E}-02$ & $8.39 \mathrm{E}-03$ & 1.49E-04 & $7.73 \mathrm{E}-08$ & $0.00 \mathrm{E}+00$ \\
\hline M0101 & 7.11E-02 & $1.71 \mathrm{E}-02$ & 4.73E-08 & $0.00 \mathrm{E}+00$ & $0.00 \mathrm{E}+00$ & $0.00 \mathrm{E}+00$ & $0.00 \mathrm{E}+00$ & $0.00 \mathrm{E}+00$ & $0.00 \mathrm{E}+00$ \\
\hline TC 99 & $3.45 \mathrm{E}-08$ & $3.46 \mathrm{E}-08$ & $3.46 \mathrm{E}-08$ & $3.48 \mathrm{E}-08$ & $3.56 \mathrm{E}-08$ & $3.58 \mathrm{E}-08$ & $3.58 \mathrm{E}-08$ & $3.58 \mathrm{E}-08$ & $3.58 \mathrm{E}-08$ \\
\hline TC100 & $3.61 \mathrm{E}-03$ & $0.00 \mathrm{E}+00$ & $0.00 \mathrm{E}+00$ & $0.00 \mathrm{E}+00$ & $0.00 \mathrm{E}+00$ & $0.00 \mathrm{E}+00$ & $0.00 \mathrm{E}+00$ & $0.00 \mathrm{E}+00$ & $0.00 \mathrm{E}+00$ \\
\hline TC101 & $7.11 \mathrm{E}-02$ & $4.10 \mathrm{E}-02$ & $5.96 \mathrm{E}-07$ & $0.00 \mathrm{E}+00$ & $0.00 \mathrm{E}+00$ & $0.00 \mathrm{E}+00$ & $0.00 \mathrm{E}+00$ & $0.00 \mathrm{E}+00$ & $0.00 \mathrm{E}+00$ \\
\hline RU103 & $1.78 \mathrm{E}-06$ & 1.77E-06 & 1.77E-06 & $1.74 \mathrm{E}-06$ & 1.57E-06 & $1.39 \mathrm{E}-06$ & $1.05 \mathrm{E}-06$ & $6.16 \mathrm{E}-07$ & $2.82 \mathrm{E}-09$ \\
\hline RU105 & $2.38 \mathrm{E}-11$ & $2.20 \mathrm{E}-11$ & $1.09 \mathrm{E}-11$ & $5.60 \mathrm{E}-13$ & $9.61 \mathrm{E}-23$ & $0.00 \mathrm{E}+00$ & $0.00 \mathrm{E}+00$ & $0.00 \mathrm{E}+00$ & $0.00 E+00$ \\
\hline RH104 & $2.10 \mathrm{E}-08$ & $1.50 \mathrm{E}-11$ & $0.00 \mathrm{E}+00$ & $0.00 E+00$ & $0.00 \mathrm{E}+00$ & $0.00 \mathrm{E}+00$ & $0.00 \mathrm{E}+00$ & $0.00 \mathrm{E}+00$ & $0.00 E+00$ \\
\hline RH104M & $1.52 \mathrm{E}-09$ & $1.26 \mathrm{E}-11$ & $0.00 \mathrm{E}+00$ & $0.00 \mathrm{E}+00$ & $0.00 \mathrm{E}+00$ & $0.00 \mathrm{E}+00$ & $0.00 \mathrm{E}+00$ & $0.00 \mathrm{E}+00$ & $0.00 E+00$ \\
\hline RH105 & $1.79 \mathrm{E}-11$ & $1.80 \mathrm{E}-11$ & $1.78 \mathrm{E}-11$ & $1.33 \mathrm{E}-11$ & $7.93 \mathrm{E}-13$ & $2.94 \mathrm{E}-14$ & $1.58 \mathrm{E}-17$ & $1.18 \mathrm{E}-23$ & $0.00 \mathrm{E}+00$ \\
\hline RH105M & $6.65 \mathrm{E}-12$ & $6.17 \mathrm{E}-12$ & $3.06 \mathrm{E}-12$ & $1.57 \mathrm{E}-13$ & $2.70 \mathrm{E}-23$ & $0.00 \mathrm{E}+00$ & $0.00 \mathrm{E}+00$ & $0.00 \mathrm{E}+00$ & $0.00 \mathrm{E}+00$ \\
\hline RH106 & $3.96 \mathrm{E}-12$ & $8.51 \mathrm{E}-18$ & $8.51 \mathrm{E}-18$ & $8.51 \mathrm{E}-18$ & $8.41 \mathrm{E}-18$ & $8.30 \mathrm{E}-18$ & $8.06 \mathrm{E}-18$ & $7.61 \mathrm{E}-18$ & $4.28 \mathrm{E}-18$ \\
\hline RH106M & $1.92 \mathrm{E}-12$ & $1.64 \mathrm{E}-12$ & $3.98 \mathrm{E}-13$ & $9.63 \mathrm{E}-16$ & $0.00 \mathrm{E}+00$ & $0.00 \mathrm{E}+00$ & $0.00 \mathrm{E}+00$ & $0.00 \mathrm{E}+00$ & $0.00 \mathrm{E}+00$ \\
\hline SUMTOT & $1.57 \mathrm{E}+01$ & $1.41 \mathrm{E}+01$ & $7.36 \mathrm{E}+00$ & $4.42 \mathrm{E}+00$ & $3.70 \mathrm{E}+00$ & $3.13 \mathrm{E}+00$ & $2.21 \mathrm{E}+00$ & $1.22 \mathrm{E}+00$ & $2.14 \mathrm{E}-01$ \\
\hline OTOTAL & $1.57 \mathrm{E}+01$ & $1.41 \mathrm{E}+01$ & $7.36 \mathrm{E}+00$ & $4.42 \mathrm{E}+00$ & $3.70 \mathrm{E}+00$ & $3.13 \mathrm{E}+00$ & $2.21 \mathrm{E}+00$ & $1.22 \mathrm{E}+00$ & $2.14 \mathrm{E}-01$ \\
\hline
\end{tabular}


TEM-10200-1

ENGINEERING CALCULATIONS AND ANALYSIS

Page 53 of 81

$12 / 19 / 17$

Rev.08

Title:

AS-RUN NEUTRONICS EVALUATION FOR THE CSM-10584 EXPERIMENT IN THE ATR

\begin{tabular}{llllllll} 
ECAR NO.: & 4496 & REV. NO.: & 1 & PROJECT NO.: & 32501 & Date: & 10/19/2020 \\
\hline
\end{tabular}

\begin{tabular}{|c|c|c|c|c|c|c|c|c|c|}
\hline \multicolumn{10}{|c|}{1 gram of inconel irradiated for 54.9 EFPDs } \\
\hline & EOC & $30 \mathrm{MINs}$ & $5 \mathrm{HRS}$ & $1 \mathrm{DAY}$ & 7 DAYS & 14 DAYS & 30 DAYS & 60 DAYS & 1 YEAR \\
\hline & $\mathrm{Ci} / \mathrm{g}$ & $\mathrm{Ci} / \mathrm{g}$ & $\mathrm{Ci} / \mathrm{g}$ & $\mathrm{Ci} / \mathrm{g}$ & $\mathrm{Ci} / \mathrm{g}$ & $\mathrm{Ci} / \mathrm{g}$ & $\mathrm{Ci} / \mathrm{g}$ & $\mathrm{Ci} / \mathrm{g}$ & $\mathrm{Ci} / \mathrm{g}$ \\
\hline H 3 & $6.16 \mathrm{E}-11$ & $6.16 \mathrm{E}-11$ & $6.16 \mathrm{E}-11$ & $6.16 \mathrm{E}-11$ & $6.16 \mathrm{E}-11$ & $6.15 \mathrm{E}-11$ & $6.14 \mathrm{E}-11$ & $6.11 \mathrm{E}-11$ & $5.83 \mathrm{E}-11$ \\
\hline H 4 & $1.51 \mathrm{E}-04$ & $0.00 \mathrm{E}+00$ & $0.00 \mathrm{E}+00$ & $0.00 \mathrm{E}+00$ & $0.00 \mathrm{E}+00$ & $0.00 \mathrm{E}+00$ & $0.00 \mathrm{E}+00$ & $0.00 \mathrm{E}+00$ & $0.00 \mathrm{E}+00$ \\
\hline HE 6 & $1.05 \mathrm{E}-07$ & $0.00 \mathrm{E}+00$ & $0.00 \mathrm{E}+00$ & $0.00 \mathrm{E}+00$ & $0.00 \mathrm{E}+00$ & $0.00 \mathrm{E}+00$ & $0.00 \mathrm{E}+00$ & $0.00 \mathrm{E}+00$ & $0.00 \mathrm{E}+00$ \\
\hline LI 8 & $6.07 \mathrm{E}-05$ & $0.00 \mathrm{E}+00$ & $0.00 \mathrm{E}+00$ & $0.00 \mathrm{E}+00$ & $0.00 \mathrm{E}+00$ & $0.00 \mathrm{E}+00$ & $0.00 \mathrm{E}+00$ & $0.00 \mathrm{E}+00$ & $0.00 \mathrm{E}+00$ \\
\hline BE 8 & $6.08 \mathrm{E}-05$ & $0.00 \mathrm{E}+00$ & $0.00 \mathrm{E}+00$ & $0.00 \mathrm{E}+00$ & $0.00 \mathrm{E}+00$ & $0.00 \mathrm{E}+00$ & $0.00 \mathrm{E}+00$ & $0.00 \mathrm{E}+00$ & $0.00 \mathrm{E}+00$ \\
\hline BE 10 & $2.11 \mathrm{E}-12$ & $2.11 \mathrm{E}-12$ & $2.11 \mathrm{E}-12$ & $2.11 \mathrm{E}-12$ & $2.11 \mathrm{E}-12$ & $2.11 \mathrm{E}-12$ & $2.11 \mathrm{E}-12$ & $2.11 \mathrm{E}-12$ & $2.11 \mathrm{E}-12$ \\
\hline BE 11 & $1.67 \mathrm{E}-11$ & $0.00 \mathrm{E}+00$ & $0.00 \mathrm{E}+00$ & $0.00 \mathrm{E}+00$ & $0.00 \mathrm{E}+00$ & $0.00 \mathrm{E}+00$ & $0.00 \mathrm{E}+00$ & $0.00 \mathrm{E}+00$ & $0.00 \mathrm{E}+00$ \\
\hline B 12 & 4.57E-05 & $0.00 \mathrm{E}+00$ & $0.00 \mathrm{E}+00$ & $0.00 \mathrm{E}+00$ & $0.00 \mathrm{E}+00$ & $0.00 \mathrm{E}+00$ & $0.00 \mathrm{E}+00$ & $0.00 \mathrm{E}+00$ & $0.00 \mathrm{E}+00$ \\
\hline C 14 & $2.71 \mathrm{E}-11$ & $2.71 \mathrm{E}-11$ & $2.71 \mathrm{E}-11$ & $2.71 \mathrm{E}-11$ & $2.71 \mathrm{E}-11$ & $2.71 \mathrm{E}-11$ & $2.71 \mathrm{E}-11$ & $2.71 \mathrm{E}-11$ & $2.71 \mathrm{E}-11$ \\
\hline NE 23 & 1.13E-09 & $3.18 \mathrm{E}-24$ & $0.00 \mathrm{E}+00$ & $0.00 \mathrm{E}+00$ & $0.00 \mathrm{E}+00$ & $0.00 \mathrm{E}+00$ & $0.00 \mathrm{E}+00$ & $0.00 \mathrm{E}+00$ & $0.00 \mathrm{E}+00$ \\
\hline NA 24 & $1.13 \mathrm{E}-03$ & $1.11 \mathrm{E}-03$ & $8.98 \mathrm{E}-04$ & $3.73 \mathrm{E}-04$ & $4.81 \mathrm{E}-07$ & $2.04 \mathrm{E}-10$ & $4.02 \mathrm{E}-18$ & $0.00 \mathrm{E}+00$ & $0.00 \mathrm{E}+00$ \\
\hline NA 24M & $1.20 \mathrm{E}-13$ & $0.00 \mathrm{E}+00$ & $0.00 \mathrm{E}+00$ & $0.00 \mathrm{E}+00$ & $0.00 \mathrm{E}+00$ & $0.00 \mathrm{E}+00$ & $0.00 \mathrm{E}+00$ & $0.00 \mathrm{E}+00$ & $0.00 \mathrm{E}+00$ \\
\hline NA 25 & $1.71 \mathrm{E}-09$ & $1.39 \mathrm{E}-18$ & $0.00 E+00$ & $0.00 \mathrm{E}+00$ & $0.00 \mathrm{E}+00$ & $0.00 \mathrm{E}+00$ & $0.00 \mathrm{E}+00$ & $0.00 \mathrm{E}+00$ & $0.00 \mathrm{E}+00$ \\
\hline MG 27 & $5.41 \mathrm{E}-03$ & $6.01 \mathrm{E}-04$ & $1.54 \mathrm{E}-12$ & $0.00 \mathrm{E}+00$ & $0.00 \mathrm{E}+00$ & $0.00 \mathrm{E}+00$ & $0.00 \mathrm{E}+00$ & $0.00 \mathrm{E}+00$ & $0.00 \mathrm{E}+00$ \\
\hline MG 28 & $1.93 \mathrm{E}-11$ & $1.89 \mathrm{E}-11$ & 1.63E-11 & $8.69 \mathrm{E}-12$ & $7.34 \mathrm{E}-14$ & $2.85 \mathrm{E}-16$ & $8.46 \mathrm{E}-22$ & $0.00 \mathrm{E}+00$ & $0.00 \mathrm{E}+00$ \\
\hline AL 28 & $1.32 \mathrm{E}-01$ & $1.23 \mathrm{E}-05$ & $1.63 \mathrm{E}-11$ & $8.70 \mathrm{E}-12$ & $7.36 \mathrm{E}-14$ & $2.86 \mathrm{E}-16$ & $8.48 \mathrm{E}-22$ & $0.00 \mathrm{E}+00$ & $0.00 \mathrm{E}+00$ \\
\hline AL 29 & $9.27 \mathrm{E}-05$ & $3.82 \mathrm{E}-06$ & $1.31 \mathrm{E}-18$ & $0.00 \mathrm{E}+00$ & $0.00 \mathrm{E}+00$ & $0.00 \mathrm{E}+00$ & $0.00 \mathrm{E}+00$ & $0.00 \mathrm{E}+00$ & $0.00 \mathrm{E}+00$ \\
\hline AL 30 & $8.98 \mathrm{E}-08$ & $0.00 \mathrm{E}+00$ & $0.00 \mathrm{E}+00$ & $0.00 \mathrm{E}+00$ & $0.00 \mathrm{E}+00$ & $0.00 \mathrm{E}+00$ & $0.00 \mathrm{E}+00$ & $0.00 \mathrm{E}+00$ & $0.00 \mathrm{E}+00$ \\
\hline SI 31 & $8.38 \mathrm{E}-04$ & $7.34 \mathrm{E}-04$ & $2.24 \mathrm{E}-04$ & 1.47E-06 & $4.29 \mathrm{E}-23$ & $0.00 \mathrm{E}+00$ & $0.00 \mathrm{E}+00$ & $0.00 \mathrm{E}+00$ & $0.00 \mathrm{E}+00$ \\
\hline SI 32 & $9.50 \mathrm{E}-14$ & $9.50 \mathrm{E}-14$ & $9.50 \mathrm{E}-14$ & $9.50 \mathrm{E}-14$ & $9.50 \mathrm{E}-14$ & $9.50 \mathrm{E}-14$ & $9.50 \mathrm{E}-14$ & $9.50 \mathrm{E}-14$ & $9.49 \mathrm{E}-14$ \\
\hline P 32 & $1.46 \mathrm{E}-03$ & $1.46 \mathrm{E}-03$ & $1.45 \mathrm{E}-03$ & $1.39 \mathrm{E}-03$ & 1.04E-03 & 7.40E-04 & $3.41 \mathrm{E}-04$ & $7.96 \mathrm{E}-05$ & $3.00 \mathrm{E}-11$ \\
\hline P 33 & $9.89 \mathrm{E}-08$ & $9.89 \mathrm{E}-08$ & $9.83 \mathrm{E}-08$ & $9.62 \mathrm{E}-08$ & $8.15 \mathrm{E}-08$ & $6.71 \mathrm{E}-08$ & $4.31 \mathrm{E}-08$ & 1.87E-08 & $3.96 \mathrm{E}-12$ \\
\hline P 34 & $7.29 \mathrm{E}-07$ & $0.00 \mathrm{E}+00$ & $0.00 \mathrm{E}+00$ & $0.00 \mathrm{E}+00$ & $0.00 \mathrm{E}+00$ & $0.00 \mathrm{E}+00$ & $0.00 \mathrm{E}+00$ & $0.00 \mathrm{E}+00$ & $0.00 \mathrm{E}+00$ \\
\hline S 35 & $2.93 \mathrm{E}-05$ & $2.93 \mathrm{E}-05$ & $2.93 \mathrm{E}-05$ & $2.91 \mathrm{E}-05$ & $2.77 \mathrm{E}-05$ & $2.63 \mathrm{E}-05$ & $2.31 \mathrm{E}-05$ & $1.83 \mathrm{E}-05$ & $1.65 \mathrm{E}-06$ \\
\hline S 37 & $2.85 \mathrm{E}-07$ & $4.68 \mathrm{E}-09$ & $4.05 \mathrm{E}-25$ & $0.00 \mathrm{E}+00$ & $0.00 \mathrm{E}+00$ & $0.00 \mathrm{E}+00$ & $0.00 \mathrm{E}+00$ & $0.00 \mathrm{E}+00$ & $0.00 \mathrm{E}+00$ \\
\hline $\mathrm{CL} 36$ & $1.34 \mathrm{E}-10$ & 1.34E-10 & $1.34 \mathrm{E}-10$ & $1.34 \mathrm{E}-10$ & $1.34 \mathrm{E}-10$ & $1.34 \mathrm{E}-10$ & $1.34 \mathrm{E}-10$ & $1.34 \mathrm{E}-10$ & $1.34 \mathrm{E}-10$ \\
\hline CL 38 & $6.58 \mathrm{E}-10$ & $3.76 \mathrm{E}-10$ & $2.46 \mathrm{E}-12$ & $1.48 \mathrm{E}-21$ & $0.00 \mathrm{E}+00$ & $0.00 \mathrm{E}+00$ & $0.00 \mathrm{E}+00$ & $0.00 \mathrm{E}+00$ & $0.00 \mathrm{E}+00$ \\
\hline CL 38M & $7.83 \mathrm{E}-12$ & $0.00 E+00$ & $0.00 E+00$ & $0.00 E+00$ & $0.00 \mathrm{E}+00$ & $0.00 \mathrm{E}+00$ & $0.00 \mathrm{E}+00$ & $0.00 E+00$ & $0.00 \mathrm{E}+00$ \\
\hline AR 37 & $4.82 \mathrm{E}-14$ & $4.82 \mathrm{E}-14$ & $4.80 \mathrm{E}-14$ & $4.73 E-14$ & $4.20 \mathrm{E}-14$ & $3.65 \mathrm{E}-14$ & $2.66 \mathrm{E}-14$ & 1.47E-14 & $3.48 \mathrm{E}-17$ \\
\hline AR 39 & $5.04 \mathrm{E}-07$ & 5.04E-07 & 5.04E-07 & 5.04E-07 & 5.04E-07 & 5.04E-07 & 5.04E-07 & $5.04 \mathrm{E}-07$ & $5.03 \mathrm{E}-07$ \\
\hline AR 41 & 8.13E-08 & $6.73 \mathrm{E}-08$ & $1.22 \mathrm{E}-08$ & $9.03 E-12$ & $0.00 \mathrm{E}+00$ & $0.00 \mathrm{E}+00$ & $0.00 \mathrm{E}+00$ & $0.00 \mathrm{E}+00$ & $0.00 \mathrm{E}+00$ \\
\hline K 40 & 1.13E-12 & 1.13E-12 & 1.13E-12 & 1.13E-12 & $1.13 \mathrm{E}-12$ & 1.13E-12 & 1.13E-12 & 1.13E-12 & 1.13E-12 \\
\hline K 42 & $7.58 \mathrm{E}-04$ & 7.37E-04 & 5.73E-04 & 1.97E-04 & $6.14 \mathrm{E}-08$ & $4.98 \mathrm{E}-12$ & $4.52 \mathrm{E}-17$ & $4.51 \mathrm{E}-17$ & $4.44 \mathrm{E}-17$ \\
\hline K 43 & 4.49E-10 & $4.42 \mathrm{E}-10$ & $3.85 \mathrm{E}-10$ & $2.15 \mathrm{E}-10$ & 2.60E-12 & $1.50 \mathrm{E}-14$ & 1.15E-19 & $2.96 \mathrm{E}-29$ & $0.00 \mathrm{E}+00$ \\
\hline K 44 & 7.94E-11 & 3.09E-11 & $6.24 \mathrm{E}-15$ & $0.00 \mathrm{E}+00$ & $0.00 \mathrm{E}+00$ & $0.00 \mathrm{E}+00$ & $0.00 \mathrm{E}+00$ & $0.00 \mathrm{E}+00$ & $0.00 \mathrm{E}+00$ \\
\hline CA 45 & 2.43E-06 & $2.43 \mathrm{E}-06$ & $2.42 \mathrm{E}-06$ & $2.42 \mathrm{E}-06$ & 2.35E-06 & $2.29 \mathrm{E}-06$ & $2.14 \mathrm{E}-06$ & $1.88 \mathrm{E}-06$ & $5.13 \mathrm{E}-07$ \\
\hline CA 47 & $4.98 \mathrm{E}-08$ & 4.96E-08 & $4.82 \mathrm{E}-08$ & 4.27E-08 & $1.71 \mathrm{E}-08$ & 5.86E-09 & $5.08 \mathrm{E}-10$ & 5.19E-12 & $0.00 \mathrm{E}+00$ \\
\hline SC 46 & $5.45 \mathrm{E}-04$ & 5.45E-04 & 5.44E-04 & $5.41 \mathrm{E}-04$ & 5.15E-04 & 4.86E-04 & 4.25E-04 & $3.32 \mathrm{E}-04$ & $2.66 \mathrm{E}-05$ \\
\hline SC 46M & $6.13 \mathrm{E}-09$ & $0.00 \mathrm{E}+00$ & $0.00 \mathrm{E}+00$ & $0.00 \mathrm{E}+00$ & $0.00 \mathrm{E}+00$ & $0.00 \mathrm{E}+00$ & $0.00 \mathrm{E}+00$ & $0.00 \mathrm{E}+00$ & $0.00 \mathrm{E}+00$ \\
\hline SC 47 & $2.32 \mathrm{E}-03$ & $2.31 \mathrm{E}-03$ & $2.22 \mathrm{E}-03$ & 1.89E-03 & 5.45E-04 & $1.28 \mathrm{E}-04$ & $4.68 \mathrm{E}-06$ & 9.44E-09 & $0.00 \mathrm{E}+00$ \\
\hline SC 48 & $2.18 \mathrm{E}-04$ & 2.16E-04 & 2.01E-04 & 1.49E-04 & $1.53 \mathrm{E}-05$ & 1.07E-06 & 2.46E-09 & $2.78 \mathrm{E}-14$ & $0.00 \mathrm{E}+00$ \\
\hline SC 49 & $1.85 \mathrm{E}-04$ & 1.29E-04 & 4.98E-06 & 5.35E-12 & $0.00 \mathrm{E}+00$ & $0.00 \mathrm{E}+00$ & $0.00 \mathrm{E}+00$ & $0.00 E+00$ & $0.00 \mathrm{E}+00$ \\
\hline SC 50 & $3.68 \mathrm{E}-06$ & $1.90 \mathrm{E}-11$ & $0.00 \mathrm{E}+00$ & $0.00 \mathrm{E}+00$ & $0.00 \mathrm{E}+00$ & $0.00 \mathrm{E}+00$ & $0.00 \mathrm{E}+00$ & $0.00 \mathrm{E}+00$ & $0.00 \mathrm{E}+00$ \\
\hline TI 51 & 4.20E-03 & $1.14 \mathrm{E}-04$ & $8.80 \mathrm{E}-19$ & $0.00 \mathrm{E}+00$ & $0.00 \mathrm{E}+00$ & $0.00 \mathrm{E}+00$ & $0.00 \mathrm{E}+00$ & $0.00 \mathrm{E}+00$ & $0.00 \mathrm{E}+00$ \\
\hline V 52 & $3.64 \mathrm{E}-02$ & $1.42 \mathrm{E}-04$ & $3.01 \mathrm{E}-26$ & $0.00 \mathrm{E}+00$ & $0.00 \mathrm{E}+00$ & $0.00 \mathrm{E}+00$ & $0.00 \mathrm{E}+00$ & $0.00 \mathrm{E}+00$ & $0.00 \mathrm{E}+00$ \\
\hline V 53 & $2.25 \mathrm{E}-04$ & 5.54E-10 & $0.00 \mathrm{E}+00$ & $0.00 \mathrm{E}+00$ & $0.00 \mathrm{E}+00$ & $0.00 \mathrm{E}+00$ & $0.00 \mathrm{E}+00$ & $0.00 \mathrm{E}+00$ & $0.00 \mathrm{E}+00$ \\
\hline V 54 & $2.33 \mathrm{E}-06$ & $3.27 \mathrm{E}-16$ & $0.00 \mathrm{E}+00$ & $0.00 \mathrm{E}+00$ & $0.00 \mathrm{E}+00$ & $0.00 \mathrm{E}+00$ & $0.00 \mathrm{E}+00$ & $0.00 \mathrm{E}+00$ & $0.00 \mathrm{E}+00$ \\
\hline CR 51 & $3.69 \mathrm{E}+00$ & $3.69 \mathrm{E}+00$ & $3.67 E+00$ & $3.60 \mathrm{E}+00$ & $3.10 \mathrm{E}+00$ & $2.60 \mathrm{E}+00$ & $1.74 \mathrm{E}+00$ & $8.23 \mathrm{E}-01$ & $3.97 \mathrm{E}-04$ \\
\hline CR 55 & $6.42 \mathrm{E}-02$ & $1.83 \mathrm{E}-04$ & $0.00 \mathrm{E}+00$ & $0.00 \mathrm{E}+00$ & $0.00 \mathrm{E}+00$ & $0.00 \mathrm{E}+00$ & $0.00 \mathrm{E}+00$ & $0.00 \mathrm{E}+00$ & $0.00 \mathrm{E}+00$ \\
\hline MN 54 & $5.13 \mathrm{E}-03$ & $5.13 \mathrm{E}-03$ & $5.13 \mathrm{E}-03$ & $5.12 \mathrm{E}-03$ & $5.05 \mathrm{E}-03$ & $4.98 \mathrm{E}-03$ & $4.80 \mathrm{E}-03$ & $4.49 \mathrm{E}-03$ & $2.28 \mathrm{E}-03$ \\
\hline MN 56 & $2.14 \mathrm{E}+00$ & $1.87 \mathrm{E}+00$ & $5.58 \mathrm{E}-01$ & $3.38 \mathrm{E}-03$ & $5.22 \mathrm{E}-20$ & $0.00 \mathrm{E}+00$ & $0.00 \mathrm{E}+00$ & $0.00 \mathrm{E}+00$ & $0.00 \mathrm{E}+00$ \\
\hline MN 57 & $1.07 \mathrm{E}-04$ & $2.64 \mathrm{E}-10$ & $0.00 \mathrm{E}+00$ & $0.00 \mathrm{E}+00$ & $0.00 \mathrm{E}+00$ & $0.00 \mathrm{E}+00$ & $0.00 \mathrm{E}+00$ & $0.00 \mathrm{E}+00$ & $0.00 \mathrm{E}+00$ \\
\hline MN 58 & $3.49 \mathrm{E}-07$ & $1.76 \mathrm{E}-15$ & $0.00 \mathrm{E}+00$ & $0.00 \mathrm{E}+00$ & $0.00 \mathrm{E}+00$ & $0.00 \mathrm{E}+00$ & $0.00 \mathrm{E}+00$ & $0.00 \mathrm{E}+00$ & $0.00 \mathrm{E}+00$ \\
\hline FE 55 & $2.50 \mathrm{E}-02$ & $2.50 \mathrm{E}-02$ & $2.50 \mathrm{E}-02$ & $2.50 \mathrm{E}-02$ & $2.49 \mathrm{E}-02$ & $2.48 \mathrm{E}-02$ & $2.45 \mathrm{E}-02$ & $2.39 \mathrm{E}-02$ & $1.92 \mathrm{E}-02$ \\
\hline FE 59 & $8.07 \mathrm{E}-03$ & 8.07E-03 & $8.05 \mathrm{E}-03$ & $7.95 \mathrm{E}-03$ & $7.25 \mathrm{E}-03$ & $6.51 \mathrm{E}-03$ & $5.08 \mathrm{E}-03$ & $3.20 \mathrm{E}-03$ & $2.91 \mathrm{E}-05$ \\
\hline
\end{tabular}


TEM-10200-1

ENGINEERING CALCULATIONS AND ANALYSIS

Page 54 of 81

$12 / 19 / 17$

Rev.08

Title:

AS-RUN NEUTRONICS EVALUATION FOR THE CSM-10584 EXPERIMENT IN THE ATR

\begin{tabular}{llllllll} 
ECAR NO.: & 4496 & REV. NO.: & 1 & PROJECT NO.: & 32501 & Date: & 10/19/2020 \\
\hline
\end{tabular}

\begin{tabular}{|c|c|c|c|c|c|c|c|c|c|}
\hline \multicolumn{10}{|c|}{1 gram of inconel irradiated for 54.9 EFPDs } \\
\hline & EOC & $30 \mathrm{MINs}$ & $5 \mathrm{HRS}$ & $1 \mathrm{DAY}$ & 7 DAYS & 14 DAYS & 30 DAYS & 60 DAYS & 1 YEAR \\
\hline & $\mathrm{Ci} / \mathrm{g}$ & $\mathrm{Ci} / \mathrm{g}$ & $\mathrm{Ci} / \mathrm{g}$ & $\mathrm{Ci} / \mathrm{g}$ & $\mathrm{Ci} / \mathrm{g}$ & $\mathrm{Ci} / \mathrm{g}$ & $\mathrm{Ci} / \mathrm{g}$ & $\mathrm{Ci} / \mathrm{g}$ & $\mathrm{Ci} / \mathrm{g}$ \\
\hline $\mathrm{CO} 58$ & 5.34E-01 & 5.34E-01 & 5.33E-01 & 5.29E-01 & 4.99E-01 & $4.66 \mathrm{E}-01$ & $3.98 \mathrm{E}-01$ & 2.97E-01 & $1.49 \mathrm{E}-02$ \\
\hline $\mathrm{CO} 60$ & 3.05E-01 & $3.05 \mathrm{E}-01$ & 3.05E-01 & 3.05E-01 & 3.04E-01 & $3.04 \mathrm{E}-01$ & $3.02 \mathrm{E}-01$ & 2.99E-01 & $2.68 \mathrm{E}-01$ \\
\hline CO 60M & $8.32 \mathrm{E}+00$ & $1.14 \mathrm{E}+00$ & $1.97 \mathrm{E}-08$ & $0.00 \mathrm{E}+00$ & $0.00 \mathrm{E}+00$ & $0.00 \mathrm{E}+00$ & $0.00 \mathrm{E}+00$ & $0.00 \mathrm{E}+00$ & $0.00 E+00$ \\
\hline CO 61 & $2.43 \mathrm{E}-02$ & $1.97 \mathrm{E}-02$ & $2.98 \mathrm{E}-03$ & $1.02 \mathrm{E}-06$ & $0.00 \mathrm{E}+00$ & $0.00 \mathrm{E}+00$ & $0.00 \mathrm{E}+00$ & $0.00 \mathrm{E}+00$ & $0.00 \mathrm{E}+00$ \\
\hline $\mathrm{CO} 62$ & $2.09 \mathrm{E}-04$ & $2.00 \mathrm{E}-10$ & $0.00 \mathrm{E}+00$ & $0.00 \mathrm{E}+00$ & $0.00 \mathrm{E}+00$ & $0.00 \mathrm{E}+00$ & $0.00 \mathrm{E}+00$ & $0.00 \mathrm{E}+00$ & $0.00 \mathrm{E}+00$ \\
\hline $\mathrm{NI} 59$ & $7.52 \mathrm{E}-05$ & $7.52 \mathrm{E}-05$ & $7.52 \mathrm{E}-05$ & $7.52 \mathrm{E}-05$ & $7.52 \mathrm{E}-05$ & $7.52 \mathrm{E}-05$ & $7.52 \mathrm{E}-05$ & $7.52 \mathrm{E}-05$ & $7.52 \mathrm{E}-05$ \\
\hline NI 63 & $9.53 \mathrm{E}-03$ & $9.53 \mathrm{E}-03$ & $9.53 \mathrm{E}-03$ & $9.53 \mathrm{E}-03$ & $9.53 \mathrm{E}-03$ & $9.53 \mathrm{E}-03$ & $9.52 \mathrm{E}-03$ & $9.52 \mathrm{E}-03$ & $9.46 \mathrm{E}-03$ \\
\hline NI 65 & $2.42 \mathrm{E}-01$ & $2.11 \mathrm{E}-01$ & $6.11 \mathrm{E}-02$ & $3.28 \mathrm{E}-04$ & $2.06 \mathrm{E}-21$ & $0.00 \mathrm{E}+00$ & $0.00 \mathrm{E}+00$ & $0.00 \mathrm{E}+00$ & $0.00 \mathrm{E}+00$ \\
\hline NI 66 & $8.35 \mathrm{E}-06$ & $8.30 \mathrm{E}-06$ & $7.83 \mathrm{E}-06$ & $6.16 \mathrm{E}-06$ & $9.90 \mathrm{E}-07$ & 1.17E-07 & $8.97 \mathrm{E}-10$ & $9.64 \mathrm{E}-14$ & $0.00 \mathrm{E}+00$ \\
\hline CU 64 & $3.03 \mathrm{E}-01$ & $2.95 \mathrm{E}-01$ & $2.31 \mathrm{E}-01$ & $8.18 \mathrm{E}-02$ & $3.16 \mathrm{E}-05$ & $3.29 \mathrm{E}-09$ & $2.60 \mathrm{E}-18$ & $0.00 \mathrm{E}+00$ & $0.00 \mathrm{E}+00$ \\
\hline CU 66 & $6.57 \mathrm{E}-02$ & $1.12 \mathrm{E}-03$ & $7.85 \mathrm{E}-06$ & $6.17 \mathrm{E}-06$ & $9.91 \mathrm{E}-07$ & $1.18 \mathrm{E}-07$ & $8.98 \mathrm{E}-10$ & $9.65 \mathrm{E}-14$ & $0.00 \mathrm{E}+00$ \\
\hline CU 67 & $4.25 \mathrm{E}-07$ & $4.23 \mathrm{E}-07$ & $4.02 \mathrm{E}-07$ & $3.25 \mathrm{E}-07$ & $6.47 \mathrm{E}-08$ & $9.85 \mathrm{E}-09$ & $1.33 \mathrm{E}-10$ & $4.18 \mathrm{E}-14$ & $0.00 \mathrm{E}+00$ \\
\hline ZN 65 & $4.51 \mathrm{E}-06$ & $4.51 \mathrm{E}-06$ & $4.51 \mathrm{E}-06$ & 4.49E-06 & $4.42 \mathrm{E}-06$ & $4.33 \mathrm{E}-06$ & 4.14E-06 & $3.80 \mathrm{E}-06$ & $1.60 \mathrm{E}-06$ \\
\hline ZN 69 & $3.08 \mathrm{E}-11$ & $2.20 \mathrm{E}-11$ & $2.47 \mathrm{E}-12$ & $6.64 \mathrm{E}-13$ & $4.70 \mathrm{E}-16$ & $9.93 \mathrm{E}-20$ & $0.00 \mathrm{E}+00$ & $0.00 \mathrm{E}+00$ & $0.00 \mathrm{E}+00$ \\
\hline ZN 69M & 2.07E-12 & $2.02 \mathrm{E}-12$ & $1.61 \mathrm{E}-12$ & $6.18 \mathrm{E}-13$ & $4.38 \mathrm{E}-16$ & $9.25 \mathrm{E}-20$ & $3.68 \mathrm{E}-28$ & $0.00 \mathrm{E}+00$ & $0.00 \mathrm{E}+00$ \\
\hline GA 70 & $2.25 \mathrm{E}-14$ & $7.67 \mathrm{E}-15$ & $1.08 \mathrm{E}-18$ & $0.00 \mathrm{E}+00$ & $0.00 \mathrm{E}+00$ & $0.00 \mathrm{E}+00$ & $0.00 \mathrm{E}+00$ & $0.00 \mathrm{E}+00$ & $0.00 \mathrm{E}+00$ \\
\hline SR 89 & $8.84 \mathrm{E}-11$ & $8.84 \mathrm{E}-11$ & $8.82 \mathrm{E}-11$ & $8.72 \mathrm{E}-11$ & $8.03 \mathrm{E}-11$ & 7.30E-11 & $5.86 \mathrm{E}-11$ & $3.88 \mathrm{E}-11$ & $5.88 \mathrm{E}-13$ \\
\hline SR 90 & $3.19 \mathrm{E}-13$ & $3.19 \mathrm{E}-13$ & $3.19 \mathrm{E}-13$ & $3.19 \mathrm{E}-13$ & $3.19 \mathrm{E}-13$ & $3.18 \mathrm{E}-13$ & $3.18 \mathrm{E}-13$ & $3.18 \mathrm{E}-13$ & $3.11 \mathrm{E}-13$ \\
\hline SR 91 & $6.26 \mathrm{E}-12$ & $6.03 \mathrm{E}-12$ & $4.34 \mathrm{E}-12$ & 1.09E-12 & 2.97E-17 & $1.41 \mathrm{E}-22$ & $0.00 \mathrm{E}+00$ & $0.00 \mathrm{E}+00$ & $0.00 \mathrm{E}+00$ \\
\hline Y 89M & $9.31 \mathrm{E}-06$ & 9.27E-06 & $8.91 \mathrm{E}-06$ & $7.54 \mathrm{E}-06$ & $2.11 \mathrm{E}-06$ & $4.78 \mathrm{E}-07$ & $1.61 \mathrm{E}-08$ & $2.78 \mathrm{E}-11$ & $0.00 \mathrm{E}+00$ \\
\hline Y 90 & $2.10 \mathrm{E}-04$ & $2.09 \mathrm{E}-04$ & 1.99E-04 & $1.62 \mathrm{E}-04$ & $3.41 \mathrm{E}-05$ & $5.53 \mathrm{E}-06$ & 8.63E-08 & $3.58 \mathrm{E}-11$ & $3.11 \mathrm{E}-13$ \\
\hline Y 90M & $4.78 \mathrm{E}-12$ & $4.27 \mathrm{E}-12$ & $1.56 \mathrm{E}-12$ & $2.23 \mathrm{E}-14$ & $2.31 \mathrm{E}-28$ & $0.00 \mathrm{E}+00$ & $0.00 \mathrm{E}+00$ & $0.00 \mathrm{E}+00$ & $0.00 \mathrm{E}+00$ \\
\hline Y91 & $1.62 \mathrm{E}-08$ & $1.62 \mathrm{E}-08$ & $1.62 \mathrm{E}-08$ & 1.60E-08 & 1.49E-08 & $1.38 \mathrm{E}-08$ & $1.14 \mathrm{E}-08$ & 7.97E-09 & $2.14 \mathrm{E}-10$ \\
\hline Y 92 & $7.23 \mathrm{E}-10$ & $6.56 \mathrm{E}-10$ & $2.72 \mathrm{E}-10$ & $6.57 \mathrm{E}-12$ & $3.70 \mathrm{E}-24$ & $0.00 \mathrm{E}+00$ & $0.00 \mathrm{E}+00$ & $0.00 \mathrm{E}+00$ & $0.00 \mathrm{E}+00$ \\
\hline Y94 & $4.45 \mathrm{E}-12$ & 1.50E-12 & $8.31 \mathrm{E}-17$ & $0.00 \mathrm{E}+00$ & $0.00 \mathrm{E}+00$ & $0.00 \mathrm{E}+00$ & $0.00 \mathrm{E}+00$ & $0.00 \mathrm{E}+00$ & $0.00 \mathrm{E}+00$ \\
\hline ZR 89 & $9.33 \mathrm{E}-06$ & $9.29 E-06$ & 8.93E-06 & 7.55E-06 & $2.11 \mathrm{E}-06$ & $4.79 \mathrm{E}-07$ & $1.61 \mathrm{E}-08$ & $2.78 \mathrm{E}-11$ & $0.00 \mathrm{E}+00$ \\
\hline ZR 93 & $1.42 \mathrm{E}-10$ & $1.42 \mathrm{E}-10$ & $1.42 \mathrm{E}-10$ & $1.42 \mathrm{E}-10$ & $1.42 \mathrm{E}-10$ & $1.42 \mathrm{E}-10$ & $1.42 \mathrm{E}-10$ & $1.42 \mathrm{E}-10$ & $1.42 \mathrm{E}-10$ \\
\hline ZR 95 & 1.44E-05 & 1.44E-05 & 1.44E-05 & 1.43E-05 & 1.34E-05 & $1.24 \mathrm{E}-05$ & 1.04E-05 & 7.53E-06 & $2.76 \mathrm{E}-07$ \\
\hline ZR 97 & $7.38 \mathrm{E}-07$ & 7.23E-07 & $6.01 \mathrm{E}-07$ & $2.76 \mathrm{E}-07$ & $7.51 \mathrm{E}-10$ & 7.57E-13 & $1.10 \mathrm{E}-19$ & $0.00 \mathrm{E}+00$ & $0.00 \mathrm{E}+00$ \\
\hline NB 92 & $1.81 \mathrm{E}-03$ & $1.81 \mathrm{E}-03$ & 1.79E-03 & 1.69E-03 & $1.12 \mathrm{E}-03$ & $6.98 \mathrm{E}-04$ & $2.34 \mathrm{E}-04$ & $3.02 \mathrm{E}-05$ & $2.73 \mathrm{E}-14$ \\
\hline NB 93M & $5.14 \mathrm{E}-13$ & 5.15E-13 & $5.18 \mathrm{E}-13$ & 5.33E-13 & $6.45 \mathrm{E}-13$ & $7.76 \mathrm{E}-13$ & 1.07E-12 & 1.63E-12 & 7.17E-12 \\
\hline NB 94 & $2.36 \mathrm{E}-05$ & $2.36 \mathrm{E}-05$ & $2.36 \mathrm{E}-05$ & $2.36 \mathrm{E}-05$ & $2.36 \mathrm{E}-05$ & $2.36 \mathrm{E}-05$ & $2.36 \mathrm{E}-05$ & $2.36 \mathrm{E}-05$ & $2.36 \mathrm{E}-05$ \\
\hline NB 95 & $4.30 \mathrm{E}-02$ & $4.30 \mathrm{E}-02$ & $4.29 \mathrm{E}-02$ & $4.22 \mathrm{E}-02$ & $3.75 \mathrm{E}-02$ & $3.27 \mathrm{E}-02$ & $2.38 \mathrm{E}-02$ & $1.32 \mathrm{E}-02$ & $3.26 \mathrm{E}-05$ \\
\hline NB 95M & 9.36E-08 & 9.36E-08 & 9.39E-08 & $9.48 \mathrm{E}-08$ & 9.57E-08 & $9.11 \mathrm{E}-08$ & 7.73E-08 & $5.59 \mathrm{E}-08$ & $2.05 \mathrm{E}-09$ \\
\hline NB 96 & $2.56 \mathrm{E}-04$ & $2.52 \mathrm{E}-04$ & $2.21 \mathrm{E}-04$ & $1.26 \mathrm{E}-04$ & $1.75 \mathrm{E}-06$ & $1.19 \mathrm{E}-08$ & $1.34 \mathrm{E}-13$ & $6.97 \mathrm{E}-23$ & $0.00 E+00$ \\
\hline NB 97 & 2.61E-05 & 1.97E-05 & 2.06E-06 & 2.77E-07 & 7.55E-10 & $7.68 \mathrm{E}-13$ & $1.18 \mathrm{E}-19$ & $0.00 \mathrm{E}+00$ & $0.00 \mathrm{E}+00$ \\
\hline NB 97M & $6.98 \mathrm{E}-07$ & $6.85 \mathrm{E}-07$ & $5.69 \mathrm{E}-07$ & $2.61 \mathrm{E}-07$ & $7.11 \mathrm{E}-10$ & $7.24 \mathrm{E}-13$ & $1.04 \mathrm{E}-19$ & $0.00 \mathrm{E}+00$ & $0.00 \mathrm{E}+00$ \\
\hline NB 98 & $3.90 \mathrm{E}-06$ & $0.00 \mathrm{E}+00$ & $0.00 \mathrm{E}+00$ & $0.00 \mathrm{E}+00$ & $0.00 \mathrm{E}+00$ & $0.00 \mathrm{E}+00$ & $0.00 \mathrm{E}+00$ & $0.00 \mathrm{E}+00$ & $0.00 \mathrm{E}+00$ \\
\hline NB100 & $1.94 \mathrm{E}-07$ & $0.00 \mathrm{E}+00$ & $0.00 \mathrm{E}+00$ & $0.00 \mathrm{E}+00$ & $0.00 \mathrm{E}+00$ & $0.00 \mathrm{E}+00$ & $0.00 \mathrm{E}+00$ & $0.00 \mathrm{E}+00$ & $0.00 \mathrm{E}+00$ \\
\hline MO 93M & 5.39E-04 & 5.13E-04 & $3.25 \mathrm{E}-04$ & 4.75E-05 & $2.23 \mathrm{E}-11$ & $9.25 \mathrm{E}-19$ & $0.00 \mathrm{E}+00$ & $0.00 \mathrm{E}+00$ & $0.00 \mathrm{E}+00$ \\
\hline MO 93 & 4.94E-07 & $4.94 \mathrm{E}-07$ & 4.94E-07 & 4.94E-07 & 4.94E-07 & 4.94E-07 & 4.94E-07 & 4.94E-07 & 4.94E-07 \\
\hline MO 99 & 3.77E-01 & 3.75E-01 & $3.58 \mathrm{E}-01$ & $2.93 \mathrm{E}-01$ & $6.46 \mathrm{E}-02$ & $1.11 \mathrm{E}-02$ & $1.96 \mathrm{E}-04$ & $1.02 \mathrm{E}-07$ & $0.00 \mathrm{E}+00$ \\
\hline MO101 & $9.39 \mathrm{E}-02$ & $2.27 \mathrm{E}-02$ & $6.25 \mathrm{E}-08$ & $0.00 \mathrm{E}+00$ & $0.00 \mathrm{E}+00$ & $0.00 \mathrm{E}+00$ & $0.00 \mathrm{E}+00$ & $0.00 \mathrm{E}+00$ & $0.00 \mathrm{E}+00$ \\
\hline TC 99 & $2.08 \mathrm{E}-08$ & $2.08 \mathrm{E}-08$ & $2.09 \mathrm{E}-08$ & $2.12 \mathrm{E}-08$ & $2.22 \mathrm{E}-08$ & $2.24 \mathrm{E}-08$ & $2.25 \mathrm{E}-08$ & $2.25 \mathrm{E}-08$ & $2.25 \mathrm{E}-08$ \\
\hline TC100 & $2.18 \mathrm{E}-03$ & $0.00 \mathrm{E}+00$ & $0.00 \mathrm{E}+00$ & $0.00 \mathrm{E}+00$ & $0.00 \mathrm{E}+00$ & $0.00 \mathrm{E}+00$ & $0.00 \mathrm{E}+00$ & $0.00 \mathrm{E}+00$ & $0.00 \mathrm{E}+00$ \\
\hline TC101 & $9.39 \mathrm{E}-02$ & $5.42 \mathrm{E}-02$ & $7.88 \mathrm{E}-07$ & $0.00 \mathrm{E}+00$ & $0.00 \mathrm{E}+00$ & $0.00 \mathrm{E}+00$ & $0.00 \mathrm{E}+00$ & $0.00 \mathrm{E}+00$ & $0.00 \mathrm{E}+00$ \\
\hline RU103 & $2.91 \mathrm{E}-07$ & $2.91 \mathrm{E}-07$ & $2.90 \mathrm{E}-07$ & $2.86 \mathrm{E}-07$ & 2.57E-07 & $2.27 \mathrm{E}-07$ & $1.71 \mathrm{E}-07$ & $1.01 \mathrm{E}-07$ & $4.62 \mathrm{E}-10$ \\
\hline RU105 & $1.72 \mathrm{E}-12$ & $1.59 \mathrm{E}-12$ & $7.88 \mathrm{E}-13$ & $4.05 \mathrm{E}-14$ & $6.95 \mathrm{E}-24$ & $0.00 \mathrm{E}+00$ & $0.00 \mathrm{E}+00$ & $0.00 \mathrm{E}+00$ & $0.00 E+00$ \\
\hline RH104 & $1.61 \mathrm{E}-09$ & $1.15 \mathrm{E}-12$ & $0.00 \mathrm{E}+00$ & $0.00 \mathrm{E}+00$ & $0.00 \mathrm{E}+00$ & $0.00 \mathrm{E}+00$ & $0.00 \mathrm{E}+00$ & $0.00 \mathrm{E}+00$ & $0.00 \mathrm{E}+00$ \\
\hline RH104M & $1.16 \mathrm{E}-10$ & $9.63 \mathrm{E}-13$ & $0.00 \mathrm{E}+00$ & $0.00 \mathrm{E}+00$ & $0.00 \mathrm{E}+00$ & $0.00 \mathrm{E}+00$ & $0.00 \mathrm{E}+00$ & $0.00 \mathrm{E}+00$ & $0.00 E+00$ \\
\hline RH105 & $1.30 \mathrm{E}-12$ & $1.30 \mathrm{E}-12$ & $1.29 \mathrm{E}-12$ & $9.60 \mathrm{E}-13$ & 5.74E-14 & $2.13 \mathrm{E}-15$ & 1.15E-18 & $8.53 \mathrm{E}-25$ & $0.00 \mathrm{E}+00$ \\
\hline RH105M & $4.82 \mathrm{E}-13$ & $4.47 \mathrm{E}-13$ & $2.21 \mathrm{E}-13$ & $1.14 \mathrm{E}-14$ & $1.95 \mathrm{E}-24$ & $0.00 \mathrm{E}+00$ & $0.00 \mathrm{E}+00$ & $0.00 \mathrm{E}+00$ & $0.00 E+00$ \\
\hline RH106 & $2.87 \mathrm{E}-13$ & $2.36 \mathrm{E}-19$ & $2.36 \mathrm{E}-19$ & $2.36 \mathrm{E}-19$ & $2.36 \mathrm{E}-19$ & $2.36 \mathrm{E}-19$ & $2.36 \mathrm{E}-19$ & $2.36 \mathrm{E}-19$ & $1.39 \mathrm{E}-19$ \\
\hline RH106M & $1.39 \mathrm{E}-13$ & $1.19 \mathrm{E}-13$ & $2.88 \mathrm{E}-14$ & $1.78 \mathrm{E}-16$ & $0.00 \mathrm{E}+00$ & $0.00 \mathrm{E}+00$ & $0.00 \mathrm{E}+00$ & $0.00 \mathrm{E}+00$ & $0.00 \mathrm{E}+00$ \\
\hline SUMTOT & $1.65 \mathrm{E}+01$ & $8.62 E+00$ & $5.82 \mathrm{E}+00$ & $4.91 \mathrm{E}+00$ & $4.05 E+00$ & $3.46 \mathrm{E}+00$ & $2.51 \mathrm{E}+00$ & $1.47 \mathrm{E}+00$ & $3.14 \mathrm{E}-01$ \\
\hline
\end{tabular}


TEM-10200-1

ENGINEERING CALCULATIONS AND ANALYSIS

Page 55 of 81

$12 / 19 / 17$

Rev.08

Title:

AS-RUN NEUTRONICS EVALUATION FOR THE CSM-10584 EXPERIMENT IN THE ATR

\begin{tabular}{llllllll} 
ECAR NO.: & 4496 & REV. NO.: & 1 & PROJECT NO.: & 32501 & Date: & 10/19/2020 \\
\hline
\end{tabular}

\begin{tabular}{|c|c|c|c|c|c|c|c|c|c|}
\hline \multicolumn{10}{|c|}{1 gram of inconel irradiated for 54.9 EFPDs } \\
\hline & EOC & $30 \mathrm{MINs}$ & $5 \mathrm{HRS}$ & $1 \mathrm{DAY}$ & $7 \mathrm{DAYS}$ & $14 \mathrm{DAYS}$ & 30 DAYS & $60 \mathrm{DAYS}$ & 1 YEAR \\
\hline & $\mathrm{Ci} / \mathrm{g}$ & $\mathrm{Ci} / \mathrm{g}$ & $\mathrm{Ci} / \mathrm{g}$ & $\mathrm{Ci} / \mathrm{g}$ & $\mathrm{Ci} / \mathrm{g}$ & $\mathrm{Ci} / \mathrm{g}$ & $\mathrm{Ci} / \mathrm{g}$ & $\mathrm{Ci} / \mathrm{g}$ & $\mathrm{Ci} / \mathrm{g}$ \\
\hline OTOTAL & $1.65 \mathrm{E}+01$ & $8.62 \mathrm{E}+00$ & $5.82 \mathrm{E}+00$ & $4.91 \mathrm{E}+00$ & $4.05 \mathrm{E}+00$ & $3.46 \mathrm{E}+00$ & $2.51 \mathrm{E}+00$ & $1.47 \mathrm{E}+00$ & $3.14 \mathrm{E}-01$ \\
\hline
\end{tabular}

\begin{tabular}{|c|c|c|c|c|c|c|c|c|c|}
\hline \multicolumn{10}{|c|}{1 gram of inconel irradiated for 119 EFPDs } \\
\hline & EOC & $30 \mathrm{MINs}$ & $5 \mathrm{HRS}$ & $1 \mathrm{DAY}$ & 7 DAYS & 14 DAYS & 30 DAYS & 60 DAYS & 1 YEAR \\
\hline & $\mathrm{Ci} / \mathrm{g}$ & $\mathrm{Ci} / \mathrm{g}$ & $\mathrm{Ci} / \mathrm{g}$ & $\mathrm{Ci} / \mathrm{g}$ & $\mathrm{Ci} / \mathrm{g}$ & $\mathrm{Ci} / \mathrm{g}$ & $\mathrm{Ci} / \mathrm{g}$ & $\mathrm{Ci} / \mathrm{g}$ & $\mathrm{Ci} / \mathrm{g}$ \\
\hline $\mathrm{H} 3$ & $5.56 \mathrm{E}-10$ & $5.56 \mathrm{E}-10$ & $5.56 \mathrm{E}-10$ & $5.56 \mathrm{E}-10$ & $5.55 \mathrm{E}-10$ & $5.54 \mathrm{E}-10$ & $5.53 \mathrm{E}-10$ & $5.51 \mathrm{E}-10$ & $5.25 \mathrm{E}-10$ \\
\hline $\mathrm{H} 4$ & $1.68 \mathrm{E}-04$ & $0.00 \mathrm{E}+00$ & $0.00 \mathrm{E}+00$ & $0.00 \mathrm{E}+00$ & $0.00 \mathrm{E}+00$ & $0.00 \mathrm{E}+00$ & $0.00 \mathrm{E}+00$ & $0.00 \mathrm{E}+00$ & $0.00 E+00$ \\
\hline HE 6 & $2.28 \mathrm{E}-07$ & $0.00 \mathrm{E}+00$ & $0.00 \mathrm{E}+00$ & $0.00 \mathrm{E}+00$ & $0.00 \mathrm{E}+00$ & $0.00 \mathrm{E}+00$ & $0.00 \mathrm{E}+00$ & $0.00 \mathrm{E}+00$ & $0.00 \mathrm{E}+00$ \\
\hline LI 8 & $6.73 \mathrm{E}-05$ & $0.00 \mathrm{E}+00$ & $0.00 \mathrm{E}+00$ & $0.00 \mathrm{E}+00$ & $0.00 \mathrm{E}+00$ & $0.00 \mathrm{E}+00$ & $0.00 \mathrm{E}+00$ & $0.00 \mathrm{E}+00$ & $0.00 \mathrm{E}+00$ \\
\hline BE 8 & $6.77 \mathrm{E}-05$ & $0.00 \mathrm{E}+00$ & $0.00 \mathrm{E}+00$ & $0.00 E+00$ & $0.00 \mathrm{E}+00$ & $0.00 E+00$ & $0.00 E+00$ & $0.00 \mathrm{E}+00$ & $0.00 \mathrm{E}+00$ \\
\hline BE 10 & $3.12 \mathrm{E}-12$ & $3.12 \mathrm{E}-12$ & $3.12 \mathrm{E}-12$ & $3.12 \mathrm{E}-12$ & $3.12 \mathrm{E}-12$ & $3.12 \mathrm{E}-12$ & $3.12 \mathrm{E}-12$ & $3.12 \mathrm{E}-12$ & $3.12 \mathrm{E}-12$ \\
\hline BE 11 & $2.47 \mathrm{E}-11$ & $0.00 \mathrm{E}+00$ & $0.00 \mathrm{E}+00$ & $0.00 \mathrm{E}+00$ & $0.00 \mathrm{E}+00$ & $0.00 \mathrm{E}+00$ & $0.00 \mathrm{E}+00$ & $0.00 \mathrm{E}+00$ & $0.00 \mathrm{E}+00$ \\
\hline B 12 & 4.57E-05 & $0.00 E+00$ & $0.00 \mathrm{E}+00$ & $0.00 \mathrm{E}+00$ & $0.00 \mathrm{E}+00$ & $0.00 \mathrm{E}+00$ & $0.00 \mathrm{E}+00$ & $0.00 \mathrm{E}+00$ & $0.00 E+00$ \\
\hline C 14 & $5.87 \mathrm{E}-11$ & $5.87 \mathrm{E}-11$ & $5.87 \mathrm{E}-11$ & 5.87E-11 & 5.87E-11 & $5.87 \mathrm{E}-11$ & $5.87 \mathrm{E}-11$ & $5.87 \mathrm{E}-11$ & $5.87 \mathrm{E}-11$ \\
\hline NE 23 & $2.45 \mathrm{E}-09$ & $6.90 \mathrm{E}-24$ & $0.00 \mathrm{E}+00$ & $0.00 \mathrm{E}+00$ & $0.00 \mathrm{E}+00$ & $0.00 \mathrm{E}+00$ & $0.00 \mathrm{E}+00$ & $0.00 \mathrm{E}+00$ & $0.00 \mathrm{E}+00$ \\
\hline NA 24 & $1.13 \mathrm{E}-03$ & $1.11 \mathrm{E}-03$ & $8.98 \mathrm{E}-04$ & 3.73E-04 & $4.83 \mathrm{E}-07$ & $2.06 \mathrm{E}-10$ & 4.04E-18 & $0.00 \mathrm{E}+00$ & $0.00 \mathrm{E}+00$ \\
\hline NA 24M & $5.66 \mathrm{E}-13$ & $0.00 \mathrm{E}+00$ & $0.00 \mathrm{E}+00$ & $0.00 E+00$ & $0.00 \mathrm{E}+00$ & $0.00 E+00$ & $0.00 E+00$ & $0.00 \mathrm{E}+00$ & $0.00 \mathrm{E}+00$ \\
\hline NA 25 & 3.71E-09 & $3.01 \mathrm{E}-18$ & $0.00 \mathrm{E}+00$ & $0.00 \mathrm{E}+00$ & $0.00 \mathrm{E}+00$ & $0.00 \mathrm{E}+00$ & $0.00 \mathrm{E}+00$ & $0.00 \mathrm{E}+00$ & $0.00 \mathrm{E}+00$ \\
\hline MG 27 & $5.41 \mathrm{E}-03$ & $6.01 \mathrm{E}-04$ & $1.54 \mathrm{E}-12$ & $0.00 \mathrm{E}+00$ & $0.00 \mathrm{E}+00$ & $0.00 \mathrm{E}+00$ & $0.00 \mathrm{E}+00$ & $0.00 \mathrm{E}+00$ & $0.00 \mathrm{E}+00$ \\
\hline MG 28 & $1.92 \mathrm{E}-11$ & $1.89 \mathrm{E}-11$ & $1.63 \mathrm{E}-11$ & $8.69 \mathrm{E}-12$ & $7.34 \mathrm{E}-14$ & $2.85 \mathrm{E}-16$ & $8.46 \mathrm{E}-22$ & $0.00 \mathrm{E}+00$ & $0.00 \mathrm{E}+00$ \\
\hline AL 28 & $1.32 \mathrm{E}-01$ & $1.23 \mathrm{E}-05$ & $1.63 \mathrm{E}-11$ & $8.70 \mathrm{E}-12$ & $7.36 \mathrm{E}-14$ & $2.86 \mathrm{E}-16$ & $8.48 \mathrm{E}-22$ & $0.00 \mathrm{E}+00$ & $0.00 \mathrm{E}+00$ \\
\hline AL 29 & $9.29 \mathrm{E}-05$ & $3.83 \mathrm{E}-06$ & $1.31 \mathrm{E}-18$ & $0.00 \mathrm{E}+00$ & $0.00 \mathrm{E}+00$ & $0.00 \mathrm{E}+00$ & $0.00 \mathrm{E}+00$ & $0.00 \mathrm{E}+00$ & $0.00 \mathrm{E}+00$ \\
\hline $\mathrm{AL} 30$ & $8.99 \mathrm{E}-08$ & $0.00 \mathrm{E}+00$ & $0.00 \mathrm{E}+00$ & $0.00 \mathrm{E}+00$ & $0.00 \mathrm{E}+00$ & $0.00 \mathrm{E}+00$ & $0.00 \mathrm{E}+00$ & $0.00 \mathrm{E}+00$ & $0.00 \mathrm{E}+00$ \\
\hline SI 31 & $8.38 \mathrm{E}-04$ & $7.35 \mathrm{E}-04$ & $2.24 \mathrm{E}-04$ & $1.47 \mathrm{E}-06$ & $4.30 \mathrm{E}-23$ & $0.00 E+00$ & $0.00 E+00$ & $0.00 \mathrm{E}+00$ & $0.00 \mathrm{E}+00$ \\
\hline SI 32 & $2.06 \mathrm{E}-13$ & $2.06 \mathrm{E}-13$ & $2.06 \mathrm{E}-13$ & $2.06 \mathrm{E}-13$ & $2.06 \mathrm{E}-13$ & $2.06 \mathrm{E}-13$ & $2.06 \mathrm{E}-13$ & $2.06 \mathrm{E}-13$ & $2.06 \mathrm{E}-13$ \\
\hline P 32 & $1.56 \mathrm{E}-03$ & $1.56 \mathrm{E}-03$ & $1.55 \mathrm{E}-03$ & $1.49 \mathrm{E}-03$ & $1.11 \mathrm{E}-03$ & 7.93E-04 & $3.65 \mathrm{E}-04$ & $8.53 \mathrm{E}-05$ & $3.22 \mathrm{E}-11$ \\
\hline P 33 & $1.26 \mathrm{E}-07$ & $1.26 \mathrm{E}-07$ & $1.25 \mathrm{E}-07$ & $1.22 \mathrm{E}-07$ & $1.04 \mathrm{E}-07$ & $8.54 \mathrm{E}-08$ & $5.48 \mathrm{E}-08$ & $2.38 \mathrm{E}-08$ & $5.03 \mathrm{E}-12$ \\
\hline P 34 & $7.29 \mathrm{E}-07$ & $0.00 \mathrm{E}+00$ & $0.00 \mathrm{E}+00$ & $0.00 \mathrm{E}+00$ & $0.00 \mathrm{E}+00$ & $0.00 \mathrm{E}+00$ & $0.00 \mathrm{E}+00$ & $0.00 \mathrm{E}+00$ & $0.00 \mathrm{E}+00$ \\
\hline S 35 & $5.08 \mathrm{E}-05$ & $5.08 \mathrm{E}-05$ & 5.07E-05 & 5.04E-05 & $4.81 \mathrm{E}-05$ & 4.55E-05 & 4.01E-05 & 3.17E-05 & $2.86 \mathrm{E}-06$ \\
\hline S 37 & $5.22 \mathrm{E}-07$ & 8.57E-09 & $7.42 \mathrm{E}-25$ & $0.00 \mathrm{E}+00$ & $0.00 \mathrm{E}+00$ & $0.00 \mathrm{E}+00$ & $0.00 \mathrm{E}+00$ & $0.00 \mathrm{E}+00$ & $0.00 \mathrm{E}+00$ \\
\hline $\mathrm{CL} 36$ & $2.89 \mathrm{E}-10$ & $2.89 \mathrm{E}-10$ & $2.89 \mathrm{E}-10$ & $2.89 \mathrm{E}-10$ & $2.89 \mathrm{E}-10$ & $2.89 \mathrm{E}-10$ & $2.89 \mathrm{E}-10$ & $2.89 \mathrm{E}-10$ & $2.89 \mathrm{E}-10$ \\
\hline CL 38 & $2.79 \mathrm{E}-09$ & $1.60 \mathrm{E}-09$ & $1.05 \mathrm{E}-11$ & $6.28 \mathrm{E}-21$ & $0.00 \mathrm{E}+00$ & $0.00 \mathrm{E}+00$ & $0.00 E+00$ & $0.00 \mathrm{E}+00$ & $0.00 \mathrm{E}+00$ \\
\hline $\mathrm{CL} 38 \mathrm{M}$ & $3.32 \mathrm{E}-11$ & $0.00 \mathrm{E}+00$ & $0.00 \mathrm{E}+00$ & $0.00 \mathrm{E}+00$ & $0.00 \mathrm{E}+00$ & $0.00 \mathrm{E}+00$ & $0.00 \mathrm{E}+00$ & $0.00 \mathrm{E}+00$ & $0.00 \mathrm{E}+00$ \\
\hline AR 37 & $3.82 \mathrm{E}-13$ & $3.82 \mathrm{E}-13$ & $3.81 \mathrm{E}-13$ & $3.75 \mathrm{E}-13$ & $3.33 \mathrm{E}-13$ & $2.90 \mathrm{E}-13$ & $2.11 \mathrm{E}-13$ & $1.17 \mathrm{E}-13$ & $2.77 \mathrm{E}-16$ \\
\hline AR 39 & $9.25 \mathrm{E}-07$ & $9.25 \mathrm{E}-07$ & $9.25 \mathrm{E}-07$ & $9.25 \mathrm{E}-07$ & $9.25 \mathrm{E}-07$ & $9.25 \mathrm{E}-07$ & $9.25 \mathrm{E}-07$ & $9.24 \mathrm{E}-07$ & $9.22 \mathrm{E}-07$ \\
\hline AR 41 & $3.41 \mathrm{E}-07$ & $2.82 \mathrm{E}-07$ & $5.12 \mathrm{E}-08$ & $3.79 \mathrm{E}-11$ & $0.00 \mathrm{E}+00$ & $0.00 \mathrm{E}+00$ & $0.00 \mathrm{E}+00$ & $0.00 \mathrm{E}+00$ & $0.00 \mathrm{E}+00$ \\
\hline $\mathrm{K} 40$ & $2.27 \mathrm{E}-12$ & $2.27 \mathrm{E}-12$ & $2.27 \mathrm{E}-12$ & $2.27 \mathrm{E}-12$ & $2.27 \mathrm{E}-12$ & $2.27 \mathrm{E}-12$ & 2.27E-12 & $2.27 \mathrm{E}-12$ & $2.27 \mathrm{E}-12$ \\
\hline $\mathrm{K} 42$ & 7.57E-04 & $7.36 \mathrm{E}-04$ & $5.72 \mathrm{E}-04$ & 1.97E-04 & $6.14 \mathrm{E}-08$ & $4.98 \mathrm{E}-12$ & $4.21 \mathrm{E}-16$ & $4.20 \mathrm{E}-16$ & $4.13 \mathrm{E}-16$ \\
\hline K 43 & $9.73 \mathrm{E}-10$ & $9.59 \mathrm{E}-10$ & $8.35 \mathrm{E}-10$ & $4.66 \mathrm{E}-10$ & $5.63 \mathrm{E}-12$ & $3.26 \mathrm{E}-14$ & $2.50 \mathrm{E}-19$ & $6.42 \mathrm{E}-29$ & $0.00 \mathrm{E}+00$ \\
\hline K 44 & $1.72 \mathrm{E}-10$ & $6.69 \mathrm{E}-11$ & $1.35 \mathrm{E}-14$ & $0.00 \mathrm{E}+00$ & $0.00 \mathrm{E}+00$ & $0.00 \mathrm{E}+00$ & $0.00 \mathrm{E}+00$ & $0.00 \mathrm{E}+00$ & $0.00 \mathrm{E}+00$ \\
\hline CA 45 & $4.69 \mathrm{E}-06$ & $4.69 \mathrm{E}-06$ & 4.69E-06 & 4.67E-06 & $4.56 \mathrm{E}-06$ & $4.42 \mathrm{E}-06$ & $4.13 \mathrm{E}-06$ & $3.64 \mathrm{E}-06$ & 9.93E-07 \\
\hline CA 47 & 7.79E-08 & $7.76 \mathrm{E}-08$ & $7.54 \mathrm{E}-08$ & $6.68 \mathrm{E}-08$ & 2.67E-08 & 9.17E-09 & $7.95 \mathrm{E}-10$ & $8.12 \mathrm{E}-12$ & $0.00 \mathrm{E}+00$ \\
\hline SC 46 & $9.34 \mathrm{E}-04$ & $9.34 \mathrm{E}-04$ & $9.32 \mathrm{E}-04$ & $9.26 \mathrm{E}-04$ & $8.81 \mathrm{E}-04$ & $8.32 \mathrm{E}-04$ & 7.29E-04 & $5.68 \mathrm{E}-04$ & $4.55 \mathrm{E}-05$ \\
\hline SC 46M & $2.66 \mathrm{E}-08$ & $0.00 \mathrm{E}+00$ & $0.00 \mathrm{E}+00$ & $0.00 \mathrm{E}+00$ & $0.00 \mathrm{E}+00$ & $0.00 \mathrm{E}+00$ & $0.00 \mathrm{E}+00$ & $0.00 \mathrm{E}+00$ & $0.00 \mathrm{E}+00$ \\
\hline SC 47 & $2.32 \mathrm{E}-03$ & $2.31 \mathrm{E}-03$ & $2.22 \mathrm{E}-03$ & $1.89 \mathrm{E}-03$ & $5.46 \mathrm{E}-04$ & $1.28 \mathrm{E}-04$ & $4.69 \mathrm{E}-06$ & $9.48 \mathrm{E}-09$ & $0.00 \mathrm{E}+00$ \\
\hline SC 48 & 2.17E-04 & $2.15 \mathrm{E}-04$ & $2.01 \mathrm{E}-04$ & $1.48 \mathrm{E}-04$ & $1.52 \mathrm{E}-05$ & 1.07E-06 & 2.45E-09 & $2.76 \mathrm{E}-14$ & $0.00 \mathrm{E}+00$ \\
\hline SC 49 & $1.96 \mathrm{E}-04$ & $1.37 \mathrm{E}-04$ & $5.27 \mathrm{E}-06$ & 5.67E-12 & $0.00 \mathrm{E}+00$ & $0.00 \mathrm{E}+00$ & $0.00 \mathrm{E}+00$ & $0.00 \mathrm{E}+00$ & $0.00 \mathrm{E}+00$ \\
\hline SC 50 & $3.68 \mathrm{E}-06$ & $1.91 \mathrm{E}-11$ & $0.00 \mathrm{E}+00$ & $0.00 \mathrm{E}+00$ & $0.00 \mathrm{E}+00$ & $0.00 \mathrm{E}+00$ & $0.00 \mathrm{E}+00$ & $0.00 \mathrm{E}+00$ & $0.00 \mathrm{E}+00$ \\
\hline TI 51 & $4.21 \mathrm{E}-03$ & $1.14 \mathrm{E}-04$ & $8.82 \mathrm{E}-19$ & $0.00 \mathrm{E}+00$ & $0.00 \mathrm{E}+00$ & $0.00 \mathrm{E}+00$ & $0.00 \mathrm{E}+00$ & $0.00 \mathrm{E}+00$ & $0.00 \mathrm{E}+00$ \\
\hline V 52 & $4.93 \mathrm{E}-02$ & $1.93 \mathrm{E}-04$ & $4.08 \mathrm{E}-26$ & $0.00 \mathrm{E}+00$ & $0.00 \mathrm{E}+00$ & $0.00 \mathrm{E}+00$ & $0.00 \mathrm{E}+00$ & $0.00 \mathrm{E}+00$ & $0.00 \mathrm{E}+00$ \\
\hline V 53 & $2.27 \mathrm{E}-04$ & $5.59 \mathrm{E}-10$ & $0.00 \mathrm{E}+00$ & $0.00 \mathrm{E}+00$ & $0.00 \mathrm{E}+00$ & $0.00 \mathrm{E}+00$ & $0.00 \mathrm{E}+00$ & $0.00 \mathrm{E}+00$ & $0.00 \mathrm{E}+00$ \\
\hline V 54 & $2.43 \mathrm{E}-06$ & $3.42 \mathrm{E}-16$ & $0.00 \mathrm{E}+00$ & $0.00 E+00$ & $0.00 \mathrm{E}+00$ & $0.00 \mathrm{E}+00$ & $0.00 E+00$ & $0.00 \mathrm{E}+00$ & $0.00 E+00$ \\
\hline CR 51 & $4.66 \mathrm{E}+00$ & $4.65 E+00$ & $4.63 E+00$ & $4.54 \mathrm{E}+00$ & $3.91 \mathrm{E}+00$ & $3.28 \mathrm{E}+00$ & $2.20 \mathrm{E}+00$ & $1.04 \mathrm{E}+00$ & $5.01 \mathrm{E}-04$ \\
\hline
\end{tabular}


TEM-10200-1

ENGINEERING CALCULATIONS AND ANALYSIS

Page 56 of 81

$12 / 19 / 17$

Rev.08

Title:

AS-RUN NEUTRONICS EVALUATION FOR THE CSM-10584 EXPERIMENT IN THE ATR

\begin{tabular}{llllllll} 
ECAR NO.: & 4496 & REV. NO.: & 1 & PROJECT NO.: & 32501 & Date: & 10/19/2020 \\
\hline
\end{tabular}

\begin{tabular}{|c|c|c|c|c|c|c|c|c|c|}
\hline \multicolumn{10}{|c|}{1 gram of inconel irradiated for 119 EFPDs } \\
\hline & EOC & $30 \mathrm{MINs}$ & $5 \mathrm{HRS}$ & $1 \mathrm{DAY}$ & 7 DAYS & 14 DAYS & 30 DAYS & 60 DAYS & 1 YEAR \\
\hline & $\mathrm{Ci} / \mathrm{g}$ & $\mathrm{Ci} / \mathrm{g}$ & $\mathrm{Ci} / \mathrm{g}$ & $\mathrm{Ci} / \mathrm{g}$ & $\mathrm{Ci} / \mathrm{g}$ & $\mathrm{Ci} / \mathrm{g}$ & $\mathrm{Ci} / \mathrm{g}$ & $\mathrm{Ci} / \mathrm{g}$ & $\mathrm{Ci} / \mathrm{g}$ \\
\hline CR 55 & $6.69 \mathrm{E}-02$ & $1.91 \mathrm{E}-04$ & $0.00 \mathrm{E}+00$ & $0.00 \mathrm{E}+00$ & $0.00 \mathrm{E}+00$ & $0.00 \mathrm{E}+00$ & $0.00 \mathrm{E}+00$ & $0.00 \mathrm{E}+00$ & $0.00 \mathrm{E}+00$ \\
\hline MN 54 & 1.04E-02 & 1.04E-02 & 1.03E-02 & 1.03E-02 & $1.02 \mathrm{E}-02$ & $1.00 \mathrm{E}-02$ & $9.68 \mathrm{E}-03$ & $9.06 \mathrm{E}-03$ & $4.60 \mathrm{E}-03$ \\
\hline MN 56 & $2.12 \mathrm{E}+00$ & $1.85 \mathrm{E}+00$ & $5.52 \mathrm{E}-01$ & $3.34 \mathrm{E}-03$ & $5.16 \mathrm{E}-20$ & $0.00 \mathrm{E}+00$ & $0.00 \mathrm{E}+00$ & $0.00 \mathrm{E}+00$ & $0.00 E+00$ \\
\hline MN 57 & $1.15 \mathrm{E}-04$ & $2.82 \mathrm{E}-10$ & $0.00 \mathrm{E}+00$ & $0.00 \mathrm{E}+00$ & $0.00 \mathrm{E}+00$ & $0.00 \mathrm{E}+00$ & $0.00 \mathrm{E}+00$ & $0.00 \mathrm{E}+00$ & $0.00 \mathrm{E}+00$ \\
\hline MN 58 & $3.64 \mathrm{E}-07$ & $1.83 \mathrm{E}-15$ & $0.00 \mathrm{E}+00$ & $0.00 \mathrm{E}+00$ & $0.00 \mathrm{E}+00$ & $0.00 \mathrm{E}+00$ & $0.00 \mathrm{E}+00$ & $0.00 \mathrm{E}+00$ & $0.00 \mathrm{E}+00$ \\
\hline FE 55 & $5.29 \mathrm{E}-02$ & $5.29 \mathrm{E}-02$ & $5.29 \mathrm{E}-02$ & $5.29 \mathrm{E}-02$ & $5.26 \mathrm{E}-02$ & $5.24 \mathrm{E}-02$ & $5.18 \mathrm{E}-02$ & $5.06 \mathrm{E}-02$ & $4.05 \mathrm{E}-02$ \\
\hline FE 59 & $1.22 \mathrm{E}-02$ & $1.22 \mathrm{E}-02$ & $1.22 \mathrm{E}-02$ & $1.20 \mathrm{E}-02$ & $1.10 \mathrm{E}-02$ & $9.85 \mathrm{E}-03$ & $7.70 \mathrm{E}-03$ & $4.85 \mathrm{E}-03$ & $4.40 \mathrm{E}-05$ \\
\hline CO 58 & $5.86 \mathrm{E}-01$ & 5.86E-01 & $5.84 \mathrm{E}-01$ & $5.80 \mathrm{E}-01$ & 5.47E-01 & $5.11 \mathrm{E}-01$ & 4.37E-01 & $3.25 \mathrm{E}-01$ & $1.64 \mathrm{E}-02$ \\
\hline $\mathrm{CO} 60$ & $6.44 \mathrm{E}-01$ & $6.44 \mathrm{E}-01$ & $6.44 \mathrm{E}-01$ & $6.44 \mathrm{E}-01$ & $6.43 \mathrm{E}-01$ & $6.41 \mathrm{E}-01$ & $6.37 \mathrm{E}-01$ & $6.30 \mathrm{E}-01$ & $5.65 \mathrm{E}-01$ \\
\hline CO 60M & $8.09 \mathrm{E}+00$ & $1.11 \mathrm{E}+00$ & $1.92 \mathrm{E}-08$ & $0.00 \mathrm{E}+00$ & $0.00 \mathrm{E}+00$ & $0.00 \mathrm{E}+00$ & $0.00 \mathrm{E}+00$ & $0.00 \mathrm{E}+00$ & $0.00 \mathrm{E}+00$ \\
\hline $\mathrm{CO} 61$ & $5.00 \mathrm{E}-02$ & $4.05 \mathrm{E}-02$ & $6.12 \mathrm{E}-03$ & $2.09 \mathrm{E}-06$ & $0.00 \mathrm{E}+00$ & $0.00 \mathrm{E}+00$ & $0.00 \mathrm{E}+00$ & $0.00 \mathrm{E}+00$ & $0.00 \mathrm{E}+00$ \\
\hline $\mathrm{CO} 62$ & $2.08 \mathrm{E}-04$ & $1.98 \mathrm{E}-10$ & $0.00 \mathrm{E}+00$ & $0.00 \mathrm{E}+00$ & $0.00 \mathrm{E}+00$ & $0.00 \mathrm{E}+00$ & $0.00 \mathrm{E}+00$ & $0.00 \mathrm{E}+00$ & $0.00 \mathrm{E}+00$ \\
\hline NI 59 & 1.57E-04 & 1.57E-04 & 1.57E-04 & 1.57E-04 & 1.57E-04 & 1.57E-04 & 1.57E-04 & 1.57E-04 & 1.57E-04 \\
\hline NI 63 & $2.04 \mathrm{E}-02$ & $2.04 \mathrm{E}-02$ & $2.04 \mathrm{E}-02$ & $2.04 \mathrm{E}-02$ & $2.04 \mathrm{E}-02$ & $2.04 \mathrm{E}-02$ & $2.04 \mathrm{E}-02$ & $2.04 \mathrm{E}-02$ & $2.03 \mathrm{E}-02$ \\
\hline NI 65 & $2.42 \mathrm{E}-01$ & $2.11 \mathrm{E}-01$ & $6.11 \mathrm{E}-02$ & $3.28 \mathrm{E}-04$ & $2.06 \mathrm{E}-21$ & $0.00 \mathrm{E}+00$ & $0.00 E+00$ & $0.00 \mathrm{E}+00$ & $0.00 \mathrm{E}+00$ \\
\hline NI 66 & $8.28 \mathrm{E}-06$ & $8.23 \mathrm{E}-06$ & $7.77 \mathrm{E}-06$ & $6.11 \mathrm{E}-06$ & $9.82 \mathrm{E}-07$ & $1.16 \mathrm{E}-07$ & $8.90 \mathrm{E}-10$ & $9.56 \mathrm{E}-14$ & $0.00 \mathrm{E}+00$ \\
\hline CU 64 & $3.02 \mathrm{E}-01$ & $2.94 \mathrm{E}-01$ & $2.30 \mathrm{E}-01$ & $8.16 \mathrm{E}-02$ & $3.15 \mathrm{E}-05$ & $3.28 \mathrm{E}-09$ & $2.59 \mathrm{E}-18$ & $0.00 \mathrm{E}+00$ & $0.00 \mathrm{E}+00$ \\
\hline CU 66 & $6.60 \mathrm{E}-02$ & $1.13 \mathrm{E}-03$ & $7.78 \mathrm{E}-06$ & $6.12 \mathrm{E}-06$ & $9.83 \mathrm{E}-07$ & 1.17E-07 & $8.91 \mathrm{E}-10$ & 9.57E-14 & $0.00 \mathrm{E}+00$ \\
\hline CU 67 & 4.27E-07 & $4.24 \mathrm{E}-07$ & $4.03 \mathrm{E}-07$ & $3.26 \mathrm{E}-07$ & $6.50 \mathrm{E}-08$ & $9.89 \mathrm{E}-09$ & $1.34 \mathrm{E}-10$ & $4.20 \mathrm{E}-14$ & $0.00 \mathrm{E}+00$ \\
\hline ZN 65 & $2.01 \mathrm{E}-05$ & $2.01 \mathrm{E}-05$ & $2.01 \mathrm{E}-05$ & $2.01 \mathrm{E}-05$ & 1.97E-05 & $1.93 \mathrm{E}-05$ & $1.85 \mathrm{E}-05$ & $1.70 \mathrm{E}-05$ & $7.12 \mathrm{E}-06$ \\
\hline ZN 69 & $3.08 \mathrm{E}-10$ & $2.20 \mathrm{E}-10$ & $2.48 \mathrm{E}-11$ & $6.64 \mathrm{E}-12$ & $4.70 \mathrm{E}-15$ & $9.94 \mathrm{E}-19$ & $0.00 \mathrm{E}+00$ & $0.00 \mathrm{E}+00$ & $0.00 \mathrm{E}+00$ \\
\hline ZN 69M & 2.07E-11 & $2.02 \mathrm{E}-11$ & $1.61 \mathrm{E}-11$ & $6.19 \mathrm{E}-12$ & $4.38 \mathrm{E}-15$ & $9.25 \mathrm{E}-19$ & $3.68 \mathrm{E}-27$ & $0.00 \mathrm{E}+00$ & $0.00 \mathrm{E}+00$ \\
\hline GA 70 & $4.88 \mathrm{E}-13$ & $1.82 \mathrm{E}-13$ & $2.55 \mathrm{E}-17$ & $0.00 \mathrm{E}+00$ & $0.00 \mathrm{E}+00$ & $0.00 \mathrm{E}+00$ & $0.00 \mathrm{E}+00$ & $0.00 \mathrm{E}+00$ & $0.00 \mathrm{E}+00$ \\
\hline SR 89 & $3.74 \mathrm{E}-10$ & $3.74 \mathrm{E}-10$ & $3.73 \mathrm{E}-10$ & $3.69 \mathrm{E}-10$ & $3.40 \mathrm{E}-10$ & $3.09 \mathrm{E}-10$ & $2.48 \mathrm{E}-10$ & $1.64 \mathrm{E}-10$ & 2.49E-12 \\
\hline SR 90 & 1.49E-12 & 1.49E-12 & 1.49E-12 & 1.49E-12 & 1.49E-12 & 1.49E-12 & 1.49E-12 & 1.49E-12 & $1.46 \mathrm{E}-12$ \\
\hline SR 91 & $1.41 \mathrm{E}-11$ & $1.36 \mathrm{E}-11$ & $9.76 \mathrm{E}-12$ & $2.44 \mathrm{E}-12$ & 6.67E-17 & 3.17E-22 & $0.00 \mathrm{E}+00$ & $0.00 \mathrm{E}+00$ & $0.00 \mathrm{E}+00$ \\
\hline Y 89M & 9.31E-06 & 9.27E-06 & $8.91 \mathrm{E}-06$ & 7.53E-06 & 2.11E-06 & $4.78 \mathrm{E}-07$ & $1.61 \mathrm{E}-08$ & $2.78 \mathrm{E}-11$ & $0.00 \mathrm{E}+00$ \\
\hline Y 90 & 2.10E-04 & 2.09E-04 & 1.99E-04 & 1.62E-04 & 3.40E-05 & $5.51 \mathrm{E}-06$ & $8.61 \mathrm{E}-08$ & $3.68 \mathrm{E}-11$ & $1.46 \mathrm{E}-12$ \\
\hline Y 90M & $1.09 \mathrm{E}-11$ & $9.72 \mathrm{E}-12$ & $3.56 \mathrm{E}-12$ & $5.08 \mathrm{E}-14$ & $5.28 \mathrm{E}-28$ & $0.00 \mathrm{E}+00$ & $0.00 \mathrm{E}+00$ & $0.00 \mathrm{E}+00$ & $0.00 \mathrm{E}+00$ \\
\hline Y 91 & 2.64E-08 & 2.64E-08 & 2.63E-08 & $2.61 \mathrm{E}-08$ & $2.43 \mathrm{E}-08$ & $2.23 \mathrm{E}-08$ & $1.85 \mathrm{E}-08$ & $1.30 \mathrm{E}-08$ & $3.48 \mathrm{E}-10$ \\
\hline Y92 & 1.70E-09 & 1.54E-09 & $6.38 \mathrm{E}-10$ & $1.54 \mathrm{E}-11$ & 8.69E-24 & $0.00 \mathrm{E}+00$ & $0.00 \mathrm{E}+00$ & $0.00 \mathrm{E}+00$ & $0.00 \mathrm{E}+00$ \\
\hline Y 94 & $9.91 \mathrm{E}-12$ & 3.34E-12 & 1.85E-16 & $0.00 \mathrm{E}+00$ & $0.00 \mathrm{E}+00$ & $0.00 \mathrm{E}+00$ & $0.00 \mathrm{E}+00$ & $0.00 \mathrm{E}+00$ & $0.00 \mathrm{E}+00$ \\
\hline ZR 89 & 9.33E-06 & $9.29 \mathrm{E}-06$ & 8.93E-06 & 7.55E-06 & $2.11 \mathrm{E}-06$ & 4.79E-07 & $1.61 \mathrm{E}-08$ & $2.78 \mathrm{E}-11$ & $0.00 \mathrm{E}+00$ \\
\hline ZR 93 & $3.05 \mathrm{E}-10$ & $3.05 \mathrm{E}-10$ & $3.05 \mathrm{E}-10$ & $3.05 \mathrm{E}-10$ & $3.05 \mathrm{E}-10$ & 3.05E-10 & 3.05E-10 & $3.05 \mathrm{E}-10$ & $3.05 \mathrm{E}-10$ \\
\hline ZR 95 & $2.33 \mathrm{E}-05$ & $2.33 \mathrm{E}-05$ & $2.33 \mathrm{E}-05$ & $2.31 \mathrm{E}-05$ & $2.16 \mathrm{E}-05$ & $2.01 \mathrm{E}-05$ & 1.69E-05 & $1.22 \mathrm{E}-05$ & $4.46 \mathrm{E}-07$ \\
\hline ZR 97 & 7.37E-07 & $7.22 \mathrm{E}-07$ & $6.01 \mathrm{E}-07$ & $2.76 \mathrm{E}-07$ & $7.49 \mathrm{E}-10$ & 7.59E-13 & $1.10 \mathrm{E}-19$ & $0.00 \mathrm{E}+00$ & $0.00 \mathrm{E}+00$ \\
\hline NB 92 & $1.86 \mathrm{E}-03$ & $1.85 \mathrm{E}-03$ & $1.83 \mathrm{E}-03$ & $1.73 \mathrm{E}-03$ & 1.15E-03 & 7.14E-04 & $2.40 \mathrm{E}-04$ & $3.10 \mathrm{E}-05$ & $2.80 \mathrm{E}-14$ \\
\hline NB 93M & $2.40 \mathrm{E}-12$ & $2.40 \mathrm{E}-12$ & $2.41 \mathrm{E}-12$ & 2.44E-12 & $2.68 \mathrm{E}-12$ & $2.96 \mathrm{E}-12$ & 3.60E-12 & 4.80E-12 & 1.67E-11 \\
\hline NB 94 & 5.03E-05 & $5.03 \mathrm{E}-05$ & $5.03 \mathrm{E}-05$ & $5.03 \mathrm{E}-05$ & 5.03E-05 & 5.03E-05 & 5.03E-05 & 5.03E-05 & 5.03E-05 \\
\hline NB 95 & $1.45 \mathrm{E}-01$ & $1.45 \mathrm{E}-01$ & $1.45 \mathrm{E}-01$ & $1.42 \mathrm{E}-01$ & 1.27E-01 & 1.10E-01 & 8.04E-02 & 4.45E-02 & $1.09 \mathrm{E}-04$ \\
\hline NB 95M & 1.60E-07 & 1.60E-07 & 1.60E-07 & 1.60E-07 & 1.57E-07 & $1.48 \mathrm{E}-07$ & $1.25 \mathrm{E}-07$ & 9.04E-08 & $3.31 \mathrm{E}-09$ \\
\hline NB 96 & $7.15 \mathrm{E}-04$ & $7.05 \mathrm{E}-04$ & $6.17 \mathrm{E}-04$ & $3.51 \mathrm{E}-04$ & $4.88 \mathrm{E}-06$ & $3.33 \mathrm{E}-08$ & $3.74 \mathrm{E}-13$ & $1.95 \mathrm{E}-22$ & $0.00 \mathrm{E}+00$ \\
\hline NB 97 & $2.62 \mathrm{E}-05$ & $1.98 \mathrm{E}-05$ & $2.06 \mathrm{E}-06$ & $2.77 \mathrm{E}-07$ & $7.54 \mathrm{E}-10$ & $7.66 \mathrm{E}-13$ & $1.18 \mathrm{E}-19$ & $0.00 \mathrm{E}+00$ & $0.00 \mathrm{E}+00$ \\
\hline NB 97M & $6.98 \mathrm{E}-07$ & $6.84 \mathrm{E}-07$ & $5.69 \mathrm{E}-07$ & $2.61 \mathrm{E}-07$ & $7.11 \mathrm{E}-10$ & $7.22 \mathrm{E}-13$ & 1.04E-19 & $0.00 \mathrm{E}+00$ & $0.00 \mathrm{E}+00$ \\
\hline NB 98 & $3.90 \mathrm{E}-06$ & $0.00 \mathrm{E}+00$ & $0.00 \mathrm{E}+00$ & $0.00 \mathrm{E}+00$ & $0.00 \mathrm{E}+00$ & $0.00 \mathrm{E}+00$ & $0.00 \mathrm{E}+00$ & $0.00 \mathrm{E}+00$ & $0.00 \mathrm{E}+00$ \\
\hline NB100 & 1.94E-07 & $0.00 \mathrm{E}+00$ & $0.00 \mathrm{E}+00$ & $0.00 \mathrm{E}+00$ & $0.00 \mathrm{E}+00$ & $0.00 \mathrm{E}+00$ & $0.00 \mathrm{E}+00$ & $0.00 \mathrm{E}+00$ & $0.00 \mathrm{E}+00$ \\
\hline MO 93M & $5.39 \mathrm{E}-04$ & $5.13 \mathrm{E}-04$ & $3.25 \mathrm{E}-04$ & $4.75 \mathrm{E}-05$ & $2.23 \mathrm{E}-11$ & $9.24 \mathrm{E}-19$ & $0.00 \mathrm{E}+00$ & $0.00 \mathrm{E}+00$ & $0.00 E+00$ \\
\hline MO 93 & 1.07E-06 & $1.07 \mathrm{E}-06$ & 1.07E-06 & 1.07E-06 & 1.07E-06 & 1.07E-06 & 1.07E-06 & 1.07E-06 & 1.07E-06 \\
\hline MO 99 & 3.77E-01 & $3.75 \mathrm{E}-01$ & $3.58 \mathrm{E}-01$ & $2.93 \mathrm{E}-01$ & $6.46 \mathrm{E}-02$ & $1.11 \mathrm{E}-02$ & 1.96E-04 & $1.02 \mathrm{E}-07$ & $0.00 \mathrm{E}+00$ \\
\hline M0101 & $9.38 \mathrm{E}-02$ & $2.26 \mathrm{E}-02$ & $6.24 \mathrm{E}-08$ & $0.00 \mathrm{E}+00$ & $0.00 \mathrm{E}+00$ & $0.00 \mathrm{E}+00$ & $0.00 \mathrm{E}+00$ & $0.00 \mathrm{E}+00$ & $0.00 E+00$ \\
\hline TC 99 & $4.56 \mathrm{E}-08$ & $4.56 \mathrm{E}-08$ & 4.57E-08 & $4.60 \mathrm{E}-08$ & 4.70E-08 & $4.72 \mathrm{E}-08$ & $4.73 \mathrm{E}-08$ & $4.73 \mathrm{E}-08$ & $4.73 \mathrm{E}-08$ \\
\hline TC100 & $4.77 \mathrm{E}-03$ & $0.00 \mathrm{E}+00$ & $0.00 \mathrm{E}+00$ & $0.00 \mathrm{E}+00$ & $0.00 \mathrm{E}+00$ & $0.00 \mathrm{E}+00$ & $0.00 \mathrm{E}+00$ & $0.00 \mathrm{E}+00$ & $0.00 E+00$ \\
\hline TC101 & $9.38 \mathrm{E}-02$ & $5.42 \mathrm{E}-02$ & 7.87E-07 & $0.00 \mathrm{E}+00$ & $0.00 \mathrm{E}+00$ & $0.00 \mathrm{E}+00$ & $0.00 \mathrm{E}+00$ & $0.00 \mathrm{E}+00$ & $0.00 E+00$ \\
\hline RU103 & $2.34 \mathrm{E}-06$ & $2.34 \mathrm{E}-06$ & $2.33 \mathrm{E}-06$ & $2.30 \mathrm{E}-06$ & 2.07E-06 & $1.83 \mathrm{E}-06$ & $1.38 \mathrm{E}-06$ & $8.13 \mathrm{E}-07$ & $3.72 \mathrm{E}-09$ \\
\hline RU105 & $3.14 \mathrm{E}-11$ & $2.90 \mathrm{E}-11$ & $1.44 \mathrm{E}-11$ & $7.39 \mathrm{E}-13$ & 1.27E-22 & $0.00 \mathrm{E}+00$ & $0.00 \mathrm{E}+00$ & $0.00 \mathrm{E}+00$ & $0.00 E+00$ \\
\hline
\end{tabular}


TEM-10200-1

ENGINEERING CALCULATIONS AND ANALYSIS

Page 57 of 81

$12 / 19 / 17$

Rev.08

Title:

AS-RUN NEUTRONICS EVALUATION FOR THE CSM-10584 EXPERIMENT IN THE ATR

\begin{tabular}{llllllll} 
ECAR NO.: & 4496 & REV. NO.: & 1 & PROJECT NO.: & 32501 & Date: & 10/19/2020 \\
\hline
\end{tabular}

\begin{tabular}{|c|c|c|c|c|c|c|c|c|c|}
\hline \multicolumn{9}{|c|}{1 gram of inconel irradiated for 119 EFPDs } \\
\hline & EOC & $30 \mathrm{MINs}$ & $5 \mathrm{HRS}$ & $1 \mathrm{DAY}$ & $7 \mathrm{DAYS}$ & $14 \mathrm{DAYS}$ & 30 DAYS & 60 DAYS & 1 YEAR \\
\hline & $\mathrm{Ci} / \mathrm{g}$ & $\mathrm{Ci} / \mathrm{g}$ & $\mathrm{Ci} / \mathrm{g}$ & Ci/g & $\mathrm{Ci} / \mathrm{g}$ & $\mathrm{Ci} / \mathrm{g}$ & $\mathrm{Ci} / \mathrm{g}$ & $\mathrm{Ci} / \mathrm{g}$ & $\mathrm{Ci} / \mathrm{g}$ \\
\hline RH104 & $2.77 \mathrm{E}-08$ & $1.99 \mathrm{E}-11$ & $0.00 \mathrm{E}+00$ & $0.00 \mathrm{E}+00$ & $0.00 \mathrm{E}+00$ & $0.00 \mathrm{E}+00$ & $0.00 \mathrm{E}+00$ & $0.00 \mathrm{E}+00$ & $0.00 \mathrm{E}+00$ \\
\hline RH104M & $2.01 \mathrm{E}-09$ & $1.66 \mathrm{E}-11$ & $0.00 \mathrm{E}+00$ & $0.00 \mathrm{E}+00$ & $0.00 \mathrm{E}+00$ & $0.00 \mathrm{E}+00$ & $0.00 \mathrm{E}+00$ & $0.00 \mathrm{E}+00$ & $0.00 \mathrm{E}+00$ \\
\hline RH105 & $2.37 \mathrm{E}-11$ & $2.37 \mathrm{E}-11$ & $2.35 \mathrm{E}-11$ & $1.75 \mathrm{E}-11$ & $1.05 \mathrm{E}-12$ & $3.89 \mathrm{E}-14$ & $2.09 \mathrm{E}-17$ & $1.55 \mathrm{E}-23$ & $0.00 \mathrm{E}+00$ \\
\hline RH105M & $8.78 \mathrm{E}-12$ & $8.14 \mathrm{E}-12$ & $4.03 \mathrm{E}-12$ & $2.08 \mathrm{E}-13$ & $3.56 \mathrm{E}-23$ & $0.00 \mathrm{E}+00$ & $0.00 \mathrm{E}+00$ & $0.00 \mathrm{E}+00$ & $0.00 \mathrm{E}+00$ \\
\hline RH106 & $5.22 \mathrm{E}-12$ & $1.12 \mathrm{E}-17$ & $1.12 \mathrm{E}-17$ & $1.12 \mathrm{E}-17$ & $1.11 \mathrm{E}-17$ & $1.10 \mathrm{E}-17$ & $1.06 \mathrm{E}-17$ & $1.00 \mathrm{E}-17$ & $5.65 \mathrm{E}-18$ \\
\hline RH106M & $2.54 \mathrm{E}-12$ & $2.17 \mathrm{E}-12$ & $5.25 \mathrm{E}-13$ & $1.27 \mathrm{E}-15$ & $0.00 \mathrm{E}+00$ & $0.00 \mathrm{E}+00$ & $0.00 \mathrm{E}+00$ & $0.00 \mathrm{E}+00$ & $0.00 \mathrm{E}+00$ \\
\hline SUMTOT & $1.78 \mathrm{E}+01$ & $1.01 \mathrm{E}+01$ & $7.32 \mathrm{E}+00$ & $6.39 \mathrm{E}+00$ & $5.39 \mathrm{E}+00$ & $4.65 \mathrm{E}+00$ & $3.44 \mathrm{E}+00$ & $2.12 \mathrm{E}+00$ & $6.47 \mathrm{E}-01$ \\
\hline OTOTAL & $1.78 \mathrm{E}+01$ & $1.01 \mathrm{E}+01$ & $7.32 \mathrm{E}+00$ & $6.39 \mathrm{E}+00$ & $5.39 \mathrm{E}+00$ & $4.65 \mathrm{E}+00$ & $3.44 \mathrm{E}+00$ & $2.12 \mathrm{E}+00$ & $6.47 \mathrm{E}-01$ \\
\hline
\end{tabular}

\begin{tabular}{|c|c|c|c|c|c|c|c|c|c|}
\hline \multicolumn{10}{|c|}{1 gram of inconel irradiated for 54.9 EFPDs } \\
\hline & EOC & $30 \mathrm{MINs}$ & $5 \mathrm{HRS}$ & 1 DAY & 7 DAYS & 14 DAYS & 30 DAYS & 60 DAYS & 1 YEAR \\
\hline & $\begin{array}{l}\mathrm{g} / 1 \mathrm{~g} \text { of } \\
\text { inconel }\end{array}$ & $\begin{array}{l}\mathrm{g} / 1 \mathrm{~g} \text { of } \\
\text { inconel }\end{array}$ & $\begin{array}{l}\mathrm{g} / 1 \mathrm{~g} \text { of } \\
\text { inconel }\end{array}$ & $\begin{array}{l}\mathrm{g} / 1 \mathrm{~g} \text { of } \\
\text { inconel }\end{array}$ & $\begin{array}{l}\mathrm{g} / 1 \mathrm{~g} \text { of } \\
\text { inconel }\end{array}$ & $\begin{array}{l}\mathrm{g} / 1 \mathrm{~g} \text { of } \\
\text { inconel }\end{array}$ & $\begin{array}{l}\mathrm{g} / 1 \mathrm{~g} \text { of } \\
\text { inconel }\end{array}$ & $\begin{array}{l}\mathrm{g} / 1 \mathrm{~g} \text { of } \\
\text { inconel }\end{array}$ & $\begin{array}{l}\mathrm{g} / 1 \mathrm{~g} \text { of } \\
\text { inconel }\end{array}$ \\
\hline H 1 & $8.11 \mathrm{E}-07$ & $8.11 \mathrm{E}-07$ & $8.11 \mathrm{E}-07$ & $8.11 \mathrm{E}-07$ & $8.11 \mathrm{E}-07$ & $8.11 \mathrm{E}-07$ & $8.11 \mathrm{E}-07$ & $8.11 \mathrm{E}-07$ & $8.11 \mathrm{E}-07$ \\
\hline H 2 & $1.58 \mathrm{E}-10$ & $1.58 \mathrm{E}-10$ & $1.58 \mathrm{E}-10$ & $1.58 \mathrm{E}-10$ & $1.58 \mathrm{E}-10$ & $1.58 \mathrm{E}-10$ & $1.58 \mathrm{E}-10$ & $1.58 \mathrm{E}-10$ & $1.58 \mathrm{E}-10$ \\
\hline H 3 & $6.38 \mathrm{E}-15$ & $6.38 \mathrm{E}-15$ & $6.38 \mathrm{E}-15$ & $6.38 \mathrm{E}-15$ & $6.38 \mathrm{E}-15$ & $6.37 \mathrm{E}-15$ & $6.36 \mathrm{E}-15$ & $6.33 \mathrm{E}-15$ & $6.04 \mathrm{E}-15$ \\
\hline HE 4 & $4.71 \mathrm{E}-06$ & 4.71E-06 & $4.71 \mathrm{E}-06$ & $4.71 \mathrm{E}-06$ & 4.71E-06 & $4.71 \mathrm{E}-06$ & $4.71 \mathrm{E}-06$ & $4.71 \mathrm{E}-06$ & $4.71 \mathrm{E}-06$ \\
\hline LI 6 & $7.94 \mathrm{E}-14$ & $7.94 \mathrm{E}-14$ & $7.94 \mathrm{E}-14$ & $7.94 \mathrm{E}-14$ & $7.94 \mathrm{E}-14$ & $7.94 \mathrm{E}-14$ & $7.94 \mathrm{E}-14$ & $7.94 \mathrm{E}-14$ & $7.94 \mathrm{E}-14$ \\
\hline LI 7 & $6.96 \mathrm{E}-06$ & $6.96 \mathrm{E}-06$ & $6.96 \mathrm{E}-06$ & $6.96 \mathrm{E}-06$ & $6.96 \mathrm{E}-06$ & $6.96 \mathrm{E}-06$ & $6.96 \mathrm{E}-06$ & $6.96 \mathrm{E}-06$ & $6.96 \mathrm{E}-06$ \\
\hline BE 9 & $1.29 \mathrm{E}-09$ & $1.29 \mathrm{E}-09$ & $1.29 \mathrm{E}-09$ & $1.29 \mathrm{E}-09$ & $1.29 \mathrm{E}-09$ & $1.29 \mathrm{E}-09$ & $1.29 \mathrm{E}-09$ & 1.29E-09 & $1.29 \mathrm{E}-09$ \\
\hline BE 10 & $9.44 \mathrm{E}-11$ & $9.44 \mathrm{E}-11$ & 9.44E-11 & $9.44 \mathrm{E}-11$ & $9.44 \mathrm{E}-11$ & 9.44E-11 & $9.44 \mathrm{E}-11$ & 9.44E-11 & $9.44 \mathrm{E}-11$ \\
\hline B 10 & $1.17 \mathrm{E}-06$ & 1.17E-06 & 1.17E-06 & $1.17 \mathrm{E}-06$ & 1.17E-06 & 1.17E-06 & 1.17E-06 & 1.17E-06 & $1.17 \mathrm{E}-06$ \\
\hline B 11 & $4.89 \mathrm{E}-05$ & 4.89E-05 & $4.89 \mathrm{E}-05$ & $4.89 \mathrm{E}-05$ & 4.89E-05 & $4.89 \mathrm{E}-05$ & $4.89 \mathrm{E}-05$ & $4.89 \mathrm{E}-05$ & $4.89 \mathrm{E}-05$ \\
\hline C 12 & $7.90 \mathrm{E}-04$ & $7.90 \mathrm{E}-04$ & $7.90 \mathrm{E}-04$ & $7.90 \mathrm{E}-04$ & $7.90 \mathrm{E}-04$ & $7.90 \mathrm{E}-04$ & $7.90 \mathrm{E}-04$ & $7.90 \mathrm{E}-04$ & $7.90 \mathrm{E}-04$ \\
\hline C 13 & $9.61 \mathrm{E}-06$ & 9.61E-06 & $9.61 \mathrm{E}-06$ & $9.61 \mathrm{E}-06$ & $9.61 \mathrm{E}-06$ & $9.61 \mathrm{E}-06$ & $9.61 \mathrm{E}-06$ & $9.61 \mathrm{E}-06$ & $9.61 \mathrm{E}-06$ \\
\hline C 14 & $6.07 \mathrm{E}-12$ & $6.07 \mathrm{E}-12$ & 6.07E-12 & $6.07 \mathrm{E}-12$ & $6.07 \mathrm{E}-12$ & $6.07 \mathrm{E}-12$ & 6.07E-12 & 6.07E-12 & $6.07 \mathrm{E}-12$ \\
\hline NE 21 & $8.76 \mathrm{E}-15$ & $8.76 \mathrm{E}-15$ & $8.76 \mathrm{E}-15$ & $8.76 \mathrm{E}-15$ & $8.76 \mathrm{E}-15$ & $8.76 \mathrm{E}-15$ & $8.76 \mathrm{E}-15$ & $8.76 \mathrm{E}-15$ & $8.76 \mathrm{E}-15$ \\
\hline NE 22 & $4.67 E-14$ & $4.67 \mathrm{E}-14$ & 4.67E-14 & $4.67 \mathrm{E}-14$ & $4.67 \mathrm{E}-14$ & $4.67 E-14$ & 4.67E-14 & 4.67E-14 & $4.67 \mathrm{E}-14$ \\
\hline NA 23 & $3.78 \mathrm{E}-15$ & $3.78 \mathrm{E}-15$ & $3.78 \mathrm{E}-15$ & $3.78 \mathrm{E}-15$ & $3.78 \mathrm{E}-15$ & $3.78 \mathrm{E}-15$ & $3.78 \mathrm{E}-15$ & $3.78 \mathrm{E}-15$ & $3.78 \mathrm{E}-15$ \\
\hline NA 24 & $1.30 \mathrm{E}-10$ & $1.27 \mathrm{E}-10$ & $1.03 \mathrm{E}-10$ & $4.29 \mathrm{E}-11$ & $5.52 \mathrm{E}-14$ & $2.35 \mathrm{E}-17$ & $4.62 \mathrm{E}-25$ & $0.00 \mathrm{E}+00$ & $0.00 \mathrm{E}+00$ \\
\hline MG 24 & $7.78 \mathrm{E}-09$ & 7.79E-09 & 7.81E-09 & 7.87E-09 & 7.91E-09 & 7.91E-09 & 7.91E-09 & 7.91E-09 & $7.91 \mathrm{E}-09$ \\
\hline MG 25 & $3.64 \mathrm{E}-09$ & $3.64 \mathrm{E}-09$ & $3.64 \mathrm{E}-09$ & $3.64 \mathrm{E}-09$ & $3.64 \mathrm{E}-09$ & $3.64 \mathrm{E}-09$ & $3.64 \mathrm{E}-09$ & $3.64 \mathrm{E}-09$ & $3.64 \mathrm{E}-09$ \\
\hline MG 26 & $1.66 \mathrm{E}-09$ & 1.66E-09 & 1.66E-09 & $1.66 \mathrm{E}-09$ & 1.66E-09 & 1.66E-09 & 1.66E-09 & $1.66 \mathrm{E}-09$ & $1.66 \mathrm{E}-09$ \\
\hline MG 27 & $7.35 \mathrm{E}-12$ & $8.16 \mathrm{E}-13$ & $2.10 \mathrm{E}-21$ & $0.00 \mathrm{E}+00$ & $0.00 \mathrm{E}+00$ & $0.00 \mathrm{E}+00$ & $0.00 \mathrm{E}+00$ & $0.00 \mathrm{E}+00$ & $0.00 \mathrm{E}+00$ \\
\hline AL 27 & $8.00 \mathrm{E}-03$ & $8.00 \mathrm{E}-03$ & $8.00 \mathrm{E}-03$ & $8.00 \mathrm{E}-03$ & $8.00 \mathrm{E}-03$ & $8.00 \mathrm{E}-03$ & $8.00 \mathrm{E}-03$ & $8.00 \mathrm{E}-03$ & $8.00 \mathrm{E}-03$ \\
\hline AL 28 & $4.42 \mathrm{E}-11$ & $4.11 \mathrm{E}-15$ & $5.45 \mathrm{E}-21$ & $2.90 \mathrm{E}-21$ & $2.45 \mathrm{E}-23$ & $9.53 \mathrm{E}-26$ & $2.83 \mathrm{E}-31$ & $0.00 \mathrm{E}+00$ & $0.00 \mathrm{E}+00$ \\
\hline AL 29 & $9.32 \mathrm{E}-14$ & $3.84 \mathrm{E}-15$ & $1.31 \mathrm{E}-27$ & $0.00 \mathrm{E}+00$ & $0.00 \mathrm{E}+00$ & $0.00 \mathrm{E}+00$ & $0.00 \mathrm{E}+00$ & $0.00 \mathrm{E}+00$ & $0.00 \mathrm{E}+00$ \\
\hline $\mathrm{SI} 28$ & $3.22 \mathrm{E}-03$ & $3.22 \mathrm{E}-03$ & $3.22 \mathrm{E}-03$ & $3.22 \mathrm{E}-03$ & $3.22 \mathrm{E}-03$ & $3.22 \mathrm{E}-03$ & $3.22 \mathrm{E}-03$ & $3.22 \mathrm{E}-03$ & $3.22 \mathrm{E}-03$ \\
\hline SI 29 & $1.69 \mathrm{E}-04$ & $1.69 \mathrm{E}-04$ & $1.69 \mathrm{E}-04$ & $1.69 \mathrm{E}-04$ & $1.69 \mathrm{E}-04$ & $1.69 \mathrm{E}-04$ & $1.69 \mathrm{E}-04$ & 1.69E-04 & $1.69 \mathrm{E}-04$ \\
\hline $\mathrm{SI} 30$ & $1.16 \mathrm{E}-04$ & 1.16E-04 & 1.16E-04 & $1.16 \mathrm{E}-04$ & 1.16E-04 & 1.16E-04 & 1.16E-04 & $1.16 \mathrm{E}-04$ & $1.16 \mathrm{E}-04$ \\
\hline SI 31 & $2.17 \mathrm{E}-11$ & $1.90 \mathrm{E}-11$ & $5.79 \mathrm{E}-12$ & $3.81 \mathrm{E}-14$ & $1.11 \mathrm{E}-30$ & $0.00 \mathrm{E}+00$ & $0.00 \mathrm{E}+00$ & $0.00 \mathrm{E}+00$ & $0.00 \mathrm{E}+00$ \\
\hline $\mathrm{SI} 32$ & $5.53 \mathrm{E}-15$ & $5.53 \mathrm{E}-15$ & $5.53 \mathrm{E}-15$ & $5.53 \mathrm{E}-15$ & $5.53 \mathrm{E}-15$ & $5.53 \mathrm{E}-15$ & $5.53 \mathrm{E}-15$ & $5.53 \mathrm{E}-15$ & $5.52 \mathrm{E}-15$ \\
\hline P 31 & $7.55 \mathrm{E}-09$ & $7.55 \mathrm{E}-09$ & $7.56 \mathrm{E}-09$ & 7.57E-09 & 7.57E-09 & $7.57 \mathrm{E}-09$ & 7.57E-09 & $7.57 \mathrm{E}-09$ & $7.57 \mathrm{E}-09$ \\
\hline P 32 & $5.11 \mathrm{E}-09$ & $5.11 \mathrm{E}-09$ & 5.06E-09 & 4.87E-09 & 3.64E-09 & $2.59 \mathrm{E}-09$ & 1.19E-09 & $2.79 \mathrm{E}-10$ & $1.05 \mathrm{E}-16$ \\
\hline P 33 & $6.25 \mathrm{E}-13$ & $6.24 \mathrm{E}-13$ & $6.21 \mathrm{E}-13$ & $6.08 \mathrm{E}-13$ & $5.15 \mathrm{E}-13$ & $4.24 \mathrm{E}-13$ & $2.72 \mathrm{E}-13$ & $1.18 \mathrm{E}-13$ & $2.50 \mathrm{E}-17$ \\
\hline S 32 & $1.42 \mathrm{E}-04$ & 1.42E-04 & 1.42E-04 & $1.42 \mathrm{E}-04$ & 1.42E-04 & 1.42E-04 & 1.42E-04 & $1.42 \mathrm{E}-04$ & $1.42 \mathrm{E}-04$ \\
\hline S 33 & $1.20 \mathrm{E}-06$ & $1.20 \mathrm{E}-06$ & $1.20 \mathrm{E}-06$ & $1.20 \mathrm{E}-06$ & $1.20 \mathrm{E}-06$ & $1.20 \mathrm{E}-06$ & $1.20 \mathrm{E}-06$ & $1.20 \mathrm{E}-06$ & $1.20 \mathrm{E}-06$ \\
\hline S 34 & $6.69 \mathrm{E}-06$ & 6.69E-06 & $6.69 \mathrm{E}-06$ & $6.69 \mathrm{E}-06$ & 6.69E-06 & $6.69 \mathrm{E}-06$ & $6.69 \mathrm{E}-06$ & $6.69 \mathrm{E}-06$ & $6.69 \mathrm{E}-06$ \\
\hline S 35 & $6.91 \mathrm{E}-10$ & $6.91 \mathrm{E}-10$ & $6.90 \mathrm{E}-10$ & $6.86 \mathrm{E}-10$ & $6.54 \mathrm{E}-10$ & $6.19 \mathrm{E}-10$ & $5.46 \mathrm{E}-10$ & $4.31 \mathrm{E}-10$ & $3.89 \mathrm{E}-11$ \\
\hline S 36 & $2.86 \mathrm{E}-08$ & $2.86 \mathrm{E}-08$ & $2.86 \mathrm{E}-08$ & $2.86 \mathrm{E}-08$ & $2.86 \mathrm{E}-08$ & $2.86 \mathrm{E}-08$ & $2.86 \mathrm{E}-08$ & $2.86 \mathrm{E}-08$ & $2.86 \mathrm{E}-08$ \\
\hline CL 35 & $1.59 \mathrm{E}-10$ & $1.59 \mathrm{E}-10$ & 1.60E-10 & $1.64 \mathrm{E}-10$ & $1.96 \mathrm{E}-10$ & $2.31 \mathrm{E}-10$ & $3.04 \mathrm{E}-10$ & $4.19 \mathrm{E}-10$ & $8.11 \mathrm{E}-10$ \\
\hline CL 36 & $4.05 \mathrm{E}-09$ & 4.05E-09 & 4.05E-09 & $4.05 \mathrm{E}-09$ & 4.05E-09 & 4.05E-09 & 4.05E-09 & $4.05 \mathrm{E}-09$ & $4.05 \mathrm{E}-09$ \\
\hline CL 37 & $3.00 \mathrm{E}-11$ & $3.00 \mathrm{E}-11$ & $3.00 \mathrm{E}-11$ & $3.00 \mathrm{E}-11$ & 3.00E-11 & $3.00 \mathrm{E}-11$ & $3.00 \mathrm{E}-11$ & $3.00 \mathrm{E}-11$ & $3.00 \mathrm{E}-11$ \\
\hline
\end{tabular}


TEM-10200-1

ENGINEERING CALCULATIONS AND ANALYSIS

Page 58 of 81

$12 / 19 / 17$

Rev.08

Title:

AS-RUN NEUTRONICS EVALUATION FOR THE CSM-10584 EXPERIMENT IN THE ATR

\begin{tabular}{llllllll} 
ECAR NO.: & 4496 & REV. NO.: & 1 & PROJECT NO.: & 32501 & Date: & 10/19/2020 \\
\hline
\end{tabular}

\begin{tabular}{|c|c|c|c|c|c|c|c|c|c|}
\hline \multicolumn{10}{|c|}{1 gram of inconel irradiated for 54.9 EFPDs } \\
\hline & EOC & $30 \mathrm{MINs}$ & $5 \mathrm{HRS}$ & $1 \mathrm{DAY}$ & 7 DAYS & 14 DAYS & 30 DAYS & 60 DAYS & 1 YEAR \\
\hline & $\begin{array}{l}\mathrm{g} / 1 \mathrm{~g} \text { of } \\
\text { inconel }\end{array}$ & $\begin{array}{l}\mathrm{g} / 1 \mathrm{~g} \text { of } \\
\text { inconel }\end{array}$ & $\begin{array}{l}\mathrm{g} / 1 \mathrm{~g} \text { of } \\
\text { inconel }\end{array}$ & $\begin{array}{l}\mathrm{g} / 1 \mathrm{~g} \text { of } \\
\text { inconel }\end{array}$ & $\begin{array}{l}\mathrm{g} / 1 \mathrm{~g} \text { of } \\
\text { inconel }\end{array}$ & $\begin{array}{l}\mathrm{g} / 1 \mathrm{~g} \text { of } \\
\text { inconel }\end{array}$ & $\begin{array}{l}\mathrm{g} / 1 \mathrm{~g} \text { of } \\
\text { inconel }\end{array}$ & $\begin{array}{l}\mathrm{g} / 1 \mathrm{~g} \text { of } \\
\text { inconel }\end{array}$ & $\begin{array}{l}\mathrm{g} / 1 \mathrm{~g} \text { of } \\
\text { inconel }\end{array}$ \\
\hline AR 36 & $6.87 \mathrm{E}-16$ & $6.88 \mathrm{E}-16$ & $6.92 \mathrm{E}-16$ & $7.12 \mathrm{E}-16$ & $8.62 \mathrm{E}-16$ & $1.04 \mathrm{E}-15$ & $1.44 \mathrm{E}-15$ & $2.19 \mathrm{E}-15$ & $9.83 \mathrm{E}-15$ \\
\hline AR 38 & $2.65 \mathrm{E}-15$ & $2.65 \mathrm{E}-15$ & $2.66 \mathrm{E}-15$ & $2.66 \mathrm{E}-15$ & $2.66 \mathrm{E}-15$ & $2.66 \mathrm{E}-15$ & $2.66 \mathrm{E}-15$ & $2.66 \mathrm{E}-15$ & $2.66 \mathrm{E}-15$ \\
\hline AR 39 & $1.48 \mathrm{E}-08$ & $1.48 \mathrm{E}-08$ & $1.48 \mathrm{E}-08$ & $1.48 \mathrm{E}-08$ & $1.48 \mathrm{E}-08$ & $1.48 \mathrm{E}-08$ & $1.48 \mathrm{E}-08$ & $1.48 \mathrm{E}-08$ & $1.48 \mathrm{E}-08$ \\
\hline AR 40 & $2.67 \mathrm{E}-09$ & $2.67 \mathrm{E}-09$ & 2.67E-09 & 2.67E-09 & 2.67E-09 & $2.67 \mathrm{E}-09$ & $2.67 \mathrm{E}-09$ & $2.67 \mathrm{E}-09$ & $2.67 \mathrm{E}-09$ \\
\hline AR 41 & $1.94 \mathrm{E}-15$ & $1.61 \mathrm{E}-15$ & $2.92 \mathrm{E}-16$ & $2.16 \mathrm{E}-19$ & $0.00 \mathrm{E}+00$ & $0.00 E+00$ & $0.00 \mathrm{E}+00$ & $0.00 \mathrm{E}+00$ & $0.00 \mathrm{E}+00$ \\
\hline K 39 & $1.39 \mathrm{E}-04$ & $1.39 \mathrm{E}-04$ & $1.39 \mathrm{E}-04$ & $1.39 \mathrm{E}-04$ & $1.39 \mathrm{E}-04$ & $1.39 \mathrm{E}-04$ & $1.39 \mathrm{E}-04$ & $1.39 \mathrm{E}-04$ & $1.39 \mathrm{E}-04$ \\
\hline K 40 & $1.61 \mathrm{E}-07$ & $1.61 \mathrm{E}-07$ & $1.61 \mathrm{E}-07$ & $1.61 \mathrm{E}-07$ & $1.61 \mathrm{E}-07$ & $1.61 \mathrm{E}-07$ & $1.61 \mathrm{E}-07$ & $1.61 \mathrm{E}-07$ & $1.61 \mathrm{E}-07$ \\
\hline K 41 & 1.06E-05 & $1.06 \mathrm{E}-05$ & 1.06E-05 & 1.06E-05 & 1.06E-05 & $1.06 \mathrm{E}-05$ & 1.06E-05 & 1.06E-05 & $1.06 \mathrm{E}-05$ \\
\hline K 42 & $1.26 \mathrm{E}-10$ & $1.22 \mathrm{E}-10$ & $9.48 \mathrm{E}-11$ & 3.27E-11 & $1.02 \mathrm{E}-14$ & $8.24 \mathrm{E}-19$ & $7.49 \mathrm{E}-24$ & $7.48 \mathrm{E}-24$ & $7.35 \mathrm{E}-24$ \\
\hline CA 42 & $9.15 \mathrm{E}-09$ & $9.15 \mathrm{E}-09$ & $9.18 \mathrm{E}-09$ & $9.24 \mathrm{E}-09$ & 9.27E-09 & $9.27 \mathrm{E}-09$ & $9.27 \mathrm{E}-09$ & 9.27E-09 & $9.27 \mathrm{E}-09$ \\
\hline CA 43 & $9.66 \mathrm{E}-10$ & $9.66 \mathrm{E}-10$ & $9.66 \mathrm{E}-10$ & $9.66 \mathrm{E}-10$ & $9.66 \mathrm{E}-10$ & $9.66 \mathrm{E}-10$ & $9.66 \mathrm{E}-10$ & $9.66 \mathrm{E}-10$ & $9.66 \mathrm{E}-10$ \\
\hline CA 44 & $7.42 \mathrm{E}-09$ & $7.42 \mathrm{E}-09$ & $7.42 \mathrm{E}-09$ & $7.42 \mathrm{E}-09$ & $7.42 \mathrm{E}-09$ & $7.42 \mathrm{E}-09$ & $7.42 \mathrm{E}-09$ & $7.42 \mathrm{E}-09$ & $7.42 \mathrm{E}-09$ \\
\hline CA 45 & $1.36 \mathrm{E}-10$ & $1.36 \mathrm{E}-10$ & $1.36 \mathrm{E}-10$ & $1.36 \mathrm{E}-10$ & $1.32 \mathrm{E}-10$ & $1.28 \mathrm{E}-10$ & $1.20 \mathrm{E}-10$ & $1.06 \mathrm{E}-10$ & $2.88 \mathrm{E}-11$ \\
\hline CA 46 & $8.49 \mathrm{E}-10$ & $8.49 \mathrm{E}-10$ & $8.49 \mathrm{E}-10$ & $8.49 \mathrm{E}-10$ & $8.49 \mathrm{E}-10$ & $8.49 \mathrm{E}-10$ & $8.49 \mathrm{E}-10$ & $8.49 \mathrm{E}-10$ & $8.49 \mathrm{E}-10$ \\
\hline CA 47 & $8.12 \mathrm{E}-14$ & $8.09 \mathrm{E}-14$ & $7.87 \mathrm{E}-14$ & $6.97 \mathrm{E}-14$ & $2.79 \mathrm{E}-14$ & $9.56 \mathrm{E}-15$ & $8.29 \mathrm{E}-16$ & $8.46 \mathrm{E}-18$ & $0.00 \mathrm{E}+00$ \\
\hline SC 45 & $1.64 \mathrm{E}-11$ & $1.64 \mathrm{E}-11$ & $1.65 \mathrm{E}-11$ & $1.70 \mathrm{E}-11$ & $2.04 \mathrm{E}-11$ & $2.42 \mathrm{E}-11$ & $3.27 \mathrm{E}-11$ & $4.70 \mathrm{E}-11$ & $1.24 \mathrm{E}-10$ \\
\hline SC 46 & $1.61 \mathrm{E}-08$ & $1.61 \mathrm{E}-08$ & $1.61 \mathrm{E}-08$ & $1.60 \mathrm{E}-08$ & $1.52 \mathrm{E}-08$ & $1.43 \mathrm{E}-08$ & $1.26 \mathrm{E}-08$ & $9.80 \mathrm{E}-09$ & $7.84 \mathrm{E}-10$ \\
\hline SC 47 & $2.80 \mathrm{E}-09$ & $2.78 \mathrm{E}-09$ & $2.68 \mathrm{E}-09$ & $2.27 \mathrm{E}-09$ & $6.57 \mathrm{E}-10$ & $1.55 \mathrm{E}-10$ & $5.64 \mathrm{E}-12$ & $1.14 \mathrm{E}-14$ & $0.00 \mathrm{E}+00$ \\
\hline SC 48 & $1.46 \mathrm{E}-10$ & $1.45 \mathrm{E}-10$ & $1.35 \mathrm{E}-10$ & $9.99 \mathrm{E}-11$ & $1.02 \mathrm{E}-11$ & 7.17E-13 & $1.65 \mathrm{E}-15$ & $1.86 \mathrm{E}-20$ & $0.00 \mathrm{E}+00$ \\
\hline SC 49 & $2.77 \mathrm{E}-12$ & $1.93 \mathrm{E}-12$ & $7.45 \mathrm{E}-14$ & $8.02 \mathrm{E}-20$ & $0.00 \mathrm{E}+00$ & $0.00 \mathrm{E}+00$ & $0.00 \mathrm{E}+00$ & $0.00 \mathrm{E}+00$ & $0.00 \mathrm{E}+00$ \\
\hline SC 50 & $1.67 \mathrm{E}-15$ & $8.64 \mathrm{E}-21$ & $0.00 \mathrm{E}+00$ & $0.00 \mathrm{E}+00$ & $0.00 \mathrm{E}+00$ & $0.00 \mathrm{E}+00$ & $0.00 \mathrm{E}+00$ & $0.00 \mathrm{E}+00$ & $0.00 \mathrm{E}+00$ \\
\hline TI 46 & $9.11 \mathrm{E}-04$ & $9.11 \mathrm{E}-04$ & $9.11 \mathrm{E}-04$ & $9.11 \mathrm{E}-04$ & $9.11 \mathrm{E}-04$ & $9.11 \mathrm{E}-04$ & $9.11 \mathrm{E}-04$ & $9.11 \mathrm{E}-04$ & $9.11 \mathrm{E}-04$ \\
\hline TI 47 & $8.40 \mathrm{E}-04$ & $8.40 \mathrm{E}-04$ & 8.40E-04 & 8.40E-04 & 8.40E-04 & $8.40 \mathrm{E}-04$ & $8.40 \mathrm{E}-04$ & $8.40 \mathrm{E}-04$ & $8.40 \mathrm{E}-04$ \\
\hline TI 48 & $8.46 \mathrm{E}-03$ & $8.46 \mathrm{E}-03$ & $8.46 \mathrm{E}-03$ & $8.46 \mathrm{E}-03$ & $8.46 \mathrm{E}-03$ & $8.46 \mathrm{E}-03$ & $8.46 \mathrm{E}-03$ & $8.46 \mathrm{E}-03$ & $8.46 \mathrm{E}-03$ \\
\hline TI 49 & $6.69 \mathrm{E}-04$ & $6.69 \mathrm{E}-04$ & $6.69 \mathrm{E}-04$ & $6.69 \mathrm{E}-04$ & 6.69E-04 & $6.69 \mathrm{E}-04$ & $6.69 \mathrm{E}-04$ & $6.69 \mathrm{E}-04$ & $6.69 \mathrm{E}-04$ \\
\hline TI 50 & $6.25 \mathrm{E}-04$ & $6.25 \mathrm{E}-04$ & $6.25 \mathrm{E}-04$ & $6.25 \mathrm{E}-04$ & $6.25 \mathrm{E}-04$ & $6.25 \mathrm{E}-04$ & $6.25 \mathrm{E}-04$ & $6.25 \mathrm{E}-04$ & $6.25 \mathrm{E}-04$ \\
\hline TI 51 & $6.56 \mathrm{E}-12$ & $1.77 \mathrm{E}-13$ & $1.38 \mathrm{E}-27$ & $0.00 \mathrm{E}+00$ & $0.00 \mathrm{E}+00$ & $0.00 \mathrm{E}+00$ & $0.00 \mathrm{E}+00$ & $0.00 \mathrm{E}+00$ & $0.00 \mathrm{E}+00$ \\
\hline V 50 & $3.72 \mathrm{E}-07$ & $3.72 \mathrm{E}-07$ & $3.72 \mathrm{E}-07$ & $3.72 \mathrm{E}-07$ & $3.72 \mathrm{E}-07$ & $3.72 \mathrm{E}-07$ & $3.72 \mathrm{E}-07$ & $3.72 \mathrm{E}-07$ & $3.72 \mathrm{E}-07$ \\
\hline V 51 & $3.36 \mathrm{E}-05$ & $3.36 \mathrm{E}-05$ & $3.38 \mathrm{E}-05$ & $3.46 \mathrm{E}-05$ & 4.00E-05 & $4.54 \mathrm{E}-05$ & 5.47E-05 & 6.47E-05 & $7.36 \mathrm{E}-05$ \\
\hline V 52 & $3.78 \mathrm{E}-11$ & $1.48 \mathrm{E}-13$ & $3.12 \mathrm{E}-35$ & $0.00 \mathrm{E}+00$ & $0.00 \mathrm{E}+00$ & $0.00 \mathrm{E}+00$ & $0.00 \mathrm{E}+00$ & $0.00 \mathrm{E}+00$ & $0.00 \mathrm{E}+00$ \\
\hline V 53 & $1.02 \mathrm{E}-13$ & $2.51 \mathrm{E}-19$ & $0.00 \mathrm{E}+00$ & $0.00 \mathrm{E}+00$ & $0.00 \mathrm{E}+00$ & $0.00 \mathrm{E}+00$ & $0.00 \mathrm{E}+00$ & $0.00 \mathrm{E}+00$ & $0.00 \mathrm{E}+00$ \\
\hline CR 50 & $8.70 \mathrm{E}-03$ & $8.70 \mathrm{E}-03$ & $8.70 \mathrm{E}-03$ & $8.70 \mathrm{E}-03$ & $8.70 \mathrm{E}-03$ & $8.70 \mathrm{E}-03$ & $8.70 \mathrm{E}-03$ & $8.70 \mathrm{E}-03$ & $8.70 \mathrm{E}-03$ \\
\hline CR 51 & $3.99 \mathrm{E}-05$ & $3.99 \mathrm{E}-05$ & $3.97 \mathrm{E}-05$ & $3.90 \mathrm{E}-05$ & $3.35 \mathrm{E}-05$ & $2.81 \mathrm{E}-05$ & $1.89 \mathrm{E}-05$ & $8.90 \mathrm{E}-06$ & $4.30 \mathrm{E}-09$ \\
\hline CR 52 & $1.75 \mathrm{E}-01$ & $1.75 \mathrm{E}-01$ & $1.75 \mathrm{E}-01$ & $1.75 \mathrm{E}-01$ & $1.75 \mathrm{E}-01$ & $1.75 \mathrm{E}-01$ & $1.75 \mathrm{E}-01$ & $1.75 \mathrm{E}-01$ & $1.75 \mathrm{E}-01$ \\
\hline CR 53 & $2.05 \mathrm{E}-02$ & $2.05 \mathrm{E}-02$ & $2.05 \mathrm{E}-02$ & $2.05 \mathrm{E}-02$ & $2.05 \mathrm{E}-02$ & $2.05 \mathrm{E}-02$ & $2.05 \mathrm{E}-02$ & $2.05 \mathrm{E}-02$ & $2.05 \mathrm{E}-02$ \\
\hline CR 54 & $5.34 \mathrm{E}-03$ & $5.34 \mathrm{E}-03$ & $5.34 \mathrm{E}-03$ & 5.34E-03 & $5.34 \mathrm{E}-03$ & 5.34E-03 & $5.34 \mathrm{E}-03$ & $5.34 \mathrm{E}-03$ & $5.34 \mathrm{E}-03$ \\
\hline CR 55 & $6.66 \mathrm{E}-11$ & $1.90 \mathrm{E}-13$ & $0.00 \mathrm{E}+00$ & $0.00 \mathrm{E}+00$ & $0.00 \mathrm{E}+00$ & $0.00 E+00$ & $0.00 \mathrm{E}+00$ & $0.00 \mathrm{E}+00$ & $0.00 \mathrm{E}+00$ \\
\hline MN 54 & $6.63 \mathrm{E}-07$ & $6.63 \mathrm{E}-07$ & $6.63 \mathrm{E}-07$ & 6.61E-07 & $6.53 \mathrm{E}-07$ & $6.43 \mathrm{E}-07$ & $6.20 \mathrm{E}-07$ & $5.80 \mathrm{E}-07$ & $2.95 \mathrm{E}-07$ \\
\hline MN 55 & $3.47 \mathrm{E}-03$ & $3.47 \mathrm{E}-03$ & $3.47 \mathrm{E}-03$ & 3.47E-03 & 3.47E-03 & $3.47 \mathrm{E}-03$ & $3.47 \mathrm{E}-03$ & $3.47 \mathrm{E}-03$ & $3.47 \mathrm{E}-03$ \\
\hline MN 56 & $9.86 \mathrm{E}-08$ & $8.62 \mathrm{E}-08$ & $2.57 \mathrm{E}-08$ & $1.56 \mathrm{E}-10$ & $2.41 \mathrm{E}-27$ & $0.00 \mathrm{E}+00$ & $0.00 \mathrm{E}+00$ & $0.00 \mathrm{E}+00$ & $0.00 \mathrm{E}+00$ \\
\hline MN 57 & $5.23 \mathrm{E}-14$ & $1.29 \mathrm{E}-19$ & $0.00 \mathrm{E}+00$ & $0.00 \mathrm{E}+00$ & $0.00 \mathrm{E}+00$ & $0.00 \mathrm{E}+00$ & $0.00 \mathrm{E}+00$ & $0.00 \mathrm{E}+00$ & $0.00 \mathrm{E}+00$ \\
\hline FE 54 & $6.22 \mathrm{E}-03$ & $6.22 \mathrm{E}-03$ & $6.22 \mathrm{E}-03$ & $6.22 \mathrm{E}-03$ & $6.22 \mathrm{E}-03$ & $6.22 \mathrm{E}-03$ & $6.22 \mathrm{E}-03$ & $6.22 \mathrm{E}-03$ & $6.22 \mathrm{E}-03$ \\
\hline FE 55 & $1.00 \mathrm{E}-05$ & $1.00 \mathrm{E}-05$ & 1.00E-05 & $9.99 \mathrm{E}-06$ & $9.95 \mathrm{E}-06$ & $9.90 \mathrm{E}-06$ & $9.78 \mathrm{E}-06$ & 9.57E-06 & $7.66 \mathrm{E}-06$ \\
\hline FE 56 & $1.02 \mathrm{E}-01$ & $1.02 \mathrm{E}-01$ & 1.02E-01 & $1.02 \mathrm{E}-01$ & $1.02 \mathrm{E}-01$ & $1.02 \mathrm{E}-01$ & $1.02 \mathrm{E}-01$ & $1.02 \mathrm{E}-01$ & $1.02 \mathrm{E}-01$ \\
\hline FE 57 & $2.59 \mathrm{E}-03$ & $2.59 \mathrm{E}-03$ & $2.59 \mathrm{E}-03$ & $2.59 \mathrm{E}-03$ & $2.59 \mathrm{E}-03$ & $2.59 \mathrm{E}-03$ & $2.59 \mathrm{E}-03$ & $2.59 \mathrm{E}-03$ & $2.59 \mathrm{E}-03$ \\
\hline FE 58 & $3.43 \mathrm{E}-04$ & $3.43 \mathrm{E}-04$ & $3.43 \mathrm{E}-04$ & 3.44E-04 & 3.45E-04 & $3.46 \mathrm{E}-04$ & $3.48 \mathrm{E}-04$ & $3.51 \mathrm{E}-04$ & $3.60 \mathrm{E}-04$ \\
\hline FE 59 & $1.64 \mathrm{E}-07$ & $1.64 \mathrm{E}-07$ & $1.64 \mathrm{E}-07$ & $1.62 \mathrm{E}-07$ & 1.47E-07 & $1.32 \mathrm{E}-07$ & $1.03 \mathrm{E}-07$ & $6.51 \mathrm{E}-08$ & $5.91 \mathrm{E}-10$ \\
\hline CO 58 & $1.68 \mathrm{E}-05$ & $1.68 \mathrm{E}-05$ & $1.68 \mathrm{E}-05$ & $1.66 \mathrm{E}-05$ & 1.57E-05 & $1.46 \mathrm{E}-05$ & $1.25 \mathrm{E}-05$ & $9.32 \mathrm{E}-06$ & $4.69 \mathrm{E}-07$ \\
\hline CO 59 & $9.75 \mathrm{E}-03$ & $9.75 \mathrm{E}-03$ & $9.75 \mathrm{E}-03$ & $9.75 \mathrm{E}-03$ & $9.75 \mathrm{E}-03$ & $9.75 \mathrm{E}-03$ & $9.75 \mathrm{E}-03$ & $9.75 \mathrm{E}-03$ & $9.75 \mathrm{E}-03$ \\
\hline $\mathrm{CO} 60$ & $2.70 \mathrm{E}-04$ & $2.70 \mathrm{E}-04$ & $2.70 \mathrm{E}-04$ & $2.70 \mathrm{E}-04$ & $2.69 \mathrm{E}-04$ & $2.69 \mathrm{E}-04$ & $2.67 \mathrm{E}-04$ & $2.64 \mathrm{E}-04$ & $2.37 \mathrm{E}-04$ \\
\hline $\mathrm{CO} 60 \mathrm{M}$ & $2.78 \mathrm{E}-08$ & $3.82 \mathrm{E}-09$ & $6.59 \mathrm{E}-17$ & $0.00 \mathrm{E}+00$ & $0.00 \mathrm{E}+00$ & $0.00 E+00$ & $0.00 \mathrm{E}+00$ & $0.00 \mathrm{E}+00$ & $0.00 \mathrm{E}+00$ \\
\hline CO 61 & $7.80 \mathrm{E}-10$ & $6.33 \mathrm{E}-10$ & $9.55 \mathrm{E}-11$ & $3.26 \mathrm{E}-14$ & $0.00 \mathrm{E}+00$ & $0.00 E+00$ & $0.00 E+00$ & $0.00 \mathrm{E}+00$ & $0.00 \mathrm{E}+00$ \\
\hline CO 62 & $1.04 \mathrm{E}-13$ & $9.87 \mathrm{E}-20$ & $0.00 \mathrm{E}+00$ & $0.00 \mathrm{E}+00$ & $0.00 \mathrm{E}+00$ & $0.00 \mathrm{E}+00$ & $0.00 \mathrm{E}+00$ & $0.00 \mathrm{E}+00$ & $0.00 \mathrm{E}+00$ \\
\hline NI 58 & $3.70 \mathrm{E}-01$ & $3.70 \mathrm{E}-01$ & $3.70 \mathrm{E}-01$ & $3.70 \mathrm{E}-01$ & $3.70 \mathrm{E}-01$ & $3.70 \mathrm{E}-01$ & $3.70 \mathrm{E}-01$ & $3.70 \mathrm{E}-01$ & $3.70 \mathrm{E}-01$ \\
\hline
\end{tabular}


TEM-10200-1 ENGINEERING CALCULATIONS AND ANALYSIS

Page 59 of 81

$12 / 19 / 17$

Rev.08

Title:

AS-RUN NEUTRONICS EVALUATION FOR THE CSM-10584 EXPERIMENT IN THE ATR

\begin{tabular}{llllllll} 
ECAR NO.: & 4496 & REV. NO.: & 1 & PROJECT NO.: & 32501 & Date: & 10/19/2020 \\
\hline
\end{tabular}

\begin{tabular}{|c|c|c|c|c|c|c|c|c|c|}
\hline \multicolumn{10}{|c|}{1 gram of inconel irradiated for 54.9 EFPDs } \\
\hline & EOC & $30 \mathrm{MINs}$ & $5 \mathrm{HRS}$ & $1 \mathrm{DAY}$ & 7 DAYS & 14 DAYS & 30 DAYS & 60 DAYS & 1 YEAR \\
\hline & $\begin{array}{l}\mathrm{g} / 1 \mathrm{~g} \text { of } \\
\text { inconel }\end{array}$ & $\begin{array}{l}\mathrm{g} / 1 \mathrm{~g} \text { of } \\
\text { inconel }\end{array}$ & $\begin{array}{l}\mathrm{g} / 1 \mathrm{~g} \text { of } \\
\text { inconel }\end{array}$ & $\begin{array}{l}\mathrm{g} / 1 \mathrm{~g} \text { of } \\
\text { inconel }\end{array}$ & $\begin{array}{l}\mathrm{g} / 1 \mathrm{~g} \text { of } \\
\text { inconel }\end{array}$ & $\begin{array}{l}\mathrm{g} / 1 \mathrm{~g} \text { of } \\
\text { inconel }\end{array}$ & $\begin{array}{l}\mathrm{g} / 1 \mathrm{~g} \text { of } \\
\text { inconel }\end{array}$ & $\begin{array}{l}\mathrm{g} / 1 \mathrm{~g} \text { of } \\
\text { inconel }\end{array}$ & $\begin{array}{l}\mathrm{g} / 1 \mathrm{~g} \text { of } \\
\text { inconel }\end{array}$ \\
\hline NI 59 & $9.92 \mathrm{E}-04$ & $9.92 \mathrm{E}-04$ & $9.92 \mathrm{E}-04$ & $9.92 \mathrm{E}-04$ & $9.92 \mathrm{E}-04$ & $9.92 \mathrm{E}-04$ & $9.92 \mathrm{E}-04$ & $9.92 \mathrm{E}-04$ & $9.92 \mathrm{E}-04$ \\
\hline NI 60 & $1.46 \mathrm{E}-01$ & $1.46 \mathrm{E}-01$ & $1.46 \mathrm{E}-01$ & $1.46 \mathrm{E}-01$ & $1.46 \mathrm{E}-01$ & $1.46 \mathrm{E}-01$ & $1.46 \mathrm{E}-01$ & $1.46 \mathrm{E}-01$ & $1.46 \mathrm{E}-01$ \\
\hline NI 61 & $6.66 \mathrm{E}-03$ & $6.66 \mathrm{E}-03$ & $6.66 \mathrm{E}-03$ & $6.66 \mathrm{E}-03$ & $6.66 \mathrm{E}-03$ & $6.66 \mathrm{E}-03$ & $6.66 \mathrm{E}-03$ & $6.66 \mathrm{E}-03$ & $6.66 \mathrm{E}-03$ \\
\hline NI 62 & $2.07 \mathrm{E}-02$ & 2.07E-02 & $2.07 \mathrm{E}-02$ & $2.07 \mathrm{E}-02$ & 2.07E-02 & $2.07 \mathrm{E}-02$ & $2.07 \mathrm{E}-02$ & $2.07 \mathrm{E}-02$ & 2.07E-02 \\
\hline $\mathrm{NI} 63$ & $1.54 \mathrm{E}-04$ & $1.54 \mathrm{E}-04$ & $1.54 \mathrm{E}-04$ & $1.54 \mathrm{E}-04$ & $1.54 \mathrm{E}-04$ & $1.54 \mathrm{E}-04$ & $1.54 \mathrm{E}-04$ & $1.54 \mathrm{E}-04$ & $1.53 \mathrm{E}-04$ \\
\hline NI 64 & $5.45 \mathrm{E}-03$ & $5.45 \mathrm{E}-03$ & $5.45 \mathrm{E}-03$ & $5.45 \mathrm{E}-03$ & $5.45 \mathrm{E}-03$ & $5.45 \mathrm{E}-03$ & 5.45E-03 & $5.45 \mathrm{E}-03$ & 5.45E-03 \\
\hline NI 65 & $1.26 \mathrm{E}-08$ & $1.10 \mathrm{E}-08$ & 3.19E-09 & $1.72 \mathrm{E}-11$ & $1.08 \mathrm{E}-28$ & $0.00 \mathrm{E}+00$ & $0.00 \mathrm{E}+00$ & $0.00 \mathrm{E}+00$ & $0.00 \mathrm{E}+00$ \\
\hline NI 66 & $9.60 \mathrm{E}-12$ & $9.54 \mathrm{E}-12$ & $9.01 \mathrm{E}-12$ & $7.08 \mathrm{E}-12$ & $1.14 \mathrm{E}-12$ & $1.35 \mathrm{E}-13$ & $1.03 \mathrm{E}-15$ & $1.11 \mathrm{E}-19$ & $0.00 \mathrm{E}+00$ \\
\hline CU 63 & $2.05 \mathrm{E}-03$ & $2.05 \mathrm{E}-03$ & $2.05 \mathrm{E}-03$ & $2.05 \mathrm{E}-03$ & $2.05 \mathrm{E}-03$ & $2.05 \mathrm{E}-03$ & $2.05 \mathrm{E}-03$ & $2.05 \mathrm{E}-03$ & $2.05 \mathrm{E}-03$ \\
\hline CU 64 & $7.86 \mathrm{E}-08$ & $7.65 \mathrm{E}-08$ & $5.98 \mathrm{E}-08$ & $2.12 \mathrm{E}-08$ & $8.19 \mathrm{E}-12$ & $8.53 \mathrm{E}-16$ & $6.75 \mathrm{E}-25$ & $0.00 \mathrm{E}+00$ & $0.00 \mathrm{E}+00$ \\
\hline CU 65 & 9.47E-04 & $9.47 \mathrm{E}-04$ & 9.47E-04 & 9.47E-04 & 9.47E-04 & 9.47E-04 & 9.47E-04 & 9.47E-04 & 9.47E-04 \\
\hline CU 66 & $1.18 \mathrm{E}-10$ & $2.01 \mathrm{E}-12$ & $1.40 \mathrm{E}-14$ & $1.10 \mathrm{E}-14$ & $1.77 \mathrm{E}-15$ & $2.10 \mathrm{E}-16$ & $1.61 \mathrm{E}-18$ & $1.73 \mathrm{E}-22$ & $0.00 E+00$ \\
\hline CU 67 & $5.62 \mathrm{E}-13$ & $5.59 \mathrm{E}-13$ & $5.31 \mathrm{E}-13$ & 4.29E-13 & $8.55 \mathrm{E}-14$ & $1.30 \mathrm{E}-14$ & $1.76 \mathrm{E}-16$ & $5.52 \mathrm{E}-20$ & $0.00 E+00$ \\
\hline ZN 64 & $2.08 \mathrm{E}-06$ & $2.08 \mathrm{E}-06$ & $2.08 \mathrm{E}-06$ & $2.10 \mathrm{E}-06$ & $2.10 \mathrm{E}-06$ & $2.10 \mathrm{E}-06$ & $2.10 \mathrm{E}-06$ & $2.10 \mathrm{E}-06$ & $2.10 \mathrm{E}-06$ \\
\hline ZN 65 & $5.47 \mathrm{E}-10$ & $5.47 \mathrm{E}-10$ & $5.47 \mathrm{E}-10$ & $5.45 \mathrm{E}-10$ & $5.36 \mathrm{E}-10$ & $5.26 \mathrm{E}-10$ & $5.02 \mathrm{E}-10$ & $4.61 \mathrm{E}-10$ & $1.94 \mathrm{E}-10$ \\
\hline ZN 66 & $1.26 \mathrm{E}-06$ & $1.26 \mathrm{E}-06$ & $1.26 \mathrm{E}-06$ & $1.26 \mathrm{E}-06$ & $1.26 \mathrm{E}-06$ & $1.26 \mathrm{E}-06$ & $1.26 \mathrm{E}-06$ & $1.26 \mathrm{E}-06$ & $1.26 \mathrm{E}-06$ \\
\hline ZN 67 & $3.24 \mathrm{E}-10$ & $3.24 \mathrm{E}-10$ & $3.24 \mathrm{E}-10$ & $3.24 \mathrm{E}-10$ & $3.24 \mathrm{E}-10$ & $3.24 \mathrm{E}-10$ & $3.24 \mathrm{E}-10$ & $3.24 \mathrm{E}-10$ & $3.24 \mathrm{E}-10$ \\
\hline ZN 68 & $6.50 \mathrm{E}-13$ & $6.50 \mathrm{E}-13$ & $6.50 \mathrm{E}-13$ & $6.50 \mathrm{E}-13$ & $6.50 \mathrm{E}-13$ & $6.50 \mathrm{E}-13$ & $6.50 \mathrm{E}-13$ & $6.50 \mathrm{E}-13$ & $6.50 \mathrm{E}-13$ \\
\hline SR 88 & $4.10 \mathrm{E}-15$ & $4.10 \mathrm{E}-15$ & $4.10 \mathrm{E}-15$ & $4.10 \mathrm{E}-15$ & $4.10 \mathrm{E}-15$ & $4.10 \mathrm{E}-15$ & $4.10 \mathrm{E}-15$ & $4.10 \mathrm{E}-15$ & $4.10 \mathrm{E}-15$ \\
\hline SR 89 & $3.04 \mathrm{E}-15$ & $3.04 \mathrm{E}-15$ & $3.03 \mathrm{E}-15$ & $3.00 \mathrm{E}-15$ & $2.76 \mathrm{E}-15$ & $2.51 \mathrm{E}-15$ & $2.02 \mathrm{E}-15$ & $1.34 \mathrm{E}-15$ & $2.02 \mathrm{E}-17$ \\
\hline SR 90 & $2.34 \mathrm{E}-15$ & $2.34 \mathrm{E}-15$ & $2.34 \mathrm{E}-15$ & $2.34 \mathrm{E}-15$ & $2.33 \mathrm{E}-15$ & $2.33 \mathrm{E}-15$ & $2.33 \mathrm{E}-15$ & $2.33 \mathrm{E}-15$ & $2.28 \mathrm{E}-15$ \\
\hline Y 89 & $2.21 \mathrm{E}-10$ & $2.21 \mathrm{E}-10$ & $2.22 \mathrm{E}-10$ & $2.25 \mathrm{E}-10$ & $2.37 \mathrm{E}-10$ & $2.41 \mathrm{E}-10$ & $2.42 \mathrm{E}-10$ & $2.42 \mathrm{E}-10$ & $2.42 \mathrm{E}-10$ \\
\hline Y 89M & $1.18 \mathrm{E}-15$ & 1.17E-15 & $1.13 \mathrm{E}-15$ & 9.54E-16 & $2.67 \mathrm{E}-16$ & $6.06 \mathrm{E}-17$ & $2.04 \mathrm{E}-18$ & $3.52 \mathrm{E}-21$ & $0.00 \mathrm{E}+00$ \\
\hline Y 90 & $3.86 \mathrm{E}-10$ & $3.84 \mathrm{E}-10$ & $3.66 \mathrm{E}-10$ & $2.98 \mathrm{E}-10$ & $6.26 \mathrm{E}-11$ & $1.02 \mathrm{E}-11$ & $1.59 \mathrm{E}-13$ & $6.57 \mathrm{E}-17$ & $5.72 \mathrm{E}-19$ \\
\hline Y 91 & $6.61 \mathrm{E}-13$ & $6.61 \mathrm{E}-13$ & $6.60 \mathrm{E}-13$ & $6.54 \mathrm{E}-13$ & $6.09 \mathrm{E}-13$ & $5.60 \mathrm{E}-13$ & $4.64 \mathrm{E}-13$ & $3.25 \mathrm{E}-13$ & 8.73E-15 \\
\hline ZR 89 & $2.08 \mathrm{E}-11$ & $2.07 \mathrm{E}-11$ & $1.99 \mathrm{E}-11$ & $1.68 \mathrm{E}-11$ & $4.71 \mathrm{E}-12$ & $1.07 \mathrm{E}-12$ & $3.59 \mathrm{E}-14$ & $6.19 \mathrm{E}-17$ & $0.00 E+00$ \\
\hline ZR 90 & 5.13E-09 & 5.14E-09 & 5.15E-09 & $5.22 \mathrm{E}-09$ & 5.46E-09 & 5.51E-09 & $5.52 \mathrm{E}-09$ & 5.52E-09 & 5.52E-09 \\
\hline ZR 91 & $3.29 \mathrm{E}-09$ & 3.29E-09 & $3.29 \mathrm{E}-09$ & $3.29 \mathrm{E}-09$ & $3.29 \mathrm{E}-09$ & $3.29 \mathrm{E}-09$ & $3.29 \mathrm{E}-09$ & $3.29 \mathrm{E}-09$ & 3.29E-09 \\
\hline ZR 92 & $6.83 \mathrm{E}-08$ & $6.83 \mathrm{E}-08$ & $6.85 \mathrm{E}-08$ & $6.92 \mathrm{E}-08$ & 7.32E-08 & $7.63 \mathrm{E}-08$ & $7.96 \mathrm{E}-08$ & $8.11 \mathrm{E}-08$ & 8.13E-08 \\
\hline ZR 93 & $5.63 \mathrm{E}-08$ & $5.63 \mathrm{E}-08$ & $5.63 \mathrm{E}-08$ & $5.63 \mathrm{E}-08$ & $5.63 \mathrm{E}-08$ & $5.63 \mathrm{E}-08$ & $5.63 \mathrm{E}-08$ & $5.63 \mathrm{E}-08$ & $5.63 \mathrm{E}-08$ \\
\hline ZR 94 & 7.09E-09 & 7.09E-09 & 7.09E-09 & 7.09E-09 & 7.09E-09 & 7.09E-09 & 7.09E-09 & 7.09E-09 & 7.09E-09 \\
\hline ZR 95 & $6.72 \mathrm{E}-10$ & $6.71 \mathrm{E}-10$ & $6.70 \mathrm{E}-10$ & $6.64 \mathrm{E}-10$ & $6.23 \mathrm{E}-10$ & $5.77 \mathrm{E}-10$ & $4.85 \mathrm{E}-10$ & $3.51 \mathrm{E}-10$ & $1.28 \mathrm{E}-11$ \\
\hline ZR 96 & $4.85 \mathrm{E}-13$ & $4.85 \mathrm{E}-13$ & $4.85 \mathrm{E}-13$ & $4.85 \mathrm{E}-13$ & $4.85 \mathrm{E}-13$ & $4.85 \mathrm{E}-13$ & $4.85 \mathrm{E}-13$ & $4.85 \mathrm{E}-13$ & $4.85 \mathrm{E}-13$ \\
\hline ZR 97 & $3.86 \mathrm{E}-13$ & $3.78 \mathrm{E}-13$ & $3.14 \mathrm{E}-13$ & $1.44 \mathrm{E}-13$ & $3.93 \mathrm{E}-16$ & $3.96 \mathrm{E}-19$ & $5.72 \mathrm{E}-26$ & $0.00 \mathrm{E}+00$ & $0.00 \mathrm{E}+00$ \\
\hline NB 92 & $1.30 \mathrm{E}-08$ & $1.30 \mathrm{E}-08$ & $1.28 \mathrm{E}-08$ & $1.21 \mathrm{E}-08$ & 8.05E-09 & $4.99 \mathrm{E}-09$ & $1.68 \mathrm{E}-09$ & $2.16 \mathrm{E}-10$ & $1.95 \mathrm{E}-19$ \\
\hline NB 93 & $5.48 \mathrm{E}-02$ & $5.48 \mathrm{E}-02$ & $5.48 \mathrm{E}-02$ & $5.48 \mathrm{E}-02$ & $5.48 \mathrm{E}-02$ & $5.48 \mathrm{E}-02$ & $5.48 \mathrm{E}-02$ & $5.48 \mathrm{E}-02$ & $5.48 \mathrm{E}-02$ \\
\hline NB 93M & $1.82 \mathrm{E}-15$ & $1.82 \mathrm{E}-15$ & $1.83 \mathrm{E}-15$ & $1.88 \mathrm{E}-15$ & $2.28 \mathrm{E}-15$ & $2.74 \mathrm{E}-15$ & $3.80 \mathrm{E}-15$ & 5.77E-15 & $2.54 \mathrm{E}-14$ \\
\hline NB 94 & $1.26 \mathrm{E}-04$ & $1.26 \mathrm{E}-04$ & $1.26 \mathrm{E}-04$ & $1.26 \mathrm{E}-04$ & $1.26 \mathrm{E}-04$ & $1.26 \mathrm{E}-04$ & $1.26 \mathrm{E}-04$ & $1.26 \mathrm{E}-04$ & 1.26E-04 \\
\hline NB 95 & $1.10 \mathrm{E}-06$ & $1.10 \mathrm{E}-06$ & $1.10 \mathrm{E}-06$ & $1.08 \mathrm{E}-06$ & $9.58 \mathrm{E}-07$ & $8.35 \mathrm{E}-07$ & $6.09 \mathrm{E}-07$ & 3.37E-07 & $8.34 \mathrm{E}-10$ \\
\hline NB 95M & $2.46 \mathrm{E}-13$ & $2.46 \mathrm{E}-13$ & $2.46 \mathrm{E}-13$ & $2.49 \mathrm{E}-13$ & $2.51 \mathrm{E}-13$ & $2.39 \mathrm{E}-13$ & $2.03 \mathrm{E}-13$ & $1.47 \mathrm{E}-13$ & 5.37E-15 \\
\hline NB 96 & $1.83 \mathrm{E}-10$ & $1.80 \mathrm{E}-10$ & $1.58 \mathrm{E}-10$ & $8.97 \mathrm{E}-11$ & $1.25 \mathrm{E}-12$ & $8.52 \mathrm{E}-15$ & $9.55 \mathrm{E}-20$ & $4.99 E-29$ & $0.00 \mathrm{E}+00$ \\
\hline NB 97 & $9.70 \mathrm{E}-13$ & $7.34 \mathrm{E}-13$ & $7.66 \mathrm{E}-14$ & $1.03 \mathrm{E}-14$ & $2.81 \mathrm{E}-17$ & $2.86 \mathrm{E}-20$ & $4.39 \mathrm{E}-27$ & $0.00 \mathrm{E}+00$ & $0.00 E+00$ \\
\hline MO 92 & $4.68 \mathrm{E}-03$ & $4.68 \mathrm{E}-03$ & $4.68 \mathrm{E}-03$ & $4.68 \mathrm{E}-03$ & $4.68 \mathrm{E}-03$ & $4.68 \mathrm{E}-03$ & $4.68 \mathrm{E}-03$ & $4.68 \mathrm{E}-03$ & $4.68 \mathrm{E}-03$ \\
\hline $\begin{array}{r}\mathrm{MO} \\
93 \mathrm{M} \\
\end{array}$ & $1.10 \mathrm{E}-10$ & $1.04 \mathrm{E}-10$ & $6.61 \mathrm{E}-11$ & $9.66 \mathrm{E}-12$ & $4.54 \mathrm{E}-18$ & $1.88 \mathrm{E}-25$ & $0.00 \mathrm{E}+00$ & $0.00 \mathrm{E}+00$ & $0.00 \mathrm{E}+00$ \\
\hline MO 93 & $4.50 \mathrm{E}-07$ & $4.50 \mathrm{E}-07$ & $4.50 \mathrm{E}-07$ & $4.50 \mathrm{E}-07$ & $4.50 \mathrm{E}-07$ & $4.50 \mathrm{E}-07$ & $4.50 \mathrm{E}-07$ & $4.50 \mathrm{E}-07$ & $4.50 \mathrm{E}-07$ \\
\hline MO 94 & $3.00 \mathrm{E}-03$ & $3.00 \mathrm{E}-03$ & $3.00 \mathrm{E}-03$ & $3.00 \mathrm{E}-03$ & $3.00 \mathrm{E}-03$ & $3.00 \mathrm{E}-03$ & $3.00 \mathrm{E}-03$ & $3.00 \mathrm{E}-03$ & 3.00E-03 \\
\hline MO 95 & 5.07E-03 & 5.07E-03 & 5.07E-03 & 5.07E-03 & 5.07E-03 & 5.07E-03 & 5.07E-03 & 5.07E-03 & 5.07E-03 \\
\hline MO 96 & 5.61E-03 & $5.61 \mathrm{E}-03$ & $5.61 \mathrm{E}-03$ & 5.61E-03 & $5.61 \mathrm{E}-03$ & $5.61 \mathrm{E}-03$ & 5.61E-03 & 5.61E-03 & 5.61E-03 \\
\hline MO 97 & $3.21 \mathrm{E}-03$ & $3.21 \mathrm{E}-03$ & $3.21 \mathrm{E}-03$ & $3.21 \mathrm{E}-03$ & $3.21 \mathrm{E}-03$ & $3.21 \mathrm{E}-03$ & $3.21 \mathrm{E}-03$ & $3.21 \mathrm{E}-03$ & $3.21 \mathrm{E}-03$ \\
\hline MO 98 & $8.12 \mathrm{E}-03$ & 8.12E-03 & $8.12 \mathrm{E}-03$ & $8.12 \mathrm{E}-03$ & $8.12 \mathrm{E}-03$ & $8.12 \mathrm{E}-03$ & $8.12 \mathrm{E}-03$ & $8.12 \mathrm{E}-03$ & $8.12 \mathrm{E}-03$ \\
\hline MO 99 & $7.86 \mathrm{E}-07$ & $7.82 \mathrm{E}-07$ & $7.46 \mathrm{E}-07$ & $6.11 \mathrm{E}-07$ & $1.35 \mathrm{E}-07$ & $2.31 \mathrm{E}-08$ & $4.09 \mathrm{E}-10$ & $2.13 \mathrm{E}-13$ & $0.00 \mathrm{E}+00$ \\
\hline Mo100 & $3.30 \mathrm{E}-03$ & 3.30E-03 & $3.30 \mathrm{E}-03$ & $3.30 \mathrm{E}-03$ & 3.30E-03 & $3.30 \mathrm{E}-03$ & $3.30 \mathrm{E}-03$ & $3.30 \mathrm{E}-03$ & 3.30E-03 \\
\hline M0101 & 7.37E-10 & $1.78 \mathrm{E}-10$ & $4.90 \mathrm{E}-16$ & $0.00 \mathrm{E}+00$ & $0.00 \mathrm{E}+00$ & $0.00 \mathrm{E}+00$ & $0.00 \mathrm{E}+00$ & $0.00 \mathrm{E}+00$ & $0.00 \mathrm{E}+00$ \\
\hline
\end{tabular}


TEM-10200-1 ENGINEERING CALCULATIONS AND ANALYSIS

Page 60 of 81

$12 / 19 / 17$

Rev.08

Title:

AS-RUN NEUTRONICS EVALUATION FOR THE CSM-10584 EXPERIMENT IN THE ATR

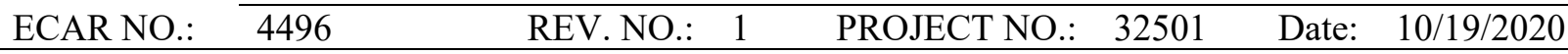

\begin{tabular}{|c|c|c|c|c|c|c|c|c|c|}
\hline \multicolumn{9}{|c|}{1 gram inconel irradiated for 54.9 EFPDs } \\
\hline & EOC & $30 \mathrm{MINs}$ & $5 \mathrm{HRS}$ & $1 \mathrm{DAY}$ & $7 \mathrm{DAYS}$ & $14 \mathrm{DAYS}$ & 30 DAYS & 60 DAYS & 1 YEAR \\
\hline & $\begin{array}{c}\mathrm{g} / 1 \mathrm{~g} \text { of } \\
\text { inconel }\end{array}$ & $\begin{array}{c}\mathrm{g} / 1 \mathrm{~g} \text { of } \\
\text { inconel }\end{array}$ & $\begin{array}{c}\mathrm{g} / 1 \mathrm{~g} \text { of } \\
\text { inconel }\end{array}$ & $\begin{array}{c}\mathrm{g} / 1 \mathrm{~g} \text { of } \\
\text { inconel }\end{array}$ & $\begin{array}{c}\mathrm{g} / 1 \mathrm{~g} \text { of } \\
\text { inconel }\end{array}$ & $\begin{array}{c}\mathrm{g} / 1 \mathrm{~g} \text { of } \\
\text { inconel }\end{array}$ & $\begin{array}{c}\mathrm{g} / 1 \mathrm{~g} \text { of } \\
\text { inconel }\end{array}$ & $\begin{array}{c}\mathrm{g} / 1 \mathrm{~g} \text { of } \\
\text { inconel }\end{array}$ & $\begin{array}{c}\mathrm{g} / 1 \mathrm{~g} \text { of } \\
\text { inconel }\end{array}$ \\
\hline TC 99 & $1.23 \mathrm{E}-06$ & $1.23 \mathrm{E}-06$ & $1.23 \mathrm{E}-06$ & $1.25 \mathrm{E}-06$ & $1.31 \mathrm{E}-06$ & $1.32 \mathrm{E}-06$ & $1.33 \mathrm{E}-06$ & $1.33 \mathrm{E}-06$ & $1.33 \mathrm{E}-06$ \\
\hline TC100 & $3.05 \mathrm{E}-13$ & $0.00 \mathrm{E}+00$ & $0.00 \mathrm{E}+00$ & $0.00 \mathrm{E}+00$ & $0.00 \mathrm{E}+00$ & $0.00 \mathrm{E}+00$ & $0.00 \mathrm{E}+00$ & $0.00 \mathrm{E}+00$ & $0.00 \mathrm{E}+00$ \\
\hline TC101 & $7.16 \mathrm{E}-10$ & $4.13 \mathrm{E}-10$ & $6.01 \mathrm{E}-15$ & $0.00 \mathrm{E}+00$ & $0.00 \mathrm{E}+00$ & $0.00 \mathrm{E}+00$ & $0.00 \mathrm{E}+00$ & $0.00 \mathrm{E}+00$ & $0.00 \mathrm{E}+00$ \\
\hline RU 99 & $2.80 \mathrm{E}-13$ & $2.80 \mathrm{E}-13$ & $2.82 \mathrm{E}-13$ & $2.91 \mathrm{E}-13$ & $3.60 \mathrm{E}-13$ & $4.42 \mathrm{E}-13$ & $6.31 \mathrm{E}-13$ & $9.85 \mathrm{E}-13$ & $4.59 \mathrm{E}-12$ \\
\hline RU100 & $2.98 \mathrm{E}-08$ & $2.98 \mathrm{E}-08$ & $2.98 \mathrm{E}-08$ & $2.98 \mathrm{E}-08$ & $2.98 \mathrm{E}-08$ & $2.98 \mathrm{E}-08$ & $2.98 \mathrm{E}-08$ & $2.98 \mathrm{E}-08$ & $2.98 \mathrm{E}-08$ \\
\hline RU101 & $2.74 \mathrm{E}-06$ & $2.74 \mathrm{E}-06$ & $2.74 \mathrm{E}-06$ & $2.74 \mathrm{E}-06$ & $2.74 \mathrm{E}-06$ & $2.74 \mathrm{E}-06$ & $2.74 \mathrm{E}-06$ & $2.74 \mathrm{E}-06$ & $2.74 \mathrm{E}-06$ \\
\hline RU102 & $2.29 \mathrm{E}-08$ & $2.29 \mathrm{E}-08$ & $2.29 \mathrm{E}-08$ & $2.29 \mathrm{E}-08$ & $2.29 \mathrm{E}-08$ & $2.29 \mathrm{E}-08$ & $2.29 \mathrm{E}-08$ & $2.29 \mathrm{E}-08$ & $2.29 \mathrm{E}-08$ \\
\hline RU103 & $9.01 \mathrm{E}-12$ & $9.01 \mathrm{E}-12$ & $8.98 \mathrm{E}-12$ & $8.85 \mathrm{E}-12$ & $7.96 \mathrm{E}-12$ & $7.04 \mathrm{E}-12$ & $5.31 \mathrm{E}-12$ & $3.13 \mathrm{E}-12$ & $1.43 \mathrm{E}-14$ \\
\hline RU104 & $3.55 \mathrm{E}-14$ & $3.55 \mathrm{E}-14$ & $3.55 \mathrm{E}-14$ & $3.55 \mathrm{E}-14$ & $3.55 \mathrm{E}-14$ & $3.55 \mathrm{E}-14$ & $3.55 \mathrm{E}-14$ & $3.55 \mathrm{E}-14$ & $3.55 \mathrm{E}-14$ \\
\hline RH103 & $2.17 \mathrm{E}-13$ & $2.17 \mathrm{E}-13$ & $2.20 \mathrm{E}-13$ & $2.33 \mathrm{E}-13$ & $3.21 \mathrm{E}-13$ & $4.13 \mathrm{E}-13$ & $5.85 \mathrm{E}-13$ & $8.02 \mathrm{E}-13$ & $1.11 \mathrm{E}-12$ \\
\hline PD104 & $1.01 \mathrm{E}-14$ & $1.01 \mathrm{E}-14$ & $1.01 \mathrm{E}-14$ & $1.01 \mathrm{E}-14$ & $1.01 \mathrm{E}-14$ & $1.01 \mathrm{E}-14$ & $1.01 \mathrm{E}-14$ & $1.01 \mathrm{E}-14$ & $1.01 \mathrm{E}-14$ \\
\hline & & & & & & & & & \\
SUMTOT & $1.00 \mathrm{E}+00$ & $1.00 \mathrm{E}+00$ & $1.00 \mathrm{E}+00$ & $1.00 \mathrm{E}+00$ & $1.00 \mathrm{E}+00$ & $1.00 \mathrm{E}+00$ & $1.00 \mathrm{E}+00$ & $1.00 \mathrm{E}+00$ & $1.00 \mathrm{E}+00$ \\
\hline 0TOTAL & $1.00 \mathrm{E}+00$ & $1.00 \mathrm{E}+00$ & $1.00 \mathrm{E}+00$ & $1.00 \mathrm{E}+00$ & $1.00 \mathrm{E}+00$ & $1.00 \mathrm{E}+00$ & $1.00 \mathrm{E}+00$ & $1.00 \mathrm{E}+00$ & $1.00 \mathrm{E}+00$ \\
\hline
\end{tabular}

\begin{tabular}{|c|c|c|c|c|c|c|c|c|c|}
\hline \multicolumn{10}{|c|}{1 gram of inconel irradiated for 119 EFPDs } \\
\hline & EOC & $30 \mathrm{MINs}$ & $5 \mathrm{HRS}$ & $1 \mathrm{DAY}$ & 7 DAYS & 14 DAYS & 30 DAYS & 60 DAYS & 1 YEAR \\
\hline & $\begin{array}{l}\mathrm{g} / 1 \mathrm{~g} \text { of } \\
\text { inconel }\end{array}$ & $\begin{array}{l}\mathrm{g} / 1 \mathrm{~g} \text { of } \\
\text { inconel }\end{array}$ & $\begin{array}{l}\mathrm{g} / 1 \mathrm{~g} \text { of } \\
\text { inconel }\end{array}$ & $\begin{array}{l}\mathrm{g} / 1 \mathrm{~g} \text { of } \\
\text { inconel }\end{array}$ & $\begin{array}{l}\mathrm{g} / 1 \mathrm{~g} \text { of } \\
\text { inconel }\end{array}$ & $\begin{array}{l}\mathrm{g} / 1 \mathrm{~g} \text { of } \\
\text { inconel }\end{array}$ & $\begin{array}{l}\mathrm{g} / 1 \mathrm{~g} \text { of } \\
\text { inconel }\end{array}$ & $\begin{array}{l}\mathrm{g} / 1 \mathrm{~g} \text { of } \\
\text { inconel }\end{array}$ & $\begin{array}{l}\mathrm{g} / 1 \mathrm{~g} \text { of } \\
\text { inconel }\end{array}$ \\
\hline H 1 & $1.76 \mathrm{E}-06$ & $1.76 \mathrm{E}-06$ & $1.76 \mathrm{E}-06$ & $1.76 \mathrm{E}-06$ & $1.76 \mathrm{E}-06$ & $1.76 \mathrm{E}-06$ & $1.76 \mathrm{E}-06$ & $1.76 \mathrm{E}-06$ & $1.76 \mathrm{E}-06$ \\
\hline $\mathrm{H} 2$ & $7.40 \mathrm{E}-10$ & $7.40 \mathrm{E}-10$ & $7.40 \mathrm{E}-10$ & $7.40 \mathrm{E}-10$ & $7.40 \mathrm{E}-10$ & $7.40 \mathrm{E}-10$ & $7.40 \mathrm{E}-10$ & $7.40 \mathrm{E}-10$ & $7.40 \mathrm{E}-10$ \\
\hline H 3 & $5.76 \mathrm{E}-14$ & $5.76 \mathrm{E}-14$ & $5.76 \mathrm{E}-14$ & $5.75 \mathrm{E}-14$ & $5.75 \mathrm{E}-14$ & $5.74 \mathrm{E}-14$ & $5.73 \mathrm{E}-14$ & $5.70 \mathrm{E}-14$ & $5.44 \mathrm{E}-14$ \\
\hline HE 3 & $1.07 \mathrm{E}-16$ & $1.08 \mathrm{E}-16$ & 1.09E-16 & $1.16 \mathrm{E}-16$ & 1.69E-16 & $2.31 \mathrm{E}-16$ & $3.72 \mathrm{E}-16$ & $6.35 \mathrm{E}-16$ & $3.25 \mathrm{E}-15$ \\
\hline HE 4 & $6.50 \mathrm{E}-06$ & $6.50 \mathrm{E}-06$ & $6.50 \mathrm{E}-06$ & $6.50 \mathrm{E}-06$ & $6.50 \mathrm{E}-06$ & $6.50 \mathrm{E}-06$ & $6.50 \mathrm{E}-06$ & $6.50 \mathrm{E}-06$ & $6.50 \mathrm{E}-06$ \\
\hline LI 6 & $3.18 \mathrm{E}-13$ & $3.18 \mathrm{E}-13$ & $3.18 \mathrm{E}-13$ & $3.18 \mathrm{E}-13$ & $3.18 \mathrm{E}-13$ & $3.18 \mathrm{E}-13$ & $3.18 \mathrm{E}-13$ & $3.18 \mathrm{E}-13$ & $3.18 \mathrm{E}-13$ \\
\hline LI 7 & $7.72 \mathrm{E}-06$ & $7.72 \mathrm{E}-06$ & $7.72 \mathrm{E}-06$ & $7.72 \mathrm{E}-06$ & $7.72 \mathrm{E}-06$ & $7.72 \mathrm{E}-06$ & $7.72 \mathrm{E}-06$ & $7.72 \mathrm{E}-06$ & 7.72E-06 \\
\hline BE 9 & $2.80 \mathrm{E}-09$ & $2.80 \mathrm{E}-09$ & $2.80 \mathrm{E}-09$ & 2.80E-09 & $2.80 \mathrm{E}-09$ & 2.80E-09 & $2.80 \mathrm{E}-09$ & $2.80 \mathrm{E}-09$ & $2.80 \mathrm{E}-09$ \\
\hline BE 10 & $1.40 \mathrm{E}-10$ & $1.40 \mathrm{E}-10$ & $1.40 \mathrm{E}-10$ & $1.40 \mathrm{E}-10$ & $1.40 \mathrm{E}-10$ & $1.40 \mathrm{E}-10$ & $1.40 \mathrm{E}-10$ & $1.40 \mathrm{E}-10$ & $1.40 \mathrm{E}-10$ \\
\hline B 10 & $8.51 \mathrm{E}-08$ & $8.51 \mathrm{E}-08$ & $8.51 \mathrm{E}-08$ & $8.51 \mathrm{E}-08$ & $8.51 \mathrm{E}-08$ & $8.51 \mathrm{E}-08$ & $8.51 \mathrm{E}-08$ & $8.51 \mathrm{E}-08$ & $8.51 \mathrm{E}-08$ \\
\hline B 11 & 4.89E-05 & $4.89 \mathrm{E}-05$ & $4.89 \mathrm{E}-05$ & 4.89E-05 & $4.89 \mathrm{E}-05$ & $4.89 \mathrm{E}-05$ & $4.89 \mathrm{E}-05$ & $4.89 \mathrm{E}-05$ & $4.89 \mathrm{E}-05$ \\
\hline C 12 & $7.90 \mathrm{E}-04$ & $7.90 \mathrm{E}-04$ & $7.90 \mathrm{E}-04$ & $7.90 \mathrm{E}-04$ & $7.90 \mathrm{E}-04$ & 7.90E-04 & $7.90 \mathrm{E}-04$ & $7.90 \mathrm{E}-04$ & $7.90 \mathrm{E}-04$ \\
\hline C 13 & 9.61E-06 & $9.61 \mathrm{E}-06$ & 9.61E-06 & $9.61 \mathrm{E}-06$ & $9.61 \mathrm{E}-06$ & $9.61 \mathrm{E}-06$ & $9.61 \mathrm{E}-06$ & $9.61 \mathrm{E}-06$ & $9.61 \mathrm{E}-06$ \\
\hline C 14 & $1.32 \mathrm{E}-11$ & $1.32 \mathrm{E}-11$ & $1.32 \mathrm{E}-11$ & $1.32 \mathrm{E}-11$ & $1.32 \mathrm{E}-11$ & $1.32 \mathrm{E}-11$ & $1.32 \mathrm{E}-11$ & $1.32 \mathrm{E}-11$ & $1.32 \mathrm{E}-11$ \\
\hline N 14 & $2.59 \mathrm{E}-16$ & $2.59 \mathrm{E}-16$ & $2.60 \mathrm{E}-16$ & $2.64 \mathrm{E}-16$ & $2.90 \mathrm{E}-16$ & $3.20 \mathrm{E}-16$ & $3.90 \mathrm{E}-16$ & $5.21 \mathrm{E}-16$ & $1.85 \mathrm{E}-15$ \\
\hline NE 21 & $4.15 \mathrm{E}-14$ & $4.15 \mathrm{E}-14$ & $4.15 \mathrm{E}-14$ & $4.15 \mathrm{E}-14$ & $4.15 \mathrm{E}-14$ & $4.15 \mathrm{E}-14$ & $4.15 \mathrm{E}-14$ & $4.15 \mathrm{E}-14$ & $4.15 \mathrm{E}-14$ \\
\hline NE 22 & $2.20 \mathrm{E}-13$ & $2.20 \mathrm{E}-13$ & $2.20 \mathrm{E}-13$ & $2.20 \mathrm{E}-13$ & $2.20 \mathrm{E}-13$ & $2.20 \mathrm{E}-13$ & $2.20 \mathrm{E}-13$ & $2.20 \mathrm{E}-13$ & $2.20 \mathrm{E}-13$ \\
\hline NA 23 & $1.78 \mathrm{E}-14$ & $1.78 \mathrm{E}-14$ & $1.78 \mathrm{E}-14$ & $1.78 \mathrm{E}-14$ & $1.78 \mathrm{E}-14$ & $1.78 \mathrm{E}-14$ & $1.78 \mathrm{E}-14$ & $1.78 \mathrm{E}-14$ & $1.78 \mathrm{E}-14$ \\
\hline NA 24 & $1.30 \mathrm{E}-10$ & $1.27 \mathrm{E}-10$ & $1.03 \mathrm{E}-10$ & $4.29 \mathrm{E}-11$ & $5.55 \mathrm{E}-14$ & $2.36 \mathrm{E}-17$ & $4.64 \mathrm{E}-25$ & $0.00 \mathrm{E}+00$ & $0.00 E+00$ \\
\hline MG 24 & $1.70 \mathrm{E}-08$ & $1.70 \mathrm{E}-08$ & $1.71 \mathrm{E}-08$ & $1.71 \mathrm{E}-08$ & $1.72 \mathrm{E}-08$ & $1.72 \mathrm{E}-08$ & $1.72 \mathrm{E}-08$ & $1.72 \mathrm{E}-08$ & $1.72 \mathrm{E}-08$ \\
\hline MG 25 & 7.90E-09 & 7.90E-09 & 7.90E-09 & 7.90E-09 & 7.90E-09 & 7.90E-09 & 7.90E-09 & 7.90E-09 & 7.90E-09 \\
\hline MG 26 & $3.60 \mathrm{E}-09$ & $3.60 \mathrm{E}-09$ & 3.60E-09 & 3.60E-09 & 3.60E-09 & $3.60 \mathrm{E}-09$ & $3.60 \mathrm{E}-09$ & $3.60 \mathrm{E}-09$ & $3.60 \mathrm{E}-09$ \\
\hline MG 27 & $7.35 \mathrm{E}-12$ & $8.16 \mathrm{E}-13$ & $2.10 \mathrm{E}-21$ & $0.00 E+00$ & $0.00 E+00$ & $0.00 \mathrm{E}+00$ & $0.00 \mathrm{E}+00$ & $0.00 E+00$ & $0.00 \mathrm{E}+00$ \\
\hline AL 27 & 8.00E-03 & $8.00 \mathrm{E}-03$ & 8.00E-03 & 8.00E-03 & 8.00E-03 & $8.00 \mathrm{E}-03$ & $8.00 \mathrm{E}-03$ & 8.00E-03 & $8.00 \mathrm{E}-03$ \\
\hline AL 28 & $4.42 \mathrm{E}-11$ & $4.11 \mathrm{E}-15$ & $5.45 \mathrm{E}-21$ & 2.90E-21 & $2.45 \mathrm{E}-23$ & $9.53 \mathrm{E}-26$ & $2.83 \mathrm{E}-31$ & $0.00 E+00$ & $0.00 \mathrm{E}+00$ \\
\hline AL 29 & $9.34 \mathrm{E}-14$ & $3.85 \mathrm{E}-15$ & $1.32 \mathrm{E}-27$ & $0.00 \mathrm{E}+00$ & $0.00 E+00$ & $0.00 \mathrm{E}+00$ & $0.00 \mathrm{E}+00$ & $0.00 E+00$ & $0.00 \mathrm{E}+00$ \\
\hline SI 28 & $3.22 \mathrm{E}-03$ & $3.22 \mathrm{E}-03$ & $3.22 \mathrm{E}-03$ & $3.22 \mathrm{E}-03$ & $3.22 \mathrm{E}-03$ & $3.22 \mathrm{E}-03$ & $3.22 \mathrm{E}-03$ & $3.22 \mathrm{E}-03$ & $3.22 \mathrm{E}-03$ \\
\hline SI 29 & 1.69E-04 & $1.69 \mathrm{E}-04$ & 1.69E-04 & 1.69E-04 & 1.69E-04 & $1.69 \mathrm{E}-04$ & $1.69 \mathrm{E}-04$ & $1.69 \mathrm{E}-04$ & $1.69 \mathrm{E}-04$ \\
\hline SI 30 & 1.16E-04 & $1.16 \mathrm{E}-04$ & 1.16E-04 & 1.16E-04 & 1.16E-04 & 1.16E-04 & $1.16 \mathrm{E}-04$ & $1.16 \mathrm{E}-04$ & $1.16 \mathrm{E}-04$ \\
\hline SI 31 & $2.17 \mathrm{E}-11$ & $1.90 \mathrm{E}-11$ & 5.79E-12 & $3.81 \mathrm{E}-14$ & $1.11 \mathrm{E}-30$ & $0.00 \mathrm{E}+00$ & $0.00 \mathrm{E}+00$ & $0.00 E+00$ & $0.00 \mathrm{E}+00$ \\
\hline SI 32 & $1.20 \mathrm{E}-14$ & $1.20 \mathrm{E}-14$ & 1.20E-14 & $1.20 \mathrm{E}-14$ & $1.20 \mathrm{E}-14$ & $1.20 \mathrm{E}-14$ & $1.20 \mathrm{E}-14$ & $1.20 \mathrm{E}-14$ & $1.20 \mathrm{E}-14$ \\
\hline P 31 & $1.64 \mathrm{E}-08$ & $1.64 \mathrm{E}-08$ & $1.64 \mathrm{E}-08$ & $1.64 \mathrm{E}-08$ & $1.64 \mathrm{E}-08$ & $1.64 \mathrm{E}-08$ & $1.64 \mathrm{E}-08$ & $1.64 \mathrm{E}-08$ & $1.64 \mathrm{E}-08$ \\
\hline P 32 & $5.48 \mathrm{E}-09$ & 5.47E-09 & $5.42 \mathrm{E}-09$ & 5.22E-09 & 3.90E-09 & $2.78 \mathrm{E}-09$ & $1.28 \mathrm{E}-09$ & $2.99 \mathrm{E}-10$ & $1.13 \mathrm{E}-16$ \\
\hline
\end{tabular}




\section{TEM-10200-1 ENGINEERING CALCULATIONS AND ANALYSIS}

Page 61 of 81

$12 / 19 / 17$

Rev.08

Title:

AS-RUN NEUTRONICS EVALUATION FOR THE CSM-10584 EXPERIMENT IN THE ATR

\begin{tabular}{llllllll} 
ECAR NO.: & 4496 & REV. NO.: & 1 & PROJECT NO.: & 32501 & Date: & 10/19/2020 \\
\hline
\end{tabular}

\begin{tabular}{|c|c|c|c|c|c|c|c|c|c|}
\hline \multicolumn{10}{|c|}{1 gram of inconel irradiated for 119 EFPDs } \\
\hline & EOC & $30 \mathrm{MINs}$ & $5 \mathrm{HRS}$ & $1 \mathrm{DAY}$ & 7 DAYS & 14 DAYS & 30 DAYS & 60 DAYS & 1 YEAR \\
\hline & $\begin{array}{l}\mathrm{g} / 1 \mathrm{~g} \text { of } \\
\text { inconel }\end{array}$ & $\begin{array}{l}\mathrm{g} / 1 \mathrm{~g} \text { of } \\
\text { inconel }\end{array}$ & $\begin{array}{l}\mathrm{g} / 1 \mathrm{~g} \text { of } \\
\text { inconel }\end{array}$ & $\begin{array}{l}\mathrm{g} / 1 \mathrm{~g} \text { of } \\
\text { inconel }\end{array}$ & $\begin{array}{l}\mathrm{g} / 1 \mathrm{~g} \text { of } \\
\text { inconel }\end{array}$ & $\begin{array}{l}\mathrm{g} / 1 \mathrm{~g} \text { of } \\
\text { inconel }\end{array}$ & $\begin{array}{l}\mathrm{g} / 1 \mathrm{~g} \text { of } \\
\text { inconel }\end{array}$ & $\begin{array}{l}\mathrm{g} / 1 \mathrm{~g} \text { of } \\
\text { inconel }\end{array}$ & $\begin{array}{l}\mathrm{g} / 1 \mathrm{~g} \text { of } \\
\text { inconel }\end{array}$ \\
\hline P 33 & $7.95 \mathrm{E}-13$ & $7.94 \mathrm{E}-13$ & $7.90 \mathrm{E}-13$ & $7.73 \mathrm{E}-13$ & $6.55 \mathrm{E}-13$ & $5.39 \mathrm{E}-13$ & $3.46 \mathrm{E}-13$ & $1.51 \mathrm{E}-13$ & $3.18 \mathrm{E}-17$ \\
\hline S 32 & $1.42 \mathrm{E}-04$ & $1.42 \mathrm{E}-04$ & $1.42 \mathrm{E}-04$ & $1.42 \mathrm{E}-04$ & $1.42 \mathrm{E}-04$ & $1.42 \mathrm{E}-04$ & $1.42 \mathrm{E}-04$ & $1.42 \mathrm{E}-04$ & $1.42 \mathrm{E}-04$ \\
\hline S 33 & $1.24 \mathrm{E}-06$ & $1.24 \mathrm{E}-06$ & $1.24 \mathrm{E}-06$ & $1.24 \mathrm{E}-06$ & $1.24 \mathrm{E}-06$ & $1.24 \mathrm{E}-06$ & $1.24 \mathrm{E}-06$ & $1.24 \mathrm{E}-06$ & $1.24 \mathrm{E}-06$ \\
\hline S 34 & $6.69 \mathrm{E}-06$ & $6.69 \mathrm{E}-06$ & $6.69 \mathrm{E}-06$ & $6.69 \mathrm{E}-06$ & $6.69 \mathrm{E}-06$ & $6.69 \mathrm{E}-06$ & $6.69 \mathrm{E}-06$ & $6.69 \mathrm{E}-06$ & $6.69 \mathrm{E}-06$ \\
\hline S 35 & $1.20 \mathrm{E}-09$ & $1.20 \mathrm{E}-09$ & $1.20 \mathrm{E}-09$ & $1.19 \mathrm{E}-09$ & $1.13 \mathrm{E}-09$ & 1.07E-09 & $9.46 \mathrm{E}-10$ & 7.47E-10 & $6.74 \mathrm{E}-11$ \\
\hline S 36 & $2.86 \mathrm{E}-08$ & $2.86 \mathrm{E}-08$ & $2.86 \mathrm{E}-08$ & $2.86 \mathrm{E}-08$ & $2.86 \mathrm{E}-08$ & $2.86 \mathrm{E}-08$ & $2.86 \mathrm{E}-08$ & $2.86 \mathrm{E}-08$ & $2.86 \mathrm{E}-08$ \\
\hline $\mathrm{CL} 35$ & $6.37 \mathrm{E}-10$ & $6.37 \mathrm{E}-10$ & $6.39 \mathrm{E}-10$ & $6.46 \mathrm{E}-10$ & $7.01 \mathrm{E}-10$ & $7.62 \mathrm{E}-10$ & $8.89 \mathrm{E}-10$ & 1.09E-09 & $1.77 \mathrm{E}-09$ \\
\hline $\mathrm{CL} 36$ & $8.75 \mathrm{E}-09$ & $8.75 \mathrm{E}-09$ & $8.75 \mathrm{E}-09$ & $8.75 \mathrm{E}-09$ & $8.75 \mathrm{E}-09$ & $8.75 \mathrm{E}-09$ & $8.75 \mathrm{E}-09$ & $8.75 \mathrm{E}-09$ & $8.75 \mathrm{E}-09$ \\
\hline CL 37 & $1.27 \mathrm{E}-10$ & $1.27 \mathrm{E}-10$ & $1.27 \mathrm{E}-10$ & $1.27 \mathrm{E}-10$ & $1.27 \mathrm{E}-10$ & $1.27 \mathrm{E}-10$ & $1.27 \mathrm{E}-10$ & $1.27 \mathrm{E}-10$ & $1.27 \mathrm{E}-10$ \\
\hline AR 36 & $3.22 \mathrm{E}-15$ & $3.22 \mathrm{E}-15$ & $3.23 \mathrm{E}-15$ & $3.27 \mathrm{E}-15$ & $3.60 \mathrm{E}-15$ & $3.98 \mathrm{E}-15$ & $4.84 \mathrm{E}-15$ & $6.47 \mathrm{E}-15$ & $2.30 \mathrm{E}-14$ \\
\hline AR 38 & $2.33 \mathrm{E}-14$ & $2.33 \mathrm{E}-14$ & $2.33 \mathrm{E}-14$ & $2.33 \mathrm{E}-14$ & $2.33 \mathrm{E}-14$ & $2.33 \mathrm{E}-14$ & $2.33 \mathrm{E}-14$ & $2.33 \mathrm{E}-14$ & $2.33 \mathrm{E}-14$ \\
\hline AR 39 & $2.71 \mathrm{E}-08$ & $2.71 \mathrm{E}-08$ & $2.71 \mathrm{E}-08$ & $2.71 \mathrm{E}-08$ & $2.71 \mathrm{E}-08$ & $2.71 \mathrm{E}-08$ & $2.71 \mathrm{E}-08$ & $2.71 \mathrm{E}-08$ & $2.71 \mathrm{E}-08$ \\
\hline AR 40 & $1.12 \mathrm{E}-08$ & $1.12 \mathrm{E}-08$ & $1.12 \mathrm{E}-08$ & $1.12 \mathrm{E}-08$ & $1.12 \mathrm{E}-08$ & $1.12 \mathrm{E}-08$ & $1.12 \mathrm{E}-08$ & $1.12 \mathrm{E}-08$ & $1.12 \mathrm{E}-08$ \\
\hline AR 41 & $8.16 \mathrm{E}-15$ & $6.75 \mathrm{E}-15$ & $1.22 \mathrm{E}-15$ & $9.06 \mathrm{E}-19$ & $0.00 \mathrm{E}+00$ & $0.00 \mathrm{E}+00$ & $0.00 \mathrm{E}+00$ & $0.00 \mathrm{E}+00$ & $0.00 \mathrm{E}+00$ \\
\hline K 39 & $1.39 \mathrm{E}-04$ & $1.39 \mathrm{E}-04$ & $1.39 \mathrm{E}-04$ & $1.39 \mathrm{E}-04$ & $1.39 \mathrm{E}-04$ & $1.39 \mathrm{E}-04$ & $1.39 \mathrm{E}-04$ & $1.39 \mathrm{E}-04$ & $1.39 \mathrm{E}-04$ \\
\hline K 40 & $3.25 \mathrm{E}-07$ & $3.25 \mathrm{E}-07$ & $3.25 \mathrm{E}-07$ & $3.25 \mathrm{E}-07$ & $3.25 \mathrm{E}-07$ & $3.25 \mathrm{E}-07$ & $3.25 \mathrm{E}-07$ & $3.25 \mathrm{E}-07$ & $3.25 \mathrm{E}-07$ \\
\hline K 41 & $1.06 \mathrm{E}-05$ & $1.06 \mathrm{E}-05$ & $1.06 \mathrm{E}-05$ & $1.06 \mathrm{E}-05$ & $1.06 \mathrm{E}-05$ & $1.06 \mathrm{E}-05$ & $1.06 \mathrm{E}-05$ & $1.06 \mathrm{E}-05$ & $1.06 \mathrm{E}-05$ \\
\hline K 42 & $1.25 \mathrm{E}-10$ & $1.22 \mathrm{E}-10$ & $9.48 \mathrm{E}-11$ & $3.27 \mathrm{E}-11$ & $1.02 \mathrm{E}-14$ & $8.24 \mathrm{E}-19$ & $6.96 \mathrm{E}-23$ & $6.95 \mathrm{E}-23$ & $6.83 \mathrm{E}-23$ \\
\hline CA 42 & $2.00 \mathrm{E}-08$ & $2.00 \mathrm{E}-08$ & 2.00E-08 & $2.01 \mathrm{E}-08$ & $2.01 \mathrm{E}-08$ & $2.01 \mathrm{E}-08$ & $2.01 \mathrm{E}-08$ & $2.01 \mathrm{E}-08$ & $2.01 \mathrm{E}-08$ \\
\hline CA 43 & $2.09 \mathrm{E}-09$ & $2.09 \mathrm{E}-09$ & $2.09 \mathrm{E}-09$ & $2.09 \mathrm{E}-09$ & $2.09 \mathrm{E}-09$ & $2.09 \mathrm{E}-09$ & $2.09 \mathrm{E}-09$ & $2.09 \mathrm{E}-09$ & $2.09 \mathrm{E}-09$ \\
\hline CA 44 & $1.61 \mathrm{E}-08$ & $1.61 \mathrm{E}-08$ & $1.61 \mathrm{E}-08$ & $1.61 \mathrm{E}-08$ & $1.61 \mathrm{E}-08$ & $1.61 \mathrm{E}-08$ & $1.61 \mathrm{E}-08$ & $1.61 \mathrm{E}-08$ & $1.61 \mathrm{E}-08$ \\
\hline CA 45 & $2.64 \mathrm{E}-10$ & $2.64 \mathrm{E}-10$ & $2.63 \mathrm{E}-10$ & $2.62 \mathrm{E}-10$ & $2.56 \mathrm{E}-10$ & $2.48 \mathrm{E}-10$ & $2.32 \mathrm{E}-10$ & $2.04 \mathrm{E}-10$ & $5.57 \mathrm{E}-11$ \\
\hline CA 46 & $1.90 \mathrm{E}-09$ & $1.90 \mathrm{E}-09$ & 1.90E-09 & 1.90E-09 & 1.90E-09 & 1.90E-09 & 1.90E-09 & 1.90E-09 & $1.90 \mathrm{E}-09$ \\
\hline CA 47 & $1.27 \mathrm{E}-13$ & $1.27 \mathrm{E}-13$ & $1.23 \mathrm{E}-13$ & $1.09 \mathrm{E}-13$ & $4.36 \mathrm{E}-14$ & $1.50 \mathrm{E}-14$ & $1.30 \mathrm{E}-15$ & $1.32 \mathrm{E}-17$ & $0.00 \mathrm{E}+00$ \\
\hline SC 45 & $7.09 \mathrm{E}-11$ & 7.09E-11 & $7.11 \mathrm{E}-11$ & $7.20 \mathrm{E}-11$ & $7.86 \mathrm{E}-11$ & $8.61 \mathrm{E}-11$ & 1.03E-10 & $1.30 \mathrm{E}-10$ & $2.79 \mathrm{E}-10$ \\
\hline SC 46 & $2.76 \mathrm{E}-08$ & $2.76 \mathrm{E}-08$ & $2.75 \mathrm{E}-08$ & $2.73 \mathrm{E}-08$ & $2.60 \mathrm{E}-08$ & $2.45 \mathrm{E}-08$ & $2.15 \mathrm{E}-08$ & $1.68 \mathrm{E}-08$ & $1.34 \mathrm{E}-09$ \\
\hline SC 47 & $2.80 \mathrm{E}-09$ & $2.79 \mathrm{E}-09$ & $2.68 \mathrm{E}-09$ & $2.28 \mathrm{E}-09$ & $6.58 \mathrm{E}-10$ & $1.55 \mathrm{E}-10$ & $5.65 \mathrm{E}-12$ & $1.14 \mathrm{E}-14$ & $0.00 \mathrm{E}+00$ \\
\hline SC 48 & $1.46 \mathrm{E}-10$ & $1.44 \mathrm{E}-10$ & $1.35 \mathrm{E}-10$ & $9.96 \mathrm{E}-11$ & $1.02 \mathrm{E}-11$ & $7.15 \mathrm{E}-13$ & $1.64 \mathrm{E}-15$ & $1.85 \mathrm{E}-20$ & $0.00 \mathrm{E}+00$ \\
\hline SC 49 & $2.94 \mathrm{E}-12$ & $2.05 \mathrm{E}-12$ & $7.89 \mathrm{E}-14$ & $8.49 \mathrm{E}-20$ & $0.00 \mathrm{E}+00$ & $0.00 \mathrm{E}+00$ & $0.00 \mathrm{E}+00$ & $0.00 \mathrm{E}+00$ & $0.00 \mathrm{E}+00$ \\
\hline SC 50 & $1.67 \mathrm{E}-15$ & $8.65 \mathrm{E}-21$ & $0.00 \mathrm{E}+00$ & $0.00 \mathrm{E}+00$ & $0.00 \mathrm{E}+00$ & $0.00 \mathrm{E}+00$ & $0.00 \mathrm{E}+00$ & $0.00 \mathrm{E}+00$ & $0.00 \mathrm{E}+00$ \\
\hline TI 46 & $9.10 \mathrm{E}-04$ & $9.10 \mathrm{E}-04$ & $9.10 \mathrm{E}-04$ & $9.10 \mathrm{E}-04$ & $9.10 \mathrm{E}-04$ & $9.10 \mathrm{E}-04$ & $9.10 \mathrm{E}-04$ & $9.10 \mathrm{E}-04$ & $9.10 \mathrm{E}-04$ \\
\hline TI 47 & $8.39 \mathrm{E}-04$ & $8.39 \mathrm{E}-04$ & $8.39 \mathrm{E}-04$ & $8.39 \mathrm{E}-04$ & 8.39E-04 & 8.39E-04 & 8.39E-04 & 8.39E-04 & 8.39E-04 \\
\hline TI 48 & $8.42 \mathrm{E}-03$ & $8.42 \mathrm{E}-03$ & $8.42 \mathrm{E}-03$ & $8.42 \mathrm{E}-03$ & $8.42 \mathrm{E}-03$ & $8.42 \mathrm{E}-03$ & $8.42 \mathrm{E}-03$ & $8.42 \mathrm{E}-03$ & $8.42 \mathrm{E}-03$ \\
\hline TI 49 & $7.09 \mathrm{E}-04$ & $7.09 \mathrm{E}-04$ & 7.09E-04 & 7.09E-04 & 7.09E-04 & 7.09E-04 & 7.09E-04 & 7.09E-04 & $7.09 \mathrm{E}-04$ \\
\hline TI 50 & $6.26 \mathrm{E}-04$ & $6.26 \mathrm{E}-04$ & $6.26 \mathrm{E}-04$ & $6.26 \mathrm{E}-04$ & $6.26 \mathrm{E}-04$ & $6.26 \mathrm{E}-04$ & $6.26 \mathrm{E}-04$ & $6.26 \mathrm{E}-04$ & $6.26 \mathrm{E}-04$ \\
\hline TI 51 & 6.57E-12 & $1.78 \mathrm{E}-13$ & $1.38 \mathrm{E}-27$ & $0.00 \mathrm{E}+00$ & $0.00 \mathrm{E}+00$ & $0.00 E+00$ & $0.00 \mathrm{E}+00$ & $0.00 \mathrm{E}+00$ & $0.00 \mathrm{E}+00$ \\
\hline V 50 & $7.84 \mathrm{E}-07$ & $7.84 \mathrm{E}-07$ & $7.84 \mathrm{E}-07$ & 7.84E-07 & $7.84 \mathrm{E}-07$ & 7.84E-07 & $7.84 \mathrm{E}-07$ & $7.84 \mathrm{E}-07$ & $7.84 \mathrm{E}-07$ \\
\hline V 51 & $1.08 \mathrm{E}-04$ & $1.08 \mathrm{E}-04$ & $1.08 \mathrm{E}-04$ & $1.09 \mathrm{E}-04$ & $1.16 \mathrm{E}-04$ & $1.23 \mathrm{E}-04$ & $1.35 \mathrm{E}-04$ & 1.47E-04 & $1.59 \mathrm{E}-04$ \\
\hline V 52 & $5.11 \mathrm{E}-11$ & $2.00 \mathrm{E}-13$ & $4.23 \mathrm{E}-35$ & $0.00 \mathrm{E}+00$ & $0.00 \mathrm{E}+00$ & $0.00 \mathrm{E}+00$ & $0.00 \mathrm{E}+00$ & $0.00 \mathrm{E}+00$ & $0.00 \mathrm{E}+00$ \\
\hline V 53 & $1.03 \mathrm{E}-13$ & $2.54 \mathrm{E}-19$ & $0.00 \mathrm{E}+00$ & $0.00 \mathrm{E}+00$ & $0.00 \mathrm{E}+00$ & $0.00 E+00$ & $0.00 \mathrm{E}+00$ & $0.00 \mathrm{E}+00$ & $0.00 \mathrm{E}+00$ \\
\hline CR 50 & $8.62 \mathrm{E}-03$ & $8.62 \mathrm{E}-03$ & $8.62 \mathrm{E}-03$ & $8.62 \mathrm{E}-03$ & $8.62 \mathrm{E}-03$ & $8.62 \mathrm{E}-03$ & $8.62 \mathrm{E}-03$ & $8.62 \mathrm{E}-03$ & $8.62 \mathrm{E}-03$ \\
\hline CR 51 & $5.04 \mathrm{E}-05$ & 5.03E-05 & $5.01 \mathrm{E}-05$ & $4.91 \mathrm{E}-05$ & $4.23 \mathrm{E}-05$ & $3.55 \mathrm{E}-05$ & $2.38 \mathrm{E}-05$ & $1.12 \mathrm{E}-05$ & $5.42 \mathrm{E}-09$ \\
\hline CR 52 & $1.75 \mathrm{E}-01$ & $1.75 \mathrm{E}-01$ & $1.75 \mathrm{E}-01$ & $1.75 \mathrm{E}-01$ & $1.75 \mathrm{E}-01$ & $1.75 \mathrm{E}-01$ & $1.75 \mathrm{E}-01$ & $1.75 \mathrm{E}-01$ & $1.75 \mathrm{E}-01$ \\
\hline CR 53 & $2.06 \mathrm{E}-02$ & $2.06 \mathrm{E}-02$ & $2.06 \mathrm{E}-02$ & $2.06 \mathrm{E}-02$ & 2.06E-02 & 2.06E-02 & 2.06E-02 & 2.06E-02 & $2.06 \mathrm{E}-02$ \\
\hline CR 54 & $5.57 \mathrm{E}-03$ & 5.57E-03 & 5.57E-03 & 5.57E-03 & 5.57E-03 & $5.57 \mathrm{E}-03$ & 5.57E-03 & 5.57E-03 & $5.57 \mathrm{E}-03$ \\
\hline CR 55 & $6.94 \mathrm{E}-11$ & $1.99 \mathrm{E}-13$ & $0.00 \mathrm{E}+00$ & $0.00 \mathrm{E}+00$ & $0.00 E+00$ & $0.00 E+00$ & $0.00 E+00$ & $0.00 E+00$ & $0.00 E+00$ \\
\hline MN 54 & $1.34 \mathrm{E}-06$ & $1.34 \mathrm{E}-06$ & $1.34 \mathrm{E}-06$ & $1.33 \mathrm{E}-06$ & $1.32 \mathrm{E}-06$ & $1.30 \mathrm{E}-06$ & $1.25 \mathrm{E}-06$ & 1.17E-06 & $5.95 \mathrm{E}-07$ \\
\hline MN 55 & $3.44 \mathrm{E}-03$ & $3.44 \mathrm{E}-03$ & $3.44 \mathrm{E}-03$ & $3.44 \mathrm{E}-03$ & $3.44 \mathrm{E}-03$ & $3.44 \mathrm{E}-03$ & $3.44 \mathrm{E}-03$ & $3.44 \mathrm{E}-03$ & $3.44 \mathrm{E}-03$ \\
\hline MN 56 & $9.75 \mathrm{E}-08$ & $8.52 \mathrm{E}-08$ & $2.54 \mathrm{E}-08$ & $1.54 \mathrm{E}-10$ & $2.38 \mathrm{E}-27$ & $0.00 E+00$ & $0.00 \mathrm{E}+00$ & $0.00 \mathrm{E}+00$ & $0.00 \mathrm{E}+00$ \\
\hline MN 57 & $5.60 \mathrm{E}-14$ & $1.38 \mathrm{E}-19$ & $0.00 \mathrm{E}+00$ & $0.00 \mathrm{E}+00$ & $0.00 \mathrm{E}+00$ & $0.00 E+00$ & $0.00 E+00$ & $0.00 \mathrm{E}+00$ & $0.00 \mathrm{E}+00$ \\
\hline FE 54 & $6.21 \mathrm{E}-03$ & $6.21 \mathrm{E}-03$ & $6.21 \mathrm{E}-03$ & $6.21 \mathrm{E}-03$ & $6.21 \mathrm{E}-03$ & $6.21 \mathrm{E}-03$ & $6.21 \mathrm{E}-03$ & $6.21 \mathrm{E}-03$ & $6.21 \mathrm{E}-03$ \\
\hline FE 55 & $2.12 \mathrm{E}-05$ & $2.12 \mathrm{E}-05$ & $2.12 \mathrm{E}-05$ & $2.11 \mathrm{E}-05$ & $2.10 \mathrm{E}-05$ & $2.09 \mathrm{E}-05$ & 2.07E-05 & $2.03 \mathrm{E}-05$ & $1.62 \mathrm{E}-05$ \\
\hline FE 56 & $1.02 \mathrm{E}-01$ & $1.02 \mathrm{E}-01$ & $1.02 \mathrm{E}-01$ & $1.02 \mathrm{E}-01$ & $1.02 \mathrm{E}-01$ & $1.02 \mathrm{E}-01$ & $1.02 \mathrm{E}-01$ & $1.02 \mathrm{E}-01$ & $1.02 \mathrm{E}-01$ \\
\hline FE 57 & $2.76 \mathrm{E}-03$ & $2.76 \mathrm{E}-03$ & $2.76 \mathrm{E}-03$ & $2.76 \mathrm{E}-03$ & $2.76 \mathrm{E}-03$ & $2.76 \mathrm{E}-03$ & $2.76 \mathrm{E}-03$ & $2.76 \mathrm{E}-03$ & $2.76 \mathrm{E}-03$ \\
\hline
\end{tabular}


TEM-10200-1 ENGINEERING CALCULATIONS AND ANALYSIS

Page 62 of 81

$12 / 19 / 17$

Rev.08

Title:

AS-RUN NEUTRONICS EVALUATION FOR THE CSM-10584 EXPERIMENT IN THE ATR

\begin{tabular}{llllllll} 
ECAR NO.: & 4496 & REV. NO.: & 1 & PROJECT NO.: & 32501 & Date: & 10/19/2020 \\
\hline
\end{tabular}

\begin{tabular}{|c|c|c|c|c|c|c|c|c|c|}
\hline \multicolumn{10}{|c|}{1 gram of inconel irradiated for 119 EFPDs } \\
\hline & EOC & $30 \mathrm{MINs}$ & $5 \mathrm{HRS}$ & $1 \mathrm{DAY}$ & 7 DAYS & 14 DAYS & 30 DAYS & 60 DAYS & 1 YEAR \\
\hline & $\begin{array}{l}\mathrm{g} / 1 \mathrm{~g} \text { of } \\
\text { inconel }\end{array}$ & $\begin{array}{l}\mathrm{g} / 1 \mathrm{~g} \text { of } \\
\text { inconel }\end{array}$ & $\begin{array}{l}\mathrm{g} / 1 \mathrm{~g} \text { of } \\
\text { inconel }\end{array}$ & $\begin{array}{l}\mathrm{g} / 1 \mathrm{~g} \text { of } \\
\text { inconel }\end{array}$ & $\begin{array}{l}\mathrm{g} / 1 \mathrm{~g} \text { of } \\
\text { inconel }\end{array}$ & $\begin{array}{l}\mathrm{g} / 1 \mathrm{~g} \text { of } \\
\text { inconel }\end{array}$ & $\begin{array}{l}\mathrm{g} / 1 \mathrm{~g} \text { of } \\
\text { inconel }\end{array}$ & $\begin{array}{l}\mathrm{g} / 1 \mathrm{~g} \text { of } \\
\text { inconel }\end{array}$ & $\begin{array}{l}\mathrm{g} / 1 \mathrm{~g} \text { of } \\
\text { inconel }\end{array}$ \\
\hline FE 58 & $3.59 \mathrm{E}-04$ & $3.59 \mathrm{E}-04$ & $3.59 \mathrm{E}-04$ & $3.59 \mathrm{E}-04$ & $3.60 \mathrm{E}-04$ & $3.61 \mathrm{E}-04$ & $3.63 \mathrm{E}-04$ & $3.67 \mathrm{E}-04$ & 3.77E-04 \\
\hline FE 59 & $2.48 \mathrm{E}-07$ & $2.48 \mathrm{E}-07$ & $2.48 \mathrm{E}-07$ & $2.45 \mathrm{E}-07$ & $2.23 \mathrm{E}-07$ & $2.00 \mathrm{E}-07$ & $1.56 \mathrm{E}-07$ & $9.86 \mathrm{E}-08$ & $8.95 \mathrm{E}-10$ \\
\hline CO 58 & $1.84 \mathrm{E}-05$ & $1.84 \mathrm{E}-05$ & $1.84 \mathrm{E}-05$ & $1.82 \mathrm{E}-05$ & $1.72 \mathrm{E}-05$ & $1.60 \mathrm{E}-05$ & 1.37E-05 & $1.02 \mathrm{E}-05$ & $5.14 \mathrm{E}-07$ \\
\hline CO 59 & $9.48 \mathrm{E}-03$ & $9.48 \mathrm{E}-03$ & $9.48 \mathrm{E}-03$ & $9.48 \mathrm{E}-03$ & $9.48 \mathrm{E}-03$ & $9.48 \mathrm{E}-03$ & $9.48 \mathrm{E}-03$ & $9.48 \mathrm{E}-03$ & $9.48 \mathrm{E}-03$ \\
\hline CO 60 & $5.69 \mathrm{E}-04$ & $5.69 \mathrm{E}-04$ & 5.69E-04 & 5.69E-04 & $5.68 \mathrm{E}-04$ & $5.67 \mathrm{E}-04$ & $5.63 \mathrm{E}-04$ & $5.57 \mathrm{E}-04$ & $4.99 \mathrm{E}-04$ \\
\hline $\mathrm{CO} 60 \mathrm{M}$ & $2.70 \mathrm{E}-08$ & $3.71 \mathrm{E}-09$ & $6.40 \mathrm{E}-17$ & $0.00 \mathrm{E}+00$ & $0.00 \mathrm{E}+00$ & $0.00 \mathrm{E}+00$ & $0.00 \mathrm{E}+00$ & $0.00 \mathrm{E}+00$ & $0.00 \mathrm{E}+00$ \\
\hline CO 61 & $1.61 \mathrm{E}-09$ & $1.30 \mathrm{E}-09$ & $1.97 \mathrm{E}-10$ & $6.72 \mathrm{E}-14$ & $0.00 \mathrm{E}+00$ & $0.00 \mathrm{E}+00$ & $0.00 \mathrm{E}+00$ & $0.00 \mathrm{E}+00$ & $0.00 \mathrm{E}+00$ \\
\hline $\mathrm{CO} 62$ & $1.03 \mathrm{E}-13$ & $9.80 \mathrm{E}-20$ & $0.00 \mathrm{E}+00$ & $0.00 \mathrm{E}+00$ & $0.00 \mathrm{E}+00$ & $0.00 \mathrm{E}+00$ & $0.00 \mathrm{E}+00$ & $0.00 \mathrm{E}+00$ & $0.00 \mathrm{E}+00$ \\
\hline NI 58 & $3.68 \mathrm{E}-01$ & $3.68 \mathrm{E}-01$ & $3.68 \mathrm{E}-01$ & $3.68 \mathrm{E}-01$ & $3.68 \mathrm{E}-01$ & $3.68 \mathrm{E}-01$ & $3.68 \mathrm{E}-01$ & $3.68 \mathrm{E}-01$ & $3.68 \mathrm{E}-01$ \\
\hline NI 59 & $2.07 \mathrm{E}-03$ & $2.07 \mathrm{E}-03$ & 2.07E-03 & 2.07E-03 & $2.07 \mathrm{E}-03$ & $2.07 \mathrm{E}-03$ & 2.07E-03 & $2.07 \mathrm{E}-03$ & $2.07 \mathrm{E}-03$ \\
\hline $\mathrm{NI} 60$ & $1.46 \mathrm{E}-01$ & $1.46 \mathrm{E}-01$ & $1.46 \mathrm{E}-01$ & $1.46 \mathrm{E}-01$ & $1.46 \mathrm{E}-01$ & $1.46 \mathrm{E}-01$ & $1.46 \mathrm{E}-01$ & $1.46 \mathrm{E}-01$ & $1.46 \mathrm{E}-01$ \\
\hline NI 61 & $6.91 \mathrm{E}-03$ & $6.91 \mathrm{E}-03$ & $6.91 \mathrm{E}-03$ & $6.91 \mathrm{E}-03$ & $6.91 \mathrm{E}-03$ & $6.91 \mathrm{E}-03$ & $6.91 \mathrm{E}-03$ & $6.91 \mathrm{E}-03$ & $6.91 \mathrm{E}-03$ \\
\hline NI 62 & $2.05 \mathrm{E}-02$ & $2.05 \mathrm{E}-02$ & $2.05 \mathrm{E}-02$ & $2.05 \mathrm{E}-02$ & $2.05 \mathrm{E}-02$ & $2.05 \mathrm{E}-02$ & $2.05 \mathrm{E}-02$ & $2.05 \mathrm{E}-02$ & $2.05 \mathrm{E}-02$ \\
\hline NI 63 & $3.31 \mathrm{E}-04$ & $3.31 \mathrm{E}-04$ & $3.31 \mathrm{E}-04$ & $3.31 \mathrm{E}-04$ & $3.31 \mathrm{E}-04$ & $3.31 \mathrm{E}-04$ & $3.31 \mathrm{E}-04$ & $3.31 \mathrm{E}-04$ & $3.28 \mathrm{E}-04$ \\
\hline NI 64 & $5.45 \mathrm{E}-03$ & $5.45 \mathrm{E}-03$ & $5.45 \mathrm{E}-03$ & $5.45 \mathrm{E}-03$ & $5.45 \mathrm{E}-03$ & $5.45 \mathrm{E}-03$ & $5.45 \mathrm{E}-03$ & $5.45 \mathrm{E}-03$ & $5.45 \mathrm{E}-03$ \\
\hline NI 65 & $1.26 \mathrm{E}-08$ & $1.10 \mathrm{E}-08$ & 3.19E-09 & $1.72 \mathrm{E}-11$ & $1.08 \mathrm{E}-28$ & $0.00 \mathrm{E}+00$ & $0.00 \mathrm{E}+00$ & $0.00 \mathrm{E}+00$ & $0.00 \mathrm{E}+00$ \\
\hline $\mathrm{NI} 66$ & $9.52 \mathrm{E}-12$ & $9.46 \mathrm{E}-12$ & $8.94 \mathrm{E}-12$ & $7.02 \mathrm{E}-12$ & $1.13 \mathrm{E}-12$ & $1.34 \mathrm{E}-13$ & $1.02 \mathrm{E}-15$ & $1.10 \mathrm{E}-19$ & $0.00 \mathrm{E}+00$ \\
\hline CU 63 & $2.04 \mathrm{E}-03$ & $2.04 \mathrm{E}-03$ & $2.04 \mathrm{E}-03$ & $2.04 \mathrm{E}-03$ & $2.04 \mathrm{E}-03$ & $2.04 \mathrm{E}-03$ & $2.04 \mathrm{E}-03$ & $2.05 \mathrm{E}-03$ & $2.05 \mathrm{E}-03$ \\
\hline CU 64 & $7.83 \mathrm{E}-08$ & $7.62 \mathrm{E}-08$ & $5.96 \mathrm{E}-08$ & $2.11 \mathrm{E}-08$ & $8.16 \mathrm{E}-12$ & $8.51 \mathrm{E}-16$ & $6.73 \mathrm{E}-25$ & $0.00 \mathrm{E}+00$ & $0.00 \mathrm{E}+00$ \\
\hline CU 65 & $9.51 \mathrm{E}-04$ & $9.51 \mathrm{E}-04$ & $9.51 \mathrm{E}-04$ & $9.51 \mathrm{E}-04$ & $9.51 \mathrm{E}-04$ & $9.51 \mathrm{E}-04$ & $9.51 \mathrm{E}-04$ & $9.51 \mathrm{E}-04$ & $9.51 \mathrm{E}-04$ \\
\hline CU 66 & $1.18 \mathrm{E}-10$ & $2.02 \mathrm{E}-12$ & $1.39 \mathrm{E}-14$ & 1.09E-14 & $1.76 \mathrm{E}-15$ & $2.09 \mathrm{E}-16$ & $1.60 \mathrm{E}-18$ & $1.71 \mathrm{E}-22$ & $0.00 \mathrm{E}+00$ \\
\hline CU 67 & $5.64 \mathrm{E}-13$ & $5.61 \mathrm{E}-13$ & $5.33 \mathrm{E}-13$ & $4.31 \mathrm{E}-13$ & $8.59 \mathrm{E}-14$ & $1.31 \mathrm{E}-14$ & $1.77 \mathrm{E}-16$ & $5.55 \mathrm{E}-20$ & $0.00 \mathrm{E}+00$ \\
\hline ZN 64 & $4.52 \mathrm{E}-06$ & $4.53 \mathrm{E}-06$ & $4.53 \mathrm{E}-06$ & $4.55 \mathrm{E}-06$ & $4.55 \mathrm{E}-06$ & $4.55 \mathrm{E}-06$ & $4.55 \mathrm{E}-06$ & $4.55 \mathrm{E}-06$ & $4.55 \mathrm{E}-06$ \\
\hline ZN 65 & $2.44 \mathrm{E}-09$ & $2.44 \mathrm{E}-09$ & $2.44 \mathrm{E}-09$ & 2.43E-09 & $2.39 \mathrm{E}-09$ & $2.34 \mathrm{E}-09$ & $2.24 \mathrm{E}-09$ & $2.06 \mathrm{E}-09$ & $8.64 \mathrm{E}-10$ \\
\hline ZN 66 & $2.74 \mathrm{E}-06$ & $2.74 \mathrm{E}-06$ & $2.74 \mathrm{E}-06$ & $2.74 \mathrm{E}-06$ & $2.74 \mathrm{E}-06$ & $2.74 \mathrm{E}-06$ & $2.74 \mathrm{E}-06$ & $2.74 \mathrm{E}-06$ & $2.74 \mathrm{E}-06$ \\
\hline ZN 67 & $1.50 \mathrm{E}-09$ & $1.50 \mathrm{E}-09$ & $1.50 \mathrm{E}-09$ & 1.50E-09 & $1.50 \mathrm{E}-09$ & $1.50 \mathrm{E}-09$ & 1.50E-09 & $1.50 \mathrm{E}-09$ & $1.50 \mathrm{E}-09$ \\
\hline ZN 68 & $6.50 \mathrm{E}-12$ & $6.50 \mathrm{E}-12$ & $6.50 \mathrm{E}-12$ & $6.50 \mathrm{E}-12$ & $6.50 \mathrm{E}-12$ & $6.50 \mathrm{E}-12$ & $6.50 \mathrm{E}-12$ & $6.50 \mathrm{E}-12$ & $6.50 \mathrm{E}-12$ \\
\hline GA 69 & 3.37E-15 & $3.37 \mathrm{E}-15$ & $3.38 \mathrm{E}-15$ & $3.38 \mathrm{E}-15$ & $3.38 \mathrm{E}-15$ & $3.38 \mathrm{E}-15$ & $3.38 \mathrm{E}-15$ & $3.38 \mathrm{E}-15$ & $3.38 \mathrm{E}-15$ \\
\hline SR 88 & $1.93 \mathrm{E}-14$ & $1.93 \mathrm{E}-14$ & $1.93 \mathrm{E}-14$ & 1.93E-14 & $1.93 \mathrm{E}-14$ & $1.93 \mathrm{E}-14$ & 1.93E-14 & $1.93 \mathrm{E}-14$ & $1.93 \mathrm{E}-14$ \\
\hline SR 89 & $1.29 \mathrm{E}-14$ & $1.29 \mathrm{E}-14$ & $1.28 \mathrm{E}-14$ & 1.27E-14 & $1.17 \mathrm{E}-14$ & $1.06 \mathrm{E}-14$ & $8.53 \mathrm{E}-15$ & $5.65 \mathrm{E}-15$ & $8.56 \mathrm{E}-17$ \\
\hline SR 90 & $1.09 \mathrm{E}-14$ & $1.09 \mathrm{E}-14$ & $1.09 \mathrm{E}-14$ & $1.09 \mathrm{E}-14$ & $1.09 \mathrm{E}-14$ & $1.09 \mathrm{E}-14$ & $1.09 \mathrm{E}-14$ & $1.09 \mathrm{E}-14$ & $1.07 \mathrm{E}-14$ \\
\hline Y 89 & $5.03 \mathrm{E}-10$ & $5.03 \mathrm{E}-10$ & $5.04 \mathrm{E}-10$ & 5.07E-10 & $5.19 \mathrm{E}-10$ & $5.23 \mathrm{E}-10$ & $5.24 \mathrm{E}-10$ & $5.24 \mathrm{E}-10$ & $5.24 \mathrm{E}-10$ \\
\hline Y 89M & $1.18 \mathrm{E}-15$ & $1.17 \mathrm{E}-15$ & $1.13 \mathrm{E}-15$ & $9.54 \mathrm{E}-16$ & $2.67 \mathrm{E}-16$ & $6.06 \mathrm{E}-17$ & $2.04 \mathrm{E}-18$ & $3.52 \mathrm{E}-21$ & $0.00 \mathrm{E}+00$ \\
\hline Y90 & $3.85 \mathrm{E}-10$ & $3.83 \mathrm{E}-10$ & $3.65 \mathrm{E}-10$ & $2.97 \mathrm{E}-10$ & $6.24 \mathrm{E}-11$ & $1.01 \mathrm{E}-11$ & $1.58 \mathrm{E}-13$ & $6.77 \mathrm{E}-17$ & $2.68 \mathrm{E}-18$ \\
\hline Y 91 & $1.08 \mathrm{E}-12$ & $1.07 \mathrm{E}-12$ & 1.07E-12 & $1.06 \mathrm{E}-12$ & $9.89 \mathrm{E}-13$ & $9.10 \mathrm{E}-13$ & $7.53 \mathrm{E}-13$ & $5.28 \mathrm{E}-13$ & $1.42 \mathrm{E}-14$ \\
\hline ZR 89 & $2.08 \mathrm{E}-11$ & $2.07 \mathrm{E}-11$ & 1.99E-11 & $1.68 \mathrm{E}-11$ & $4.71 \mathrm{E}-12$ & $1.07 \mathrm{E}-12$ & 3.59E-14 & $6.19 \mathrm{E}-17$ & $0.00 \mathrm{E}+00$ \\
\hline ZR 90 & $1.16 \mathrm{E}-08$ & $1.16 \mathrm{E}-08$ & $1.16 \mathrm{E}-08$ & 1.17E-08 & $1.19 \mathrm{E}-08$ & 1.19E-08 & 1.19E-08 & $1.19 \mathrm{E}-08$ & $1.19 \mathrm{E}-08$ \\
\hline ZR 91 & 7.13E-09 & 7.13E-09 & 7.13E-09 & 7.13E-09 & 7.13E-09 & 7.13E-09 & 7.13E-09 & 7.13E-09 & 7.13E-09 \\
\hline ZR 92 & $1.62 \mathrm{E}-07$ & $1.62 \mathrm{E}-07$ & $1.62 \mathrm{E}-07$ & $1.63 \mathrm{E}-07$ & $1.67 \mathrm{E}-07$ & $1.70 \mathrm{E}-07$ & $1.74 \mathrm{E}-07$ & $1.75 \mathrm{E}-07$ & $1.75 \mathrm{E}-07$ \\
\hline ZR 93 & $1.22 \mathrm{E}-07$ & $1.22 \mathrm{E}-07$ & $1.22 \mathrm{E}-07$ & $1.22 \mathrm{E}-07$ & $1.22 \mathrm{E}-07$ & $1.22 \mathrm{E}-07$ & $1.22 \mathrm{E}-07$ & $1.22 \mathrm{E}-07$ & $1.22 \mathrm{E}-07$ \\
\hline ZR 94 & $1.58 \mathrm{E}-08$ & $1.58 \mathrm{E}-08$ & $1.58 \mathrm{E}-08$ & $1.58 \mathrm{E}-08$ & $1.58 \mathrm{E}-08$ & $1.58 \mathrm{E}-08$ & $1.58 \mathrm{E}-08$ & $1.58 \mathrm{E}-08$ & $1.58 \mathrm{E}-08$ \\
\hline ZR 95 & 1.09E-09 & 1.09E-09 & $1.08 \mathrm{E}-09$ & 1.07E-09 & $1.01 \mathrm{E}-09$ & $9.33 \mathrm{E}-10$ & $7.84 \mathrm{E}-10$ & $5.67 \mathrm{E}-10$ & $2.08 \mathrm{E}-11$ \\
\hline ZR 96 & $1.87 \mathrm{E}-12$ & $1.87 \mathrm{E}-12$ & $1.87 \mathrm{E}-12$ & $1.87 \mathrm{E}-12$ & $1.87 \mathrm{E}-12$ & $1.87 \mathrm{E}-12$ & $1.87 \mathrm{E}-12$ & $1.87 \mathrm{E}-12$ & $1.87 \mathrm{E}-12$ \\
\hline ZR 97 & $3.86 \mathrm{E}-13$ & $3.78 \mathrm{E}-13$ & $3.14 \mathrm{E}-13$ & $1.44 \mathrm{E}-13$ & $3.92 \mathrm{E}-16$ & 3.97E-19 & $5.73 \mathrm{E}-26$ & $0.00 \mathrm{E}+00$ & $0.00 \mathrm{E}+00$ \\
\hline NB 92 & $1.33 \mathrm{E}-08$ & $1.33 \mathrm{E}-08$ & $1.31 \mathrm{E}-08$ & $1.24 \mathrm{E}-08$ & $8.24 \mathrm{E}-09$ & 5.11E-09 & $1.72 \mathrm{E}-09$ & $2.22 \mathrm{E}-10$ & $2.00 \mathrm{E}-19$ \\
\hline NB 93 & 5.47E-02 & $5.47 \mathrm{E}-02$ & 5.47E-02 & 5.47E-02 & $5.47 \mathrm{E}-02$ & 5.47E-02 & 5.47E-02 & 5.47E-02 & 5.47E-02 \\
\hline NB 93M & $8.49 \mathrm{E}-15$ & $8.50 \mathrm{E}-15$ & $8.52 \mathrm{E}-15$ & $8.64 \mathrm{E}-15$ & $9.49 \mathrm{E}-15$ & $1.05 \mathrm{E}-14$ & $1.28 \mathrm{E}-14$ & $1.70 \mathrm{E}-14$ & $5.91 \mathrm{E}-14$ \\
\hline NB 94 & $2.69 \mathrm{E}-04$ & $2.69 \mathrm{E}-04$ & $2.69 \mathrm{E}-04$ & $2.69 \mathrm{E}-04$ & $2.69 \mathrm{E}-04$ & $2.69 \mathrm{E}-04$ & $2.69 \mathrm{E}-04$ & $2.69 \mathrm{E}-04$ & $2.69 \mathrm{E}-04$ \\
\hline NB 95 & $3.71 \mathrm{E}-06$ & $3.71 \mathrm{E}-06$ & $3.70 \mathrm{E}-06$ & $3.64 \mathrm{E}-06$ & $3.23 \mathrm{E}-06$ & $2.82 \mathrm{E}-06$ & $2.06 \mathrm{E}-06$ & $1.14 \mathrm{E}-06$ & $2.79 \mathrm{E}-09$ \\
\hline NB 95M & $4.19 \mathrm{E}-13$ & $4.19 \mathrm{E}-13$ & $4.19 \mathrm{E}-13$ & $4.20 \mathrm{E}-13$ & $4.12 \mathrm{E}-13$ & $3.88 \mathrm{E}-13$ & $3.28 \mathrm{E}-13$ & $2.37 \mathrm{E}-13$ & $8.69 \mathrm{E}-15$ \\
\hline NB 96 & $5.12 \mathrm{E}-10$ & $5.04 \mathrm{E}-10$ & $4.41 \mathrm{E}-10$ & $2.51 \mathrm{E}-10$ & $3.49 \mathrm{E}-12$ & $2.38 \mathrm{E}-14$ & 2.67E-19 & $1.39 \mathrm{E}-28$ & $0.00 \mathrm{E}+00$ \\
\hline NB 97 & $9.73 \mathrm{E}-13$ & $7.36 \mathrm{E}-13$ & 7.67E-14 & 1.03E-14 & $2.80 \mathrm{E}-17$ & $2.85 \mathrm{E}-20$ & 4.39E-27 & $0.00 \mathrm{E}+00$ & $0.00 \mathrm{E}+00$ \\
\hline MO 92 & $4.68 \mathrm{E}-03$ & $4.68 \mathrm{E}-03$ & $4.68 \mathrm{E}-03$ & $4.68 \mathrm{E}-03$ & $4.68 \mathrm{E}-03$ & $4.68 \mathrm{E}-03$ & $4.68 \mathrm{E}-03$ & $4.68 \mathrm{E}-03$ & $4.68 \mathrm{E}-03$ \\
\hline
\end{tabular}


TEM-10200-1 ENGINEERING CALCULATIONS AND ANALYSIS

Page 63 of 81

$12 / 19 / 17$

Rev.08

Title:

AS-RUN NEUTRONICS EVALUATION FOR THE CSM-10584 EXPERIMENT IN THE ATR

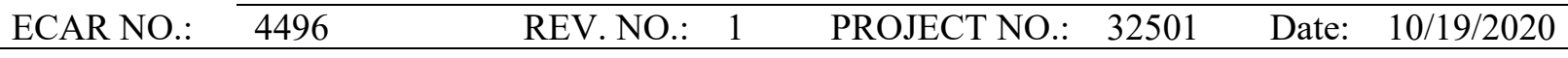

\begin{tabular}{|c|c|c|c|c|c|c|c|c|c|}
\hline \multicolumn{10}{|c|}{1 gram of inconel irradiated for 119 EFPDs } \\
\hline & EOC & $30 \mathrm{MINs}$ & $5 \mathrm{HRS}$ & $1 \mathrm{DAY}$ & 7 DAYS & 14 DAYS & 30 DAYS & 60 DAYS & 1 YEAR \\
\hline & $\begin{array}{l}\mathrm{g} / 1 \mathrm{~g} \text { of } \\
\text { inconel }\end{array}$ & $\begin{array}{l}\mathrm{g} / 1 \mathrm{~g} \text { of } \\
\text { inconel }\end{array}$ & $\begin{array}{l}\mathrm{g} / 1 \mathrm{~g} \text { of } \\
\text { inconel }\end{array}$ & $\begin{array}{l}\mathrm{g} / 1 \mathrm{~g} \text { of } \\
\text { inconel }\end{array}$ & $\begin{array}{l}\mathrm{g} / 1 \mathrm{~g} \text { of } \\
\text { inconel }\end{array}$ & $\begin{array}{l}\mathrm{g} / 1 \mathrm{~g} \text { of } \\
\text { inconel }\end{array}$ & $\begin{array}{l}\mathrm{g} / 1 \mathrm{~g} \text { of } \\
\text { inconel }\end{array}$ & $\begin{array}{l}\mathrm{g} / 1 \mathrm{~g} \text { of } \\
\text { inconel }\end{array}$ & $\begin{array}{l}\text { g/1g of } \\
\text { inconel }\end{array}$ \\
\hline $\mathrm{MO}$ & & & & & & & & & \\
\hline $93 \mathrm{M}$ & $1.10 \mathrm{E}-10$ & $1.04 \mathrm{E}-10$ & $6.61 \mathrm{E}-11$ & $9.66 \mathrm{E}-12$ & 4.54E-18 & $1.88 \mathrm{E}-25$ & $0.00 \mathrm{E}+00$ & $0.00 \mathrm{E}+00$ & $0.00 \mathrm{E}+00$ \\
\hline MO 93 & $9.74 \mathrm{E}-07$ & $9.74 \mathrm{E}-07$ & $9.74 \mathrm{E}-07$ & $9.74 \mathrm{E}-07$ & $9.74 \mathrm{E}-07$ & $9.74 \mathrm{E}-07$ & $9.74 \mathrm{E}-07$ & $9.74 \mathrm{E}-07$ & $9.74 \mathrm{E}-07$ \\
\hline MO 94 & $3.00 \mathrm{E}-03$ & $3.00 \mathrm{E}-03$ & $3.00 \mathrm{E}-03$ & $3.00 \mathrm{E}-03$ & $3.00 \mathrm{E}-03$ & $3.00 \mathrm{E}-03$ & $3.00 \mathrm{E}-03$ & $3.00 \mathrm{E}-03$ & $3.00 \mathrm{E}-03$ \\
\hline MO 95 & $4.94 \mathrm{E}-03$ & $4.94 \mathrm{E}-03$ & $4.94 \mathrm{E}-03$ & $4.94 \mathrm{E}-03$ & $4.94 \mathrm{E}-03$ & $4.94 \mathrm{E}-03$ & $4.94 \mathrm{E}-03$ & $4.94 \mathrm{E}-03$ & $4.94 \mathrm{E}-03$ \\
\hline MO 96 & $5.73 \mathrm{E}-03$ & $5.73 \mathrm{E}-03$ & $5.73 \mathrm{E}-03$ & $5.73 \mathrm{E}-03$ & $5.73 \mathrm{E}-03$ & $5.73 \mathrm{E}-03$ & $5.73 \mathrm{E}-03$ & $5.73 \mathrm{E}-03$ & $5.73 \mathrm{E}-03$ \\
\hline MO 97 & $3.22 \mathrm{E}-03$ & $3.22 \mathrm{E}-03$ & $3.22 \mathrm{E}-03$ & $3.22 \mathrm{E}-03$ & $3.22 \mathrm{E}-03$ & $3.22 \mathrm{E}-03$ & $3.22 \mathrm{E}-03$ & $3.22 \mathrm{E}-03$ & $3.22 \mathrm{E}-03$ \\
\hline MO 98 & $8.12 \mathrm{E}-03$ & $8.12 \mathrm{E}-03$ & $8.12 \mathrm{E}-03$ & $8.12 \mathrm{E}-03$ & $8.12 \mathrm{E}-03$ & $8.12 \mathrm{E}-03$ & $8.12 \mathrm{E}-03$ & $8.12 \mathrm{E}-03$ & $8.12 \mathrm{E}-03$ \\
\hline MO 99 & $7.86 \mathrm{E}-07$ & $7.82 \mathrm{E}-07$ & $7.46 \mathrm{E}-07$ & $6.11 \mathrm{E}-07$ & $1.35 \mathrm{E}-07$ & $2.31 \mathrm{E}-08$ & $4.09 \mathrm{E}-10$ & $2.13 \mathrm{E}-13$ & $0.00 \mathrm{E}+00$ \\
\hline MO100 & $3.29 \mathrm{E}-03$ & $3.29 \mathrm{E}-03$ & $3.29 \mathrm{E}-03$ & $3.29 \mathrm{E}-03$ & $3.29 \mathrm{E}-03$ & $3.29 \mathrm{E}-03$ & $3.29 \mathrm{E}-03$ & $3.29 \mathrm{E}-03$ & $3.29 \mathrm{E}-03$ \\
\hline MO101 & $7.37 \mathrm{E}-10$ & $1.78 \mathrm{E}-10$ & $4.90 \mathrm{E}-16$ & $0.00 \mathrm{E}+00$ & $0.00 \mathrm{E}+00$ & $0.00 \mathrm{E}+00$ & $0.00 \mathrm{E}+00$ & $0.00 \mathrm{E}+00$ & $0.00 \mathrm{E}+00$ \\
\hline TC 99 & $2.69 \mathrm{E}-06$ & $2.69 \mathrm{E}-06$ & $2.69 \mathrm{E}-06$ & $2.71 \mathrm{E}-06$ & $2.77 \mathrm{E}-06$ & $2.78 \mathrm{E}-06$ & $2.79 \mathrm{E}-06$ & $2.79 \mathrm{E}-06$ & $2.79 \mathrm{E}-06$ \\
\hline TC100 & $6.67 \mathrm{E}-13$ & $0.00 \mathrm{E}+00$ & $0.00 \mathrm{E}+00$ & $0.00 \mathrm{E}+00$ & $0.00 \mathrm{E}+00$ & $0.00 \mathrm{E}+00$ & $0.00 \mathrm{E}+00$ & $0.00 \mathrm{E}+00$ & $0.00 \mathrm{E}+00$ \\
\hline TC101 & $7.16 \mathrm{E}-10$ & $4.13 \mathrm{E}-10$ & $6.00 \mathrm{E}-15$ & $0.00 \mathrm{E}+00$ & $0.00 \mathrm{E}+00$ & $0.00 \mathrm{E}+00$ & $0.00 \mathrm{E}+00$ & $0.00 \mathrm{E}+00$ & $0.00 \mathrm{E}+00$ \\
\hline RU 99 & $1.41 \mathrm{E}-12$ & $1.41 \mathrm{E}-12$ & $1.42 \mathrm{E}-12$ & $1.43 \mathrm{E}-12$ & $1.58 \mathrm{E}-12$ & $1.75 \mathrm{E}-12$ & $2.15 \mathrm{E}-12$ & $2.90 \mathrm{E}-12$ & 1.05E-11 \\
\hline RU100 & $1.51 \mathrm{E}-07$ & $1.51 \mathrm{E}-07$ & $1.51 \mathrm{E}-07$ & 1.51E-07 & $1.51 \mathrm{E}-07$ & $1.51 \mathrm{E}-07$ & $1.51 \mathrm{E}-07$ & $1.51 \mathrm{E}-07$ & $1.51 \mathrm{E}-07$ \\
\hline RU101 & $5.88 \mathrm{E}-06$ & $5.88 \mathrm{E}-06$ & $5.88 \mathrm{E}-06$ & $5.88 \mathrm{E}-06$ & $5.88 \mathrm{E}-06$ & $5.88 \mathrm{E}-06$ & $5.88 \mathrm{E}-06$ & $5.88 \mathrm{E}-06$ & $5.88 \mathrm{E}-06$ \\
\hline RU102 & $1.07 \mathrm{E}-07$ & $1.07 \mathrm{E}-07$ & 1.07E-07 & 1.07E-07 & 1.07E-07 & 1.07E-07 & $1.07 \mathrm{E}-07$ & $1.07 \mathrm{E}-07$ & 1.07E-07 \\
\hline RU103 & $7.26 \mathrm{E}-11$ & $7.25 \mathrm{E}-11$ & $7.23 \mathrm{E}-11$ & $7.13 \mathrm{E}-11$ & $6.41 \mathrm{E}-11$ & $5.67 \mathrm{E}-11$ & $4.27 \mathrm{E}-11$ & $2.52 \mathrm{E}-11$ & $1.15 \mathrm{E}-13$ \\
\hline RU104 & $6.48 \mathrm{E}-13$ & $6.48 \mathrm{E}-13$ & $6.48 \mathrm{E}-13$ & $6.48 \mathrm{E}-13$ & $6.48 \mathrm{E}-13$ & $6.48 \mathrm{E}-13$ & $6.48 \mathrm{E}-13$ & $6.48 \mathrm{E}-13$ & $6.48 \mathrm{E}-13$ \\
\hline $\mathrm{RH} 103$ & $3.74 \mathrm{E}-12$ & $3.75 \mathrm{E}-12$ & $3.77 \mathrm{E}-12$ & 3.87E-12 & $4.58 \mathrm{E}-12$ & $5.32 \mathrm{E}-12$ & $6.71 \mathrm{E}-12$ & $8.45 \mathrm{E}-12$ & 1.09E-11 \\
\hline PD104 & $3.91 \mathrm{E}-13$ & $3.91 \mathrm{E}-13$ & $3.91 \mathrm{E}-13$ & $3.91 \mathrm{E}-13$ & $3.91 \mathrm{E}-13$ & $3.91 \mathrm{E}-13$ & $3.91 \mathrm{E}-13$ & $3.91 \mathrm{E}-13$ & $3.91 \mathrm{E}-13$ \\
\hline SUMTOT & $1.00 \mathrm{E}+00$ & $1.00 \mathrm{E}+00$ & $1.00 \mathrm{E}+00$ & $1.00 \mathrm{E}+00$ & $1.00 \mathrm{E}+00$ & $1.00 \mathrm{E}+00$ & $1.00 \mathrm{E}+00$ & $1.00 \mathrm{E}+00$ & $1.00 \mathrm{E}+00$ \\
\hline OTOTAL & $1.00 \mathrm{E}+00$ & $1.00 \mathrm{E}+00$ & $1.00 \mathrm{E}+00$ & $1.00 \mathrm{E}+00$ & $1.00 \mathrm{E}+00$ & $1.00 \mathrm{E}+00$ & $1.00 \mathrm{E}+00$ & $1.00 \mathrm{E}+00$ & $1.00 \mathrm{E}+00$ \\
\hline
\end{tabular}

\begin{tabular}{|c|c|c|c|c|c|c|c|c|c|}
\hline \multicolumn{10}{|c|}{1 gram of $3165 S$ irradiated for 54.9 EFPDs } \\
\hline & EOC & $30 \mathrm{MINs}$ & $5 \mathrm{HRS}$ & $1 \mathrm{DAY}$ & 7 DAYS & 14 DAYS & 30 DAYS & 60 DAYS & 1 YEAR \\
\hline & $\begin{array}{l}/ 1 g \text { of } \\
316 S S\end{array}$ & $\begin{array}{c}\mathrm{g} / 1 \mathrm{~g} \text { of } \\
316 \mathrm{SS}\end{array}$ & $\begin{array}{l}\mathrm{g} / 1 \mathrm{~g} \text { of } \\
316 \mathrm{SS}\end{array}$ & $\begin{array}{c}\mathrm{g} / 1 \mathrm{~g} \text { of } \\
316 \mathrm{SS}\end{array}$ & $\begin{array}{l}\mathrm{g} / 1 \mathrm{~g} \text { of } \\
316 \mathrm{SS}\end{array}$ & $\begin{array}{c}\mathrm{g} / 1 \mathrm{~g} \text { of } \\
316 \mathrm{SS}\end{array}$ & $\begin{array}{l}/ 1 \mathrm{~g} \text { of } \\
316 \mathrm{SS}\end{array}$ & $\begin{array}{l}\mathrm{g} / 1 \mathrm{~g} \text { of } \\
316 \mathrm{SS}\end{array}$ & $\begin{array}{l}\mathrm{g} / 1 \mathrm{~g} \text { of } \\
316 \mathrm{SS}\end{array}$ \\
\hline H 1 & $3.61 \mathrm{E}-07$ & $3.61 \mathrm{E}-07$ & $3.61 \mathrm{E}-07$ & $3.61 \mathrm{E}-07$ & $3.61 \mathrm{E}-07$ & $3.61 \mathrm{E}-07$ & $3.61 \mathrm{E}-07$ & $3.61 \mathrm{E}-07$ & $3.61 \mathrm{E}-07$ \\
\hline H 2 & $7.03 \mathrm{E}-11$ & $7.03 \mathrm{E}-11$ & $7.03 \mathrm{E}-11$ & 7.03E-11 & $7.03 \mathrm{E}-11$ & $7.03 \mathrm{E}-11$ & $7.03 \mathrm{E}-11$ & $7.03 \mathrm{E}-11$ & $7.03 \mathrm{E}-11$ \\
\hline H 3 & $6.37 \mathrm{E}-15$ & $6.37 E-15$ & $6.37 \mathrm{E}-15$ & $6.37 \mathrm{E}-15$ & $6.37 \mathrm{E}-15$ & $6.36 \mathrm{E}-15$ & $6.34 \mathrm{E}-15$ & $6.31 \mathrm{E}-15$ & $6.03 \mathrm{E}-15$ \\
\hline HE 4 & $1.48 \mathrm{E}-07$ & $1.48 \mathrm{E}-07$ & $1.48 \mathrm{E}-07$ & $1.48 \mathrm{E}-07$ & $1.48 \mathrm{E}-07$ & $1.48 \mathrm{E}-07$ & $1.48 \mathrm{E}-07$ & $1.48 \mathrm{E}-07$ & $1.48 \mathrm{E}-07$ \\
\hline LI 6 & 7.94E-14 & $7.94 \mathrm{E}-14$ & 7.94E-14 & $7.94 \mathrm{E}-14$ & $7.94 \mathrm{E}-14$ & 7.94E-14 & $7.94 \mathrm{E}-14$ & $7.94 \mathrm{E}-14$ & $7.94 \mathrm{E}-14$ \\
\hline BE 9 & $1.29 \mathrm{E}-09$ & $1.29 \mathrm{E}-09$ & $1.29 \mathrm{E}-09$ & $1.29 \mathrm{E}-09$ & $1.29 \mathrm{E}-09$ & $1.29 \mathrm{E}-09$ & $1.29 \mathrm{E}-09$ & $1.29 \mathrm{E}-09$ & $1.29 \mathrm{E}-09$ \\
\hline BE 10 & $3.30 \mathrm{E}-11$ & $3.30 \mathrm{E}-11$ & $3.30 \mathrm{E}-11$ & $3.30 \mathrm{E}-11$ & $3.30 \mathrm{E}-11$ & $3.30 \mathrm{E}-11$ & 3.30E-11 & $3.30 \mathrm{E}-11$ & $3.30 \mathrm{E}-11$ \\
\hline C 12 & $7.90 \mathrm{E}-04$ & $7.90 \mathrm{E}-04$ & $7.90 \mathrm{E}-04$ & $7.90 \mathrm{E}-04$ & $7.90 \mathrm{E}-04$ & $7.90 \mathrm{E}-04$ & $7.90 \mathrm{E}-04$ & $7.90 \mathrm{E}-04$ & $7.90 \mathrm{E}-04$ \\
\hline C 13 & $9.61 \mathrm{E}-06$ & $9.61 \mathrm{E}-06$ & $9.61 \mathrm{E}-06$ & $9.61 \mathrm{E}-06$ & $9.61 \mathrm{E}-06$ & $9.61 \mathrm{E}-06$ & $9.61 \mathrm{E}-06$ & $9.61 \mathrm{E}-06$ & $9.61 \mathrm{E}-06$ \\
\hline C 14 & $6.07 \mathrm{E}-12$ & $6.07 E-12$ & $6.07 \mathrm{E}-12$ & $6.07 \mathrm{E}-12$ & $6.07 \mathrm{E}-12$ & $6.07 \mathrm{E}-12$ & $6.07 \mathrm{E}-12$ & $6.07 \mathrm{E}-12$ & $6.07 \mathrm{E}-12$ \\
\hline NE 22 & $1.34 \mathrm{E}-13$ & $1.34 \mathrm{E}-13$ & $1.34 \mathrm{E}-13$ & $1.34 \mathrm{E}-13$ & $1.34 \mathrm{E}-13$ & $1.34 \mathrm{E}-13$ & $1.34 \mathrm{E}-13$ & $1.34 \mathrm{E}-13$ & $1.34 \mathrm{E}-13$ \\
\hline NA 23 & $1.08 \mathrm{E}-14$ & $1.08 \mathrm{E}-14$ & $1.08 \mathrm{E}-14$ & $1.08 \mathrm{E}-14$ & $1.08 \mathrm{E}-14$ & $1.08 \mathrm{E}-14$ & $1.08 \mathrm{E}-14$ & $1.08 \mathrm{E}-14$ & $1.08 \mathrm{E}-14$ \\
\hline MG 25 & $1.04 \mathrm{E}-08$ & 1.04E-08 & $1.04 \mathrm{E}-08$ & $1.04 \mathrm{E}-08$ & $1.04 \mathrm{E}-08$ & $1.04 \mathrm{E}-08$ & $1.04 \mathrm{E}-08$ & $1.04 \mathrm{E}-08$ & $1.04 \mathrm{E}-08$ \\
\hline MG 26 & $4.75 \mathrm{E}-09$ & 4.75E-09 & $4.75 \mathrm{E}-09$ & $4.75 \mathrm{E}-09$ & $4.75 \mathrm{E}-09$ & $4.75 \mathrm{E}-09$ & 4.75E-09 & $4.75 \mathrm{E}-09$ & $4.75 \mathrm{E}-09$ \\
\hline MG 27 & $8.84 \mathrm{E}-15$ & $9.82 \mathrm{E}-16$ & $2.52 \mathrm{E}-24$ & $0.00 \mathrm{E}+00$ & $0.00 \mathrm{E}+00$ & $0.00 \mathrm{E}+00$ & $0.00 \mathrm{E}+00$ & $0.00 \mathrm{E}+00$ & $0.00 \mathrm{E}+00$ \\
\hline AL 27 & $5.11 \mathrm{E}-11$ & $5.11 \mathrm{E}-11$ & $5.11 \mathrm{E}-11$ & $5.11 \mathrm{E}-11$ & $5.11 \mathrm{E}-11$ & $5.11 \mathrm{E}-11$ & $5.11 \mathrm{E}-11$ & $5.11 \mathrm{E}-11$ & $5.11 \mathrm{E}-11$ \\
\hline AL 28 & $2.61 \mathrm{E}-12$ & $2.43 \mathrm{E}-16$ & $6.55 \mathrm{E}-24$ & $3.49 \mathrm{E}-24$ & $3.22 \mathrm{E}-26$ & $2.91 \mathrm{E}-27$ & $8.65 \mathrm{E}-33$ & $0.00 \mathrm{E}+00$ & $0.00 \mathrm{E}+00$ \\
\hline AL 29 & $2.66 \mathrm{E}-13$ & $1.10 \mathrm{E}-14$ & $3.75 \mathrm{E}-27$ & $0.00 \mathrm{E}+00$ & $0.00 \mathrm{E}+00$ & $0.00 \mathrm{E}+00$ & $0.00 \mathrm{E}+00$ & $0.00 \mathrm{E}+00$ & $0.00 \mathrm{E}+00$ \\
\hline SI 28 & $9.19 \mathrm{E}-03$ & $9.19 \mathrm{E}-03$ & $9.19 \mathrm{E}-03$ & $9.19 \mathrm{E}-03$ & $9.19 \mathrm{E}-03$ & $9.19 \mathrm{E}-03$ & $9.19 \mathrm{E}-03$ & $9.19 \mathrm{E}-03$ & $9.19 \mathrm{E}-03$ \\
\hline SI 29 & 4.83E-04 & 4.83E-04 & $4.83 \mathrm{E}-04$ & $4.83 \mathrm{E}-04$ & 4.83E-04 & $4.83 \mathrm{E}-04$ & 4.83E-04 & 4.83E-04 & $4.83 \mathrm{E}-04$ \\
\hline $\mathrm{SI} 30$ & 3.31E-04 & 3.31E-04 & $3.31 \mathrm{E}-04$ & $3.31 \mathrm{E}-04$ & $3.31 \mathrm{E}-04$ & $3.31 \mathrm{E}-04$ & 3.31E-04 & 3.31E-04 & $3.31 \mathrm{E}-04$ \\
\hline SI 31 & $1.31 \mathrm{E}-10$ & $1.15 \mathrm{E}-10$ & $3.49 \mathrm{E}-11$ & $2.29 \mathrm{E}-13$ & $6.70 \mathrm{E}-30$ & $0.00 \mathrm{E}+00$ & $0.00 \mathrm{E}+00$ & $0.00 \mathrm{E}+00$ & $0.00 \mathrm{E}+00$ \\
\hline
\end{tabular}


TEM-10200-1

ENGINEERING CALCULATIONS AND ANALYSIS

Page 64 of 81

$12 / 19 / 17$

Rev.08

Title:

AS-RUN NEUTRONICS EVALUATION FOR THE CSM-10584 EXPERIMENT IN THE ATR

\begin{tabular}{llllllll} 
ECAR NO.: & 4496 & REV. NO.: & 1 & PROJECT NO.: & 32501 & Date: & 10/19/2020 \\
\hline
\end{tabular}

\begin{tabular}{|c|c|c|c|c|c|c|c|c|c|}
\hline \multicolumn{10}{|c|}{1 gram of $316 \mathrm{SS}$ irradiated for 54.9 EFPDs } \\
\hline & EOC & $30 \mathrm{MINs}$ & $5 \mathrm{HRS}$ & 1 DAY & 7 DAYS & 14 DAYS & 30 DAYS & 60 DAYS & 1 YEAR \\
\hline & $\begin{array}{l}\mathrm{g} / 1 \mathrm{~g} \text { of } \\
316 \mathrm{SS}\end{array}$ & $\begin{array}{l}\mathrm{g} / 1 \mathrm{~g} \text { of } \\
316 \mathrm{SS}\end{array}$ & $\begin{array}{l}\mathrm{g} / 1 \mathrm{~g} \text { of } \\
316 \mathrm{SS}\end{array}$ & $\begin{array}{l}\mathrm{g} / 1 \mathrm{~g} \text { of } \\
316 \mathrm{SS}\end{array}$ & $\begin{array}{l}\mathrm{g} / 1 \mathrm{~g} \text { of } \\
316 \mathrm{SS}\end{array}$ & $\begin{array}{l}\mathrm{g} / 1 \mathrm{~g} \text { of } \\
316 \mathrm{SS}\end{array}$ & $\begin{array}{l}\mathrm{g} / 1 \mathrm{~g} \text { of } \\
316 \mathrm{SS}\end{array}$ & $\begin{array}{l}g / 1 \mathrm{~g} \text { of } \\
316 \mathrm{SS}\end{array}$ & $\begin{array}{l}\mathrm{g} / 1 \mathrm{~g} \text { of } \\
316 \mathrm{SS}\end{array}$ \\
\hline SI 32 & $3.33 \mathrm{E}-14$ & $3.33 \mathrm{E}-14$ & $3.33 \mathrm{E}-14$ & $3.33 \mathrm{E}-14$ & $3.33 \mathrm{E}-14$ & $3.33 \mathrm{E}-14$ & $3.33 \mathrm{E}-14$ & $3.32 \mathrm{E}-14$ & $3.32 \mathrm{E}-14$ \\
\hline P 31 & $4.50 \mathrm{E}-04$ & $4.50 \mathrm{E}-04$ & $4.50 \mathrm{E}-04$ & $4.50 \mathrm{E}-04$ & $4.50 \mathrm{E}-04$ & $4.50 \mathrm{E}-04$ & $4.50 \mathrm{E}-04$ & 4.50E-04 & $4.50 \mathrm{E}-04$ \\
\hline P 32 & $2.53 \mathrm{E}-08$ & $2.52 \mathrm{E}-08$ & $2.50 \mathrm{E}-08$ & $2.41 \mathrm{E}-08$ & $1.80 \mathrm{E}-08$ & $1.28 \mathrm{E}-08$ & $5.90 \mathrm{E}-09$ & $1.38 \mathrm{E}-09$ & $5.19 \mathrm{E}-16$ \\
\hline P 33 & $1.25 \mathrm{E}-12$ & $1.25 \mathrm{E}-12$ & $1.24 \mathrm{E}-12$ & $1.22 \mathrm{E}-12$ & $1.03 \mathrm{E}-12$ & $8.47 \mathrm{E}-13$ & $5.44 \mathrm{E}-13$ & $2.37 \mathrm{E}-13$ & $5.00 \mathrm{E}-17$ \\
\hline S 32 & $2.84 \mathrm{E}-04$ & $2.84 \mathrm{E}-04$ & $2.84 \mathrm{E}-04$ & $2.84 \mathrm{E}-04$ & $2.84 \mathrm{E}-04$ & $2.84 \mathrm{E}-04$ & $2.84 \mathrm{E}-04$ & $2.84 \mathrm{E}-04$ & $2.84 \mathrm{E}-04$ \\
\hline 533 & $2.39 \mathrm{E}-06$ & $2.39 \mathrm{E}-06$ & $2.39 \mathrm{E}-06$ & $2.39 \mathrm{E}-06$ & $2.39 \mathrm{E}-06$ & $2.39 \mathrm{E}-06$ & $2.39 \mathrm{E}-06$ & $2.39 \mathrm{E}-06$ & $2.39 \mathrm{E}-06$ \\
\hline S 34 & $1.34 \mathrm{E}-05$ & $1.34 \mathrm{E}-05$ & $1.34 \mathrm{E}-05$ & $1.34 \mathrm{E}-05$ & $1.34 \mathrm{E}-05$ & $1.34 \mathrm{E}-05$ & $1.34 \mathrm{E}-05$ & $1.34 \mathrm{E}-05$ & $1.34 \mathrm{E}-05$ \\
\hline S 35 & $1.38 \mathrm{E}-09$ & $1.38 \mathrm{E}-09$ & $1.38 \mathrm{E}-09$ & $1.37 \mathrm{E}-09$ & $1.31 \mathrm{E}-09$ & $1.24 \mathrm{E}-09$ & 1.09E-09 & $8.62 \mathrm{E}-10$ & $7.78 \mathrm{E}-11$ \\
\hline S 36 & $5.72 \mathrm{E}-08$ & $5.72 \mathrm{E}-08$ & $5.72 \mathrm{E}-08$ & $5.72 \mathrm{E}-08$ & $5.72 \mathrm{E}-08$ & $5.72 \mathrm{E}-08$ & $5.72 \mathrm{E}-08$ & $5.72 \mathrm{E}-08$ & $5.72 \mathrm{E}-08$ \\
\hline CL 35 & $3.18 \mathrm{E}-10$ & $3.18 \mathrm{E}-10$ & $3.20 \mathrm{E}-10$ & $3.29 \mathrm{E}-10$ & $3.92 \mathrm{E}-10$ & $4.62 \mathrm{E}-10$ & $6.09 \mathrm{E}-10$ & $8.39 \mathrm{E}-10$ & $1.62 \mathrm{E}-09$ \\
\hline CL 36 & $2.46 \mathrm{E}-12$ & $2.46 \mathrm{E}-12$ & $2.46 \mathrm{E}-12$ & $2.46 \mathrm{E}-12$ & $2.46 \mathrm{E}-12$ & $2.46 \mathrm{E}-12$ & $2.46 \mathrm{E}-12$ & $2.46 \mathrm{E}-12$ & $2.46 \mathrm{E}-12$ \\
\hline CL 37 & $4.54 \mathrm{E}-12$ & $4.54 \mathrm{E}-12$ & $4.54 \mathrm{E}-12$ & $4.54 \mathrm{E}-12$ & $4.54 \mathrm{E}-12$ & 4.54E-12 & $4.54 \mathrm{E}-12$ & $4.54 \mathrm{E}-12$ & $4.54 \mathrm{E}-12$ \\
\hline CA 44 & $3.28 \mathrm{E}-14$ & $3.28 \mathrm{E}-14$ & $3.28 \mathrm{E}-14$ & $3.28 \mathrm{E}-14$ & $3.28 \mathrm{E}-14$ & $3.28 \mathrm{E}-14$ & $3.28 \mathrm{E}-14$ & $3.28 \mathrm{E}-14$ & $3.28 \mathrm{E}-14$ \\
\hline CA 46 & $1.31 \mathrm{E}-14$ & $1.31 \mathrm{E}-14$ & $1.31 \mathrm{E}-14$ & $1.31 \mathrm{E}-14$ & $1.31 \mathrm{E}-14$ & $1.31 \mathrm{E}-14$ & $1.31 \mathrm{E}-14$ & $1.31 \mathrm{E}-14$ & $1.31 \mathrm{E}-14$ \\
\hline SC 47 & $2.25 \mathrm{E}-14$ & $2.24 \mathrm{E}-14$ & $2.15 \mathrm{E}-14$ & $1.83 \mathrm{E}-14$ & $5.28 \mathrm{E}-15$ & $1.24 \mathrm{E}-15$ & $4.55 \mathrm{E}-17$ & $9.32 \mathrm{E}-20$ & $0.00 \mathrm{E}+00$ \\
\hline SC 48 & $7.46 \mathrm{E}-15$ & $7.40 \mathrm{E}-15$ & $6.89 \mathrm{E}-15$ & $5.10 \mathrm{E}-15$ & $5.23 \mathrm{E}-16$ & $3.66 \mathrm{E}-17$ & $8.42 \mathrm{E}-20$ & $9.49 \mathrm{E}-25$ & $0.00 \mathrm{E}+00$ \\
\hline TI 47 & $7.41 \mathrm{E}-09$ & $7.41 \mathrm{E}-09$ & $7.41 \mathrm{E}-09$ & $7.41 \mathrm{E}-09$ & $7.41 \mathrm{E}-09$ & $7.41 \mathrm{E}-09$ & $7.41 \mathrm{E}-09$ & $7.41 \mathrm{E}-09$ & $7.41 \mathrm{E}-09$ \\
\hline TI 48 & $3.90 \mathrm{E}-12$ & $3.90 \mathrm{E}-12$ & $3.90 \mathrm{E}-12$ & $3.91 \mathrm{E}-12$ & $3.91 \mathrm{E}-12$ & $3.91 \mathrm{E}-12$ & $3.91 \mathrm{E}-12$ & $3.91 \mathrm{E}-12$ & $3.91 \mathrm{E}-12$ \\
\hline TI 49 & $2.01 \mathrm{E}-08$ & $2.01 \mathrm{E}-08$ & $2.01 \mathrm{E}-08$ & $2.01 \mathrm{E}-08$ & $2.01 \mathrm{E}-08$ & $2.01 \mathrm{E}-08$ & $2.01 \mathrm{E}-08$ & $2.01 \mathrm{E}-08$ & $2.01 \mathrm{E}-08$ \\
\hline TI 50 & $8.02 \mathrm{E}-08$ & $8.02 \mathrm{E}-08$ & $8.02 \mathrm{E}-08$ & $8.02 \mathrm{E}-08$ & $8.02 \mathrm{E}-08$ & $8.02 \mathrm{E}-08$ & $8.02 \mathrm{E}-08$ & $8.02 \mathrm{E}-08$ & $8.02 \mathrm{E}-08$ \\
\hline TI 51 & $3.52 \mathrm{E}-14$ & $9.52 \mathrm{E}-16$ & $7.37 \mathrm{E}-30$ & $0.00 \mathrm{E}+00$ & $0.00 \mathrm{E}+00$ & $0.00 \mathrm{E}+00$ & $0.00 \mathrm{E}+00$ & $0.00 \mathrm{E}+00$ & $0.00 \mathrm{E}+00$ \\
\hline V 50 & $3.01 \mathrm{E}-07$ & $3.01 \mathrm{E}-07$ & $3.01 \mathrm{E}-07$ & $3.01 \mathrm{E}-07$ & $3.01 \mathrm{E}-07$ & $3.01 \mathrm{E}-07$ & $3.01 \mathrm{E}-07$ & $3.01 \mathrm{E}-07$ & $3.01 \mathrm{E}-07$ \\
\hline V 51 & $2.72 \mathrm{E}-05$ & $2.72 \mathrm{E}-05$ & $2.74 \mathrm{E}-05$ & $2.80 \mathrm{E}-05$ & $3.24 \mathrm{E}-05$ & $3.68 \mathrm{E}-05$ & $4.43 \mathrm{E}-05$ & $5.24 \mathrm{E}-05$ & $5.96 \mathrm{E}-05$ \\
\hline V 52 & $3.08 \mathrm{E}-11$ & $1.20 \mathrm{E}-13$ & $2.54 \mathrm{E}-35$ & $0.00 \mathrm{E}+00$ & $0.00 \mathrm{E}+00$ & $0.00 \mathrm{E}+00$ & $0.00 \mathrm{E}+00$ & $0.00 \mathrm{E}+00$ & $0.00 \mathrm{E}+00$ \\
\hline V 53 & $8.28 \mathrm{E}-14$ & $2.04 \mathrm{E}-19$ & $0.00 E+00$ & $0.00 \mathrm{E}+00$ & $0.00 \mathrm{E}+00$ & $0.00 \mathrm{E}+00$ & $0.00 \mathrm{E}+00$ & $0.00 \mathrm{E}+00$ & $0.00 \mathrm{E}+00$ \\
\hline CR 50 & $7.04 \mathrm{E}-03$ & $7.04 \mathrm{E}-03$ & $7.04 \mathrm{E}-03$ & $7.04 \mathrm{E}-03$ & $7.04 \mathrm{E}-03$ & $7.04 \mathrm{E}-03$ & $7.04 \mathrm{E}-03$ & $7.04 \mathrm{E}-03$ & $7.04 \mathrm{E}-03$ \\
\hline CR 51 & $3.24 \mathrm{E}-05$ & $3.24 \mathrm{E}-05$ & $3.22 \mathrm{E}-05$ & $3.16 \mathrm{E}-05$ & $2.72 \mathrm{E}-05$ & $2.28 \mathrm{E}-05$ & $1.53 \mathrm{E}-05$ & $7.22 \mathrm{E}-06$ & 3.49E-09 \\
\hline CR 52 & $1.42 \mathrm{E}-01$ & $1.42 \mathrm{E}-01$ & $1.42 \mathrm{E}-01$ & $1.42 \mathrm{E}-01$ & $1.42 \mathrm{E}-01$ & $1.42 \mathrm{E}-01$ & $1.42 \mathrm{E}-01$ & $1.42 \mathrm{E}-01$ & $1.42 \mathrm{E}-01$ \\
\hline CR 53 & $1.66 \mathrm{E}-02$ & $1.66 \mathrm{E}-02$ & $1.66 \mathrm{E}-02$ & $1.66 \mathrm{E}-02$ & $1.66 \mathrm{E}-02$ & $1.66 \mathrm{E}-02$ & $1.66 \mathrm{E}-02$ & $1.66 \mathrm{E}-02$ & $1.66 \mathrm{E}-02$ \\
\hline CR 54 & $4.32 \mathrm{E}-03$ & $4.32 \mathrm{E}-03$ & $4.32 \mathrm{E}-03$ & $4.32 \mathrm{E}-03$ & $4.32 \mathrm{E}-03$ & $4.32 \mathrm{E}-03$ & $4.32 \mathrm{E}-03$ & $4.32 \mathrm{E}-03$ & $4.32 \mathrm{E}-03$ \\
\hline CR 55 & $5.62 \mathrm{E}-11$ & $1.61 \mathrm{E}-13$ & $0.00 \mathrm{E}+00$ & $0.00 \mathrm{E}+00$ & $0.00 \mathrm{E}+00$ & $0.00 \mathrm{E}+00$ & $0.00 \mathrm{E}+00$ & $0.00 \mathrm{E}+00$ & $0.00 \mathrm{E}+00$ \\
\hline MN 54 & $3.90 \mathrm{E}-06$ & $3.90 \mathrm{E}-06$ & $3.90 \mathrm{E}-06$ & $3.89 \mathrm{E}-06$ & $3.84 \mathrm{E}-06$ & $3.78 \mathrm{E}-06$ & $3.65 \mathrm{E}-06$ & $3.41 \mathrm{E}-06$ & $1.74 \mathrm{E}-06$ \\
\hline MN 55 & $1.98 \mathrm{E}-02$ & $1.98 \mathrm{E}-02$ & $1.98 \mathrm{E}-02$ & $1.98 \mathrm{E}-02$ & $1.98 \mathrm{E}-02$ & $1.98 \mathrm{E}-02$ & $1.98 \mathrm{E}-02$ & $1.98 \mathrm{E}-02$ & $1.98 \mathrm{E}-02$ \\
\hline MN 56 & $5.02 \mathrm{E}-07$ & 4.39E-07 & $1.31 \mathrm{E}-07$ & $7.92 \mathrm{E}-10$ & $1.23 \mathrm{E}-26$ & $0.00 \mathrm{E}+00$ & $0.00 \mathrm{E}+00$ & $0.00 \mathrm{E}+00$ & $0.00 \mathrm{E}+00$ \\
\hline MN 57 & $3.08 \mathrm{E}-13$ & $7.57 \mathrm{E}-19$ & $0.00 \mathrm{E}+00$ & $0.00 \mathrm{E}+00$ & $0.00 \mathrm{E}+00$ & $0.00 \mathrm{E}+00$ & $0.00 \mathrm{E}+00$ & $0.00 \mathrm{E}+00$ & $0.00 \mathrm{E}+00$ \\
\hline FE 54 & $3.66 \mathrm{E}-02$ & $3.66 \mathrm{E}-02$ & $3.66 \mathrm{E}-02$ & $3.66 \mathrm{E}-02$ & $3.66 \mathrm{E}-02$ & $3.66 \mathrm{E}-02$ & $3.66 \mathrm{E}-02$ & $3.66 \mathrm{E}-02$ & $3.66 \mathrm{E}-02$ \\
\hline FE 55 & 4.39E-05 & 4.39E-05 & 4.39E-05 & $4.38 \mathrm{E}-05$ & 4.36E-05 & $4.34 \mathrm{E}-05$ & 4.29E-05 & $4.20 \mathrm{E}-05$ & $3.36 \mathrm{E}-05$ \\
\hline FE 56 & 5.99E-01 & 5.99E-01 & 5.99E-01 & 5.99E-01 & 5.99E-01 & 5.99E-01 & 5.99E-01 & 5.99E-01 & 5.99E-01 \\
\hline FE 57 & $1.52 \mathrm{E}-02$ & $1.52 \mathrm{E}-02$ & $1.52 \mathrm{E}-02$ & $1.52 \mathrm{E}-02$ & $1.52 \mathrm{E}-02$ & $1.52 \mathrm{E}-02$ & $1.52 \mathrm{E}-02$ & $1.52 \mathrm{E}-02$ & $1.52 \mathrm{E}-02$ \\
\hline FE 58 & $1.98 \mathrm{E}-03$ & $1.98 \mathrm{E}-03$ & $1.98 \mathrm{E}-03$ & $1.98 \mathrm{E}-03$ & $1.98 \mathrm{E}-03$ & 1.99E-03 & 1.99E-03 & $1.99 \mathrm{E}-03$ & 1.99E-03 \\
\hline FE 59 & $9.29 \mathrm{E}-07$ & $9.28 \mathrm{E}-07$ & $9.26 \mathrm{E}-07$ & $9.15 \mathrm{E}-07$ & $8.34 \mathrm{E}-07$ & 7.49E-07 & $5.85 \mathrm{E}-07$ & $3.69 \mathrm{E}-07$ & 3.35E-09 \\
\hline CO 58 & $3.66 \mathrm{E}-06$ & $3.66 \mathrm{E}-06$ & $3.65 \mathrm{E}-06$ & $3.63 \mathrm{E}-06$ & $3.42 \mathrm{E}-06$ & $3.19 \mathrm{E}-06$ & $2.73 \mathrm{E}-06$ & $2.03 \mathrm{E}-06$ & $1.02 \mathrm{E}-07$ \\
\hline $\mathrm{CO} 59$ & 4.95E-06 & 4.95E-06 & 4.95E-06 & 4.97E-06 & $5.05 \mathrm{E}-06$ & 5.13E-06 & $5.29 \mathrm{E}-06$ & $5.51 \mathrm{E}-06$ & $5.88 \mathrm{E}-06$ \\
\hline $\mathrm{CO} 60$ & $1.61 \mathrm{E}-07$ & $1.61 \mathrm{E}-07$ & $1.61 \mathrm{E}-07$ & $1.61 \mathrm{E}-07$ & 1.60E-07 & $1.60 \mathrm{E}-07$ & 1.59E-07 & $1.57 \mathrm{E}-07$ & $1.41 \mathrm{E}-07$ \\
\hline $\mathrm{CO} 60 \mathrm{M}$ & $1.41 \mathrm{E}-11$ & $1.94 \mathrm{E}-12$ & $3.34 \mathrm{E}-20$ & $0.00 \mathrm{E}+00$ & $0.00 \mathrm{E}+00$ & $0.00 \mathrm{E}+00$ & $0.00 \mathrm{E}+00$ & $0.00 \mathrm{E}+00$ & $0.00 \mathrm{E}+00$ \\
\hline $\mathrm{CO} 61$ & $8.11 \mathrm{E}-12$ & $6.57 \mathrm{E}-12$ & 9.93E-13 & $3.39 \mathrm{E}-16$ & $0.00 \mathrm{E}+00$ & $0.00 \mathrm{E}+00$ & $0.00 \mathrm{E}+00$ & $0.00 \mathrm{E}+00$ & $0.00 \mathrm{E}+00$ \\
\hline $\mathrm{CO} 62$ & $2.12 \mathrm{E}-14$ & $2.02 \mathrm{E}-20$ & $0.00 \mathrm{E}+00$ & $0.00 \mathrm{E}+00$ & $0.00 \mathrm{E}+00$ & $0.00 \mathrm{E}+00$ & $0.00 \mathrm{E}+00$ & $0.00 \mathrm{E}+00$ & $0.00 \mathrm{E}+00$ \\
\hline $\mathrm{NI} 58$ & $8.06 \mathrm{E}-02$ & $8.06 \mathrm{E}-02$ & $8.06 \mathrm{E}-02$ & $8.06 \mathrm{E}-02$ & $8.06 \mathrm{E}-02$ & $8.06 \mathrm{E}-02$ & $8.06 \mathrm{E}-02$ & $8.06 \mathrm{E}-02$ & $8.06 \mathrm{E}-02$ \\
\hline NI 59 & $2.16 \mathrm{E}-04$ & $2.16 \mathrm{E}-04$ & $2.16 \mathrm{E}-04$ & $2.16 \mathrm{E}-04$ & $2.16 \mathrm{E}-04$ & $2.16 \mathrm{E}-04$ & $2.16 \mathrm{E}-04$ & 2.16E-04 & $2.16 \mathrm{E}-04$ \\
\hline $\mathrm{NI} 60$ & $3.19 \mathrm{E}-02$ & $3.19 \mathrm{E}-02$ & $3.19 \mathrm{E}-02$ & $3.19 \mathrm{E}-02$ & $3.19 \mathrm{E}-02$ & 3.19E-02 & $3.19 \mathrm{E}-02$ & 3.19E-02 & $3.19 \mathrm{E}-02$ \\
\hline NI 61 & 1.45E-03 & $1.45 \mathrm{E}-03$ & $1.45 \mathrm{E}-03$ & $1.45 \mathrm{E}-03$ & $1.45 \mathrm{E}-03$ & $1.45 \mathrm{E}-03$ & $1.45 \mathrm{E}-03$ & $1.45 \mathrm{E}-03$ & $1.45 \mathrm{E}-03$ \\
\hline NI 62 & $4.52 \mathrm{E}-03$ & $4.52 \mathrm{E}-03$ & $4.52 \mathrm{E}-03$ & 4.52E-03 & $4.52 \mathrm{E}-03$ & $4.52 \mathrm{E}-03$ & 4.52E-03 & $4.52 \mathrm{E}-03$ & $4.52 \mathrm{E}-03$ \\
\hline NI 63 & 3.37E-05 & $3.37 \mathrm{E}-05$ & 3.37E-05 & 3.37E-05 & 3.37E-05 & $3.37 \mathrm{E}-05$ & 3.37E-05 & $3.37 \mathrm{E}-05$ & $3.34 \mathrm{E}-05$ \\
\hline NI 64 & $1.19 \mathrm{E}-03$ & $1.19 \mathrm{E}-03$ & $1.19 \mathrm{E}-03$ & $1.19 \mathrm{E}-03$ & $1.19 \mathrm{E}-03$ & $1.19 \mathrm{E}-03$ & $1.19 \mathrm{E}-03$ & $1.19 \mathrm{E}-03$ & $1.19 \mathrm{E}-03$ \\
\hline
\end{tabular}


TEM-10200-1

ENGINEERING CALCULATIONS AND ANALYSIS

Page 65 of 81

$12 / 19 / 17$

Rev.08

Title:

AS-RUN NEUTRONICS EVALUATION FOR THE CSM-10584 EXPERIMENT IN THE ATR

\begin{tabular}{llllllll} 
ECAR NO.: & 4496 & REV. NO.: & 1 & PROJECT NO.: & 32501 & Date: & 10/19/2020 \\
\hline
\end{tabular}

\begin{tabular}{|c|c|c|c|c|c|c|c|c|c|}
\hline \multicolumn{10}{|c|}{1 gram of $3165 S$ irradiated for 54.9 EFPDs } \\
\hline & EOC & $30 \mathrm{MINs}$ & $5 \mathrm{HRS}$ & 1 DAY & 7 DAYS & 14 DAYS & 30 DAYS & 60 DAYS & 1 YEAR \\
\hline & $\begin{array}{l}\mathrm{g} / 1 \mathrm{~g} \text { of } \\
316 \mathrm{SS}\end{array}$ & $\begin{array}{l}\mathrm{g} / 1 \mathrm{~g} \text { of } \\
316 \mathrm{SS}\end{array}$ & $\begin{array}{l}\mathrm{g} / 1 \mathrm{~g} \text { of } \\
316 \mathrm{SS}\end{array}$ & $\begin{array}{l}\mathrm{g} / 1 \mathrm{~g} \text { of } \\
316 \mathrm{SS}\end{array}$ & $\begin{array}{c}\mathrm{g} / 1 \mathrm{~g} \text { of } \\
316 \mathrm{SS}\end{array}$ & $\begin{array}{l}\mathrm{g} / 1 \mathrm{~g} \text { of } \\
316 \mathrm{SS}\end{array}$ & $\begin{array}{l}\mathrm{g} / 1 \mathrm{~g} \text { of } \\
316 \mathrm{SS}\end{array}$ & $\begin{array}{c}\mathrm{g} / 1 \mathrm{~g} \text { of } \\
316 \mathrm{SS}\end{array}$ & $\begin{array}{c}\mathrm{g} / 1 \mathrm{~g} \text { of } \\
316 \mathrm{SS}\end{array}$ \\
\hline NI 65 & $2.75 \mathrm{E}-09$ & $2.40 \mathrm{E}-09$ & $6.96 \mathrm{E}-10$ & $3.74 \mathrm{E}-12$ & $2.35 \mathrm{E}-29$ & $0.00 \mathrm{E}+00$ & $0.00 \mathrm{E}+00$ & $0.00 \mathrm{E}+00$ & $0.00 \mathrm{E}+00$ \\
\hline NI 66 & $2.09 \mathrm{E}-12$ & $2.08 \mathrm{E}-12$ & $1.96 \mathrm{E}-12$ & $1.54 \mathrm{E}-12$ & $2.48 \mathrm{E}-13$ & $2.94 \mathrm{E}-14$ & $2.25 \mathrm{E}-16$ & $2.42 \mathrm{E}-20$ & $0.00 \mathrm{E}+00$ \\
\hline CU 63 & $1.91 \mathrm{E}-08$ & $1.91 \mathrm{E}-08$ & $1.93 \mathrm{E}-08$ & $1.98 \mathrm{E}-08$ & $2.40 \mathrm{E}-08$ & $2.89 \mathrm{E}-08$ & $4.00 \mathrm{E}-08$ & $6.08 \mathrm{E}-08$ & $2.72 \mathrm{E}-07$ \\
\hline CU 64 & $7.49 \mathrm{E}-13$ & $7.29 \mathrm{E}-13$ & $5.70 \mathrm{E}-13$ & $2.02 \mathrm{E}-13$ & $7.81 \mathrm{E}-17$ & $8.14 \mathrm{E}-21$ & $6.43 \mathrm{E}-30$ & $0.00 \mathrm{E}+00$ & $0.00 \mathrm{E}+00$ \\
\hline CU 65 & $9.94 \mathrm{E}-07$ & $9.95 \mathrm{E}-07$ & $9.96 \mathrm{E}-07$ & 9.97E-07 & 9.97E-07 & 9.97E-07 & 9.97E-07 & 9.97E-07 & 9.97E-07 \\
\hline CU 66 & $1.27 \mathrm{E}-13$ & $5.33 \mathrm{E}-15$ & $3.06 \mathrm{E}-15$ & $2.41 \mathrm{E}-15$ & $3.87 \mathrm{E}-16$ & 4.59E-17 & $3.51 \mathrm{E}-19$ & $3.77 \mathrm{E}-23$ & $0.00 \mathrm{E}+00$ \\
\hline ZN 64 & $6.64 \mathrm{E}-12$ & $6.65 \mathrm{E}-12$ & $6.71 \mathrm{E}-12$ & $6.84 \mathrm{E}-12$ & $6.92 \mathrm{E}-12$ & $6.92 \mathrm{E}-12$ & $6.92 \mathrm{E}-12$ & $6.92 \mathrm{E}-12$ & $6.92 \mathrm{E}-12$ \\
\hline ZN 66 & $6.96 \mathrm{E}-10$ & $6.96 \mathrm{E}-10$ & $6.96 \mathrm{E}-10$ & $6.96 \mathrm{E}-10$ & $6.98 \mathrm{E}-10$ & $6.98 \mathrm{E}-10$ & $6.98 \mathrm{E}-10$ & $6.98 \mathrm{E}-10$ & $6.98 \mathrm{E}-10$ \\
\hline ZN 67 & $1.23 \mathrm{E}-13$ & $1.23 \mathrm{E}-13$ & $1.23 \mathrm{E}-13$ & $1.23 \mathrm{E}-13$ & $1.23 \mathrm{E}-13$ & $1.23 \mathrm{E}-13$ & $1.23 \mathrm{E}-13$ & $1.23 \mathrm{E}-13$ & $1.23 \mathrm{E}-13$ \\
\hline SR 88 & $3.11 \mathrm{E}-15$ & $3.11 \mathrm{E}-15$ & $3.11 \mathrm{E}-15$ & $3.11 \mathrm{E}-15$ & $3.11 \mathrm{E}-15$ & $3.11 \mathrm{E}-15$ & $3.11 \mathrm{E}-15$ & $3.11 \mathrm{E}-15$ & $3.11 \mathrm{E}-15$ \\
\hline SR 89 & $2.31 \mathrm{E}-15$ & $2.30 \mathrm{E}-15$ & $2.30 \mathrm{E}-15$ & $2.27 \mathrm{E}-15$ & $2.09 \mathrm{E}-15$ & $1.90 \mathrm{E}-15$ & $1.53 \mathrm{E}-15$ & $1.01 \mathrm{E}-15$ & $1.53 \mathrm{E}-17$ \\
\hline Y 89 & $1.67 \mathrm{E}-10$ & $1.68 \mathrm{E}-10$ & $1.68 \mathrm{E}-10$ & $1.71 \mathrm{E}-10$ & $1.80 \mathrm{E}-10$ & $1.82 \mathrm{E}-10$ & $1.83 \mathrm{E}-10$ & $1.83 \mathrm{E}-10$ & $1.83 \mathrm{E}-10$ \\
\hline Y 90 & $8.17 \mathrm{E}-15$ & $8.12 \mathrm{E}-15$ & $7.74 \mathrm{E}-15$ & $6.30 \mathrm{E}-15$ & $1.32 \mathrm{E}-15$ & $2.15 \mathrm{E}-16$ & $3.38 \mathrm{E}-18$ & $2.31 \mathrm{E}-20$ & $2.13 \mathrm{E}-20$ \\
\hline ZR 89 & $1.57 \mathrm{E}-11$ & $1.57 \mathrm{E}-11$ & $1.51 \mathrm{E}-11$ & $1.27 \mathrm{E}-11$ & $3.57 \mathrm{E}-12$ & $8.08 \mathrm{E}-13$ & $2.72 \mathrm{E}-14$ & $4.69 \mathrm{E}-17$ & $0.00 \mathrm{E}+00$ \\
\hline ZR 90 & $5.00 \mathrm{E}-14$ & $5.01 \mathrm{E}-14$ & $5.05 \mathrm{E}-14$ & $5.19 \mathrm{E}-14$ & $5.69 \mathrm{E}-14$ & $5.80 \mathrm{E}-14$ & $5.82 \mathrm{E}-14$ & $5.82 \mathrm{E}-14$ & $5.82 \mathrm{E}-14$ \\
\hline ZR 91 & $2.49 \mathrm{E}-09$ & $2.49 \mathrm{E}-09$ & $2.49 \mathrm{E}-09$ & $2.49 \mathrm{E}-09$ & $2.49 \mathrm{E}-09$ & $2.49 \mathrm{E}-09$ & $2.49 \mathrm{E}-09$ & $2.49 \mathrm{E}-09$ & $2.49 \mathrm{E}-09$ \\
\hline ZR 92 & $5.18 \mathrm{E}-08$ & $5.18 \mathrm{E}-08$ & $5.19 \mathrm{E}-08$ & $5.24 \mathrm{E}-08$ & $5.55 \mathrm{E}-08$ & $5.78 \mathrm{E}-08$ & $6.03 \mathrm{E}-08$ & $6.14 \mathrm{E}-08$ & $6.16 \mathrm{E}-08$ \\
\hline ZR 93 & $2.10 \mathrm{E}-09$ & $2.10 \mathrm{E}-09$ & $2.10 \mathrm{E}-09$ & $2.10 \mathrm{E}-09$ & $2.10 \mathrm{E}-09$ & $2.10 \mathrm{E}-09$ & $2.10 \mathrm{E}-09$ & $2.10 \mathrm{E}-09$ & $2.10 \mathrm{E}-09$ \\
\hline ZR 94 & $5.25 \mathrm{E}-09$ & $5.25 \mathrm{E}-09$ & $5.25 \mathrm{E}-09$ & $5.25 \mathrm{E}-09$ & $5.25 \mathrm{E}-09$ & $5.25 \mathrm{E}-09$ & $5.25 \mathrm{E}-09$ & $5.25 \mathrm{E}-09$ & $5.25 \mathrm{E}-09$ \\
\hline ZR 95 & $5.09 \mathrm{E}-10$ & $5.09 \mathrm{E}-10$ & $5.08 \mathrm{E}-10$ & $5.03 \mathrm{E}-10$ & $4.72 \mathrm{E}-10$ & $4.37 \mathrm{E}-10$ & $3.68 \mathrm{E}-10$ & $2.66 \mathrm{E}-10$ & $9.73 \mathrm{E}-12$ \\
\hline ZR 96 & $3.67 \mathrm{E}-13$ & $3.67 \mathrm{E}-13$ & $3.67 \mathrm{E}-13$ & $3.67 \mathrm{E}-13$ & $3.67 \mathrm{E}-13$ & $3.67 \mathrm{E}-13$ & $3.67 \mathrm{E}-13$ & 3.67E-13 & 3.67E-13 \\
\hline ZR 97 & $2.92 \mathrm{E}-13$ & $2.86 \mathrm{E}-13$ & $2.38 \mathrm{E}-13$ & $1.09 \mathrm{E}-13$ & $2.97 \mathrm{E}-16$ & $2.98 \mathrm{E}-19$ & $4.31 \mathrm{E}-26$ & $0.00 \mathrm{E}+00$ & $0.00 \mathrm{E}+00$ \\
\hline NB 92 & $9.83 \mathrm{E}-09$ & $9.81 \mathrm{E}-09$ & 9.69E-09 & $9.18 \mathrm{E}-09$ & $6.10 \mathrm{E}-09$ & $3.78 \mathrm{E}-09$ & $1.27 \mathrm{E}-09$ & $1.64 \mathrm{E}-10$ & 1.48E-19 \\
\hline NB 93 & $5.07 \mathrm{E}-12$ & $5.07 E-12$ & $5.10 \mathrm{E}-12$ & $5.25 \mathrm{E}-12$ & $6.36 \mathrm{E}-12$ & $7.65 \mathrm{E}-12$ & $1.06 \mathrm{E}-11$ & $1.62 \mathrm{E}-11$ & $7.25 \mathrm{E}-11$ \\
\hline NB 94 & $3.20 \mathrm{E}-09$ & $3.20 \mathrm{E}-09$ & $3.20 \mathrm{E}-09$ & $3.20 \mathrm{E}-09$ & $3.20 \mathrm{E}-09$ & $3.20 \mathrm{E}-09$ & $3.20 \mathrm{E}-09$ & $3.20 \mathrm{E}-09$ & 3.20E-09 \\
\hline NB 95 & $6.74 \mathrm{E}-10$ & $6.73 \mathrm{E}-10$ & $6.72 \mathrm{E}-10$ & $6.66 \mathrm{E}-10$ & $6.21 \mathrm{E}-10$ & $5.73 \mathrm{E}-10$ & $4.78 \mathrm{E}-10$ & $3.40 \mathrm{E}-10$ & 1.19E-11 \\
\hline NB 95M & $1.86 \mathrm{E}-13$ & $1.86 \mathrm{E}-13$ & $1.87 \mathrm{E}-13$ & $1.89 \mathrm{E}-13$ & $1.90 \mathrm{E}-13$ & $1.81 \mathrm{E}-13$ & $1.54 \mathrm{E}-13$ & $1.11 \mathrm{E}-13$ & 4.07E-15 \\
\hline NB 96 & $3.55 \mathrm{E}-11$ & $3.50 \mathrm{E}-11$ & $3.06 \mathrm{E}-11$ & $1.74 \mathrm{E}-11$ & $2.43 \mathrm{E}-13$ & $1.66 \mathrm{E}-15$ & $1.86 \mathrm{E}-20$ & $9.69 \mathrm{E}-30$ & $0.00 \mathrm{E}+00$ \\
\hline NB 97 & $7.35 \mathrm{E}-13$ & $5.56 \mathrm{E}-13$ & $5.80 \mathrm{E}-14$ & $7.81 \mathrm{E}-15$ & $2.13 \mathrm{E}-17$ & $2.16 \mathrm{E}-20$ & $3.31 \mathrm{E}-27$ & $0.00 \mathrm{E}+00$ & $0.00 \mathrm{E}+00$ \\
\hline MO 92 & $3.55 \mathrm{E}-03$ & $3.55 \mathrm{E}-03$ & $3.55 \mathrm{E}-03$ & $3.55 \mathrm{E}-03$ & $3.55 \mathrm{E}-03$ & $3.55 \mathrm{E}-03$ & $3.55 \mathrm{E}-03$ & $3.55 \mathrm{E}-03$ & $3.55 \mathrm{E}-03$ \\
\hline $\mathrm{MO}$ & & & & & & & & & \\
\hline $93 \mathrm{M}$ & 8.30E-11 & 7.89E-11 & $5.00 \mathrm{E}-11$ & $7.32 \mathrm{E}-12$ & 3.44E-18 & $1.42 \mathrm{E}-25$ & $0.00 \mathrm{E}+00$ & $0.00 E+00$ & $0.00 E+00$ \\
\hline MO 93 & $3.41 \mathrm{E}-07$ & $3.41 \mathrm{E}-07$ & $3.41 \mathrm{E}-07$ & $3.41 \mathrm{E}-07$ & $3.41 \mathrm{E}-07$ & $3.41 \mathrm{E}-07$ & $3.41 \mathrm{E}-07$ & $3.41 \mathrm{E}-07$ & $3.41 \mathrm{E}-07$ \\
\hline MO 94 & $2.28 \mathrm{E}-03$ & $2.28 \mathrm{E}-03$ & $2.28 \mathrm{E}-03$ & $2.28 \mathrm{E}-03$ & $2.28 \mathrm{E}-03$ & $2.28 \mathrm{E}-03$ & $2.28 \mathrm{E}-03$ & $2.28 \mathrm{E}-03$ & $2.28 \mathrm{E}-03$ \\
\hline MO 95 & $3.84 \mathrm{E}-03$ & $3.84 \mathrm{E}-03$ & $3.84 \mathrm{E}-03$ & $3.84 \mathrm{E}-03$ & $3.84 \mathrm{E}-03$ & $3.84 \mathrm{E}-03$ & $3.84 \mathrm{E}-03$ & $3.84 \mathrm{E}-03$ & $3.84 \mathrm{E}-03$ \\
\hline MO 96 & $4.25 \mathrm{E}-03$ & $4.25 \mathrm{E}-03$ & $4.25 \mathrm{E}-03$ & $4.25 \mathrm{E}-03$ & $4.25 \mathrm{E}-03$ & $4.25 \mathrm{E}-03$ & 4.25E-03 & $4.25 \mathrm{E}-03$ & 4.25E-03 \\
\hline MO 97 & $2.43 \mathrm{E}-03$ & $2.43 \mathrm{E}-03$ & $2.43 \mathrm{E}-03$ & $2.43 \mathrm{E}-03$ & $2.43 \mathrm{E}-03$ & $2.43 \mathrm{E}-03$ & $2.43 \mathrm{E}-03$ & $2.43 \mathrm{E}-03$ & $2.43 \mathrm{E}-03$ \\
\hline MO 98 & $6.15 \mathrm{E}-03$ & $6.15 \mathrm{E}-03$ & $6.15 \mathrm{E}-03$ & $6.15 \mathrm{E}-03$ & $6.15 \mathrm{E}-03$ & $6.15 \mathrm{E}-03$ & $6.15 \mathrm{E}-03$ & $6.15 \mathrm{E}-03$ & $6.15 \mathrm{E}-03$ \\
\hline MO 99 & $5.96 \mathrm{E}-07$ & 5.93E-07 & $5.65 \mathrm{E}-07$ & $4.63 \mathrm{E}-07$ & $1.02 \mathrm{E}-07$ & $1.75 \mathrm{E}-08$ & $3.10 \mathrm{E}-10$ & $1.61 \mathrm{E}-13$ & $0.00 \mathrm{E}+00$ \\
\hline MO100 & $2.50 \mathrm{E}-03$ & $2.50 \mathrm{E}-03$ & $2.50 \mathrm{E}-03$ & $2.50 \mathrm{E}-03$ & $2.50 \mathrm{E}-03$ & $2.50 \mathrm{E}-03$ & $2.50 \mathrm{E}-03$ & $2.50 \mathrm{E}-03$ & $2.50 \mathrm{E}-03$ \\
\hline M0101 & $5.59 \mathrm{E}-10$ & $1.35 \mathrm{E}-10$ & $3.72 \mathrm{E}-16$ & $0.00 \mathrm{E}+00$ & $0.00 \mathrm{E}+00$ & $0.00 \mathrm{E}+00$ & $0.00 \mathrm{E}+00$ & $0.00 \mathrm{E}+00$ & $0.00 \mathrm{E}+00$ \\
\hline TC 99 & $9.30 \mathrm{E}-07$ & $9.30 \mathrm{E}-07$ & $9.33 \mathrm{E}-07$ & $9.46 \mathrm{E}-07$ & $9.91 \mathrm{E}-07$ & $1.00 \mathrm{E}-06$ & $1.00 \mathrm{E}-06$ & 1.00E-06 & $1.00 \mathrm{E}-06$ \\
\hline TC100 & $2.31 \mathrm{E}-13$ & $0.00 \mathrm{E}+00$ & $0.00 E+00$ & $0.00 \mathrm{E}+00$ & $0.00 \mathrm{E}+00$ & $0.00 \mathrm{E}+00$ & $0.00 \mathrm{E}+00$ & $0.00 \mathrm{E}+00$ & $0.00 \mathrm{E}+00$ \\
\hline TC101 & $5.43 \mathrm{E}-10$ & $3.13 \mathrm{E}-10$ & $4.55 \mathrm{E}-15$ & $0.00 \mathrm{E}+00$ & $0.00 \mathrm{E}+00$ & $0.00 \mathrm{E}+00$ & $0.00 \mathrm{E}+00$ & $0.00 \mathrm{E}+00$ & $0.00 \mathrm{E}+00$ \\
\hline RU 99 & $2.12 \mathrm{E}-13$ & $2.12 \mathrm{E}-13$ & $2.14 \mathrm{E}-13$ & $2.21 \mathrm{E}-13$ & $2.73 \mathrm{E}-13$ & $3.35 \mathrm{E}-13$ & $4.78 \mathrm{E}-13$ & $7.46 \mathrm{E}-13$ & $3.48 \mathrm{E}-12$ \\
\hline RU100 & $2.26 \mathrm{E}-08$ & $2.26 \mathrm{E}-08$ & $2.26 \mathrm{E}-08$ & $2.26 \mathrm{E}-08$ & $2.26 \mathrm{E}-08$ & $2.26 \mathrm{E}-08$ & $2.26 \mathrm{E}-08$ & $2.26 \mathrm{E}-08$ & $2.26 \mathrm{E}-08$ \\
\hline RU101 & $2.08 \mathrm{E}-06$ & $2.08 \mathrm{E}-06$ & $2.08 \mathrm{E}-06$ & $2.08 \mathrm{E}-06$ & $2.08 \mathrm{E}-06$ & $2.08 \mathrm{E}-06$ & $2.08 \mathrm{E}-06$ & $2.08 \mathrm{E}-06$ & $2.08 \mathrm{E}-06$ \\
\hline RU102 & $1.74 \mathrm{E}-08$ & $1.74 \mathrm{E}-08$ & $1.74 \mathrm{E}-08$ & $1.74 \mathrm{E}-08$ & $1.74 \mathrm{E}-08$ & $1.74 \mathrm{E}-08$ & $1.74 \mathrm{E}-08$ & $1.74 \mathrm{E}-08$ & $1.74 \mathrm{E}-08$ \\
\hline RU103 & $6.83 \mathrm{E}-12$ & $6.82 \mathrm{E}-12$ & $6.80 \mathrm{E}-12$ & $6.71 \mathrm{E}-12$ & $6.03 \mathrm{E}-12$ & $5.33 \mathrm{E}-12$ & $4.02 \mathrm{E}-12$ & $2.37 \mathrm{E}-12$ & $1.08 \mathrm{E}-14$ \\
\hline RU104 & $2.69 \mathrm{E}-14$ & $2.69 \mathrm{E}-14$ & $2.69 \mathrm{E}-14$ & $2.69 \mathrm{E}-14$ & $2.69 \mathrm{E}-14$ & $2.69 \mathrm{E}-14$ & $2.69 \mathrm{E}-14$ & $2.69 \mathrm{E}-14$ & $2.69 \mathrm{E}-14$ \\
\hline RH103 & $1.64 \mathrm{E}-13$ & $1.65 \mathrm{E}-13$ & $1.67 \mathrm{E}-13$ & $1.76 \mathrm{E}-13$ & $2.43 \mathrm{E}-13$ & $3.13 \mathrm{E}-13$ & $4.43 \mathrm{E}-13$ & $6.07 \mathrm{E}-13$ & $8.42 \mathrm{E}-13$ \\
\hline PD104 & $7.61 \mathrm{E}-15$ & $7.61 \mathrm{E}-15$ & $7.61 \mathrm{E}-15$ & $7.61 \mathrm{E}-15$ & $7.61 \mathrm{E}-15$ & $7.61 \mathrm{E}-15$ & $7.61 \mathrm{E}-15$ & $7.61 \mathrm{E}-15$ & $7.61 \mathrm{E}-15$ \\
\hline SUMTOT & $1.00 \mathrm{E}+00$ & $1.00 \mathrm{E}+00$ & $1.00 \mathrm{E}+00$ & $1.00 \mathrm{E}+00$ & $1.00 \mathrm{E}+00$ & $1.00 E+00$ & $1.00 \mathrm{E}+00$ & $1.00 \mathrm{E}+00$ & $1.00 E+00$ \\
\hline
\end{tabular}




\section{TEM-10200-1 ENGINEERING CALCULATIONS AND ANALYSIS}

Title: AS-RUN NEUTRONICS EVALUATION FOR THE CSM-10584 EXPERIMENT IN THE ATR

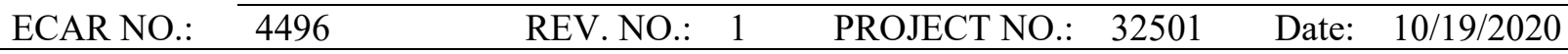

\begin{tabular}{|c|c|c|c|c|c|c|c|c|c|}
\hline \multicolumn{10}{|c|}{1 gram of 316SS irradiated for 54.9 EFPDs } \\
\hline & EOC & $30 \mathrm{MINs}$ & $5 \mathrm{HRS}$ & $1 \mathrm{DAY}$ & $7 \mathrm{DAYS}$ & $14 \mathrm{DAYS}$ & 30 DAYS & 60 DAYS & 1 YEAR \\
\hline & $\mathrm{g} / 1 \mathrm{~g}$ of & $\mathrm{g} / 1 \mathrm{~g}$ of & $\mathrm{g} / 1 \mathrm{~g}$ of & $\mathrm{g} / 1 \mathrm{~g}$ of & $\mathrm{g} / 1 \mathrm{~g}$ of & $\mathrm{g} / 1 \mathrm{~g}$ of & $\mathrm{g} / 1 \mathrm{~g}$ of & $\mathrm{g} / 1 \mathrm{~g}$ of & $\mathrm{g} / 1 \mathrm{~g}$ of \\
& $316 \mathrm{SS}$ & $316 \mathrm{SS}$ & $316 \mathrm{SS}$ & $316 \mathrm{SS}$ & $316 \mathrm{SS}$ & $316 \mathrm{SS}$ & $316 \mathrm{SS}$ & $316 \mathrm{SS}$ & $316 \mathrm{SS}$ \\
\hline OTOTAL & $1.00 \mathrm{E}+00$ & $1.00 \mathrm{E}+00$ & $1.00 \mathrm{E}+00$ & $1.00 \mathrm{E}+00$ & $1.00 \mathrm{E}+00$ & $1.00 \mathrm{E}+00$ & $1.00 \mathrm{E}+00$ & $1.00 \mathrm{E}+00$ & $1.00 \mathrm{E}+00$ \\
\hline
\end{tabular}

\begin{tabular}{|c|c|c|c|c|c|c|c|c|c|}
\hline \multicolumn{10}{|c|}{1 gram of $316 \mathrm{SS}$ irradiated for $119 \mathrm{EFPDs}$} \\
\hline & EOC & $30 \mathrm{MINs}$ & $5 \mathrm{HRS}$ & $1 \mathrm{DAY}$ & 7 DAYS & 14 DAYS & 30 DAYS & 60 DAYS & 1 Year \\
\hline & $\begin{array}{l}\mathrm{g} / 1 \mathrm{~g} \text { of } \\
316 \mathrm{SS}\end{array}$ & $\begin{array}{c}\mathrm{g} / 1 \mathrm{~g} \text { of } \\
316 \mathrm{SS}\end{array}$ & $\begin{array}{c}\mathrm{g} / 1 \mathrm{~g} \text { of } \\
316 \mathrm{SS}\end{array}$ & $\begin{array}{l}\mathrm{g} / 1 \mathrm{~g} \text { of } \\
316 \mathrm{SS}\end{array}$ & $\begin{array}{c}\mathrm{g} / 1 \mathrm{~g} \text { of } \\
316 \mathrm{SS}\end{array}$ & $\begin{array}{c}\mathrm{g} / 1 \mathrm{~g} \text { of } \\
316 \mathrm{SS}\end{array}$ & $\begin{array}{c}\mathrm{g} / 1 \mathrm{~g} \text { of } \\
316 \mathrm{SS}\end{array}$ & $\begin{array}{c}\mathrm{g} / 1 \mathrm{~g} \text { of } \\
316 \mathrm{SS}\end{array}$ & $\begin{array}{c}\mathrm{g} / 1 \mathrm{~g} \text { of } \\
316 \mathrm{SS}\end{array}$ \\
\hline H 1 & $7.82 \mathrm{E}-07$ & $7.82 \mathrm{E}-07$ & $7.82 \mathrm{E}-07$ & $7.82 \mathrm{E}-07$ & $7.82 \mathrm{E}-07$ & $7.82 \mathrm{E}-07$ & $7.82 \mathrm{E}-07$ & $7.82 \mathrm{E}-07$ & $7.82 \mathrm{E}-07$ \\
\hline H 2 & $3.30 \mathrm{E}-10$ & $3.30 \mathrm{E}-10$ & $3.30 \mathrm{E}-10$ & $3.30 \mathrm{E}-10$ & $3.30 \mathrm{E}-10$ & $3.30 \mathrm{E}-10$ & $3.30 \mathrm{E}-10$ & $3.30 \mathrm{E}-10$ & $3.30 \mathrm{E}-10$ \\
\hline H 3 & $5.74 \mathrm{E}-14$ & $5.74 \mathrm{E}-14$ & $5.74 \mathrm{E}-14$ & $5.74 \mathrm{E}-14$ & $5.74 \mathrm{E}-14$ & $5.73 \mathrm{E}-14$ & $5.72 \mathrm{E}-14$ & $5.69 \mathrm{E}-14$ & $5.43 \mathrm{E}-14$ \\
\hline HE 3 & $1.07 \mathrm{E}-16$ & $1.07 \mathrm{E}-16$ & $1.09 \mathrm{E}-16$ & $1.16 \mathrm{E}-16$ & $1.69 \mathrm{E}-16$ & $2.31 \mathrm{E}-16$ & $3.71 \mathrm{E}-16$ & $6.34 \mathrm{E}-16$ & $3.24 \mathrm{E}-15$ \\
\hline HE 4 & $4.31 \mathrm{E}-07$ & $4.31 \mathrm{E}-07$ & $4.31 \mathrm{E}-07$ & $4.31 \mathrm{E}-07$ & $4.31 \mathrm{E}-07$ & $4.31 \mathrm{E}-07$ & $4.31 \mathrm{E}-07$ & $4.31 \mathrm{E}-07$ & $4.31 \mathrm{E}-07$ \\
\hline LI 6 & $3.18 \mathrm{E}-13$ & $3.18 \mathrm{E}-13$ & $3.18 \mathrm{E}-13$ & $3.18 \mathrm{E}-13$ & $3.18 \mathrm{E}-13$ & $3.18 \mathrm{E}-13$ & $3.18 \mathrm{E}-13$ & $3.18 \mathrm{E}-13$ & $3.18 \mathrm{E}-13$ \\
\hline BE 9 & $2.80 \mathrm{E}-09$ & $2.80 \mathrm{E}-09$ & $2.80 \mathrm{E}-09$ & 2.80E-09 & $2.80 \mathrm{E}-09$ & $2.80 \mathrm{E}-09$ & 2.80E-09 & $2.80 \mathrm{E}-09$ & $2.80 \mathrm{E}-09$ \\
\hline BE 10 & $7.15 \mathrm{E}-11$ & $7.15 \mathrm{E}-11$ & $7.15 \mathrm{E}-11$ & 7.15E-11 & $7.15 \mathrm{E}-11$ & $7.15 \mathrm{E}-11$ & $7.15 \mathrm{E}-11$ & $7.15 \mathrm{E}-11$ & $7.15 \mathrm{E}-11$ \\
\hline C 12 & 7.90E-04 & 7.90E-04 & 7.90E-04 & 7.90E-04 & 7.90E-04 & 7.90E-04 & 7.90E-04 & 7.90E-04 & $7.90 \mathrm{E}-04$ \\
\hline C 13 & $9.61 \mathrm{E}-06$ & $9.61 \mathrm{E}-06$ & $9.61 \mathrm{E}-06$ & $9.61 \mathrm{E}-06$ & $9.61 \mathrm{E}-06$ & $9.61 \mathrm{E}-06$ & 9.61E-06 & $9.61 \mathrm{E}-06$ & $9.61 \mathrm{E}-06$ \\
\hline C 14 & $1.32 \mathrm{E}-11$ & $1.32 \mathrm{E}-11$ & $1.32 \mathrm{E}-11$ & $1.32 \mathrm{E}-11$ & $1.32 \mathrm{E}-11$ & $1.32 \mathrm{E}-11$ & $1.32 \mathrm{E}-11$ & $1.32 \mathrm{E}-11$ & $1.32 \mathrm{E}-11$ \\
\hline N 14 & $2.59 \mathrm{E}-16$ & $2.59 \mathrm{E}-16$ & $2.60 \mathrm{E}-16$ & $2.64 \mathrm{E}-16$ & $2.90 \mathrm{E}-16$ & $3.20 \mathrm{E}-16$ & $3.90 \mathrm{E}-16$ & $5.21 \mathrm{E}-16$ & $1.85 \mathrm{E}-15$ \\
\hline NE 22 & $6.27 \mathrm{E}-13$ & $6.27 \mathrm{E}-13$ & $6.27 \mathrm{E}-13$ & $6.27 \mathrm{E}-13$ & $6.27 \mathrm{E}-13$ & $6.27 \mathrm{E}-13$ & $6.27 \mathrm{E}-13$ & $6.27 \mathrm{E}-13$ & $6.27 \mathrm{E}-13$ \\
\hline NA 23 & $5.08 \mathrm{E}-14$ & $5.08 \mathrm{E}-14$ & $5.08 \mathrm{E}-14$ & $5.08 \mathrm{E}-14$ & $5.08 \mathrm{E}-14$ & $5.08 \mathrm{E}-14$ & $5.08 \mathrm{E}-14$ & $5.08 \mathrm{E}-14$ & $5.08 \mathrm{E}-14$ \\
\hline MG 25 & $2.26 \mathrm{E}-08$ & $2.26 \mathrm{E}-08$ & $2.26 \mathrm{E}-08$ & $2.26 \mathrm{E}-08$ & $2.26 \mathrm{E}-08$ & $2.26 \mathrm{E}-08$ & 2.26E-08 & $2.26 \mathrm{E}-08$ & $2.26 \mathrm{E}-08$ \\
\hline MG 26 & 1.03E-08 & $1.03 \mathrm{E}-08$ & $1.03 \mathrm{E}-08$ & 1.03E-08 & 1.03E-08 & $1.03 \mathrm{E}-08$ & 1.03E-08 & 1.03E-08 & $1.03 \mathrm{E}-08$ \\
\hline MG 27 & $8.86 \mathrm{E}-15$ & $9.84 \mathrm{E}-16$ & $2.53 \mathrm{E}-24$ & $0.00 \mathrm{E}+00$ & $0.00 \mathrm{E}+00$ & $0.00 \mathrm{E}+00$ & $0.00 \mathrm{E}+00$ & $0.00 \mathrm{E}+00$ & $0.00 \mathrm{E}+00$ \\
\hline AL 27 & $1.11 \mathrm{E}-10$ & $1.11 \mathrm{E}-10$ & $1.11 \mathrm{E}-10$ & $1.11 \mathrm{E}-10$ & $1.11 \mathrm{E}-10$ & $1.11 \mathrm{E}-10$ & $1.11 \mathrm{E}-10$ & $1.11 \mathrm{E}-10$ & $1.11 \mathrm{E}-10$ \\
\hline AL 28 & 2.61E-12 & $2.43 \mathrm{E}-16$ & $6.57 \mathrm{E}-24$ & $3.50 \mathrm{E}-24$ & $3.23 \mathrm{E}-26$ & $2.92 \mathrm{E}-27$ & 8.67E-33 & $0.00 \mathrm{E}+00$ & $0.00 \mathrm{E}+00$ \\
\hline AL 29 & 2.67E-13 & $1.10 \mathrm{E}-14$ & $3.76 \mathrm{E}-27$ & $0.00 \mathrm{E}+00$ & $0.00 \mathrm{E}+00$ & $0.00 \mathrm{E}+00$ & $0.00 \mathrm{E}+00$ & $0.00 \mathrm{E}+00$ & $0.00 \mathrm{E}+00$ \\
\hline SI 28 & $9.19 \mathrm{E}-03$ & $9.19 \mathrm{E}-03$ & $9.19 \mathrm{E}-03$ & 9.19E-03 & $9.19 \mathrm{E}-03$ & $9.19 \mathrm{E}-03$ & 9.19E-03 & 9.19E-03 & $9.19 \mathrm{E}-03$ \\
\hline SI 29 & 4.84E-04 & $4.84 \mathrm{E}-04$ & $4.84 \mathrm{E}-04$ & 4.84E-04 & 4.84E-04 & $4.84 \mathrm{E}-04$ & 4.84E-04 & 4.84E-04 & $4.84 \mathrm{E}-04$ \\
\hline SI 30 & 3.31E-04 & $3.31 \mathrm{E}-04$ & $3.31 \mathrm{E}-04$ & $3.31 \mathrm{E}-04$ & $3.31 \mathrm{E}-04$ & $3.31 \mathrm{E}-04$ & $3.31 \mathrm{E}-04$ & $3.31 \mathrm{E}-04$ & $3.31 \mathrm{E}-04$ \\
\hline SI 31 & $1.31 \mathrm{E}-10$ & $1.15 \mathrm{E}-10$ & 3.49E-11 & $2.29 \mathrm{E}-13$ & $6.70 \mathrm{E}-30$ & $0.00 \mathrm{E}+00$ & $0.00 \mathrm{E}+00$ & $0.00 \mathrm{E}+00$ & $0.00 \mathrm{E}+00$ \\
\hline SI 32 & 7.22E-14 & $7.22 \mathrm{E}-14$ & $7.22 \mathrm{E}-14$ & $7.22 \mathrm{E}-14$ & $7.22 \mathrm{E}-14$ & $7.22 \mathrm{E}-14$ & $7.22 \mathrm{E}-14$ & 7.22E-14 & $7.21 \mathrm{E}-14$ \\
\hline P 31 & $4.50 \mathrm{E}-04$ & 4.50E-04 & 4.50E-04 & $4.50 \mathrm{E}-04$ & 4.50E-04 & 4.50E-04 & $4.50 \mathrm{E}-04$ & 4.50E-04 & 4.50E-04 \\
\hline P 32 & 2.71E-08 & $2.70 \mathrm{E}-08$ & $2.68 \mathrm{E}-08$ & $2.58 \mathrm{E}-08$ & 1.93E-08 & $1.37 \mathrm{E}-08$ & $6.32 \mathrm{E}-09$ & $1.48 \mathrm{E}-09$ & $5.58 \mathrm{E}-16$ \\
\hline P 33 & 1.59E-12 & 1.59E-12 & $1.58 \mathrm{E}-12$ & $1.55 \mathrm{E}-12$ & $1.31 \mathrm{E}-12$ & $1.08 \mathrm{E}-12$ & $6.92 \mathrm{E}-13$ & $3.01 \mathrm{E}-13$ & $6.36 \mathrm{E}-17$ \\
\hline S 32 & $2.84 \mathrm{E}-04$ & $2.84 \mathrm{E}-04$ & $2.84 \mathrm{E}-04$ & $2.84 \mathrm{E}-04$ & $2.84 \mathrm{E}-04$ & $2.84 \mathrm{E}-04$ & $2.84 \mathrm{E}-04$ & $2.84 \mathrm{E}-04$ & $2.84 \mathrm{E}-04$ \\
\hline S 33 & $2.49 \mathrm{E}-06$ & 2.49E-06 & 2.49E-06 & $2.49 \mathrm{E}-06$ & $2.49 \mathrm{E}-06$ & 2.49E-06 & $2.49 \mathrm{E}-06$ & $2.49 \mathrm{E}-06$ & $2.49 \mathrm{E}-06$ \\
\hline S 34 & $1.34 \mathrm{E}-05$ & $1.34 \mathrm{E}-05$ & $1.34 \mathrm{E}-05$ & $1.34 \mathrm{E}-05$ & $1.34 \mathrm{E}-05$ & $1.34 \mathrm{E}-05$ & $1.34 \mathrm{E}-05$ & $1.34 \mathrm{E}-05$ & $1.34 \mathrm{E}-05$ \\
\hline S 35 & $2.40 \mathrm{E}-09$ & $2.40 \mathrm{E}-09$ & 2.39E-09 & $2.38 \mathrm{E}-09$ & $2.27 \mathrm{E}-09$ & 2.15E-09 & $1.89 \mathrm{E}-09$ & $1.49 \mathrm{E}-09$ & $1.35 \mathrm{E}-10$ \\
\hline S 36 & $5.72 \mathrm{E}-08$ & $5.72 \mathrm{E}-08$ & $5.72 \mathrm{E}-08$ & $5.72 \mathrm{E}-08$ & $5.72 \mathrm{E}-08$ & $5.72 \mathrm{E}-08$ & $5.72 \mathrm{E}-08$ & $5.72 \mathrm{E}-08$ & $5.72 \mathrm{E}-08$ \\
\hline CL 35 & 1.27E-09 & 1.27E-09 & $1.28 \mathrm{E}-09$ & 1.29E-09 & 1.40E-09 & $1.52 \mathrm{E}-09$ & $1.78 \mathrm{E}-09$ & $2.18 \mathrm{E}-09$ & $3.53 \mathrm{E}-09$ \\
\hline $\mathrm{CL} 36$ & $2.22 \mathrm{E}-11$ & $2.22 \mathrm{E}-11$ & $2.22 \mathrm{E}-11$ & $2.22 \mathrm{E}-11$ & 2.22E-11 & $2.22 \mathrm{E}-11$ & $2.22 \mathrm{E}-11$ & $2.22 \mathrm{E}-11$ & $2.22 \mathrm{E}-11$ \\
\hline CL 37 & $9.91 \mathrm{E}-12$ & $9.91 \mathrm{E}-12$ & $9.91 \mathrm{E}-12$ & $9.91 \mathrm{E}-12$ & $9.91 \mathrm{E}-12$ & $9.91 \mathrm{E}-12$ & $9.91 \mathrm{E}-12$ & $9.91 \mathrm{E}-12$ & $9.91 \mathrm{E}-12$ \\
\hline AR 38 & $2.60 \mathrm{E}-15$ & $2.60 \mathrm{E}-15$ & $2.60 \mathrm{E}-15$ & $2.60 \mathrm{E}-15$ & $2.60 \mathrm{E}-15$ & $2.60 \mathrm{E}-15$ & $2.60 \mathrm{E}-15$ & $2.60 \mathrm{E}-15$ & $2.60 \mathrm{E}-15$ \\
\hline CA 44 & $1.53 \mathrm{E}-13$ & $1.53 \mathrm{E}-13$ & $1.53 \mathrm{E}-13$ & $1.53 \mathrm{E}-13$ & $1.53 \mathrm{E}-13$ & $1.53 \mathrm{E}-13$ & $1.53 \mathrm{E}-13$ & $1.53 \mathrm{E}-13$ & $1.53 \mathrm{E}-13$ \\
\hline CA 46 & $6.15 E-14$ & $6.15 E-14$ & $6.15 \mathrm{E}-14$ & $6.15 \mathrm{E}-14$ & $6.15 E-14$ & $6.15 \mathrm{E}-14$ & $6.15 \mathrm{E}-14$ & $6.15 E-14$ & $6.15 \mathrm{E}-14$ \\
\hline SC 47 & $5.10 \mathrm{E}-14$ & $5.07 \mathrm{E}-14$ & $4.88 \mathrm{E}-14$ & $4.14 \mathrm{E}-14$ & $1.20 \mathrm{E}-14$ & $2.82 \mathrm{E}-15$ & $1.03 \mathrm{E}-16$ & $2.12 \mathrm{E}-19$ & $0.00 \mathrm{E}+00$ \\
\hline SC 48 & $2.40 \mathrm{E}-14$ & $2.38 \mathrm{E}-14$ & $2.22 \mathrm{E}-14$ & $1.64 \mathrm{E}-14$ & $1.68 \mathrm{E}-15$ & $1.18 \mathrm{E}-16$ & $2.71 \mathrm{E}-19$ & $3.05 E-24$ & $0.00 \mathrm{E}+00$ \\
\hline TI 47 & $1.60 \mathrm{E}-08$ & $1.60 \mathrm{E}-08$ & 1.60E-08 & $1.60 \mathrm{E}-08$ & $1.60 \mathrm{E}-08$ & 1.60E-08 & $1.60 \mathrm{E}-08$ & $1.60 \mathrm{E}-08$ & $1.60 \mathrm{E}-08$ \\
\hline TI 48 & $1.84 \mathrm{E}-11$ & $1.84 \mathrm{E}-11$ & $1.84 \mathrm{E}-11$ & $1.84 \mathrm{E}-11$ & $1.84 \mathrm{E}-11$ & $1.84 \mathrm{E}-11$ & $1.84 \mathrm{E}-11$ & $1.84 \mathrm{E}-11$ & $1.84 \mathrm{E}-11$ \\
\hline TI 49 & $4.35 \mathrm{E}-08$ & $4.35 \mathrm{E}-08$ & $4.35 \mathrm{E}-08$ & $4.35 \mathrm{E}-08$ & $4.35 \mathrm{E}-08$ & $4.35 \mathrm{E}-08$ & $4.35 \mathrm{E}-08$ & $4.35 \mathrm{E}-08$ & $4.35 \mathrm{E}-08$ \\
\hline TI 50 & $1.75 \mathrm{E}-07$ & $1.75 \mathrm{E}-07$ & $1.75 \mathrm{E}-07$ & $1.75 \mathrm{E}-07$ & $1.75 \mathrm{E}-07$ & $1.75 \mathrm{E}-07$ & $1.75 \mathrm{E}-07$ & $1.75 \mathrm{E}-07$ & $1.75 \mathrm{E}-07$ \\
\hline TI 51 & $3.87 \mathrm{E}-14$ & $1.05 \mathrm{E}-15$ & $8.12 \mathrm{E}-30$ & $0.00 E+00$ & $0.00 E+00$ & $0.00 E+00$ & $0.00 E+00$ & $0.00 \mathrm{E}+00$ & $0.00 E+00$ \\
\hline
\end{tabular}


TEM-10200-1

ENGINEERING CALCULATIONS AND ANALYSIS

Page 67 of 81

$12 / 19 / 17$

Rev.08

Title:

AS-RUN NEUTRONICS EVALUATION FOR THE CSM-10584 EXPERIMENT IN THE ATR

\begin{tabular}{llllllll} 
ECAR NO.: & 4496 & REV. NO.: & 1 & PROJECT NO.: & 32501 & Date: & 10/19/2020 \\
\hline
\end{tabular}

\begin{tabular}{|c|c|c|c|c|c|c|c|c|c|}
\hline \multicolumn{10}{|c|}{1 gram of $3165 S$ irradiated for 119 EFPDs } \\
\hline & EOC & $30 \mathrm{MINs}$ & $5 \mathrm{HRS}$ & 1 DAY & 7 DAYS & 14 DAYS & 30 DAYS & 60 DAYS & 1 Year \\
\hline & $\begin{array}{l}\mathrm{g} / 1 \mathrm{~g} \text { of } \\
316 \mathrm{SS}\end{array}$ & $\begin{array}{l}\mathrm{g} / 1 \mathrm{~g} \text { of } \\
316 \mathrm{SS}\end{array}$ & $\begin{array}{l}\mathrm{g} / 1 \mathrm{~g} \text { of } \\
316 \mathrm{SS}\end{array}$ & $\begin{array}{l}\mathrm{g} / 1 \mathrm{~g} \text { of } \\
316 \mathrm{SS}\end{array}$ & $\begin{array}{c}\mathrm{g} / 1 \mathrm{~g} \text { of } \\
316 \mathrm{SS}\end{array}$ & $\begin{array}{l}\mathrm{g} / 1 \mathrm{~g} \text { of } \\
316 \mathrm{SS}\end{array}$ & $\begin{array}{l}\mathrm{g} / 1 \mathrm{~g} \text { of } \\
316 \mathrm{SS}\end{array}$ & $\begin{array}{c}\mathrm{g} / 1 \mathrm{~g} \text { of } \\
316 \mathrm{SS}\end{array}$ & $\begin{array}{c}\mathrm{g} / 1 \mathrm{~g} \text { of } \\
316 \mathrm{SS}\end{array}$ \\
\hline V 50 & $6.35 \mathrm{E}-07$ & $6.35 \mathrm{E}-07$ & $6.35 \mathrm{E}-07$ & $6.35 \mathrm{E}-07$ & $6.35 \mathrm{E}-07$ & $6.35 \mathrm{E}-07$ & $6.35 \mathrm{E}-07$ & $6.35 \mathrm{E}-07$ & $6.35 \mathrm{E}-07$ \\
\hline V51 & $8.76 \mathrm{E}-05$ & $8.77 \mathrm{E}-05$ & $8.79 \mathrm{E}-05$ & $8.86 \mathrm{E}-05$ & $9.42 \mathrm{E}-05$ & 9.97E-05 & 1.09E-04 & 1.19E-04 & $1.29 \mathrm{E}-04$ \\
\hline V 52 & $4.16 \mathrm{E}-11$ & $1.63 \mathrm{E}-13$ & $3.44 \mathrm{E}-35$ & $0.00 \mathrm{E}+00$ & $0.00 \mathrm{E}+00$ & $0.00 \mathrm{E}+00$ & $0.00 \mathrm{E}+00$ & $0.00 \mathrm{E}+00$ & $0.00 \mathrm{E}+00$ \\
\hline V53 & $8.35 \mathrm{E}-14$ & $2.05 \mathrm{E}-19$ & $0.00 \mathrm{E}+00$ & $0.00 \mathrm{E}+00$ & $0.00 \mathrm{E}+00$ & $0.00 \mathrm{E}+00$ & $0.00 \mathrm{E}+00$ & $0.00 \mathrm{E}+00$ & $0.00 \mathrm{E}+00$ \\
\hline CR 50 & $6.98 \mathrm{E}-03$ & $6.98 \mathrm{E}-03$ & $6.98 \mathrm{E}-03$ & $6.98 \mathrm{E}-03$ & $6.98 \mathrm{E}-03$ & $6.98 \mathrm{E}-03$ & $6.98 \mathrm{E}-03$ & $6.98 \mathrm{E}-03$ & $6.98 \mathrm{E}-03$ \\
\hline CR 51 & 4.09E-05 & $4.08 \mathrm{E}-05$ & 4.06E-05 & $3.98 \mathrm{E}-05$ & $3.43 \mathrm{E}-05$ & $2.88 \mathrm{E}-05$ & $1.93 \mathrm{E}-05$ & $9.11 \mathrm{E}-06$ & $4.40 \mathrm{E}-09$ \\
\hline CR 52 & $1.42 \mathrm{E}-01$ & $1.42 \mathrm{E}-01$ & $1.42 \mathrm{E}-01$ & $1.42 \mathrm{E}-01$ & $1.42 \mathrm{E}-01$ & $1.42 \mathrm{E}-01$ & $1.42 \mathrm{E}-01$ & $1.42 \mathrm{E}-01$ & $1.42 \mathrm{E}-01$ \\
\hline CR 53 & $1.67 \mathrm{E}-02$ & $1.67 \mathrm{E}-02$ & 1.67E-02 & $1.67 \mathrm{E}-02$ & $1.67 \mathrm{E}-02$ & $1.67 \mathrm{E}-02$ & 1.67E-02 & 1.67E-02 & 1.67E-02 \\
\hline CR 54 & $4.51 \mathrm{E}-03$ & $4.51 \mathrm{E}-03$ & $4.51 \mathrm{E}-03$ & $4.51 \mathrm{E}-03$ & $4.51 \mathrm{E}-03$ & $4.51 \mathrm{E}-03$ & $4.51 \mathrm{E}-03$ & $4.51 \mathrm{E}-03$ & $4.51 \mathrm{E}-03$ \\
\hline CR 55 & $5.85 \mathrm{E}-11$ & $1.67 \mathrm{E}-13$ & $0.00 \mathrm{E}+00$ & $0.00 \mathrm{E}+00$ & $0.00 \mathrm{E}+00$ & $0.00 \mathrm{E}+00$ & $0.00 \mathrm{E}+00$ & $0.00 \mathrm{E}+00$ & $0.00 \mathrm{E}+00$ \\
\hline MN 54 & $7.86 \mathrm{E}-06$ & $7.86 \mathrm{E}-06$ & $7.86 \mathrm{E}-06$ & $7.85 \mathrm{E}-06$ & $7.74 \mathrm{E}-06$ & $7.62 \mathrm{E}-06$ & $7.36 \mathrm{E}-06$ & $6.88 \mathrm{E}-06$ & $3.50 \mathrm{E}-06$ \\
\hline MN 55 & $1.96 \mathrm{E}-02$ & $1.96 \mathrm{E}-02$ & $1.96 \mathrm{E}-02$ & $1.96 \mathrm{E}-02$ & $1.96 \mathrm{E}-02$ & $1.96 \mathrm{E}-02$ & $1.96 \mathrm{E}-02$ & 1.96E-02 & $1.97 \mathrm{E}-02$ \\
\hline MN 56 & $4.97 \mathrm{E}-07$ & $4.35 \mathrm{E}-07$ & $1.30 \mathrm{E}-07$ & $7.85 \mathrm{E}-10$ & $1.21 \mathrm{E}-26$ & $0.00 \mathrm{E}+00$ & $0.00 \mathrm{E}+00$ & $0.00 \mathrm{E}+00$ & $0.00 \mathrm{E}+00$ \\
\hline MN 57 & $3.29 \mathrm{E}-13$ & $8.09 \mathrm{E}-19$ & $0.00 E+00$ & $0.00 \mathrm{E}+00$ & $0.00 \mathrm{E}+00$ & $0.00 \mathrm{E}+00$ & $0.00 \mathrm{E}+00$ & $0.00 \mathrm{E}+00$ & $0.00 \mathrm{E}+00$ \\
\hline FE 54 & $3.65 \mathrm{E}-02$ & $3.65 \mathrm{E}-02$ & $3.65 \mathrm{E}-02$ & $3.65 \mathrm{E}-02$ & $3.65 \mathrm{E}-02$ & $3.65 \mathrm{E}-02$ & $3.65 \mathrm{E}-02$ & $3.65 \mathrm{E}-02$ & $3.65 \mathrm{E}-02$ \\
\hline FE 55 & $9.28 \mathrm{E}-05$ & $9.28 \mathrm{E}-05$ & $9.28 \mathrm{E}-05$ & $9.27 \mathrm{E}-05$ & $9.23 \mathrm{E}-05$ & $9.19 \mathrm{E}-05$ & $9.08 \mathrm{E}-05$ & $8.88 \mathrm{E}-05$ & $7.11 \mathrm{E}-05$ \\
\hline FE 56 & $5.99 \mathrm{E}-01$ & $5.99 \mathrm{E}-01$ & $5.99 \mathrm{E}-01$ & $5.99 \mathrm{E}-01$ & $5.99 \mathrm{E}-01$ & $5.99 \mathrm{E}-01$ & $5.99 \mathrm{E}-01$ & $5.99 \mathrm{E}-01$ & $5.99 \mathrm{E}-01$ \\
\hline FE 57 & $1.63 \mathrm{E}-02$ & $1.63 \mathrm{E}-02$ & $1.63 \mathrm{E}-02$ & $1.63 \mathrm{E}-02$ & $1.63 \mathrm{E}-02$ & $1.63 \mathrm{E}-02$ & $1.63 \mathrm{E}-02$ & $1.63 \mathrm{E}-02$ & $1.63 \mathrm{E}-02$ \\
\hline FE 58 & $2.01 \mathrm{E}-03$ & $2.01 \mathrm{E}-03$ & $2.01 \mathrm{E}-03$ & $2.01 \mathrm{E}-03$ & $2.01 \mathrm{E}-03$ & $2.01 \mathrm{E}-03$ & $2.01 \mathrm{E}-03$ & $2.01 \mathrm{E}-03$ & $2.01 \mathrm{E}-03$ \\
\hline FE 59 & $1.38 \mathrm{E}-06$ & $1.38 \mathrm{E}-06$ & $1.37 \mathrm{E}-06$ & $1.36 \mathrm{E}-06$ & $1.24 \mathrm{E}-06$ & $1.11 \mathrm{E}-06$ & $8.69 \mathrm{E}-07$ & 5.47E-07 & 4.97E-09 \\
\hline CO 58 & $4.02 \mathrm{E}-06$ & $4.01 \mathrm{E}-06$ & $4.01 \mathrm{E}-06$ & $3.98 \mathrm{E}-06$ & $3.75 \mathrm{E}-06$ & $3.50 \mathrm{E}-06$ & $2.99 \mathrm{E}-06$ & $2.23 \mathrm{E}-06$ & $1.12 \mathrm{E}-07$ \\
\hline CO 59 & $1.42 \mathrm{E}-05$ & $1.42 \mathrm{E}-05$ & $1.42 \mathrm{E}-05$ & $1.42 \mathrm{E}-05$ & $1.43 \mathrm{E}-05$ & $1.45 \mathrm{E}-05$ & $1.47 \mathrm{E}-05$ & $1.50 \mathrm{E}-05$ & $1.56 \mathrm{E}-05$ \\
\hline $\mathrm{CO} 60$ & $5.86 \mathrm{E}-07$ & $5.86 \mathrm{E}-07$ & $5.86 \mathrm{E}-07$ & $5.86 \mathrm{E}-07$ & $5.84 \mathrm{E}-07$ & $5.83 \mathrm{E}-07$ & $5.80 \mathrm{E}-07$ & $5.73 \mathrm{E}-07$ & $5.14 \mathrm{E}-07$ \\
\hline $\mathrm{CO} 60 \mathrm{M}$ & $4.05 \mathrm{E}-11$ & $5.55 \mathrm{E}-12$ & $9.58 \mathrm{E}-20$ & $0.00 \mathrm{E}+00$ & $0.00 \mathrm{E}+00$ & $0.00 \mathrm{E}+00$ & $0.00 \mathrm{E}+00$ & $0.00 \mathrm{E}+00$ & $0.00 \mathrm{E}+00$ \\
\hline $\mathrm{CO} 61$ & $9.56 \mathrm{E}-12$ & $7.75 \mathrm{E}-12$ & $1.17 \mathrm{E}-12$ & $4.00 \mathrm{E}-16$ & $0.00 \mathrm{E}+00$ & $0.00 \mathrm{E}+00$ & $0.00 \mathrm{E}+00$ & $0.00 \mathrm{E}+00$ & $0.00 \mathrm{E}+00$ \\
\hline $\mathrm{CO} 62$ & $2.10 \mathrm{E}-14$ & $2.01 \mathrm{E}-20$ & $0.00 \mathrm{E}+00$ & $0.00 \mathrm{E}+00$ & $0.00 \mathrm{E}+00$ & $0.00 \mathrm{E}+00$ & $0.00 \mathrm{E}+00$ & $0.00 \mathrm{E}+00$ & $0.00 \mathrm{E}+00$ \\
\hline NI 58 & $8.04 \mathrm{E}-02$ & $8.04 \mathrm{E}-02$ & $8.04 \mathrm{E}-02$ & $8.04 \mathrm{E}-02$ & $8.04 \mathrm{E}-02$ & $8.04 \mathrm{E}-02$ & $8.04 \mathrm{E}-02$ & 8.04E-02 & $8.04 \mathrm{E}-02$ \\
\hline NI 59 & $4.51 \mathrm{E}-04$ & $4.51 \mathrm{E}-04$ & $4.51 \mathrm{E}-04$ & $4.51 \mathrm{E}-04$ & $4.51 \mathrm{E}-04$ & $4.51 \mathrm{E}-04$ & $4.51 \mathrm{E}-04$ & $4.51 \mathrm{E}-04$ & $4.51 \mathrm{E}-04$ \\
\hline $\mathrm{NI} 60$ & $3.19 \mathrm{E}-02$ & $3.19 \mathrm{E}-02$ & $3.19 \mathrm{E}-02$ & $3.19 \mathrm{E}-02$ & $3.19 \mathrm{E}-02$ & $3.19 \mathrm{E}-02$ & $3.19 \mathrm{E}-02$ & $3.19 \mathrm{E}-02$ & $3.19 \mathrm{E}-02$ \\
\hline NI 61 & $1.51 \mathrm{E}-03$ & $1.51 \mathrm{E}-03$ & $1.51 \mathrm{E}-03$ & $1.51 \mathrm{E}-03$ & $1.51 \mathrm{E}-03$ & $1.51 \mathrm{E}-03$ & $1.51 \mathrm{E}-03$ & $1.51 \mathrm{E}-03$ & $1.51 \mathrm{E}-03$ \\
\hline NI 62 & $4.48 \mathrm{E}-03$ & $4.48 \mathrm{E}-03$ & $4.48 \mathrm{E}-03$ & $4.48 \mathrm{E}-03$ & $4.48 \mathrm{E}-03$ & $4.48 \mathrm{E}-03$ & $4.48 \mathrm{E}-03$ & $4.48 \mathrm{E}-03$ & $4.48 \mathrm{E}-03$ \\
\hline NI 63 & $7.22 \mathrm{E}-05$ & $7.22 \mathrm{E}-05$ & $7.22 \mathrm{E}-05$ & $7.22 \mathrm{E}-05$ & $7.22 \mathrm{E}-05$ & $7.22 \mathrm{E}-05$ & $7.21 \mathrm{E}-05$ & $7.21 \mathrm{E}-05$ & $7.16 \mathrm{E}-05$ \\
\hline NI 64 & $1.19 \mathrm{E}-03$ & $1.19 \mathrm{E}-03$ & $1.19 \mathrm{E}-03$ & $1.19 \mathrm{E}-03$ & $1.19 \mathrm{E}-03$ & $1.19 \mathrm{E}-03$ & $1.19 \mathrm{E}-03$ & $1.19 \mathrm{E}-03$ & $1.19 \mathrm{E}-03$ \\
\hline NI 65 & $2.75 \mathrm{E}-09$ & $2.40 \mathrm{E}-09$ & $6.95 \mathrm{E}-10$ & $3.74 \mathrm{E}-12$ & $2.35 \mathrm{E}-29$ & $0.00 \mathrm{E}+00$ & $0.00 \mathrm{E}+00$ & $0.00 \mathrm{E}+00$ & $0.00 \mathrm{E}+00$ \\
\hline NI 66 & $2.07 \mathrm{E}-12$ & $2.06 \mathrm{E}-12$ & $1.95 \mathrm{E}-12$ & $1.53 \mathrm{E}-12$ & $2.46 \mathrm{E}-13$ & $2.92 \mathrm{E}-14$ & $2.23 \mathrm{E}-16$ & $2.39 \mathrm{E}-20$ & $0.00 \mathrm{E}+00$ \\
\hline CU 63 & 8.91E-08 & $8.91 \mathrm{E}-08$ & $8.94 \mathrm{E}-08$ & 9.05E-08 & $9.95 \mathrm{E}-08$ & $1.10 \mathrm{E}-07$ & 1.34E-07 & $1.78 \mathrm{E}-07$ & 6.31E-07 \\
\hline CU 64 & $3.45 \mathrm{E}-12$ & $3.36 \mathrm{E}-12$ & $2.63 \mathrm{E}-12$ & $9.31 \mathrm{E}-13$ & $3.59 \mathrm{E}-16$ & $3.74 \mathrm{E}-20$ & $2.96 \mathrm{E}-29$ & $0.00 \mathrm{E}+00$ & $0.00 \mathrm{E}+00$ \\
\hline CU 65 & $2.16 \mathrm{E}-06$ & $2.16 \mathrm{E}-06$ & $2.16 \mathrm{E}-06$ & $2.16 \mathrm{E}-06$ & $2.16 \mathrm{E}-06$ & $2.16 \mathrm{E}-06$ & $2.16 \mathrm{E}-06$ & $2.16 \mathrm{E}-06$ & 2.16E-06 \\
\hline CU 66 & $2.71 \mathrm{E}-13$ & $7.75 \mathrm{E}-15$ & $3.03 E-15$ & $2.38 \mathrm{E}-15$ & $3.83 E-16$ & $4.55 \mathrm{E}-17$ & 3.47E-19 & $3.73 E-23$ & $0.00 E+00$ \\
\hline CU 67 & $1.25 \mathrm{E}-15$ & $1.25 \mathrm{E}-15$ & 1.19E-15 & $9.59 \mathrm{E}-16$ & $1.91 \mathrm{E}-16$ & $2.91 \mathrm{E}-17$ & 3.93E-19 & 1.23E-22 & $0.00 \mathrm{E}+00$ \\
\hline ZN 64 & $6.67 \mathrm{E}-11$ & $6.67 \mathrm{E}-11$ & $6.70 \mathrm{E}-11$ & $6.76 \mathrm{E}-11$ & $6.80 \mathrm{E}-11$ & $6.80 \mathrm{E}-11$ & $6.80 \mathrm{E}-11$ & $6.80 \mathrm{E}-11$ & $6.80 \mathrm{E}-11$ \\
\hline ZN 65 & $1.90 \mathrm{E}-14$ & $1.90 \mathrm{E}-14$ & $1.90 \mathrm{E}-14$ & $1.90 \mathrm{E}-14$ & $1.87 \mathrm{E}-14$ & $1.83 \mathrm{E}-14$ & $1.75 \mathrm{E}-14$ & $1.60 \mathrm{E}-14$ & $6.74 \mathrm{E}-15$ \\
\hline ZN 66 & $3.19 \mathrm{E}-09$ & 3.19E-09 & 3.19E-09 & $3.19 \mathrm{E}-09$ & 3.19E-09 & 3.19E-09 & $3.19 \mathrm{E}-09$ & 3.19E-09 & $3.19 \mathrm{E}-09$ \\
\hline ZN 67 & 1.19E-12 & 1.19E-12 & 1.19E-12 & 1.19E-12 & 1.19E-12 & 1.19E-12 & 1.19E-12 & $1.19 \mathrm{E}-12$ & 1.19E-12 \\
\hline ZN 68 & $3.90 \mathrm{E}-15$ & $3.90 \mathrm{E}-15$ & $3.90 \mathrm{E}-15$ & $3.90 \mathrm{E}-15$ & $3.90 \mathrm{E}-15$ & $3.90 \mathrm{E}-15$ & $3.90 \mathrm{E}-15$ & $3.90 \mathrm{E}-15$ & 3.90E-15 \\
\hline SR 88 & $1.46 \mathrm{E}-14$ & $1.46 \mathrm{E}-14$ & $1.46 \mathrm{E}-14$ & $1.46 \mathrm{E}-14$ & $1.46 \mathrm{E}-14$ & $1.46 \mathrm{E}-14$ & $1.46 \mathrm{E}-14$ & $1.46 \mathrm{E}-14$ & 1.46E-14 \\
\hline SR 89 & $9.76 \mathrm{E}-15$ & $9.75 E-15$ & $9.73 \mathrm{E}-15$ & $9.62 \mathrm{E}-15$ & $8.86 \mathrm{E}-15$ & $8.05 E-15$ & $6.46 \mathrm{E}-15$ & $4.28 \mathrm{E}-15$ & $6.49 \mathrm{E}-17$ \\
\hline Y 89 & $3.81 \mathrm{E}-10$ & $3.81 \mathrm{E}-10$ & $3.82 \mathrm{E}-10$ & $3.84 \mathrm{E}-10$ & $3.93 \mathrm{E}-10$ & $3.96 \mathrm{E}-10$ & $3.97 \mathrm{E}-10$ & 3.97E-10 & 3.97E-10 \\
\hline Y 90 & 1.95E-14 & $1.94 \mathrm{E}-14$ & $1.84 \mathrm{E}-14$ & $1.50 \mathrm{E}-14$ & $3.15 \mathrm{E}-15$ & $5.11 \mathrm{E}-16$ & $8.09 \mathrm{E}-18$ & $1.06 \mathrm{E}-19$ & 1.01E-19 \\
\hline Y 91 & $1.96 \mathrm{E}-15$ & $1.96 \mathrm{E}-15$ & $1.95 \mathrm{E}-15$ & $1.94 \mathrm{E}-15$ & $1.80 \mathrm{E}-15$ & $1.66 \mathrm{E}-15$ & 1.37E-15 & $9.62 \mathrm{E}-16$ & 2.59E-17 \\
\hline ZR 89 & $1.57 \mathrm{E}-11$ & $1.57 \mathrm{E}-11$ & $1.51 \mathrm{E}-11$ & $1.27 \mathrm{E}-11$ & $3.57 \mathrm{E}-12$ & $8.08 \mathrm{E}-13$ & $2.72 \mathrm{E}-14$ & $4.70 \mathrm{E}-17$ & $0.00 E+00$ \\
\hline ZR 90 & $2.94 \mathrm{E}-13$ & $2.94 \mathrm{E}-13$ & $2.95 \mathrm{E}-13$ & $2.98 \mathrm{E}-13$ & $3.10 \mathrm{E}-13$ & $3.13 \mathrm{E}-13$ & $3.13 \mathrm{E}-13$ & 3.13E-13 & $3.14 \mathrm{E}-13$ \\
\hline ZR 91 & $5.40 \mathrm{E}-09$ & 5.40E-09 & 5.40E-09 & $5.40 \mathrm{E}-09$ & $5.40 \mathrm{E}-09$ & $5.40 \mathrm{E}-09$ & $5.40 \mathrm{E}-09$ & $5.40 \mathrm{E}-09$ & 5.40E-09 \\
\hline ZR 92 & $1.23 \mathrm{E}-07$ & $1.23 \mathrm{E}-07$ & $1.23 \mathrm{E}-07$ & $1.23 \mathrm{E}-07$ & $1.27 \mathrm{E}-07$ & $1.29 \mathrm{E}-07$ & $1.32 \mathrm{E}-07$ & $1.33 \mathrm{E}-07$ & $1.33 \mathrm{E}-07$ \\
\hline
\end{tabular}


TEM-10200-1

ENGINEERING CALCULATIONS AND ANALYSIS

Page 68 of 81

$12 / 19 / 17$

Rev.08

Title:

AS-RUN NEUTRONICS EVALUATION FOR THE CSM-10584 EXPERIMENT IN THE ATR

\begin{tabular}{llllllll} 
ECAR NO.: & 4496 & REV. NO.: & 1 & PROJECT NO.: & 32501 & Date: & 10/19/2020 \\
\hline
\end{tabular}

\begin{tabular}{|c|c|c|c|c|c|c|c|c|c|}
\hline \multicolumn{10}{|c|}{1 gram of 316SS irradiated for 119 EFPDs } \\
\hline & EOC & $30 \mathrm{MINs}$ & $5 \mathrm{HRS}$ & $1 \mathrm{DAY}$ & 7 DAYS & 14 DAYS & 30 DAYS & 60 DAYS & 1 Year \\
\hline & $\begin{array}{l}\mathrm{g} / 1 \mathrm{~g} \text { of } \\
316 \mathrm{SS}\end{array}$ & $\begin{array}{l}\mathrm{g} / 1 \mathrm{~g} \text { of } \\
316 \mathrm{SS}\end{array}$ & $\begin{array}{l}\mathrm{g} / 1 \mathrm{~g} \text { of } \\
316 \mathrm{SS}\end{array}$ & $\begin{array}{l}\mathrm{g} / 1 \mathrm{~g} \text { of } \\
316 \mathrm{SS}\end{array}$ & $\begin{array}{c}\mathrm{g} / 1 \mathrm{~g} \text { of } \\
316 \mathrm{SS}\end{array}$ & $\begin{array}{c}\mathrm{g} / 1 \mathrm{~g} \text { of } \\
316 \mathrm{SS}\end{array}$ & $\begin{array}{c}\mathrm{g} / 1 \mathrm{~g} \text { of } \\
316 \mathrm{SS}\end{array}$ & $\begin{array}{c}\mathrm{g} / 1 \mathrm{~g} \text { of } \\
316 \mathrm{SS}\end{array}$ & $\begin{array}{l}\mathrm{g} / 1 \mathrm{~g} \text { of } \\
316 \mathrm{SS}\end{array}$ \\
\hline ZR 94 & 1.14E-08 & $1.14 \mathrm{E}-08$ & $1.14 \mathrm{E}-08$ & $1.14 \mathrm{E}-08$ & 1.14E-08 & $1.14 \mathrm{E}-08$ & $1.14 \mathrm{E}-08$ & $1.14 \mathrm{E}-08$ & $1.14 \mathrm{E}-08$ \\
\hline ZR 95 & $8.22 \mathrm{E}-10$ & $8.22 \mathrm{E}-10$ & $8.21 \mathrm{E}-10$ & $8.14 \mathrm{E}-10$ & $7.62 \mathrm{E}-10$ & $7.07 \mathrm{E}-10$ & $5.94 \mathrm{E}-10$ & $4.29 \mathrm{E}-10$ & $1.57 \mathrm{E}-11$ \\
\hline ZR 96 & $1.42 \mathrm{E}-12$ & $1.42 \mathrm{E}-12$ & $1.42 \mathrm{E}-12$ & $1.42 \mathrm{E}-12$ & $1.42 \mathrm{E}-12$ & $1.42 \mathrm{E}-12$ & $1.42 \mathrm{E}-12$ & $1.42 \mathrm{E}-12$ & $1.42 \mathrm{E}-12$ \\
\hline NB 92 & $1.01 \mathrm{E}-08$ & $1.01 \mathrm{E}-08$ & $9.92 \mathrm{E}-09$ & $9.40 \mathrm{E}-09$ & $6.24 \mathrm{E}-09$ & 3.87E-09 & $1.30 \mathrm{E}-09$ & $1.68 \mathrm{E}-10$ & $1.52 \mathrm{E}-19$ \\
\hline NB 93 & $2.38 \mathrm{E}-11$ & $2.38 \mathrm{E}-11$ & $2.39 \mathrm{E}-11$ & $2.42 \mathrm{E}-11$ & $2.66 \mathrm{E}-11$ & $2.94 \mathrm{E}-11$ & $3.58 \mathrm{E}-11$ & $4.78 \mathrm{E}-11$ & $1.70 \mathrm{E}-10$ \\
\hline NB 93M & $3.19 \mathrm{E}-16$ & $3.19 \mathrm{E}-16$ & $3.20 \mathrm{E}-16$ & $3.24 \mathrm{E}-16$ & 3.57E-16 & $3.94 \mathrm{E}-16$ & $4.80 \mathrm{E}-16$ & $6.41 \mathrm{E}-16$ & $2.24 \mathrm{E}-15$ \\
\hline NB 94 & $6.84 \mathrm{E}-09$ & $6.84 \mathrm{E}-09$ & $6.84 \mathrm{E}-09$ & $6.84 \mathrm{E}-09$ & $6.84 \mathrm{E}-09$ & $6.84 \mathrm{E}-09$ & $6.84 \mathrm{E}-09$ & $6.84 \mathrm{E}-09$ & $6.84 \mathrm{E}-09$ \\
\hline NB 95 & $1.12 \mathrm{E}-09$ & $1.12 \mathrm{E}-09$ & $1.11 \mathrm{E}-09$ & $1.10 \mathrm{E}-09$ & 1.03E-09 & $9.47 \mathrm{E}-10$ & $7.87 \mathrm{E}-10$ & $5.58 \mathrm{E}-10$ & $1.93 \mathrm{E}-11$ \\
\hline NB 95M & $3.17 \mathrm{E}-13$ & $3.17 \mathrm{E}-13$ & $3.18 \mathrm{E}-13$ & $3.18 \mathrm{E}-13$ & $3.12 \mathrm{E}-13$ & $2.94 \mathrm{E}-13$ & $2.49 \mathrm{E}-13$ & $1.80 \mathrm{E}-13$ & $6.58 \mathrm{E}-15$ \\
\hline NB 96 & $3.63 \mathrm{E}-11$ & $3.58 \mathrm{E}-11$ & $3.13 \mathrm{E}-11$ & $1.78 \mathrm{E}-11$ & $2.48 \mathrm{E}-13$ & $1.69 \mathrm{E}-15$ & $1.90 \mathrm{E}-20$ & $9.90 \mathrm{E}-30$ & $0.00 \mathrm{E}+00$ \\
\hline NB 97 & $7.37 \mathrm{E}-13$ & $5.58 \mathrm{E}-13$ & $5.81 \mathrm{E}-14$ & $7.80 \mathrm{E}-15$ & $2.12 \mathrm{E}-17$ & $2.16 \mathrm{E}-20$ & $3.35 \mathrm{E}-27$ & $0.00 \mathrm{E}+00$ & $0.00 \mathrm{E}+00$ \\
\hline $\begin{array}{l}\mathrm{MO} \\
93 \mathrm{M}\end{array}$ & $8.30 \mathrm{E}-11$ & $7.89 E-11$ & $5.00 E-11$ & $7.32 \mathrm{E}-12$ & $3.44 \mathrm{E}-18$ & $1.42 \mathrm{E}-25$ & $0.00 E+00$ & $0.00 E+00$ & $0.00 E+00$ \\
\hline MO 93 & $7.38 \mathrm{E}-07$ & $7.38 \mathrm{E}-07$ & $7.38 \mathrm{E}-07$ & $7.38 \mathrm{E}-07$ & $7.38 \mathrm{E}-07$ & $7.38 \mathrm{E}-07$ & $7.38 \mathrm{E}-07$ & $7.38 \mathrm{E}-07$ & $7.38 \mathrm{E}-07$ \\
\hline MO 94 & $2.28 \mathrm{E}-03$ & $2.28 \mathrm{E}-03$ & $2.28 \mathrm{E}-03$ & $2.28 \mathrm{E}-03$ & $2.28 \mathrm{E}-03$ & $2.28 \mathrm{E}-03$ & $2.28 \mathrm{E}-03$ & $2.28 \mathrm{E}-03$ & $2.28 \mathrm{E}-03$ \\
\hline MO 95 & $3.74 \mathrm{E}-03$ & $3.74 \mathrm{E}-03$ & $3.74 \mathrm{E}-03$ & $3.74 \mathrm{E}-03$ & $3.74 \mathrm{E}-03$ & $3.74 \mathrm{E}-03$ & $3.74 \mathrm{E}-03$ & $3.74 \mathrm{E}-03$ & $3.74 \mathrm{E}-03$ \\
\hline MO 96 & $4.34 \mathrm{E}-03$ & $4.34 \mathrm{E}-03$ & $4.34 \mathrm{E}-03$ & $4.34 \mathrm{E}-03$ & $4.34 \mathrm{E}-03$ & $4.34 \mathrm{E}-03$ & $4.34 \mathrm{E}-03$ & $4.34 \mathrm{E}-03$ & $4.34 \mathrm{E}-03$ \\
\hline MO 97 & $2.44 \mathrm{E}-03$ & $2.44 \mathrm{E}-03$ & $2.44 \mathrm{E}-03$ & $2.44 \mathrm{E}-03$ & $2.44 \mathrm{E}-03$ & $2.44 \mathrm{E}-03$ & $2.44 \mathrm{E}-03$ & $2.44 \mathrm{E}-03$ & $2.44 \mathrm{E}-03$ \\
\hline MO 98 & $6.15 \mathrm{E}-03$ & $6.15 \mathrm{E}-03$ & $6.15 \mathrm{E}-03$ & $6.15 \mathrm{E}-03$ & $6.15 \mathrm{E}-03$ & $6.15 \mathrm{E}-03$ & $6.15 \mathrm{E}-03$ & $6.15 \mathrm{E}-03$ & $6.15 \mathrm{E}-03$ \\
\hline MO 99 & $5.96 \mathrm{E}-07$ & $5.93 \mathrm{E}-07$ & $5.65 \mathrm{E}-07$ & $4.63 \mathrm{E}-07$ & $1.02 \mathrm{E}-07$ & $1.75 \mathrm{E}-08$ & $3.10 \mathrm{E}-10$ & $1.61 \mathrm{E}-13$ & $0.00 \mathrm{E}+00$ \\
\hline M0100 & $2.50 \mathrm{E}-03$ & $2.50 \mathrm{E}-03$ & $2.50 \mathrm{E}-03$ & $2.50 \mathrm{E}-03$ & $2.50 \mathrm{E}-03$ & $2.50 \mathrm{E}-03$ & $2.50 \mathrm{E}-03$ & $2.50 \mathrm{E}-03$ & $2.50 \mathrm{E}-03$ \\
\hline M0101 & $5.58 \mathrm{E}-10$ & $1.35 \mathrm{E}-10$ & $3.71 \mathrm{E}-16$ & $0.00 \mathrm{E}+00$ & $0.00 E+00$ & $0.00 \mathrm{E}+00$ & $0.00 \mathrm{E}+00$ & $0.00 \mathrm{E}+00$ & $0.00 \mathrm{E}+00$ \\
\hline TC 99 & $2.04 \mathrm{E}-06$ & $2.04 \mathrm{E}-06$ & $2.04 \mathrm{E}-06$ & $2.05 \mathrm{E}-06$ & $2.10 \mathrm{E}-06$ & $2.11 \mathrm{E}-06$ & $2.11 \mathrm{E}-06$ & $2.11 \mathrm{E}-06$ & $2.11 \mathrm{E}-06$ \\
\hline TC100 & $5.06 \mathrm{E}-13$ & $0.00 \mathrm{E}+00$ & $0.00 \mathrm{E}+00$ & $0.00 \mathrm{E}+00$ & $0.00 E+00$ & $0.00 \mathrm{E}+00$ & $0.00 \mathrm{E}+00$ & $0.00 \mathrm{E}+00$ & $0.00 E+00$ \\
\hline TC101 & $5.42 \mathrm{E}-10$ & $3.13 \mathrm{E}-10$ & $4.55 \mathrm{E}-15$ & $0.00 \mathrm{E}+00$ & $0.00 E+00$ & $0.00 \mathrm{E}+00$ & $0.00 \mathrm{E}+00$ & $0.00 \mathrm{E}+00$ & $0.00 \mathrm{E}+00$ \\
\hline RU 99 & $1.07 \mathrm{E}-12$ & $1.07 \mathrm{E}-12$ & $1.07 \mathrm{E}-12$ & $1.09 \mathrm{E}-12$ & $1.20 \mathrm{E}-12$ & $1.33 \mathrm{E}-12$ & $1.63 \mathrm{E}-12$ & $2.19 \mathrm{E}-12$ & $7.94 \mathrm{E}-12$ \\
\hline RU102 & $8.10 \mathrm{E}-08$ & $8.10 \mathrm{E}-08$ & $8.10 \mathrm{E}-08$ & $8.10 \mathrm{E}-08$ & 8.10E-08 & $8.10 \mathrm{E}-08$ & $8.10 \mathrm{E}-08$ & $8.10 \mathrm{E}-08$ & $8.10 \mathrm{E}-08$ \\
\hline RU103 & $5.50 \mathrm{E}-11$ & $5.50 \mathrm{E}-11$ & $5.48 \mathrm{E}-11$ & $5.40 \mathrm{E}-11$ & $4.86 \mathrm{E}-11$ & $4.29 \mathrm{E}-11$ & $3.24 \mathrm{E}-11$ & $1.91 \mathrm{E}-11$ & $8.73 \mathrm{E}-14$ \\
\hline RU104 & $4.91 \mathrm{E}-13$ & $4.91 \mathrm{E}-13$ & $4.91 \mathrm{E}-13$ & $4.91 \mathrm{E}-13$ & $4.91 \mathrm{E}-13$ & $4.91 \mathrm{E}-13$ & $4.91 \mathrm{E}-13$ & $4.91 \mathrm{E}-13$ & $4.91 \mathrm{E}-13$ \\
\hline RH103 & $2.84 \mathrm{E}-12$ & $2.84 \mathrm{E}-12$ & $2.86 \mathrm{E}-12$ & $2.93 \mathrm{E}-12$ & $3.47 \mathrm{E}-12$ & $4.03 \mathrm{E}-12$ & $5.08 \mathrm{E}-12$ & $6.40 \mathrm{E}-12$ & $8.29 \mathrm{E}-12$ \\
\hline PD104 & $2.96 \mathrm{E}-13$ & $2.96 \mathrm{E}-13$ & $2.96 \mathrm{E}-13$ & $2.96 \mathrm{E}-13$ & $2.96 \mathrm{E}-13$ & $2.96 \mathrm{E}-13$ & $2.96 \mathrm{E}-13$ & $2.96 \mathrm{E}-13$ & $2.96 \mathrm{E}-13$ \\
\hline SUMTOT & $1.00 \mathrm{E}+00$ & $1.00 \mathrm{E}+00$ & $1.00 \mathrm{E}+00$ & $1.00 \mathrm{E}+00$ & $1.00 \mathrm{E}+00$ & $1.00 \mathrm{E}+00$ & $1.00 \mathrm{E}+00$ & $1.00 \mathrm{E}+00$ & $1.00 \mathrm{E}+00$ \\
\hline OTOTAL & $1.00 \mathrm{E}+00$ & $1.00 \mathrm{E}+00$ & $1.00 \mathrm{E}+00$ & $1.00 \mathrm{E}+00$ & $1.00 \mathrm{E}+00$ & $1.00 \mathrm{E}+00$ & $1.00 \mathrm{E}+00$ & $1.00 \mathrm{E}+00$ & $1.00 \mathrm{E}+00$ \\
\hline
\end{tabular}

The tables below can be used for determining the source term for the flux wires used in the CSM-10584 experiment.

\begin{tabular}{|c|c|c|c|c|c|c|c|c|c|}
\hline \multicolumn{9}{|c|}{1 gram of Fe (CSM flux wire) irradiated for 54.9 EFPDs } \\
\hline & EOC & 30 MINs & 5 HRS & 1 DAY & 7 DAYS & 14 DAYS & 30 DAYS & 60 DAYS & 1 YEAR \\
\hline & Ci/g & Ci/g & Ci/g & Ci/g & Ci/g & Ci/g & Ci/g & Ci/g & Ci/g \\
\hline H 3 & $6.94 \mathrm{E}-14$ & $6.94 \mathrm{E}-14$ & $6.94 \mathrm{E}-14$ & $6.94 \mathrm{E}-14$ & $6.93 \mathrm{E}-14$ & $6.93 \mathrm{E}-14$ & $6.91 \mathrm{E}-14$ & $6.88 \mathrm{E}-14$ & $6.56 \mathrm{E}-14$ \\
\hline SC 48 & $4.06 \mathrm{E}-11$ & $4.02 \mathrm{E}-11$ & $3.75 \mathrm{E}-11$ & $2.77 \mathrm{E}-11$ & $2.84 \mathrm{E}-12$ & $1.99 \mathrm{E}-13$ & $4.56 \mathrm{E}-16$ & $5.14 \mathrm{E}-21$ & $0.00 \mathrm{E}+00$ \\
\hline SC 50 & $6.12 \mathrm{E}-15$ & $3.17 \mathrm{E}-20$ & $0.00 \mathrm{E}+00$ & $0.00 \mathrm{E}+00$ & $0.00 \mathrm{E}+00$ & $0.00 \mathrm{E}+00$ & $0.00 \mathrm{E}+00$ & $0.00 \mathrm{E}+00$ & $0.00 \mathrm{E}+00$ \\
\hline TI 51 & $3.68 \mathrm{E}-09$ & $9.95 \mathrm{E}-11$ & $7.71 \mathrm{E}-25$ & $0.00 \mathrm{E}+00$ & $0.00 \mathrm{E}+00$ & $0.00 \mathrm{E}+00$ & $0.00 \mathrm{E}+00$ & $0.00 \mathrm{E}+00$ & $0.00 \mathrm{E}+00$ \\
\hline V 52 & $1.73 \mathrm{E}-05$ & $6.75 \mathrm{E}-08$ & $0.00 \mathrm{E}+00$ & $0.00 \mathrm{E}+00$ & $0.00 \mathrm{E}+00$ & $0.00 \mathrm{E}+00$ & $0.00 \mathrm{E}+00$ & $0.00 \mathrm{E}+00$ & $0.00 \mathrm{E}+00$ \\
\hline
\end{tabular}


TEM-10200-1

ENGINEERING CALCULATIONS AND ANALYSIS

Page 69 of 81

$12 / 19 / 17$

Rev.08

Title:

AS-RUN NEUTRONICS EVALUATION FOR THE CSM-10584 EXPERIMENT IN THE ATR

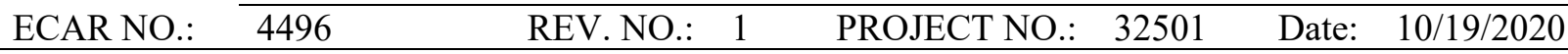

\begin{tabular}{|c|c|c|c|c|c|c|c|c|c|}
\hline V 53 & 4.71E-09 & 1.16E-14 & $0.00 E+00$ & $0.00 E+00$ & $0.00 \mathrm{E}+00$ & $0.00 \mathrm{E}+00$ & $0.00 \mathrm{E}+00$ & $0.00 E+00$ & $0.00 E+00$ \\
\hline V 54 & $2.19 \mathrm{E}-10$ & $3.08 \mathrm{E}-20$ & $0.00 \mathrm{E}+00$ & $0.00 E+00$ & $0.00 E+00$ & $0.00 \mathrm{E}+00$ & $0.00 E+00$ & $0.00 \mathrm{E}+00$ & $0.00 E+00$ \\
\hline CR 51 & $1.09 \mathrm{E}-02$ & 1.09E-02 & $1.09 \mathrm{E}-02$ & 1.07E-02 & $9.19 \mathrm{E}-03$ & $7.71 \mathrm{E}-03$ & 5.17E-03 & $2.44 \mathrm{E}-03$ & $1.18 \mathrm{E}-06$ \\
\hline CR 55 & $9.25 \mathrm{E}-06$ & $2.65 \mathrm{E}-08$ & $0.00 \mathrm{E}+00$ & $0.00 \mathrm{E}+00$ & $0.00 E+00$ & $0.00 E+00$ & $0.00 \mathrm{E}+00$ & $0.00 E+00$ & $0.00 \mathrm{E}+00$ \\
\hline MN 54 & $4.62 \mathrm{E}-02$ & $4.62 \mathrm{E}-02$ & $4.62 \mathrm{E}-02$ & $4.61 \mathrm{E}-02$ & $4.55 \mathrm{E}-02$ & $4.48 \mathrm{E}-02$ & $4.33 \mathrm{E}-02$ & $4.05 \mathrm{E}-02$ & $2.06 \mathrm{E}-02$ \\
\hline MN 56 & $5.27 \mathrm{E}-01$ & $4.60 \mathrm{E}-01$ & $1.37 \mathrm{E}-01$ & $8.31 \mathrm{E}-04$ & $1.29 \mathrm{E}-20$ & $0.00 \mathrm{E}+00$ & $0.00 \mathrm{E}+00$ & $0.00 \mathrm{E}+00$ & $0.00 \mathrm{E}+00$ \\
\hline MN 57 & 9.67E-04 & $2.38 \mathrm{E}-09$ & $0.00 \mathrm{E}+00$ & $0.00 \mathrm{E}+00$ & $0.00 \mathrm{E}+00$ & $0.00 \mathrm{E}+00$ & $0.00 \mathrm{E}+00$ & $0.00 \mathrm{E}+00$ & $0.00 \mathrm{E}+00$ \\
\hline MN 58 & $3.08 \mathrm{E}-06$ & $1.55 \mathrm{E}-14$ & $0.00 \mathrm{E}+00$ & $0.00 E+00$ & $0.00 E+00$ & $0.00 E+00$ & $0.00 \mathrm{E}+00$ & $0.00 E+00$ & $0.00 \mathrm{E}+00$ \\
\hline FE 59 & $6.99 \mathrm{E}-02$ & $6.99 \mathrm{E}-02$ & $6.97 \mathrm{E}-02$ & $6.89 \mathrm{E}-02$ & $6.28 \mathrm{E}-02$ & $5.64 \mathrm{E}-02$ & $4.41 \mathrm{E}-02$ & $2.78 \mathrm{E}-02$ & $2.52 \mathrm{E}-04$ \\
\hline $\mathrm{CO} 60$ & 7.47E-06 & 7.47E-06 & 7.47E-06 & 7.47E-06 & $7.46 \mathrm{E}-06$ & $7.44 \mathrm{E}-06$ & $7.39 \mathrm{E}-06$ & 7.31E-06 & $6.55 \mathrm{E}-06$ \\
\hline $\mathrm{CO} 60 \mathrm{M}$ & $5.78 \mathrm{E}-04$ & 7.93E-05 & $1.37 \mathrm{E}-12$ & $0.00 \mathrm{E}+00$ & $0.00 E+00$ & $0.00 \mathrm{E}+00$ & $0.00 \mathrm{E}+00$ & $0.00 \mathrm{E}+00$ & $0.00 E+00$ \\
\hline CO 61 & $5.72 \mathrm{E}-07$ & $4.64 \mathrm{E}-07$ & 7.01E-08 & $2.39 \mathrm{E}-11$ & $0.00 \mathrm{E}+00$ & $0.00 \mathrm{E}+00$ & $0.00 \mathrm{E}+00$ & $0.00 \mathrm{E}+00$ & $0.00 \mathrm{E}+00$ \\
\hline SUMTOT & $8.21 \mathrm{E}-01$ & $7.53 \mathrm{E}-01$ & $4.30 \mathrm{E}-01$ & $2.92 \mathrm{E}-01$ & $2.82 \mathrm{E}-01$ & $2.73 \mathrm{E}-01$ & $2.55 \mathrm{E}-01$ & $2.29 \mathrm{E}-01$ & $1.48 \mathrm{E}-01$ \\
\hline OTOTAL & $8.21 \mathrm{E}-01$ & $7.53 \mathrm{E}-01$ & $4.30 \mathrm{E}-01$ & $2.92 \mathrm{E}-01$ & $2.82 \mathrm{E}-01$ & $2.73 \mathrm{E}-01$ & $2.55 \mathrm{E}-01$ & $2.29 \mathrm{E}-01$ & $1.48 \mathrm{E}-01$ \\
\hline
\end{tabular}

\begin{tabular}{|c|c|c|c|c|c|c|c|c|c|}
\hline \multicolumn{10}{|c|}{1 gram of Fe (CSM flux wire) irradiated for 54.9 EFPDs } \\
\hline & EOC & $30 \mathrm{MINs}$ & $5 \mathrm{HRS}$ & $1 \mathrm{DAY}$ & 7 DAYS & 14 DAYS & 30 DAYS & 60 DAYS & 1 YEAR \\
\hline & $\mathrm{g} / 1 \mathrm{~g}$ of $\mathrm{Fe}$ & $\mathrm{g} / 1 \mathrm{~g}$ of $\mathrm{Fe}$ & $\mathrm{g} / \mathrm{g}$ of $\mathrm{Fe}$ & $\mathrm{g} / \mathrm{g}$ of $\mathrm{Fe}$ & $\mathrm{g} / 1 \mathrm{~g}$ of $\mathrm{Fe}$ & $\mathrm{g} / 1 \mathrm{~g}$ of $\mathrm{Fe}$ & $\mathrm{g} / 1 \mathrm{~g}$ of $\mathrm{Fe}$ & $\mathrm{g} / \mathrm{g}$ of $\mathrm{Fe}$ & $\mathrm{g} / 1 \mathrm{~g}$ of $\mathrm{Fe}$ \\
\hline H 1 & $2.71 \mathrm{E}-07$ & $2.71 \mathrm{E}-07$ & $2.71 \mathrm{E}-07$ & $2.71 \mathrm{E}-07$ & $2.71 \mathrm{E}-07$ & $2.71 \mathrm{E}-07$ & $2.71 \mathrm{E}-07$ & $2.71 \mathrm{E}-07$ & $2.71 \mathrm{E}-07$ \\
\hline $\mathrm{H} 2$ & $5.28 \mathrm{E}-11$ & $5.28 \mathrm{E}-11$ & $5.28 \mathrm{E}-11$ & $5.28 \mathrm{E}-11$ & $5.28 \mathrm{E}-11$ & $5.28 \mathrm{E}-11$ & $5.28 \mathrm{E}-11$ & $5.28 \mathrm{E}-11$ & $5.28 \mathrm{E}-11$ \\
\hline HE 4 & $5.90 \mathrm{E}-08$ & $5.90 \mathrm{E}-08$ & $5.90 \mathrm{E}-08$ & $5.90 \mathrm{E}-08$ & $5.90 \mathrm{E}-08$ & $5.90 \mathrm{E}-08$ & $5.90 \mathrm{E}-08$ & $5.90 \mathrm{E}-08$ & $5.90 \mathrm{E}-08$ \\
\hline TI 50 & $1.04 \mathrm{E}-12$ & $1.04 \mathrm{E}-12$ & $1.04 \mathrm{E}-12$ & $1.04 \mathrm{E}-12$ & $1.04 \mathrm{E}-12$ & $1.04 \mathrm{E}-12$ & $1.04 \mathrm{E}-12$ & $1.04 \mathrm{E}-12$ & $1.04 \mathrm{E}-12$ \\
\hline V 51 & $9.93 \mathrm{E}-08$ & 9.94E-08 & $9.99 \mathrm{E}-08$ & $1.02 \mathrm{E}-07$ & $1.18 \mathrm{E}-07$ & $1.34 \mathrm{E}-07$ & $1.62 \mathrm{E}-07$ & $1.91 \mathrm{E}-07$ & $2.18 \mathrm{E}-07$ \\
\hline CR 51 & $1.18 \mathrm{E}-07$ & $1.18 \mathrm{E}-07$ & $1.18 \mathrm{E}-07$ & 1.16E-07 & $9.94 \mathrm{E}-08$ & $8.34 \mathrm{E}-08$ & $5.59 \mathrm{E}-08$ & $2.64 \mathrm{E}-08$ & $1.27 \mathrm{E}-11$ \\
\hline CR 52 & $1.27 \mathrm{E}-10$ & $1.27 \mathrm{E}-10$ & $1.27 \mathrm{E}-10$ & $1.27 \mathrm{E}-10$ & $1.27 \mathrm{E}-10$ & $1.27 \mathrm{E}-10$ & $1.27 \mathrm{E}-10$ & $1.27 \mathrm{E}-10$ & $1.27 \mathrm{E}-10$ \\
\hline CR 53 & $4.28 \mathrm{E}-07$ & $4.28 \mathrm{E}-07$ & $4.28 \mathrm{E}-07$ & $4.28 \mathrm{E}-07$ & $4.28 \mathrm{E}-07$ & $4.28 \mathrm{E}-07$ & $4.28 \mathrm{E}-07$ & $4.28 \mathrm{E}-07$ & $4.28 \mathrm{E}-07$ \\
\hline CR 54 & $5.02 \mathrm{E}-07$ & $5.02 \mathrm{E}-07$ & $5.05 \mathrm{E}-07$ & $5.15 \mathrm{E}-07$ & $5.94 \mathrm{E}-07$ & $6.85 \mathrm{E}-07$ & $8.86 \mathrm{E}-07$ & $1.25 \mathrm{E}-06$ & $3.82 \mathrm{E}-06$ \\
\hline CR 55 & $9.60 \mathrm{E}-15$ & $2.75 \mathrm{E}-17$ & $0.00 \mathrm{E}+00$ & $0.00 E+00$ & $0.00 \mathrm{E}+00$ & $0.00 \mathrm{E}+00$ & $0.00 E+00$ & $0.00 \mathrm{E}+00$ & $0.00 \mathrm{E}+00$ \\
\hline MN 54 & 5.97E-06 & 5.97E-06 & 5.97E-06 & $5.96 \mathrm{E}-06$ & $5.88 \mathrm{E}-06$ & $5.79 \mathrm{E}-06$ & $5.59 \mathrm{E}-06$ & $5.23 \mathrm{E}-06$ & $2.66 \mathrm{E}-06$ \\
\hline MN 55 & $1.35 \mathrm{E}-06$ & $1.35 \mathrm{E}-06$ & $1.36 \mathrm{E}-06$ & $1.40 \mathrm{E}-06$ & $1.69 \mathrm{E}-06$ & $2.02 \mathrm{E}-06$ & $2.79 \mathrm{E}-06$ & 4.19E-06 & $1.69 \mathrm{E}-05$ \\
\hline MN 57 & $4.72 \mathrm{E}-13$ & $1.16 \mathrm{E}-18$ & $0.00 \mathrm{E}+00$ & $0.00 \mathrm{E}+00$ & $0.00 \mathrm{E}+00$ & $0.00 \mathrm{E}+00$ & $0.00 \mathrm{E}+00$ & $0.00 \mathrm{E}+00$ & $0.00 \mathrm{E}+00$ \\
\hline MN 58 & $1.04 \mathrm{E}-15$ & $5.21 \mathrm{E}-24$ & $0.00 \mathrm{E}+00$ & $0.00 \mathrm{E}+00$ & $0.00 \mathrm{E}+00$ & $0.00 \mathrm{E}+00$ & $0.00 \mathrm{E}+00$ & $0.00 \mathrm{E}+00$ & $0.00 \mathrm{E}+00$ \\
\hline FE 54 & $5.60 \mathrm{E}-02$ & $5.60 \mathrm{E}-02$ & $5.60 \mathrm{E}-02$ & $5.60 \mathrm{E}-02$ & $5.60 \mathrm{E}-02$ & $5.60 \mathrm{E}-02$ & $5.60 \mathrm{E}-02$ & $5.60 \mathrm{E}-02$ & $5.60 \mathrm{E}-02$ \\
\hline FE 55 & $6.63 \mathrm{E}-05$ & $6.63 \mathrm{E}-05$ & $6.63 \mathrm{E}-05$ & $6.62 \mathrm{E}-05$ & $6.59 \mathrm{E}-05$ & $6.56 \mathrm{E}-05$ & $6.48 \mathrm{E}-05$ & $6.34 \mathrm{E}-05$ & $5.08 \mathrm{E}-05$ \\
\hline FE 56 & $9.18 \mathrm{E}-01$ & $9.18 \mathrm{E}-01$ & $9.18 \mathrm{E}-01$ & $9.18 \mathrm{E}-01$ & $9.18 \mathrm{E}-01$ & $9.18 \mathrm{E}-01$ & $9.18 \mathrm{E}-01$ & $9.18 \mathrm{E}-01$ & $9.18 \mathrm{E}-01$ \\
\hline FE 57 & $2.33 \mathrm{E}-02$ & $2.33 \mathrm{E}-02$ & $2.33 \mathrm{E}-02$ & $2.33 \mathrm{E}-02$ & $2.33 \mathrm{E}-02$ & $2.33 \mathrm{E}-02$ & $2.33 \mathrm{E}-02$ & $2.33 \mathrm{E}-02$ & $2.33 \mathrm{E}-02$ \\
\hline FE 58 & $3.04 \mathrm{E}-03$ & 3.04E-03 & 3.04E-03 & $3.04 \mathrm{E}-03$ & $3.04 \mathrm{E}-03$ & $3.04 \mathrm{E}-03$ & $3.04 \mathrm{E}-03$ & $3.04 \mathrm{E}-03$ & $3.04 \mathrm{E}-03$ \\
\hline FE 59 & $1.42 \mathrm{E}-06$ & $1.42 \mathrm{E}-06$ & $1.42 \mathrm{E}-06$ & $1.40 \mathrm{E}-06$ & $1.28 \mathrm{E}-06$ & $1.15 \mathrm{E}-06$ & $8.95 \mathrm{E}-07$ & $5.64 \mathrm{E}-07$ & $5.12 \mathrm{E}-09$ \\
\hline CO 59 & $6.77 \mathrm{E}-07$ & $6.77 \mathrm{E}-07$ & $6.81 \mathrm{E}-07$ & $6.98 \mathrm{E}-07$ & $8.22 \mathrm{E}-07$ & $9.52 \mathrm{E}-07$ & $1.20 \mathrm{E}-06$ & $1.53 \mathrm{E}-06$ & $2.09 \mathrm{E}-06$ \\
\hline $\mathrm{CO} 60$ & 6.61E-09 & 6.61E-09 & 6.61E-09 & 6.61E-09 & 6.59E-09 & 6.57E-09 & $6.54 \mathrm{E}-09$ & $6.47 \mathrm{E}-09$ & 5.79E-09 \\
\hline $\mathrm{CO} 60 \mathrm{M}$ & $1.93 \mathrm{E}-12$ & $2.65 \mathrm{E}-13$ & $4.57 \mathrm{E}-21$ & $0.00 E+00$ & $0.00 \mathrm{E}+00$ & $0.00 \mathrm{E}+00$ & $0.00 \mathrm{E}+00$ & $0.00 \mathrm{E}+00$ & $0.00 E+00$ \\
\hline CO 61 & $1.84 \mathrm{E}-14$ & $1.49 \mathrm{E}-14$ & $2.25 \mathrm{E}-15$ & 7.69E-19 & $0.00 \mathrm{E}+00$ & $0.00 \mathrm{E}+00$ & $0.00 \mathrm{E}+00$ & $0.00 \mathrm{E}+00$ & $0.00 \mathrm{E}+00$ \\
\hline NI 60 & $4.30 \mathrm{E}-11$ & $4.30 \mathrm{E}-11$ & $4.35 \mathrm{E}-11$ & $4.54 \mathrm{E}-11$ & $5.96 \mathrm{E}-11$ & $7.62 \mathrm{E}-11$ & $1.14 \mathrm{E}-10$ & $1.84 \mathrm{E}-10$ & $8.57 \mathrm{E}-10$ \\
\hline NI 61 & $2.67 \mathrm{E}-12$ & 2.67E-12 & $2.68 \mathrm{E}-12$ & $2.68 \mathrm{E}-12$ & $2.68 \mathrm{E}-12$ & $2.68 \mathrm{E}-12$ & $2.68 \mathrm{E}-12$ & $2.68 \mathrm{E}-12$ & $2.68 \mathrm{E}-12$ \\
\hline SUMTOT & $1.00 \mathrm{E}+00$ & $1.00 \mathrm{E}+00$ & $1.00 \mathrm{E}+00$ & $1.00 \mathrm{E}+00$ & $1.00 \mathrm{E}+00$ & $1.00 \mathrm{E}+00$ & $1.00 \mathrm{E}+00$ & $1.00 \mathrm{E}+00$ & $1.00 \mathrm{E}+00$ \\
\hline OTOTAL & $1.00 \mathrm{E}+00$ & $1.00 \mathrm{E}+00$ & $1.00 \mathrm{E}+00$ & $1.00 \mathrm{E}+00$ & $1.00 \mathrm{E}+00$ & $1.00 \mathrm{E}+00$ & $1.00 \mathrm{E}+00$ & $1.00 \mathrm{E}+00$ & $1.00 \mathrm{E}+00$ \\
\hline
\end{tabular}

\begin{tabular}{|c|c|c|c|c|c|c|c|c|c|}
\hline \multicolumn{10}{|c|}{1 gram of Fe (CSM flux wire) irradiated for 119 EFPDs } \\
\hline & EOC & $30 \mathrm{MINs}$ & $5 \mathrm{HRS}$ & $1 \mathrm{DAY}$ & 7 DAYS & 14 DAYS & 30 DAYS & 60 DAYS & 1 YEAR \\
\hline & $\mathrm{Ci} / \mathrm{g}$ & $\mathrm{Ci} / \mathrm{g}$ & $\mathrm{Ci} / \mathrm{g}$ & $\mathrm{Ci} / \mathrm{g}$ & $\mathrm{Ci} / \mathrm{g}$ & $\mathrm{Ci} / \mathrm{g}$ & $\mathrm{Ci} / \mathrm{g}$ & $\mathrm{Ci} / \mathrm{g}$ & $\mathrm{Ci} / \mathrm{g}$ \\
\hline $\mathrm{H} 3$ & 7.06E-13 & 7.06E-13 & $7.06 \mathrm{E}-13$ & $7.06 \mathrm{E}-13$ & $7.05 \mathrm{E}-13$ & $7.04 \mathrm{E}-13$ & 7.03E-13 & $6.99 \mathrm{E}-13$ & $6.67 \mathrm{E}-13$ \\
\hline
\end{tabular}


TEM-10200-1

ENGINEERING CALCULATIONS AND ANALYSIS

Page 70 of 81

$12 / 19 / 17$

Rev.08

Title:

AS-RUN NEUTRONICS EVALUATION FOR THE CSM-10584 EXPERIMENT IN THE ATR

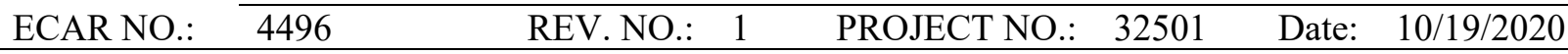

\begin{tabular}{|c|c|c|c|c|c|c|c|c|c|}
\hline SC 48 & $1.31 \mathrm{E}-10$ & $1.30 \mathrm{E}-10$ & $1.21 \mathrm{E}-10$ & $8.96 \mathrm{E}-11$ & $9.18 \mathrm{E}-12$ & $6.43 \mathrm{E}-13$ & $1.47 \mathrm{E}-15$ & $1.66 \mathrm{E}-20$ & $0.00 E+00$ \\
\hline SC 50 & $2.87 \mathrm{E}-14$ & $1.48 \mathrm{E}-19$ & $0.00 \mathrm{E}+00$ & $0.00 \mathrm{E}+00$ & $0.00 \mathrm{E}+00$ & $0.00 \mathrm{E}+00$ & $0.00 E+00$ & $0.00 \mathrm{E}+00$ & $0.00 E+00$ \\
\hline TI 51 & $1.36 \mathrm{E}-08$ & $3.68 \mathrm{E}-10$ & $2.85 \mathrm{E}-24$ & $0.00 \mathrm{E}+00$ & $0.00 \mathrm{E}+00$ & $0.00 \mathrm{E}+00$ & $0.00 \mathrm{E}+00$ & $0.00 \mathrm{E}+00$ & $0.00 \mathrm{E}+00$ \\
\hline V 52 & $5.58 \mathrm{E}-05$ & $2.18 \mathrm{E}-07$ & $0.00 \mathrm{E}+00$ & $0.00 \mathrm{E}+00$ & $0.00 \mathrm{E}+00$ & $0.00 \mathrm{E}+00$ & $0.00 \mathrm{E}+00$ & $0.00 \mathrm{E}+00$ & $0.00 \mathrm{E}+00$ \\
\hline V 53 & $1.02 \mathrm{E}-08$ & $2.50 \mathrm{E}-14$ & $0.00 \mathrm{E}+00$ & $0.00 \mathrm{E}+00$ & $0.00 \mathrm{E}+00$ & $0.00 \mathrm{E}+00$ & $0.00 \mathrm{E}+00$ & $0.00 \mathrm{E}+00$ & $0.00 \mathrm{E}+00$ \\
\hline V 54 & $8.55 \mathrm{E}-10$ & $1.20 \mathrm{E}-19$ & $0.00 \mathrm{E}+00$ & $0.00 \mathrm{E}+00$ & $0.00 \mathrm{E}+00$ & $0.00 \mathrm{E}+00$ & $0.00 \mathrm{E}+00$ & $0.00 \mathrm{E}+00$ & $0.00 \mathrm{E}+00$ \\
\hline CR 51 & $1.39 \mathrm{E}-02$ & $1.39 \mathrm{E}-02$ & $1.38 \mathrm{E}-02$ & $1.36 \mathrm{E}-02$ & $1.17 \mathrm{E}-02$ & $9.79 \mathrm{E}-03$ & $6.56 \mathrm{E}-03$ & $3.10 \mathrm{E}-03$ & $1.50 \mathrm{E}-06$ \\
\hline CR 55 & 2.73E-05 & $7.82 \mathrm{E}-08$ & $0.00 \mathrm{E}+00$ & $0.00 \mathrm{E}+00$ & $0.00 \mathrm{E}+00$ & $0.00 \mathrm{E}+00$ & $0.00 \mathrm{E}+00$ & $0.00 \mathrm{E}+00$ & $0.00 \mathrm{E}+00$ \\
\hline MN 54 & $9.32 \mathrm{E}-02$ & $9.32 \mathrm{E}-02$ & $9.32 \mathrm{E}-02$ & $9.30 \mathrm{E}-02$ & $9.18 \mathrm{E}-02$ & $9.04 \mathrm{E}-02$ & $8.72 \mathrm{E}-02$ & $8.16 \mathrm{E}-02$ & $4.15 \mathrm{E}-02$ \\
\hline MN 56 & $5.28 \mathrm{E}-01$ & 4.62E-01 & $1.38 \mathrm{E}-01$ & 8.34E-04 & $1.29 \mathrm{E}-20$ & $0.00 \mathrm{E}+00$ & $0.00 \mathrm{E}+00$ & $0.00 \mathrm{E}+00$ & $0.00 \mathrm{E}+00$ \\
\hline MN 57 & $1.03 \mathrm{E}-03$ & $2.54 \mathrm{E}-09$ & $0.00 \mathrm{E}+00$ & $0.00 \mathrm{E}+00$ & $0.00 \mathrm{E}+00$ & $0.00 \mathrm{E}+00$ & $0.00 \mathrm{E}+00$ & $0.00 \mathrm{E}+00$ & $0.00 \mathrm{E}+00$ \\
\hline MN 58 & $3.12 \mathrm{E}-06$ & 1.57E-14 & $0.00 \mathrm{E}+00$ & $0.00 \mathrm{E}+00$ & $0.00 \mathrm{E}+00$ & $0.00 \mathrm{E}+00$ & $0.00 \mathrm{E}+00$ & $0.00 \mathrm{E}+00$ & $0.00 \mathrm{E}+00$ \\
\hline FE 55 & $3.51 \mathrm{E}-01$ & 3.51E-01 & 3.51E-01 & 3.51E-01 & $3.49 \mathrm{E}-01$ & 3.47E-01 & 3.43E-01 & 3.36E-01 & 2.69E-01 \\
\hline FE 59 & 1.04E-01 & $1.04 \mathrm{E}-01$ & $1.03 \mathrm{E}-01$ & $1.02 \mathrm{E}-01$ & $9.31 \mathrm{E}-02$ & $8.36 \mathrm{E}-02$ & $6.54 \mathrm{E}-02$ & 4.12E-02 & 3.74E-04 \\
\hline $\mathrm{CO} 60$ & $6.13 \mathrm{E}-05$ & $6.13 \mathrm{E}-05$ & $6.13 \mathrm{E}-05$ & $6.13 \mathrm{E}-05$ & $6.12 \mathrm{E}-05$ & $6.10 \mathrm{E}-05$ & 6.07E-05 & $6.00 \mathrm{E}-05$ & $5.38 \mathrm{E}-05$ \\
\hline $\mathrm{CO} 60 \mathrm{M}$ & $2.07 \mathrm{E}-03$ & $2.84 \mathrm{E}-04$ & $4.90 \mathrm{E}-12$ & $0.00 \mathrm{E}+00$ & $0.00 \mathrm{E}+00$ & $0.00 \mathrm{E}+00$ & $0.00 \mathrm{E}+00$ & $0.00 \mathrm{E}+00$ & $0.00 \mathrm{E}+00$ \\
\hline CO 61 & $4.67 \mathrm{E}-06$ & $3.79 \mathrm{E}-06$ & $5.72 \mathrm{E}-07$ & $1.95 \mathrm{E}-10$ & $0.00 \mathrm{E}+00$ & $0.00 \mathrm{E}+00$ & $0.00 \mathrm{E}+00$ & $0.00 \mathrm{E}+00$ & $0.00 \mathrm{E}+00$ \\
\hline $\mathrm{NI} 63$ & $5.35 \mathrm{E}-15$ & $5.35 \mathrm{E}-15$ & $5.35 \mathrm{E}-15$ & $5.35 \mathrm{E}-15$ & $5.35 \mathrm{E}-15$ & $5.35 \mathrm{E}-15$ & $5.35 \mathrm{E}-15$ & $5.34 \mathrm{E}-15$ & 5.31E-15 \\
\hline SUMTOT & $1.09 \mathrm{E}+00$ & $1.02 \mathrm{E}+00$ & $6.99 \mathrm{E}-01$ & $5.60 \mathrm{E}-01$ & $5.46 \mathrm{E}-01$ & $5.31 \mathrm{E}-01$ & $5.02 \mathrm{E}-01$ & $4.62 \mathrm{E}-01$ & $3.11 \mathrm{E}-01$ \\
\hline OTOTAL & $1.09 \mathrm{E}+00$ & $1.02 \mathrm{E}+00$ & $6.99 \mathrm{E}-01$ & $5.60 \mathrm{E}-01$ & $5.46 \mathrm{E}-01$ & $5.31 \mathrm{E}-01$ & $5.02 \mathrm{E}-01$ & 4.62E-01 & $3.11 \mathrm{E}-01$ \\
\hline
\end{tabular}

\begin{tabular}{|c|c|c|c|c|c|c|c|c|c|}
\hline \multicolumn{10}{|c|}{1 gram of $\mathrm{Fe}$ (CSM flux wire) irradiated for 119 EFPDs } \\
\hline & EOC & $30 \mathrm{MINs}$ & $5 \mathrm{HRS}$ & $1 \mathrm{DAY}$ & 7 DAYS & 14 DAYS & 30 DAYS & 60 DAYS & 1 YEAR \\
\hline & $\mathrm{g} / 1 \mathrm{~g}$ of $\mathrm{Fe}$ & $\mathrm{g} / 1 \mathrm{~g}$ of $\mathrm{Fe}$ & $\mathrm{g} / 1 \mathrm{~g}$ of $\mathrm{Fe}$ & $\mathrm{g} / 1 \mathrm{~g}$ of $\mathrm{Fe}$ & $\mathrm{g} / 1 \mathrm{~g}$ of $\mathrm{Fe}$ & $\mathrm{g} / 1 \mathrm{~g}$ of $\mathrm{Fe}$ & $\mathrm{g} / 1 \mathrm{~g}$ of $\mathrm{Fe}$ & $\mathrm{g} / 1 \mathrm{~g}$ of $\mathrm{Fe}$ & $\mathrm{g} / 1 \mathrm{~g}$ of $\mathrm{Fe}$ \\
\hline H 1 & $5.88 \mathrm{E}-07$ & $5.88 \mathrm{E}-07$ & $5.88 \mathrm{E}-07$ & $5.88 \mathrm{E}-07$ & $5.88 \mathrm{E}-07$ & $5.88 \mathrm{E}-07$ & $5.88 \mathrm{E}-07$ & $5.88 \mathrm{E}-07$ & $5.88 \mathrm{E}-07$ \\
\hline H 2 & $2.48 \mathrm{E}-10$ & $2.48 \mathrm{E}-10$ & $2.48 \mathrm{E}-10$ & $2.48 \mathrm{E}-10$ & $2.48 \mathrm{E}-10$ & $2.48 \mathrm{E}-10$ & $2.48 \mathrm{E}-10$ & $2.48 \mathrm{E}-10$ & $2.48 \mathrm{E}-10$ \\
\hline HE 4 & $1.29 \mathrm{E}-07$ & $1.29 \mathrm{E}-07$ & $1.29 \mathrm{E}-07$ & $1.29 \mathrm{E}-07$ & $1.29 \mathrm{E}-07$ & $1.29 \mathrm{E}-07$ & $1.29 \mathrm{E}-07$ & $1.29 \mathrm{E}-07$ & $1.29 \mathrm{E}-07$ \\
\hline TI 48 & $1.54 \mathrm{E}-15$ & $1.54 \mathrm{E}-15$ & $1.54 \mathrm{E}-15$ & $1.57 \mathrm{E}-15$ & $1.62 \mathrm{E}-15$ & $1.63 \mathrm{E}-15$ & $1.63 \mathrm{E}-15$ & $1.63 \mathrm{E}-15$ & $1.63 \mathrm{E}-15$ \\
\hline TI 50 & 4.87E-12 & $4.87 \mathrm{E}-12$ & $4.87 \mathrm{E}-12$ & $4.87 \mathrm{E}-12$ & $4.87 \mathrm{E}-12$ & $4.87 \mathrm{E}-12$ & $4.87 \mathrm{E}-12$ & $4.87 \mathrm{E}-12$ & $4.87 \mathrm{E}-12$ \\
\hline V 51 & $3.21 \mathrm{E}-07$ & $3.21 \mathrm{E}-07$ & $3.21 \mathrm{E}-07$ & $3.24 \mathrm{E}-07$ & $3.45 \mathrm{E}-07$ & $3.65 \mathrm{E}-07$ & $4.00 \mathrm{E}-07$ & 4.37E-07 & $4.71 \mathrm{E}-07$ \\
\hline V 52 & $5.78 \mathrm{E}-14$ & $2.26 \mathrm{E}-16$ & $0.00 \mathrm{E}+00$ & $0.00 \mathrm{E}+00$ & $0.00 \mathrm{E}+00$ & $0.00 \mathrm{E}+00$ & $0.00 \mathrm{E}+00$ & $0.00 \mathrm{E}+00$ & $0.00 E+00$ \\
\hline CR 51 & $1.50 \mathrm{E}-07$ & $1.50 \mathrm{E}-07$ & $1.50 \mathrm{E}-07$ & $1.47 \mathrm{E}-07$ & $1.26 \mathrm{E}-07$ & $1.06 \mathrm{E}-07$ & $7.09 \mathrm{E}-08$ & $3.35 \mathrm{E}-08$ & $1.62 \mathrm{E}-11$ \\
\hline CR 52 & $8.27 \mathrm{E}-10$ & $8.27 \mathrm{E}-10$ & $8.27 \mathrm{E}-10$ & $8.27 \mathrm{E}-10$ & $8.27 \mathrm{E}-10$ & $8.27 \mathrm{E}-10$ & $8.27 \mathrm{E}-10$ & $8.27 \mathrm{E}-10$ & $8.27 \mathrm{E}-10$ \\
\hline CR 53 & $9.21 \mathrm{E}-07$ & $9.21 \mathrm{E}-07$ & $9.21 \mathrm{E}-07$ & $9.21 \mathrm{E}-07$ & $9.21 \mathrm{E}-07$ & $9.21 \mathrm{E}-07$ & $9.21 \mathrm{E}-07$ & $9.21 \mathrm{E}-07$ & $9.21 \mathrm{E}-07$ \\
\hline CR 54 & $1.96 \mathrm{E}-06$ & $1.96 \mathrm{E}-06$ & $1.97 \mathrm{E}-06$ & $1.99 \mathrm{E}-06$ & $2.15 \mathrm{E}-06$ & $2.33 \mathrm{E}-06$ & $2.74 \mathrm{E}-06$ & $3.46 \mathrm{E}-06$ & $8.65 \mathrm{E}-06$ \\
\hline CR 55 & $2.84 \mathrm{E}-14$ & $8.12 \mathrm{E}-17$ & $0.00 \mathrm{E}+00$ & $0.00 \mathrm{E}+00$ & $0.00 \mathrm{E}+00$ & $0.00 \mathrm{E}+00$ & $0.00 \mathrm{E}+00$ & $0.00 \mathrm{E}+00$ & $0.00 E+00$ \\
\hline MN 54 & $1.20 \mathrm{E}-05$ & $1.20 \mathrm{E}-05$ & $1.20 \mathrm{E}-05$ & $1.20 \mathrm{E}-05$ & $1.19 \mathrm{E}-05$ & $1.17 \mathrm{E}-05$ & $1.13 \mathrm{E}-05$ & $1.05 \mathrm{E}-05$ & $5.36 \mathrm{E}-06$ \\
\hline MN 55 & $6.22 \mathrm{E}-06$ & $6.22 \mathrm{E}-06$ & $6.24 \mathrm{E}-06$ & $6.32 \mathrm{E}-06$ & $6.93 \mathrm{E}-06$ & $7.64 \mathrm{E}-06$ & $9.25 \mathrm{E}-06$ & $1.22 \mathrm{E}-05$ & $3.90 \mathrm{E}-05$ \\
\hline MN 56 & $2.43 \mathrm{E}-08$ & $2.13 \mathrm{E}-08$ & $6.35 \mathrm{E}-09$ & $3.84 \mathrm{E}-11$ & $5.94 \mathrm{E}-28$ & $0.00 \mathrm{E}+00$ & $0.00 \mathrm{E}+00$ & $0.00 \mathrm{E}+00$ & $0.00 \mathrm{E}+00$ \\
\hline MN 57 & $5.04 \mathrm{E}-13$ & $1.24 \mathrm{E}-18$ & $0.00 \mathrm{E}+00$ & $0.00 \mathrm{E}+00$ & $0.00 \mathrm{E}+00$ & $0.00 \mathrm{E}+00$ & $0.00 \mathrm{E}+00$ & $0.00 \mathrm{E}+00$ & $0.00 E+00$ \\
\hline MN 58 & $1.05 \mathrm{E}-15$ & $5.27 \mathrm{E}-24$ & $0.00 \mathrm{E}+00$ & $0.00 \mathrm{E}+00$ & $0.00 \mathrm{E}+00$ & $0.00 \mathrm{E}+00$ & $0.00 \mathrm{E}+00$ & $0.00 \mathrm{E}+00$ & $0.00 E+00$ \\
\hline FE 54 & $5.60 \mathrm{E}-02$ & $5.60 \mathrm{E}-02$ & $5.60 \mathrm{E}-02$ & $5.60 \mathrm{E}-02$ & $5.60 \mathrm{E}-02$ & $5.60 \mathrm{E}-02$ & $5.60 \mathrm{E}-02$ & $5.60 \mathrm{E}-02$ & $5.60 \mathrm{E}-02$ \\
\hline FE 55 & 1.40E-04 & $1.40 \mathrm{E}-04$ & $1.40 \mathrm{E}-04$ & $1.40 \mathrm{E}-04$ & 1.40E-04 & $1.39 \mathrm{E}-04$ & $1.37 \mathrm{E}-04$ & $1.34 \mathrm{E}-04$ & 1.07E-04 \\
\hline FE 56 & $9.16 \mathrm{E}-01$ & $9.16 \mathrm{E}-01$ & $9.16 \mathrm{E}-01$ & $9.16 \mathrm{E}-01$ & $9.16 \mathrm{E}-01$ & $9.16 \mathrm{E}-01$ & $9.16 \mathrm{E}-01$ & $9.16 \mathrm{E}-01$ & $9.16 \mathrm{E}-01$ \\
\hline FE 57 & $2.49 \mathrm{E}-02$ & $2.49 \mathrm{E}-02$ & $2.49 \mathrm{E}-02$ & $2.49 \mathrm{E}-02$ & $2.49 \mathrm{E}-02$ & $2.49 \mathrm{E}-02$ & $2.49 \mathrm{E}-02$ & $2.49 \mathrm{E}-02$ & $2.49 \mathrm{E}-02$ \\
\hline FE 58 & $3.07 \mathrm{E}-03$ & $3.07 \mathrm{E}-03$ & $3.07 \mathrm{E}-03$ & $3.07 \mathrm{E}-03$ & 3.07E-03 & $3.07 \mathrm{E}-03$ & $3.07 \mathrm{E}-03$ & 3.07E-03 & 3.07E-03 \\
\hline FE 59 & $2.11 \mathrm{E}-06$ & $2.11 \mathrm{E}-06$ & $2.10 \mathrm{E}-06$ & $2.08 \mathrm{E}-06$ & $1.89 \mathrm{E}-06$ & $1.70 \mathrm{E}-06$ & $1.33 \mathrm{E}-06$ & $8.37 \mathrm{E}-07$ & 7.60E-09 \\
\hline CO 59 & $2.42 \mathrm{E}-06$ & $2.42 \mathrm{E}-06$ & $2.43 \mathrm{E}-06$ & $2.46 \mathrm{E}-06$ & $2.64 \mathrm{E}-06$ & $2.83 \mathrm{E}-06$ & $3.20 \mathrm{E}-06$ & $3.70 \mathrm{E}-06$ & $4.52 \mathrm{E}-06$ \\
\hline $\mathrm{CO} 60$ & $5.42 \mathrm{E}-08$ & $5.42 \mathrm{E}-08$ & $5.42 \mathrm{E}-08$ & $5.42 \mathrm{E}-08$ & 5.41E-08 & $5.40 \mathrm{E}-08$ & 5.37E-08 & $5.31 \mathrm{E}-08$ & $4.76 \mathrm{E}-08$ \\
\hline $\mathrm{CO} 60 \mathrm{M}$ & $6.91 \mathrm{E}-12$ & $9.48 \mathrm{E}-13$ & $1.64 \mathrm{E}-20$ & $0.00 \mathrm{E}+00$ & $0.00 \mathrm{E}+00$ & $0.00 \mathrm{E}+00$ & $0.00 \mathrm{E}+00$ & $0.00 \mathrm{E}+00$ & $0.00 \mathrm{E}+00$ \\
\hline $\mathrm{CO} 61$ & 1.50E-13 & $1.22 \mathrm{E}-13$ & $1.84 \mathrm{E}-14$ & $6.27 \mathrm{E}-18$ & $0.00 \mathrm{E}+00$ & $0.00 \mathrm{E}+00$ & $0.00 \mathrm{E}+00$ & $0.00 \mathrm{E}+00$ & $0.00 \mathrm{E}+00$ \\
\hline NI 60 & 7.03E-10 & $7.04 \mathrm{E}-10$ & 7.07E-10 & $7.23 \mathrm{E}-10$ & $8.40 \mathrm{E}-10$ & $9.76 \mathrm{E}-10$ & $1.29 \mathrm{E}-09$ & $1.86 \mathrm{E}-09$ & $7.39 \mathrm{E}-09$ \\
\hline NI 61 & $4.92 \mathrm{E}-11$ & $4.93 \mathrm{E}-11$ & $4.94 \mathrm{E}-11$ & $4.94 \mathrm{E}-11$ & $4.94 \mathrm{E}-11$ & 4.94E-11 & 4.94E-11 & $4.94 \mathrm{E}-11$ & 4.94E-11 \\
\hline NI 62 & 3.09E-14 & $3.09 \mathrm{E}-14$ & $3.09 \mathrm{E}-14$ & $3.09 \mathrm{E}-14$ & 3.09E-14 & $3.09 \mathrm{E}-14$ & 3.09E-14 & 3.09E-14 & $3.09 \mathrm{E}-14$ \\
\hline SUMTOT & $1.00 \mathrm{E}+00$ & $1.00 \mathrm{E}+00$ & $1.00 \mathrm{E}+00$ & $1.00 \mathrm{E}+00$ & $1.00 \mathrm{E}+00$ & $1.00 \mathrm{E}+00$ & $1.00 \mathrm{E}+00$ & $1.00 \mathrm{E}+00$ & $1.00 \mathrm{E}+00$ \\
\hline OTOTAL & $1.00 \mathrm{E}+00$ & $1.00 \mathrm{E}+00$ & $1.00 \mathrm{E}+00$ & $1.00 \mathrm{E}+00$ & $1.00 \mathrm{E}+00$ & $1.00 \mathrm{E}+00$ & $1.00 \mathrm{E}+00$ & $1.00 \mathrm{E}+00$ & $1.00 E+00$ \\
\hline
\end{tabular}


TEM-10200-1

ENGINEERING CALCULATIONS AND ANALYSIS

Page 71 of 81

$12 / 19 / 17$

Rev.08

Title:

AS-RUN NEUTRONICS EVALUATION FOR THE CSM-10584 EXPERIMENT IN THE ATR

\begin{tabular}{llllllll} 
ECAR NO.: & 4496 & REV. NO.: & 1 & PROJECT NO.: & 32501 & Date: & 10/19/2020 \\
\hline
\end{tabular}

\begin{tabular}{|c|c|c|c|c|c|c|c|c|c|}
\hline \multicolumn{10}{|c|}{1 gram of Ti (CSM flux wire) irradiated for 54.9 EFPDs } \\
\hline & EOC & $30 \mathrm{MINs}$ & $5 \mathrm{HRS}$ & 1 DAY & 7 DAYS & 14 DAYS & 30 DAYS & 60 DAYS & 1 YEAR \\
\hline & $\mathrm{Ci} / \mathrm{g}$ & $\mathrm{Ci} / \mathrm{g}$ & $\mathrm{Ci} / \mathrm{g}$ & $\mathrm{Ci} / \mathrm{g}$ & $\mathrm{Ci} / \mathrm{g}$ & $\mathrm{Ci} / \mathrm{g}$ & $\mathrm{Ci} / \mathrm{g}$ & $\mathrm{Ci} / \mathrm{g}$ & $\mathrm{Ci} / \mathrm{g}$ \\
\hline H 3 & $2.73 \mathrm{E}-14$ & $2.73 \mathrm{E}-14$ & $2.73 \mathrm{E}-14$ & $2.73 \mathrm{E}-14$ & $2.73 \mathrm{E}-14$ & $2.72 \mathrm{E}-14$ & $2.72 \mathrm{E}-14$ & $2.70 \mathrm{E}-14$ & $2.58 \mathrm{E}-14$ \\
\hline S 37 & 1.57E-11 & $2.58 \mathrm{E}-13$ & $0.00 \mathrm{E}+00$ & $0.00 \mathrm{E}+00$ & $0.00 \mathrm{E}+00$ & $0.00 \mathrm{E}+00$ & $0.00 \mathrm{E}+00$ & $0.00 \mathrm{E}+00$ & $0.00 \mathrm{E}+00$ \\
\hline CL 38 & $1.23 \mathrm{E}-15$ & $5.44 \mathrm{E}-16$ & $0.00 \mathrm{E}+00$ & $0.00 \mathrm{E}+00$ & $0.00 \mathrm{E}+00$ & $0.00 \mathrm{E}+00$ & $0.00 \mathrm{E}+00$ & $0.00 \mathrm{E}+00$ & $0.00 \mathrm{E}+00$ \\
\hline AR 41 & 3.04E-09 & $2.51 \mathrm{E}-09$ & $4.56 \mathrm{E}-10$ & 3.37E-13 & $0.00 \mathrm{E}+00$ & $0.00 \mathrm{E}+00$ & $0.00 \mathrm{E}+00$ & $0.00 \mathrm{E}+00$ & $0.00 \mathrm{E}+00$ \\
\hline AR 42 & $2.46 \mathrm{E}-18$ & $2.46 \mathrm{E}-18$ & $2.46 \mathrm{E}-18$ & $2.46 \mathrm{E}-18$ & $2.46 \mathrm{E}-18$ & $2.46 \mathrm{E}-18$ & $2.45 \mathrm{E}-18$ & $2.45 \mathrm{E}-18$ & $2.41 \mathrm{E}-18$ \\
\hline K 42 & $8.23 \mathrm{E}-12$ & $8.00 \mathrm{E}-12$ & $6.22 \mathrm{E}-12$ & $2.14 \mathrm{E}-12$ & $6.69 \mathrm{E}-16$ & $2.51 \mathrm{E}-18$ & $2.45 \mathrm{E}-18$ & $2.45 \mathrm{E}-18$ & $2.41 \mathrm{E}-18$ \\
\hline $\mathrm{K} 43$ & $3.90 \mathrm{E}-08$ & $3.84 \mathrm{E}-08$ & $3.35 \mathrm{E}-08$ & $1.87 \mathrm{E}-08$ & $2.26 \mathrm{E}-10$ & $1.31 \mathrm{E}-12$ & $1.00 \mathrm{E}-17$ & $2.57 \mathrm{E}-27$ & $0.00 \mathrm{E}+00$ \\
\hline $\mathrm{K} 44$ & $6.90 \mathrm{E}-09$ & $2.68 \mathrm{E}-09$ & $5.42 \mathrm{E}-13$ & 1.37E-28 & $0.00 \mathrm{E}+00$ & $0.00 \mathrm{E}+00$ & $0.00 \mathrm{E}+00$ & $0.00 \mathrm{E}+00$ & $0.00 \mathrm{E}+00$ \\
\hline CA 45 & $2.11 \mathrm{E}-04$ & $2.11 \mathrm{E}-04$ & $2.11 \mathrm{E}-04$ & $2.10 \mathrm{E}-04$ & $2.05 \mathrm{E}-04$ & $1.99 \mathrm{E}-04$ & $1.86 \mathrm{E}-04$ & $1.63 \mathrm{E}-04$ & $4.46 \mathrm{E}-05$ \\
\hline CA 47 & $4.33 \mathrm{E}-06$ & $4.31 \mathrm{E}-06$ & $4.19 \mathrm{E}-06$ & $3.71 \mathrm{E}-06$ & $1.48 \mathrm{E}-06$ & $5.09 \mathrm{E}-07$ & $4.42 \mathrm{E}-08$ & $4.51 \mathrm{E}-10$ & $2.49 \mathrm{E}-30$ \\
\hline SC 46 & $4.74 \mathrm{E}-02$ & $4.74 \mathrm{E}-02$ & $4.73 \mathrm{E}-02$ & $4.70 \mathrm{E}-02$ & $4.48 \mathrm{E}-02$ & $4.22 \mathrm{E}-02$ & $3.70 \mathrm{E}-02$ & $2.89 \mathrm{E}-02$ & $2.31 \mathrm{E}-03$ \\
\hline SC 46M & $5.33 \mathrm{E}-07$ & $0.00 \mathrm{E}+00$ & $0.00 \mathrm{E}+00$ & $0.00 \mathrm{E}+00$ & $0.00 \mathrm{E}+00$ & $0.00 \mathrm{E}+00$ & $0.00 \mathrm{E}+00$ & $0.00 \mathrm{E}+00$ & $0.00 \mathrm{E}+00$ \\
\hline SC 47 & $2.02 \mathrm{E}-01$ & $2.01 \mathrm{E}-01$ & $1.93 \mathrm{E}-01$ & $1.64 \mathrm{E}-01$ & $4.74 \mathrm{E}-02$ & $1.11 \mathrm{E}-02$ & 4.07E-04 & $8.20 \mathrm{E}-07$ & $0.00 \mathrm{E}+00$ \\
\hline SC 48 & $1.89 \mathrm{E}-02$ & $1.88 \mathrm{E}-02$ & $1.75 \mathrm{E}-02$ & $1.30 \mathrm{E}-02$ & $1.33 \mathrm{E}-03$ & $9.30 \mathrm{E}-05$ & $2.14 \mathrm{E}-07$ & $2.41 \mathrm{E}-12$ & $0.00 \mathrm{E}+00$ \\
\hline SC 49 & $1.61 \mathrm{E}-02$ & $1.12 \mathrm{E}-02$ & $4.33 \mathrm{E}-04$ & $4.65 \mathrm{E}-10$ & $0.00 \mathrm{E}+00$ & $0.00 \mathrm{E}+00$ & $0.00 \mathrm{E}+00$ & $0.00 \mathrm{E}+00$ & $0.00 \mathrm{E}+00$ \\
\hline SC 50 & $3.20 \mathrm{E}-04$ & 1.65E-09 & $0.00 \mathrm{E}+00$ & $0.00 \mathrm{E}+00$ & $0.00 \mathrm{E}+00$ & $0.00 \mathrm{E}+00$ & $0.00 \mathrm{E}+00$ & $0.00 \mathrm{E}+00$ & $0.00 \mathrm{E}+00$ \\
\hline TI 51 & $3.63 \mathrm{E}-01$ & $9.81 \mathrm{E}-03$ & $7.60 \mathrm{E}-17$ & $0.00 \mathrm{E}+00$ & $0.00 \mathrm{E}+00$ & $0.00 \mathrm{E}+00$ & $0.00 \mathrm{E}+00$ & $0.00 \mathrm{E}+00$ & $0.00 \mathrm{E}+00$ \\
\hline V 52 & 9.35E-04 & 3.65E-06 & $7.73 \mathrm{E}-28$ & $0.00 \mathrm{E}+00$ & $0.00 \mathrm{E}+00$ & $0.00 \mathrm{E}+00$ & $0.00 \mathrm{E}+00$ & $0.00 \mathrm{E}+00$ & $0.00 \mathrm{E}+00$ \\
\hline V 53 & 4.96E-14 & $1.22 \mathrm{E}-19$ & $0.00 \mathrm{E}+00$ & $0.00 \mathrm{E}+00$ & $0.00 \mathrm{E}+00$ & $0.00 \mathrm{E}+00$ & $0.00 \mathrm{E}+00$ & $0.00 \mathrm{E}+00$ & $0.00 \mathrm{E}+00$ \\
\hline CR 51 & 2.63E-11 & $2.63 \mathrm{E}-11$ & $2.61 \mathrm{E}-11$ & $2.56 \mathrm{E}-11$ & $2.21 \mathrm{E}-11$ & $1.85 \mathrm{E}-11$ & $1.24 \mathrm{E}-11$ & $5.86 \mathrm{E}-12$ & $2.83 \mathrm{E}-15$ \\
\hline CR 55 & $1.29 \mathrm{E}-13$ & $3.45 \mathrm{E}-15$ & $0.00 \mathrm{E}+00$ & $0.00 \mathrm{E}+00$ & $0.00 \mathrm{E}+00$ & $0.00 \mathrm{E}+00$ & $0.00 \mathrm{E}+00$ & $0.00 \mathrm{E}+00$ & $0.00 \mathrm{E}+00$ \\
\hline SUMTOT & $6.48 \mathrm{E}-01$ & $2.88 \mathrm{E}-01$ & $2.59 \mathrm{E}-01$ & $2.24 \mathrm{E}-01$ & $9.37 \mathrm{E}-02$ & $5.37 \mathrm{E}-02$ & $3.76 \mathrm{E}-02$ & $2.90 \mathrm{E}-02$ & $2.36 \mathrm{E}-03$ \\
\hline OTOTAL & $6.48 \mathrm{E}-01$ & $2.88 \mathrm{E}-01$ & $2.59 \mathrm{E}-01$ & $2.24 \mathrm{E}-01$ & $9.37 \mathrm{E}-02$ & $5.37 \mathrm{E}-02$ & $3.76 \mathrm{E}-02$ & $2.90 \mathrm{E}-02$ & $2.36 \mathrm{E}-03$ \\
\hline
\end{tabular}

\begin{tabular}{|c|c|c|c|c|c|c|c|c|c|}
\hline \multicolumn{10}{|c|}{1 gram of Ti (CSM flux wire) irradiated for 54.9 EFPDs } \\
\hline & EOC & $30 \mathrm{MINs}$ & $5 \mathrm{HRS}$ & 1 DAY & 7 DAYS & 14 DAYS & 30 DAYS & 60 DAYS & 1 YEAR \\
\hline & $\mathrm{g} / 1 \mathrm{~g}$ of $\mathrm{Ti}$ & $\mathrm{g} / 1 \mathrm{~g}$ of $\mathrm{Ti}$ & $\mathrm{g} / 1 \mathrm{~g}$ of $\mathrm{Ti}$ & $\mathrm{g} / 1 \mathrm{~g}$ of $\mathrm{Ti}$ & $\mathrm{g} / 1 \mathrm{~g}$ of $\mathrm{Ti}$ & $\mathrm{g} / 1 \mathrm{~g}$ of $\mathrm{Ti}$ & $\mathrm{g} / 1 \mathrm{~g}$ of $\mathrm{Ti}$ & $\mathrm{g} / 1 \mathrm{~g}$ of $\mathrm{Ti}$ & $\mathrm{g} / 1 \mathrm{~g}$ of $\mathrm{Ti}$ \\
\hline H 1 & 1.07E-07 & 1.07E-07 & 1.07E-07 & $1.07 \mathrm{E}-07$ & 1.07E-07 & 1.07E-07 & $1.07 \mathrm{E}-07$ & $1.07 \mathrm{E}-07$ & $1.07 \mathrm{E}-07$ \\
\hline H 2 & $2.08 \mathrm{E}-11$ & $2.08 \mathrm{E}-11$ & $2.08 \mathrm{E}-11$ & $2.08 \mathrm{E}-11$ & $2.08 \mathrm{E}-11$ & $2.08 \mathrm{E}-11$ & $2.08 \mathrm{E}-11$ & $2.08 \mathrm{E}-11$ & $2.08 \mathrm{E}-11$ \\
\hline HE 4 & $7.41 \mathrm{E}-08$ & $7.41 \mathrm{E}-08$ & $7.41 \mathrm{E}-08$ & $7.41 \mathrm{E}-08$ & $7.41 \mathrm{E}-08$ & $7.41 \mathrm{E}-08$ & $7.41 \mathrm{E}-08$ & $7.41 \mathrm{E}-08$ & $7.41 \mathrm{E}-08$ \\
\hline AR 40 & $5.62 \mathrm{E}-13$ & $5.62 \mathrm{E}-13$ & $5.62 \mathrm{E}-13$ & $5.62 \mathrm{E}-13$ & $5.62 \mathrm{E}-13$ & $5.62 \mathrm{E}-13$ & $5.62 \mathrm{E}-13$ & $5.62 \mathrm{E}-13$ & $5.62 \mathrm{E}-13$ \\
\hline K 41 & $1.81 \mathrm{E}-14$ & $1.81 \mathrm{E}-14$ & $1.81 \mathrm{E}-14$ & $1.81 \mathrm{E}-14$ & $1.81 \mathrm{E}-14$ & $1.81 \mathrm{E}-14$ & $1.81 \mathrm{E}-14$ & $1.81 \mathrm{E}-14$ & $1.81 \mathrm{E}-14$ \\
\hline K 43 & $1.21 \mathrm{E}-14$ & $1.19 \mathrm{E}-14$ & $1.04 \mathrm{E}-14$ & $5.79 \mathrm{E}-15$ & $6.99 \mathrm{E}-17$ & 4.04E-19 & $3.10 \mathrm{E}-24$ & $7.97 \mathrm{E}-34$ & $0.00 \mathrm{E}+00$ \\
\hline CA 43 & $8.38 \mathrm{E}-08$ & $8.38 \mathrm{E}-08$ & $8.38 \mathrm{E}-08$ & $8.38 \mathrm{E}-08$ & $8.38 \mathrm{E}-08$ & $8.38 \mathrm{E}-08$ & $8.38 \mathrm{E}-08$ & $8.38 \mathrm{E}-08$ & $8.38 \mathrm{E}-08$ \\
\hline CA 44 & $6.45 \mathrm{E}-07$ & $6.45 \mathrm{E}-07$ & $6.45 \mathrm{E}-07$ & $6.45 \mathrm{E}-07$ & $6.45 \mathrm{E}-07$ & $6.45 \mathrm{E}-07$ & $6.45 \mathrm{E}-07$ & $6.45 \mathrm{E}-07$ & $6.45 \mathrm{E}-07$ \\
\hline CA 45 & $1.18 \mathrm{E}-08$ & $1.18 \mathrm{E}-08$ & $1.18 \mathrm{E}-08$ & $1.18 \mathrm{E}-08$ & $1.15 \mathrm{E}-08$ & $1.12 \mathrm{E}-08$ & $1.04 \mathrm{E}-08$ & $9.17 \mathrm{E}-09$ & $2.50 \mathrm{E}-09$ \\
\hline CA 46 & $7.38 \mathrm{E}-08$ & $7.38 \mathrm{E}-08$ & $7.38 \mathrm{E}-08$ & $7.38 \mathrm{E}-08$ & $7.38 \mathrm{E}-08$ & $7.38 \mathrm{E}-08$ & $7.38 \mathrm{E}-08$ & $7.38 \mathrm{E}-08$ & $7.38 \mathrm{E}-08$ \\
\hline CA 47 & $7.06 \mathrm{E}-12$ & 7.04E-12 & $6.84 \mathrm{E}-12$ & $6.06 \mathrm{E}-12$ & $2.42 \mathrm{E}-12$ & $8.31 \mathrm{E}-13$ & $7.21 \mathrm{E}-14$ & $7.36 \mathrm{E}-16$ & $4.06 E-36$ \\
\hline SC 45 & $1.42 \mathrm{E}-09$ & $1.43 \mathrm{E}-09$ & $1.43 \mathrm{E}-09$ & 1.47E-09 & 1.77E-09 & $2.11 \mathrm{E}-09$ & $2.84 \mathrm{E}-09$ & 4.09E-09 & $1.08 \mathrm{E}-08$ \\
\hline SC 46 & $1.40 \mathrm{E}-06$ & 1.40E-06 & 1.40E-06 & $1.39 \mathrm{E}-06$ & $1.32 \mathrm{E}-06$ & $1.25 \mathrm{E}-06$ & $1.09 \mathrm{E}-06$ & $8.52 \mathrm{E}-07$ & $6.82 \mathrm{E}-08$ \\
\hline SC 47 & $2.43 \mathrm{E}-07$ & $2.42 \mathrm{E}-07$ & $2.33 \mathrm{E}-07$ & $1.98 \mathrm{E}-07$ & $5.72 \mathrm{E}-08$ & $1.34 \mathrm{E}-08$ & $4.91 \mathrm{E}-10$ & $9.88 \mathrm{E}-13$ & $0.00 \mathrm{E}+00$ \\
\hline SC 48 & 1.27E-08 & $1.26 \mathrm{E}-08$ & 1.17E-08 & $8.69 \mathrm{E}-09$ & $8.90 \mathrm{E}-10$ & $6.24 \mathrm{E}-11$ & $1.43 \mathrm{E}-13$ & $1.61 \mathrm{E}-18$ & $0.00 \mathrm{E}+00$ \\
\hline SC 49 & $2.41 \mathrm{E}-10$ & $1.68 \mathrm{E}-10$ & $6.48 \mathrm{E}-12$ & $6.97 \mathrm{E}-18$ & $0.00 \mathrm{E}+00$ & $0.00 \mathrm{E}+00$ & $0.00 \mathrm{E}+00$ & $0.00 \mathrm{E}+00$ & $0.00 \mathrm{E}+00$ \\
\hline SC 50 & $1.45 \mathrm{E}-13$ & $7.51 \mathrm{E}-19$ & $0.00 \mathrm{E}+00$ & $0.00 \mathrm{E}+00$ & $0.00 \mathrm{E}+00$ & $0.00 \mathrm{E}+00$ & $0.00 \mathrm{E}+00$ & $0.00 \mathrm{E}+00$ & $0.00 \mathrm{E}+00$ \\
\hline TI 46 & $7.92 \mathrm{E}-02$ & $7.92 \mathrm{E}-02$ & $7.92 \mathrm{E}-02$ & $7.92 \mathrm{E}-02$ & $7.92 \mathrm{E}-02$ & $7.92 \mathrm{E}-02$ & $7.92 \mathrm{E}-02$ & $7.92 \mathrm{E}-02$ & $7.92 \mathrm{E}-02$ \\
\hline TI 47 & $7.30 \mathrm{E}-02$ & $7.30 \mathrm{E}-02$ & $7.30 \mathrm{E}-02$ & $7.30 \mathrm{E}-02$ & $7.30 \mathrm{E}-02$ & $7.30 \mathrm{E}-02$ & $7.30 \mathrm{E}-02$ & $7.30 \mathrm{E}-02$ & $7.30 \mathrm{E}-02$ \\
\hline TI 48 & $7.35 \mathrm{E}-01$ & $7.35 \mathrm{E}-01$ & $7.35 \mathrm{E}-01$ & $7.35 \mathrm{E}-01$ & $7.35 \mathrm{E}-01$ & $7.35 \mathrm{E}-01$ & $7.35 \mathrm{E}-01$ & $7.35 \mathrm{E}-01$ & $7.35 \mathrm{E}-01$ \\
\hline TI 49 & $5.82 \mathrm{E}-02$ & $5.82 \mathrm{E}-02$ & $5.82 \mathrm{E}-02$ & $5.82 \mathrm{E}-02$ & $5.82 \mathrm{E}-02$ & $5.82 \mathrm{E}-02$ & $5.82 \mathrm{E}-02$ & $5.82 \mathrm{E}-02$ & $5.82 \mathrm{E}-02$ \\
\hline TI 50 & $5.43 \mathrm{E}-02$ & $5.43 \mathrm{E}-02$ & $5.43 \mathrm{E}-02$ & $5.43 \mathrm{E}-02$ & $5.43 \mathrm{E}-02$ & $5.43 \mathrm{E}-02$ & $5.43 \mathrm{E}-02$ & $5.43 \mathrm{E}-02$ & $5.43 \mathrm{E}-02$ \\
\hline TI 51 & $5.67 \mathrm{E}-10$ & $1.53 \mathrm{E}-11$ & $1.19 \mathrm{E}-25$ & $0.00 E+00$ & $0.00 \mathrm{E}+00$ & $0.00 \mathrm{E}+00$ & $0.00 \mathrm{E}+00$ & $0.00 \mathrm{E}+00$ & $0.00 \mathrm{E}+00$ \\
\hline
\end{tabular}


TEM-10200-1

ENGINEERING CALCULATIONS AND ANALYSIS

Page 72 of 81

$12 / 19 / 17$

Rev.08

Title:

AS-RUN NEUTRONICS EVALUATION FOR THE CSM-10584 EXPERIMENT IN THE ATR

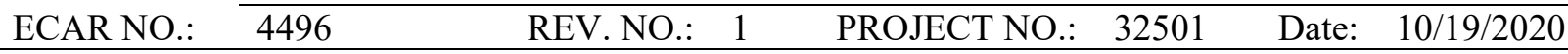

\begin{tabular}{|c|c|c|c|c|c|c|c|c|c|}
\hline V 51 & $5.38 \mathrm{E}-06$ & $5.38 \mathrm{E}-06$ & 5.38E-06 & 5.38E-06 & $5.38 \mathrm{E}-06$ & $5.38 \mathrm{E}-06$ & $5.38 \mathrm{E}-06$ & $5.38 \mathrm{E}-06$ & $5.38 \mathrm{E}-06$ \\
\hline V 52 & $9.69 \mathrm{E}-13$ & $3.78 \mathrm{E}-15$ & 8.01E-37 & $0.00 E+00$ & $0.00 E+00$ & $0.00 E+00$ & $0.00 E+00$ & $0.00 \mathrm{E}+00$ & $0.00 \mathrm{E}+00$ \\
\hline CR 52 & $7.08 \mathrm{E}-09$ & $7.08 \mathrm{E}-09$ & $7.08 \mathrm{E}-09$ & $7.08 \mathrm{E}-09$ & $7.08 \mathrm{E}-09$ & $7.08 \mathrm{E}-09$ & $7.08 \mathrm{E}-09$ & $7.08 \mathrm{E}-09$ & $7.08 \mathrm{E}-09$ \\
\hline CR 53 & $4.50 \mathrm{E}-12$ & $4.50 \mathrm{E}-12$ & $4.50 \mathrm{E}-12$ & $4.50 \mathrm{E}-12$ & $4.50 \mathrm{E}-12$ & $4.50 \mathrm{E}-12$ & $4.50 \mathrm{E}-12$ & $4.50 \mathrm{E}-12$ & $4.50 \mathrm{E}-12$ \\
\hline CR 54 & $1.08 \mathrm{E}-14$ & $1.08 \mathrm{E}-14$ & $1.08 \mathrm{E}-14$ & $1.08 \mathrm{E}-14$ & $1.08 \mathrm{E}-14$ & $1.08 \mathrm{E}-14$ & $1.08 \mathrm{E}-14$ & $1.08 \mathrm{E}-14$ & $1.08 \mathrm{E}-14$ \\
\hline SUMTOT & $1.00 \mathrm{E}+00$ & $1.00 \mathrm{E}+00$ & $1.00 \mathrm{E}+00$ & $1.00 \mathrm{E}+00$ & $1.00 \mathrm{E}+00$ & $1.00 \mathrm{E}+00$ & $1.00 \mathrm{E}+00$ & $1.00 \mathrm{E}+00$ & $1.00 \mathrm{E}+00$ \\
\hline OTOTAL & $1.00 \mathrm{E}+00$ & $1.00 \mathrm{E}+00$ & $1.00 \mathrm{E}+00$ & $1.00 \mathrm{E}+00$ & $1.00 \mathrm{E}+00$ & $1.00 \mathrm{E}+00$ & $1.00 \mathrm{E}+00$ & $1.00 \mathrm{E}+00$ & $1.00 \mathrm{E}+00$ \\
\hline
\end{tabular}

\begin{tabular}{|c|c|c|c|c|c|c|c|c|c|}
\hline \multicolumn{10}{|c|}{1 gram of Ti (CSM flux wire) irradiated for 119 EFPDs } \\
\hline & EOC & $30 \mathrm{MINs}$ & $5 \mathrm{HRS}$ & $1 \mathrm{DAY}$ & 7 DAYS & 14 DAYS & 30 DAYS & 60 DAYS & 1 YEAR \\
\hline & $\mathrm{Ci} / \mathrm{g}$ & $\mathrm{Ci} / \mathrm{g}$ & $\mathrm{Ci} / \mathrm{g}$ & $\mathrm{Ci} / \mathrm{g}$ & $\mathrm{Ci} / \mathrm{g}$ & $\mathrm{Ci} / \mathrm{g}$ & $\mathrm{Ci} / \mathrm{g}$ & $\mathrm{Ci} / \mathrm{g}$ & $\mathrm{Ci} / \mathrm{g}$ \\
\hline $\mathrm{H} 3$ & $2.78 \mathrm{E}-13$ & $2.78 \mathrm{E}-13$ & $2.78 \mathrm{E}-13$ & $2.78 \mathrm{E}-13$ & $2.78 \mathrm{E}-13$ & $2.77 \mathrm{E}-13$ & $2.77 \mathrm{E}-13$ & $2.75 \mathrm{E}-13$ & $2.63 \mathrm{E}-13$ \\
\hline $\mathrm{S} 37$ & $7.34 \mathrm{E}-11$ & $1.20 \mathrm{E}-12$ & $0.00 \mathrm{E}+00$ & $0.00 \mathrm{E}+00$ & $0.00 \mathrm{E}+00$ & $0.00 \mathrm{E}+00$ & $0.00 \mathrm{E}+00$ & $0.00 \mathrm{E}+00$ & $0.00 \mathrm{E}+00$ \\
\hline $\mathrm{CL} 38$ & $1.26 \mathrm{E}-14$ & $7.50 \mathrm{E}-15$ & $1.94 \mathrm{E}-16$ & $1.16 \mathrm{E}-25$ & $0.00 \mathrm{E}+00$ & $0.00 \mathrm{E}+00$ & $0.00 \mathrm{E}+00$ & $0.00 \mathrm{E}+00$ & $0.00 E+00$ \\
\hline AR 41 & $6.63 \mathrm{E}-09$ & $5.48 \mathrm{E}-09$ & $9.94 \mathrm{E}-10$ & $7.36 \mathrm{E}-13$ & $0.00 \mathrm{E}+00$ & $0.00 \mathrm{E}+00$ & $0.00 \mathrm{E}+00$ & $0.00 \mathrm{E}+00$ & $0.00 \mathrm{E}+00$ \\
\hline AR 42 & $1.16 \mathrm{E}-17$ & $1.16 \mathrm{E}-17$ & $1.16 \mathrm{E}-17$ & $1.16 \mathrm{E}-17$ & $1.16 \mathrm{E}-17$ & $1.16 \mathrm{E}-17$ & $1.16 \mathrm{E}-17$ & $1.16 \mathrm{E}-17$ & $1.14 \mathrm{E}-17$ \\
\hline $\mathrm{K} 43$ & $8.43 \mathrm{E}-08$ & 8.31E-08 & $7.23 \mathrm{E}-08$ & $4.04 \mathrm{E}-08$ & $4.88 \mathrm{E}-10$ & $2.82 \mathrm{E}-12$ & $2.17 \mathrm{E}-17$ & $5.56 \mathrm{E}-27$ & $0.00 \mathrm{E}+00$ \\
\hline K 44 & $1.50 \mathrm{E}-08$ & 5.81E-09 & $1.18 \mathrm{E}-12$ & $2.96 \mathrm{E}-28$ & $0.00 \mathrm{E}+00$ & $0.00 \mathrm{E}+00$ & $0.00 \mathrm{E}+00$ & $0.00 \mathrm{E}+00$ & $0.00 \mathrm{E}+00$ \\
\hline CA 45 & $4.08 \mathrm{E}-04$ & $4.08 \mathrm{E}-04$ & $4.08 \mathrm{E}-04$ & 4.06E-04 & $3.96 \mathrm{E}-04$ & $3.85 \mathrm{E}-04$ & $3.59 \mathrm{E}-04$ & $3.16 \mathrm{E}-04$ & 8.63E-05 \\
\hline CA 47 & $6.77 \mathrm{E}-06$ & $6.75 \mathrm{E}-06$ & $6.56 \mathrm{E}-06$ & $5.81 \mathrm{E}-06$ & $2.32 \mathrm{E}-06$ & 7.97E-07 & $6.91 \mathrm{E}-08$ & $7.06 \mathrm{E}-10$ & $3.89 \mathrm{E}-30$ \\
\hline SC 46 & $8.12 \mathrm{E}-02$ & $8.12 \mathrm{E}-02$ & $8.11 \mathrm{E}-02$ & $8.05 \mathrm{E}-02$ & $7.66 \mathrm{E}-02$ & $7.23 \mathrm{E}-02$ & $6.34 \mathrm{E}-02$ & $4.94 \mathrm{E}-02$ & $3.96 \mathrm{E}-03$ \\
\hline SC 46M & $2.31 \mathrm{E}-06$ & $0.00 \mathrm{E}+00$ & $0.00 \mathrm{E}+00$ & $0.00 \mathrm{E}+00$ & $0.00 \mathrm{E}+00$ & $0.00 \mathrm{E}+00$ & $0.00 \mathrm{E}+00$ & $0.00 \mathrm{E}+00$ & $0.00 \mathrm{E}+00$ \\
\hline SC 47 & $2.02 \mathrm{E}-01$ & $2.01 \mathrm{E}-01$ & $1.93 \mathrm{E}-01$ & $1.64 \mathrm{E}-01$ & $4.75 \mathrm{E}-02$ & $1.12 \mathrm{E}-02$ & $4.08 \mathrm{E}-04$ & $8.25 \mathrm{E}-07$ & $0.00 \mathrm{E}+00$ \\
\hline SC 49 & $1.71 \mathrm{E}-02$ & 1.19E-02 & $4.58 \mathrm{E}-04$ & $4.93 \mathrm{E}-10$ & $0.00 \mathrm{E}+00$ & $0.00 \mathrm{E}+00$ & $0.00 \mathrm{E}+00$ & $0.00 \mathrm{E}+00$ & $0.00 \mathrm{E}+00$ \\
\hline SC 50 & $3.20 \mathrm{E}-04$ & $1.66 \mathrm{E}-09$ & $0.00 \mathrm{E}+00$ & $0.00 \mathrm{E}+00$ & $0.00 \mathrm{E}+00$ & $0.00 \mathrm{E}+00$ & $0.00 \mathrm{E}+00$ & $0.00 \mathrm{E}+00$ & $0.00 \mathrm{E}+00$ \\
\hline TI 51 & $3.63 \mathrm{E}-01$ & $9.83 \mathrm{E}-03$ & $7.62 \mathrm{E}-17$ & $0.00 \mathrm{E}+00$ & $0.00 E+00$ & $0.00 E+00$ & $0.00 E+00$ & $0.00 E+00$ & $0.00 E+00$ \\
\hline V 52 & $2.03 \mathrm{E}-03$ & 7.91E-06 & $1.68 \mathrm{E}-27$ & $0.00 \mathrm{E}+00$ & $0.00 \mathrm{E}+00$ & $0.00 \mathrm{E}+00$ & $0.00 \mathrm{E}+00$ & $0.00 \mathrm{E}+00$ & $0.00 \mathrm{E}+00$ \\
\hline V 53 & $5.03 \mathrm{E}-13$ & $1.24 \mathrm{E}-18$ & $0.00 \mathrm{E}+00$ & $0.00 \mathrm{E}+00$ & $0.00 \mathrm{E}+00$ & $0.00 \mathrm{E}+00$ & $0.00 \mathrm{E}+00$ & $0.00 \mathrm{E}+00$ & $0.00 \mathrm{E}+00$ \\
\hline CR 51 & $1.99 \mathrm{E}-10$ & $1.99 \mathrm{E}-10$ & $1.98 \mathrm{E}-10$ & $1.94 \mathrm{E}-10$ & $1.67 \mathrm{E}-10$ & $1.40 \mathrm{E}-10$ & $9.41 \mathrm{E}-11$ & $4.44 \mathrm{E}-11$ & $2.14 \mathrm{E}-14$ \\
\hline CR 55 & $2.85 \mathrm{E}-12$ & $6.08 \mathrm{E}-15$ & $0.00 \mathrm{E}+00$ & $0.00 \mathrm{E}+00$ & $0.00 \mathrm{E}+00$ & $0.00 \mathrm{E}+00$ & $0.00 \mathrm{E}+00$ & $0.00 \mathrm{E}+00$ & $0.00 \mathrm{E}+00$ \\
\hline MN 56 & $1.05 \mathrm{E}-14$ & $9.09 \mathrm{E}-15$ & $2.68 \mathrm{E}-15$ & $8.11 \mathrm{E}-17$ & $0.00 \mathrm{E}+00$ & $0.00 \mathrm{E}+00$ & $0.00 \mathrm{E}+00$ & $0.00 \mathrm{E}+00$ & $0.00 \mathrm{E}+00$ \\
\hline SUMTOT & $6.85 \mathrm{E}-01$ & 3.23E-01 & $2.93 \mathrm{E}-01$ & $2.58 \mathrm{E}-01$ & $1.26 \mathrm{E}-01$ & 8.39E-02 & $6.41 \mathrm{E}-02$ & 4.97E-02 & 4.04E-03 \\
\hline OTOTAL & $6.85 \mathrm{E}-01$ & $3.23 \mathrm{E}-01$ & $2.93 \mathrm{E}-01$ & $2.58 \mathrm{E}-01$ & $1.26 \mathrm{E}-01$ & $8.39 \mathrm{E}-02$ & $6.41 \mathrm{E}-02$ & $4.97 \mathrm{E}-02$ & $4.04 \mathrm{E}-03$ \\
\hline
\end{tabular}

\begin{tabular}{|c|c|c|c|c|c|c|c|c|c|}
\hline \multicolumn{10}{|c|}{1 gram of Ti (CSM flux wire) irradiated for 119 EFPDs } \\
\hline & EOC & $30 \mathrm{MINs}$ & $5 \mathrm{HRS}$ & $1 \mathrm{DAY}$ & 7 DAYS & 14 DAYS & 30 DAYS & 60 DAYS & 1 YEAR \\
\hline & $\mathrm{g} / 1 \mathrm{~g}$ of $\mathrm{Ti}$ & $\mathrm{g} / 1 \mathrm{~g}$ of $\mathrm{Ti}$ & $\mathrm{g} / 1 \mathrm{~g}$ of $\mathrm{Ti}$ & $\mathrm{g} / 1 \mathrm{~g}$ of $\mathrm{Ti}$ & $\mathrm{g} / 1 \mathrm{~g}$ of $\mathrm{Ti}$ & $\mathrm{g} / 1 \mathrm{~g}$ of $\mathrm{Ti}$ & $\mathrm{g} / 1 \mathrm{~g}$ of $\mathrm{Ti}$ & $\mathrm{g} / 1 \mathrm{~g}$ of $\mathrm{Ti}$ & $\mathrm{g} / 1 \mathrm{~g}$ of $\mathrm{Ti}$ \\
\hline H 1 & $2.32 \mathrm{E}-07$ & $2.32 \mathrm{E}-07$ & $2.32 \mathrm{E}-07$ & $2.32 \mathrm{E}-07$ & $2.32 \mathrm{E}-07$ & $2.32 \mathrm{E}-07$ & $2.32 \mathrm{E}-07$ & $2.32 \mathrm{E}-07$ & $2.32 \mathrm{E}-07$ \\
\hline H 2 & $9.76 \mathrm{E}-11$ & $9.76 \mathrm{E}-11$ & $9.76 \mathrm{E}-11$ & $9.76 \mathrm{E}-11$ & $9.76 \mathrm{E}-11$ & $9.76 \mathrm{E}-11$ & $9.76 \mathrm{E}-11$ & $9.76 \mathrm{E}-11$ & $9.76 \mathrm{E}-11$ \\
\hline HE 4 & 1.61E-07 & 1.61E-07 & 1.61E-07 & $1.61 \mathrm{E}-07$ & $1.61 \mathrm{E}-07$ & $1.61 \mathrm{E}-07$ & 1.61E-07 & $1.61 \mathrm{E}-07$ & 1.61E-07 \\
\hline AR 40 & $2.64 \mathrm{E}-12$ & $2.64 \mathrm{E}-12$ & $2.64 \mathrm{E}-12$ & $2.64 \mathrm{E}-12$ & $2.64 \mathrm{E}-12$ & $2.64 \mathrm{E}-12$ & $2.64 \mathrm{E}-12$ & $2.64 \mathrm{E}-12$ & $2.64 \mathrm{E}-12$ \\
\hline K 41 & $8.53 \mathrm{E}-14$ & $8.53 \mathrm{E}-14$ & $8.54 \mathrm{E}-14$ & $8.55 \mathrm{E}-14$ & $8.55 \mathrm{E}-14$ & $8.55 \mathrm{E}-14$ & $8.55 \mathrm{E}-14$ & $8.55 \mathrm{E}-14$ & $8.55 \mathrm{E}-14$ \\
\hline K 43 & $2.61 \mathrm{E}-14$ & $2.57 \mathrm{E}-14$ & $2.24 \mathrm{E}-14$ & $1.25 \mathrm{E}-14$ & $1.51 \mathrm{E}-16$ & $8.75 \mathrm{E}-19$ & $6.71 \mathrm{E}-24$ & $1.72 \mathrm{E}-33$ & $0.00 \mathrm{E}+00$ \\
\hline CA 43 & $1.81 \mathrm{E}-07$ & $1.81 \mathrm{E}-07$ & $1.81 \mathrm{E}-07$ & $1.81 \mathrm{E}-07$ & $1.81 \mathrm{E}-07$ & $1.81 \mathrm{E}-07$ & $1.81 \mathrm{E}-07$ & $1.81 \mathrm{E}-07$ & $1.81 \mathrm{E}-07$ \\
\hline CA 44 & $1.40 \mathrm{E}-06$ & $1.40 \mathrm{E}-06$ & $1.40 \mathrm{E}-06$ & $1.40 \mathrm{E}-06$ & $1.40 \mathrm{E}-06$ & $1.40 \mathrm{E}-06$ & $1.40 \mathrm{E}-06$ & 1.40E-06 & $1.40 \mathrm{E}-06$ \\
\hline CA 45 & $2.29 \mathrm{E}-08$ & $2.29 \mathrm{E}-08$ & $2.29 \mathrm{E}-08$ & $2.28 \mathrm{E}-08$ & $2.22 \mathrm{E}-08$ & $2.16 \mathrm{E}-08$ & $2.02 \mathrm{E}-08$ & $1.78 \mathrm{E}-08$ & $4.85 \mathrm{E}-09$ \\
\hline CA 46 & $1.65 \mathrm{E}-07$ & $1.65 \mathrm{E}-07$ & $1.65 \mathrm{E}-07$ & $1.65 \mathrm{E}-07$ & $1.65 \mathrm{E}-07$ & $1.65 \mathrm{E}-07$ & $1.65 \mathrm{E}-07$ & $1.65 \mathrm{E}-07$ & 1.65E-07 \\
\hline CA 47 & $1.11 \mathrm{E}-11$ & $1.10 \mathrm{E}-11$ & 1.07E-11 & $9.48 \mathrm{E}-12$ & $3.79 \mathrm{E}-12$ & $1.30 \mathrm{E}-12$ & $1.13 \mathrm{E}-13$ & $1.15 \mathrm{E}-15$ & $6.35 \mathrm{E}-36$ \\
\hline SC 45 & $6.16 \mathrm{E}-09$ & 6.17E-09 & $6.19 \mathrm{E}-09$ & $6.26 \mathrm{E}-09$ & $6.84 \mathrm{E}-09$ & 7.49E-09 & 8.91E-09 & $1.13 \mathrm{E}-08$ & $2.42 \mathrm{E}-08$ \\
\hline SC 46 & $2.40 \mathrm{E}-06$ & $2.40 \mathrm{E}-06$ & $2.39 \mathrm{E}-06$ & $2.38 \mathrm{E}-06$ & $2.26 \mathrm{E}-06$ & $2.13 \mathrm{E}-06$ & $1.87 \mathrm{E}-06$ & $1.46 \mathrm{E}-06$ & 1.17E-07 \\
\hline SC 47 & $2.43 \mathrm{E}-07$ & $2.42 \mathrm{E}-07$ & $2.33 \mathrm{E}-07$ & $1.98 \mathrm{E}-07$ & $5.72 \mathrm{E}-08$ & $1.34 \mathrm{E}-08$ & $4.91 \mathrm{E}-10$ & $9.94 \mathrm{E}-13$ & $0.00 \mathrm{E}+00$ \\
\hline SC 48 & $1.27 \mathrm{E}-08$ & $1.26 \mathrm{E}-08$ & 1.17E-08 & $8.66 \mathrm{E}-09$ & $8.87 \mathrm{E}-10$ & $6.21 \mathrm{E}-11$ & $1.43 \mathrm{E}-13$ & $1.61 \mathrm{E}-18$ & $0.00 \mathrm{E}+00$ \\
\hline
\end{tabular}


TEM-10200-1

ENGINEERING CALCULATIONS AND ANALYSIS

Page 73 of 81

$12 / 19 / 17$

Rev.08

Title:

AS-RUN NEUTRONICS EVALUATION FOR THE CSM-10584 EXPERIMENT IN THE ATR

ECAR NO.: 4496

REV. NO.: $1 \quad$ PROJECT NO.: 32501

Date: $10 / 19 / 2020$

\begin{tabular}{|c|c|c|c|c|c|c|c|c|c|}
\hline SC 49 & $2.55 \mathrm{E}-10$ & $1.78 \mathrm{E}-10$ & $6.86 \mathrm{E}-12$ & 7.38E-18 & $0.00 E+00$ & $0.00 \mathrm{E}+00$ & $0.00 E+00$ & $0.00 E+00$ & $0.00 E+00$ \\
\hline SC 50 & $1.45 \mathrm{E}-13$ & $7.52 \mathrm{E}-19$ & $0.00 \mathrm{E}+00$ & $0.00 \mathrm{E}+00$ & $0.00 \mathrm{E}+00$ & $0.00 \mathrm{E}+00$ & $0.00 \mathrm{E}+00$ & $0.00 \mathrm{E}+00$ & $0.00 \mathrm{E}+00$ \\
\hline TI 46 & $7.91 \mathrm{E}-02$ & $7.91 \mathrm{E}-02$ & $7.91 \mathrm{E}-02$ & $7.91 \mathrm{E}-02$ & $7.91 \mathrm{E}-02$ & $7.91 \mathrm{E}-02$ & $7.91 \mathrm{E}-02$ & $7.91 \mathrm{E}-02$ & $7.91 \mathrm{E}-02$ \\
\hline TI 47 & $7.30 \mathrm{E}-02$ & $7.30 \mathrm{E}-02$ & $7.30 \mathrm{E}-02$ & $7.30 \mathrm{E}-02$ & $7.30 \mathrm{E}-02$ & $7.30 \mathrm{E}-02$ & $7.30 \mathrm{E}-02$ & $7.30 \mathrm{E}-02$ & $7.30 \mathrm{E}-02$ \\
\hline TI 48 & $7.32 \mathrm{E}-01$ & $7.32 \mathrm{E}-01$ & $7.32 \mathrm{E}-01$ & $7.32 \mathrm{E}-01$ & $7.32 \mathrm{E}-01$ & $7.32 \mathrm{E}-01$ & $7.32 \mathrm{E}-01$ & $7.32 \mathrm{E}-01$ & $7.32 \mathrm{E}-01$ \\
\hline TI 49 & $6.16 \mathrm{E}-02$ & $6.16 \mathrm{E}-02$ & $6.16 \mathrm{E}-02$ & $6.16 \mathrm{E}-02$ & $6.16 \mathrm{E}-02$ & $6.16 \mathrm{E}-02$ & $6.16 \mathrm{E}-02$ & $6.16 \mathrm{E}-02$ & $6.16 \mathrm{E}-02$ \\
\hline TI 50 & 5.44E-02 & 5.44E-02 & $5.44 \mathrm{E}-02$ & $5.44 \mathrm{E}-02$ & $5.44 \mathrm{E}-02$ & $5.44 \mathrm{E}-02$ & $5.44 \mathrm{E}-02$ & 5.44E-02 & 5.44E-02 \\
\hline TI 51 & 5.67E-10 & 1.54E-11 & 1.19E-25 & $0.00 \mathrm{E}+00$ & $0.00 \mathrm{E}+00$ & $0.00 \mathrm{E}+00$ & $0.00 E+00$ & $0.00 \mathrm{E}+00$ & $0.00 E+00$ \\
\hline V 51 & 1.17E-05 & 1.17E-05 & 1.17E-05 & 1.17E-05 & 1.17E-05 & 1.17E-05 & 1.17E-05 & 1.17E-05 & 1.17E-05 \\
\hline V 52 & $2.10 \mathrm{E}-12$ & $8.20 \mathrm{E}-15$ & $1.74 \mathrm{E}-36$ & $0.00 \mathrm{E}+00$ & $0.00 \mathrm{E}+00$ & $0.00 \mathrm{E}+00$ & $0.00 \mathrm{E}+00$ & $0.00 \mathrm{E}+00$ & $0.00 \mathrm{E}+00$ \\
\hline CR 51 & $2.16 \mathrm{E}-15$ & $2.16 \mathrm{E}-15$ & $2.15 \mathrm{E}-15$ & $2.10 \mathrm{E}-15$ & $1.81 \mathrm{E}-15$ & $1.52 \mathrm{E}-15$ & $1.02 \mathrm{E}-15$ & $4.81 \mathrm{E}-16$ & $2.32 \mathrm{E}-19$ \\
\hline CR 52 & $3.32 \mathrm{E}-08$ & $3.32 \mathrm{E}-08$ & $3.32 \mathrm{E}-08$ & $3.32 \mathrm{E}-08$ & $3.32 \mathrm{E}-08$ & $3.32 \mathrm{E}-08$ & $3.32 \mathrm{E}-08$ & $3.32 \mathrm{E}-08$ & $3.32 \mathrm{E}-08$ \\
\hline CR 53 & $4.56 \mathrm{E}-11$ & $4.56 \mathrm{E}-11$ & $4.56 \mathrm{E}-11$ & $4.56 \mathrm{E}-11$ & $4.56 \mathrm{E}-11$ & $4.56 \mathrm{E}-11$ & $4.56 \mathrm{E}-11$ & $4.56 \mathrm{E}-11$ & $4.56 \mathrm{E}-11$ \\
\hline CR 54 & $2.39 \mathrm{E}-13$ & $2.39 \mathrm{E}-13$ & $2.39 \mathrm{E}-13$ & $2.39 \mathrm{E}-13$ & $2.39 \mathrm{E}-13$ & $2.39 \mathrm{E}-13$ & $2.39 \mathrm{E}-13$ & $2.39 \mathrm{E}-13$ & $2.39 \mathrm{E}-13$ \\
\hline SUMTOT & $1.00 \mathrm{E}+00$ & $1.00 \mathrm{E}+00$ & $1.00 \mathrm{E}+00$ & $1.00 \mathrm{E}+00$ & $1.00 \mathrm{E}+00$ & $1.00 \mathrm{E}+00$ & $1.00 \mathrm{E}+00$ & $1.00 \mathrm{E}+00$ & $1.00 \mathrm{E}+00$ \\
\hline OTOTAL & $1.00 \mathrm{E}+00$ & $1.00 \mathrm{E}+00$ & $1.00 \mathrm{E}+00$ & $1.00 \mathrm{E}+00$ & $1.00 \mathrm{E}+00$ & $1.00 \mathrm{E}+00$ & $1.00 \mathrm{E}+00$ & $1.00 \mathrm{E}+00$ & $1.00 \mathrm{E}+00$ \\
\hline
\end{tabular}

\begin{tabular}{|c|c|c|c|c|c|c|c|c|c|}
\hline \multicolumn{10}{|c|}{1 gram of 95Zn-5Al (CSM melt wire) irradiated for 119 EFPDs } \\
\hline & EOC & $30 \mathrm{MINs}$ & $5 \mathrm{HRS}$ & 1 DAY & 7 DAYS & 14 DAYS & 30 DAYS & 60 DAYS & 1 YEAR \\
\hline & $\begin{array}{c}\mathrm{Ci} / 1 \mathrm{~g} \text { of } \\
95 \mathrm{Zn}-5 \mathrm{Al}\end{array}$ & $\begin{array}{c}\mathrm{Ci} / 1 \mathrm{~g} \text { of } \\
95 \mathrm{Zn}-5 \mathrm{Al}\end{array}$ & $\begin{array}{c}\mathrm{Ci} / 1 \mathrm{~g} \text { of } \\
95 \mathrm{Zn}-5 \mathrm{Al}\end{array}$ & $\begin{array}{c}\mathrm{Ci} / 1 \mathrm{~g} \text { of } \\
95 \mathrm{Zn}-5 \mathrm{Al}\end{array}$ & $\begin{array}{c}\mathrm{Ci} / 1 \mathrm{~g} \text { of } \\
95 \mathrm{Zn}-5 \mathrm{Al}\end{array}$ & $\begin{array}{c}\mathrm{Ci} / 1 \mathrm{~g} \text { of } \\
95 \mathrm{Zn}-5 \mathrm{Al}\end{array}$ & $\begin{array}{c}\mathrm{Ci} / 1 \mathrm{~g} \text { of } \\
95 \mathrm{Zn}-5 \mathrm{Al}\end{array}$ & $\begin{array}{c}\mathrm{Ci} / 1 \mathrm{~g} \text { of } \\
95 \mathrm{Zn}-5 \mathrm{Al}\end{array}$ & $\begin{array}{c}\mathrm{Ci} / 1 \mathrm{~g} \text { of } \\
95 \mathrm{Zn}-5 \mathrm{Al}\end{array}$ \\
\hline H 3 & $2.56 \mathrm{E}-14$ & $2.56 \mathrm{E}-14$ & $2.56 \mathrm{E}-14$ & $2.56 \mathrm{E}-14$ & $2.56 \mathrm{E}-14$ & $2.56 \mathrm{E}-14$ & $2.55 \mathrm{E}-14$ & $2.54 \mathrm{E}-14$ & $2.42 \mathrm{E}-14$ \\
\hline NA 24 & 7.07E-03 & $6.91 \mathrm{E}-03$ & $5.61 \mathrm{E}-03$ & $2.33 \mathrm{E}-03$ & $3.00 \mathrm{E}-06$ & $1.28 \mathrm{E}-09$ & $2.51 \mathrm{E}-17$ & $0.00 \mathrm{E}+00$ & $0.00 \mathrm{E}+00$ \\
\hline NA 25 & $9.83 \mathrm{E}-12$ & $7.96 \mathrm{E}-21$ & $0.00 E+00$ & $0.00 \mathrm{E}+00$ & $0.00 \mathrm{E}+00$ & $0.00 \mathrm{E}+00$ & $0.00 \mathrm{E}+00$ & $0.00 \mathrm{E}+00$ & $0.00 \mathrm{E}+00$ \\
\hline MG & & & & & & & & & \\
\hline 27 & $3.38 \mathrm{E}-02$ & $3.76 \mathrm{E}-03$ & $9.65 \mathrm{E}-12$ & $0.00 \mathrm{E}+00$ & $0.00 \mathrm{E}+00$ & $0.00 \mathrm{E}+00$ & $0.00 \mathrm{E}+00$ & $0.00 \mathrm{E}+00$ & $0.00 \mathrm{E}+00$ \\
\hline MG & & & & & & & & & \\
\hline 28 & $1.20 \mathrm{E}-10$ & $1.18 \mathrm{E}-10$ & $1.02 \mathrm{E}-10$ & $5.43 \mathrm{E}-11$ & $4.59 \mathrm{E}-13$ & $1.74 \mathrm{E}-15$ & 5.17E-21 & $0.00 \mathrm{E}+00$ & $0.00 \mathrm{E}+00$ \\
\hline AL 28 & $8.11 \mathrm{E}-01$ & 7.54E-05 & $1.02 \mathrm{E}-10$ & 5.44E-11 & $4.60 \mathrm{E}-13$ & $1.75 \mathrm{E}-15$ & $5.18 \mathrm{E}-21$ & $0.00 \mathrm{E}+00$ & $0.00 \mathrm{E}+00$ \\
\hline AL 29 & $7.75 \mathrm{E}-10$ & $3.19 \mathrm{E}-11$ & $1.09 \mathrm{E}-23$ & $0.00 \mathrm{E}+00$ & $0.00 \mathrm{E}+00$ & $0.00 \mathrm{E}+00$ & $0.00 \mathrm{E}+00$ & $0.00 \mathrm{E}+00$ & $0.00 E+00$ \\
\hline SI 31 & $1.10 \mathrm{E}-12$ & $9.61 \mathrm{E}-13$ & $2.92 \mathrm{E}-13$ & $1.96 \mathrm{E}-15$ & $0.00 \mathrm{E}+00$ & $0.00 \mathrm{E}+00$ & $0.00 \mathrm{E}+00$ & $0.00 \mathrm{E}+00$ & $0.00 \mathrm{E}+00$ \\
\hline FE 59 & $3.77 \mathrm{E}-12$ & $3.77 \mathrm{E}-12$ & $3.76 \mathrm{E}-12$ & $3.71 \mathrm{E}-12$ & $3.38 \mathrm{E}-12$ & $3.04 \mathrm{E}-12$ & $2.37 \mathrm{E}-12$ & $1.50 \mathrm{E}-12$ & $1.36 \mathrm{E}-14$ \\
\hline CO 60 & $7.23 \mathrm{E}-15$ & $7.23 \mathrm{E}-15$ & $7.23 \mathrm{E}-15$ & $7.23 \mathrm{E}-15$ & $7.22 \mathrm{E}-15$ & $7.20 \mathrm{E}-15$ & $7.16 \mathrm{E}-15$ & $7.08 \mathrm{E}-15$ & $6.34 \mathrm{E}-15$ \\
\hline $\mathrm{CO}$ & & & & & & & & & \\
\hline $60 \mathrm{M}$ & $3.19 \mathrm{E}-14$ & 4.99E-15 & $8.61 \mathrm{E}-23$ & $0.00 E+00$ & $0.00 E+00$ & $0.00 E+00$ & $0.00 E+00$ & $0.00 \mathrm{E}+00$ & $0.00 E+00$ \\
\hline CO 61 & 1.17E-09 & $9.45 \mathrm{E}-10$ & $1.43 \mathrm{E}-10$ & $4.88 \mathrm{E}-14$ & $0.00 \mathrm{E}+00$ & $0.00 \mathrm{E}+00$ & $0.00 \mathrm{E}+00$ & $0.00 \mathrm{E}+00$ & $0.00 \mathrm{E}+00$ \\
\hline CO 62 & $1.12 \mathrm{E}-06$ & $1.07 \mathrm{E}-12$ & $0.00 \mathrm{E}+00$ & $0.00 \mathrm{E}+00$ & $0.00 \mathrm{E}+00$ & $0.00 \mathrm{E}+00$ & $0.00 \mathrm{E}+00$ & $0.00 \mathrm{E}+00$ & $0.00 \mathrm{E}+00$ \\
\hline NI 63 & $3.53 \mathrm{E}-07$ & $3.53 \mathrm{E}-07$ & $3.53 \mathrm{E}-07$ & $3.53 \mathrm{E}-07$ & $3.53 \mathrm{E}-07$ & $3.53 \mathrm{E}-07$ & $3.53 \mathrm{E}-07$ & $3.53 \mathrm{E}-07$ & 3.50E-07 \\
\hline NI 65 & $1.01 \mathrm{E}-04$ & $8.79 \mathrm{E}-05$ & $2.55 \mathrm{E}-05$ & $1.37 \mathrm{E}-07$ & $8.61 \mathrm{E}-25$ & $0.00 \mathrm{E}+00$ & $0.00 \mathrm{E}+00$ & $0.00 \mathrm{E}+00$ & $0.00 \mathrm{E}+00$ \\
\hline NI 66 & $3.45 \mathrm{E}-09$ & $3.43 \mathrm{E}-09$ & $3.24 \mathrm{E}-09$ & $2.54 \mathrm{E}-09$ & 4.09E-10 & $4.85 \mathrm{E}-11$ & $3.71 \mathrm{E}-13$ & $3.98 \mathrm{E}-17$ & $0.00 \mathrm{E}+00$ \\
\hline CU 64 & $5.27 \mathrm{E}-06$ & $5.13 \mathrm{E}-06$ & $4.01 \mathrm{E}-06$ & $1.42 \mathrm{E}-06$ & $5.49 \mathrm{E}-10$ & $5.73 \mathrm{E}-14$ & $4.53 \mathrm{E}-23$ & $0.00 \mathrm{E}+00$ & $0.00 \mathrm{E}+00$ \\
\hline CU 66 & $5.69 \mathrm{E}-03$ & $9.65 \mathrm{E}-05$ & $3.24 \mathrm{E}-09$ & $2.55 \mathrm{E}-09$ & $4.10 \mathrm{E}-10$ & $4.86 \mathrm{E}-11$ & $3.71 \mathrm{E}-13$ & $3.99 \mathrm{E}-17$ & $0.00 \mathrm{E}+00$ \\
\hline CU 67 & 3.47E-08 & $3.45 \mathrm{E}-08$ & $3.28 \mathrm{E}-08$ & $2.65 \mathrm{E}-08$ & $5.28 \mathrm{E}-09$ & $8.04 \mathrm{E}-10$ & $1.09 \mathrm{E}-11$ & $3.41 \mathrm{E}-15$ & $0.00 \mathrm{E}+00$ \\
\hline ZN 65 & $3.79 \mathrm{E}+00$ & $3.79 \mathrm{E}+00$ & $3.79 \mathrm{E}+00$ & $3.78 \mathrm{E}+00$ & $3.72 \mathrm{E}+00$ & $3.64 \mathrm{E}+00$ & $3.48 \mathrm{E}+00$ & $3.20 \mathrm{E}+00$ & $1.34 \mathrm{E}+00$ \\
\hline ZN 69 & $8.80 \mathrm{E}+00$ & $6.29 \mathrm{E}+00$ & 7.06E-01 & $1.90 \mathrm{E}-01$ & $1.34 \mathrm{E}-04$ & $2.83 \mathrm{E}-08$ & $1.13 \mathrm{E}-16$ & $0.00 \mathrm{E}+00$ & $0.00 \mathrm{E}+00$ \\
\hline $\mathrm{ZN}$ & & & & & & & & & \\
\hline $69 \mathrm{M}$ & $5.91 \mathrm{E}-01$ & $5.76 \mathrm{E}-01$ & 4.60E-01 & $1.77 \mathrm{E}-01$ & $1.25 \mathrm{E}-04$ & $2.64 \mathrm{E}-08$ & $1.05 \mathrm{E}-16$ & $0.00 E+00$ & $0.00 E+00$ \\
\hline ZN 71 & $1.32 \mathrm{E}-02$ & $2.92 \mathrm{E}-06$ & $2.89 \mathrm{E}-07$ & $1.00 \mathrm{E}-08$ & $8.77 \mathrm{E}-20$ & $0.00 \mathrm{E}+00$ & $0.00 \mathrm{E}+00$ & $0.00 \mathrm{E}+00$ & $0.00 E+00$ \\
\hline $\mathrm{ZN}$ & & & & & & & & & \\
\hline $71 \mathrm{M}$ & $1.38 \mathrm{E}-03$ & $1.27 \mathrm{E}-03$ & $5.71 \mathrm{E}-04$ & $1.99 \mathrm{E}-05$ & $1.74 \mathrm{E}-16$ & $2.18 \mathrm{E}-29$ & $0.00 E+00$ & $0.00 \mathrm{E}+00$ & $0.00 \mathrm{E}+00$ \\
\hline GA 70 & $5.52 \mathrm{E}-02$ & $2.06 \mathrm{E}-02$ & $2.90 \mathrm{E}-06$ & $1.58 \mathrm{E}-22$ & $0.00 \mathrm{E}+00$ & $0.00 \mathrm{E}+00$ & $0.00 \mathrm{E}+00$ & $0.00 \mathrm{E}+00$ & $0.00 \mathrm{E}+00$ \\
\hline
\end{tabular}


TEM-10200-1

ENGINEERING CALCULATIONS AND ANALYSIS

Page 74 of 81

$12 / 19 / 17$

Rev.08

Title:

AS-RUN NEUTRONICS EVALUATION FOR THE CSM-10584 EXPERIMENT IN THE ATR

\begin{tabular}{llllllll} 
ECAR NO.: & 4496 & REV. NO.: & 1 & PROJECT NO.: & 32501 & Date: & 10/19/2020 \\
\hline
\end{tabular}

\begin{tabular}{|c|c|c|c|c|c|c|c|c|c|}
$\mathrm{GA} 72$ & $2.04 \mathrm{E}-04$ & $1.99 \mathrm{E}-04$ & $1.60 \mathrm{E}-04$ & $6.28 \mathrm{E}-05$ & $5.29 \mathrm{E}-08$ & $1.37 \mathrm{E}-11$ & $8.68 \mathrm{E}-20$ & $0.00 \mathrm{E}+00$ & $0.00 \mathrm{E}+00$ \\
\hline $\begin{array}{c}\mathrm{GA} \\
72 \mathrm{M}\end{array}$ & $6.31 \mathrm{E}-06$ & $0.00 \mathrm{E}+00$ & $0.00 \mathrm{E}+00$ & $0.00 \mathrm{E}+00$ & $0.00 \mathrm{E}+00$ & $0.00 \mathrm{E}+00$ & $0.00 \mathrm{E}+00$ & $0.00 \mathrm{E}+00$ & $0.00 \mathrm{E}+00$ \\
\hline $\mathrm{GE} 71$ & $8.51 \mathrm{E}-05$ & $8.50 \mathrm{E}-05$ & $8.40 \mathrm{E}-05$ & $8.02 \mathrm{E}-05$ & $5.64 \mathrm{E}-05$ & $3.74 \mathrm{E}-05$ & $1.46 \mathrm{E}-05$ & $2.51 \mathrm{E}-06$ & $4.09 \mathrm{E}-14$ \\
\hline $\begin{array}{c}\mathrm{GE} \\
71 \mathrm{M}\end{array}$ & $9.19 \mathrm{E}-06$ & $0.00 \mathrm{E}+00$ & $0.00 \mathrm{E}+00$ & $0.00 \mathrm{E}+00$ & $0.00 \mathrm{E}+00$ & $0.00 \mathrm{E}+00$ & $0.00 \mathrm{E}+00$ & $0.00 \mathrm{E}+00$ & $0.00 \mathrm{E}+00$ \\
\hline $\mathrm{GE} 75$ & $4.28 \mathrm{E}-13$ & $3.34 \mathrm{E}-13$ & $3.49 \mathrm{E}-14$ & $2.50 \mathrm{E}-18$ & $0.00 \mathrm{E}+00$ & $0.00 \mathrm{E}+00$ & $0.00 \mathrm{E}+00$ & $0.00 \mathrm{E}+00$ & $0.00 \mathrm{E}+00$ \\
\hline $\begin{array}{c}\mathrm{GE} \\
75 \mathrm{M}\end{array}$ & $9.42 \mathrm{E}-14$ & $7.82 \mathrm{E}-25$ & $0.00 \mathrm{E}+00$ & $0.00 \mathrm{E}+00$ & $0.00 \mathrm{E}+00$ & $0.00 \mathrm{E}+00$ & $0.00 \mathrm{E}+00$ & $0.00 \mathrm{E}+00$ & $0.00 \mathrm{E}+00$ \\
\hline $\begin{array}{c}\text { SUMT } \\
\text { OT }\end{array}$ & $1.41 \mathrm{E}+01$ & $1.07 \mathrm{E}+01$ & $4.96 \mathrm{E}+00$ & $4.15 \mathrm{E}+00$ & $3.72 \mathrm{E}+00$ & $3.64 \mathrm{E}+00$ & $3.48 \mathrm{E}+00$ & $3.20 \mathrm{E}+00$ & $1.34 \mathrm{E}+00$ \\
\hline $\begin{array}{c}\text { OTOTA } \\
\text { L }\end{array}$ & $1.41 \mathrm{E}+01$ & $1.07 \mathrm{E}+01$ & $4.96 \mathrm{E}+00$ & $4.15 \mathrm{E}+00$ & $3.72 \mathrm{E}+00$ & $3.64 \mathrm{E}+00$ & $3.48 \mathrm{E}+00$ & $3.20 \mathrm{E}+00$ & $1.34 \mathrm{E}+00$ \\
\hline
\end{tabular}

\begin{tabular}{|c|c|c|c|c|c|c|c|c|c|}
\hline \multicolumn{10}{|c|}{1 gram of $95 \mathrm{Zn}-5 \mathrm{Al}$ (CSM melt wire) irradiated for $54.9 \mathrm{EFPDs}$} \\
\hline & EOC & $30 \mathrm{MINs}$ & $5 \mathrm{HRS}$ & $1 \mathrm{DAY}$ & 7 DAYS & 14 DAYS & 30 DAYS & 60 DAYS & 1 YEAR \\
\hline & $\begin{array}{c}\mathrm{Ci} / 1 \mathrm{~g} \text { of } \\
95 \mathrm{Zn}-5 \mathrm{Al}\end{array}$ & $\begin{array}{c}\mathrm{Ci} / 1 \mathrm{~g} \text { of } \\
95 \mathrm{Zn}-5 \mathrm{Al}\end{array}$ & $\begin{array}{c}\mathrm{Ci} / 1 \mathrm{~g} \text { of } \\
95 \mathrm{Zn}-5 \mathrm{Al}\end{array}$ & $\begin{array}{c}\mathrm{Ci} / 1 \mathrm{~g} \text { of } \\
95 \mathrm{Zn}-5 \mathrm{Al}\end{array}$ & $\begin{array}{c}\mathrm{Ci} / 1 \mathrm{~g} \text { of } \\
95 \mathrm{Zn}-5 \mathrm{Al}\end{array}$ & $\begin{array}{c}\mathrm{Ci} / 1 \mathrm{~g} \text { of } \\
95 \mathrm{Zn}-5 \mathrm{Al}\end{array}$ & $\begin{array}{c}\mathrm{Ci} / 1 \mathrm{~g} \text { of } \\
95 \mathrm{Zn}-5 \mathrm{Al}\end{array}$ & $\begin{array}{c}\mathrm{Ci} / 1 \mathrm{~g} \text { of } \\
95 \mathrm{Zn}-5 \mathrm{Al}\end{array}$ & $\begin{array}{c}\mathrm{Ci} / 1 \mathrm{~g} \text { of } \\
95 \mathrm{Zn}-5 \mathrm{Al}\end{array}$ \\
\hline H 3 & $2.52 \mathrm{E}-15$ & $2.52 \mathrm{E}-15$ & $2.52 \mathrm{E}-15$ & $2.52 \mathrm{E}-15$ & $2.52 \mathrm{E}-15$ & $2.51 \mathrm{E}-15$ & $2.51 \mathrm{E}-15$ & $2.50 \mathrm{E}-15$ & $2.38 \mathrm{E}-15$ \\
\hline NA 24 & 7.07E-03 & $6.91 \mathrm{E}-03$ & $5.61 \mathrm{E}-03$ & $2.33 \mathrm{E}-03$ & $3.00 \mathrm{E}-06$ & $1.28 \mathrm{E}-09$ & $2.51 \mathrm{E}-17$ & $0.00 \mathrm{E}+00$ & $0.00 \mathrm{E}+00$ \\
\hline NA 25 & $2.09 \mathrm{E}-12$ & $1.69 \mathrm{E}-21$ & $0.00 \mathrm{E}+00$ & $0.00 \mathrm{E}+00$ & $0.00 \mathrm{E}+00$ & $0.00 \mathrm{E}+00$ & $0.00 \mathrm{E}+00$ & $0.00 \mathrm{E}+00$ & $0.00 \mathrm{E}+00$ \\
\hline $\begin{array}{l}\text { MG } \\
27 \\
\end{array}$ & $3.38 \mathrm{E}-02$ & $3.76 \mathrm{E}-03$ & $9.65 \mathrm{E}-12$ & $0.00 \mathrm{E}+00$ & $0.00 E+00$ & $0.00 \mathrm{E}+00$ & $0.00 \mathrm{E}+00$ & $0.00 \mathrm{E}+00$ & $0.00 \mathrm{E}+00$ \\
\hline $\begin{array}{l}M G \\
28\end{array}$ & $1.20 \mathrm{E}-10$ & $1.18 \mathrm{E}-10$ & $1.02 \mathrm{E}-10$ & $5.43 \mathrm{E}-11$ & $4.59 \mathrm{E}-13$ & $1.74 \mathrm{E}-15$ & $5.16 \mathrm{E}-21$ & $0.00 \mathrm{E}+00$ & $0.00 \mathrm{E}+00$ \\
\hline AL 28 & $8.11 \mathrm{E}-01$ & 7.54E-05 & $1.02 \mathrm{E}-10$ & $5.44 \mathrm{E}-11$ & $4.60 \mathrm{E}-13$ & $1.75 \mathrm{E}-15$ & $5.17 \mathrm{E}-21$ & $0.00 \mathrm{E}+00$ & $0.00 \mathrm{E}+00$ \\
\hline AL 29 & $1.65 \mathrm{E}-10$ & $6.79 \mathrm{E}-12$ & $2.32 \mathrm{E}-24$ & $0.00 \mathrm{E}+00$ & $0.00 \mathrm{E}+00$ & $0.00 \mathrm{E}+00$ & $0.00 \mathrm{E}+00$ & $0.00 \mathrm{E}+00$ & $0.00 \mathrm{E}+00$ \\
\hline SI 31 & $1.08 \mathrm{E}-13$ & $9.44 \mathrm{E}-14$ & $2.88 \mathrm{E}-14$ & $2.42 \mathrm{E}-16$ & $0.00 \mathrm{E}+00$ & $0.00 \mathrm{E}+00$ & $0.00 \mathrm{E}+00$ & $0.00 \mathrm{E}+00$ & $0.00 \mathrm{E}+00$ \\
\hline FE 59 & $4.52 \mathrm{E}-13$ & $4.52 \mathrm{E}-13$ & $4.50 \mathrm{E}-13$ & $4.45 \mathrm{E}-13$ & $4.06 \mathrm{E}-13$ & $3.64 \mathrm{E}-13$ & $2.85 \mathrm{E}-13$ & $1.79 \mathrm{E}-13$ & $1.63 \mathrm{E}-15$ \\
\hline CO 61 & 5.39E-10 & $4.36 \mathrm{E}-10$ & $6.59 \mathrm{E}-11$ & $2.25 \mathrm{E}-14$ & $0.00 \mathrm{E}+00$ & $0.00 \mathrm{E}+00$ & $0.00 \mathrm{E}+00$ & $0.00 \mathrm{E}+00$ & $0.00 \mathrm{E}+00$ \\
\hline CO 62 & $2.52 \mathrm{E}-07$ & $2.41 \mathrm{E}-13$ & $0.00 \mathrm{E}+00$ & $0.00 \mathrm{E}+00$ & $0.00 \mathrm{E}+00$ & $0.00 \mathrm{E}+00$ & $0.00 \mathrm{E}+00$ & $0.00 \mathrm{E}+00$ & $0.00 \mathrm{E}+00$ \\
\hline $\mathrm{NI} 63$ & $1.64 \mathrm{E}-07$ & $1.64 \mathrm{E}-07$ & $1.64 \mathrm{E}-07$ & $1.64 \mathrm{E}-07$ & $1.64 \mathrm{E}-07$ & $1.64 \mathrm{E}-07$ & $1.64 \mathrm{E}-07$ & $1.64 \mathrm{E}-07$ & 1.63E-07 \\
\hline NI 65 & 9.87E-05 & $8.60 \mathrm{E}-05$ & $2.50 \mathrm{E}-05$ & $1.34 \mathrm{E}-07$ & $8.43 \mathrm{E}-25$ & $0.00 \mathrm{E}+00$ & $0.00 \mathrm{E}+00$ & $0.00 \mathrm{E}+00$ & $0.00 \mathrm{E}+00$ \\
\hline NI 66 & 3.41E-09 & 3.39E-09 & $3.20 \mathrm{E}-09$ & $2.52 \mathrm{E}-09$ & 4.04E-10 & $4.79 \mathrm{E}-11$ & $3.66 \mathrm{E}-13$ & $3.94 \mathrm{E}-17$ & $0.00 \mathrm{E}+00$ \\
\hline CU 64 & 1.19E-06 & $1.16 \mathrm{E}-06$ & $9.06 \mathrm{E}-07$ & $3.21 \mathrm{E}-07$ & $1.24 \mathrm{E}-10$ & $1.29 \mathrm{E}-14$ & $1.02 \mathrm{E}-23$ & $0.00 \mathrm{E}+00$ & $0.00 \mathrm{E}+00$ \\
\hline CU 66 & 1.29E-03 & $2.18 \mathrm{E}-05$ & $3.21 \mathrm{E}-09$ & $2.52 \mathrm{E}-09$ & $4.05 \mathrm{E}-10$ & $4.80 \mathrm{E}-11$ & $3.67 \mathrm{E}-13$ & $3.94 \mathrm{E}-17$ & $0.00 \mathrm{E}+00$ \\
\hline CU 67 & 7.29E-09 & $7.25 \mathrm{E}-09$ & $6.89 \mathrm{E}-09$ & 5.57E-09 & 1.11E-09 & $1.69 \mathrm{E}-10$ & $2.29 \mathrm{E}-12$ & 7.17E-16 & $0.00 \mathrm{E}+00$ \\
\hline ZN 65 & $1.91 \mathrm{E}+00$ & $1.91 \mathrm{E}+00$ & $1.91 \mathrm{E}+00$ & $1.90 \mathrm{E}+00$ & $1.87 \mathrm{E}+00$ & $1.84 \mathrm{E}+00$ & $1.75 \mathrm{E}+00$ & $1.61 \mathrm{E}+00$ & $6.76 \mathrm{E}-01$ \\
\hline ZN 69 & $8.79 \mathrm{E}+00$ & $6.28 \mathrm{E}+00$ & $7.06 \mathrm{E}-01$ & $1.89 \mathrm{E}-01$ & $1.34 \mathrm{E}-04$ & $2.83 \mathrm{E}-08$ & $1.13 \mathrm{E}-16$ & $0.00 \mathrm{E}+00$ & $0.00 \mathrm{E}+00$ \\
\hline $\begin{array}{r}\text { ZN } \\
69 \mathrm{M}\end{array}$ & 5.91E-01 & $5.76 \mathrm{E}-01$ & 4.59E-01 & $1.76 \mathrm{E}-01$ & $1.25 \mathrm{E}-04$ & $2.64 \mathrm{E}-08$ & $1.05 \mathrm{E}-16$ & $0.00 E+00$ & $0.00 E+00$ \\
\hline ZN 71 & $1.32 \mathrm{E}-02$ & $2.92 \mathrm{E}-06$ & $2.89 \mathrm{E}-07$ & $1.00 \mathrm{E}-08$ & 8.77E-20 & $0.00 \mathrm{E}+00$ & $0.00 \mathrm{E}+00$ & $0.00 \mathrm{E}+00$ & $0.00 \mathrm{E}+00$ \\
\hline $\begin{array}{r}\mathrm{ZN} \\
71 \mathrm{M}\end{array}$ & $1.38 \mathrm{E}-03$ & 1.27E-03 & $5.71 \mathrm{E}-04$ & $1.99 \mathrm{E}-05$ & $1.74 \mathrm{E}-16$ & $2.18 \mathrm{E}-29$ & $0.00 E+00$ & $0.00 \mathrm{E}+00$ & $0.00 \mathrm{E}+00$ \\
\hline GA 70 & $2.55 \mathrm{E}-02$ & $9.51 \mathrm{E}-03$ & $1.34 \mathrm{E}-06$ & $7.28 \mathrm{E}-23$ & $0.00 \mathrm{E}+00$ & $0.00 \mathrm{E}+00$ & $0.00 \mathrm{E}+00$ & $0.00 \mathrm{E}+00$ & $0.00 \mathrm{E}+00$ \\
\hline GA 72 & $9.44 \mathrm{E}-05$ & $9.21 \mathrm{E}-05$ & $7.38 \mathrm{E}-05$ & $2.90 \mathrm{E}-05$ & $2.45 \mathrm{E}-08$ & $6.33 \mathrm{E}-12$ & $4.01 \mathrm{E}-20$ & $0.00 \mathrm{E}+00$ & $0.00 \mathrm{E}+00$ \\
\hline $\begin{array}{c}\mathrm{GA} \\
72 \mathrm{M}\end{array}$ & 2.92E-06 & $0.00 \mathrm{E}+00$ & $0.00 E+00$ & $0.00 \mathrm{E}+00$ & $0.00 E+00$ & $0.00 \mathrm{E}+00$ & $0.00 E+00$ & $0.00 \mathrm{E}+00$ & $0.00 \mathrm{E}+00$ \\
\hline GE 71 & $1.35 \mathrm{E}-05$ & $1.35 \mathrm{E}-05$ & $1.34 \mathrm{E}-05$ & $1.28 \mathrm{E}-05$ & $8.98 \mathrm{E}-06$ & $5.95 \mathrm{E}-06$ & $2.33 \mathrm{E}-06$ & $3.99 \mathrm{E}-07$ & $6.51 \mathrm{E}-15$ \\
\hline $\begin{array}{c}\mathrm{GE} \\
71 \mathrm{M}\end{array}$ & 1.96E-06 & $0.00 E+00$ & $0.00 E+00$ & $0.00 E+00$ & $0.00 E+00$ & $0.00 E+00$ & $0.00 E+00$ & $0.00 E+00$ & $0.00 E+00$ \\
\hline GE 75 & $1.95 \mathrm{E}-14$ & $1.53 \mathrm{E}-14$ & $1.65 \mathrm{E}-15$ & $1.18 \mathrm{E}-19$ & $0.00 \mathrm{E}+00$ & $0.00 \mathrm{E}+00$ & $0.00 \mathrm{E}+00$ & $0.00 \mathrm{E}+00$ & $0.00 \mathrm{E}+00$ \\
\hline $\begin{array}{c}\text { SUMT } \\
\text { OT }\end{array}$ & $1.22 \mathrm{E}+01$ & $8.79 E+00$ & $3.08 E+00$ & $2.27 E+00$ & $1.87 \mathrm{E}+00$ & $1.84 \mathrm{E}+00$ & $1.75 E+00$ & $1.61 \mathrm{E}+00$ & $6.76 \mathrm{E}-01$ \\
\hline
\end{tabular}


TEM-10200-1

ENGINEERING CALCULATIONS AND ANALYSIS

Page 75 of 81

$12 / 19 / 17$

Rev.08

Title:

AS-RUN NEUTRONICS EVALUATION FOR THE CSM-10584 EXPERIMENT IN THE ATR

\begin{tabular}{llllllll} 
ECAR NO.: & 4496 & REV. NO.: & 1 & PROJECT NO.: & 32501 & Date: & 10/19/2020 \\
\hline
\end{tabular}

\begin{tabular}{|c|c|c|c|c|c|c|c|c|c|}
\hline \multicolumn{10}{|c|}{1 gram of $95 \mathrm{Zn}-5 \mathrm{Al}$ (CSM melt wire) irradiated for 54.9 EFPDs } \\
\hline & EOC & $30 \mathrm{MINs}$ & $5 \mathrm{HRS}$ & $1 \mathrm{DAY}$ & 7 DAYS & 14 DAYS & 30 DAYS & 60 DAYS & 1 YEAR \\
\hline & $\begin{array}{c}\mathrm{Ci} / 1 \mathrm{~g} \text { of } \\
95 \mathrm{Zn}-5 \mathrm{Al}\end{array}$ & $\begin{array}{c}\mathrm{Ci} / 1 \mathrm{~g} \text { of } \\
95 \mathrm{Zn}-5 \mathrm{Al}\end{array}$ & $\begin{array}{c}\mathrm{Ci} / 1 \mathrm{~g} \text { of } \\
95 \mathrm{Zn}-5 \mathrm{Al}\end{array}$ & $\begin{array}{c}\mathrm{Ci} / 1 \mathrm{~g} \text { of } \\
95 \mathrm{Zn}-5 \mathrm{Al}\end{array}$ & $\begin{array}{c}\mathrm{Ci} / 1 \mathrm{~g} \text { of } \\
95 \mathrm{Zn}-5 \mathrm{Al}\end{array}$ & $\begin{array}{c}\mathrm{Ci} / 1 \mathrm{~g} \text { of } \\
95 \mathrm{Zn}-5 \mathrm{Al}\end{array}$ & $\begin{array}{c}\mathrm{Ci} / 1 \mathrm{~g} \text { of } \\
95 \mathrm{Zn}-5 \mathrm{Al}\end{array}$ & $\begin{array}{c}\mathrm{Ci} / 1 \mathrm{~g} \text { of } \\
95 \mathrm{Zn}-5 \mathrm{Al}\end{array}$ & $\begin{array}{c}\mathrm{Ci} / 1 \mathrm{~g} \text { of } \\
95 \mathrm{Zn}-5 \mathrm{~A}\end{array}$ \\
\hline $\begin{array}{c}\text { OTOTA } \\
\mathrm{L}\end{array}$ & $1.22 \mathrm{E}+01$ & $8.79 \mathrm{E}+00$ & $3.08 \mathrm{E}+00$ & $2.27 \mathrm{E}+00$ & $1.87 \mathrm{E}+00$ & $1.84 \mathrm{E}+00$ & $1.75 \mathrm{E}+00$ & $1.61 \mathrm{E}+00$ & $6.76 \mathrm{E}-01$ \\
\hline
\end{tabular}

\begin{tabular}{|c|c|c|c|c|c|c|c|c|c|}
\hline \multicolumn{10}{|c|}{1 gram of $95 \mathrm{Zn}-5 \mathrm{Al}$ (CSM melt wire) irradiated for 119 EFPDs } \\
\hline & EOC & $30 \mathrm{MINs}$ & $5 \mathrm{HRS}$ & $1 \mathrm{DAY}$ & 7 DAYS & 14 DAYS & 30 DAYS & 60 DAYS & 1 YEAR \\
\hline & $\begin{array}{c}\mathrm{g} / 1 \mathrm{~g} \text { of } \\
95 \mathrm{Zn}-5 \mathrm{Al}\end{array}$ & $\begin{array}{c}\mathrm{g} / 1 \mathrm{~g} \text { of } \\
95 \mathrm{Zn}-5 \mathrm{Al}\end{array}$ & $\begin{array}{c}\text { g/1g of } \\
95 \mathrm{Zn}-5 \mathrm{Al}\end{array}$ & $\begin{array}{c}\mathrm{g} / 1 \mathrm{~g} \text { of } \\
95 \mathrm{Zn}-5 \mathrm{Al}\end{array}$ & $\begin{array}{c}\mathrm{g} / 1 \mathrm{~g} \text { of } \\
95 \mathrm{Zn}-5 \mathrm{Al}\end{array}$ & $\begin{array}{c}\mathrm{g} / 1 \mathrm{~g} \text { of } \\
95 \mathrm{Zn}-5 \mathrm{Al}\end{array}$ & $\begin{array}{c}\mathrm{g} / 1 \mathrm{~g} \text { of } \\
95 \mathrm{Zn}-5 \mathrm{Al}\end{array}$ & $\begin{array}{c}\mathrm{g} / 1 \mathrm{~g} \text { of } \\
95 \mathrm{Zn}-5 \mathrm{Al}\end{array}$ & $\begin{array}{c}\mathrm{g} / 1 \mathrm{~g} \text { of } \\
95 \mathrm{Zn}-5 \mathrm{Al}\end{array}$ \\
\hline H 1 & $2.14 \mathrm{E}-08$ & $2.14 \mathrm{E}-08$ & $2.14 \mathrm{E}-08$ & $2.14 \mathrm{E}-08$ & $2.14 \mathrm{E}-08$ & $2.14 \mathrm{E}-08$ & $2.14 \mathrm{E}-08$ & $2.14 \mathrm{E}-08$ & $2.14 \mathrm{E}-08$ \\
\hline $\mathrm{H} 2$ & $9.00 \mathrm{E}-12$ & $9.00 \mathrm{E}-12$ & $9.00 \mathrm{E}-12$ & $9.00 \mathrm{E}-12$ & $9.00 \mathrm{E}-12$ & $9.00 \mathrm{E}-12$ & $9.00 \mathrm{E}-12$ & $9.00 \mathrm{E}-12$ & $9.00 \mathrm{E}-12$ \\
\hline $\mathrm{HE} 4$ & $1.90 \mathrm{E}-08$ & $1.90 \mathrm{E}-08$ & $1.90 \mathrm{E}-08$ & $1.90 \mathrm{E}-08$ & $1.90 \mathrm{E}-08$ & $1.90 \mathrm{E}-08$ & $1.90 \mathrm{E}-08$ & $1.90 \mathrm{E}-08$ & $1.90 \mathrm{E}-08$ \\
\hline NE 21 & $2.60 \mathrm{E}-13$ & $2.60 \mathrm{E}-13$ & $2.60 \mathrm{E}-13$ & $2.60 \mathrm{E}-13$ & $2.60 \mathrm{E}-13$ & $2.60 \mathrm{E}-13$ & $2.60 \mathrm{E}-13$ & $2.60 \mathrm{E}-13$ & $2.60 \mathrm{E}-13$ \\
\hline NA 24 & $8.12 \mathrm{E}-10$ & $7.94 \mathrm{E}-10$ & $6.45 \mathrm{E}-10$ & $2.68 \mathrm{E}-10$ & $3.45 \mathrm{E}-13$ & $1.47 \mathrm{E}-16$ & $2.88 \mathrm{E}-24$ & $0.00 \mathrm{E}+00$ & $0.00 \mathrm{E}+00$ \\
\hline $\begin{array}{c}M G \\
24 \\
\end{array}$ & $1.06 \mathrm{E}-07$ & $1.06 \mathrm{E}-07$ & 1.07E-07 & 1.07E-07 & 1.07E-07 & 1.07E-07 & $1.07 \mathrm{E}-07$ & 1.07E-07 & 1.07E-07 \\
\hline $\begin{array}{l}\text { MG } \\
25 \\
\end{array}$ & $2.09 \mathrm{E}-11$ & $2.09 \mathrm{E}-11$ & 2.09E-11 & $2.09 \mathrm{E}-11$ & 2.09E-11 & $2.09 \mathrm{E}-11$ & 2.09E-11 & $2.09 \mathrm{E}-11$ & 2.09E-11 \\
\hline $\begin{array}{l}\text { MG } \\
26 \\
\end{array}$ & $1.16 \mathrm{E}-14$ & $1.16 \mathrm{E}-14$ & 1.16E-14 & $1.16 \mathrm{E}-14$ & $1.16 \mathrm{E}-14$ & $1.16 \mathrm{E}-14$ & $1.16 \mathrm{E}-14$ & $1.16 \mathrm{E}-14$ & $1.16 \mathrm{E}-14$ \\
\hline $\begin{array}{l}\text { MG } \\
27\end{array}$ & $4.59 \mathrm{E}-11$ & $5.10 \mathrm{E}-12$ & $1.31 \mathrm{E}-20$ & $0.00 \mathrm{E}+00$ & $0.00 \mathrm{E}+00$ & $0.00 \mathrm{E}+00$ & $0.00 \mathrm{E}+00$ & $0.00 \mathrm{E}+00$ & $0.00 \mathrm{E}+00$ \\
\hline AL 27 & 5.00E-02 & $5.00 \mathrm{E}-02$ & 5.00E-02 & 5.00E-02 & 5.00E-02 & $5.00 \mathrm{E}-02$ & $5.00 \mathrm{E}-02$ & $5.00 \mathrm{E}-02$ & $5.00 \mathrm{E}-02$ \\
\hline AL 28 & $2.70 \mathrm{E}-10$ & $2.51 \mathrm{E}-14$ & $3.40 \mathrm{E}-20$ & $1.81 \mathrm{E}-20$ & $1.53 \mathrm{E}-22$ & $5.85 \mathrm{E}-25$ & $1.73 \mathrm{E}-30$ & $0.00 \mathrm{E}+00$ & $0.00 \mathrm{E}+00$ \\
\hline SI 28 & $1.43 \mathrm{E}-05$ & $1.43 \mathrm{E}-05$ & $1.43 \mathrm{E}-05$ & $1.43 \mathrm{E}-05$ & $1.43 \mathrm{E}-05$ & $1.43 \mathrm{E}-05$ & $1.43 \mathrm{E}-05$ & $1.43 \mathrm{E}-05$ & $1.43 \mathrm{E}-05$ \\
\hline SI 29 & $1.41 \mathrm{E}-09$ & $1.41 \mathrm{E}-09$ & $1.41 \mathrm{E}-09$ & $1.41 \mathrm{E}-09$ & $1.41 \mathrm{E}-09$ & $1.41 \mathrm{E}-09$ & $1.41 \mathrm{E}-09$ & $1.41 \mathrm{E}-09$ & $1.41 \mathrm{E}-09$ \\
\hline $\mathrm{SI} 30$ & $1.52 \mathrm{E}-13$ & $1.52 \mathrm{E}-13$ & $1.52 \mathrm{E}-13$ & $1.52 \mathrm{E}-13$ & $1.52 \mathrm{E}-13$ & $1.52 \mathrm{E}-13$ & $1.52 \mathrm{E}-13$ & $1.52 \mathrm{E}-13$ & $1.52 \mathrm{E}-13$ \\
\hline FE 58 & $2.24 \mathrm{E}-13$ & $2.24 \mathrm{E}-13$ & $2.24 \mathrm{E}-13$ & $2.24 \mathrm{E}-13$ & $2.24 \mathrm{E}-13$ & $2.24 \mathrm{E}-13$ & $2.24 \mathrm{E}-13$ & $2.24 \mathrm{E}-13$ & $2.24 \mathrm{E}-13$ \\
\hline $\mathrm{NI} 61$ & 7.10E-09 & 7.10E-09 & 7.10E-09 & 7.10E-09 & 7.10E-09 & $7.10 \mathrm{E}-09$ & 7.10E-09 & 7.10E-09 & 7.10E-09 \\
\hline $\mathrm{NI} 62$ & $2.54 \mathrm{E}-11$ & $2.54 \mathrm{E}-11$ & $2.54 \mathrm{E}-11$ & $2.54 \mathrm{E}-11$ & $2.54 \mathrm{E}-11$ & $2.54 \mathrm{E}-11$ & $2.54 \mathrm{E}-11$ & $2.54 \mathrm{E}-11$ & $2.54 \mathrm{E}-11$ \\
\hline $\mathrm{NI} 63$ & $5.72 \mathrm{E}-09$ & $5.72 \mathrm{E}-09$ & $5.72 \mathrm{E}-09$ & $5.72 \mathrm{E}-09$ & $5.72 \mathrm{E}-09$ & $5.72 \mathrm{E}-09$ & $5.72 \mathrm{E}-09$ & 5.71E-09 & $5.68 \mathrm{E}-09$ \\
\hline NI 64 & $3.74 \mathrm{E}-10$ & $3.75 \mathrm{E}-10$ & $3.75 \mathrm{E}-10$ & $3.75 \mathrm{E}-10$ & $3.75 \mathrm{E}-10$ & $3.75 \mathrm{E}-10$ & $3.75 \mathrm{E}-10$ & $3.75 \mathrm{E}-10$ & $3.75 \mathrm{E}-10$ \\
\hline $\mathrm{NI} 65$ & $5.27 \mathrm{E}-12$ & $4.59 \mathrm{E}-12$ & $1.33 \mathrm{E}-12$ & $7.16 \mathrm{E}-15$ & $4.50 \mathrm{E}-32$ & $0.00 \mathrm{E}+00$ & $0.00 \mathrm{E}+00$ & $0.00 \mathrm{E}+00$ & $0.00 \mathrm{E}+00$ \\
\hline NI 66 & 3.97E-15 & $3.94 \mathrm{E}-15$ & $3.72 \mathrm{E}-15$ & $2.93 \mathrm{E}-15$ & 4.70E-16 & $5.58 \mathrm{E}-17$ & $4.26 \mathrm{E}-19$ & $4.58 \mathrm{E}-23$ & $0.00 \mathrm{E}+00$ \\
\hline CU 63 & $7.04 \mathrm{E}-12$ & $7.05 \mathrm{E}-12$ & 7.07E-12 & 7.16E-12 & 7.87E-12 & $8.69 \mathrm{E}-12$ & $1.06 \mathrm{E}-11$ & $1.41 \mathrm{E}-11$ & $5.00 \mathrm{E}-11$ \\
\hline CU 64 & 1.37E-12 & $1.33 \mathrm{E}-12$ & $1.04 \mathrm{E}-12$ & $3.69 \mathrm{E}-13$ & $1.42 \mathrm{E}-16$ & $1.48 \mathrm{E}-20$ & 1.17E-29 & $0.00 \mathrm{E}+00$ & $0.00 \mathrm{E}+00$ \\
\hline CU 65 & $8.21 \mathrm{E}-05$ & $8.21 \mathrm{E}-05$ & $8.24 \mathrm{E}-05$ & $8.34 \mathrm{E}-05$ & $9.12 \mathrm{E}-05$ & $1.00 \mathrm{E}-04$ & $1.20 \mathrm{E}-04$ & $1.54 \mathrm{E}-04$ & 3.79E-04 \\
\hline CU 66 & $1.02 \mathrm{E}-11$ & $1.73 \mathrm{E}-13$ & $5.80 \mathrm{E}-18$ & 4.56E-18 & 7.33E-19 & $8.69 \mathrm{E}-20$ & $6.64 \mathrm{E}-22$ & $7.14 \mathrm{E}-26$ & $0.00 \mathrm{E}+00$ \\
\hline CU 67 & $4.59 \mathrm{E}-14$ & $4.56 \mathrm{E}-14$ & $4.34 \mathrm{E}-14$ & $3.51 \mathrm{E}-14$ & $6.99 \mathrm{E}-15$ & $1.06 \mathrm{E}-15$ & $1.44 \mathrm{E}-17$ & $4.51 \mathrm{E}-21$ & $0.00 \mathrm{E}+00$ \\
\hline ZN 64 & 4.51E-01 & $4.51 \mathrm{E}-01$ & 4.51E-01 & $4.51 \mathrm{E}-01$ & $4.51 \mathrm{E}-01$ & $4.51 \mathrm{E}-01$ & $4.51 \mathrm{E}-01$ & $4.51 \mathrm{E}-01$ & $4.51 \mathrm{E}-01$ \\
\hline ZN 65 & $4.60 \mathrm{E}-04$ & $4.60 \mathrm{E}-04$ & $4.60 \mathrm{E}-04$ & $4.59 \mathrm{E}-04$ & $4.51 \mathrm{E}-04$ & $4.42 \mathrm{E}-04$ & $4.22 \mathrm{E}-04$ & $3.88 \mathrm{E}-04$ & $1.63 \mathrm{E}-04$ \\
\hline ZN 66 & 2.67E-01 & 2.67E-01 & 2.67E-01 & 2.67E-01 & 2.67E-01 & 2.67E-01 & 2.67E-01 & 2.67E-01 & 2.67E-01 \\
\hline ZN 67 & 3.97E-02 & $3.97 \mathrm{E}-02$ & 3.97E-02 & 3.97E-02 & 3.97E-02 & $3.97 \mathrm{E}-02$ & $3.97 \mathrm{E}-02$ & $3.97 \mathrm{E}-02$ & $3.97 \mathrm{E}-02$ \\
\hline ZN 68 & $1.85 \mathrm{E}-01$ & $1.85 \mathrm{E}-01$ & $1.85 \mathrm{E}-01$ & $1.85 \mathrm{E}-01$ & $1.85 \mathrm{E}-01$ & $1.85 \mathrm{E}-01$ & $1.85 \mathrm{E}-01$ & $1.85 \mathrm{E}-01$ & $1.85 \mathrm{E}-01$ \\
\hline ZN 69 & $1.84 \mathrm{E}-07$ & $1.31 \mathrm{E}-07$ & $1.48 \mathrm{E}-08$ & 3.96E-09 & $2.80 \mathrm{E}-12$ & $5.93 \mathrm{E}-16$ & $2.36 \mathrm{E}-24$ & $0.00 \mathrm{E}+00$ & $0.00 \mathrm{E}+00$ \\
\hline $\begin{array}{r}\text { ZN } \\
69 M \\
\end{array}$ & $1.79 \mathrm{E}-07$ & $1.75 \mathrm{E}-07$ & $1.39 \mathrm{E}-07$ & $5.34 \mathrm{E}-08$ & $3.78 \mathrm{E}-11$ & $7.99 \mathrm{E}-15$ & $3.18 \mathrm{E}-23$ & $0.00 \mathrm{E}+00$ & $0.00 \mathrm{E}+00$ \\
\hline ZN 70 & $6.30 \mathrm{E}-03$ & $6.30 \mathrm{E}-03$ & $6.30 \mathrm{E}-03$ & $6.30 \mathrm{E}-03$ & $6.30 \mathrm{E}-03$ & $6.30 \mathrm{E}-03$ & $6.30 \mathrm{E}-03$ & $6.30 \mathrm{E}-03$ & $6.30 \mathrm{E}-03$ \\
\hline ZN 71 & $1.20 \mathrm{E}-11$ & $2.64 \mathrm{E}-15$ & 2.62E-16 & $9.09 \mathrm{E}-18$ & 7.95E-29 & $0.00 \mathrm{E}+00$ & $0.00 \mathrm{E}+00$ & $0.00 \mathrm{E}+00$ & $0.00 \mathrm{E}+00$ \\
\hline $\begin{array}{c}\mathrm{ZN} \\
71 \mathrm{M}\end{array}$ & $1.23 \mathrm{E}-10$ & $1.12 \mathrm{E}-10$ & 5.07E-11 & $1.76 \mathrm{E}-12$ & $1.54 \mathrm{E}-23$ & $1.94 \mathrm{E}-36$ & $0.00 E+00$ & $0.00 \mathrm{E}+00$ & $0.00 \mathrm{E}+00$ \\
\hline GA 69 & $3.82 \mathrm{E}-04$ & $3.82 \mathrm{E}-04$ & $3.82 \mathrm{E}-04$ & $3.82 \mathrm{E}-04$ & $3.82 \mathrm{E}-04$ & $3.82 \mathrm{E}-04$ & $3.82 \mathrm{E}-04$ & $3.82 \mathrm{E}-04$ & $3.82 \mathrm{E}-04$ \\
\hline GA 70 & $4.34 \mathrm{E}-10$ & $1.62 \mathrm{E}-10$ & $2.28 \mathrm{E}-14$ & $1.24 \mathrm{E}-30$ & $0.00 \mathrm{E}+00$ & $0.00 \mathrm{E}+00$ & $0.00 \mathrm{E}+00$ & $0.00 \mathrm{E}+00$ & $0.00 \mathrm{E}+00$ \\
\hline GA 71 & $6.50 \mathrm{E}-07$ & $6.50 \mathrm{E}-07$ & $6.50 \mathrm{E}-07$ & $6.50 \mathrm{E}-07$ & $6.50 \mathrm{E}-07$ & $6.50 \mathrm{E}-07$ & $6.51 \mathrm{E}-07$ & $6.51 \mathrm{E}-07$ & 6.51E-07 \\
\hline
\end{tabular}


TEM-10200-1

ENGINEERING CALCULATIONS AND ANALYSIS

Page 76 of 81

$12 / 19 / 17$

Rev.08

Title:

AS-RUN NEUTRONICS EVALUATION FOR THE CSM-10584 EXPERIMENT IN THE ATR

\begin{tabular}{llllllll} 
ECAR NO.: & 4496 & REV. NO.: & 1 & PROJECT NO.: & 32501 & Date: & 10/19/2020 \\
\hline
\end{tabular}

\begin{tabular}{|c|c|c|c|c|c|c|c|c|c|}
\hline \multicolumn{10}{|c|}{1 gram of $95 \mathrm{Zn}-5 \mathrm{Al}$ (CSM melt wire) irradiated for 119 EFPDs } \\
\hline & EOC & $30 \mathrm{MINs}$ & $5 \mathrm{HRS}$ & $1 \mathrm{DAY}$ & 7 DAYS & 14 DAYS & 30 DAYS & 60 DAYS & 1 YEAR \\
\hline & $\begin{array}{c}\mathrm{g} / 1 \mathrm{~g} \text { of } \\
95 \mathrm{Zn}-5 \mathrm{Al}\end{array}$ & $\begin{array}{c}\mathrm{g} / 1 \mathrm{~g} \text { of } \\
95 \mathrm{Zn}-5 \mathrm{Al}\end{array}$ & $\begin{array}{c}\mathrm{g} / 1 \mathrm{~g} \text { of } \\
95 \mathrm{Zn}-5 \mathrm{Al}\end{array}$ & $\begin{array}{c}\mathrm{g} / 1 \mathrm{~g} \text { of } \\
95 \mathrm{Zn}-5 \mathrm{Al}\end{array}$ & $\begin{array}{c}\mathrm{g} / 1 \mathrm{~g} \text { of } \\
95 \mathrm{Zn}-5 \mathrm{Al}\end{array}$ & $\begin{array}{c}\mathrm{g} / 1 \mathrm{~g} \text { of } \\
95 \mathrm{Zn}-5 \mathrm{Al}\end{array}$ & $\begin{array}{c}\mathrm{g} / 1 \mathrm{~g} \text { of } \\
95 \mathrm{Zn}-5 \mathrm{Al}\end{array}$ & $\begin{array}{c}\mathrm{g} / 1 \mathrm{~g} \text { of } \\
95 \mathrm{Zn}-5 \mathrm{Al}\end{array}$ & $\begin{array}{c}\mathrm{g} / 1 \mathrm{~g} \text { of } \\
95 \mathrm{Zn}-5 \mathrm{Al}\end{array}$ \\
\hline GA 72 & $6.61 \mathrm{E}-11$ & $6.45 \mathrm{E}-11$ & 5.17E-11 & $2.03 \mathrm{E}-11$ & $1.71 \mathrm{E}-14$ & $4.44 \mathrm{E}-18$ & $2.81 \mathrm{E}-26$ & $0.00 \mathrm{E}+00$ & $0.00 \mathrm{E}+00$ \\
\hline GE 70 & $1.22 \mathrm{E}-06$ & $1.22 \mathrm{E}-06$ & $1.22 \mathrm{E}-06$ & $1.22 \mathrm{E}-06$ & $1.22 \mathrm{E}-06$ & $1.22 \mathrm{E}-06$ & $1.22 \mathrm{E}-06$ & $1.22 \mathrm{E}-06$ & $1.22 \mathrm{E}-06$ \\
\hline GE 71 & $5.46 \mathrm{E}-10$ & $5.45 \mathrm{E}-10$ & $5.39 \mathrm{E}-10$ & $5.15 \mathrm{E}-10$ & $3.62 \mathrm{E}-10$ & $2.40 \mathrm{E}-10$ & $9.37 \mathrm{E}-11$ & $1.61 \mathrm{E}-11$ & $2.62 \mathrm{E}-19$ \\
\hline GE 72 & 4.61E-09 & 4.61E-09 & 4.63E-09 & 4.66E-09 & $4.68 \mathrm{E}-09$ & $4.68 \mathrm{E}-09$ & $4.68 \mathrm{E}-09$ & 4.68E-09 & 4.68E-09 \\
\hline GE 73 & $2.32 \mathrm{E}-12$ & $2.32 \mathrm{E}-12$ & $2.32 \mathrm{E}-12$ & $2.32 \mathrm{E}-12$ & $2.32 \mathrm{E}-12$ & $2.32 \mathrm{E}-12$ & $2.32 \mathrm{E}-12$ & $2.32 \mathrm{E}-12$ & $2.32 \mathrm{E}-12$ \\
\hline GE 74 & $2.34 \mathrm{E}-14$ & $2.34 \mathrm{E}-14$ & $2.34 \mathrm{E}-14$ & $2.34 \mathrm{E}-14$ & $2.34 \mathrm{E}-14$ & $2.34 \mathrm{E}-14$ & $2.34 \mathrm{E}-14$ & $2.34 \mathrm{E}-14$ & $2.34 \mathrm{E}-14$ \\
\hline $\begin{array}{c}\text { SUMT } \\
\text { OT }\end{array}$ & $1.00 \mathrm{E}+00$ & $1.00 \mathrm{E}+00$ & $1.00 \mathrm{E}+00$ & $1.00 \mathrm{E}+00$ & $1.00 \mathrm{E}+00$ & $1.00 \mathrm{E}+00$ & $1.00 \mathrm{E}+00$ & $1.00 \mathrm{E}+00$ & $1.00 \mathrm{E}+00$ \\
\hline $\begin{array}{c}\text { OTOTA } \\
\mathrm{L}\end{array}$ & $1.00 \mathrm{E}+00$ & $1.00 \mathrm{E}+00$ & $1.00 \mathrm{E}+00$ & $1.00 \mathrm{E}+00$ & $1.00 \mathrm{E}+00$ & $1.00 \mathrm{E}+00$ & $1.00 \mathrm{E}+00$ & $1.00 \mathrm{E}+00$ & $1.00 \mathrm{E}+00$ \\
\hline
\end{tabular}

\begin{tabular}{|c|c|c|c|c|c|c|c|c|c|}
\hline \multicolumn{10}{|c|}{1 gram of $95 \mathrm{Zn}-5 \mathrm{Al}$ (CSM melt wire) irradiated for $54.9 \mathrm{EFPDs}$} \\
\hline & EOC & $30 \mathrm{MINs}$ & 5 HRS & $1 \mathrm{DAY}$ & 7 DAYS & 14 DAYS & 30 DAYS & 60 DAYS & 1 YEAR \\
\hline & $\begin{array}{c}\mathrm{g} / 1 \mathrm{~g} \text { of } \\
95 \mathrm{Zn}-5 \mathrm{Al}\end{array}$ & $\begin{array}{c}\mathrm{g} / 1 \mathrm{~g} \text { of } \\
95 \mathrm{Zn}-5 \mathrm{Al}\end{array}$ & $\begin{array}{c}\mathrm{g} / 1 \mathrm{~g} \text { of } \\
95 \mathrm{Zn}-5 \mathrm{Al}\end{array}$ & $\begin{array}{c}\mathrm{g} / 1 \mathrm{~g} \text { of } \\
95 \mathrm{Zn}-5 \mathrm{Al}\end{array}$ & $\begin{array}{c}\mathrm{g} / 1 \mathrm{~g} \text { of } \\
95 \mathrm{Zn}-5 \mathrm{Al}\end{array}$ & $\begin{array}{c}\mathrm{g} / 1 \mathrm{~g} \text { of } \\
95 \mathrm{Zn}-5 \mathrm{Al}\end{array}$ & $\begin{array}{c}\mathrm{g} / 1 \mathrm{~g} \text { of } \\
95 \mathrm{Zn}-5 \mathrm{Al}\end{array}$ & $\begin{array}{c}\text { g/1g of } \\
95 \mathrm{Zn}-5 \mathrm{Al}\end{array}$ & $\begin{array}{c}\mathrm{g} / 1 \mathrm{~g} \text { of } \\
95 \mathrm{Zn}-5 \mathrm{Al}\end{array}$ \\
\hline H 1 & $9.85 \mathrm{E}-09$ & $9.85 \mathrm{E}-09$ & $9.85 \mathrm{E}-09$ & $9.85 \mathrm{E}-09$ & $9.85 \mathrm{E}-09$ & $9.85 \mathrm{E}-09$ & $9.85 \mathrm{E}-09$ & $9.85 \mathrm{E}-09$ & $9.85 \mathrm{E}-09$ \\
\hline $\mathrm{H} 2$ & $1.92 \mathrm{E}-12$ & $1.92 \mathrm{E}-12$ & 1.92E-12 & $1.92 \mathrm{E}-12$ & $1.92 \mathrm{E}-12$ & $1.92 \mathrm{E}-12$ & $1.92 \mathrm{E}-12$ & $1.92 \mathrm{E}-12$ & $1.92 \mathrm{E}-12$ \\
\hline $\mathrm{HE} 4$ & $8.75 \mathrm{E}-09$ & $8.75 E-09$ & $8.75 E-09$ & $8.75 \mathrm{E}-09$ & $8.75 \mathrm{E}-09$ & $8.75 \mathrm{E}-09$ & $8.75 \mathrm{E}-09$ & $8.75 \mathrm{E}-09$ & 8.75E-09 \\
\hline NE 21 & $5.48 \mathrm{E}-14$ & $5.48 \mathrm{E}-14$ & $5.48 \mathrm{E}-14$ & $5.48 \mathrm{E}-14$ & $5.48 \mathrm{E}-14$ & $5.48 \mathrm{E}-14$ & $5.48 \mathrm{E}-14$ & $5.48 \mathrm{E}-14$ & $5.48 \mathrm{E}-14$ \\
\hline NA 24 & $8.12 \mathrm{E}-10$ & $7.94 \mathrm{E}-10$ & $6.45 \mathrm{E}-10$ & $2.68 \mathrm{E}-10$ & $3.45 \mathrm{E}-13$ & $1.47 \mathrm{E}-16$ & $2.88 \mathrm{E}-24$ & $0.00 \mathrm{E}+00$ & $0.00 \mathrm{E}+00$ \\
\hline $\begin{array}{l}\text { MG } \\
24\end{array}$ & 4.86E-08 & 4.87E-08 & 4.88E-08 & $4.92 \mathrm{E}-08$ & $4.95 \mathrm{E}-08$ & $4.95 \mathrm{E}-08$ & $4.95 \mathrm{E}-08$ & 4.95E-08 & 4.95E-08 \\
\hline $\begin{array}{l}\text { MG } \\
25\end{array}$ & $4.45 \mathrm{E}-12$ & $4.45 \mathrm{E}-12$ & $4.45 \mathrm{E}-12$ & $4.45 \mathrm{E}-12$ & $4.45 \mathrm{E}-12$ & $4.45 \mathrm{E}-12$ & $4.45 \mathrm{E}-12$ & $4.45 \mathrm{E}-12$ & $4.45 \mathrm{E}-12$ \\
\hline $\begin{array}{l}M G \\
26\end{array}$ & $1.13 \mathrm{E}-15$ & $1.13 \mathrm{E}-15$ & $1.13 \mathrm{E}-15$ & $1.13 \mathrm{E}-15$ & $1.13 \mathrm{E}-15$ & $1.13 \mathrm{E}-15$ & $1.13 \mathrm{E}-15$ & $1.13 \mathrm{E}-15$ & $1.13 \mathrm{E}-15$ \\
\hline $\begin{array}{l}\text { MG } \\
27\end{array}$ & 4.59E-11 & $5.10 \mathrm{E}-12$ & $1.31 \mathrm{E}-20$ & $0.00 E+00$ & $0.00 E+00$ & $0.00 E+00$ & $0.00 E+00$ & $0.00 E+00$ & $0.00 E+00$ \\
\hline AL 27 & $5.00 \mathrm{E}-02$ & $5.00 \mathrm{E}-02$ & $5.00 \mathrm{E}-02$ & $5.00 \mathrm{E}-02$ & $5.00 \mathrm{E}-02$ & $5.00 \mathrm{E}-02$ & $5.00 \mathrm{E}-02$ & $5.00 \mathrm{E}-02$ & $5.00 \mathrm{E}-02$ \\
\hline AL 28 & $2.70 \mathrm{E}-10$ & $2.51 \mathrm{E}-14$ & $3.40 \mathrm{E}-20$ & $1.81 \mathrm{E}-20$ & 1.53E-22 & $5.85 \mathrm{E}-25$ & $1.72 \mathrm{E}-30$ & $0.00 \mathrm{E}+00$ & $0.00 E+00$ \\
\hline SI 28 & $6.61 \mathrm{E}-06$ & $6.61 \mathrm{E}-06$ & $6.61 \mathrm{E}-06$ & $6.61 \mathrm{E}-06$ & $6.61 \mathrm{E}-06$ & $6.61 \mathrm{E}-06$ & $6.61 \mathrm{E}-06$ & $6.61 \mathrm{E}-06$ & $6.61 \mathrm{E}-06$ \\
\hline SI 29 & $3.00 \mathrm{E}-10$ & $3.00 \mathrm{E}-10$ & $3.00 \mathrm{E}-10$ & $3.00 \mathrm{E}-10$ & $3.00 \mathrm{E}-10$ & $3.00 \mathrm{E}-10$ & $3.00 \mathrm{E}-10$ & $3.00 \mathrm{E}-10$ & $3.00 \mathrm{E}-10$ \\
\hline $\mathrm{SI} 30$ & $1.49 \mathrm{E}-14$ & $1.49 \mathrm{E}-14$ & $1.49 \mathrm{E}-14$ & $1.49 \mathrm{E}-14$ & $1.49 \mathrm{E}-14$ & $1.49 \mathrm{E}-14$ & $1.49 \mathrm{E}-14$ & $1.49 \mathrm{E}-14$ & $1.49 \mathrm{E}-14$ \\
\hline FE 58 & 4.77E-14 & $4.77 \mathrm{E}-14$ & 4.77E-14 & $4.77 \mathrm{E}-14$ & 4.77E-14 & $4.77 \mathrm{E}-14$ & $4.77 \mathrm{E}-14$ & $4.77 \mathrm{E}-14$ & 4.77E-14 \\
\hline NI 61 & $3.28 \mathrm{E}-09$ & $3.28 \mathrm{E}-09$ & $3.28 \mathrm{E}-09$ & $3.28 \mathrm{E}-09$ & $3.28 \mathrm{E}-09$ & $3.28 \mathrm{E}-09$ & $3.28 \mathrm{E}-09$ & $3.28 \mathrm{E}-09$ & $3.28 \mathrm{E}-09$ \\
\hline $\mathrm{NI} 62$ & $3.78 \mathrm{E}-12$ & $3.78 \mathrm{E}-12$ & $3.78 \mathrm{E}-12$ & $3.78 \mathrm{E}-12$ & $3.78 \mathrm{E}-12$ & $3.78 \mathrm{E}-12$ & $3.78 \mathrm{E}-12$ & $3.78 \mathrm{E}-12$ & $3.78 \mathrm{E}-12$ \\
\hline $\mathrm{NI} 63$ & 2.66E-09 & $2.66 \mathrm{E}-09$ & $2.66 \mathrm{E}-09$ & $2.66 \mathrm{E}-09$ & $2.66 \mathrm{E}-09$ & $2.66 \mathrm{E}-09$ & $2.66 \mathrm{E}-09$ & $2.66 \mathrm{E}-09$ & 2.64E-09 \\
\hline $\mathrm{NI} 64$ & $1.38 \mathrm{E}-10$ & $1.38 \mathrm{E}-10$ & $1.38 \mathrm{E}-10$ & $1.38 \mathrm{E}-10$ & $1.38 \mathrm{E}-10$ & $1.38 \mathrm{E}-10$ & $1.38 \mathrm{E}-10$ & $1.38 \mathrm{E}-10$ & $1.38 \mathrm{E}-10$ \\
\hline $\mathrm{NI} 65$ & $5.16 \mathrm{E}-12$ & $4.50 \mathrm{E}-12$ & $1.30 \mathrm{E}-12$ & 7.01E-15 & $4.40 \mathrm{E}-32$ & $0.00 \mathrm{E}+00$ & $0.00 E+00$ & $0.00 \mathrm{E}+00$ & $0.00 \mathrm{E}+00$ \\
\hline NI 66 & $3.92 \mathrm{E}-15$ & $3.90 \mathrm{E}-15$ & $3.68 \mathrm{E}-15$ & $2.89 \mathrm{E}-15$ & $4.65 \mathrm{E}-16$ & $5.51 \mathrm{E}-17$ & $4.21 \mathrm{E}-19$ & $4.53 \mathrm{E}-23$ & $0.00 E+00$ \\
\hline CU 63 & $1.51 \mathrm{E}-12$ & $1.51 \mathrm{E}-12$ & $1.52 \mathrm{E}-12$ & $1.56 \mathrm{E}-12$ & $1.89 \mathrm{E}-12$ & $2.28 \mathrm{E}-12$ & $3.15 \mathrm{E}-12$ & $4.80 \mathrm{E}-12$ & $2.15 \mathrm{E}-11$ \\
\hline CU 64 & $3.09 \mathrm{E}-13$ & $3.00 \mathrm{E}-13$ & $2.35 \mathrm{E}-13$ & $8.33 \mathrm{E}-14$ & $3.22 \mathrm{E}-17$ & $3.35 \mathrm{E}-21$ & $2.65 \mathrm{E}-30$ & $0.00 \mathrm{E}+00$ & $0.00 \mathrm{E}+00$ \\
\hline CU 65 & $1.85 \mathrm{E}-05$ & $1.86 \mathrm{E}-05$ & 1.87E-05 & $1.92 \mathrm{E}-05$ & $2.31 \mathrm{E}-05$ & $2.76 \mathrm{E}-05$ & $3.75 \mathrm{E}-05$ & $5.49 \mathrm{E}-05$ & $1.68 \mathrm{E}-04$ \\
\hline CU 66 & $2.30 \mathrm{E}-12$ & $3.90 \mathrm{E}-14$ & $5.74 \mathrm{E}-18$ & $4.51 \mathrm{E}-18$ & $7.25 \mathrm{E}-19$ & $8.59 \mathrm{E}-20$ & $6.57 \mathrm{E}-22$ & $7.06 \mathrm{E}-26$ & $0.00 \mathrm{E}+00$ \\
\hline CU 67 & $9.63 \mathrm{E}-15$ & $9.58 \mathrm{E}-15$ & $9.11 \mathrm{E}-15$ & $7.36 \mathrm{E}-15$ & $1.47 \mathrm{E}-15$ & $2.23 \mathrm{E}-16$ & $3.02 \mathrm{E}-18$ & $9.47 \mathrm{E}-22$ & $0.00 \mathrm{E}+00$ \\
\hline ZN 64 & 4.51E-01 & $4.51 \mathrm{E}-01$ & 4.51E-01 & $4.51 \mathrm{E}-01$ & $4.51 \mathrm{E}-01$ & 4.51E-01 & $4.51 \mathrm{E}-01$ & $4.51 \mathrm{E}-01$ & 4.51E-01 \\
\hline ZN 65 & $2.32 \mathrm{E}-04$ & $2.32 \mathrm{E}-04$ & $2.32 \mathrm{E}-04$ & $2.31 \mathrm{E}-04$ & $2.27 \mathrm{E}-04$ & $2.23 \mathrm{E}-04$ & $2.13 \mathrm{E}-04$ & $1.95 \mathrm{E}-04$ & $8.20 \mathrm{E}-05$ \\
\hline ZN 66 & 2.67E-01 & $2.67 \mathrm{E}-01$ & 2.67E-01 & 2.67E-01 & 2.67E-01 & $2.67 \mathrm{E}-01$ & 2.67E-01 & 2.67E-01 & 2.67E-01 \\
\hline ZN 67 & $3.98 \mathrm{E}-02$ & $3.98 \mathrm{E}-02$ & $3.98 \mathrm{E}-02$ & $3.98 \mathrm{E}-02$ & $3.98 \mathrm{E}-02$ & $3.98 \mathrm{E}-02$ & $3.98 \mathrm{E}-02$ & $3.98 \mathrm{E}-02$ & $3.98 \mathrm{E}-02$ \\
\hline ZN 68 & $1.85 \mathrm{E}-01$ & $1.85 \mathrm{E}-01$ & $1.85 \mathrm{E}-01$ & $1.85 \mathrm{E}-01$ & $1.85 \mathrm{E}-01$ & $1.85 \mathrm{E}-01$ & $1.85 \mathrm{E}-01$ & $1.85 \mathrm{E}-01$ & $1.85 \mathrm{E}-01$ \\
\hline ZN 69 & $1.84 \mathrm{E}-07$ & $1.31 \mathrm{E}-07$ & $1.48 \mathrm{E}-08$ & 3.96E-09 & $2.80 \mathrm{E}-12$ & $5.92 \mathrm{E}-16$ & $2.36 \mathrm{E}-24$ & $0.00 \mathrm{E}+00$ & $0.00 \mathrm{E}+00$ \\
\hline
\end{tabular}


TEM-10200-1

ENGINEERING CALCULATIONS AND ANALYSIS

Page 77 of 81

$12 / 19 / 17$

Rev.08

Title:

AS-RUN NEUTRONICS EVALUATION FOR THE CSM-10584 EXPERIMENT IN THE ATR

$\begin{array}{llllllll}\text { ECAR NO.: } & 4496 & \text { REV. NO.: } & 1 & \text { PROJECT NO.: } & 32501 & \text { Date: } & \text { 10/19/2020 }\end{array}$

\begin{tabular}{|c|c|c|c|c|c|c|c|c|c|}
\hline \multicolumn{10}{|c|}{1 gram of $95 \mathrm{Zn}-5 \mathrm{Al}$ (CSM melt wire) irradiated for 54.9 EFPDs } \\
\hline & EOC & $30 \mathrm{MINs}$ & $5 \mathrm{HRS}$ & $1 \mathrm{DAY}$ & 7 DAYS & 14 DAYS & 30 DAYS & 60 DAYS & 1 YEAR \\
\hline & $\begin{array}{c}\mathrm{g} / 1 \mathrm{~g} \text { of } \\
95 \mathrm{Zn}-5 \mathrm{Al}\end{array}$ & $\begin{array}{c}\mathrm{g} / 1 \mathrm{~g} \text { of } \\
95 \mathrm{Zn}-5 \mathrm{Al}\end{array}$ & $\begin{array}{c}\mathrm{g} / 1 \mathrm{~g} \text { of } \\
95 \mathrm{Zn}-5 \mathrm{Al}\end{array}$ & $\begin{array}{c}\mathrm{g} / 1 \mathrm{~g} \text { of } \\
95 \mathrm{Zn}-5 \mathrm{Al}\end{array}$ & $\begin{array}{c}\mathrm{g} / 1 \mathrm{~g} \text { of } \\
95 \mathrm{Zn}-5 \mathrm{Al}\end{array}$ & $\begin{array}{c}\mathrm{g} / 1 \mathrm{~g} \text { of } \\
95 \mathrm{Zn}-5 \mathrm{Al}\end{array}$ & $\begin{array}{c}\mathrm{g} / 1 \mathrm{~g} \text { of } \\
95 \mathrm{Zn}-5 \mathrm{Al}\end{array}$ & $\begin{array}{c}\mathrm{g} / 1 \mathrm{~g} \text { of } \\
95 \mathrm{Zn}-5 \mathrm{Al}\end{array}$ & $\begin{array}{c}\text { g/1g of } \\
95 \mathrm{Zn}-5 \mathrm{Al}\end{array}$ \\
\hline ZN & & & & & & & & & \\
\hline $69 \mathrm{M}$ & $1.79 \mathrm{E}-07$ & $1.75 \mathrm{E}-07$ & $1.39 \mathrm{E}-07$ & $5.34 \mathrm{E}-08$ & $3.78 \mathrm{E}-11$ & 7.99E-15 & $3.18 \mathrm{E}-23$ & $0.00 \mathrm{E}+00$ & $0.00 \mathrm{E}+00$ \\
\hline ZN 70 & $6.30 \mathrm{E}-03$ & $6.30 \mathrm{E}-03$ & $6.30 \mathrm{E}-03$ & $6.30 \mathrm{E}-03$ & $6.30 \mathrm{E}-03$ & $6.30 \mathrm{E}-03$ & $6.30 \mathrm{E}-03$ & $6.30 \mathrm{E}-03$ & $6.30 \mathrm{E}-03$ \\
\hline ZN 71 & $1.20 \mathrm{E}-11$ & $2.64 \mathrm{E}-15$ & $2.62 \mathrm{E}-16$ & $9.09 \mathrm{E}-18$ & $7.95 \mathrm{E}-29$ & $0.00 \mathrm{E}+00$ & $0.00 \mathrm{E}+00$ & $0.00 \mathrm{E}+00$ & $0.00 \mathrm{E}+00$ \\
\hline $\mathrm{ZN}$ & & & & & & & & & \\
\hline $71 \mathrm{M}$ & $1.23 \mathrm{E}-10$ & $1.12 \mathrm{E}-10$ & 5.07E-11 & $1.76 \mathrm{E}-12$ & $1.54 \mathrm{E}-23$ & $1.94 \mathrm{E}-36$ & $0.00 E+00$ & $0.00 E+00$ & $0.00 \mathrm{E}+00$ \\
\hline GA 69 & $1.76 \mathrm{E}-04$ & $1.76 \mathrm{E}-04$ & 1.76E-04 & 1.76E-04 & 1.77E-04 & 1.77E-04 & 1.77E-04 & 1.77E-04 & 1.77E-04 \\
\hline GA 70 & $2.00 \mathrm{E}-10$ & 7.47E-11 & $1.05 \mathrm{E}-14$ & $5.72 \mathrm{E}-31$ & $0.00 \mathrm{E}+00$ & $0.00 \mathrm{E}+00$ & $0.00 \mathrm{E}+00$ & $0.00 \mathrm{E}+00$ & $0.00 \mathrm{E}+00$ \\
\hline GA 71 & $3.01 \mathrm{E}-07$ & $3.01 \mathrm{E}-07$ & $3.01 \mathrm{E}-07$ & $3.01 \mathrm{E}-07$ & $3.01 \mathrm{E}-07$ & $3.01 \mathrm{E}-07$ & $3.01 \mathrm{E}-07$ & $3.01 \mathrm{E}-07$ & $3.01 \mathrm{E}-07$ \\
\hline GA 72 & $3.06 \mathrm{E}-11$ & $2.98 \mathrm{E}-11$ & $2.39 \mathrm{E}-11$ & $9.39 \mathrm{E}-12$ & $7.92 \mathrm{E}-15$ & $2.05 \mathrm{E}-18$ & $1.30 \mathrm{E}-26$ & $0.00 \mathrm{E}+00$ & $0.00 \mathrm{E}+00$ \\
\hline GE 70 & $2.60 \mathrm{E}-07$ & $2.60 \mathrm{E}-07$ & $2.60 \mathrm{E}-07$ & $2.60 \mathrm{E}-07$ & $2.60 \mathrm{E}-07$ & $2.60 \mathrm{E}-07$ & $2.60 \mathrm{E}-07$ & $2.60 \mathrm{E}-07$ & $2.60 \mathrm{E}-07$ \\
\hline GE 71 & $8.69 \mathrm{E}-11$ & $8.68 \mathrm{E}-11$ & $8.58 \mathrm{E}-11$ & $8.19 \mathrm{E}-11$ & $5.76 \mathrm{E}-11$ & $3.82 \mathrm{E}-11$ & $1.49 \mathrm{E}-11$ & $2.56 \mathrm{E}-12$ & $4.18 \mathrm{E}-20$ \\
\hline GE 72 & $9.75 \mathrm{E}-10$ & $9.76 \mathrm{E}-10$ & $9.82 \mathrm{E}-10$ & $9.97 \mathrm{E}-10$ & $1.01 \mathrm{E}-09$ & $1.01 \mathrm{E}-09$ & $1.01 \mathrm{E}-09$ & $1.01 \mathrm{E}-09$ & $1.01 \mathrm{E}-09$ \\
\hline GE 73 & $2.28 \mathrm{E}-13$ & $2.28 \mathrm{E}-13$ & $2.28 \mathrm{E}-13$ & $2.28 \mathrm{E}-13$ & $2.28 \mathrm{E}-13$ & $2.28 \mathrm{E}-13$ & $2.28 \mathrm{E}-13$ & $2.28 \mathrm{E}-13$ & $2.28 \mathrm{E}-13$ \\
\hline GE 74 & $1.07 \mathrm{E}-15$ & $1.07 \mathrm{E}-15$ & $1.07 \mathrm{E}-15$ & $1.07 \mathrm{E}-15$ & $1.07 \mathrm{E}-15$ & $1.07 \mathrm{E}-15$ & $1.07 \mathrm{E}-15$ & $1.07 \mathrm{E}-15$ & $1.07 \mathrm{E}-15$ \\
\hline SUMT & & & & & & & & & \\
\hline OT & $1.00 \mathrm{E}+00$ & $1.00 \mathrm{E}+00$ & $1.00 \mathrm{E}+00$ & $1.00 \mathrm{E}+00$ & $1.00 E+00$ & $1.00 \mathrm{E}+00$ & $1.00 \mathrm{E}+00$ & $1.00 \mathrm{E}+00$ & $1.00 \mathrm{E}+00$ \\
\hline ОTOTA & & & & & & & & & \\
\hline L & $1.00 \mathrm{E}+00$ & $1.00 \mathrm{E}+00$ & $1.00 \mathrm{E}+00$ & $1.00 \mathrm{E}+00$ & $1.00 E+00$ & $1.00 \mathrm{E}+00$ & $1.00 \mathrm{E}+00$ & $1.00 \mathrm{E}+00$ & $1.00 \mathrm{E}+00$ \\
\hline
\end{tabular}


TEM-10200-1

ENGINEERING CALCULATIONS AND ANALYSIS

Page 78 of 81

$12 / 19 / 17$

Rev.08

Title:

AS-RUN NEUTRONICS EVALUATION FOR THE CSM-10584 EXPERIMENT IN THE ATR

\begin{tabular}{llllllll} 
ECAR NO.: & 4496 & REV. NO.: & 1 & PROJECT NO.: & 32501 & Date: & 10/19/2020 \\
\hline
\end{tabular}

\begin{tabular}{|c|c|c|c|c|c|c|c|c|c|}
\hline \multicolumn{10}{|c|}{1 gram of $\mathrm{Pb}$ (CSM melt wire) irradiated for 119 EFPDs } \\
\hline & EOC & $30 \mathrm{MINs}$ & $5 \mathrm{HRS}$ & $1 \mathrm{DAY}$ & 7 DAYS & 14 DAYS & 30 DAYS & 60 DAYS & 1 YEAR \\
\hline & $\mathrm{Ci} / 1 \mathrm{~g}$ of $\mathrm{Pb}$ & $\mathrm{Ci} / 1 \mathrm{~g}$ of $\mathrm{Pb}$ & $\mathrm{Ci} / 1 \mathrm{~g}$ of $\mathrm{Pb}$ & $\mathrm{Ci} / 1 \mathrm{~g}$ of $\mathrm{Pb}$ & $\mathrm{Ci} / 1 \mathrm{~g}$ of $\mathrm{Pb}$ & $\mathrm{Ci} / 1 \mathrm{~g}$ of $\mathrm{Pb}$ & $\mathrm{Ci} / 1 \mathrm{~g}$ of $\mathrm{Pb}$ & $\mathrm{Ci} / 1 \mathrm{~g}$ of $\mathrm{Pb}$ & $\mathrm{Ci} / 1 \mathrm{~g}$ of $\mathrm{Pb}$ \\
\hline TL206 & $1.46 \mathrm{E}-13$ & $6.36 \mathrm{E}-15$ & $3.10 \mathrm{E}-15$ & $3.10 \mathrm{E}-15$ & $3.10 \mathrm{E}-15$ & $3.10 \mathrm{E}-15$ & $3.10 \mathrm{E}-15$ & $3.10 \mathrm{E}-15$ & $3.10 \mathrm{E}-15$ \\
\hline PB204 & $1.72 \mathrm{E}-16$ & $1.72 \mathrm{E}-16$ & $1.72 \mathrm{E}-16$ & $1.72 \mathrm{E}-16$ & $1.72 \mathrm{E}-16$ & $1.72 \mathrm{E}-16$ & $1.72 \mathrm{E}-16$ & $1.72 \mathrm{E}-16$ & $1.72 \mathrm{E}-16$ \\
\hline PB205 & $9.31 \mathrm{E}-10$ & $9.31 \mathrm{E}-10$ & $9.31 \mathrm{E}-10$ & $9.31 \mathrm{E}-10$ & $9.31 \mathrm{E}-10$ & $9.31 \mathrm{E}-10$ & $9.31 \mathrm{E}-10$ & $9.31 \mathrm{E}-10$ & $9.31 \mathrm{E}-10$ \\
\hline PB209 & $2.18 \mathrm{E}-03$ & $1.96 \mathrm{E}-03$ & $7.62 \mathrm{E}-04$ & $1.41 \mathrm{E}-05$ & $1.03 \mathrm{E}-18$ & $0.00 \mathrm{E}+00$ & $0.00 \mathrm{E}+00$ & $0.00 \mathrm{E}+00$ & $0.00 \mathrm{E}+00$ \\
\hline $\mathrm{B} 1208$ & $4.72 \mathrm{E}-15$ & $4.72 \mathrm{E}-15$ & $4.72 \mathrm{E}-15$ & $4.72 \mathrm{E}-15$ & $4.72 \mathrm{E}-15$ & $4.72 \mathrm{E}-15$ & $4.72 \mathrm{E}-15$ & $4.72 \mathrm{E}-15$ & $4.72 \mathrm{E}-15$ \\
\hline $\mathrm{BI} 210 \mathrm{M}$ & $3.11 \mathrm{E}-15$ & $3.11 \mathrm{E}-15$ & $3.11 \mathrm{E}-15$ & $3.11 \mathrm{E}-15$ & $3.11 \mathrm{E}-15$ & $3.11 \mathrm{E}-15$ & $3.11 \mathrm{E}-15$ & $3.11 \mathrm{E}-15$ & $3.11 \mathrm{E}-15$ \\
\hline $\mathrm{BI} 211$ & $3.86 \mathrm{E}-13$ & $2.22 \mathrm{E}-17$ & $0.00 \mathrm{E}+00$ & $0.00 \mathrm{E}+00$ & $0.00 \mathrm{E}+00$ & $0.00 \mathrm{E}+00$ & $0.00 \mathrm{E}+00$ & $0.00 \mathrm{E}+00$ & $0.00 \mathrm{E}+00$ \\
\hline PO210 & $2.47 \mathrm{E}-08$ & $2.47 \mathrm{E}-08$ & $2.48 \mathrm{E}-08$ & $2.51 \mathrm{E}-08$ & $2.62 \mathrm{E}-08$ & $2.62 \mathrm{E}-08$ & $2.46 \mathrm{E}-08$ & $2.12 \mathrm{E}-08$ & 4.61E-09 \\
\hline PO211 & $1.39 \mathrm{E}-12$ & $6.25 \mathrm{E}-20$ & $0.00 E+00$ & $0.00 E+00$ & $0.00 E+00$ & $0.00 E+00$ & $0.00 \mathrm{E}+00$ & $0.00 E+00$ & $0.00 E+00$ \\
\hline PO211M & $2.32 \mathrm{E}-14$ & $0.00 \mathrm{E}+00$ & $0.00 \mathrm{E}+00$ & $0.00 \mathrm{E}+00$ & $0.00 \mathrm{E}+00$ & $0.00 \mathrm{E}+00$ & $0.00 \mathrm{E}+00$ & $0.00 \mathrm{E}+00$ & $0.00 \mathrm{E}+00$ \\
\hline SUMTOT & $2.18 \mathrm{E}-03$ & $1.96 \mathrm{E}-03$ & $7.62 \mathrm{E}-04$ & $1.42 \mathrm{E}-05$ & $6.71 \mathrm{E}-08$ & $4.23 \mathrm{E}-08$ & $2.72 \mathrm{E}-08$ & $2.22 \mathrm{E}-08$ & 5.54E-09 \\
\hline OTOTAL & $2.18 \mathrm{E}-03$ & $1.96 \mathrm{E}-03$ & 7.62E-04 & $1.42 \mathrm{E}-05$ & $6.71 \mathrm{E}-08$ & $4.23 \mathrm{E}-08$ & $2.72 \mathrm{E}-08$ & $2.22 \mathrm{E}-08$ & 5.54E-09 \\
\hline & EOC & $30 \mathrm{MINs}$ & $5 \mathrm{HRS}$ & $1 \mathrm{DAY}$ & 7 DAYS & 14 DAYS & 30 DAYS & 60 DAYS & 1 YEAR \\
\hline & $\mathrm{Ci} / 1 \mathrm{~g}$ of $\mathrm{Pb}$ & $\mathrm{Ci} / 1 \mathrm{~g}$ of $\mathrm{Pb}$ & $\mathrm{Ci} / 1 \mathrm{~g}$ of $\mathrm{Pb}$ & $\mathrm{Ci} / 1 \mathrm{~g}$ of $\mathrm{Pb}$ & $\mathrm{Ci} / 1 \mathrm{~g}$ of $\mathrm{Pb}$ & $\mathrm{Ci} / 1 \mathrm{~g}$ of $\mathrm{Pb}$ & $\mathrm{Ci} / 1 \mathrm{~g}$ of $\mathrm{Pb}$ & $\mathrm{Ci} / 1 \mathrm{~g}$ of $\mathrm{Pb}$ & $\mathrm{Ci} / 1 \mathrm{~g}$ of $\mathrm{Pb}$ \\
\hline TL206 & $3.11 \mathrm{E}-14$ & $0.00 E+00$ & $6.65 \mathrm{E}-16$ & $6.65 \mathrm{E}-16$ & $6.65 \mathrm{E}-16$ & $6.65 \mathrm{E}-16$ & $6.65 \mathrm{E}-16$ & $6.65 \mathrm{E}-16$ & $6.65 \mathrm{E}-16$ \\
\hline PB204 & $1.73 \mathrm{E}-16$ & $1.73 \mathrm{E}-16$ & $1.73 \mathrm{E}-16$ & $1.73 \mathrm{E}-16$ & $1.73 \mathrm{E}-16$ & $1.73 \mathrm{E}-16$ & $1.73 \mathrm{E}-16$ & $1.73 \mathrm{E}-16$ & $1.73 \mathrm{E}-16$ \\
\hline PB205 & $4.29 \mathrm{E}-10$ & 4.29E-10 & $4.29 \mathrm{E}-10$ & $4.29 \mathrm{E}-10$ & 4.29E-10 & 4.29E-10 & 4.29E-10 & $4.29 \mathrm{E}-10$ & $4.29 \mathrm{E}-10$ \\
\hline PB209 & $2.18 \mathrm{E}-03$ & $1.96 \mathrm{E}-03$ & 7.61E-04 & $1.41 \mathrm{E}-05$ & $1.03 \mathrm{E}-18$ & $0.00 \mathrm{E}+00$ & $0.00 \mathrm{E}+00$ & $0.00 \mathrm{E}+00$ & $0.00 \mathrm{E}+00$ \\
\hline $\mathrm{BI} 208$ & $1.02 \mathrm{E}-15$ & $1.02 \mathrm{E}-15$ & $1.02 \mathrm{E}-15$ & $1.02 \mathrm{E}-15$ & $1.02 \mathrm{E}-15$ & $1.02 \mathrm{E}-15$ & $1.02 \mathrm{E}-15$ & $1.02 \mathrm{E}-15$ & $1.02 \mathrm{E}-15$ \\
\hline $\mathrm{B} 1210$ & $4.53 \mathrm{E}-08$ & $4.51 \mathrm{E}-08$ & $4.40 \mathrm{E}-08$ & $3.94 \mathrm{E}-08$ & $1.72 \mathrm{E}-08$ & $6.53 \mathrm{E}-09$ & $7.14 \mathrm{E}-10$ & $1.13 \mathrm{E}-11$ & $5.20 \mathrm{E}-30$ \\
\hline $\mathrm{BI} 210 \mathrm{M}$ & $6.68 \mathrm{E}-16$ & $6.68 \mathrm{E}-16$ & $6.68 \mathrm{E}-16$ & $6.68 \mathrm{E}-16$ & $6.68 \mathrm{E}-16$ & $6.68 \mathrm{E}-16$ & $6.68 \mathrm{E}-16$ & $6.68 \mathrm{E}-16$ & $6.68 \mathrm{E}-16$ \\
\hline $\mathrm{BI} 211$ & $1.66 \mathrm{E}-13$ & $9.54 \mathrm{E}-18$ & $0.00 E+00$ & $0.00 \mathrm{E}+00$ & $0.00 \mathrm{E}+00$ & $0.00 \mathrm{E}+00$ & $0.00 \mathrm{E}+00$ & $0.00 \mathrm{E}+00$ & $0.00 \mathrm{E}+00$ \\
\hline PO210 & 5.11E-09 & 5.11E-09 & 5.15E-09 & 5.29E-09 & 5.93E-09 & $6.10 \mathrm{E}-09$ & $5.83 \mathrm{E}-09$ & $5.04 \mathrm{E}-09$ & 1.09E-09 \\
\hline P0211 & $2.87 \mathrm{E}-13$ & $2.68 \mathrm{E}-20$ & $0.00 \mathrm{E}+00$ & $0.00 \mathrm{E}+00$ & $0.00 \mathrm{E}+00$ & $0.00 \mathrm{E}+00$ & $0.00 \mathrm{E}+00$ & $0.00 \mathrm{E}+00$ & $0.00 \mathrm{E}+00$ \\
\hline PO211M & $4.78 \mathrm{E}-15$ & $0.00 \mathrm{E}+00$ & $0.00 \mathrm{E}+00$ & $0.00 \mathrm{E}+00$ & $0.00 \mathrm{E}+00$ & $0.00 \mathrm{E}+00$ & $0.00 \mathrm{E}+00$ & $0.00 \mathrm{E}+00$ & $0.00 \mathrm{E}+00$ \\
\hline SUMTOT & $2.18 \mathrm{E}-03$ & $1.96 \mathrm{E}-03$ & 7.62E-04 & $1.41 \mathrm{E}-05$ & $2.36 \mathrm{E}-08$ & $1.31 \mathrm{E}-08$ & $6.98 \mathrm{E}-09$ & $5.48 \mathrm{E}-09$ & $1.52 \mathrm{E}-09$ \\
\hline OTOTAL & $2.18 \mathrm{E}-03$ & $1.96 \mathrm{E}-03$ & $7.62 \mathrm{E}-04$ & $1.41 \mathrm{E}-05$ & $2.36 \mathrm{E}-08$ & $1.31 \mathrm{E}-08$ & $6.98 \mathrm{E}-09$ & 5.48E-09 & $1.52 \mathrm{E}-09$ \\
\hline
\end{tabular}


TEM-10200-1

ENGINEERING CALCULATIONS AND ANALYSIS

Page 79 of 81

$12 / 19 / 17$

Rev.08

Title:

AS-RUN NEUTRONICS EVALUATION FOR THE CSM-10584 EXPERIMENT IN THE ATR

\begin{tabular}{llllllll} 
ECAR NO.: & 4496 & REV. NO.: & 1 & PROJECT NO.: & 32501 & Date: & 10/19/2020 \\
\hline
\end{tabular}

\begin{tabular}{|c|c|c|c|c|c|c|c|c|c|}
\hline \multicolumn{10}{|c|}{1 gram of $\mathrm{Pb}$ (CSM melt wire) irradiated for 119 EFPDs } \\
\hline & EOC & $30 \mathrm{MINs}$ & $5 \mathrm{HRS}$ & $1 \mathrm{DAY}$ & 7 DAYS & 14 DAYS & 30 DAYS & 60 DAYS & 1 YEAR \\
\hline & $\mathrm{g} / 1 \mathrm{~g}$ of $\mathrm{Pb}$ & $\mathrm{g} / 1 \mathrm{~g}$ of $\mathrm{Pb}$ & $\mathrm{g} / 1 \mathrm{~g}$ of $\mathrm{Pb}$ & $\mathrm{g} / 1 \mathrm{~g}$ of $\mathrm{Pb}$ & $\mathrm{g} / 1 \mathrm{~g}$ of $\mathrm{Pb}$ & $\mathrm{g} / 1 \mathrm{~g}$ of $\mathrm{Pb}$ & $\mathrm{g} / 1 \mathrm{~g}$ of $\mathrm{Pb}$ & $\mathrm{g} / 1 \mathrm{~g}$ of $\mathrm{Pb}$ & $\mathrm{g} / 1 \mathrm{~g}$ of $\mathrm{Pb}$ \\
\hline $\mathrm{HE} 4$ & $2.06 \mathrm{E}-14$ & $2.06 \mathrm{E}-14$ & $2.07 \mathrm{E}-14$ & $2.11 \mathrm{E}-14$ & $2.44 \mathrm{E}-14$ & $2.83 \mathrm{E}-14$ & $3.69 \mathrm{E}-14$ & $5.15 \mathrm{E}-14$ & $1.22 \mathrm{E}-13$ \\
\hline TL205 & $6.02 \mathrm{E}-14$ & $6.03 \mathrm{E}-14$ & $6.04 \mathrm{E}-14$ & $6.12 \mathrm{E}-14$ & $6.73 E-14$ & $7.44 \mathrm{E}-14$ & $9.06 \mathrm{E}-14$ & $1.21 \mathrm{E}-13$ & $4.30 \mathrm{E}-13$ \\
\hline PB204 & $1.38 \mathrm{E}-02$ & $1.38 \mathrm{E}-02$ & $1.38 \mathrm{E}-02$ & $1.38 \mathrm{E}-02$ & $1.38 \mathrm{E}-02$ & $1.38 \mathrm{E}-02$ & $1.38 \mathrm{E}-02$ & $1.38 \mathrm{E}-02$ & $1.38 \mathrm{E}-02$ \\
\hline PB205 & $1.60 \mathrm{E}-05$ & $1.60 \mathrm{E}-05$ & $1.60 \mathrm{E}-05$ & $1.60 \mathrm{E}-05$ & $1.60 \mathrm{E}-05$ & $1.60 \mathrm{E}-05$ & $1.60 \mathrm{E}-05$ & $1.60 \mathrm{E}-05$ & $1.60 \mathrm{E}-05$ \\
\hline PB206 & $2.33 \mathrm{E}-01$ & $2.33 \mathrm{E}-01$ & $2.33 \mathrm{E}-01$ & $2.33 \mathrm{E}-01$ & $2.33 \mathrm{E}-01$ & $2.33 \mathrm{E}-01$ & $2.33 \mathrm{E}-01$ & $2.33 \mathrm{E}-01$ & $2.33 \mathrm{E}-01$ \\
\hline PB207 & $2.28 \mathrm{E}-01$ & $2.28 \mathrm{E}-01$ & $2.28 \mathrm{E}-01$ & $2.28 \mathrm{E}-01$ & $2.28 \mathrm{E}-01$ & $2.28 \mathrm{E}-01$ & $2.28 \mathrm{E}-01$ & $2.28 \mathrm{E}-01$ & $2.28 \mathrm{E}-01$ \\
\hline PB208 & $5.26 \mathrm{E}-01$ & $5.26 \mathrm{E}-01$ & $5.26 \mathrm{E}-01$ & $5.26 \mathrm{E}-01$ & $5.26 \mathrm{E}-01$ & $5.26 \mathrm{E}-01$ & $5.26 \mathrm{E}-01$ & $5.26 \mathrm{E}-01$ & $5.26 \mathrm{E}-01$ \\
\hline PB209 & $4.79 \mathrm{E}-10$ & $4.31 \mathrm{E}-10$ & $1.68 \mathrm{E}-10$ & $3.10 \mathrm{E}-12$ & $2.27 \mathrm{E}-25$ & $0.00 \mathrm{E}+00$ & $0.00 \mathrm{E}+00$ & $0.00 \mathrm{E}+00$ & $0.00 \mathrm{E}+00$ \\
\hline $\mathrm{BI} 208$ & $1.01 \mathrm{E}-12$ & $1.01 \mathrm{E}-12$ & $1.01 \mathrm{E}-12$ & $1.01 \mathrm{E}-12$ & $1.01 \mathrm{E}-12$ & $1.01 \mathrm{E}-12$ & $1.01 \mathrm{E}-12$ & $1.01 \mathrm{E}-12$ & $1.01 \mathrm{E}-12$ \\
\hline $\mathrm{BI} 209$ & $2.89 \mathrm{E}-07$ & $2.89 \mathrm{E}-07$ & $2.89 \mathrm{E}-07$ & $2.89 \mathrm{E}-07$ & $2.89 \mathrm{E}-07$ & $2.89 \mathrm{E}-07$ & $2.89 \mathrm{E}-07$ & $2.89 \mathrm{E}-07$ & $2.89 \mathrm{E}-07$ \\
\hline $\mathrm{B} 1210$ & $8.49 \mathrm{E}-13$ & $8.47 \mathrm{E}-13$ & $8.25 \mathrm{E}-13$ & $7.39 \mathrm{E}-13$ & $3.23 \mathrm{E}-13$ & $1.23 \mathrm{E}-13$ & $1.34 \mathrm{E}-14$ & $2.11 \mathrm{E}-16$ & $9.76 \mathrm{E}-35$ \\
\hline BI210M & $5.47 \mathrm{E}-12$ & $5.47 \mathrm{E}-12$ & 5.47E-12 & $5.47 \mathrm{E}-12$ & $5.47 \mathrm{E}-12$ & $5.47 \mathrm{E}-12$ & $5.47 \mathrm{E}-12$ & 5.47E-12 & $5.47 \mathrm{E}-12$ \\
\hline $\mathrm{PO} 210$ & $5.50 \mathrm{E}-12$ & $5.50 \mathrm{E}-12$ & $5.52 \mathrm{E}-12$ & $5.58 \mathrm{E}-12$ & $5.83 \mathrm{E}-12$ & $5.82 \mathrm{E}-12$ & $5.48 \mathrm{E}-12$ & $4.73 \mathrm{E}-12$ & $1.03 \mathrm{E}-12$ \\
\hline SUMTOT & $1.00 \mathrm{E}+00$ & $1.00 \mathrm{E}+00$ & $1.00 \mathrm{E}+00$ & $1.00 \mathrm{E}+00$ & $1.00 \mathrm{E}+00$ & $1.00 \mathrm{E}+00$ & $1.00 \mathrm{E}+00$ & $1.00 \mathrm{E}+00$ & $1.00 \mathrm{E}+00$ \\
\hline OTOTAL & $1.00 \mathrm{E}+00$ & $1.00 \mathrm{E}+00$ & $1.00 \mathrm{E}+00$ & $1.00 \mathrm{E}+00$ & $1.00 \mathrm{E}+00$ & $1.00 \mathrm{E}+00$ & $1.00 \mathrm{E}+00$ & $1.00 \mathrm{E}+00$ & $1.00 \mathrm{E}+00$ \\
\hline \multicolumn{10}{|c|}{1 gram of $\mathrm{Pb}$ (CSM melt wire) irradiated for 54.9 EFPDs } \\
\hline & EOC & $30 \mathrm{MINs}$ & 5 HRS & $1 \mathrm{DAY}$ & 7 DAYS & 14 DAYS & 30 DAYS & 60 DAYS & 1 YEAR \\
\hline & $\mathrm{g} / 1 \mathrm{~g}$ of $\mathrm{Pb}$ & $\mathrm{g} / 1 \mathrm{~g}$ of $\mathrm{Pb}$ & $\mathrm{g} / 1 \mathrm{~g}$ of $\mathrm{Pb}$ & $\mathrm{g} / 1 \mathrm{~g}$ of $\mathrm{Pb}$ & $\mathrm{g} / 1 \mathrm{~g}$ of $\mathrm{Pb}$ & $\mathrm{g} / 1 \mathrm{~g}$ of $\mathrm{Pb}$ & $\mathrm{g} / 1 \mathrm{~g}$ of $\mathrm{Pb}$ & $\mathrm{g} / 1 \mathrm{~g}$ of $\mathrm{Pb}$ & $\mathrm{g} / 1 \mathrm{~g}$ of $\mathrm{Pb}$ \\
\hline $\mathrm{HE} 4$ & $1.82 \mathrm{E}-15$ & $1.83 \mathrm{E}-15$ & $1.85 \mathrm{E}-15$ & $1.93 \mathrm{E}-15$ & $2.65 \mathrm{E}-15$ & $3.55 \mathrm{E}-15$ & 5.59E-15 & $9.05 \mathrm{E}-15$ & $2.58 \mathrm{E}-14$ \\
\hline TL205 & $1.28 \mathrm{E}-14$ & $1.28 \mathrm{E}-14$ & $1.29 \mathrm{E}-14$ & $1.33 \mathrm{E}-14$ & $1.61 \mathrm{E}-14$ & $1.94 \mathrm{E}-14$ & $2.68 \mathrm{E}-14$ & $4.09 \mathrm{E}-14$ & $1.83 \mathrm{E}-13$ \\
\hline PB204 & $1.38 \mathrm{E}-02$ & $1.38 \mathrm{E}-02$ & $1.38 \mathrm{E}-02$ & $1.38 \mathrm{E}-02$ & $1.38 \mathrm{E}-02$ & $1.38 \mathrm{E}-02$ & $1.38 \mathrm{E}-02$ & $1.38 \mathrm{E}-02$ & $1.38 \mathrm{E}-02$ \\
\hline PB205 & $7.38 \mathrm{E}-06$ & $7.38 \mathrm{E}-06$ & $7.38 \mathrm{E}-06$ & $7.38 \mathrm{E}-06$ & $7.38 \mathrm{E}-06$ & $7.38 \mathrm{E}-06$ & $7.38 \mathrm{E}-06$ & $7.38 \mathrm{E}-06$ & $7.38 \mathrm{E}-06$ \\
\hline PB206 & $2.36 \mathrm{E}-01$ & $2.36 \mathrm{E}-01$ & $2.36 \mathrm{E}-01$ & $2.36 \mathrm{E}-01$ & $2.36 \mathrm{E}-01$ & $2.36 \mathrm{E}-01$ & $2.36 \mathrm{E}-01$ & $2.36 \mathrm{E}-01$ & $2.36 \mathrm{E}-01$ \\
\hline PB207 & $2.24 \mathrm{E}-01$ & $2.24 \mathrm{E}-01$ & $2.24 \mathrm{E}-01$ & $2.24 \mathrm{E}-01$ & $2.24 \mathrm{E}-01$ & $2.24 \mathrm{E}-01$ & $2.24 \mathrm{E}-01$ & $2.24 \mathrm{E}-01$ & $2.24 \mathrm{E}-01$ \\
\hline PB208 & $5.26 \mathrm{E}-01$ & $5.26 \mathrm{E}-01$ & $5.26 \mathrm{E}-01$ & $5.26 \mathrm{E}-01$ & $5.26 \mathrm{E}-01$ & $5.26 \mathrm{E}-01$ & $5.26 \mathrm{E}-01$ & $5.26 \mathrm{E}-01$ & $5.26 \mathrm{E}-01$ \\
\hline PB209 & $4.79 \mathrm{E}-10$ & $4.31 \mathrm{E}-10$ & $1.68 \mathrm{E}-10$ & $3.10 \mathrm{E}-12$ & $2.26 \mathrm{E}-25$ & $0.00 \mathrm{E}+00$ & $0.00 \mathrm{E}+00$ & $0.00 \mathrm{E}+00$ & $0.00 \mathrm{E}+00$ \\
\hline $\mathrm{BI} 208$ & $2.17 \mathrm{E}-13$ & $2.17 \mathrm{E}-13$ & $2.17 \mathrm{E}-13$ & $2.17 \mathrm{E}-13$ & $2.17 \mathrm{E}-13$ & $2.17 \mathrm{E}-13$ & $2.17 \mathrm{E}-13$ & $2.17 \mathrm{E}-13$ & $2.17 \mathrm{E}-13$ \\
\hline BI209 & $1.34 \mathrm{E}-07$ & $1.34 \mathrm{E}-07$ & $1.34 \mathrm{E}-07$ & $1.34 \mathrm{E}-07$ & $1.34 \mathrm{E}-07$ & $1.34 \mathrm{E}-07$ & $1.34 \mathrm{E}-07$ & $1.34 \mathrm{E}-07$ & $1.34 \mathrm{E}-07$ \\
\hline $\mathrm{BI} 210$ & $3.65 \mathrm{E}-13$ & $3.64 \mathrm{E}-13$ & $3.54 \mathrm{E}-13$ & $3.18 \mathrm{E}-13$ & $1.39 \mathrm{E}-13$ & $5.26 \mathrm{E}-14$ & $5.75 \mathrm{E}-15$ & $9.08 \mathrm{E}-17$ & $4.19 \mathrm{E}-35$ \\
\hline $\mathrm{BI} 210 \mathrm{M}$ & $1.18 \mathrm{E}-12$ & $1.18 \mathrm{E}-12$ & $1.18 \mathrm{E}-12$ & $1.18 \mathrm{E}-12$ & $1.18 \mathrm{E}-12$ & $1.18 \mathrm{E}-12$ & $1.18 \mathrm{E}-12$ & $1.18 \mathrm{E}-12$ & $1.18 \mathrm{E}-12$ \\
\hline PO210 & $1.14 \mathrm{E}-12$ & $1.14 \mathrm{E}-12$ & $1.15 \mathrm{E}-12$ & $1.18 \mathrm{E}-12$ & $1.32 \mathrm{E}-12$ & $1.36 \mathrm{E}-12$ & $1.30 \mathrm{E}-12$ & $1.12 \mathrm{E}-12$ & $2.43 \mathrm{E}-13$ \\
\hline SUMTOT & $1.00 \mathrm{E}+00$ & $1.00 \mathrm{E}+00$ & $1.00 \mathrm{E}+00$ & $1.00 \mathrm{E}+00$ & $1.00 \mathrm{E}+00$ & $1.00 \mathrm{E}+00$ & $1.00 \mathrm{E}+00$ & $1.00 \mathrm{E}+00$ & $1.00 \mathrm{E}+00$ \\
\hline OTOTAL & $1.00 \mathrm{E}+00$ & $1.00 \mathrm{E}+00$ & $1.00 \mathrm{E}+00$ & $1.00 \mathrm{E}+00$ & $1.00 \mathrm{E}+00$ & $1.00 \mathrm{E}+00$ & $1.00 \mathrm{E}+00$ & $1.00 \mathrm{E}+00$ & $1.00 \mathrm{E}+00$ \\
\hline
\end{tabular}


TEM-10200-1

ENGINEERING CALCULATIONS AND ANALYSIS

Page 80 of 81

$12 / 19 / 17$

Rev.08

Title:

AS-RUN NEUTRONICS EVALUATION FOR THE CSM-10584 EXPERIMENT IN THE ATR

$\begin{array}{llllllll}\text { ECAR NO.: } & 4496 & \text { REV. NO.: } & 1 & \text { PROJECT NO.: } & 32501 & \text { Date: } & 10 / 19 / 2020\end{array}$

\begin{tabular}{|c|c|c|c|c|c|c|c|c|c|}
\hline \multicolumn{10}{|c|}{1 gram of $\mathrm{Bi}$ (CSM melt wire) irradiated for 119 EFPDs } \\
\hline & EOC & $30 \mathrm{MINs}$ & $5 \mathrm{HRS}$ & $1 \mathrm{DAY}$ & 7 DAYS & 14 DAYS & 30 DAYS & 60 DAYS & 1 YEAR \\
\hline & $\mathrm{Ci} / 1 \mathrm{~g}$ of $\mathrm{Bi}$ & $\mathrm{Ci} / 1 \mathrm{~g}$ of $\mathrm{Bi}$ & $\mathrm{Ci} / 1 \mathrm{~g}$ of $\mathrm{Bi}$ & $\mathrm{Ci} / 1 \mathrm{~g}$ of $\mathrm{Bi}$ & $\mathrm{Ci} / 1 \mathrm{~g}$ of $\mathrm{Bi}$ & $\mathrm{Ci} / 1 \mathrm{~g}$ of $\mathrm{Bi}$ & $\mathrm{Ci} / 1 \mathrm{~g}$ of $\mathrm{Bi}$ & $\mathrm{Ci} / 1 \mathrm{~g}$ of $\mathrm{Bi}$ & $\mathrm{Ci} / 1 \mathrm{~g}$ of $\mathrm{Bi}$ \\
\hline $\mathrm{H} 3$ & $1.22 \mathrm{E}-16$ & $1.22 \mathrm{E}-16$ & $1.22 \mathrm{E}-16$ & $1.22 \mathrm{E}-16$ & $1.22 \mathrm{E}-16$ & $1.22 \mathrm{E}-16$ & $1.21 \mathrm{E}-16$ & $1.21 \mathrm{E}-16$ & $1.15 \mathrm{E}-16$ \\
\hline TL206 & $2.15 \mathrm{E}-08$ & $2.15 \mathrm{E}-08$ & $2.15 \mathrm{E}-08$ & $2.15 \mathrm{E}-08$ & $2.15 \mathrm{E}-08$ & $2.15 \mathrm{E}-08$ & $2.15 \mathrm{E}-08$ & $2.15 \mathrm{E}-08$ & $2.15 \mathrm{E}-08$ \\
\hline PB209 & $1.61 \mathrm{E}-04$ & $1.45 \mathrm{E}-04$ & $5.62 \mathrm{E}-05$ & $1.04 \mathrm{E}-06$ & $7.60 \mathrm{E}-20$ & $0.00 \mathrm{E}+00$ & $0.00 \mathrm{E}+00$ & $0.00 \mathrm{E}+00$ & $0.00 \mathrm{E}+00$ \\
\hline $\mathrm{B} 1208$ & $3.28 \mathrm{E}-08$ & $3.28 \mathrm{E}-08$ & $3.28 \mathrm{E}-08$ & $3.28 \mathrm{E}-08$ & $3.28 \mathrm{E}-08$ & $3.28 \mathrm{E}-08$ & $3.28 \mathrm{E}-08$ & $3.28 \mathrm{E}-08$ & $3.28 \mathrm{E}-08$ \\
\hline $\mathrm{B} 1210$ & $3.89 \mathrm{E}-01$ & $3.88 \mathrm{E}-01$ & $3.78 \mathrm{E}-01$ & 3.39E-01 & $1.48 \mathrm{E}-01$ & $5.61 \mathrm{E}-02$ & $6.13 \mathrm{E}-03$ & 9.67E-05 & 4.47E-23 \\
\hline $\mathrm{B} \mid 211$ & $1.42 \mathrm{E}-06$ & $8.19 \mathrm{E}-11$ & $0.00 \mathrm{E}+00$ & $0.00 \mathrm{E}+00$ & $0.00 \mathrm{E}+00$ & $0.00 \mathrm{E}+00$ & $0.00 \mathrm{E}+00$ & $0.00 \mathrm{E}+00$ & $0.00 \mathrm{E}+00$ \\
\hline PO210 & $1.66 \mathrm{E}-01$ & $1.67 \mathrm{E}-01$ & $1.67 \mathrm{E}-01$ & $1.67 \mathrm{E}-01$ & $1.69 \mathrm{E}-01$ & $1.67 \mathrm{E}-01$ & $1.56 \mathrm{E}-01$ & $1.34 \mathrm{E}-01$ & $2.91 \mathrm{E}-02$ \\
\hline P0211 & $9.35 \mathrm{E}-06$ & $2.30 \mathrm{E}-13$ & $0.00 \mathrm{E}+00$ & $0.00 \mathrm{E}+00$ & $0.00 \mathrm{E}+00$ & $0.00 \mathrm{E}+00$ & $0.00 \mathrm{E}+00$ & $0.00 \mathrm{E}+00$ & $0.00 \mathrm{E}+00$ \\
\hline PO211M & $1.56 \mathrm{E}-07$ & $0.00 \mathrm{E}+00$ & $0.00 \mathrm{E}+00$ & $0.00 \mathrm{E}+00$ & $0.00 \mathrm{E}+00$ & $0.00 \mathrm{E}+00$ & $0.00 \mathrm{E}+00$ & $0.00 \mathrm{E}+00$ & $0.00 \mathrm{E}+00$ \\
\hline SUMTOT & 5.55E-01 & 5.54E-01 & $5.44 \mathrm{E}-01$ & $5.06 \mathrm{E}-01$ & 3.17E-01 & $2.23 \mathrm{E}-01$ & $1.62 \mathrm{E}-01$ & $1.34 \mathrm{E}-01$ & $2.91 \mathrm{E}-02$ \\
\hline OTOTAL & 5.55E-01 & 5.54E-01 & $5.44 \mathrm{E}-01$ & $5.06 \mathrm{E}-01$ & 3.17E-01 & $2.23 \mathrm{E}-01$ & $1.62 \mathrm{E}-01$ & $1.34 \mathrm{E}-01$ & $2.91 \mathrm{E}-02$ \\
\hline \multicolumn{10}{|c|}{1 gram of $\mathrm{Bi}$ (CSM melt wire) irradiated for 54.9 EFPDs } \\
\hline & $\mathrm{Ci} / 1 \mathrm{~g}$ of $\mathrm{Bi}$ & $\mathrm{Ci} / 1 \mathrm{~g}$ of $\mathrm{Bi}$ & $\mathrm{Ci} / 1 \mathrm{~g}$ of $\mathrm{Bi}$ & $\mathrm{Ci} / 1 \mathrm{~g}$ of $\mathrm{Bi}$ & $\mathrm{Ci} / 1 \mathrm{~g}$ of $\mathrm{Bi}$ & $\mathrm{Ci} / 1 \mathrm{~g}$ of $\mathrm{Bi}$ & $\mathrm{Ci} / 1 \mathrm{~g}$ of $\mathrm{Bi}$ & $\mathrm{Ci} / 1 \mathrm{~g}$ of $\mathrm{Bi}$ & $\mathrm{Ci} / 1 \mathrm{~g}$ of $\mathrm{Bi}$ \\
\hline TL206 & $9.91 \mathrm{E}-09$ & $9.91 \mathrm{E}-09$ & $9.91 \mathrm{E}-09$ & $9.91 \mathrm{E}-09$ & $9.91 \mathrm{E}-09$ & $9.91 \mathrm{E}-09$ & $9.91 \mathrm{E}-09$ & $9.91 \mathrm{E}-09$ & $9.91 \mathrm{E}-09$ \\
\hline PB209 & $1.61 \mathrm{E}-04$ & $1.45 \mathrm{E}-04$ & $5.62 \mathrm{E}-05$ & $1.04 \mathrm{E}-06$ & $7.60 \mathrm{E}-20$ & $0.00 \mathrm{E}+00$ & $0.00 \mathrm{E}+00$ & $0.00 \mathrm{E}+00$ & $0.00 \mathrm{E}+00$ \\
\hline $\mathrm{BI} 208$ & $1.51 \mathrm{E}-08$ & $1.51 \mathrm{E}-08$ & $1.51 \mathrm{E}-08$ & $1.51 \mathrm{E}-08$ & $1.51 \mathrm{E}-08$ & $1.51 \mathrm{E}-08$ & $1.51 \mathrm{E}-08$ & $1.51 \mathrm{E}-08$ & $1.51 \mathrm{E}-08$ \\
\hline $\mathrm{B} 1210$ & $3.89 \mathrm{E}-01$ & 3.87E-01 & 3.77E-01 & $3.38 \mathrm{E}-01$ & $1.48 \mathrm{E}-01$ & $5.60 \mathrm{E}-02$ & $6.13 \mathrm{E}-03$ & 9.67E-05 & 4.47E-23 \\
\hline $\mathrm{BI} 210 \mathrm{M}$ & 9.95E-09 & 9.95E-09 & 9.95E-09 & 9.95E-09 & 9.95E-09 & 9.95E-09 & 9.95E-09 & 9.95E-09 & $9.95 \mathrm{E}-09$ \\
\hline $\mathrm{B} \mid 211$ & $1.42 \mathrm{E}-06$ & 8.19E-11 & $0.00 \mathrm{E}+00$ & $0.00 \mathrm{E}+00$ & $0.00 \mathrm{E}+00$ & $0.00 \mathrm{E}+00$ & $0.00 \mathrm{E}+00$ & $0.00 \mathrm{E}+00$ & $0.00 \mathrm{E}+00$ \\
\hline PO210 & $8.23 \mathrm{E}-02$ & $8.24 \mathrm{E}-02$ & $8.27 \mathrm{E}-02$ & $8.37 \mathrm{E}-02$ & $8.81 \mathrm{E}-02$ & $8.83 \mathrm{E}-02$ & $8.32 \mathrm{E}-02$ & $7.18 \mathrm{E}-02$ & $1.56 \mathrm{E}-02$ \\
\hline PO211 & $4.63 \mathrm{E}-06$ & $2.30 \mathrm{E}-13$ & $0.00 \mathrm{E}+00$ & $0.00 \mathrm{E}+00$ & $0.00 \mathrm{E}+00$ & $0.00 \mathrm{E}+00$ & $0.00 \mathrm{E}+00$ & $0.00 \mathrm{E}+00$ & $0.00 \mathrm{E}+00$ \\
\hline P0211M & 7.71E-08 & $0.00 \mathrm{E}+00$ & $0.00 \mathrm{E}+00$ & $0.00 \mathrm{E}+00$ & $0.00 \mathrm{E}+00$ & $0.00 \mathrm{E}+00$ & $0.00 \mathrm{E}+00$ & $0.00 \mathrm{E}+00$ & $0.00 \mathrm{E}+00$ \\
\hline SUMTOT & 4.71E-01 & 4.70E-01 & $4.60 \mathrm{E}-01$ & $4.22 \mathrm{E}-01$ & $2.36 \mathrm{E}-01$ & $1.44 \mathrm{E}-01$ & $8.93 \mathrm{E}-02$ & 7.19E-02 & $1.56 \mathrm{E}-02$ \\
\hline OTOTAL & 4.71E-01 & 4.70E-01 & 4.60E-01 & 4.22E-01 & $2.36 \mathrm{E}-01$ & $1.44 \mathrm{E}-01$ & 8.93E-02 & 7.19E-02 & $1.56 \mathrm{E}-02$ \\
\hline
\end{tabular}


TEM-10200-1

ENGINEERING CALCULATIONS AND ANALYSIS

Page 81 of 81

$12 / 19 / 17$

Rev.08

Title:

AS-RUN NEUTRONICS EVALUATION FOR THE CSM-10584 EXPERIMENT IN THE ATR

\begin{tabular}{llllllll} 
ECAR NO.: & 4496 & REV. NO.: & 1 & PROJECT NO.: & 32501 & Date: & 10/19/2020 \\
\hline
\end{tabular}

\begin{tabular}{|c|c|c|c|c|c|c|c|c|c|}
\hline \multicolumn{10}{|c|}{1 gram of $\mathrm{Bi}$ (CSM melt wire) irradiated for 119 EFPDs } \\
\hline & EOC & $30 \mathrm{MINs}$ & $5 \mathrm{HRS}$ & $1 \mathrm{DAY}$ & 7 DAYS & 14 DAYS & 30 DAYS & 60 DAYS & 1 YEAR \\
\hline & $\mathrm{g} / 1 \mathrm{~g}$ of $\mathrm{Bi}$ & $\mathrm{g} / 1 \mathrm{~g}$ of $\mathrm{Bi}$ & $\mathrm{g} / 1 \mathrm{~g}$ of $\mathrm{Bi}$ & $\mathrm{g} / 1 \mathrm{~g}$ of $\mathrm{Bi}$ & $\mathrm{g} / 1 \mathrm{~g}$ of $\mathrm{Bi}$ & $\mathrm{g} / 1 \mathrm{~g}$ of $\mathrm{Bi}$ & $\mathrm{g} / 1 \mathrm{~g}$ of $\mathrm{Bi}$ & $\mathrm{g} / 1 \mathrm{~g}$ of $\mathrm{Bi}$ & $\mathrm{g} / 1 \mathrm{~g}$ of $\mathrm{Bi}$ \\
\hline H 1 & $1.01 \mathrm{E}-10$ & $1.01 \mathrm{E}-10$ & $1.01 \mathrm{E}-10$ & $1.01 \mathrm{E}-10$ & $1.01 \mathrm{E}-10$ & $1.01 \mathrm{E}-10$ & $1.01 \mathrm{E}-10$ & $1.01 \mathrm{E}-10$ & $1.01 \mathrm{E}-10$ \\
\hline $\mathrm{H} 2$ & 4.27E-14 & 4.27E-14 & 4.27E-14 & 4.27E-14 & 4.27E-14 & 4.27E-14 & 4.27E-14 & 4.27E-14 & 4.27E-14 \\
\hline HE 4 & 2.17E-07 & 2.17E-07 & 2.17E-07 & $2.20 \mathrm{E}-07$ & $2.42 \mathrm{E}-07$ & 2.67E-07 & $3.21 \mathrm{E}-07$ & $4.14 \mathrm{E}-07$ & $8.59 \mathrm{E}-07$ \\
\hline PB206 & $1.10 \mathrm{E}-05$ & $1.11 \mathrm{E}-05$ & $1.11 \mathrm{E}-05$ & $1.12 \mathrm{E}-05$ & $1.23 \mathrm{E}-05$ & $1.36 \mathrm{E}-05$ & $1.64 \mathrm{E}-05$ & $2.12 \mathrm{E}-05$ & 4.41E-05 \\
\hline PB207 & $1.11 \mathrm{E}-07$ & $1.11 \mathrm{E}-07$ & $1.11 \mathrm{E}-07$ & $1.11 \mathrm{E}-07$ & $1.11 \mathrm{E}-07$ & $1.11 \mathrm{E}-07$ & $1.11 \mathrm{E}-07$ & $1.11 \mathrm{E}-07$ & $1.11 \mathrm{E}-07$ \\
\hline PB209 & $3.53 \mathrm{E}-11$ & $3.18 \mathrm{E}-11$ & $1.24 \mathrm{E}-11$ & $2.29 \mathrm{E}-13$ & 1.67E-26 & $0.00 \mathrm{E}+00$ & $0.00 \mathrm{E}+00$ & $0.00 \mathrm{E}+00$ & $0.00 \mathrm{E}+00$ \\
\hline $\mathrm{BI} 208$ & 7.01E-06 & 7.01E-06 & 7.01E-06 & 7.01E-06 & 7.01E-06 & 7.01E-06 & 7.01E-06 & 7.01E-06 & 7.01E-06 \\
\hline BI209 & $1.00 \mathrm{E}+00$ & $1.00 \mathrm{E}+00$ & $1.00 \mathrm{E}+00$ & $1.00 \mathrm{E}+00$ & $1.00 \mathrm{E}+00$ & $1.00 \mathrm{E}+00$ & $1.00 \mathrm{E}+00$ & $1.00 \mathrm{E}+00$ & $1.00 \mathrm{E}+00$ \\
\hline $\mathrm{BI} 210$ & $3.13 \mathrm{E}-06$ & $3.12 \mathrm{E}-06$ & $3.04 \mathrm{E}-06$ & $2.73 \mathrm{E}-06$ & $1.19 \mathrm{E}-06$ & $4.52 \mathrm{E}-07$ & $4.94 \mathrm{E}-08$ & $7.79 \mathrm{E}-10$ & $3.60 \mathrm{E}-28$ \\
\hline $\mathrm{BI} 210 \mathrm{M}$ & $3.80 \mathrm{E}-05$ & $3.80 \mathrm{E}-05$ & $3.80 \mathrm{E}-05$ & $3.80 \mathrm{E}-05$ & $3.80 \mathrm{E}-05$ & $3.80 \mathrm{E}-05$ & $3.80 \mathrm{E}-05$ & $3.80 \mathrm{E}-05$ & $3.80 \mathrm{E}-05$ \\
\hline $\mathrm{B} 1211$ & $3.40 \mathrm{E}-15$ & $1.96 \mathrm{E}-19$ & $0.00 \mathrm{E}+00$ & $0.00 \mathrm{E}+00$ & $0.00 \mathrm{E}+00$ & $0.00 \mathrm{E}+00$ & $0.00 \mathrm{E}+00$ & $0.00 \mathrm{E}+00$ & $0.00 \mathrm{E}+00$ \\
\hline PO210 & $3.70 \mathrm{E}-05$ & $3.70 \mathrm{E}-05$ & $3.71 \mathrm{E}-05$ & $3.73 \mathrm{E}-05$ & 3.77E-05 & 3.71E-05 & $3.46 \mathrm{E}-05$ & $2.98 \mathrm{E}-05$ & 6.47E-06 \\
\hline OTOTAL & $1.00 \mathrm{E}+00$ & $1.00 \mathrm{E}+00$ & $1.00 \mathrm{E}+00$ & $1.00 \mathrm{E}+00$ & $1.00 \mathrm{E}+00$ & $1.00 \mathrm{E}+00$ & $1.00 \mathrm{E}+00$ & $1.00 \mathrm{E}+00$ & $1.00 \mathrm{E}+00$ \\
\hline \multicolumn{10}{|c|}{1 gram of $\mathrm{Bi}$ (CSM melt wire) irradiated for 54.9 EFPDs } \\
\hline & EOC & $30 \mathrm{MINs}$ & $5 \mathrm{HRS}$ & $1 \mathrm{DAY}$ & 7 DAYS & 14 DAYS & 30 DAYS & 60 DAYS & 1 YEAR \\
\hline & $\mathrm{g} / 1 \mathrm{~g}$ of $\mathrm{Bi}$ & $\mathrm{g} / 1 \mathrm{~g}$ of $\mathrm{Bi}$ & $\mathrm{g} / 1 \mathrm{~g}$ of $\mathrm{Bi}$ & $\mathrm{g} / 1 \mathrm{~g}$ of $\mathrm{Bi}$ & $\mathrm{g} / 1 \mathrm{~g}$ of $\mathrm{Bi}$ & $\mathrm{g} / 1 \mathrm{~g}$ of $\mathrm{Bi}$ & $\mathrm{g} / 1 \mathrm{~g}$ of $\mathrm{Bi}$ & $\mathrm{g} / 1 \mathrm{~g}$ of $\mathrm{Bi}$ & $\mathrm{g} / 1 \mathrm{~g}$ of $\mathrm{Bi}$ \\
\hline $\mathrm{H} 1$ & $4.68 \mathrm{E}-11$ & $4.68 \mathrm{E}-11$ & $4.68 \mathrm{E}-11$ & $4.68 \mathrm{E}-11$ & $4.68 \mathrm{E}-11$ & $4.68 \mathrm{E}-11$ & $4.68 \mathrm{E}-11$ & $4.68 \mathrm{E}-11$ & $4.68 \mathrm{E}-11$ \\
\hline $\mathrm{H} 2$ & $9.10 \mathrm{E}-15$ & $9.10 \mathrm{E}-15$ & $9.10 \mathrm{E}-15$ & $9.10 \mathrm{E}-15$ & $9.10 \mathrm{E}-15$ & $9.10 \mathrm{E}-15$ & $9.10 \mathrm{E}-15$ & $9.10 \mathrm{E}-15$ & $9.10 \mathrm{E}-15$ \\
\hline HE 4 & $4.43 \mathrm{E}-08$ & $4.43 \mathrm{E}-08$ & 4.47E-08 & $4.61 \mathrm{E}-08$ & $5.71 \mathrm{E}-08$ & $7.02 \mathrm{E}-08$ & 9.94E-08 & $1.49 \mathrm{E}-07$ & 3.87E-07 \\
\hline PB206 & 2.27E-06 & 2.27E-06 & $2.29 \mathrm{E}-06$ & $2.36 \mathrm{E}-06$ & $2.93 \mathrm{E}-06$ & $3.61 \mathrm{E}-06$ & $5.11 \mathrm{E}-06$ & $7.65 \mathrm{E}-06$ & $1.99 \mathrm{E}-05$ \\
\hline PB207 & $9.81 \mathrm{E}-09$ & $9.81 \mathrm{E}-09$ & 9.81E-09 & $9.81 \mathrm{E}-09$ & $9.81 \mathrm{E}-09$ & $9.81 \mathrm{E}-09$ & 9.81E-09 & 9.81E-09 & 9.81E-09 \\
\hline PB208 & $1.32 \mathrm{E}-12$ & $1.32 \mathrm{E}-12$ & $1.32 \mathrm{E}-12$ & $1.33 \mathrm{E}-12$ & $1.43 \mathrm{E}-12$ & $1.55 \mathrm{E}-12$ & $1.82 \mathrm{E}-12$ & $2.32 \mathrm{E}-12$ & $7.41 \mathrm{E}-12$ \\
\hline PB209 & $3.53 \mathrm{E}-11$ & $3.18 \mathrm{E}-11$ & $1.24 \mathrm{E}-11$ & $2.29 \mathrm{E}-13$ & 1.67E-26 & $0.00 \mathrm{E}+00$ & $0.00 \mathrm{E}+00$ & $0.00 \mathrm{E}+00$ & $0.00 \mathrm{E}+00$ \\
\hline $\mathrm{BI} 208$ & $3.23 \mathrm{E}-06$ & $3.23 \mathrm{E}-06$ & $3.23 \mathrm{E}-06$ & $3.23 \mathrm{E}-06$ & $3.23 \mathrm{E}-06$ & $3.23 \mathrm{E}-06$ & $3.23 \mathrm{E}-06$ & $3.23 \mathrm{E}-06$ & $3.23 \mathrm{E}-06$ \\
\hline $\mathrm{B} 1209$ & $1.00 \mathrm{E}+00$ & $1.00 \mathrm{E}+00$ & $1.00 \mathrm{E}+00$ & $1.00 \mathrm{E}+00$ & $1.00 \mathrm{E}+00$ & $1.00 \mathrm{E}+00$ & $1.00 \mathrm{E}+00$ & $1.00 \mathrm{E}+00$ & $1.00 \mathrm{E}+00$ \\
\hline $\mathrm{B} \mid 210$ & $3.13 \mathrm{E}-06$ & $3.12 \mathrm{E}-06$ & $3.04 \mathrm{E}-06$ & $2.73 \mathrm{E}-06$ & $1.19 \mathrm{E}-06$ & $4.51 \mathrm{E}-07$ & 4.94E-08 & 7.79E-10 & $3.60 \mathrm{E}-28$ \\
\hline $\mathrm{BI} 210 \mathrm{M}$ & $1.75 \mathrm{E}-05$ & $1.75 \mathrm{E}-05$ & $1.75 \mathrm{E}-05$ & $1.75 \mathrm{E}-05$ & $1.75 \mathrm{E}-05$ & $1.75 \mathrm{E}-05$ & $1.75 \mathrm{E}-05$ & $1.75 \mathrm{E}-05$ & $1.75 \mathrm{E}-05$ \\
\hline $\mathrm{B} \mid 211$ & $3.40 \mathrm{E}-15$ & $1.96 \mathrm{E}-19$ & $0.00 \mathrm{E}+00$ & $0.00 \mathrm{E}+00$ & $0.00 \mathrm{E}+00$ & $0.00 \mathrm{E}+00$ & $0.00 \mathrm{E}+00$ & $0.00 \mathrm{E}+00$ & $0.00 \mathrm{E}+00$ \\
\hline OTOTAL & $1.00 \mathrm{E}+00$ & $1.00 \mathrm{E}+00$ & $1.00 \mathrm{E}+00$ & $1.00 \mathrm{E}+00$ & $1.00 \mathrm{E}+00$ & $1.00 \mathrm{E}+00$ & $1.00 \mathrm{E}+00$ & $1.00 \mathrm{E}+00$ & $1.00 \mathrm{E}+00$ \\
\hline
\end{tabular}

